\title{
Antidepressants and benzodiazepines for panic disorder in adults
} (Review)

Bighelli I, Trespidi C, Castellazzi M, Cipriani A, Furukawa TA, Girlanda F, Guaiana G, Koesters M, Barbui C

Bighelli I, Trespidi C, Castellazzi M, Cipriani A, Furukawa TA, Girlanda F, Guaiana G, Koesters M, Barbui C. Antidepressants and benzodiazepines for panic disorder in adults.

Cochrane Database of Systematic Reviews 2016, Issue 9. Art. No.: CD011567.

DOI: 10.1002/14651858.CD011567.pub2.

www.cochranelibrary.com 
TABLE OF CONTENTS

ABSTRAC

PLAIN LANGUAGE SUMMARY

SUMMARY OF FINDINGS

BACKGROUND

OBJECTIVES

METHODS

Figure 1.

RESULTS

Figure 2.

Figure 3.

Figure 4.

Figure 5.

DISCUSSION

AUTHORS' CONCLUSIONS

ACKNOWLEDGEMENTS

REFERENCES

CHARACTERISTICS OF STUDIES

DATA AND ANALYSES

Analysis 1.1. Comparison 1 Antidepressants versus benzodiazepines, Outcome 1 Failure to respond.

Analysis 1.2. Comparison 1 Antidepressants versus benzodiazepines, Outcome 2 Total number of dropouts.

Analysis 1.3. Comparison 1 Antidepressants versus benzodiazepines, Outcome 3 Failure to remit.

Analysis 1.4. Comparison 1 Antidepressants versus benzodiazepines, Outcome 4 Panic symptoms - endpoint score. ..............

Analysis 1.5. Comparison 1 Antidepressants versus benzodiazepines, Outcome 5 Panic symptoms - mean change. .................

Analysis 1.6. Comparison 1 Antidepressants versus benzodiazepines, Outcome 6 Frequency of panic attacks. ........................

Analysis 1.7. Comparison 1 Antidepressants versus benzodiazepines, Outcome 7 Agoraphobia.

Analysis 1.8. Comparison 1 Antidepressants versus benzodiazepines, Outcome 8 General anxiety.

Analysis 1.9. Comparison 1 Antidepressants versus benzodiazepines, Outcome 9 Depression.

Analysis 1.10. Comparison 1 Antidepressants versus benzodiazepines, Outcome 10 Social functioning.

Analysis 1.14. Comparison 1 Antidepressants versus benzodiazepines, Outcome 14 Number of dropouts due to adverse effects.

Analysis 1.15. Comparison 1 Antidepressants versus benzodiazepines, Outcome 15 Number of patients experiencing at least one adverse effect.

Analysis 2.1. Comparison 2 TCAs versus benzodiazepines, Outcome 1 Failure to respond.

Analysis 2.2. Comparison 2 TCAs versus benzodiazepines, Outcome 2 Total number of dropouts.

Analysis 2.3. Comparison 2 TCAs versus benzodiazepines, Outcome 3 Failure to remit.

Analysis 2.4. Comparison 2 TCAs versus benzodiazepines, Outcome 4 Panic symptoms - endpoint score.

Analysis 2.5. Comparison 2 TCAs versus benzodiazepines, Outcome 5 Panic symptoms - mean change.

Analysis 2.6. Comparison 2 TCAs versus benzodiazepines, Outcome 6 Frequency of panic attacks.

Analysis 2.7. Comparison 2 TCAs versus benzodiazepines, Outcome 7 Agoraphobia.

Analysis 2.8. Comparison 2 TCAs versus benzodiazepines, Outcome 8 General anxiety.

Analysis 2.9. Comparison 2 TCAs versus benzodiazepines, Outcome 9 Depression.

Analysis 2.10. Comparison 2 TCAs versus benzodiazepines, Outcome 10 Social functioning.

Analysis 2.14. Comparison 2 TCAs versus benzodiazepines, Outcome 14 Number of dropouts due to adverse effects. ..............

Analysis 3.1. Comparison 3 SSRIs versus benzodiazepines, Outcome 1 Failure to respond.

Analysis 3.2. Comparison 3 SSRIs versus benzodiazepines, Outcome 2 Total number of dropouts.

Analysis 3.3. Comparison 3 SSRIs versus benzodiazepines, Outcome 3 Failure to remit.

Analysis 3.4. Comparison 3 SSRIs versus benzodiazepines, Outcome 4 Panic symptoms.

Analysis 3.5. Comparison 3 SSRIs versus benzodiazepines, Outcome 5 Frequency of panic attacks.

Analysis 3.6. Comparison 3 SSRIs versus benzodiazepines, Outcome 6 Agoraphobia.

Analysis 3.7. Comparison 3 SSRIs versus benzodiazepines, Outcome 7 General anxiety.

Analysis 3.8. Comparison 3 SSRIs versus benzodiazepines, Outcome 8 Depression. 
Analysis 3.9. Comparison 3 SSRIs versus benzodiazepines, Outcome 9 Social functioning.

Analysis 3.13. Comparison 3 SSRIs versus benzodiazepines, Outcome 13 Number of dropouts due to adverse effects. ............

Analysis 3.14. Comparison 3 SSRIs versus benzodiazepines, Outcome 14 Number of patients experiencing at least one adverse effect.

Analysis 4.1. Comparison 4 TCAs versus SSRIs, Outcome 1 Failure to respond.

Analysis 4.2. Comparison 4 TCAs versus SSRIs, Outcome 2 Total number of dropouts.

Analysis 4.3. Comparison 4 TCAs versus SSRIs, Outcome 3 Failure to remit.

Analysis 4.4. Comparison 4 TCAs versus SSRIs, Outcome 4 Panic symptoms.

Analysis 4.5. Comparison 4 TCAs versus SSRIs, Outcome 5 Frequency of panic attacks.

Analysis 4.6. Comparison 4 TCAs versus SSRIs, Outcome 6 Agoraphobia.

Analysis 4.7. Comparison 4 TCAs versus SSRIs, Outcome 7 General anxiety - endpoint score.

Analysis 4.8. Comparison 4 TCAs versus SSRIs, Outcome 8 General anxiety - mean change.

Analysis 4.9. Comparison 4 TCAs versus SSRIs, Outcome 9 Depression - endpoint score.

Analysis 4.10. Comparison 4 TCAs versus SSRIs, Outcome 10 Depression - mean change.

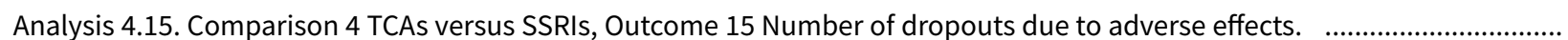
Analysis 4.16. Comparison 4 TCAs versus SSRIs, Outcome 16 Number of patients experiencing at least one adverse effect. ...... Analysis 5.1. Comparison 5 TCAs versus MAOIs, Outcome 1 Failure to respond.

Analysis 5.2. Comparison 5 TCAs versus MAOIs, Outcome 2 Total number of dropouts.

Analysis 5.4. Comparison 5 TCAs versus MAOls, Outcome 4 Panic symptoms.

Analysis 5.5. Comparison 5 TCAs versus MAOIs, Outcome 5 Frequency of panic attacks.

Analysis 5.7. Comparison 5 TCAs versus MAOIs, Outcome 7 General anxiety.

Analysis 5.8. Comparison 5 TCAs versus MAOIs, Outcome 8 Depression.

Analysis 5.13. Comparison 5 TCAs versus MAOIs, Outcome 13 Number of dropouts due to adverse effects.

Analysis 5.14. Comparison 5 TCAs versus MAOIs, Outcome 14 Number of patients experiencing at least one adverse effect. ....

Analysis 6.1. Comparison 6 SSRIs versus MAOIs, Outcome 1 Failure to respond.

Analysis 6.2. Comparison 6 SSRIs versus MAOIs, Outcome 2 Total number of dropouts.

Analysis 6.3. Comparison 6 SSRIs versus MAOIs, Outcome 3 Failure to remit.

Analysis 6.6. Comparison 6 SSRIs versus MAOIs, Outcome 6 Agoraphobia.

Analysis 6.7. Comparison 6 SSRIs versus MAOIs, Outcome 7 General anxiety.

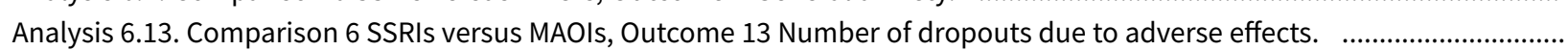

Analysis 6.14. Comparison 6 SSRIs versus MAOIs, Outcome 14 Number of patients experiencing at least one adverse effect. .....

Analysis 7.1. Comparison 7 SSRIs versus SNRIs, Outcome 1 Failure to respond.

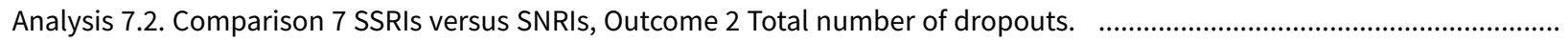

Analysis 7.3. Comparison 7 SSRIs versus SNRIs, Outcome 3 Failure to remit.

Analysis 7.4. Comparison 7 SSRIs versus SNRIs, Outcome 4 Panic symptoms.

Analysis 7.6. Comparison 7 SSRIs versus SNRIs, Outcome 6 Agoraphobia.

Analysis 7.7. Comparison 7 SSRIs versus SNRIs, Outcome 7 General anxiety.

Analysis 7.9. Comparison 7 SSRIs versus SNRIs, Outcome 9 Social functioning.

Analysis 7.10. Comparison 7 SSRIs versus SNRIs, Outcome 10 Quality of life.

Analysis 7.13. Comparison 7 SSRIs versus SNRIs, Outcome 13 Number of dropouts due to adverse effects. .............................

Analysis 7.14. Comparison 7 SSRIs versus SNRIs, Outcome 14 Number of patients experiencing at least one adverse effect. .....

Analysis 8.2. Comparison 8 SSRIs versus NaSSAs, Outcome 2 Total number of dropouts.

Analysis 8.4. Comparison 8 SSRIs versus NaSSAs, Outcome 4 Panic symptoms.

Analysis 8.7. Comparison 8 SSRIs versus NaSSAs, Outcome 7 General anxiety.

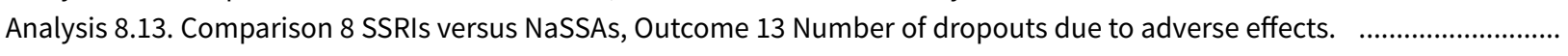

Analysis 9.1. Comparison 9 SSRIs versus Other Antidepressants, Outcome 1 Failure to respond.

Analysis 9.2. Comparison 9 SSRIs versus Other Antidepressants, Outcome 2 Total number of dropouts.

Analysis 9.6. Comparison 9 SSRIs versus Other Antidepressants, Outcome 6 Agoraphobia.

Analysis 9.7. Comparison 9 SSRIs versus Other Antidepressants, Outcome 7 General anxiety. 
Analysis 10.1. Comparison 10 Individual Antidepressants versus another antidepressant within the same class, Outcome 1 Failure to respond.

Analysis 10.2. Comparison 10 Individual Antidepressants versus another antidepressant within the same class, Outcome 2 Total number of dropouts.

Analysis 10.3. Comparison 10 Individual Antidepressants versus another antidepressant within the same class, Outcome 3 Failure to remit.

Analysis 10.4. Comparison 10 Individual Antidepressants versus another antidepressant within the same class, Outcome 4 Panic symptoms.

Analysis 10.5. Comparison 10 Individual Antidepressants versus another antidepressant within the same class, Outcome 5 Frequency of panic attacks.

Analysis 10.6. Comparison 10 Individual Antidepressants versus another antidepressant within the same class, Outcome 6 Agoraphobia.

Analysis 10.7. Comparison 10 Individual Antidepressants versus another antidepressant within the same class, Outcome 7 General anxiety.

Analysis 10.8. Comparison 10 Individual Antidepressants versus another antidepressant within the same class, Outcome 8 Depression.

Analysis 10.10. Comparison 10 Individual Antidepressants versus another antidepressant within the same class, Outcome 10 Quality of life.

Analysis 10.13. Comparison 10 Individual Antidepressants versus another antidepressant within the same class, Outcome 13 Number of dropouts due to adverse effects.

Analysis 10.14. Comparison 10 Individual Antidepressants versus another antidepressant within the same class, Outcome 14 Number of patients experiencing at least one adverse effect.

Analysis 11.1. Comparison 11 Individual benzodiazepines versus another benzodiazepine, Outcome 1 Failure to respond. ..... Analysis 11.2. Comparison 11 Individual benzodiazepines versus another benzodiazepine, Outcome 2 Total number of dropouts.

Analysis 11.3. Comparison 11 Individual benzodiazepines versus another benzodiazepine, Outcome 3 Failure to remit. .......... Analysis 11.4. Comparison 11 Individual benzodiazepines versus another benzodiazepine, Outcome 4 Panic symptoms. ....... Analysis 11.5. Comparison 11 Individual benzodiazepines versus another benzodiazepine, Outcome 5 Frequency of panic attacks.

Analysis 11.7. Comparison 11 Individual benzodiazepines versus another benzodiazepine, Outcome 7 General anxiety. ......... Analysis 11.8. Comparison 11 Individual benzodiazepines versus another benzodiazepine, Outcome 8 Depression. ................ Analysis 11.9. Comparison 11 Individual benzodiazepines versus another benzodiazepine, Outcome 9 Social functioning. ..... Analysis 11.10. Comparison 11 Individual benzodiazepines versus another benzodiazepine, Outcome 10 Quality of life. ......... Analysis 11.13. Comparison 11 Individual benzodiazepines versus another benzodiazepine, Outcome 13 Number of dropouts due to adverse effects.

Analysis 12.1. Comparison 12 High risk of bias excluded - Antidepressants versus benzodiazepines, Outcome 1 Total number of dropouts.

Analysis 13.1. Comparison 13 High risk of bias excluded - TCAs versus benzodiazepines, Outcome 1 Total number of dropouts. Analysis 14.1. Comparison 14 High risk of bias excluded - TCAs versus SSRIs, Outcome 1 Total number of dropouts. ............... Analysis 15.1. Comparison 15 High risk of bias excluded - TCAs versus MAOls, Outcome 1 Total number of dropouts. ............... Analysis 16.1. Comparison 16 High risk of bias excluded - SSRIs versus MAOIs, Outcome 1 Failure to respond.

Analysis 17.1. Comparison 17 High dropout rates excluded - Antidepressants versus benzodiazepines, Outcome 1 Total number of dropouts.

Analysis 18.1. Comparison 18 High dropout rates excluded - TCAs versus benzodiazepines, Outcome 1 Total number of dropouts.

Analysis 19.1. Comparison 19 High dropout rates excluded - TCAs versus SSRIs, Outcome 1 Failure to respond.

Analysis 19.2. Comparison 19 High dropout rates excluded - TCAs versus SSRIs, Outcome 2 Total number of dropouts. ........... Analysis 20.1. Comparison 20 Funded excluded - Antidepressants versus benzodiazepines, Outcome 1 Total number of dropouts.

Analysis 21.1. Comparison 21 Funded excluded - TCAs versus benzodiazepines, Outcome 1 Total number of dropouts. .......... Analysis 22.1. Comparison 22 Funded excluded - TCAs versus SSRIs, Outcome 1 Failure to respond.

Analysis 22.2. Comparison 22 Funded excluded - TCAs versus SSRIs, Outcome 2 Total number of dropouts.

Analysis 23.1. Comparison 23 Funded excluded - SSRIs versus MAOIs, Outcome 1 Failure to respond. 
Analysis 24.2. Comparison 24 Psychiatric comorbidities excluded - Antidepressants versus benzodiazepines, Outcome 2 Total number of dropouts.

Analysis 25.1. Comparison 25 Psychiatric comorbidities excluded - TCAs versus benzodiazepines, Outcome 1 Total number of dropouts.

Analysis 26.1. Comparison 26 Psychiatric comorbidities excluded - TCAs versus SSRIs, Outcome 1 Failure to respond. ............ Analysis 26.2. Comparison 26 Psychiatric comorbidities excluded - TCAs versus SSRIs, Outcome 2 Total number of dropouts. .. Analysis 27.1. Comparison 27 Psychiatric comorbidities excluded - Individual antidepressants versus individual antidepressants (within the same class), Outcome 1 Failure to respond.

Analysis 27.2. Comparison 27 Psychiatric comorbidities excluded - Individual antidepressants versus individual antidepressants (within the same class), Outcome 2 Total number of dropouts.

Analysis 28.1. Comparison 28 Psychiatric comorbidities excluded - Individual benzodiazepines versus individual benzodiazepines, Outcome 1 Total number of dropouts.

Analysis 29.1. Comparison 29 Imputation excluded - TCAs versus SSRIs, Outcome 1 Failure to respond.

Analysis 29.2. Comparison 29 Imputation excluded - TCAs versus SSRIs, Outcome 2 Total number of dropouts. ...................... Analysis 30.1. Comparison 30 Imputation excluded - Individual antidepressants versus individual antidepressants (within the same class), Outcome 1 Failure to respond.

Analysis 30.2. Comparison 30 Imputation excluded - Individual antidepressants versus individual antidepressants (within the same class), Outcome 2 Total number of dropouts.

Analysis 31.1. Comparison 31 Irregular benzodiazepines use excluded - TCAs versus SSRIs, Outcome 1 Failure to respond. ..... Analysis 31.2. Comparison 31 Irregular benzodiazepines use excluded - TCAs versus SSRIs, Outcome 2 Total number of dropouts.

Analysis 32.1. Comparison 32 Irregular benzodiazepines use excluded - SSRIs versus MAOIs, Outcome 1 Failure to respond. ...

Analysis 33.1. Comparison 33 Irregular benzodiazepines use excluded - Individual antidepressants versus individual antidepressants (within the same class), Outcome 1 Failure to respond.

Analysis 33.2. Comparison 33 Irregular benzodiazepines use excluded - Individual antidepressants versus individual antidepressants (within the same class), Outcome 2 Total number of dropouts. 
[Intervention Review]

\section{Antidepressants and benzodiazepines for panic disorder in adults}

Irene Bighelli1 ${ }^{1}$, Carlotta Trespidi¹, Mariasole Castellazzi ${ }^{1}$, Andrea Cipriani², Toshi A Furukawa ${ }^{3}$, Francesca Girlanda4 ${ }^{4}$, Giuseppe Guaiana 5 , Markus Koesters 6 , Corrado Barbui ${ }^{1}$

${ }^{1}$ Neuroscience, Biomedicine and Movement Sciences, Section of Psychiatry, University of Verona, Verona, Italy. ${ }^{2}$ Department of Psychiatry, University of Oxford, Oxford, UK. ${ }^{3}$ Department of Health Promotion and Human Behavior, Kyoto University Graduate School of Medicine/School of Public Health, Kyoto, Japan. ${ }^{4}$ Department of Public Health and Community Medicine, Section of Psychiatry, University of Verona, Verona, Italy. ${ }^{5}$ Department of Psychiatry, Western University, St Thomas, Canada. ${ }^{6}$ Department of Psychiatry II, Ulm University, Guenzburg, Germany

Contact: Irene Bighelli, Neuroscience, Biomedicine and Movement Sciences, Section of Psychiatry, University of Verona, Verona, Italy. irene.bighelli@univr.it.

Editorial group: Cochrane Common Mental Disorders Group.

Publication status and date: New, published in Issue 9, 2016.

Citation: Bighelli I, Trespidi C, Castellazzi M, Cipriani A, Furukawa TA, Girlanda F, Guaiana G, Koesters M, Barbui C. Antidepressants and benzodiazepines for panic disorder in adults. Cochrane Database of Systematic Reviews 2016, Issue 9. Art. No.: CD011567. DOI: 10.1002/14651858.CD011567.pub2.

Copyright @ 2016 The Cochrane Collaboration. Published by John Wiley \& Sons, Ltd.

\section{A B S T R A C T}

\section{Background}

A panic attack is a discrete period of fear or anxiety that has a rapid onset, reaches a peak within 10 minutes and in which at least four of 13 characteristic symptoms are experienced, including racing heart, chest pain, sweating, shaking, dizziness, flushing, stomach churning, faintness and breathlessness. Panic disorder is common in the general population with a lifetime prevalence of $1 \%$ to $4 \%$. The treatment of panic disorder includes psychological and pharmacological interventions. Amongst pharmacological agents, antidepressants and benzodiazepines are the mainstay of treatment for panic disorder. Different classes of antidepressants have been compared; and the British Association for Psychopharmacology, and National Institute for Health and Care Excellence (NICE) consider antidepressants (mainly selective serotonin reuptake inhibitors (SSRIs)) as the first-line treatment for panic disorder, due to their more favourable adverse effect profile over monoamine oxidase inhibitors (MAOIs) and tricyclic antidepressants (TCAs). In addition to antidepressants, benzodiazepines are widely prescribed for the treatment of panic disorder.

\section{Objectives}

To assess the evidence for the effects of antidepressants and benzodiazepines for panic disorder in adults.

\section{Search methods}

The Specialised Register of the Cochrane Common Mental Disorders Group (CCMDCTR) to 11 September 2015. This register includes relevant randomised controlled trials from the Cochrane Central Register of Controlled Trials (CENTRAL), MEDLINE (1950-), Embase (1974-) and PsycINFO (1967-). Reference lists of relevant papers and previous systematic reviews were handsearched. We contacted experts in this field for supplemental data.

\section{Selection criteria}

All double-blind randomised controlled trials allocating adult patients with panic disorder to antidepressants or benzodiazepines versus any other active treatment with antidepressants or benzodiazepines. 


\section{Data collection and analysis}

Two review authors independently checked eligibility and extracted data using a standard form. Data were entered in RevMan 5.3 using a double-check procedure. Information extracted included study characteristics, participant characteristics, intervention details, settings and outcome measures in terms of efficacy, acceptability and tolerability.

\section{Main results}

Thirty-five studies, including 6785 participants overall (of which 5365 in the arms of interest (antidepressant and benzodiazepines as monotherapy)) were included in this review; however, since studies addressed many different comparisons, only a few trials provided data for primary outcomes. We found low-quality evidence suggesting no difference between antidepressants and benzodiazepines in terms of response rate (risk ratio $(\mathrm{RR}) 0.99,95 \%$ confidence interval $(\mathrm{Cl}) 0.67$ to 1.47 ; participants $=215$; studies $=2$ ). Very low-quality evidence suggested a benefit for benzodiazepines compared to antidepressants in terms of dropouts due to any cause, even if confidence interval $(\mathrm{Cl})$ ranges from almost no difference to benefit with benzodiazepines (RR 1.64, 95\% $\mathrm{Cl} 1.03$ to 2.63; participants = 1449; studies $=7$ ). We found some evidence suggesting that serotonin reuptake inhibitors (SSRIs) are better tolerated than TCAs (when looking at the number of patients experiencing adverse effects). We failed to find clinically significant differences between individual benzodiazepines. The majority of studies did not report details on random sequence generation and allocation concealment; similarly, no details were provided about strategies to ensure blinding. The study protocol was not available for almost all studies so it is difficult to make a judgment on the possibility of outcome reporting bias. Information on adverse effects was very limited.

\section{Authors' conclusions}

The identified studies are not sufficient to comprehensively address the objectives of the present review. The majority of studies enrolled a small number of participants and did not provide data for all the outcomes specified in the protocol. For these reasons most of the analyses were underpowered and this limits the overall completeness of evidence. In general, based on the results of the current review, the possible role of antidepressants and benzodiazepines should be assessed by the clinician on an individual basis. The choice of which antidepressant and/or benzodiazepine is prescribed can not be made on the basis of this review only, and should be based on evidence of antidepressants and benzodiazepines efficacy and tolerability, including data from placebo-controlled studies, as a whole. Data on longterm tolerability issues associated with antidepressants and benzodiazepines exposure should also be carefully considered.

The present review highlights the need for further higher-quality studies comparing antidepressants with benzodiazepines, which should be conducted with high-methodological standards and including pragmatic outcome measures to provide clinicians with useful and practical data. Data from the present review will be included in a network meta-analysis of psychopharmacological treatment in panic disorder, which will hopefully provide further useful information on this issue.

\section{PLAIN LANGUAGE SUMMARY}

\section{Antidepressants and benzodiazepines for panic disorder in adults}

\section{Why is this review important?}

Panic disorder is common in the general population. It is characterised by panic attacks, periods of fear or anxiety with a rapid onset in which other symptoms are experienced (involving bodily feelings and fearful thoughts). The treatment of panic disorder includes talking therapy and medicines, often used in combination. The most commonly prescribed medicines are antidepressants and benzodiazepines. Evidence for their efficacy in comparison is unclear. It is important to find out if antidepressants and benzodiazepines are effective and acceptable in the treatment of panic disorder.

\section{Who will be interested in this review?}

Patients and practitioners.

\section{What questions does this review aim to answer?}

This review aims to answer the following questions.

What is the efficacy of antidepressants and benzodiazepines compared to other antidepressants and other benzodiazepines?

What is the acceptability of antidepressants and benzodiazepines compared to other antidepressants and other benzodiazepines?

How many adverse effects do antidepressants and benzodiazepines have compared to other antidepressants and other benzodiazepines?

\section{Which studies were included in the review?}

We searched electronic databases to find all relevant studies conducted up to September 2015. To be included in the review, studies had to be randomised controlled trials that compared treatments with antidepressants and benzodiazepines in adults with a diagnosis of panic disorder. We included 35 studies involving a total of 5365 participants in the review. 


\section{What does the evidence from the review tell us?}

We did not find substantial differences between antidepressants and benzodiazepines in terms of efficacy and tolerability. There was not enough information to compare any differences in adverse effects. However, our findings are limited in the following ways: few studies contributed to each analysis, some studies were funded by pharmaceutical companies, and only short-term outcomes were assessed. The quality of the available evidence was mainly low, meaning that further research would be very likely to have an important impact on these results.

\section{What should happen next?}

Studies with larger sample sizes and fewer risks of bias should be carried out, with head-to-head comparisons. Longer-term outcomes need to be addressed to establish whether the effect is transient or durable. Trials should better report any harms experienced by participants during the trial. In addition, a network meta-analysis of psychopharmacological treatment in panic disorder will likely shed further light on this compelling issue, also being able to provide more information with regard to comparative efficacy. 
SUMMARY OF FINDINGS

Summary of findings for the main comparison. Antidepressants compared to benzodiazepines for adults with panic disorder

Antidepressants compared to benzodiazepines for adults with panic disorder

Patient or population: adults with panic disorder

Settings: outpatient

Intervention: Antidepressants

Comparison: benzodiazepines

\begin{tabular}{|c|c|c|c|c|c|c|}
\hline \multirow[t]{3}{*}{ Outcomes } & \multicolumn{2}{|c|}{ Illustrative comparative risks* $(95 \% \mathrm{CI})$} & \multirow{3}{*}{$\begin{array}{l}\text { Relative effect } \\
(95 \% \mathrm{Cl})\end{array}$} & \multirow{3}{*}{$\begin{array}{l}\text { No of Partici- } \\
\text { pants } \\
\text { (studies) }\end{array}$} & \multirow{3}{*}{$\begin{array}{l}\text { Quality of the } \\
\text { evidence } \\
\text { (GRADE) }\end{array}$} & \multirow[t]{3}{*}{ Comments } \\
\hline & Assumed risk & Corresponding risk & & & & \\
\hline & $\begin{array}{l}\text { Benzodi- } \\
\text { azepines }\end{array}$ & Antidepressants & & & & \\
\hline $\begin{array}{l}\text { failure to respond } \\
\text { Follow-up: 8-10 weeks }\end{array}$ & 314 per 1000 & $\begin{array}{l}\mathbf{3 1 0} \text { per } \mathbf{1 0 0 0} \\
(210 \text { to } 461)\end{array}$ & $\begin{array}{l}\text { RR } 0.99 \\
(0.67 \text { to } 1.47)\end{array}$ & $\begin{array}{l}215 \\
\text { ( } 2 \text { studies) }\end{array}$ & $\begin{array}{l}\oplus \oplus \oplus \ominus \\
\text { low } 1,2\end{array}$ & \\
\hline $\begin{array}{l}\text { total number of dropouts } \\
\text { Follow-up: } 8-24 \text { weeks }\end{array}$ & 213 per 1000 & $\begin{array}{l}\mathbf{3 5 0} \text { per } 1000 \\
\text { (220 to } 561)\end{array}$ & $\begin{array}{l}\text { RR } 1.64 \\
\text { (1.03 to } 2.63)\end{array}$ & $\begin{array}{l}1449 \\
\text { (7 studies) }\end{array}$ & $\begin{array}{l}\oplus \ominus \ominus \ominus \\
\text { very low } 1,3,4\end{array}$ & \\
\hline $\begin{array}{l}\text { failure to remit } \\
\text { Follow-up: 8-10 weeks }\end{array}$ & 360 per 1000 & $\begin{array}{l}\mathbf{4 2 5} \text { per } \mathbf{1 0 0 0} \\
\text { (364 to } 493)\end{array}$ & $\begin{array}{l}\text { RR } 1.18 \\
\text { (1.01 to } 1.37)\end{array}$ & $\begin{array}{l}1002 \\
\text { (3 studies) }\end{array}$ & $\begin{array}{l}\oplus \oplus \oplus \ominus \\
\text { moderate } 1\end{array}$ & \\
\hline $\begin{array}{l}\text { panic symptoms - endpoint } \\
\text { score } \\
\text { Follow-up: } 8-24 \text { weeks }\end{array}$ & & $\begin{array}{l}\text { The mean panic symptoms - endpoint } \\
\text { score in the intervention groups was } \\
\mathbf{0 . 1 3} \text { lower } \\
\text { ( } 0.72 \text { to } 0.47 \text { lower) }\end{array}$ & & $\begin{array}{l}1144 \\
\text { (3 studies) }\end{array}$ & $\begin{array}{l}\oplus \odot \odot \odot \\
\text { very low 1,5 }\end{array}$ & \\
\hline $\begin{array}{l}\text { panic symptoms -mean } \\
\text { change } \\
\text { Follow-up: } 8 \text { weeks }\end{array}$ & & $\begin{array}{l}\text { The mean panic symptoms - mean } \\
\text { change in the intervention groups was } \\
\mathbf{0 . 4 0} \text { higher } \\
\text { ( } 0.83 \text { lower to } 1.63 \text { higher) }\end{array}$ & & $\begin{array}{l}44 \\
\text { (1 study) }\end{array}$ & $\begin{array}{l}\oplus \oplus \odot \odot \\
\text { low } 6\end{array}$ & \\
\hline $\begin{array}{l}\text { number of dropouts due to } \\
\text { adverse effects } \\
\text { Follow-up: } 8-10 \text { weeks }\end{array}$ & 52 per 1000 & $\begin{array}{l}89 \text { per } 1000 \\
(54 \text { to } 149)\end{array}$ & $\begin{array}{l}\text { RR } 1.72 \\
\text { (1.03 to } 2.87 \text { ) }\end{array}$ & $\begin{array}{l}1002 \\
\text { (3 studies) }\end{array}$ & $\begin{array}{l}\oplus \oplus \ominus \ominus \\
\text { low } 1,4\end{array}$ & \\
\hline
\end{tabular}

*The basis for the assumed risk (e.g. the median control group risk across studies) is provided in footnotes. The corresponding risk (and its $95 \%$ confidence interval) is based on the assumed risk in the comparison group and the relative effect of the intervention (and its $95 \% \mathrm{Cl}$ ). 
GRADE Working Group grades of evidence

High quality: Further research is very unlikely to change our confidence in the estimate of effect.

Moderate quality: Further research is likely to have an important impact on our confidence in the estimate of effect and may change the estimate.

Low quality: Further research is very likely to have an important impact on our confidence in the estimate of effect and is likely to change the estimate.

Very low quality: We are very uncertain about the estimate.

1 Downgraded one point due to high dropout rates (30\%)

2 Downgraded one point due to imprecision: $95 \% \mathrm{Cl}$ interval ranges from possible benefit with antidepressants to possible benefit with benzodiazepines

3 Downgraded one point due to substantial heterogeneity $(12=75 \%)$

4 Downgraded one point due to imprecision: $95 \% \mathrm{Cl}$ interval ranges from no difference to possible benefit associated with benzodiazepines

5 Downgraded two points due to substantial heterogeneity $(12=95 \%)$

6 Downgraded two points due to imprecision: number of individuals included in the trial is low (44) and 95\% Cl ranges from appreciable benefit with TCAs to appreciable benefit with benzodiazepines

\section{Summary of findings 2 . TCAs compared to benzodiazepines for adults with panic disorder}

\section{TCAs compared to benzodiazepines for adults with panic disorder}

Patient or population: adults with panic disorder

Settings: outpatient

Intervention: TCAs

Comparison: benzodiazepines

\begin{tabular}{|c|c|c|c|c|c|c|}
\hline \multirow[t]{3}{*}{ Outcomes } & \multicolumn{2}{|c|}{ Illustrative comparative risks* $(95 \% \mathrm{CI})$} & \multirow{3}{*}{$\begin{array}{l}\text { Relative effect } \\
(95 \% \mathrm{CI})\end{array}$} & \multirow{3}{*}{$\begin{array}{l}\text { No of Partici- } \\
\text { pants } \\
\text { (studies) }\end{array}$} & \multirow{3}{*}{$\begin{array}{l}\text { Quality of the } \\
\text { evidence } \\
\text { (GRADE) }\end{array}$} & \multirow[t]{3}{*}{ Comments } \\
\hline & Assumed risk & Corresponding risk & & & & \\
\hline & $\begin{array}{l}\text { Benzodi- } \\
\text { azepines }\end{array}$ & TCAs & & & & \\
\hline $\begin{array}{l}\text { failure to respond } \\
\text { Follow-up: } 8 \text { weeks }\end{array}$ & 537 per 1000 & $\begin{array}{l}\mathbf{5 4 7} \text { per } \mathbf{1 0 0 0} \\
\text { (338 to } 896)\end{array}$ & $\begin{array}{l}\text { RR } 1.02 \\
\text { (0.63 to } 1.67)\end{array}$ & $\begin{array}{l}61 \\
\text { (1 study) }\end{array}$ & $\begin{array}{l}\oplus \ominus \ominus \ominus \\
\text { very low } 1,2\end{array}$ & \\
\hline $\begin{array}{l}\text { total number of dropouts } \\
\text { Follow-up: 8-24 weeks }\end{array}$ & 212 per 1000 & $\begin{array}{l}\mathbf{3 5 5} \text { per } \mathbf{1 0 0 0} \\
\text { (197 to } 635)\end{array}$ & $\begin{array}{l}\text { RR } 1.67 \\
\text { (0.93 to } 2.99)\end{array}$ & $\begin{array}{l}1295 \\
\text { (6 studies) }\end{array}$ & $\begin{array}{l}\oplus \ominus \ominus \ominus \\
\text { very low } 1,3,4\end{array}$ & \\
\hline $\begin{array}{l}\text { failure to remit } \\
\text { Follow-up: } 8 \text { weeks }\end{array}$ & 348 per 1000 & $\begin{array}{l}\mathbf{4 1 4} \text { per } 1000 \\
\text { (348 to } 490)\end{array}$ & $\begin{array}{l}\text { RR } 1.19 \\
\text { (1 to } 1.41)\end{array}$ & $\begin{array}{l}848 \\
\text { ( } 2 \text { studies) }\end{array}$ & $\begin{array}{l}\oplus \oplus \oplus \ominus \\
\text { moderate } 1\end{array}$ & \\
\hline $\begin{array}{l}\text { panic symptoms - endpoint } \\
\text { score }\end{array}$ & & $\begin{array}{l}\text { The mean panic symptoms - endpoint } \\
\text { score in the intervention groups was }\end{array}$ & & $\begin{array}{l}998 \\
\text { (2 studies) }\end{array}$ & $\begin{array}{l}\oplus \ominus \ominus \ominus \\
\text { very low } 1,5,6\end{array}$ & \\
\hline
\end{tabular}




\section{panic symptoms - mean}

change

(1.72 lower to 1.22 higher)

Follow-up: 8 weeks

The mean panic symptoms - mean

change in the intervention groups was

44

$\mathbf{0 . 4 0}$ higher

$44 \quad \oplus \oplus \odot \odot$

( 0.83 lower to 1.63 higher)

\begin{tabular}{llllll}
\hline $\begin{array}{l}\text { number of dropouts due to } \\
\text { adverse effects }\end{array}$ & $\mathbf{3 8}$ per $\mathbf{1 0 0 0}$ & $\begin{array}{l}\mathbf{7 9} \text { per } \mathbf{1 0 0 0} \\
(43 \text { to } 149)\end{array}$ & $\begin{array}{l}\text { RR 2.10 } \\
\text { (1.13 to 3.93) }\end{array}$ & $\begin{array}{l}848 \\
\text { (2 studies) }\end{array}$ & low 1,4
\end{tabular}

Follow-up: 8 weeks

79 per 1000
(43 to 149$)$

(1.13 to 3.93$)$

(2 studies)

low 1,4

*The basis for the assumed risk (e.g. the median control group risk across studies) is provided in footnotes. The corresponding risk (and its $95 \%$ confidence interval) is based on the assumed risk in the comparison group and the relative effect of the intervention (and its $95 \% \mathrm{Cl}$ ).

Cl: Confidence interval; RR: Risk ratio;

GRADE Working Group grades of evidence

High quality: Further research is very unlikely to change our confidence in the estimate of effect.

Moderate quality: Further research is likely to have an important impact on our confidence in the estimate of effect and may change the estimate.

Low quality: Further research is very likely to have an important impact on our confidence in the estimate of effect and is likely to change the estimate.

Very low quality: We are very uncertain about the estimate.

1 Downgraded one point due to high dropout rates (around 30\%)

2 Downgraded two points due to imprecision: number of individuals included in the trial is low (61) and 95\% CI ranges from appreciable benefit with TCAs to appreciable benefit with benzodiazepines

3 Downgraded two points due to substantial heterogeneity $(12=79 \%)$

4 Downgraded one point due to imprecision: $95 \% \mathrm{Cl}$ ranges from no difference to appreciable superiority of benzodiazepines in lowering the number of dropouts

5 Downgraded two points due to substantial heterogeneity $(12=97 \%)$

6 Downgraded one point due to imprecision: $95 \% \mathrm{Cl}$ is very wide and ranges from benefit with TCAs and benefit with benzodiazepines

7 Downgraded two points due to imprecision: number of individuals included in the trial is low (44) and $95 \% \mathrm{Cl}$ ranges from appreciable benefit with TCAs to appreciable benefit with benzodiazepines

\section{Summary of findings 3. SSRIs compared to benzodiazepines for adults with panic disorder}

\section{SSRIs compared to benzodiazepines for adults with panic disorder}

Patient or population: adults with panic disorder

Settings: outpatient

Intervention: SSRI

Comparison: benzodiazepines 


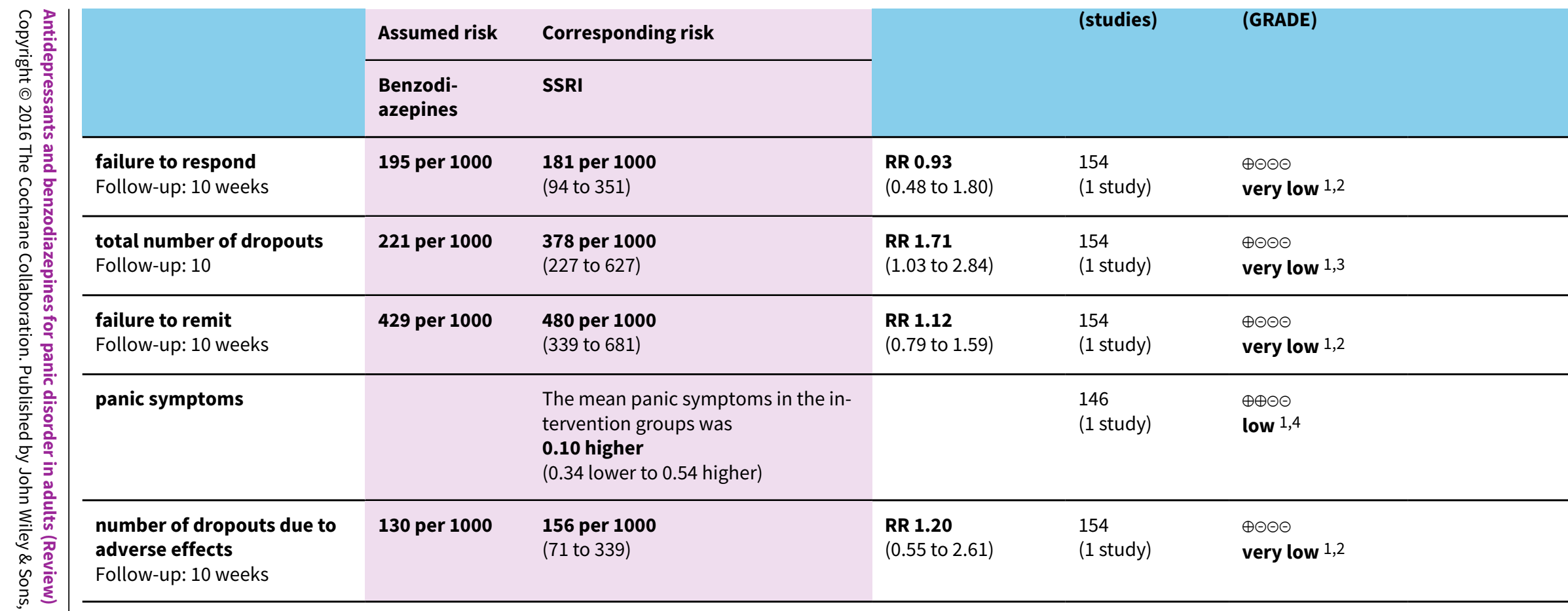

*The basis for the assumed risk (e.g. the median control group risk across studies) is provided in footnotes. The corresponding risk (and its $95 \%$ confidence interval) is based on the assumed risk in the comparison group and the relative effect of the intervention (and its $95 \% \mathrm{Cl}$ ).

Cl: Confidence interval; RR: Risk ratio;

GRADE Working Group grades of evidence

High quality: Further research is very unlikely to change our confidence in the estimate of effect.

Moderate quality: Further research is likely to have an important impact on our confidence in the estimate of effect and may change the estimate.

Low quality: Further research is very likely to have an important impact on our confidence in the estimate of effect and is likely to change the estimate.

Very low quality: We are very uncertain about the estimate.

1 Downgraded one point due to high dropout rates $(30 \%)$

2 Downgraded two points due to imprecision: number of individuals included in the trial is low $(=154)$ and $95 \% \mathrm{Cl}$ is wide, including benefit with SSRIs and benefit with benzodiazepines; only one study provides data

3 Downgraded two points due to imprecision: number of individuals included in the trial is low $(=154)$ and $95 \% \mathrm{Cl}$ ranges from no difference to appreciable superiority of with

benzodiazepines in lowering the number of dropouts; only one study provides data

4 Downgraded one point due to imprecision: number of individuals included in the trial is low 
Summary of findings 4. TCAs compared to SSRI for adults with panic disorder

TCAs compared to SSRI for adults with panic disorder

Patient or population: adults with panic disorder

Settings: outpatient

Intervention: TCA

Comparison: SSR

\begin{tabular}{|c|c|c|c|c|c|c|}
\hline \multirow[t]{3}{*}{ Outcomes } & \multicolumn{2}{|c|}{ Illustrative comparative risks* $(95 \% \mathrm{CI})$} & \multirow{3}{*}{$\begin{array}{l}\text { Relative effect } \\
(95 \% \mathrm{Cl})\end{array}$} & \multirow{3}{*}{$\begin{array}{l}\text { No of Partici- } \\
\text { pants } \\
\text { (studies) }\end{array}$} & \multirow{3}{*}{$\begin{array}{l}\text { Quality of the } \\
\text { evidence } \\
\text { (GRADE) }\end{array}$} & \multirow[t]{3}{*}{ Comments } \\
\hline & Assumed risk & Corresponding risk & & & & \\
\hline & SSRI & TCA & & & & \\
\hline $\begin{array}{l}\text { failure to respond } \\
\text { Follow-up: 8-12 weeks }\end{array}$ & 378 per 1000 & $\begin{array}{l}\mathbf{4 0 9} \text { per } 1000 \\
\text { (269 to } 628)\end{array}$ & $\begin{array}{l}\text { RR } 1.08 \\
\text { (0.71 to } 1.66)\end{array}$ & $\begin{array}{l}438 \\
\text { (4 studies) }\end{array}$ & $\begin{array}{l}\oplus \oplus \ominus \ominus \\
\text { low } 1,2\end{array}$ & \\
\hline $\begin{array}{l}\text { failure to remit } \\
\text { Follow-up: 8-24 weeks }\end{array}$ & 502 per 1000 & $\begin{array}{l}\mathbf{4 4 7} \text { per } \mathbf{1 0 0 0} \\
\text { (316 to } 633)\end{array}$ & $\begin{array}{l}\text { RR } 0.89 \\
(0.63 \text { to } 1.26)\end{array}$ & $\begin{array}{l}475 \\
\text { (5 studies) }\end{array}$ & $\begin{array}{l}\oplus \oplus \oplus \ominus \\
\text { moderate } 4\end{array}$ & \\
\hline $\begin{array}{l}\text { panic symptoms } \\
\text { Follow-up: } 8-10 \text { weeks }\end{array}$ & & $\begin{array}{l}\text { The mean panic symptoms in the in- } \\
\text { tervention groups was } \\
\mathbf{0 . 2 0} \text { lower } \\
\text { ( } 0.88 \text { lower to } 0.48 \text { higher) }\end{array}$ & & $\begin{array}{l}243 \\
\text { (4 studies) }\end{array}$ & $\begin{array}{l}\oplus \odot \odot \ominus \\
\text { very low } 5,6,7\end{array}$ & \\
\hline $\begin{array}{l}\text { number of dropouts due to } \\
\text { adverse effects } \\
\text { Follow-up: } 8-24 \text { weeks }\end{array}$ & 104 per 1000 & $\begin{array}{l}148 \text { per } 1000 \\
\text { (85 to } 257)\end{array}$ & $\begin{array}{l}\text { RR } 1.43 \\
\text { (0.82 to } 2.48 \text { ) }\end{array}$ & $\begin{array}{l}476 \\
\text { (5 studies) }\end{array}$ & $\begin{array}{l}\oplus \oplus \ominus \ominus \\
\text { low } 5,8\end{array}$ & \\
\hline
\end{tabular}

*The basis for the assumed risk (e.g. the median control group risk across studies) is provided in footnotes. The corresponding risk (and its $95 \%$ confidence interval) is based on the assumed risk in the comparison group and the relative effect of the intervention (and its $95 \% \mathrm{Cl}$ ).

Cl: Confidence interval; RR: Risk ratio;

GRADE Working Group grades of evidence

High quality: Further research is very unlikely to change our confidence in the estimate of effect.

Moderate quality: Further research is likely to have an important impact on our confidence in the estimate of effect and may change the estimate.

Low quality: Further research is very likely to have an important impact on our confidence in the estimate of effect and is likely to change the estimate.

Very low quality: We are very uncertain about the estimate.

1 Downgraded one point due to moderate heterogeneity (I squared $=61 \%)$ 


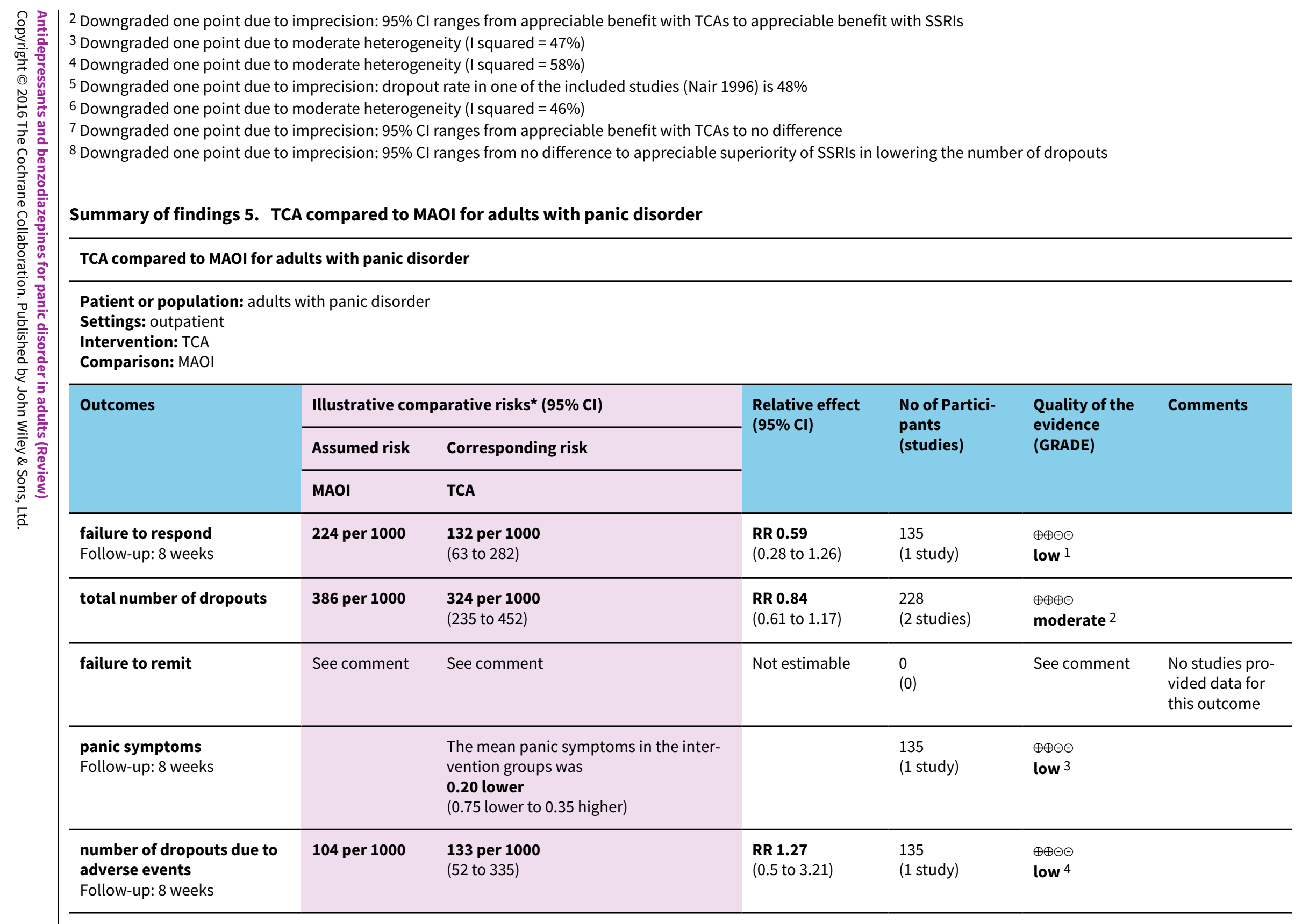


*The basis for the assumed risk (e.g. the median control group risk across studies) is provided in footnotes. The corresponding risk (and its $95 \%$ confidence interval) is based on the assumed risk in the comparison group and the relative effect of the intervention (and its $95 \% \mathrm{Cl}$ ).

Cl: Confidence interval; RR: Risk ratio;

GRADE Working Group grades of evidence

High quality: Further research is very unlikely to change our confidence in the estimate of effect.

Moderate quality: Further research is likely to have an important impact on our confidence in the estimate of effect and may change the estimate.

Low quality: Further research is very likely to have an important impact on our confidence in the estimate of effect and is likely to change the estimate.

Very low quality: We are very uncertain about the estimate.

1 Downgraded two points due to imprecision: number of individuals included in the study is low (= 135) and 95\% CI ranges from appreciable benefit with TCAs to no difference only one study provides data

2 Downgraded one point due high dropout rates

3 Downgraded two points due to imprecision: number of individuals included in the study is low (= 135) and $95 \% \mathrm{Cl}$ ranges from appreciable benefit with TCAs to no difference; only one study provides data.

4 Downgrded two points due to imprecision: number of individuals included in the study is low (= 135) and $95 \% \mathrm{Cl}$ ranges from appreciable benefit with TCAs to appreciable benefit with MAOIs; only one study provides data.

\section{Summary of findings 6 . SSRIs compared to MAOIs for adults with panic disorder}

\section{SSRIs compared to MAOIs for adults with panic disorder}

Patient or population: adults with panic disorder

Settings: outpatient

Intervention: SSRI

Comparison: MAO

\begin{tabular}{|c|c|c|c|c|c|c|}
\hline \multirow[t]{2}{*}{ Outcomes } & \multicolumn{2}{|c|}{ Illustrative comparative risks ${ }^{\star}(95 \% \mathrm{Cl})$} & \multirow{2}{*}{$\begin{array}{l}\text { Relative effect } \\
(95 \% \mathrm{CI})\end{array}$} & \multirow{2}{*}{$\begin{array}{l}\text { No of Partici- } \\
\text { pants } \\
\text { (studies) }\end{array}$} & \multirow{2}{*}{$\begin{array}{l}\text { Quality of the } \\
\text { evidence } \\
\text { (GRADE) }\end{array}$} & \multirow[t]{2}{*}{ Comments } \\
\hline & Assumed risk & Corresponding risk & & & & \\
\hline $\begin{array}{l}\text { failure to respond } \\
\text { Follow-up: 8-12 weeks }\end{array}$ & 264 per 1000 & $\begin{array}{l}\mathbf{2 9 6} \text { per } \mathbf{1 0 0 0} \\
\text { (219 to } 401)\end{array}$ & $\begin{array}{l}\text { RR } 1.12 \\
\text { (0.83 to } 1.52)\end{array}$ & $\begin{array}{l}396 \\
\text { (2 studies) }\end{array}$ & $\begin{array}{l}\oplus \oplus \oplus \ominus \\
\text { moderate } 1\end{array}$ & \\
\hline $\begin{array}{l}\text { failure to remit } \\
\text { Follow-up: } 8 \text { weeks }\end{array}$ & 467 per 1000 & $\begin{array}{l}\mathbf{4 3 4} \text { per } 1000 \\
\text { (346 to } 546 \text { ) }\end{array}$ & $\begin{array}{l}\text { RR } 0.93 \\
\text { (0.74 to } 1.17 \text { ) }\end{array}$ & $\begin{array}{l}366 \\
\text { (1 study) }\end{array}$ & $\begin{array}{l}\oplus \oplus \oplus \ominus \\
\text { moderate } 3\end{array}$ & \\
\hline
\end{tabular}




\begin{tabular}{|c|c|c|c|c|c|c|c|}
\hline 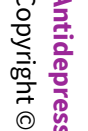 & panic symptoms & See comment & See comment & Not estimable & $\begin{array}{l}0 \\
(0)\end{array}$ & See comment & $\begin{array}{l}\text { No studies pro- } \\
\text { vided data for } \\
\text { this outcome }\end{array}$ \\
\hline 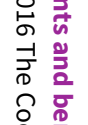 & $\begin{array}{l}\text { number of dropouts due to adverse } \\
\text { events } \\
\text { Follow-up: } 8 \text { weeks }\end{array}$ & 60 per 1000 & $\begin{array}{l}76 \text { per } 1000 \\
\text { (36 to } 163 \text { ) }\end{array}$ & $\begin{array}{l}\text { RR } 1.26 \\
(0.59 \text { to } 2.70)\end{array}$ & $\begin{array}{l}366 \\
\text { (1 study) }\end{array}$ & $\begin{array}{l}\oplus \oplus \ominus \odot \\
\text { low } 4\end{array}$ & \\
\hline
\end{tabular}

*The basis for the assumed risk (e.g. the median control group risk across studies) is provided in footnotes. The corresponding risk (and its $95 \%$ confidence interval) is based on the assumed risk in the comparison group and the relative effect of the intervention (and its $95 \% \mathrm{Cl}$ ).

CI: Confidence interval; RR: Risk ratio;

GRADE Working Group grades of evidence

High quality: Further research is very unlikely to change our confidence in the estimate of effect.

Moderate quality: Further research is likely to have an important impact on our confidence in the estimate of effect and may change the estimate.

Low quality: Further research is very likely to have an important impact on our confidence in the estimate of effect and is likely to change the estimate.

Very low quality: We are very uncertain about the estimate.

1 Downgraded one point due to imprecision: $95 \% \mathrm{Cl}$ ranges from no difference to appreciable benefit with MAOIs

2 Downgraded two points due to imprecision: number of individuals included in the trial is very low $(=30)$ and $95 \% \mathrm{Cl}$ is very wide, ranging from appreciable benefit with SSRIs to appreciable benefit with MAOIs; only one study provides data

3 Downgraded one point due to imprecision: even though the number of individuals included in the analysis is not low, only one study provides data

4 Downgrded two points due to imprecision: $95 \% \mathrm{CI}$ ranges from appreciable benefit with SSRIs to appreciable benefit with MAOIs; only one study provides data.

\section{Summary of findings 7. SSRIs compared to SNRIs for adults with panic disorder}

\section{SSRIs compared to SNRIs for adults with panic disorder}

Patient or population: adults with panic disorder

Settings: outpatient

Intervention: SSRI

Comparison: SNRI

\begin{tabular}{|c|c|c|c|c|c|c|}
\hline \multirow[t]{3}{*}{ Outcomes } & \multicolumn{2}{|c|}{ Illustrative comparative risks ${ }^{\star}(95 \% \mathrm{Cl})$} & \multirow{3}{*}{$\begin{array}{l}\text { Relative effect } \\
(95 \% \mathrm{CI})\end{array}$} & \multirow{3}{*}{$\begin{array}{l}\text { No of Partici- } \\
\text { pants } \\
\text { (studies) }\end{array}$} & \multirow{3}{*}{$\begin{array}{l}\text { Quality of the } \\
\text { evidence } \\
\text { (GRADE) }\end{array}$} & \multirow[t]{3}{*}{ Comments } \\
\hline & Assumed risk & Corresponding risk & & & & \\
\hline & SNRI & SSRI & & & & \\
\hline $\begin{array}{l}\text { failure to respond } \\
\text { Follow-up: } 12 \text { weeks }\end{array}$ & 232 per 1000 & $\begin{array}{l}\mathbf{2 2 3} \text { per } \mathbf{1 0 0 0} \\
\text { (174 to } 285)\end{array}$ & $\begin{array}{l}\text { RR } 0.96 \\
\text { (0.75 to } 1.23)\end{array}$ & $\begin{array}{l}991 \\
\text { (2 studies) }\end{array}$ & $\begin{array}{l}\oplus \oplus \oplus \oplus \\
\text { high }\end{array}$ & \\
\hline total number of dropouts & 181 per 1000 & 201 per 1000 & RR 1.11 & 991 & $\oplus \oplus \ominus \ominus$ & \\
\hline
\end{tabular}




\begin{tabular}{|c|c|c|c|c|c|c|}
\hline ᄋํㄹ & Follow-up: 2 weeks & & (134 to 298 ) & (0.74 to 1.65$)$ & (2 studies) & low 1,2 \\
\hline 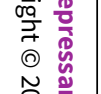 & $\begin{array}{l}\text { failure to remit } \\
\text { Follow-up: } 12 \text { weeks }\end{array}$ & 577 per 1000 & $\begin{array}{l}\mathbf{6 0 6} \text { per } \mathbf{1 0 0 0} \\
(525 \text { to } 698)\end{array}$ & $\begin{array}{l}\text { RR } 1.05 \\
(0.91 \text { to } 1.21)\end{array}$ & $\begin{array}{l}991 \\
\text { (2 studies) }\end{array}$ & $\begin{array}{l}\oplus \oplus \oplus \oplus \\
\text { high }\end{array}$ \\
\hline 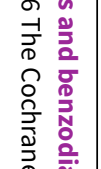 & $\begin{array}{l}\text { panic symptoms } \\
\text { Follow-up: } 12 \text { weeks }\end{array}$ & & $\begin{array}{l}\text { The mean panic symptoms in the in- } \\
\text { tervention groups was } \\
\mathbf{0 . 1 2} \text { lower } \\
\text { ( } 0.33 \text { lower to } 0.10 \text { higher) }\end{array}$ & & $\begin{array}{l}945 \\
\text { (2 studies) }\end{array}$ & $\begin{array}{l}\oplus \oplus \oplus \oplus \\
\text { high }\end{array}$ \\
\hline 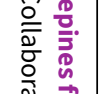 & $\begin{array}{l}\text { number of dropouts due to } \\
\text { adverse effects }\end{array}$ & 56 per 1000 & $\begin{array}{l}104 \text { per } 1000 \\
(27 \text { to } 393)\end{array}$ & $\begin{array}{l}\text { RR } 1.86 \\
(0.49 \text { to } 7.05)\end{array}$ & $\begin{array}{l}991 \\
\text { (2 studies) }\end{array}$ & $\begin{array}{l}\oplus \oplus \odot \odot \\
\text { low } 3,4\end{array}$ \\
\hline
\end{tabular}

*The basis for the assumed risk (e.g. the median control group risk across studies) is provided in footnotes. The corresponding risk (and its $95 \%$ confidence interval) is based on the assumed risk in the comparison group and the relative effect of the intervention (and its $95 \% \mathrm{Cl}$ ).

Cl: Confidence interval; RR: Risk ratio;

GRADE Working Group grades of evidence

High quality: Further research is very unlikely to change our confidence in the estimate of effect.

Moderate quality: Further research is likely to have an important impact on our confidence in the estimate of effect and may change the estimate.

Low quality: Further research is very likely to have an important impact on our confidence in the estimate of effect and is likely to change the estimate.

Very low quality: We are very uncertain about the estimate.

1 Downgraded one point due to moderate heterogeneity ( $12=53 \%)$

2 Downgraded one point due to imprecision: $95 \% \mathrm{CI}$ ranges from appreciable benefit with SSRIs to appreciable benefit with SNRIs

3 Downgraded one point due to substantial heterogeneity $(12=76 \%)$

4 Downgraded one point due to serious imprecision: $95 \% \mathrm{Cl}$ ranges from no difference to benefit with SNRIs

\section{Summary of findings 8. SSRIs compared to NaSSAs for adults with panic disorder}

\section{SSRIs compared to NaSSAs for adults with panic disorder}

Patient or population: adults with panic disorder

Settings: outpatient

Intervention: SSRI

Comparison: NaSSA

\begin{tabular}{|c|c|c|c|c|c|c|}
\hline \multirow[t]{3}{*}{ Outcomes } & \multicolumn{2}{|c|}{ Illustrative comparative risks ${ }^{\star}(95 \% \mathrm{Cl})$} & \multirow{3}{*}{$\begin{array}{l}\text { Relative effect } \\
(95 \% \mathrm{Cl})\end{array}$} & \multirow{3}{*}{$\begin{array}{l}\text { No of Partici- } \\
\text { pants } \\
\text { (studies) }\end{array}$} & \multirow{3}{*}{$\begin{array}{l}\text { Quality of the } \\
\text { evidence } \\
\text { (GRADE) }\end{array}$} & \multirow[t]{3}{*}{ Comments } \\
\hline & Assumed risk & Corresponding risk & & & & \\
\hline & NaSSA & SSRI & & & & \\
\hline
\end{tabular}




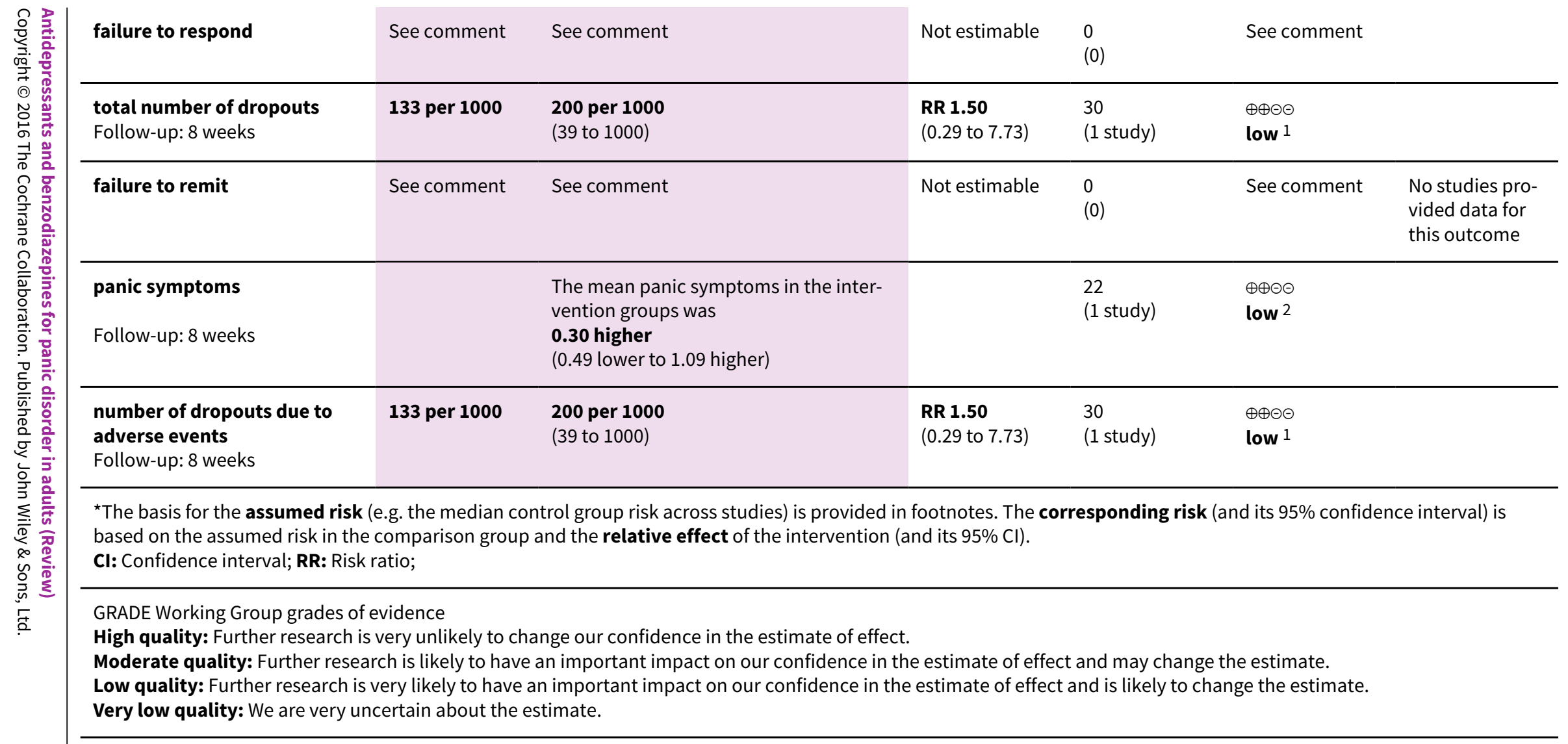

1 Downgraded two points due to imprecision: number of individuals included in the trial is very low $(=30)$ and $95 \% \mathrm{Cl}$ is very wide, ranging from appreciable benefit with SSRIS to appreciable benefit with NaSSAs; only one study provides data

2 Downgraded two points due to imprecision: number of individuals included in the trial is very low $(=22)$ and $95 \% \mathrm{Cl}$ is very wide, ranging from appreciable benefit with SSRIs to appreciable benefit with NaSSAs; only one study provides data 


\section{B A C K G R O U N D}

\section{Description of the condition}

A panic attack is a discrete period of fear or anxiety that has a rapid onset, reaches a peak within 10 minutes and in which at least four of 13 characteristic symptoms are experienced. Many of these symptoms involve bodily systems, such as racing heart, chest pain, sweating, shaking, dizziness, flushing, stomach churning, faintness and breathlessness. Further recognised panic attack symptoms involve fearful cognitions, such as the fear of collapse, going mad or dying, and derealisation (APA 1994).

Panic disorder first entered diagnostic classification systems in 1980 with the publication of the Diagnostic and Statistical Manual of Mental Disorders, Third Edition (DSM-III) (APA 1980), following observations that patients with panic attacks responded to treatment with the tricyclic antidepressant (TCA), imipramine (Klein 1964). To diagnose panic disorder, further conditions must be met relating to the frequency of attacks, the need for some panic attacks to come on 'out of the blue' rather than in a predictable, externally-triggered situation, and exclusions where attacks are attributable solely to medical causes or panic-inducing substances, notably caffeine. The Diagnostic and Statistical Manual of Mental Disorders, Fourth Edition (DSM-IV) also requires that at least one panic attack has been followed by one of the following:

1. persistent concern about having additional attacks;

2. worry about the implications of the attack or its consequences;

3. a significant change in behaviour related to the attacks (APA 1994).

The core features of panic attacks remained unchanged in the Diagnostic and Statistical Manual of Mental Disorders, Fifth Edition (DSM-5) (APA 2013a), but in DSM-5 panic disorder and agoraphobia are no longer linked and are now coded in two diagnoses (APA 2013b).

Panic disorder is common in the general population with a lifetime prevalence of $1 \%$ to $4 \%$ (Bijl 1998; Eaton 1994). In primary care settings, panic syndromes have been reported to have a prevalence of around 10\% (King 2008). Its aetiology is not fully understood and is probably heterogeneous. Biological theories incorporate the faulty triggering of an inbuilt anxiety response. Evidence for this comes from biological challenge tests (lactate and carbon dioxide trigger panic in those with the disorder) and from animal experiments and neuroimaging studies in humans that show activation of fear circuits, such as those involving the periaqueductal grey matter (Gorman 2000).

Agoraphobia is anxiety about being in places or situations from which escape might be difficult or embarrassing, or in which help may not be available in the event of having a panic attack (APA 1994). About one-fourth of people suffering from panic disorder also have agoraphobia (Kessler 2006). The presence of agoraphobia is associated with increased severity and worse outcome (Kessler 2006). There are several risk factors that predict the development of agoraphobia in people suffering from panic disorder: female gender, more severe dizziness during panic attacks, cognitive factors, dependent personality traits and social anxiety disorder (Starcevic 2009).
Panic disorder, with or without agoraphobia, is highly co-morbid with other psychiatric disorders, such as drug dependence, major depression, bipolar I disorder, social phobia, specific phobia, and generalised anxiety disorder (Grant 2006). It is estimated that generalised anxiety disorder co-occurs in $68 \%$ of people with panic disorder, whilst major depression has a prevalence of $24 \%$ to $88 \%$ among people with panic disorder (Starcevic 2009).

\section{Description of the intervention}

The treatment of panic disorder includes psychological and pharmacological interventions, often used in combination (Furukawa 2007). Historically, pharmacological interventions for panic disorder have been based on the use of monoamine oxidase inhibitors (MAOIs) and TCAs (Bruce 2003). However, MAOIs and TCAs are associated with severe adverse effects, such as the risk of hypertensive crisis for MAOIs, and anticholinergic, arrhythmogenic and overall poor tolerability for TCAs (Wade 1999). Recent guidelines by the American Psychiatric Association (APA 2009), British Association for Psychopharmacology (BAP 2005), and National Institute for Health and Care Excellence (NICE) (NICE 2011) recommend antidepressants, mainly selective serotonin reuptake inhibitors (SSRIs), as the first-line treatment for panic disorder, due to their more favourable adverse effect profile over MAOIs and TCAs. A meta-analysis of 43 studies (randomised and non randomised) comparing SSRIs and TCAs in panic disorder showed that SSRIs were as effective as TCAs, and were better tolerated (Bakker 2002), although other studies showed a possible overestimation of the efficacy of SSRIs over older antidepressants in panic disorder, finding no difference in their efficacy (Otto 2001, effect-size analysis of 12 placebo-controlled, efficacy trials of SSRIs for panic disorder). It appears that TCAs can still have a role in the treatment of panic disorder.

Benzodiazepines, particularly high-potency ones, have also been used in panic disorder (Stein 2010). Benzodiazepines have a higher incidence of dependence and withdrawal reaction when compared to antidepressants (Wade 1999). Moreover, benzodiazepines alone may not be effective in treating panic disorder co-morbid with depression (Ballenger 1998), and have less favourable long-term outcomes (NICE 2011). In spite of these caveats, it appears that benzodiazepines continue to be widely prescribed for the treatment of panic disorder (Bruce 2003).

\section{How the intervention might work}

The main classes with evidence of efficacy in panic disorder are antidepressant drugs that augment the function of the monoamines serotonin and/or noradrenaline. With regard to the serotonergic antidepressants (SSRIs such as fluoxetine, paroxetine, sertraline and citalopram), these drugs promote the transmission of the neurotransmitter serotonin across brain synapses; most notably in the dorsal raphe nucleus (Briley 1993). They prevent reuptake of serotonin into nerve terminals by inhibiting serotonin transporters, thus allowing more to be available for neurotransmission. In panic disorder, imaging studies have showed reduced expression of the $5 \mathrm{H} 1 \mathrm{~A}$ receptor (Nash 2008), which has an inhibitory function, so the increased serotonin throughput may in part serve to overcome this deficit of inhibition. Noradrenergic antidepressants can similarly increase transmission of the catecholamine noradrenaline. Some antidepressants such as the serotonin-norepinephrine reuptake inhibitors (SNRIs) (e.g. 
venlafaxine, duloxetine) and TCAs can enhance both serotonin and noradrenaline transmission by inhibiting both transporters.

Benzodiazepines moderate the gamma-aminobutyric acid (GABA) neurotransmitter system, which is the brain's main inhibitory neurotransmitter. They act as agonists at the GABA-A benzodiazepines receptor. This complex contains a chloride channel which can be opened by agonists and ultimately produces an anxiolytic effect and sedation. It is known through imaging studies that the inhibitory GABA system is deficient in panic disorder (Malizia 1998; Cameron 2007), and thus the ability of benzodiazepines to act as agonists at the GABA-A benzodiazepine receptor can counteract this. It is likely that both monoaminebased systems and GABA-based systems converge allowing both antidepressants and benzodiazepines to have efficacy in panic disorder despite their differing actions on neurotransmitter systems. One possibility is via serotonergic neurons that modulate GABA input to the periaqueductal grey matter.

\section{Why it is important to do this review}

Antidepressants and benzodiazepines are widely used in panic disorder. Published randomised controlled trials (RCTs) have shown some evidence of efficacy. However, no systematic review that includes comparisons between antidepressants and benzodiazepines in panic disorder has been conducted recently, to our knowledge. A meta-analysis published in 2007 focused on combined psychotherapy and antidepressants in panic disorder (Furukawa 2007), and a more recent systematic review focused on psychological treatments only (SanchezMeca 2010). One meta-analysis of interventions for panic disorder has been published in recent years, focusing on combined psychotherapy and benzodiazepines in panic disorder (Watanabe 2009). Furukawa 2007 concluded that either combined psychotherapy or psychotherapy alone may be chosen as firstline treatment for panic disorder. Sanchez-Meca 2010 reported that exposure, relaxation training and breathing retraining have the most robust evidence. A network meta-analysis was also performed to compare different psychological therapies for panic disorder (Pompoli 2016). To our knowledge, the most recent metaanalysis specifically focused on antidepressants in panic disorder was published in 2002 (Bakker 2002), and included 43 studies comparing SSRIs and TCAs. The authors concluded that SSRIs and TCAs were of equal efficacy in the treatment of panic disorder, with a better tolerability profile for SSRIs. Benzodiazepines are widely used in panic disorder. Available RCTs have shown some evidence of efficacy (Batelaan 2012). The last meta-analysis specifically focused on benzodiazepines in panic disorder was published in 1991 (Wilkinson 1991), and included 19 double-blind, placebocontrolled trials. This meta-analysis compared antidepressants and benzodiazepines with placebo. The authors concluded that active treatment had a $25 \%$ greater success rate than placebo over 14 weeks. Another meta-analysis has been recently published (Offidani 2013), including 22 studies comparing benzodiazepines and antidepressants in various anxiety disorders including panic disorder. The authors found that benzodiazepines were more effective in reducing the number of panic attacks, than TCAs. Additionally, benzodiazepines were better tolerated than TCAs and they were as effective as the newer antidepressants. The authors concluded that the role of benzodiazepines should be reappraised. The review mostly focused on tricyclic antidepressants and did not include studies comparing benzodiazepines with SSRIs.
An up-to-date systematic review is needed to help practitioners identify the relative efficacy of antidepressants and benzodiazepines, compared to each other, in order to be better guided in the choice of the pharmacological agent.

Two other Cochrane reviews in patients with panic disorder, one on antidepressants versus placebo and one on benzodiazepines versus placebo, respectively, are in progress, which will be of further help to identify effective treatments in panic disorder (Guaiana 2013a; Guaiana 2013b). A Cochrane network metaanalysis of psychopharmacological treatment in panic disorder is in progress (Guaiana 2014).

This review followed the methods set out in the protocol (Guaiana 2015)

\section{OB JECTIVES}

To assess the effects of antidepressants or benzodiazepines for panic disorder in adults, specifically:

1. to determine the efficacy of antidepressants and benzodiazepines in alleviating symptoms of panic disorder, with or without agoraphobia, in comparison to other antidepressants and other benzodiazepines;

2. to review the acceptability of antidepressants and benzodiazepines in panic disorder, with or without agoraphobia, in comparison to other antidepressants and other benzodiazepines; and

3. to investigate the adverse effects of antidepressants and benzodiazepines in panic disorder, with or without agoraphobia, including the general prevalence of adverse effects, compared to other antidepressants and other benzodiazepines.

\section{METHODS}

\section{Criteria for considering studies for this review}

\section{Types of studies}

Double-blind randomised controlled trials (RCTs) using a parallelgroup design that compare antidepressants as monotherapy with benzodiazepines as monotherapy, antidepressants with antidepressants and benzodiazepines with benzodiazepines. We included one cross-over trial, and RCTs with more than two arms. Cluster-RCTs were eligible for inclusion but none were identified.

We excluded quasi-randomised trials, such as those allocated by using alternate days of the week.

\section{Types of participants}

\section{Participants}

Participants aged 18 years or older.

\section{Diagnosis}

Patient with primary diagnosis of panic disorder, with or without agoraphobia, diagnosed according to any of the following criteria: Feighner criteria, Research Diagnostic Criteria, Diagnostic and Statistical Manual of Mental Disorders (DSM-III, DSM-III-R, DSMIV, DSM-V) or International Statistical Classification of Diseases and Related Health Problems, 10th revision (ICD-10). In cases where study eligibility focused on agoraphobia, rather than 
panic disorder, we included studies if operationally diagnosed according to the above-named criteria and when it was possible to safely assume that some of the participants were suffering from panic disorder as defined by the above criteria. In other words, we excluded studies that focused on agoraphobia without panic disorder. There is evidence that over $95 \%$ of patients with agoraphobia seen clinically suffer from panic disorder as well (Goisman 1995). However, we planned to further examine the effects of the inclusion of these studies (i.e. studies in which some, but possibly not all, of the patients had panic disorder) in a sensitivity analysis (Sensitivity analysis).

\section{Co-morbidities}

We excluded participants with serious co-morbid physical disorders (e.g. myocardial infarction, chronic obstructive pulmonary disorder, uncontrolled diabetes, electrolyte disturbances) as they may confound treatment effectiveness and tolerability. Studies where panic is induced were excluded. We included participants with co-morbid mental disorders, but the effect of including these participants was examined in sensitivity analyses (Sensitivity analysis).

\section{Settings}

We included all types of settings (inpatient, outpatient, primary care). We included only studies recruiting patients acutely ill. Longterm, relapse prevention studies were therefore excluded.

\section{Types of interventions}

Trials comparing antidepressants as monotherapy with benzodiazepines as monotherapy, antidepressants with antidepressants, and benzodiazepines with benzodiazepines in the treatment of panic disorder, with or without agoraphobia.

We included only acute treatment studies treating participants for less than six months. If a study treated participants for more than six months but reported outcome data within six months, we included the data.

We included the following antidepressants as classified by the World Health Organization (WHO) Anatomical, Therapeutic and Chemical (ATC) classification system and the Defined Daily Dose (DDD) (WHO ATC/DDD) system (WHO 2003).

1. Tricyclic antidepressants (TCAs): amitriptyline, amoxapine, clomipramine, desipramine, dosulepin/dothiepin, doxepin, imipramine, iofepramine, maprotiline, nortriptyline, protriptyline, trimipramine, any other agent.

2. Selective serotonin reuptake inhibitors (SSRIs): fluoxetine, fluvoxamine, sertraline, citalopram, paroxetine, escitalopram, any other agent.

3. Monomine oxidase inhibitors (MAOls: phenelzine, isocarboxazide, tranylcypromine, moclobemide, brofaromine, any other agent.

4. Serotonin-noradrenaline reuptake Inhibitors (SNRIs): venlafaxine, desvenlafaxine, duloxetine, milnacipran, any other agent.

5. Noradrenergic and specific serotonergic antidepressants (NaSSAs): mirtazapine, any other agent

6. Noradrenergic and dopaminergic reuptake inhibitors: bupropion, any other agent.
7. Noradrenergic reuptake inhibitors: reboxetine, any other agent.

8. Others: agomelatine, trazodone, nefazodone, mianserin, maprotiline, any other agent classified as antidepressants according to the WHO ATC/DDD system of non-conventional herbal products (e.g. Hypericum).

We included the following benzodiazepines: alprazolam, bretazenil, bromazepam, chlordiazepoxide, cinolazepam, clonazepam, cloxazolam, clorazepate, diazepam, estazolam, fludiazepam, flunitrazepam, flurazepam, flutoprazepam, halazepam, ketazolam, loprazolam, lorazepam, medazepam, nimatazepam, nitrazepam, nodazepam, oxazepam, phenazepam, pinazepam, prazepam, premazepam, quazepam, temazepam, tetrazepam, triazolam.

We excluded studies in which regular use of benzodiazepines was allowed in addition to the study medications; studies where irregular use of benzodiazepines was allowed were included, but the effect of including these participants was examined in sensitivity analyses (Sensitivity analysis). We applied no restrictions on dose, frequency, intensity or duration.

We also excluded studies administering psychosocial therapies targeted at panic disorder delivered concurrently with medication.

\section{Types of outcome measures}

\section{Primary outcomes}

1. Failure to respond, i.e. lacking of substantial improvement from baseline as defined by the original investigators. Examples of improvement would be "very much or much improved" according to the Clinical Global Impressions Scale, more than $40 \%$ reduction in the Panic Disorder Severity Scale score (which is considered as equivalent to "very much or much improved" on the Clinical Global Impression of Improvement Scale (Furukawa 2009), and more than 50\% reduction in the Fear Questionnaire Agoraphobia Subscale (which is considered as an appropriate rate of response according to Bandelow 2006). For this outcome we calculated the number of patients who fail to meet these improvement criteria.

2. Total number of dropouts for any reason as a proxy measure of treatment acceptability.

\section{Secondary outcomes}

1. Failure to remit, i.e. lacking of satisfactory end-state as defined by global judgment of the original investigators. Examples of satisfactory end-state would be "panic free" and "no or minimal symptoms" according to the Clinical Global Impression Severity Scale. For this outcome, we calculated the number of patients who failed to meet these remission criteria.

2. Panic symptom scales and global judgment on a continuous scale. Examples include Panic Disorder Severity Scale (PDSS) total score (0 to 28), Clinical Global Impression Severity Scale (CGI-S) (1 to 7 ), and Clinical Global Impression Change Scale (1 to 7 ). When multiple measures are used, preference was given in the order as above, with preference given to panic symptom scales. The actual measure entered into meta-analyses will be indicated at the top of the listings in the 'Characteristics of included studies' section

3. Frequency of panic attacks, as recorded, for example, by a panic diary 
4. Agoraphobia, as measured, for example, by the Fear Questionnaire (FQ), Mobility Inventory, or behavioural avoidance test

5. General anxiety, as measured, for example, by the Hamilton Rating Scale for Anxiety (HAMA), Beck Anxiety Inventory, StateTrait Anxiety Index, Sheehan Patient-Rated Anxiety Scale, or Anxiety Subscale of Symptom Checklist-90-Revised (SCL-90-R)

6. Depression, as measured, for example, by the Hamilton Rating Scale for Depression (HRSD), Beck Depression Inventory (BDI), or Depression Subscale of SCL-90-R

7. Social functioning, as measured, for example, by the Sheehan Disability Scale (SDS), Global Assessment Scale, or Social Adjustment Scale-Self Report

8. Quality of life, as measured for example by the 36-Item Short Form Health Survey (SF-36) or the 12-Item Short Form Health Survey (SF-12)

9. Patient satisfaction with treatment

10. Economic costs

11.Number of dropouts due to adverse effects

12. Number of patients experiencing at least one adverse effect

\section{Timing of outcome assessment}

All outcomes are short-term outcomes, which we defined as acute phase treatment which normally would last two to six months.

When studies reported outcome data at different time points within two to six months, the time point closest to 12 weeks was given preference.

\section{Hierarchy of outcome measures}

When multiple measures were used, preference was given in the order listed above for each outcome.

\section{Search methods for identification of studies}

The Cochrane Common Mental Disorders Group maintains a specialised register of randomised controlled trials, the CCMDCTR. This register contains over 40,000 reference records (reports of RCTs) for anxiety disorders, depression, bipolar disorder, eating disorders, self-harm and other mental disorders within the scope of this Group. The CCMDCTR is a partially studies based register with $>50 \%$ of reference records tagged to $c 12,500$ individually PICO coded study records. Reports of trials for inclusion in the register are collated from (weekly) generic searches of MEDLINE (1950-), Embase (1974-) and PsycINFO (1967-), quarterly searches of the Cochrane Central Register of Controlled Trials (CENTRAL) and review specific searches of additional databases. Reports of trials are also sourced from international trial registries, drug companies, the hand-searching of key journals, conference proceedings and other (non-Cochrane) systematic reviews and meta-analyses. Details of CCMD's core search strategies (used to identify RCTs) can be found on the Group's web site with an example of the core MEDLINE search displayed in Appendix 1.

\section{Electronic searches}

\section{Cochrane Common Mental Disorders Group's specialised register (CCMDCTR)}

The Group's Information Specialist searched the CCMDCTR (Studies and References) register to 11 September 2015, using the following search strategy:
CCDANCTR-Studies

Diagnosis $=$ panic

Records were screened for randomised-controlled trials comparing antidepressants with benzodiazepines, antidepressants with antidepressants or benzodiazepine with benzodiazepine in this population.

\section{CCDANCTR-References}

A search of the references register (to identify additional untagged, uncoded reports of RCTs) included a comprehensive list of terms for panic, anxiety disorders not otherwise specified (ADNOS), antidepressants and benzodiazepines, (Appendix 2).

We screened all abstracts for 'antidepressants' as classified by the WHO ATC/DDD system as well as relevant benzodiazepine trials. We obtained full-text articles, where necessary.

\section{National and international trials registers}

We conducted complementary searches on the WHO International Clinical Trials Registry Platform (ICTRP) and ClinicalTrials.gov

\section{Searching other resources}

We checked the reference lists of all included studies, nonCochrane systematic reviews and major textbooks of affective disorders (written in English), for published reports and citations of unpublished research. We also pursued a citation search via Web of Science (included studies only) to identify additional works. We also contacted experts in the field.

\section{Data collection and analysis}

\section{Selection of studies}

Two review authors (IB (clinical expertise), CT (methodological expertise)) independently selected studies for inclusion in this systematic review.

Review authors IB and CT inspected the search results by reading the titles and abstracts to see if they met the inclusion criteria. Disagreement was resolved by consultation with the other co-authors. We obtained full-text articles for the potentially relevant studies located in the search and both review authors independently assessed them for inclusion and, in the case of discordance, resolution was sought by discussion between all review authors. Where it was not be possible to evaluate the study because of language problems or missing information, we categorised the study as 'study awaiting classification' until a translation or further information could be obtained. We reported the reasons for the exclusion of trials in the Characteristics of excluded studies table.

We documented and presented all decisions made during the selection process, with numbers of studies and references, in a Preferred Reporting Items for Systematic Reviews and MetaAnalyses (PRISMA) flow diagram (Moher 2009) (Figure 1). 
Figure 1. Study flow diagram.

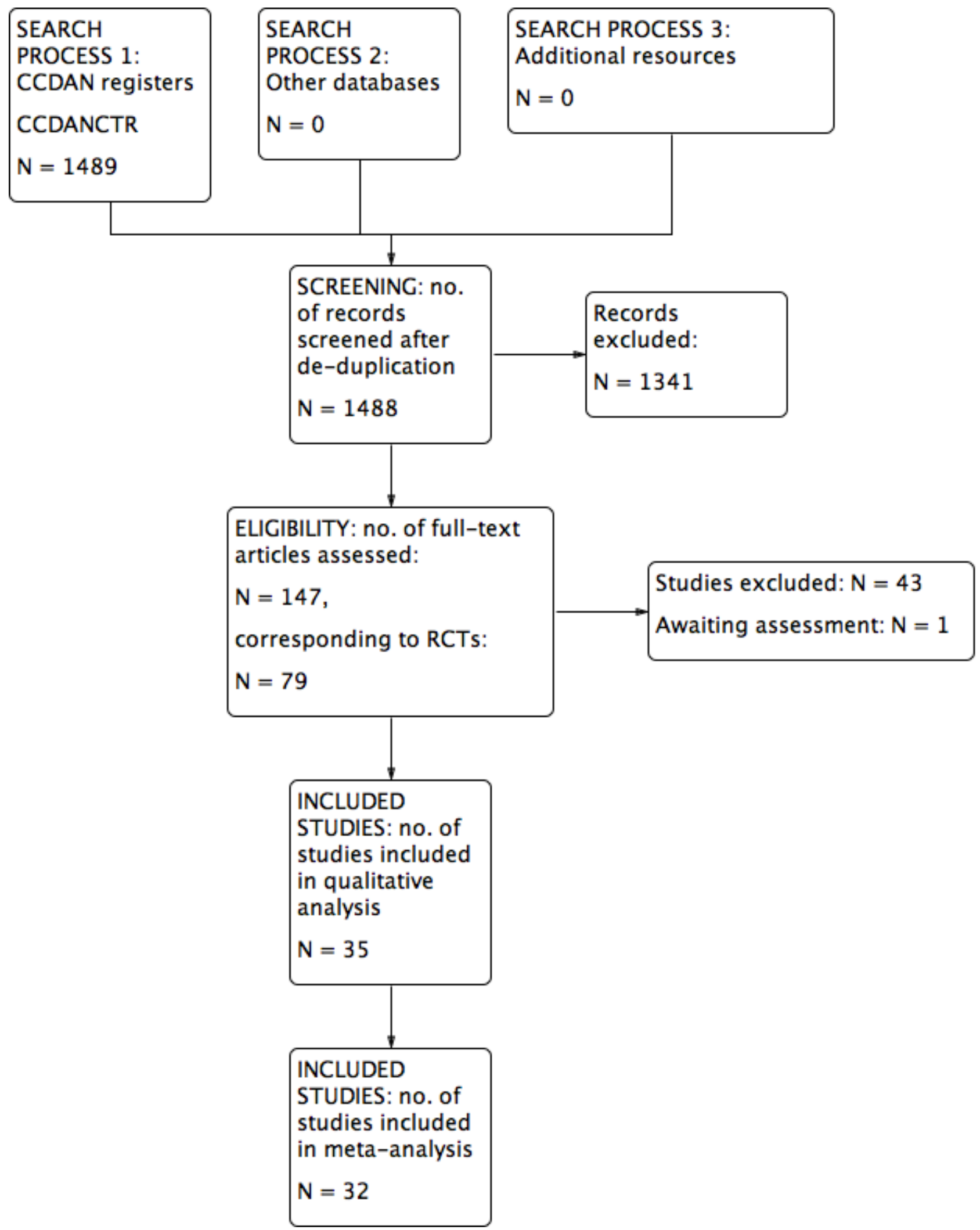




\section{Data extraction and management}

Two review authors (IB and CT) used a standardised data extraction form to independently extract data from included studies concerning participant characteristics (age, sex, study setting), intervention details (dosage, duration of study, sponsorship), study characteristics (blinding, allocation etc) and outcome measures of interest. We first piloted this data extraction sheet on a sample of $10 \%$ of the included studies. Again, any disagreement was resolved by consensus or by consulting a third review author (CB). If necessary, we contacted authors of studies to obtain clarification.

Our comparisons were as follows (Types of interventions):

A. antidepressants versus benzodiazepines;

B. antidepressants versus antidepressants;

\section{C. individual benzodiazepines versus another benzodiazepine.}

We stratified comparison A by class of antidepressant, as per the categories provided in Types of interventions. We also included comparisons of antidepressant classes versus benzodiazepines, to allow the presentation of data about individual antidepressants of each class versus benzodiazepines. For the second comparison, we made the decision to conduct separate comparisons for each class of antidepressant rather than stratify an overall comparison of antidepressant versus antidepressant according to class of drug. For the second comparison we also included individual antidepressants versus another antidepressant of the same class (see Differences between protocol and review.)

\section{Assessment of risk of bias in included studies}

Two review authors (IB and CT) independently assessed the risk of bias in included studies using the Cochrane Collaboration's tool for assessing risk of bias as described in the Cochrane Handbook for Systematic Reviews of Interventions (Higgins 2011). This tool encourages consideration of the following domains:

1. random sequence generation and allocation concealment (selection bias);

2. blinding of participants and personnel (performance bias);

3. blinding of outcome assessors (detection bias);

4. incomplete outcome data reporting (attrition bias);

5. selective reporting (reporting bias);

6. other bias (within this domain we will consider things like sponsorship bias).

We assessed and categorised risk of bias, in each domain and overall, as follows:

1. Low risk of bias, plausible bias unlikely to seriously alter the results;

2. high risk of bias, plausible bias that seriously weakens confidence in the results;

3. unclear risk of bias, plausible bias that raises some doubt about the results.

In case of disagreement, the final rating was made by consensus, if needed with the involvement of a third author (CB). Where inadequate details of randomisation and other characteristics of trials were provided, we contacted authors of the studies in order to obtain further information. Non-concurrence in assessment of bias was also reported.

\section{Measures of treatment effect}

Our main outcome measure is reduction of severity of panic and agoraphobia symptoms. Symptomatic improvement was usually presented as a change in a panic disorder scale(s) (mean and standard deviation) or as a dichotomous outcome (responder or non-responder, remitted or not-remitted), or both.

\section{Dichotomous data}

For binary or dichotomous outcomes, we calculated a standard estimation of the risk ratios (RRs) and the corresponding 95\% confidence intervals ( $\mathrm{Cls}$ ) using a random-effects model. It has been shown that a random-effects model has a good generalisability (Furukawa 2002), and that RRs are easier to understand than odds ratios (ORs) (Boissel 1999). Furthermore, ORs tend to be interpreted as RRs by clinicians (Deeks 2002). This may lead to an overestimation of the impression of the effect (Higgins 2011). For all primary outcomes, we calculated the number needed to treat for an additional beneficial outcome (NNTB) or number needed to treat for an additional harmful outcome (NNTH) and their $95 \% \mathrm{Cls}$ using Visual Rx (www.nntonline.net/), taking account of the event rate in the control group.

\section{Continuous data}

\section{Summary statistics}

We assumed a priori that the included studies used varied rating scales; therefore we used standardised mean differences (SMDs). If all included studies used the same measuring instrument, we used mean differences (MDs). We also calculated 95\% Cls for each continuous measure.

\section{Endpoint versus change data}

Trials usually report results either using endpoint means and standard deviation of scales or using change in mean values from baseline of assessment rating scales. We prefer to use scale endpoint data, which typically cannot have negative values and are easier to interpret from a clinical point of view. If endpoint data were unavailable, we used the change data in separate analyses. In the case we used MDs, we pooled results based on change data and endpoint data in the same analysis.

\section{Unit of analysis issues}

\section{Cross-over trials}

Cross-over trials are trials in which all participants receive both the control and intervention treatment but in a different order. The major problem is a carry-over effect from the first phase to the second phase of the study, especially if the condition of interest is unstable (Elbourne 2002). As this is the case with panic disorder, we planned to include cross-over studies, but to use only data up to the point of first cross-over phase.

\section{Studies with multiple treatment groups}

Where a study involved more than two treatment arms, when there were two appropriate dose groups of the same drug, we pooled different dose arms and considered them as one. If the arms involved one or more benzodiazepine arms and/or two or more arms of different classes of antidepressants, we planned 
to compare each arm separately. To avoid the of unit-of-analysis error by double counting the patients of the shared group (Higgins 2011), we planned to divide the shared group evenly among the treatment arms. In accordance to the recommendations in the Cochrane Handbook for Systematic Reviews of Interventions (Higgins 2011), we divided events and number of patients for dichotomous outcomes, while only divided the number of patients for continuous outcomes.

\section{Cluster-randomised trials}

In cluster-randomised trials, groups of individuals rather than individuals are randomised to different interventions. Had we identified cluster-RCTs, we planned to use the generic inverse variance technique. We would have checked if such trials have been appropriately analysed taking into account intraclass correlation coefficients to adjust for cluster effects. If trialists have not adjusted for the effects of clustering, we would have attempted to do this by obtaining an intracluster correlation coefficient and then following the guidance given in the Cochrane Handbook for Systematic Reviews of Interventions (Higgins 2011).

\section{Dealing with missing data}

We tried to contact the study authors for all relevant missing data.

\section{Dichotomous outcomes}

We calculated response, or remission on treatment, using an intention-to-treat analysis. We followed the principle of 'once randomised always analysed'. Where participants left the study before the intended endpoint, it was assumed that they would have experienced the negative outcome. The validity of the above assumption was planned to be tested by sensitivity analysis, applying worst and best case scenarios. When dichotomous outcomes were not reported, but the baseline mean and standard deviation on a panic disorder scale were reported, we calculated the number of responding or remitted participants according to a validated imputation method (Furukawa 2005). We analysed the validity of the above approach by sensitivity analysis (Sensitivity analysis). If necessary, we contacted authors of studies to obtain data and/or clarification.

\section{Continuous outcomes}

Concerning continuous data, the Cochrane Handbook for Systematic Reviews of Interventions recommends avoiding imputation of continuous data and suggests using the data as presented by the original authors (Higgins 2011). Where intentionto-treat data were available, they have been preferred to 'perprotocol analysis'. If necessary, we contacted authors of studies to obtain data and/or clarification.

\section{Skewed or qualitative data}

We planned to present skewed and qualitative data descriptively. We considered several strategies for skewed data. If papers reported a mean and standard deviation and there was also an absolute minimum possible value for the outcome, we planned to divide the mean by the standard deviation. If this was less than two then we concluded that there was some indication of skewness. If it was less than one (that is the standard deviation was bigger than the mean), then there was almost certainly skewness. If papers had not reported the skewness and simply reported means, standard deviations and sample sizes, these numbers were used. Because there was a possibility that these data might not have been properly analysed, and could also be misleading, they were descriptively reported in the results. If the data had been log-transformed for analysis, and the geometric means were reported, skewness was reduced. This is the recommended method of analysis of skewed data (Higgins 2011). If studies used non-parametric tests and described averages using medians, they could not be formally pooled in the analysis. We followed the recommendation made in the Cochrane Handbook for Systematic Reviews of Interventions that results of these studies should be reported in a table in our review, along with all other papers. This means that the data were not lost from the review and the results could be considered when drawing conclusions, even if they could not be formally pooled in the analyses.

\section{Missing statistics}

When only $\mathrm{P}$ or standard error values were reported, we calculated standard deviations (Altman 1996). In the absence of supplementary data after requests to the authors, we calculated the standard deviations according to a validated imputation method (Furukawa 2006). We examined the validity of these imputations in the sensitivity analyses.

\section{Assessment of heterogeneity}

We quantified heterogeneity using the $\mathrm{I}^{2}$ statistic as per the guidance by the Cochrane Handbook for Systematic Reviews of Interventions (Higgins 2011). We interpreted the $\mathrm{I}^{2}$ as follows:

1. $0 \%$ to $40 \%$ : might not be important;

2. $30 \%$ to $60 \%$ : may represent moderate heterogeneity;

3. $50 \%$ to $90 \%$ : may represent substantial heterogeneity;

4. $75 \%$ to $100 \%$ : considerable heterogeneity.

We also used the $\mathrm{Chi}^{2}$ test and its $\mathrm{P}$ value to determine the direction and magnitude of the treatment effects. In a meta-analysis of few trials, $\mathrm{Chi}^{2}$ will be underpowered to detect heterogeneity, if it exists. A P value of $<0.10$ was used as a threshold of statistical significance for the $\mathrm{Chl}^{2^{2}}$ test. We also visually assessed the forest plots for evidence of heterogeneity.

\section{Assessment of reporting biases}

Reporting biases arise when the dissemination of research findings is influenced by the nature and direction of results (Higgins 2011). A funnel plot is usually used to investigate publication bias. However, it has a limited role when there are only few studies of similar size. Secondly, asymmetry of a funnel plot does not always reflect publication bias. We visually inspected funnel plots to assess publication bias as well as performed statistical test for funnel plot asymmetry as proposed by the Cochrane Handbook for Systematic Reviews of Interventions (Higgins 2011). We did not use funnel plots for outcomes if there were 10 or fewer studies included in the metaanalysis in question, or if all studies were of similar size.

\section{Data synthesis}

We used a random-effects model to calculate the treatment effects. We preferred the random-effects model as it takes into account differences between studies even when there is no evidence of statistical heterogeneity It gives a more conservative estimate than the fixed-effect model. We noted that the random-effects model gives added weight to small studies, which can either increase 
or decrease the effect size. We applied a fixed-effect model, on primary outcomes only, to see whether it markedly changed the effect size.

\section{Subgroup analysis and investigation of heterogeneity}

Subgroup analyses are often exploratory in nature and should be interpreted cautiously: firstly, because they often involve multiple analyses leading to false positive results; and secondly, these analyses lack power and are more likely to result in false positive results. Keeping in mind these reservations, and also taking into account the number of available studies, we planned to perform the following subgroup analyses:

1. participants with agoraphobia versus participants without agoraphobia (because the same treatment may have differential effectiveness with regard to panic and agoraphobia);

2. acute phase treatment studies that lasted for less than four months versus those that lasted for four months or more.

\section{Sensitivity analysis}

We planned to conduct the following sensitivity analyses for the primary outcomes only in order to examine if the results changed and check for the robustness of the observed findings.

1. Excluding trials with high risk of bias (i.e. trials with inadequate allocation concealment and blinding, with incomplete data reporting and/or with high probability of selective reporting).

2. Excluding trials with dropout rates greater than $20 \%$.

3. Excluding studies funded by the pharmaceutical company marketing each antidepressant or benzodiazepine (This sensitivity analysis is particularly important in view of the repeated findings that funding strongly affects outcomes of research studies (Als-Nielsen 2003; Lexchin 2003; Bhandari 2004), and because industry sponsorship and authorship of clinical trial reports have increased over the last 20 years (Buchkowsky 2004)).

4. Excluding studies whose participants clearly have significant psychiatric co-morbidities including primary or secondary depressive disorders.

5. Excluding studies mostly focused on agoraphobia.

6. Applying best and worst case scenarios to studies where participants left the study before the endpoint.

7. Excluding studies where responding or remitted participants are calculated according to an imputation method.

8. Excluding studies where irregular benzodiazepine use was allowed after the first 15 days after random allocation.

\section{Summary of findings}

We summarised the findings using a 'Summary of findings' table, applying the Grading of Recommendations Assessment, Development and Evaluation (GRADE) approach (Higgins 2011).

We included the following outcomes in the 'Summary of findings' tables:

1. response;

2. total number of dropouts;

3. remission;

4. panic symptom scales and global judgment on a continuous scale;
5. number of dropouts due to adverse effects.

\section{RE S U L T S}

\section{Description of studies}

\section{Results of the search}

The number of references identified by the searches (last update September 2015) was 1489, of which 1488 remained after deduplication. We excluded 1341 references after assessment of titles and abstracts. We retrieved 147 full-text articles for full inspection, describing 79 randomised controlled trials (RCTs). Of these, 43 studies were excluded, and one study was placed in the awaiting assessment group. Finally, 35 studies including 6785 participants, 5365 for the arms of interest (described in 84 reports) were included in the review. In case of missing information, we contacted authors of the included studies for additional information, and three of them responded (Drs. Bandelow, Lavori, and Stahl). See Figure 1 for a PRISMA flow diagram (Moher 2009) depicting the study selection process.

\section{Included studies}

Thirty-five studies were included in this review, with characteristics as follows (see also Characteristics of included studies).

\section{Design}

Thirty-four of the 35 included studies were parallel-group, individually-randomised controlled trials. One study (Sasson 1999) with a cross-over design reported only aggregated results on the two active treatment phases (before and after cross-over); therefore these data could not be included in the meta-analysis.

\section{Sample sizes}

The sample sizes ranged between seven (Sheikh 1999) and 391 (CNCPS 1992) participants in each arm. Fifteen studies included overall sample sizes over 100: Bandelow $2004(n=225)$, CNCPS 1992 $(n=1168)$, GSK-29060/1 ( $n=226)$, Holland $1999(n=315)$, Krueger 1999 ( $n=135)$, Lecrubier $1997(n=368)$, Nair $1996(n=148)$, Noyes $1996(n=238)$, Pfizer $2008(n=319)$, Pollack 2007a $(n=653)$, Pollack 2007b ( $n=663)$, Schweizer $1993(n=106)$; Stahl $2003(n=380)$, Tiller $1999(n=366)$, Wade $1997(n=379)$.

\section{Setting}

A total of 19 trials enrolled only outpatients, one trial enrolled only inpatients, and both inpatients and outpatients were enrolled in three trials. For the remaining 12 trials the setting was unclear. Nine trials were conducted in the USA, four in the Netherlands, three in Italy, two in Canada, two in Brazil, two in China, one in UK, one in Finland, one in Japan, and one in Israel; seven trials were multinational, and two did not provide information about the country.

\section{Participants}

The proportion of women ranged from $36 \%$ to $92 \%$. Mean age ranged from 30.5 to 61.6 years.

\section{Interventions}

Eighteen studies included two arms, while the remaining studies had three or more arms. Nine trials included a comparison between antidepressants and benzodiazepines, 22 between 
individual antidepressants (including four comparisons between antidepressants of the same class), and four trials between individual benzodiazepines. Fifteen studies had a placebo arm.

Duration of the intervention ranged from three to 24 weeks.

\section{Outcomes}

Seventeen studies reported data on response rates, while the number of dropout for any reason was reported in 28 studies. Fourteen studies reported on remission rates. Sixteen studies reported data on panic symptoms (using Panic and Anticipatory Anxiety Scale (PAAS), Panic Disorder Severity Scale (PDSS), Clinical Global Impression Severity of Illness Score (CGI-S)), 15 on frequency of panic attacks, 10 on agoraphobia (using Fear Questionnaire (FQ) and Marks-Sheehan Phobia Scale), 26 on general anxiety (using Hamilton Anxiety Rating Scale (HAS) and Hamilton Rating Scale for Anxiety (HAMA)), 17 on depression (using Hamilton Rating Scale for Depression (HRSD), Beck Depression Inventory (BDI), MontgomeryÅsberg Depression Rating Scale (MADRS)). Slx studies reported data on social functioning (using Sheehan Disability Scale (SDS)), three on quality of life. None of the studies reported on patient satisfaction and economic costs. Twenty-one studies had data on dropouts due to adverse effects, and 11 on the number of patients experiencing at least one adverse effect.

\section{Excluded studies}

See: Characteristics of excluded studies.
Sixty-seven articles, initially selected (corresponding to 43 studies), did not meet our inclusion criteria and were excluded, for the following reasons: 19 had a wrong study design; four trials included patients who were not primarily diagnosed with panic disorder; five studies included participants with anxiety disorders in general, but the randomisation was not stratified by the presence of panic disorder; 15 studies had a wrong comparison group.

\section{Ongoing studies}

See: Characteristics of ongoing studies.

Our search identified no ongoing studies.

\section{Studies awaiting classification}

See Characteristics of studies awaiting classification.

Only one study (Minutentag 2001) was classified as "awaiting classification".

\section{Risk of bias in included studies}

For details of the 'Risk of bias' judgements for each study, see Characteristics of included studies. Graphical representations of the overall risk of bias in included studies are presented in Figure 2 and Figure 3. 
Figure 2. 'Risk of bias' summary: review authors' judgements about each risk of bias item for each included study.

\begin{tabular}{|c|c|c|c|c|c|c|c|}
\hline & 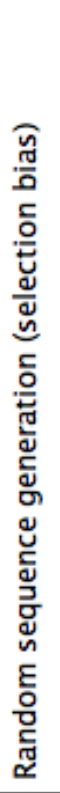 & 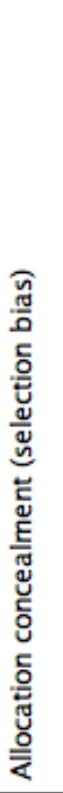 & 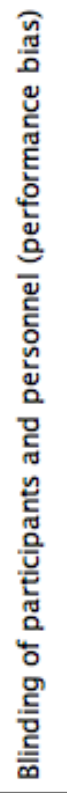 & 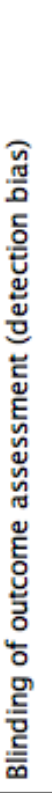 & 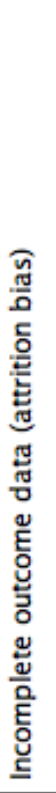 & 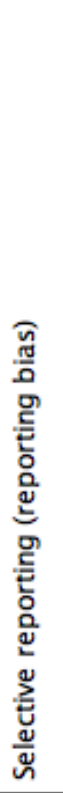 & 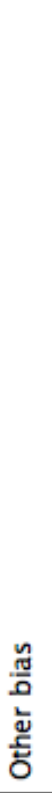 \\
\hline Amore 1999 & $?$ & $?$ & $?$ & $?$ & $?$ & - & $?$ \\
\hline Amore 1999 bis & $?$ & $?$ & $?$ & $?$ & $?$ & $\odot$ & $?$ \\
\hline Bakish 1993 & $?$ & $?$ & $?$ & $?$ & $?$ & - & $?$ \\
\hline Bandelow 2004 & $?$ & $?$ & $?$ & $?$ & 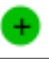 & + & $\odot$ \\
\hline Bystritsky 1995 & $?$ & $?$ & $\odot$ & + & $?$ & $\odot$ & $?$ \\
\hline CNCPS 1992 & $?$ & $?$ & $?$ & ? & $?$ & $\odot$ & - \\
\hline Den Boer 1988 & $?$ & $?$ & $?$ & ? & - & $\odot$ & $?$ \\
\hline Den Boer 1990 & $?$ & $?$ & $?$ & ? & $?$ & $\odot$ & $?$ \\
\hline Garvey 1989 & $?$ & $?$ & $?$ & $?$ & $?$ & $?$ & $?$ \\
\hline Gentil 1993 & $?$ & $?$ & $\odot$ & $\odot$ & $?$ & $\odot$ & 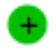 \\
\hline CSK-29060/1 & $?$ & $?$ & $?$ & ? & 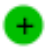 & 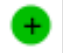 & - \\
\hline GSK-29060/525 & $?$ & $?$ & $?$ & $?$ & $?$ & 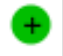 & - \\
\hline Holland 1999 & $?$ & $?$ & $?$ & $?$ & $\odot$ & $?$ & $?$ \\
\hline Krueger 1999 & $?$ & $?$ & $?$ & $?$ & 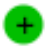 & $?$ & - \\
\hline Lecrubier 1997 & $?$ & $?$ & $?$ & ? & $?$ & $?$ & P \\
\hline Lepola 1990 & $?$ & $?$ & $?$ & ? & $?$ & 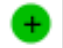 & $?$ \\
\hline Meco 1989 & $?$ & $?$ & $?$ & ? & $?$ & $\odot$ & $?$ \\
\hline \multirow[t]{2}{*}{ Nair 1996} & $?$ & $?$ & $\odot$ & $\odot$ & 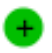 & 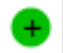 & \\
\hline & $?$ & $?$ & $?$ & 3 & 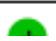 & ? & \\
\hline
\end{tabular}


Figure 2. (Continued)

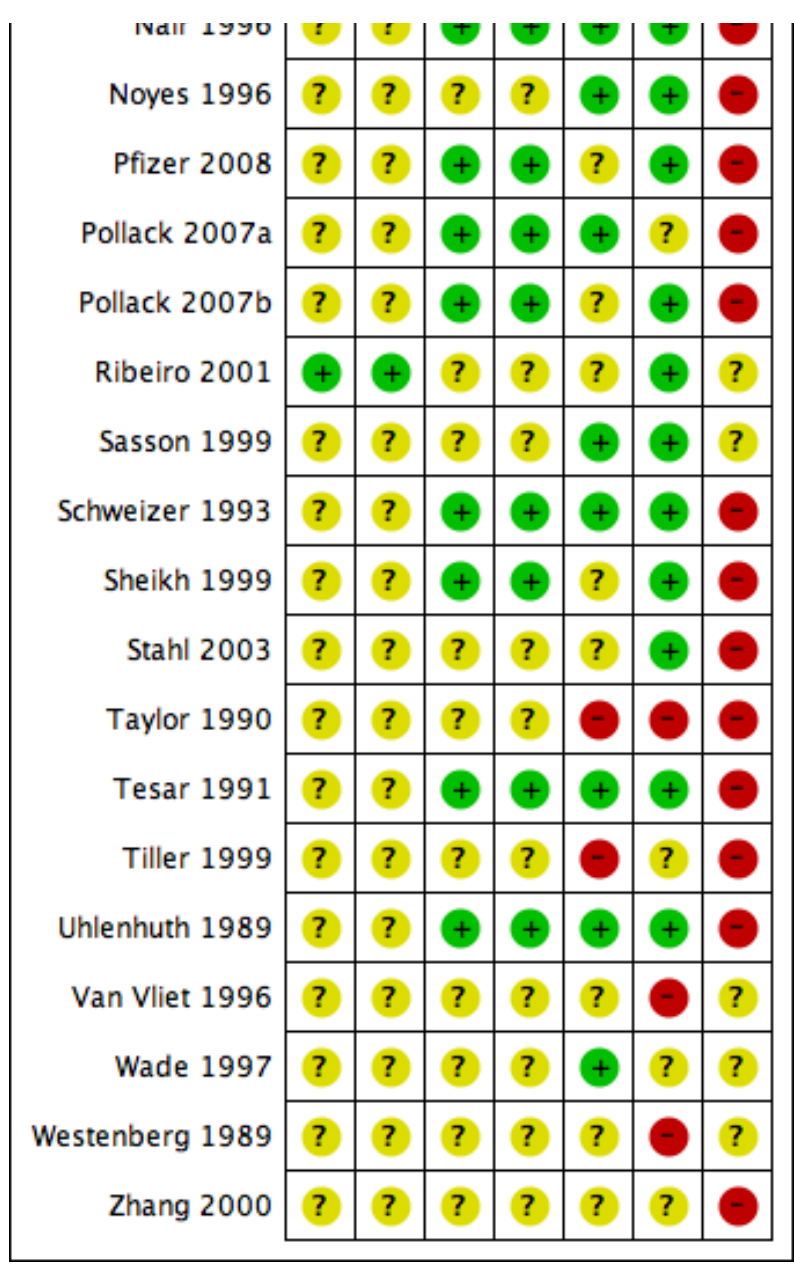

Figure 3. 'Risk of bias' graph: review authors' judgements about each risk of bias item presented as percentages across all included studies.

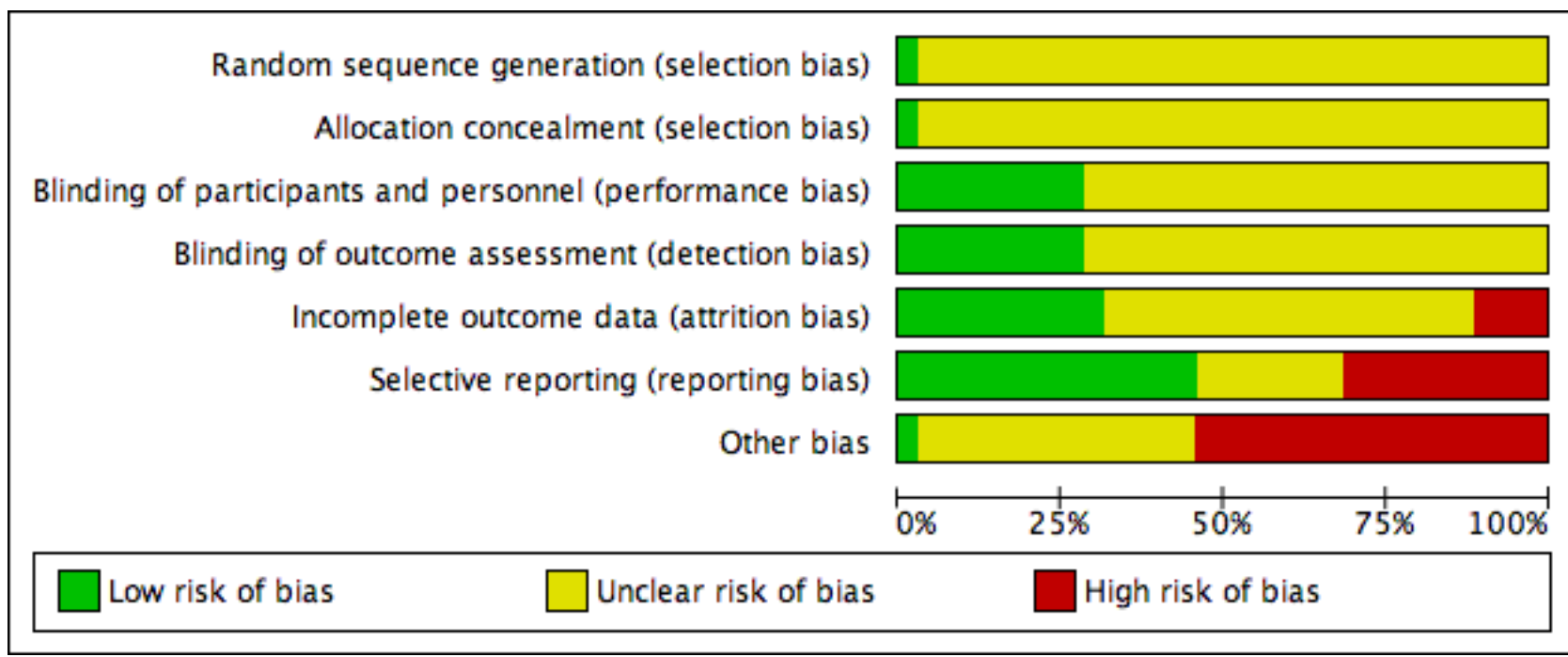

\section{Allocation}

\section{Random sequence generation}

Antidepressants and benzodiazepines for panic disorder in adults (Review) 
Thirty-four of the studies did not report the methods of random sequence generation; only one study (Ribeiro 2001) specified this information, and it was classified as "low risk".

\section{Allocation concealment}

Only one study (Ribeiro 2001) reported details on allocation concealment and was classified as "low risk".

\section{Blinding}

Twenty-five RCTs were reported to be double-blind, without providing any further detail; $10 \mathrm{RCTs}$ reported details on strategies to ensure blinding of participants and key study personnel, and were classified as "low risk".

\section{Incomplete outcome data}

Eleven trials were rated as adequate in terms of addressing incomplete outcome data, while 20 studies were classified as "unclear risk" and four as "high risk".

\section{Selective reporting}

The study protocol was not available for almost all studies so it is difficult to make a judgment on the possibility of outcome reporting bias. However, in 16 studies results were consistent with the outcomes pre-specified in the methods section, and they were evaluated as "low risk"; using the same criterion, 11 studies were judged to be at "high risk".

\section{Other potential sources of bias}

Nineteen of the included studies were funded by the pharmaceutical industry, and they did not report details on the role of the funder in planning, conducting and writing the study; for this reason they were rated as "high risk". Fifteen did not specify the source of funding. One study (Gentil 1993) was classified as "low risk".

\section{Effects of interventions}

See: Summary of findings for the main comparison Antidepressants compared to benzodiazepines for adults with panic disorder; Summary of findings 2 TCAs compared to benzodiazepines for adults with panic disorder; Summary of findings 3 SSRIs compared to benzodiazepines for adults with panic disorder; Summary of findings 4 TCAs compared to SSRI for adults with panic disorder; Summary of findings 5 TCA compared to MAOI for adults with panic disorder; Summary of findings 6 SSRIs compared to MAOIs for adults with panic disorder; Summary of findings 7 SSRIs compared to SNRIs for adults with panic disorder; Summary of findings 8 SSRIs compared to NaSSAs for adults with panic disorder

\section{A: Antidepressants versus benzodiazepines}

We found data only for the comparisons reported below.

\section{Comparison 1: Antidepressants versus benzodiazepines}

Eight studies including 2055 participants (CNCPS 1992; GSK-29060/1; Holland 1999; Lepola 1990; Schweizer 1993; Sheikh 1999; Taylor 1990; Uhlenhuth 1989) provided data for at least one outcome for this comparison. See also: Summary of findings for the main comparison; Summary of findings 2; Summary of findings 3.

\section{Primary outcomes}

\subsection{Failure to respond}

We found low-quality evidence that cannot inform us as to whether there is a difference between antidepressants and benzodiazepines in terms of response rate. Confidence interval ranges from appreciable benefit with antidepressants to appreciable benefit with benzodiazepines (risk ratio (RR) $0.99,95 \%$ confidence interval (CI) 0.67 to 1.47 ; participants $=215$; studies $=2 ; 1^{2}=0 \%$ ) (Analysis 1.1). There was consistency between study results. Risk of bias was considered unclear for this outcome. Additionally, the comparisons of tricyclic antidepressants (TCAs) versus benzodiazepines (RR $1.02,95 \% \mathrm{Cl} 0.63$ to 1.67 ; participants $=61$; studies $=1$; very low-quality evidence) and selective serotonin reuptake inhibitors (SSRIs) versus benzodiazepines (RR $0.93,95 \% \mathrm{Cl} 0.48$ to 1.80 ; participants $=154$; studies $=1$; very low-quality evidence) did not show any difference (Figure 4 ). 
Figure 4. Forest plot of comparison: 1 Antidepressants versus benzodiazepines, outcome: 1.1 Failure to respond.

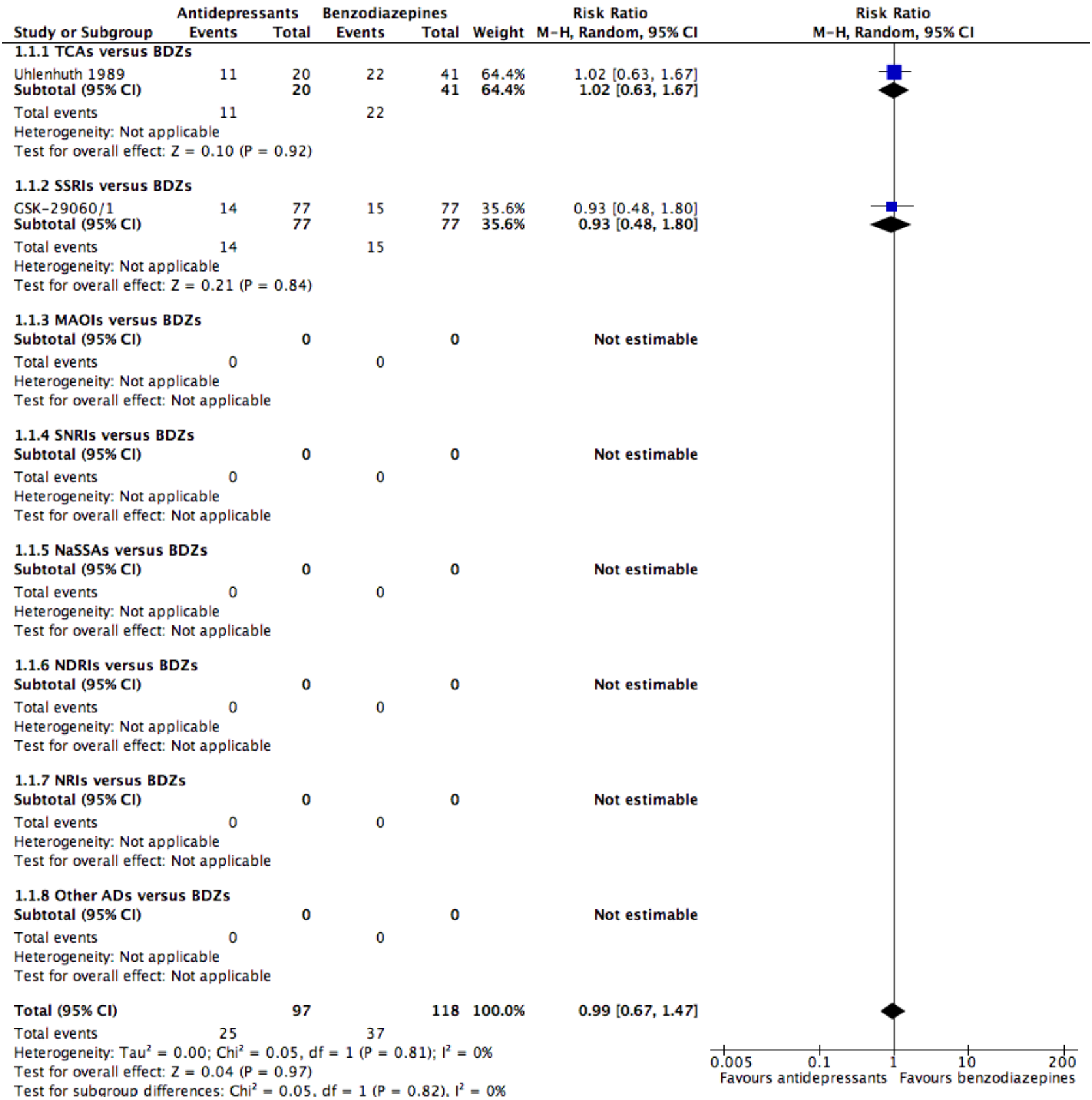

\subsection{Total number of dropouts}

In terms of participants who dropped out due to any cause, very low-quality evidence suggested a benefit of benzodiazepines compared to antidepressants. However, the wide confidence interval ranges from almost no difference to benefit with benzodiazepines (RR 1.64, 95\% Cl 1.03 to 2.63; participants $=1449$; studies $=7$ ), and the degree of heterogeneity in the studies considered was considerable $\left(I^{2}=75 \%\right.$ ) (Analysis 1.2$)$. In comparison with benzodiazepines, more participants receiving the

SSRIs dropped out due to any cause (RR $1.71,95 \% \mathrm{Cl} 1.03$ to 2.84; participants $=154$; studies $=1$; very low-quality evidence) (the magnitude of effect corresponds to a NNTH of $7,95 \% \mathrm{Cl}$ 152 to 3), while no difference was detected between TCAs and benzodiazepines ( $\mathrm{RR} 1.67,95 \% \mathrm{Cl} 0.93$ to 2.99; participants = 1295; studies $=6$; very low-quality evidence), with a considerable heterogeneity $\left(I^{2}=79 \%\right)$, (Analysis 1.2$)$. Risk of bias was considered unclear for this outcome, but the majority of the studies were rated as high risk for "other bias" because they were funded by pharmaceutical companies (Figure 5). 
Figure 5. Forest plot of comparison: 1 Antidepressants versus benzodiazepines, outcome: 1.2 Total number of dropouts.

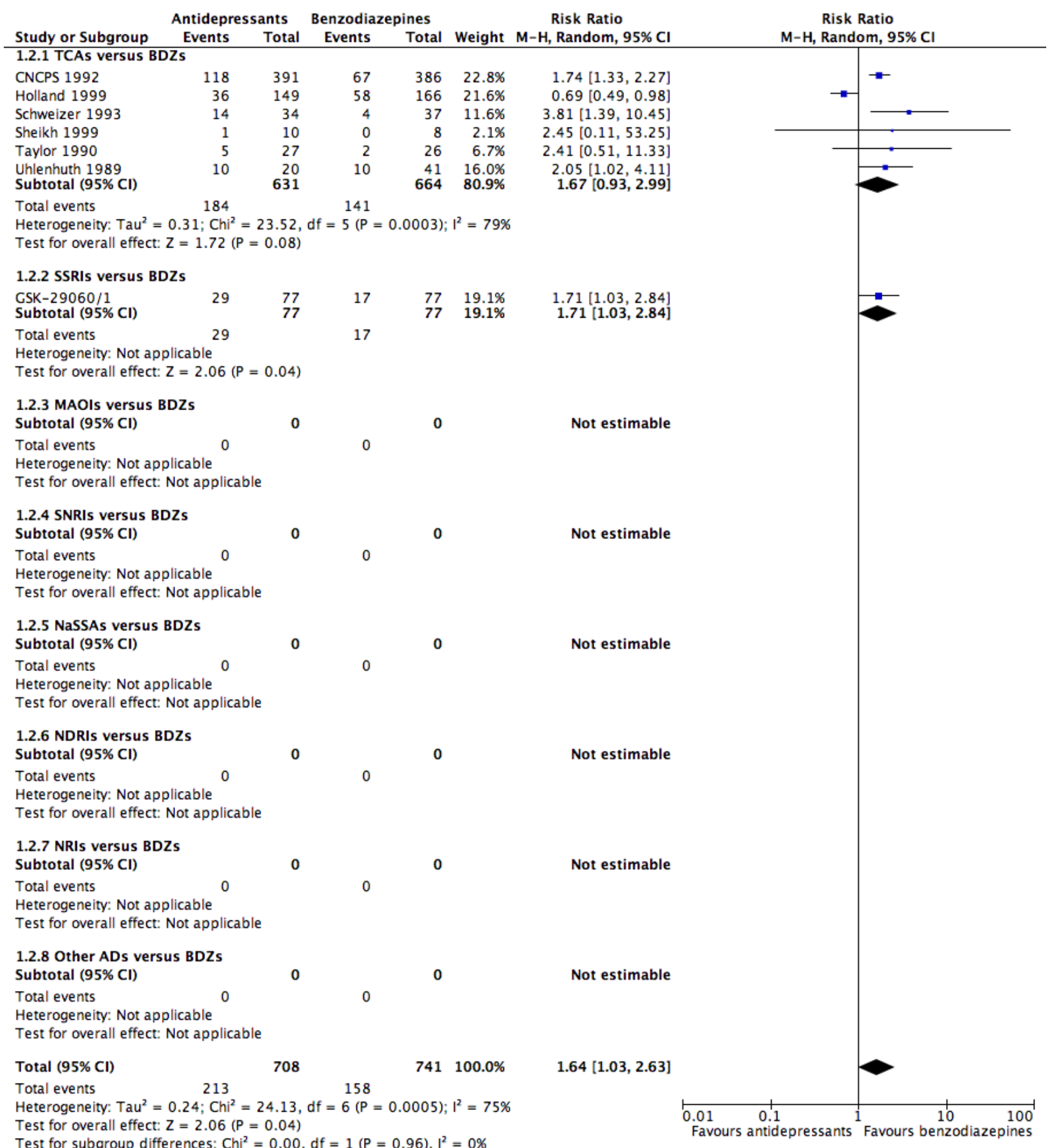

\section{Secondary outcomes}

\subsection{Failure to remit}

We found moderate-quality evidence suggesting no difference in terms of remission rate between antidepressants and benzodiazepines (RR 1.18, 95\% Cl 1.01 to 1.37; participants = 1002; studies $=3$ ), TCAs and benzodiazepines (RR $1.19,95 \% \mathrm{Cl} 1.00$ to 1.41 ; participants $=848$; studies $=2$ moderate-quality evidence) and SSRIs versus benzodiazepines (RR $1.12,95 \% \mathrm{Cl} 0.79$ to 1.59 ;

participants $=154 ;$ studies $=1 ; I^{2}=0 \%$, very low-quality evidence) (Analysis 1.3). Risk of bias was considered unclear for this outcome, but the majority of the studies were rated as high risk for "other bias" because they were funded by pharmaceutical companies.

\subsection{Panic symptoms - endpoint score}

We found very low-quality evidence suggesting no difference between antidepressants and benzodiazepines (mean difference (MD) $-0.13,95 \% \mathrm{Cl}-1.06$ to 0.80 ; participants = 1144 ; studies $=3$ ), 
with a substantial degree of heterogeneity $\left(I^{2}=95 \%\right)$, no difference between TCAs and benzodiazepines (mean difference (MD) -0.25 , $95 \% \mathrm{Cl}-1.72$ to 1.22 ; participants $=998$; studies $=2 ; 1^{2}=97 \%$, low-quality evidence) and between SSRIs and benzodiazepines (mean difference (MD) $0.10,95 \% \mathrm{Cl}-0.34$ to 0.54 ; participants $=$ 146; studies $=1$, low-quality evidence) (Analysis 1.4). Risk of bias was considered between unclear and high for this outcome, with two studies rated as high risk for "other bias" because they were funded by pharmaceutical companies. For one study included in the analysis there was some indication of skewness (Holland 1999).

\subsection{Panic symptoms - mean change}

We found low-quality evidence showing a benefit for benzodiazepines over placebo in reducing panic symptoms measured as change from baseline on a continuous measure (MD $0.40,95 \% \mathrm{Cl}-0.83$ to 1.63 ; participants $=44$; studies $=1$ ) (Analysis 1.5). The only study contributing to this analysis was about TCAs.

\subsection{Frequency of panic attacks}

We did not find any difference between antidepressants and benzodiazepines (MD 0.33, $95 \% \mathrm{Cl}-0.72$ to 1.38 ; participants $=595$ studies $=7)$, with a substantial heterogeneity $\left(I^{2}=65 \%\right)$, TCAs and benzodiazepines (MD 0.30, 95\% $\mathrm{Cl}-0.81$ to 1.41 ; participants $=458$; studies $=6)$, also with substantial heterogeneity $\left(I^{2}=71 \%\right)$, SSRIs and benzodiazepines (MD 1.20, 95\% Cl -3.31 to 5.71; participants $=137$; studies $=1$ ) in terms of frequency of panic attacks (Analysis 1.6). Risk of bias was considered unclear for this outcome, but the majority of the studies were rated as high risk for "other bias" because they were funded by pharmaceutical companies. For some studies included in the analysis there was some indication of skewness (Holland 1999; Lepola 1990; Schweizer 1993; Sheikh 1999; Uhlenhuth 1989).

\subsection{Agoraphobia}

For this outcome, we observed no difference between antidepressants and benzodiazepines (SMD $0.04,95 \% \mathrm{Cl}-0.08$ to 0.16 ; participants $=1061$; studies $=5 ;\left.\right|^{2}=0 \%$ ) (Analysis 1.7$)$. Risk of bias was considered unclear for this outcome, but all the studies were rated as high risk for "other bias" because they were funded by pharmaceutical companies.

\subsection{General anxiety}

We found no difference between antidepressants and benzodiazepines (SMD $0.06,95 \% \mathrm{Cl}-0.25$ to 0.37 ; participants = 1365; studies = 8), TCAs and benzodiazepines (SMD 0.08, 95\% $\mathrm{Cl}-0.28$ to 0.45 ; participants $=1238$; studies $=7)$, SSRIs and benzodiazepines $(\mathrm{SMD}-0.08,95 \% \mathrm{Cl}-0.42$ to 0.27 ; participants = 127; studies $=1$ ) on general anxiety (Analysis 1.8). Heterogeneity for antidepressants compared to benzodiazepines and TCAs compared to benzodiazepines was considerable $\left(\mathrm{I}^{2}=81 \%\right.$ and $\mathrm{I}^{2}$ $=83 \%)$. Risk of bias was considered unclear for this outcome, but the majority of the studies were rated as high risk for "other bias" because they were funded by pharmaceutical companies. For some studies included in the analysis, there was some indication of skewness (CNCPS 1992; GSK-29060/1; Holland 1999; Lepola 1990; Schweizer 1993; Uhlenhuth 1989).

\subsection{Depression}

The analyses did not show a difference in favour of antidepressants over benzodiazepines (SMD $-0.14,95 \% \mathrm{Cl}-0.29$ to 0.02 ; participants
$=892 ;$ studies $\left.=6 ; 1^{2}=7 \%\right)$. The SSRIs were more effective than benzodiazepines $(\mathrm{SMD}-0.46,95 \% \mathrm{Cl}-0.81$ to -0.11 ; participants $=$ 127 ; studies $=1$ ) in terms of depressive measures, while the TCAs were not different from benzodiazepines (SMD $-0.08,95 \% \mathrm{Cl}-0.22$ to 0.06 ; participants $=765$; studies $=5 ; I^{2}=0 \%$ ) (Analysis 1.9). Risk of bias was considered unclear for this outcome, but the majority of the studies were rated as high risk for "other bias" because they were funded by pharmaceutical companies. For some studies included in the analysis there was some indication of skewness (CNCPS 1992; GSK-29060/1; Lepola 1990; Sheikh 1999; Uhlenhuth 1989). This analysis found a substantial subgroup difference $\left(I^{2}=\right.$ $73.7 \%, \mathrm{P}=0.05$ ) between TCAs and SSRIs.

\subsection{Social functioning}

We found no difference between antidepressants and benzodiazepines in this outcome, with a moderate heterogeneity $\left(I^{2}=57 \%\right)($ SMD $0.16,95 \% \mathrm{Cl}-0.12$ to 0.44 ; participants = 1007 ; studies $=4)$. In terms of drug classes, benzodiazepines were more effective than TCAs (SMD $0.23,95 \% \mathrm{Cl} 0.03$ to 0.42; participants $=882 ;$ studies $=3 ; 1^{2}=14 \%$ ), while SSRIs were not different from benzodiazepines (SMD $-0.22,95 \% \mathrm{Cl}-0.57$ to 0.13 ; participants $=125$; studies $=1$ ) (Analysis 1.10). Risk of bias was considered unclear for this outcome, but all the studies were rated as high risk for "other bias" because they were funded by pharmaceutical companies. This analysis found a substantial subgroup difference $\left(I^{2}=73.4 \%, P=0.05\right)$ between TCAs and SSRIs.

\subsection{Quality of life}

No studies provided data for this outcome.

\subsection{Patient satisfaction}

No studies provided data for this outcome.

\subsection{Economic costs}

No studies provided data for this outcome.

\subsection{Number of dropouts due to adverse effects}

In terms of dropouts due to adverse effects, we found lowquality evidence that benzodiazepines were better tolerated than antidepressants as a whole ( $\mathrm{RR} 1.72,95 \% \mathrm{Cl} 1.03$ to 2.87 ; participants $=1002$; studies $\left.=3 ; I^{2}=13 \%\right)$. The magnitude of effect corresponds to a NNTH of 27 ( $95 \% \mathrm{Cl} 642$ to 11). In addition, lowquality evidence suggested that the TCAs were better tolerated than benzodiazepines (RR 2.10, 95\% Cl 1.13 to 3.93; participants $=848$; studies $=2 ; \mathrm{I}^{2}=8 \%$ ), with a magnitude of effect of NNTH $=24(95 \% \mathrm{Cl} 203$ to 9), while the SSRIs were similarly tolerated in comparison with benzodiazepines (RR $1.20,95 \% \mathrm{Cl} 0.55$ to 2.61 ; participants $=154$; studies $=1 ; I^{2}=0 \%$, very low-quality evidence) (Analysis 1.14). Risk of bias was considered unclear for this outcome, but all the studies were rated as high risk for "other bias" because they were funded by pharmaceutical companies.

\subsection{Number of patients experiencing at least one adverse effect}

Only one study provided data for this outcome, showing no difference between antidepressants (SSRIs) and benzodiazepines (RR $1.03,95 \% \mathrm{Cl} 0.92$ to 1.15 ; participants = 154; studies = 1) (Analysis 1.15). Risk of bias was considered unclear for this outcome, but the study was rated as high risk for "other bias" because it was funded by a pharmaceutical company. 


\section{Comparison 2: TCAs versus benzodiazepines}

Seven studies including 1829 participants provided data for this comparison. Six studies compared imipramine with alprazolam (CNCPS 1992; Lepola 1990; Schweizer 1993; Sheikh 1999; Taylor 1990; Uhlenhuth 1989), and one study compared clomipramine with adinazolam (Holland 1999). See also: Summary of findings 2.

\section{Primary outcomes}

\subsection{Failure to respond}

The comparison between imipramine and benzodiazepines did not show a difference in terms of response rate (RR 1.02, $95 \% \mathrm{Cl} 0.63$ to 1.67; participants $=61$; studies $=1$ ) (Analysis 2.1). Risk of bias was considered unclear for this outcome, but the study was rated as high risk for "other bias" because it was funded by a pharmaceutical company.

\subsection{Total number of dropouts}

Imipramine was less well tolerated than benzodiazepines (RR 1.87 , $95 \% \mathrm{Cl} 1.47$ to 2.37 ; participants $=980$; studies $=5 ; \mathrm{I}^{2}=0 \%$ ) (NNTH $=7,95 \% \mathrm{Cl} 13$ to 5), while there was a difference favouring clomipramine over benzodiazepines for this outcome (RR 0.69, 95\% $\mathrm{Cl} 0.49$ to 0.98 ; participants $=315$; studies $\left.=1 ; I^{2}=0 \%\right)(\mathrm{NNTH}=$ $10,95 \% \mathrm{Cl} 6$ to 143) (Analysis 2.2). Risk of bias was considered unclear for this outcome, but the majority of the studies were rated as high risk for "other bias" because of funding by pharmaceutical companies.

\section{Secondary outcomes}

\subsection{Failure to remit}

The analysis failed to detect a difference between imipramine and benzodiazepines on remission rates (RR $1.19,95 \% \mathrm{Cl} 1.00$ to 1.41 ; participants $=848$; studies $=2 ; 1^{2}=0 \%$ ). Risk of bias was considered unclear for this outcome, but the studies were rated as high risk for "other bias" because they were funded by pharmaceutical companies.

\subsection{Panic symptoms - endpoint score}

One study showed a benefit for benzodiazepines over imipramine in reducing panic symptoms measured as endpoint scores (MD 0.50 $95 \% \mathrm{Cl} 0.21$ to 0.79 ; participants $=777$ ), whereas one study showed a benefit for clomipramine over benzodiazepines (MD -1.00, 95\% $\mathrm{Cl}-1.37$ to -0.63 ; participants $=221$ ) (Analysis 2.4). Risk of bias was considered unclear for this outcome.

\subsection{Panic symptoms - mean change}

Measuring mean change in panic symptoms, we found no difference between imipramine and benzodiazepines (MD 0.40, $95 \% \mathrm{Cl}-0.83$ to 1.63; participants $=44$; studies $=1$ ) (Analysis 2.5) . Risk of bias was considered high for this outcome, and the study was rated as high risk for "other bias" because of funding from a pharmaceutical company.

\subsection{Frequency of panic attacks}

We found no difference in this outcome for the comparisons between imipramine and benzodiazepines, with a substantial heterogeneity $\left(I^{2}=67 \%\right)(M D 0.75,95 \% \mathrm{Cl}-0.44$ to 1.94 ; participants $=237$; studies $=5$ ), while clomipramine was more effective than benzodiazepines for this outcome (MD $-1.60,95 \% \mathrm{Cl}-3.17$ to -0.03 ; participants $=221$; studies $=1$ ) (Analysis 2.6). Risk of bias was considered unclear for this outcome, but the majority of the studies were rated as high risk for "other bias" because of funding by pharmaceutical companies.

\subsection{Agoraphobia}

In terms of agoraphobia, we found no difference between imipramine and benzodiazepines (SMD $0.04,95 \% \mathrm{Cl}-0.08$ to 0.17 ; participants $=949$; studies $=4 ; I^{2}=0 \%$ ) (Analysis 2.7). Risk of bias was considered unclear for this outcome, but all the studies were rated as high risk for "other bias" because they were funded by pharmaceutical companies.

\subsection{General anxiety}

For this outcome, we found a difference in favour of benzodiazepines over imipramine (SMD $0.22,95 \% \mathrm{Cl} 0.10$ to 0.35 ; participants $=1017$; studies $\left.=6 ; I^{2}=0 \%\right)$, and in favour of clomipramine over benzodiazepines (SMD $-0.67,95 \% \mathrm{Cl}-0.94$ to -0.40 ; participants $=221$; studies $=1$ ) (Analysis 2.8). Risk of bias was considered unclear for this outcome, but the majority of the studies were rated as high risk for "other bias" because they were funded by pharmaceutical companies.

\subsection{Depression}

We did not find any difference between imipramine and benzodiazepines in this outcome (SMD $-0.08,95 \% \mathrm{Cl}-0.22$ to 0.06 ; participants $=765$; studies $=5 ; I^{2}=0 \%$ ) (Analysis 2.9). Risk of bias was considered unclear for this outcome, but the majority of the studies were rated as high risk for "other bias" because they were funded by pharmaceutical companies.

\subsection{Social functioning}

We found a difference in favour of benzodiazepines over imipramine for this outcome (MD $0.25,95 \% \mathrm{Cl} 0.09$ to 0.42 ; participants $=882 ;$ studies $=3 ;\left.\right|^{2}=0 \%$ ) (Analysis 2.10). Risk of bias was considered between unclear and high for this outcome, and the studies were rated as high risk for "other bias" because they were funded by pharmaceutical companies.

\subsection{Quality of life}

No studies provided data for this outcome.

\subsection{Patient satisfaction}

No studies provided data for this outcome.

\subsection{Economic costs}

No studies provided data for this outcome.

\subsection{Number of dropouts due to adverse effects}

We found that benzodiazepines were better tolerated than imipramine (RR 2.10, $95 \% \mathrm{Cl} 1.13$ to 3.93; participants $=848$; studies $=2 ; I^{2}=8 \%$ ) (Analysis 2.14). Risk of bias was considered unclear for this outcome, but all the studies were rated as high risk for "other bias" because they were funded by pharmaceutical companies.

\subsection{Number of patients experiencing at least one adverse effect}

No studies provided data for this outcome. 


\section{Comparison 3: SSRIs versus benzodiazepines}

One study including 226 participants (GSK-29060/1) provided data for this comparison. The trial compared paroxetine versus alprazolam. See also: Summary of findings 3.

\section{Primary outcomes}

\subsection{Failure to respond}

This comparison did not show any difference in terms of response rate (RR $0.93,95 \% \mathrm{Cl} 0.48$ to 1.80 ; participants $=154$; studies $=1$ ) (Analysis 3.1). Risk of bias was considered unclear for this outcome, but the study was rated as high risk for "other bias" because it was funded by a pharmaceutical company.

\subsection{Total number of dropouts}

There was evidence that paroxetine is less well tolerated than benzodiazepines (RR 1.71, $95 \% \mathrm{Cl} 1.03$ to 2.84; participants = 154; studies $=1$ ) (Analysis 3.2). Risk of bias was considered unclear for this outcome, but the study was rated as high risk for "other bias" because it was funded by a pharmaceutical company.

\section{Secondary outcomes}

\subsection{Failure to remit}

We did not find any difference in terms of remission rate between paroxetine and benzodiazepines (RR $1.12,95 \% \mathrm{Cl} 0.79$ to 1.59 ; participants $=154$; studies $=1$ ) (Analysis 3.3). Risk of bias was considered unclear for this outcome, but the study was rated as high risk for "other bias" because it was funded by a pharmaceutical company.

\subsection{Panic symptoms}

We did not find any difference in terms of panic symptoms between paroxetine and benzodiazepines (MD $0.10,95 \% \mathrm{Cl}-0.34$ to 0.54 ; participants $=146$; studies $=1$ ) (Analysis 3.4). Risk of bias was considered unclear for this outcome, but the study was rated as high risk for "other bias" because it was funded by a pharmaceutical company.

\subsection{Frequency of panic attacks}

We did not find any difference in this outcome between paroxetine and benzodiazepines (MD 1.20, 95\% Cl -3.31 to 5.71; participants = 137; studies $=1$ ) (Analysis 3.5). Risk of bias was considered unclear for this outcome, but the study was rated as high risk for "other bias" because it was funded by a pharmaceutical company.

\subsection{Agoraphobia}

We did not find any difference in this outcome between paroxetine and benzodiazepines $(\mathrm{SMD} 0.00,95 \% \mathrm{Cl}-0.37$ to 0.37 ; participants = 112; studies $=1$ ) (Analysis 3.6). Risk of bias was considered unclear for this outcome, but the study was rated as high risk for "other bias" because it was funded by a pharmaceutical company.

\subsection{General anxiety}

We did not find any difference in terms of general anxiety between paroxetine and benzodiazepines (MD $-0.60,95 \% \mathrm{Cl}-3.35$ to 2.15; participants $=127$; studies $=1$ ) (Analysis 3.7). Risk of bias was considered unclear for this outcome, but the study was rated as high risk for "other bias" because it was funded by a pharmaceutical company.

\subsection{Depression}

We found a difference favouring paroxetine over benzodiazepines in terms of depression (MD $-3.60,95 \% \mathrm{Cl}-6.36$ to -0.84 ; participants $=127$; studies $=1$ ) (Analysis 3.8). Risk of bias was considered unclear for this outcome, but the study was rated as high risk for "other bias" because it was funded by a pharmaceutical company.

\subsection{Social functioning}

We did not find any difference in this outcome between paroxetine and benzodiazepines (MD -3.40, $95 \% \mathrm{Cl}-8.81$ to 2.01; participants = 125 ; studies $=1$ ) (Analysis 3.9). Risk of bias was considered unclear for this outcome, but the study was rated as high risk for "other bias" because it was funded by a pharmaceutical company.

\subsection{Quality of life}

The study did not provide data for this outcome.

\subsection{Patient satisfaction}

The study did not provide data for this outcome.

\subsection{Economic costs}

The study did not provide data for this outcome.

\subsection{Number of dropouts due to adverse effects}

We did not find any difference in terms of dropouts due to adverse effects between paroxetine and benzodiazepines (RR $1.20,95 \% \mathrm{CI}$ 0.55 to 2.61; participants $=154$; studies $=1$ ) (Analysis 3.13). Risk of bias was considered unclear for this outcome, but the study was rated as high risk for "other bias" because it was funded by a pharmaceutical company.

\subsection{Number of patients experiencing at least one adverse effect}

We did not find any difference in this outcome between paroxetine and benzodiazepines (RR 1.03, 95\% Cl 0.92 to 1.15; participants = 154; studies $=1$ ) (Analysis 3.14). Risk of bias was considered unclear for this outcome, but the study was rated as high risk for "other bias" because it was funded by a pharmaceutical company.

\section{B: Antidepressants versus antidepressants}

We found data only for the comparisons reported below.

\section{Comparison 4: TCAs versus SSRIs}

Nine studies including 1290 participants (Amore 1999; Bystritsky 1995; Den Boer 1988; GSK-29060/525; Lecrubier 1997; Nair 1996; Wade 1997; Westenberg 1989; Zhang 2000) provided data for this comparison. See also: Summary of findings 4.

\section{Primary outcomes}

\subsection{Failure to respond}

There was low-quality evidence suggesting no difference between TCAs and SSRIs on response rate, with moderate heterogeneity $\left(I^{2}\right.$ $=61 \%)(\mathrm{RR} 1.08,95 \% \mathrm{Cl} 0.71$ to $1.66 ;$ participants $=438 ;$ studies $=4)$ (Analysis 4.1). Risk of bias was considered unclear for this outcome, but the majority of studies were rated as high risk for "other bias" because they were funded by pharmaceutical companies.

\subsection{Total number of dropouts}

We found low-quality evidence suggesting no difference in terms of dropouts between TCAs and SSRIs, with moderate heterogeneity $\left(I^{2}\right.$ 
$=47 \%)(\mathrm{RR} 0.98,95 \% \mathrm{Cl} 0.65$ to 1.48; participants $=928$; studies $=7)$ (Analysis 4.2). Risk of bias was considered unclear for this outcome, but the majority of studies were rated as high risk for "other bias" because they were funded by pharmaceutical companies.

\section{Secondary outcomes}

\subsection{Failure to remit}

We found moderate-quality evidence suggesting no difference in terms of remission rate between TCAs and SSRIs, with moderate heterogeneity $\left(I^{2}=58 \%\right)(\mathrm{RR} 0.89,95 \% \mathrm{Cl} 0.63$ to 1.26 ; participants $=$ 475; studies $=5$ ) (Analysis 4.3). Risk of bias was considered unclear for this outcome, but many studies were rated as high risk for "other bias" because of funding by pharmaceutical companies.

\subsection{Panic symptoms}

There was very low-quality evidence suggesting no difference between TCAs and SSRIs on this outcome, with moderate heterogeneity $\left(I^{2}=50 \%\right)(\mathrm{MD}-0.20,95 \% \mathrm{Cl}-0.88$ to 0.48 ; participants $=243$; studies $=4$ ) (Analysis 4.4). Risk of bias was considered unclear for this outcome, but the majority of studies were rated as high risk for "other bias" because they were funded by pharmaceutical companies.

\subsection{Frequency of panic attacks}

No difference was found between SSRIs and TCAs in terms of frequency of panic attacks, with moderate heterogeneity $\left(\mathrm{I}^{2}=66 \%\right)$ (MD 2.30, 95\% Cl -0.11 to 4.71 ; participants $=329$; studies $=3$ ) (Analysis 4.5). Risk of bias was considered between unclear and high for this outcome, and the majority of studies were rated as high risk for "other bias" because of funding by pharmaceutical companies.

\subsection{Agoraphobia}

One study with 226 patients found no difference in this outcome between TCAs and SSRIs (MD $0.10,95 \% \mathrm{Cl}-0.68$ to 0.88 ) (Analysis 4.6). Risk of bias was considered unclear for this outcome, but the study was rated as high risk for "other bias" because it was funded by a pharmaceutical company.

\subsection{General anxiety - endpoint score}

We did not find any difference between TCAs and SSRIs on efficacy measured as endpoint scores (SMD $0.09,95 \% \mathrm{Cl}-0.27$ to 0.45 ; participants $=668$; studies $=7)$. The degree of heterogeneity was substantial $\left(I^{2}=71 \%\right)$ (Analysis 4.7$)$. Risk of bias was considered unclear for this outcome, and some studies were rated as high risk for "other bias" because they were funded by pharmaceutical companies.

\subsection{General anxiety - mean change}

We did not find any difference between TCAs and SSRIs on efficacy measured as mean change scores (MD 0.99, $95 \% \mathrm{Cl}-1.39$ to 3.36; participants $=309$; studies $=2 ; I^{2}=0 \%$ ) (Analysis 4.8). Risk of bias was considered unclear for this outcome, but all the studies were rated as high risk for "other bias" because they were funded by pharmaceutical companies.

\subsection{Depression - endpoint score}

We did not find any difference between TCAs and SSRIs on efficacy measured as endpoint scores (SMD $-0.04,95 \% \mathrm{Cl}-0.26$ to 0.17 ; participants $=597 ;$ studies $\left.=5 ; I^{2}=20 \%\right)($ Analysis 4.9). Risk of bias was considered unclear for this outcome, but some studies were rated as high risk for "other bias" because of funding by pharmaceutical companies.

\subsection{Depression - mean change}

We did not find any difference between TCAs and SSRIs on efficacy measured as mean change scores (SMD $-0.04,95 \% \mathrm{Cl}-0.31$ to 0.23 ; participants $=212$; studies $=1 ;\left.\right|^{2}=0 \%$ (Analysis 4.10). Risk of bias was considered between unclear and high for this outcome, and the study was rated as high risk for "other bias" because it was funded by a pharmaceutical company.

\subsection{Social functioning}

No studies provided data for this outcome.

\subsection{Quality of life}

No studies provided data for this outcome.

\subsection{Patient satisfaction}

No studies provided data for this outcome.

\subsection{Economic costs}

No studies provided data for this outcome.

\subsection{Number of dropouts due to adverse effects}

There was low-quality evidence suggesting no difference between TCAs and SSRIs on dropouts due to adverse effects (RR 1.43, 95\% $\mathrm{Cl} 0.82$ to 2.48; participants $=476$; studies $=5 ; \mathrm{I}^{2}=11 \%$ ) (Analysis 4.15). Risk of bias was considered unclear for this outcome, but the majority of studies were rated as high risk for "other bias" because they were funded by pharmaceutical companies.

\subsection{Number of patients experiencing at least one adverse effect}

Considering tolerability as the number of patients who experienced at least one adverse effect, we found a difference in favour of SSRIs compared to TCAs (RR 1.29, $95 \% \mathrm{Cl} 1.02$ to 1.65; participants = 489; studies $=4)$ (NNTH $=6,95 \% \mathrm{Cl} 84$ to 3), with a substantial degree of heterogeneity between the studies included in the analysis $\left(\mathrm{I}^{2}=\right.$ $78 \%$ ) (Analysis 4.16). Risk of bias was considered unclear for this outcome, but all the studies were rated as high risk for "other bias" because they were funded by pharmaceutical companies.

\section{Comparison 5: TCAs versus MAOIS}

Two studies including 228 participants provided data for this comparison. One compared brofaromine with clomipramine (Bakish 1993), and one compared moclobemide with clomipramine (Krueger 1999). See also: Summary of findings 5.

\section{Primary outcomes}

\subsection{Failure to respond}

The comparison between TCAs and MAOls did not find any difference in response rate ( $\mathrm{RR} 0.59,95 \% \mathrm{Cl} 0.28$ to 1.26; participants $=135$; studies $=1$; low-quality evidence) (Analysis 5.1). Risk of bias was considered unclear for this outcome, but the study was rated as high risk for "other bias" because it was funded by a pharmaceutical company. 


\subsection{Total number of dropouts}

We found moderate-quality evidence suggesting no difference between TCAs and MAOIs in terms of number of dropouts (RR 0.84, $95 \% \mathrm{Cl} 0.61$ to 1.17 ; participants $=228$; studies $=2 ; \mathrm{I}^{2}=0 \%$ ) (Analysis 5.2). Risk of bias was considered between unclear and high for this outcome, with one study rated as high risk for "other bias" because it was funded by a pharmaceutical company.

\section{Secondary outcomes}

\subsection{Failure to remit}

Neither study provided data for this outcome.

\subsection{Panic symptoms}

We found low-quality evidence suggesting no difference between TCAs and MAOIs on this outcome (MD $-0.20,95 \% \mathrm{Cl}-0.75$ to 0.35 ; participants $=135$; studies $=1$ ) (Analysis 5.4). Risk of bias was considered unclear for this outcome, but the study was rated as high risk for "other bias" because it was funded by a pharmaceutical company.

\subsection{Frequency of panic attacks}

We found no difference between TCAs and MAOIs for this outcome $(\mathrm{MD}-0.30,95 \% \mathrm{Cl}-3.35$ to 2.75 ; participants $=135$; studies $=1)$ (Analysis 5.5). Risk of bias was considered unclear for this outcome, but the study was rated as high risk for "other bias" because it was funded by a pharmaceutical company.

\subsection{Agoraphobia}

Neither study provided data for this outcome.

\subsection{General anxiety}

The comparison between TCAs and MAOIs did not find any difference in general anxiety (MD $0.30,95 \% \mathrm{Cl}-3.03$ to 3.63 participants $=135$; studies $=1$ ) (Analysis 5.7). Risk of bias was considered unclear for this outcome, but the study was rated as high risk for "other bias" because it was funded by a pharmaceutical company.

\subsection{Depression}

We found a difference in favour of TCAs over MAOIs for depression measured on a continuous scale (MD $-4.10,95 \% \mathrm{Cl}-8.14$ to -0.06 ; participants $=135$; studies $=1$ ) (Analysis 5.8). Risk of bias was considered unclear for this outcome, but the study was rated as high risk for "other bias" because it was funded by a pharmaceutical company.

\subsection{Social functioning}

Neither study provided data for this outcome.

\subsection{Quality of life}

Neither study provided data for this outcome.

\subsection{Patient satisfaction}

Neither study provided data for this outcome.

\subsection{Economic costs}

Neither study provided data for this outcome.

\subsection{Number of dropouts due to adverse effects}

The comparison between TCAs and MAOIs did not show any difference in terms of dropouts due to adverse effects (RR 1.27, $95 \% \mathrm{Cl} 0.50$ to 3.21 ; participants $=135$; studies $=1$; low-quality evidence) (Analysis 5.13). Risk of bias was considered unclear for this outcome, but the study was rated as high risk for "other bias" because it was funded by a pharmaceutical company.

\subsection{Number of patients experiencing at least one adverse effect}

We found a difference in favour of MAOIs over TCAs in tolerability in terms of number of patients who experienced at least one adverse effect (RR 1.24, 95\% Cl 1.06 to 1.45; participants = 135; studies $=1$ ) (Analysis 5.14). Risk of bias was considered unclear for this outcome, but the study was rated as high risk for "other bias" because it was funded by a pharmaceutical company.

\section{Comparison 6: SSRIs versus MAOIs}

Two studies including 396 participants; provided data for this comparison. One study compared moclobemide with fluoxetine (Tiller 1999), and the other compared brofaromine with fluvoxamine (Van Vliet 1996). See also: Summary of findings 6.

\section{Primary outcomes}

\subsection{Failure to respond}

We found moderate-quality evidence suggesting no difference in terms of response rate between SSRIs and MAOIs (RR 1.12, 95\% $\mathrm{Cl} 0.83$ to 1.52; participants $=396$; studies $=2 ; I^{2}=0 \%$ ) (Analysis 6.1). Risk of bias was considered between unclear and high for this outcome, with one study rated as high risk for "other bias" because it was funded by a pharmaceutical company.

\subsection{Total number of dropouts}

We found low-quality evidence suggesting no difference between SSRIs and MAOIs in terms of the number of dropouts (RR 1.00, 95\% $\mathrm{Cl} 0.07$ to 14.55; participants $=30$; studies $=1$ ) (Analysis 6.2). Risk of bias was considered between unclear and high for this outcome.

\section{Secondary outcomes}

\subsection{Failure to remit}

We found moderate-quality evidence suggesting no difference between SSRIs and MAOIs for this outcome (RR 0.93, 95\% Cl 0.74 to 1.17; participants $=366$; studies $=1$ ) $($ Analysis 6.3). Risk of bias was considered between unclear and high for this outcome, as the study was rated as high risk for "other bias" because it was funded by a pharmaceutical company.

\subsection{Panic symptoms}

Neither study provided data for this outcome.

\subsection{Frequency of panic attacks}

Neither study provided data for this outcome.

\subsection{Agoraphobia}

There was no evidence of a difference between SSRIs and MAOIs in this outcome (MD 1.50, 95\% Cl -4.47 to 7.47 ; participants = 30; studies $=1$ ) (Analysis 6.6). Risk of bias was considered between unclear and high for this outcome. 


\subsection{General anxiety}

We found a difference in favour of SSRIs over MAOIs in terms of efficacy on general anxiety (MD $-0.60,95 \% \mathrm{Cl}-1.10$ to -0.10 ; participants $=30$; studies $=1$ ) (Analysis 6.7). Risk of bias was considered between unclear and high for this outcome.

\subsection{Depression}

Neither study provided data for this outcome.

\subsection{Social functioning}

Neither study provided data for this outcome.

\subsection{Quality of life}

Neither study provided data for this outcome.

\subsection{Patient satisfaction}

Neither study provided data for this outcome.

\subsection{Economic costs}

Neither study provided data for this outcome.

\subsection{Number of dropouts due to adverse effects}

The comparison between SSRIs and MAOIs did not find any difference in terms of dropouts due to adverse effects (RR 1.26, 95\% $\mathrm{Cl} 0.59$ to 2.70; participants $=366$; studies $=1$, low-quality evidence) (Analysis 6.13). Risk of bias was considered between unclear and high for this outcome, with the study rated as high risk for "other bias" because it was funded by a pharmaceutical company.

\subsection{Number of patients experiencing at least one adverse effect}

We found no difference between SSRIs and MAOIs for this outcome $(\mathrm{RR} 1.09,95 \% \mathrm{Cl} 0.94$ to 1.27 ; participants $=366$; studies $=1)$ (Analysis 6.14). Risk of bias was considered between unclear and high for this outcome, with the study rated as high risk for "other bias" because it was funded by a pharmaceutical company.

\section{Comparison 7: SSRIs versus SNRIs}

Two studies including 1316 participants (Pollack 2007a; Pollack 2007b) provided data for this comparison. Both studies compared venlafaxine and paroxetine. See also: Summary of findings 7 .

\section{Primary outcomes}

\subsection{Failure to respond}

We found high-quality evidence suggesting no difference between SSRIs and serotonin-norepinephrine reuptake inhibitors (SNRIs) in terms of response rate $(\mathrm{RR} 0.96,95 \% \mathrm{Cl} 0.75$ to 1.23 ; participants $=$ 991; studies $=2 ; I^{2}=1 \%$ ) (Analysis 7.1). Risk of bias was considered unclear for this outcome, but both studies were rated as high risk for "other bias" because they were funded by a pharmaceutical company.

\subsection{Total number of dropouts}

We found low-quality evidence suggesting no difference between SSRIs and SNRIs in terms of total number of dropouts (RR 1.11, $95 \% \mathrm{Cl} 0.74$ to 1.65 ; participants $=991$; studies $=2$ ), with moderate heterogeneity $\left(I^{2}=53 \%\right)$ (Analysis 7.2$)$. Risk of bias was considered unclear for this outcome, but both studies were rated as high risk for "other bias" because they were funded by a pharmaceutical company.

\section{Secondary outcomes}

\subsection{Failure to remit}

We found high-quality evidence suggesting no difference between SSRIs and SNRIs in terms of remission rate (RR 1.05, 95\% Cl 0.91 to 1.21; participants $=991$; studies $=2 ; 1^{2}=42 \%$ ) (Analysis 7.3). Risk of bias was considered unclear for this outcome, but both studies were rated as high risk for "other bias" because they were funded by a pharmaceutical company.

\subsection{Panic symptoms}

We found no difference between SSRIs and SNRIs in terms of panic symptoms (MD $-0.12,95 \% \mathrm{Cl}-0.33$ to 0.10 ; participants = 945; studies $=2 ; I^{2}=0 \%$, high-quality evidence) (Analysis 7.4). Risk of bias was considered unclear for this outcome, but both studies were rated as high risk for "other bias" because they were funded by a pharmaceutical company.

\subsection{Frequency of panic attacks}

Neither study provided data for this outcome.

\subsection{Agoraphobia}

One study showed no difference between SSRIs and SNRIs on agoraphobia (SMD 0.00, 95\% Cl -0.19 to 0.19; participants $=478$; studies $=1$ ) (Analysis 7.6). Risk of bias was considered unclear for this outcome, but the study was rated as high risk for "other bias" because funded by pharmaceutical companies.

\subsection{General anxiety}

No difference was found between SSRIs and SNRIs for this outcome $(\mathrm{MD}-0.25,95 \% \mathrm{Cl}-1.83$ to 1.33 ; participants $=478$; studies $=1)$ (Analysis 7.7). Risk of bias was considered unclear for this outcome, but the study was rated as high risk for "other bias" because it was funded by a pharmaceutical company.

\subsection{Depression}

Neither study provided data for this outcome.

\subsection{Social functioning}

One study showed no difference between SSRIs and SNRIs for this outcome (MD 0.15, $95 \% \mathrm{Cl}-0.71$ to 1.01; participants $=478$; studies $=1$ ) (Analysis 7.9). Risk of bias was considered unclear for this outcome, but the study was rated as high risk for "other bias" because it was funded by a pharmaceutical company.

\subsection{Quality of life}

One study showed no difference between SSRIs and SNRIs for this outcome (MD 0.06, $95 \% \mathrm{Cl}-2.11$ to 2.23; participants $=478$; studies $=1$ ) (Analysis 7.10). Risk of bias was considered unclear for this outcome, but the study was rated as high risk for "other bias" because it was funded by pharmaceutical company.

\subsection{Patient satisfaction}

Neither study provided data for this outcome.

\subsection{Economic costs}

Neither study provided data for this outcome. 


\subsection{Number of dropouts due to adverse effects}

We found low-quality evidence that failed to reveal a difference between SSRIs and SNRIs in this outcome (RR 1.86, 95\% Cl 0.49 to 7.05; participants $=991$; studies $=2 ; 1^{2}=76 \%$ ) (Analysis 7.13). Risk of bias was considered unclear for this outcome, but both studies were rated as high risk for "other bias" because they were funded by a pharmaceutical company.

\subsection{Number of patients experiencing at least one adverse effect}

We found no difference between SSRIs and SNRIs in this outcome (RR 0.97, 95\% Cl 0.88 to 1.08; participants $=991$; studies $=2$ ) with moderate heterogeneity $\left(I^{2}=51 \%\right.$ ) (Analysis 7.14). Risk of bias was considered unclear for this outcome, but both studies were rated as high risk for "other bias" because of funding by a pharmaceutical company.

\section{Comparison 8: SSRIs versus NaSSAs}

One study including 30 participants (Ribeiro 2001) provided data for this comparison, comparing mirtazapine and fluoxetine. See also: Summary of findings 8.

\section{Primary outcomes}

\subsection{Failure to respond}

The study did not provide data for this outcome.

\subsection{Total number of dropouts}

We found low-quality evidence suggesting no difference between SSRIs and noradrenergic and specific serotonergic antidepressant (NaSSAs) in terms of dropouts due to any cause (RR 1.50, 95\% Cl 0.29 to 7.73 ; participants $=30$; studies $=1 ; 1^{2}=0 \%$ ) (Analysis 8.2). Risk of bias was considered between low and unclear for this outcome.

\section{Secondary outcomes}

\subsection{Failure to remit}

The study did not provide data for this outcome.

\subsection{Panic symptoms}

We found low-quality evidence suggesting no difference between SSRIs and NaSSAs for this outcome (MD $0.30,95 \% \mathrm{Cl}-0.49$ to 1.09; participants $=22$; studies $=1$ ) (Analysis 8.4). Risk of bias was considered between low and unclear for this outcome.

\subsection{Frequency of panic attacks}

The study did not provide data for this outcome.

\subsection{Agoraphobia}

The study did not provide data for this outcome.

\subsection{General anxiety}

No difference was found between SSRIs and NaSSAs on this outcome (MD 1.10, $95 \% \mathrm{Cl}-6.04$ to 8.24 ; participants $=27$; studies $=1$ ) (Analysis 8.7). Risk of bias was considered between low and unclear for this outcome.

\subsection{Depression}

The study did not provide data for this outcome.

\subsection{Social functioning}

The study did not provide data for this outcome.

\subsection{Quality of life}

The study did not provide data for this outcome.

\subsection{Patient satisfaction}

The study did not provide data for this outcome.

\subsection{Economic costs}

The study did not provide data for this outcome.

\subsection{Number of dropouts due to adverse effects}

We found low-quality evidence suggesting no difference between SSRIs and NaSSAs in terms of dropouts due to adverse effects (RR $1.50,95 \% \mathrm{Cl} 0.29$ to 7.73 ; participants $=30$; studies $=1$ ) (Analysis 8.13). Risk of bias was considered between low and unclear for this outcome.

\subsection{Number of patients experiencing at least one adverse effect}

The study did not provide data for this outcome.

\section{Comparison 9: SSRIs versus Other Antidepressants}

One study including 59 participants (Den Boer 1990) provided data for this comparison, comparing fluvoxamine and ritanserin.

\section{Primary outcomes}

\subsection{Failure to respond}

We found a difference favouring SSRIs over other antidepressants in terms of response rates ( $\mathrm{RR} 0.28,95 \% \mathrm{Cl} 0.13$ to 0.60 ; participants $=40$; studies $=1$ ) (Analysis 9.1). Risk of bias was considered between unclear for this outcome.

\subsection{Total number of dropouts}

The study reports that no patients left the study early in both groups, therefore it was not possible to calculate a RR (dropouts were $\mathrm{n}=0$ in 20 patients in both the fluvoxamine and ritanserin group).

\section{Secondary outcomes}

\subsection{Failure to remit}

The study did not provide data for this outcome.

\subsection{Panic symptoms}

The study did not provide data for this outcome.

\subsection{Frequency of panic attacks}

The study did not provide data for this outcome.

\subsection{Agoraphobia}

We found a difference in favour of SSRIs over other antidepressants (ritanserin) in terms of efficacy on agoraphobia (MD $-7.35,95 \% \mathrm{CI}$ -13.73 to -0.97 ; participants $=40$; studies $=1$ ) (Analysis 9.6). Risk of bias was considered unclear for this outcome.

\subsection{General anxiety}

We found a difference in favour of SSRIs over other antidepressants (ritanserin) in terms of efficacy on general anxiety (MD -3.55, 95\% 
$\mathrm{Cl}-5.07$ to -2.03 ; participants $=40$; studies $=1)($ Analysis 9.7). Risk of bias was considered unclear for this outcome.

\subsection{Depression}

We found a difference in favour of SSRIs over other antidepressant (ritanserin) (MD -5.38, 95\% Cl -7.90 to -2.86 ; participants $=40$; studies $=1$ ) (Analysis 9.8). Risk of bias was considered unclear for this outcome.

\subsection{Social functioning}

The study did not provide data for this outcome.

\subsection{Quality of life}

The study did not provide data for this outcome.

\subsection{Patient satisfaction}

The study did not provide data for this outcome.

\subsection{Economic costs}

The study did not provide data for this outcome.

\subsection{Number of dropouts due to adverse effects}

The study reports that no patients left the study early in both groups, therefore it was not possible to calculate a RR (dropouts were $n=0$ in patients in both the fluvoxamine and ritanserin group).

\subsection{Number of patients experiencing at least one adverse effect}

The study did not provide data for this outcome.

\section{Comparison 10: Individual antidepressants versus another antidepressant within the same class}

Five studies including 1026 participants (Amore 1999 bis; Bandelow 2004; Gentil 1993; Pfizer 2008; Stahl 2003) provided data for this comparison.

\section{Primary outcomes}

\subsection{Failure to respond}

We found no difference between escitalopram and citalopram in terms of response rate (RR $0.92,95 \% \mathrm{Cl} 0.79$ to 1.06 ; participants $=255$; studies $=1)$. The comparison between fluoxetine and citalopram (RR $0.57,95 \% \mathrm{Cl} 0.20$ to 1.66; participants $=42$; studies $=1$ ), as well as the one between sertraline and paroxetine (RR $0.97,95 \% \mathrm{Cl} 0.80$ to 1.17 ; participants $=546$; studies $=2 ; \mathrm{I}^{2}=$ $0 \%$ ) did not show any difference (Analysis 10.1). Risk of bias was considered unclear for this outcome, but the majority of studies were rated as high risk for "other bias" because they were funded by pharmaceutical companies.

\subsection{Total number of dropouts}

We found a difference in favour of sertraline over paroxetine in terms of dropouts due to any cause (RR $0.74,95 \% \mathrm{Cl} 0.55$ to 0.99 ; participants $=546$; studies $\left.=2 ;\left.\right|^{2}=2 \%\right)(N N T H=14,95 \% \mathrm{Cl} 8$ to 351$)$, while there was no difference for the other comparisons (Analysis 10.2). Risk of bias was considered unclear for this outcome, but the majority of studies were rated as high risk for "other bias" because of funding by pharmaceutical companies.

\section{Secondary outcomes}

\subsection{Failure to remit}

We found no difference between escitalopram and citalopram in terms of remission rates (RR 0.90, 95\% Cl 0.71 to 1.15; participants = 255; studies $=1$ ) (Analysis 10.3). Risk of bias was considered unclear for this outcome, but the study was rated as high risk for "other bias" because it was funded by a pharmaceutical company.

\subsection{Panic symptoms}

No difference was found between escitalopram and citalopram for this outcome (SMD $-0.09,95 \% \mathrm{Cl}-0.35$ to 0.16 ; participants $=237$; studies $=1$ ), as well as between sertraline and paroxetine (SMD $-0.05,95 \% \mathrm{Cl}-0.23$ to 0.13 ; participants $=473$; studies $=2 ; \mathrm{I}^{2}=$ $0 \%$ ) (Analysis 10.4). Risk of bias was considered unclear for this outcome, but all the studies were rated as high risk for "other bias" because they were funded by pharmaceutical companies.

\subsection{Frequency of panic attacks}

We found no difference between sertraline and paroxetine for this outcome (MD 0.48, 95\% Cl-1.02 to 1.98; participants = 461; studies $=2 ; I^{2}=0 \%$ ) (Analysis 10.5). Risk of bias was considered unclear for this outcome, but both studies were rated as high risk for "other bias" because they were funded by pharmaceutical companies.

\subsection{Agoraphobia}

In terms of agoraphobia, we found no difference between escitalopram and citalopram (MD $-1.10,95 \% \mathrm{Cl}-3.46$ to 1.26; participants $=237$; studies $=1$ ) (Analysis 10.6). Risk of bias was considered unclear for this outcome, but the study was rated as high risk for "other bias" because it was funded by a pharmaceutical company.

\subsection{General anxiety}

In terms of general anxiety, the comparison between escitalopram and citalopram did not show any difference (MD - $1.20,95 \% \mathrm{Cl}-3.14$ to 0.74 ; participants $=237$; studies $=1$ ), as well as between sertraline and paroxetine (MD $-0.72,95 \% \mathrm{Cl}-2.42$ to 0.99 ; participants $=454$; studies $=2 ; I^{2}=0 \%$ ) (Analysis 10.7). Risk of bias was considered unclear for this outcome, but all the studies were rated as high risk for "other bias" because of funding by pharmaceutical companies.

\subsection{Depression}

We found no difference between escitalopram and citalopram for this outcome (SMD $-0.11,95 \% \mathrm{Cl}-0.36$ to 0.15 ; participants $=237$; studies $=1$ ), and between sertraline and paroxetine (SMD 0.00, 95\% $\mathrm{Cl}-0.28$ to 0.29 ; participants $=189$; studies $=1)($ Analysis 10.8$)$. Risk of bias was considered unclear for this outcome, but both studies were rated as high risk for "other bias" because they were funded by pharmaceutical companies.

\subsection{Social functioning}

No studies provided data for this outcome.

\subsection{Quality of life}

We found no difference between escitalopram and citalopram for this outcome (MD 1.40, $95 \% \mathrm{Cl}-1.51$ to 4.31 ; participants = 237; studies $=1$ ) (Analysis 10.10). Risk of bias was considered unclear for this outcome, but the study was rated as high risk for "other bias" because it was funded by a pharmaceutical company. 


\subsection{Patient satisfaction}

No studies provided data for this outcome.

\subsection{Economic costs}

No studies provided data for this outcome.

\subsection{Number of dropouts due to adverse effects}

In terms of dropouts due to adverse effects there was no difference between sertraline and paroxetine (RR $0.66,95 \% \mathrm{Cl} 0.42$ to 1.04 ; participants $=546$; studies $\left.=2 ; I^{2}=0 \%\right)$. We found no difference between escitalopram and citalopram (RR $0.78,95 \% \mathrm{Cl} 0.32$ to 1.92 ; participants $=255$; studies $=1$ ), as well as between fluoxetine and citalopram (RR 1.00, 95\% Cl 0.07 to 14.95; participants $=42$; studies $=1$ ) and imipramine versus clomipramine (RR $0.33,95 \% \mathrm{Cl} 0.08$ to 1.46; participants $=40$; studies $=1$ ) (Analysis 10.13). Risk of bias was considered unclear for this outcome, but the majority of studies were rated as high risk for "other bias" because they were funded by pharmaceutical companies.

\subsection{Number of patients experiencing at least one adverse effect}

We found no difference between escitalopram and citalopram (RR $0.95,95 \% \mathrm{Cl} 0.85$ to 1.06 ; participants $=255$; studies $=1$ ), as well as between sertraline and paroxetine (RR $0.99,95 \% \mathrm{Cl} 0.89$ to 1.10 ; participants $=321$; studies $=1$ ) (Analysis 10.14). Risk of bias was considered unclear for this outcome, but both studies were rated as high risk for "other bias" because of funding by pharmaceutical companies.

\section{C: Individual benzodiazepines versus another benzodiazepine}

\section{Comparison 11: Individual benzodiazepines versus another benzodiazepine}

Two studies including 310 participants provided data for this comparison, one comparing diazepam with alprazolam (Noyes 1996) and one comparing alprazolam with clonazepam (Tesar 1991).

\section{Primary outcomes}

\subsection{Failure to respond}

The comparison between alprazolam and diazepam did not show any difference in terms of response rate (RR $0.94,95 \% \mathrm{Cl} 0.63$ to 1.40; participants $=159$; studies $=1$ ) (Analysis 11.1). Risk of bias was considered unclear for this outcome, but the study was rated as high risk for "other bias" because it was funded by a pharmaceutical company.

\subsection{Total number of dropouts}

We found no difference between alprazolam and diazepam (RR $0.84,95 \% \mathrm{Cl} 0.44$ to 1.64 ; participants $=159$; studies $=1$ ), alprazolam and clonazepam (RR $2.17,95 \% \mathrm{Cl} 0.44$ to 10.78 ; participants = 50; studies $=1$ ). The comparison between alprazolam and other benzodiazepines together did not show a difference (RR 1.01, 95\% $\mathrm{Cl} 0.49$ to 2.08; participants $=209$; studies $=2 ; \mathrm{I}^{2}=12 \%$ ) (Analysis 11.2). Risk of bias was considered unclear for this outcome, but both studies were rated as high risk for "other bias" because they were funded by a pharmaceutical company.

\section{Secondary outcome}

\subsection{Failure to remit}

We found no difference between alprazolam and diazepam (RR $0.76,95 \% \mathrm{Cl} 0.48$ to 1.20 ; participants $=159$; studies $=1$ ), alprazolam and clonazepam (RR $1.26,95 \% \mathrm{Cl} 0.73$ to 2.17 ; participants = 50; studies $=1$ ). The comparison between alprazolam and other benzodiazepines together did not show a difference (RR 0.96, 95\% $\mathrm{Cl} 0.58$ to 1.57; participants $=209$; studies $=2 ; \mathrm{I}^{2}=49 \%$ ) (Analysis 11.3). Risk of bias was considered unclear for this outcome, but both studies were rated as high risk for "other bias" because they were funded by a pharmaceutical company.

\subsection{Panic symptoms}

No difference was found between alprazolam and diazepam (MD $-0.20,95 \% \mathrm{Cl}-0.70$ to 0.30 ; participants $=159$; studies $=1$ ), and alprazolam and clonazepam (MD $0.60,95 \% \mathrm{Cl}-0.12$ to 1.32 ; participants $=50$; studies $=1$ ). The comparison between alprazolam and other benzodiazepines together did not show a difference (MD $0.16,95 \% \mathrm{Cl}-0.62$ to 0.93 ; participants $=209$; studies $=2 ; 1^{2}=$ $69 \%$ ) (Analysis 11.4). Risk of bias was considered unclear for this outcome, but both studies were rated as high risk for "other bias" because they were funded by a pharmaceutical company.

\subsection{Frequency of panic attacks}

We found no difference in terms of frequency of panic attacks between alprazolam and diazepam (MD 0.40, 95\% Cl -1.34 to 2.14; participants $=159$; studies $=1)$, alprazolam and clonazepam (MD $0.30,95 \% \mathrm{Cl}-6.75$ to 7.35 ; participants $=50$; studies $=1$ ) and between alprazolam and other benzodiazepines (MD 0.39, 95\% CI -1.29 to 2.08; participants $=209$; studies $=2 ; 1^{2}=0 \%$ ) (Analysis 11.5 ) . Risk of bias was considered unclear for this outcome, but both studies were rated as high risk for "other bias" because they were funded by a pharmaceutical company.

\subsection{Agoraphobia}

Neither study provided data for this outcome.

\subsection{General anxiety}

We found no difference between alprazolam and diazepam for this outcome (MD $0.20,95 \% \mathrm{Cl}-2.28$ to 2.68; participants = 159; studies $=1$ ) (Analysis 11.7). Risk of bias was considered unclear for this outcome, but the study was rated as high risk for "other bias" because it was funded by a pharmaceutical company.

\subsection{Depression}

We found no difference between alprazolam and clonazepam for this outcome (MD 2.30, 95\% Cl -2.83 to 7.43 ; participants $=44$; studies $=1$ ) (Analysis 11.8). Risk of bias was considered unclear for this outcome, but the study was rated as high risk for "other bias" because it was funded by a pharmaceutical company.

\subsection{Social functioning}

There was no difference between alprazolam and diazepam in terms of social functioning (MD $-0.10,95 \% \mathrm{Cl}-0.47$ to 0.27 ; participants $=159$; studies $=1$ ) (Analysis 11.9). Risk of bias was considered unclear for this outcome, but the study was rated as high risk for "other bias" because it was funded by a pharmaceutical company. 


\subsection{Quality of life}

We found no difference between alprazolam and clonazepam for this outcome (MD $-0.30,95 \% \mathrm{Cl}-1.07$ to 0.47 ; participants $=44$; studies $=1$ ) (Analysis 11.10). Risk of bias was considered unclear for this outcome, but the study was rated as high risk for "other bias" because it was funded by a pharmaceutical company.

\subsection{Patient satisfaction}

Neither study provided data for this outcome.

\subsection{Economic costs}

Neither study provided data for this outcome.

\subsection{Number of dropouts due to adverse effects}

There was no difference between alprazolam and diazepam (RR $1.04,95 \% \mathrm{Cl} 0.15$ to 7.19 ; participants $=159$; studies $=1$ ), alprazolam and clonazepam (RR 5.40, 95\% Cl 0.27 to 107.09; participants = 50; studies $=1$ ) and alprazolam versus benzodiazepines as a group (RR 1.69, 95\% Cl 0.33 to 8.58; participants = 209; studies $=2 ; \mathrm{I}^{2}=$ $0 \%)$ (Analysis 11.13). Risk of bias was considered unclear for this outcome, but the studies were rated as high risk for "other bias" because of funding by a pharmaceutical company.

\subsection{Number of patients experiencing at least one adverse effect}

Neither study provided data for this outcome.

\section{Subgroup analyses}

We could not conduct any of the preplanned subgroup analyses, as no relevant data were available: all studies included both participants with and without agoraphobia, so it was not possible to conduct subgroup analysis 1; no study reported acute data at more than four months, so it was not possible to perform subgroup analysis 2 .

\section{Sensitivity analyses}

\section{Excluding trials with high risk of bias (comparisons 12-16)}

\section{Antidepressants versus benzodiazepines}

\section{Failure to respond}

No studies were excluded, so the results did not change.

\section{Total number of dropouts}

Excluding two studies at high risk of bias (CNCPS 1992; Taylor 1990) did not substantially change the results (RR $1.63,95 \% \mathrm{Cl} 0.82$ to 3.23; participants $=619$; studies $=5$ ); heterogeneity was substantial $\left(I^{2}=79 \%\right)$ (Analysis 12.1).

\section{TCAs versus benzodiazepines}

\section{Failure to respond}

No studies were excluded, so the results did not change (Analysis 2.1).

\section{Total number of dropouts}

Excluding two studies at high risk of bias (CNCPS 1992; Taylor 1990) did not substantially change the results from the original analysis (RR $1.67,95 \% \mathrm{Cl} 0.64$ to 4.36; participants $=465$; studies $=4$ ); heterogeneity was substantial $\left(I^{2}=81 \%\right)$ (Analysis 13.1).

\section{SSRIs versus benzodiazepines}

No studies were excluded, so the results did not change (Analysis 3.1; Analysis 3.2).

\section{TCAs versus SSRIs}

\section{Failure to respond}

No studies were excluded, so the results did not change (Analysis 4.1).

\section{Total number of dropouts}

Excluding one study at high risk of bias (Amore 1999) the results did not substantially change from the original analysis, with a moderate heterogeneity $\left(I^{2}=54 \%\right)(\mathrm{RR} 0.97,95 \% \mathrm{Cl} 0.62$ to 1.50 ; participants $=890$; studies $=6)($ Analysis 14.1).

\section{TCAs versus MAOIs}

\section{Failure to respond}

No studies were excluded from this outcome for high risk of bias, so the results did not change (Analysis 5.1).

\section{Total number of dropouts}

Excluding one study at high risk of bias (Bakish 1993) did not substantially change the results from the original analysis (RR 0.87 , $95 \% \mathrm{Cl} 0.47$ to 1.59 ; participants $=135$; studies $=1)($ Analysis 15.1$)$.

\section{SSRIs versus MAOIs}

\section{Failure to respond}

Excluding one study at high risk of bias (Van Vliet 1996) did not substantially change the results from the original analysis (RR 1.08, $95 \% \mathrm{Cl} 0.76$ to 1.54 ; participants $=366$; studies $=1)($ Analysis 16.1$)$.

\section{Total number of dropouts}

Excluding studies at high risk of bias, no trials provided data for this analysis.

\section{SSRIs versus SNRIS}

No studies were excluded, so the results did not change (Analysis 7.1; Analysis 7.2).

\section{SSRIs versus NaSSAs}

Failure to respond

No data were available for this outcome.

\section{Total number of dropouts}

No studies were excluded, so the results did not change (Analysis 8.2).

\section{Individual antidepressants versus individual antidepressants}

\section{Failure to respond}

Excluding one study at high risk of bias (Amore 1999 bis) there were no data for fluoxetine versus citalopram (Analysis 17.1). No studies were excluded for the comparisons between escitalopram versus citalopram and sertraline versus paroxetine, thus the results remained unchanged (Analysis 10.1). 


\section{Total number of dropouts}

Excluding one study at high risk of bias (Amore 1999 bis) no trials provided data for fluoxetine versus citalopram . No studies were excluded for the comparisons between escitalopram versus citalopram and sertraline versus paroxetine, thus the results remained unchanged (Analysis 10.2).

\section{Individual benzodiazepines versus individual benzodiazepines}

No studies were excluded, so the results did not change (Analysis 11.1; Analysis 11.2).

\section{Excluding trials with dropout rates greater than 20\% (comparisons 17-19)}

\section{Antidepressants versus benzodiazepines}

Failure to respond

Excluding studies for high dropout rates, no trials provided data for this outcome.

\section{Total number of dropouts}

Excluding five studies for high dropout rates (CNCPS 1992; GSK-29060/1; Holland 1999; Schweizer 1993; Uhlenhuth 1989), no difference was found between antidepressants and benzodiazepines (RR 2.42, 95\% Cl 0.61 to 9.64; participants $=71$; studies $=2 ;\left.\right|^{2}=0 \%$ ) (Analysis 17.1) .

\section{TCAs versus benzodiazepines}

\section{Failure to respond}

Excluding studies for high dropout rates, no trials provided data for this analysis.

\section{Total number of dropouts}

Excluding four studies for high dropout rates (CNCPS 1992; Holland 1999; Schweizer 1993; Uhlenhuth 1989), the results did not substantially change from the original analysis (RR $2.42,95 \% \mathrm{Cl} 0.61$ to 9.64 ; participants $=71$; studies $=2 ; 12=0 \%$ ) (Analysis 18.1).

\section{SSRIs versus benzodiazepines}

Excluding studies for high dropout rates, no trials provided data for this analysis.

\section{TCAs versus SSRIs}

\section{Failure to respond}

Excluding two studies for high dropout rates (Lecrubier 1997; Nair 1996), the results did not substantially change from the original analysis (RR 1.22, 95\% Cl 0.47 to 3.15; participants $=95$; studies $=2$; $\mathrm{I}^{2}=0 \%$ ) (Analysis 19.1).

\section{Total number of dropouts}

Excluding three studies for high dropout rates (Lecrubier 1997; Nair 1996; Wade 1997), the results did not substantially change (RR 3.02, $95 \% \mathrm{Cl} 1.00$ to $9.11 ;$ participants $=206$; studies $\left.=4 ;\left.\right|^{2}=0 \%\right)($ Analysis 19.2).

\section{TCAs versus MAOIs}

Excluding studies for high dropout rates, no trials provided data for this comparison.

\section{SSRIs versus MAOIS}

No studies were excluded, so the results did not change (Analysis 6.1; Analysis 6.2).

\section{SSRIs versus SNRIS}

No studies were excluded, so the results did not change (Analysis 7.1; Analysis 7.2).

\section{SSRIs versus NaSSAs}

Failure to respond

No data were available for this outcome.

\section{Total number of dropouts}

No studies were excluded, so the results did not change (Analysis 8.2).

\section{Individual antidepressants versus individual antidepressants}

\section{Failure to respond}

Excluding three studies for high dropout rates (Bandelow 2004; Pfizer 2008; Stahl 2003), the only data available were for fluoxetine versus citalopram. The results of this sensitivity analysis did not change when compared with the primary analysis (Analysis 10.1).

\section{Total number of dropouts}

Excluding four studies for high dropout rates (Bandelow 2004; Gentil 1993; Pfizer 2008; Stahl 2003), the only data available were for fluoxetine versus citalopram. The results of this sensitivity analysis did not change when compared with the primary analysis (Analysis 10.2).

Individual benzodiazepines versus individual benzodiazepines

No studies were excluded, so the results did not change (Analysis 11.1; Analysis 11.2).

\section{Excluding studies funded by the pharmaceutical company marketing each antidepressant or benzodiazepine (comparisons 20-23)}

\section{Antidepressants versus benzodiazepines}

Failure to respond

Excluding studies funded by the pharmaceutical company, no trials provided data for this analysis.

\section{Total number of dropouts}

Excluding five studies funded by the pharmaceutical company marketing the drug (CNCPS 1992; Schweizer 1993; Sheikh 1999; Taylor 1990; Uhlenhuth 1989), no difference between antidepressants and benzodiazepines was found (RR 1.07, 95\% Cl 0.44 to 2.58; participants $=469$; studies $=2$ ), but with considerable heterogeneity $\left(I^{2}=88 \%\right)$ (Analysis 20.1).

\section{TCAs versus benzodiazepines}

\section{Failure to respond}

Excluding studies funded by the pharmaceutical company, no trials provided data for this analysis. 


\section{Total number of dropouts}

Excluding five studies funded by the pharmaceutical company marketing the drug, comparing imipramine with benzodiazepines (CNCPS 1992; Schweizer 1993; Sheikh 1999; Taylor 1990, Uhlenhuth 1989), only one study on clomipramine provided data for this comparison, showing a more favourable profile of TCAs over benzodiazepines (RR 0.69, $95 \% \mathrm{Cl} 0.49$ to 0.98; participants $=315$; studies $\left.=1 ;\left.\right|^{2}=0 \%\right)($ Analysis 21.1).

\section{SSRIs versus benzodiazepines}

Excluding studies funded by the pharmaceutical company, no trials provided data for this analysis.

\section{TCAs versus SSRIs}

\section{Failure to respond}

Excluding three studies funded by the pharmaceutical company marketing the drug (Lecrubier 1997; Nair 1996; Zhang 2000), did not substantially change the results (RR $2.00,95 \% \mathrm{Cl} 0.46$ to 8.76 ; participants $=22$; studies $=1$ ) (Analysis 22.1).

\section{Total number of dropouts}

Excluding four studies funded by the pharmaceutical company marketing the drug (GSK-29060/525; Lecrubier 1997; Nair 1996; Zhang 2000), the results did not substantially change (RR 1.14, 95\% $\mathrm{Cl} 0.77$ to 1.68; participants $=439$; studies $=3 ;\left.\right|^{2}=0 \%$ (Analysis 22.2) .

\section{TCAs versus MAOIs}

No studies were excluded, so the results did not change (Analysis 5.1; Analysis 5.2).

\section{SSRIs versus MAOIs}

\section{Failure to respond}

Excluding one study funded by the pharmaceutical company marketing the drug (Tiller 1999), the results did not substantially change (RR 1.25, 95\% Cl 0.69 to 2.26; participants $=30$; studies $=1$ ) (Analysis 23.1).

\section{Total number of dropouts}

No studies were excluded, so the results did not change (Analysis 6.1; Analysis 6.2).

\section{SSRIs versus SNRIS}

Excluding studies funded by the pharmaceutical company, no trials provided data for this analysis.

\section{SSRIS versus NaSSAS}

\section{Failure to respond}

No data were available for this analysis.

\section{Total number of dropouts}

No studies were excluded, so the results did not change (Analysis 8.2).

\section{Individual antidepressants versus individual antidepressants}

\section{Failure to respond}

Excluding three studies funded by the pharmaceutical company marketing the drug (Bandelow 2004; Pfizer 2008; Stahl 2003), the only data available were for fluoxetine versus citalopram. The results of this sensitivity analysis did not change when compared with the primary analysis (Analysis 10.1).

\section{Total number of dropouts}

Excluding three studies funded by the pharmaceutical company marketing the drug (Bandelow 2004; Pfizer 2008; Stahl 2003), the only data available were for fluoxetine versus citalopram and imipramine versus clomipramine. The results of these sensitivity analyses did not change when compared with the primary analyses (Analysis 10.2).

\section{Individual benzodiazepines versus individual benzodiazepines}

Excluding studies funded by the pharmaceutical company, no trials provided data for this analysis.

\section{Excluding studies whose participants clearly have significant psychiatric co-morbidities including primary or secondary depressive disorders (comparisons 24-28)}

Antidepressants versus benzodiazepines

\section{Failure to respond}

Excluding one study that included participants with psychiatric comorbidities (GSK-29060/1) did not substantially change the results (RR 1.02, 95\% Cl 0.63 to 1.67; participants $=61$; studies $=1$ ) (Analysis 24.1).

\section{Total number of dropouts}

Excluding three studies that included participants with psychiatric co-morbidities (CNCPS 1992, GSK-29060/1, Taylor 1990), no difference between antidepressants and benzodiazepines was found (RR 1.67, 95\% Cl 0.64 to 4.36; participants = 465; studies $=4$ ), with a substantial heterogeneity $\left(I^{2}=81 \%\right)$ (Analysis 24.2).

\section{TCAs versus benzodiazepines}

\section{Failure to respond}

No studies were excluded, so the results did not change (Analysis 2.1).

\section{Total number of dropouts}

Excluding two studies that included participants with psychiatric co-morbidities (CNCPS 1992; Taylor 1990) did not substantially change the results (RR 1.67, 95\% $\mathrm{Cl} 0.64$ to 4.36; participants $=465$; studies $=4$ ), with a substantial heterogeneity $\left(I^{2}=81 \%\right.$ ) (Analysis 25.1).

\section{SSRIs versus benzodiazepines}

Excluding studies that included participants with psychiatric comorbidities, no trials provided data for this analysis.

\section{TCAs versus SSRIs}

\section{Failure to respond}

Excluding one study that included participants with psychiatric co-morbidities (Bystritsky 1995) did not change the results (RR $1.03,95 \% \mathrm{Cl} 0.66$ to 1.61 ; participants $=416$; studies $=3$ ). The heterogeneity was moderate $\left(I^{2}=69 \%\right.$ ) (Analysis 26.1 ).

\section{Total number of dropouts}

Excluding one study that included participants with psychiatric co-morbidities (Bystritsky 1995) did not change the results (RR 
0.96, $95 \% \mathrm{Cl} 0.62$ to 1.49; participants = 906; studies =6). The heterogeneity was moderate $\left(I^{2}=54 \%\right.$ ) (Analysis 26.2).

\section{TCAs versus MAOIs}

No studies were excluded, so the results did not change (Analysis 5.1; Analysis 5.2).

\section{SSRIs versus MAOIs}

No studies were excluded, so the results did not change (Analysis 6.1; Analysis 6.2).

\section{SSRIS versus SNRIS}

No studies were excluded, so the results did not change (Analysis 7.1; Analysis 7.2).

\section{SSRIs versus NaSSAs}

Failure to respond

No data were available for this analysis.

\section{Total number of dropouts}

No studies were excluded, so the results did not change (Analysis 8.2).

\section{Individual antidepressants versus individual antidepressants}

\section{Failure to respond}

Excluding one study that included participants with psychiatric comorbidities (Pfizer 2008) did not change the results on sertraline versus paroxetine ( $\mathrm{RR} 0.98,95 \% \mathrm{Cl} 0.78$ to 1.23 ; participants $=225$; studies $=1$ ) (Analysis 27.1).

\section{Total number of dropouts}

Excluding two studies which included participants with psychiatric co-morbidities (Gentil 1993; Pfizer 2008), the conclusion changed from the main analysis, showing no difference between sertraline and paroxetine (RR $0.85,95 \% \mathrm{Cl} 0.57$ to 1.26 ; participants = 225; studies $=1$ ) (Analysis 27.2).

Individual benzodiazepines versus individual benzodiazepines

\section{Failure to respond}

No studies were excluded, so the results did not change (Analysis 11.1).

\section{Total number of dropouts}

Excluding one study that included participants with psychiatric comorbidities (Tesar 1991) the results did not change (RR 0.84, 95\% Cl 0.44 to 1.64 ; participants $=159$; studies $=1)($ Analysis 28.1) .

\section{Excluding studies mostly focused on agoraphobia}

This sensitivity analysis was not carried out as no studies were "mostly focused on agoraphobia".

\section{Applying best and worst case scenarios to studies where participants left the study before the endpoint}

The main analyses, according to the study protocol, considered participants who discontinued early as treatment failures (worstcase scenario) as this approach was considered more conservative. A sensitivity analysis based on a best-case scenario, which considers all dropouts as responders or remitters, was not feasible as the vast majority of studies carried forward, and included in the analyses, some observations on dropouts. This did not allow us to make the assumptions that all dropouts were responders.

Excluding studies where responding participants are calculated according to an imputation method (comparisons 29-30)

\section{Antidepressants versus benzodiazepines}

No studies were excluded, so the results did not change (Analysis 1.1; Analysis 1.2).

\section{TCAs versus benzodiazepines}

No studies were excluded, so the results did not change (Analysis 2.1; Analysis 2.2).

\section{SSRIs versus benzodiazepines}

No studies were excluded, so the results did not change (Analysis 3.1; Analysis 3.2).

\section{TCAs versus SSRIs}

\section{Failure to respond}

Excluding two studies where imputation methods were used (Bystritsky 1995; Nair 1996)did not substantially change the results (RR 1.33, 95\% Cl 0.95 to 1.86; participants $=318$; studies $=2 ; \mathrm{I}^{2}=0 \%$ ) (Analysis 29.1).

\section{Total number of dropouts}

Excluding two studies where imputation methods were used (Bystritsky 1995; Nair 1996)did not substantially change the results (RR 1.08, 95\% Cl 0.80 to 1.45; participants $=808$; studies $=5 ; I^{2}=4 \%$ ) (Analysis 29.2).

\section{TCAs versus MAOIs}

No studies were excluded, so the results did not change (Analysis 5.1; Analysis 5.2).

\section{SSRIs versus MAOIS}

No studies were excluded, so the results did not change (Analysis 6.1; Analysis 6.2).

\section{SSRIs versus SNRIs}

No studies were excluded, so the results did not change (Analysis 7.1; Analysis 7.2).

\section{SSRIs versus NaSSAs}

Failure to respond

No data were available for this analysis.

\section{Total number of dropouts}

No studies were excluded, so the results did not change (Analysis 8.2).

\section{Individual antidepressants versus individual antidepressants}

\section{Failure to respond}

Excluding two studies where imputation methods were used (Bandelow 2004; Stahl 2003), the results on sertraline versus paroxetine did not change (RR $0.95,95 \% \mathrm{Cl} 0.70$ to 1.30 ; participants $=321 ;$ studies $=1)($ Analysis 30.1). 


\section{Total number of dropouts}

Excluding two studies where imputation methods were used (Bandelow 2004; Stahl 2003), the results on sertraline versus paroxetine did not change (RR $0.62,95 \% \mathrm{Cl} 0.40$ to 0.97 ; participants $=321 ;$ studies $=1 ; 1^{2}=0 \%$ ) (Analysis 30.2).

\section{Individual benzodiazepines versus individual benzodiazepines}

No studies were excluded, so the results did not change (Analysis 11.1; Analysis 11.2).

\section{Excluding studies where irregular benzodiazepine use was allowed after the first 15 days after random allocation (comparisons 31-33)}

\section{Antidepressants versus benzodiazepines}

No studies were excluded, so the results did not change (Analysis 1.1; Analysis 1.2).

\section{TCAs versus benzodiazepines}

No studies were excluded from this comparison because of the irregular use of benzodiazepines, so the results did not change (Analysis 2.1; Analysis 2.2).

\section{SSRIs versus benzodiazepines}

No studies were excluded, so the results did not change (Analysis 3.1; Analysis 3.2).

\section{TCAs versus SSRIs}

\section{Failure to respond}

Excluding two studies in which irregular use of benzodiazepines was allowed (Lecrubier 1997; Nair 1996) did not change the results $\left(\right.$ RR 1.22, 95\% Cl 0.47 to 3.15; participants $=95 ;$ studies $\left.=2 ; I^{2}=0 \%\right)$ (Analysis 31.1).

\section{Total number of dropouts}

Excluding two studies in which irregular use of benzodiazepines was allowed (Lecrubier 1997; Nair 1996) did not change the results $\left(\mathrm{RR} 1.24,95 \% \mathrm{Cl} 0.85\right.$ to 1.81; participants $=585$; studies $\left.=5 ; \mathrm{I}^{2}=0 \%\right)$ (Analysis 31.2).

\section{TCAs versus MAOIs}

Excluding studies in which irregular use of benzodiazepines was allowed, no trials provided data for this analysis.

\section{SSRIs versus MAOIs}

\section{Failure to respond}

Excluding one study in which irregular use of benzodiazepines was allowed (Van Vliet 1996) did not change the results (RR 1.08, 95\% Cl 0.76 to 1.54; participants $=366$; studies $=1 ; I^{2}=0 \%$ ) (Analysis 32.1) .

\section{Total number of dropouts}

Excluding studies in which irregular use of benzodiazepines was allowed, no trials provided data for this analysis.

\section{SSRIs versus SNRIs}

No studies were excluded, so the results did not change (Analysis 7.1; Analysis 7.2)

\section{SSRIs versus NaSSAs}

\section{Failure to respond}

No data were available for this analysis.

\section{Total number of dropouts}

No studies were excluded, so the results did not change (Analysis 8.2).

Individual antidepressants versus individual antidepressants

Failure to respond

Excluding two studies in which irregular use of benzodiazepines was allowed (Bandelow 2004; Stahl 2003) did not change the results for sertraline versus paroxetine (RR $0.95,95 \% \mathrm{Cl} 0.70$ to 1.30 ; participants $=321$; studies $=1 ; 1^{2}=0 \%$ (Analysis 33.1).

\section{Total number of dropouts}

Excluding two studies in which irregular use of benzodiazepines was allowed (Bandelow 2004; Stahl 2003) did not change the results for sertraline versus paroxetine (RR $0.62,95 \% \mathrm{Cl} 0.40$ to 0.97 ; participants $=321$; studies $=1 ; I^{2}=0 \%$ ) (Analysis 33.2).

\section{Individual benzodiazepines versus individual benzodiazepines}

No studies were excluded, so the results did not change (Analysis 11.1; Analysis 11.2).

\section{Reporting Bias}

We did not visually inspect funnel plots to assess publication bias as no comparison included at least 10 studies.

\section{DISCUSSION}

\section{Summary of main results}

By systematically reviewing the comparative efficacy and tolerability of antidepressants and benzodiazepines for panic disorder in adults (see also Summary of findings for the main comparison; Summary of findings 2; Summary of findings 3; Summary of findings 4; Summary of findings 5; Summary of findings 6; Summary of findings 7; Summary of findings 8) we were able to include 35 studies with 6785 participants, 5365 of which in the arms of interest. However, considering the high number of individual medicines included, very few studies and participants contributed to individual comparisons. Even when antidepressants and benzodiazepines were considered as homogeneous groups, limited data were identified.

We found low-quality evidence that failed to find a difference between antidepressants and benzodiazepines in terms of efficacy measured as response rate. The same finding was observed when serotonin reuptake inhibitors (SSRIs) and tricyclic antidepressants (TCAs) were compared with benzodiazepines. However, only two studies with 215 participants contributed to this analysis, so it is not possible to determine whether there is a clinically important difference between antidepressants and benzodiazepines.

Analysis of remission rates showed a benefit for benzodiazepines compared to antidepressants, even if the effect was very small and close to no difference. 
There was no difference between antidepressants and benzodiazepines in terms of efficacy on panic symptoms, frequency of panic attacks, agoraphobia and general anxiety.

We observed a difference in favour of antidepressants over benzodiazepines in terms of depressive symptoms (standardised mean difference (SMD) -0.14, 95\% confidence interval (Cl) -0.29 to 0.02 ; participants $=892$; studies $=6 ; 1^{2}=7 \%$ ), although the confidence interval was of borderline statistical significance and the magnitude of effect of uncertain clinical meaning. This difference was still present when the SSRIs were compared with benzodiazepines, but not when TCAs were compared with benzodiazepines.

In terms of tolerability, we found evidence suggesting a benefit for benzodiazepines compared to antidepressants when looking at number of dropouts due to any cause (risk ratio (RR) 1.64 , $95 \% \mathrm{Cl} 1.03$ to 2.63; participants $=1449$; studies $=7$ ), even if the degree of heterogeneity between studies was substantial ( ${ }^{2}$ $=75 \%$ ) and the wide confidence interval close to no difference. In terms of dropout due to adverse effects, a meta-analysis of three studies showed that benzodiazepines were better tolerated in comparison with antidepressants as a whole (RR 1.72, 95\% $\mathrm{Cl} 1.03$ to 2.87; participants $=1002$ ) and with TCAs (RR 2.10, $95 \% \mathrm{Cl} 1.13$ to 3.93; participants = 848; two studies), but not in comparison with the SSRIs. For the SSRIs, in terms of participants who dropped out due to any cause, we found a difference in favour of benzodiazepines, although only one study contributed to this analysis (RR 1.71, 95\% Cl 1.03 to 2.84; participants $=154$ ). Only one study provided information on the number of patients experiencing adverse effect, showing no difference between antidepressants and benzodiazepines.

When looking at the comparisons between antidepressants, a meta-analysis of four studies confirmed a benefit for SSRIs compared to TCAs in terms of number of patients experiencing adverse effects (RR 1.29, $95 \% \mathrm{Cl} 1.02$ to 1.65; participants = 489; studies $\left.=4 ; 1^{2}=78 \%\right)$, although the magnitude of effect is small. Low-quality evidence suggests that there is no significant difference between the class of TCAs and the class of SSRIs in terms of response rate (four studies, 438 participants) and dropout due to any reason (seven studies, 928 participants).

When looking at the comparison between individual benzodiazepines, the available evidence, focused on a small number of medicines (alprazolam, clonazepam and diazepam), suggests that there is no significant difference between individual benzodiazepines in terms of response rate and dropout due to any reason.

\section{Overall completeness and applicability of evidence}

The identified studies are not sufficient to comprehensively address the objectives of the present review. The majority of studies enrolled a very small number of participants and did not provide data for all the outcomes specified in the protocol. For these reasons, most of the analyses were underpowered and this relevantly limits the overall completeness of evidence. For the primary efficacy outcome, pooling of results was possible in two studies, and for many secondary outcomes data were too sparse to allow reasonable conclusions to be drawn. For most comparisons, confidence intervals were very wide and could not rule out the possibility of clinically relevant differences. Therefore, the main question of whether there are differences between antidepressants and benzodiazepines, and between individual antidepressants and individual benzodiazepines, remains unanswered. This situation will unlikely change in the future as recent studies no longer focus on the efficacy of benzodiazepines.

Only short-term data on acceptability and adverse effects of antidepressants and benzodiazepines were available. Clinically, this is a major limitation as long-term use of benzodiazepines is controversial due to concerns about adverse psychological and physical effects, physical dependence and withdrawal. Similar concerns have been raised for long-term exposure to antidepressants, in particular the SSRIs.

In terms of applicability, considering the very low number of participants, it is difficult to assume that this population could reflect the complexity of people with panic disorder from a 'real world' setting.

\section{Quality of the evidence}

The overall methodological quality of the included studies was poor. No study showed an overall low risk of bias. The majority of studies showed mixed features, with a large prevalence of an unclear risk of bias in different domains, which seems to reflect the lack of exhaustive reporting rather than a clear evidence of bias. This is consistent with the finding of a general suboptimal reporting of randomised controlled trials (RCTs) in medical journals, despite the large diffusion of instruments designed to help transparent reporting, such as the CONSORT (Consolidated Standards of Reporting Trials) statement.

The GRADE (Grading of Recommendations, Assessment, Development and Evaluations) methodology allows the provision of outcome-specific information concerning the overall quality of evidence. In general, the confidence in the estimate of effect appeared to be from 'very low' to 'moderate' for most of the outcomes assessed. This judgement is primarily due to limitations in the included studies (high dropout rates), imprecision (wide confidence intervals) and inconsistency (heterogeneity between studies results). In accordance with that, any estimate of effect should be considered very uncertain, and further research is very likely to change the estimate of effect and thus the degree of confidence for its applicability in routine clinical practice.

\section{Potential biases in the review process}

Several possible limitations of this review should be highlighted. Some limitations are intrinsically related to the actual process of retrieving, collecting, selecting and extracting data. In order to reduce the potential bias of this complex process two authors independently worked on each of these steps. It has been highlighted that two independent extractors are overall more reliable than the extraction performed by a single author followed by verification by a second author (Buscemi 2006). We applied the same process for the 'Risk of bias' assessment. Furthermore, disagreements were discussed with a third author, who also checked the data extracted from RCTs when the analysis was performed. Another relevant problem concerns the 'systematic' nature of the search. We chose to include only randomised trials as they provide the strongest level of evidence available. In this type of review there is some risk of publication bias, which means that negative studies may not have been published. Although the search 
was thorough, it is possible that unpublished studies have not been identified, considering that there are no shared procedures to perform this kind of search. The impact of unpublished literature on the results of this review is uncertain, however it is expected that the analysis of only published literature would lead to overestimation of the efficacy of a given intervention. We did not check this formally with a funnel plot analysis, as less than 10 studies contributed to any analyses, thus making the funnel plot methodology less informative.

It is important to bear in mind that some of the included studies were funded by the pharmaceutical industry, and this may again introduce an overestimation of the efficacy of interventions.

\section{Agreements and disagreements with other studies or reviews}

The results of this systematic review are not completely in line with respect to previous reviews and meta-analyses. Offidani and colleagues, who compared benzodiazepines and antidepressants in panic disorder, found that benzodiazepines were more effective than TCAs in terms of response to treatment (Offidani 2013). However, the Offidani review included a single-blind study, three reports that are part of the same multicentre trial (CNCPS 1992), and studies with participants with a primary diagnosis other than panic disorder. Further, different definitions of response rate were applied.

Based on data from our review, the only evidence in terms of efficacy that favours benzodiazepines is about remission rates. We may argue that this outcome is usually measured in a more standard way than response rates, looking at patients who are free from panic attacks. In these terms, benzodiazepines may have the edge over antidepressants.

Further, we found that benzodiazepines are better tolerated than antidepressants; for example, in terms of participants who dropped out due to any cause, we found a difference in favour of benzodiazepines over antidepressants, and this difference is more significant in the comparison with SSRIs (that were not included in the previous review by Offidani). However, it should be recognised that short-term randomised studies can not provide a comprehensive assessment of the harmful consequences associated with drug treatment, including long-term exposure. This holds particularly true for benzodiazepines, as most concerns refer to long-term exposure to these agents. The role of benzodiazepines is still controversial; according to NICE guidelines benzodiazepines are not recommended for panic disorder, precisely because of less favourable long-term outcomes (NICE 2011). However, some authors claim for a reconsideration of the role of benzodiazepines, suggesting that they can be effective and safe in the long-term use for anxiety disorders, including panic disorder (Starcevic 2014).

Bakker and colleagues, who carried out a systematic review comparing TCAs and SSRIs in people with panic disorder, concluded that TCAs and SSRI were equally effective (Bakker 2002). Similarly to Bakker and colleagues, we did not find any difference between TCAs and SSRI in terms of efficacy, but we note that a power problem might limit the interpretation of this finding. For all efficacy outcomes, the confidence intervals were wide and could not exclude the possibility of a type-II error, namely not detecting a difference which might be real because too few participants contributed to the analysis. However, we agree with Bakker and colleagues in noting that there are no strong pre-defined reasons to hypothesise that these two classes of antidepressants should differ in terms of efficacy in panic disorder. We argue that only adding the result of this systematic review to the other ongoing Cochrane reviews in patients with panic disorder will help rank treatments in terms of efficacy, possibly increasing statistical power by means of indirect comparisons.

\section{AUTHORS' CONCLUSIONS}

\section{Implications for practice}

The identified studies are not sufficient to comprehensively address the objectives of the present review; the majority of studies enrolled a small number of participants and did not provide data for all the outcomes specified in the protocol. For these reasons most of the analyses were underpowered and this limits the overall completeness of evidence.

The findings of this review have few implications for people with panic disorder. Choice of treatment should be guided by patient's preference, if clinically appropriate; patients and their families should continue to reflect with clinicians about the basis of their care and the reasoning behind using a specific antidepressant or benzodiazepine, also taking into account possible psychiatric or medical co-morbidities.

In general, based on the results of the current review, the possible role of antidepressants and benzodiazepines should be assessed each time by the clinician on an individual basis. The choice of which antidepressant and/or benzodiazepine should be prescribed can hardly be made on the basis of this review only, and rather it may be based on whole amount of evidence on antidepressant and benzodiazepine efficacy and tolerability, including data from placebo-controlled studies. Data on long-term tolerability issues associated with antidepressant and benzodiazepine exposure should also be carefully considered.

\section{Implications for research}

The results described in this systematic review come from evidence of low to very low quality according to the GRADE methodology. Moreover, in many cases, studies were financially supported by pharmaceutical industries. Consequently, there is a high risk that these studies do not provide sufficient and adequate information for clinicians in real-world settings.The present review highlights the strong need for further higher-quality studies comparing benzodiazepines with antidepressants, which should be conducted with high-methodological standards and with the primary intent of providing clinicians with useful practical data on the effectiveness of antidepressant drugs and benzodiazepines, in head-to-head comparisons with sufficient statistical power to detect clinically meaningful differences. Alongside rating scales, pragmatic outcome measures, such as quality of life and social functioning, should also be considered.

In the meantime, it seems reasonable to make the best use of available evidence. The results of this systematic review will contribute to a Cochrane network meta-analysis of drug treatments for patients with panic disorder which is in progress, aiming to rank available drug treatments for efficacy and tolerability. 


\section{ACKN OWLEDGEMENTS}

We would like to thank the CCMD Editorial Team for their support, information and advice.

We would like to thank all authors who answered our requests for additional data, and especially Drs. Bandelow, Lavori, and Stahl.

We also thank Dr. Peiyao Chen for the help provided with Chinese papers.

\section{CRG funding}

The National Institute for Health Research (NIHR) is the largest single funder of the Cochrane Common Mental Disorders Review Group.

\section{Disclaimer}

The views and opinions expressed herein are those of the authors and do not necessarily reflect those of the NIHR, NHS or the Department of Health. 


\section{RE F E R E N C E S}

\section{References to studies included in this review}

Amore 1999 \{published data only\}

Amore M, Magnani K, Cerisoli M, Casagrande C, Ferrari G. Panic disorder. A long-term treatment study: fluoxetine vs imipramine. Human Psychopharmacology: Clinical and Experimental 1999;14(6):429-34.

\section{Amore 1999 bis \{published data only\}}

Amore M, Magnani K, Cerisoli M, Ferrari G. Short-term and long-term evaluation of selective serotonin reuptake inhibitors in the treatment of panic disorder: fluoxetine vs citalopram. Human Psychopharmacology: Clinical and Experimental 1999;14(6):435-40.

\section{Bakish 1993 \{published data only\}}

* Bakish D, Saxena BM, Bowen R, D'Souza J. Reversible monoamine oxidase-A inhibitors in panic disorder. Clinical Neuropharmacology 1993;16(Suppl 2):S77-S82.

Saxena B, Bakish D, Bowen R, D'Souza J. Brofaromine and clomipramine in panic disorder: a double-blind study. Clinical Neuropharmacology 1992;15(1 Pt B):60.

\section{Bandelow 2004 \{published data only\}}

Bandelow B, Behnke K, Lenoir S, Hendriks GJ, Alkin T, Dombrowski A, et al. Sertraline versus paroxetine in the treatment of panic disorder: a multinational randomized double-blind 15 week study. European Neuropsychopharmacology 2002;12(Suppl 3):S363.

* Bandelow B, Behnke K, Lenoir S, Hendriks GJ, Alkin T, Goebel $C$, et al. Sertraline versus paroxetine in the treatment of panic disorder: an acute, double-blind noninferiority comparison. Journal of Clinical Psychiatry 2004;65(3):405-13.

\section{Bystritsky 1995 \{published data only\}}

Bystritsky A, Rosen RM, Murphy KJ, Bohn P, Keys SA, Vapnik T. Double-blind pilot trial of desipramine versus fluoxetine in panic patients. Anxiety 1994-1995;1(6):287-90.

\section{CNCPS 1992 \{published data only\}}

Albus M, Lecrubier Y, Maier W, Buller R, Rosenberg R, Hippius H. Drug treatment of panic disorder: early response to treatment as a predictor of final outcome. Acta Psychiatrica Scandinavica 1990;82(5):359-65.

Albus M, Maier W, Shera D, Bech P. Consistencies and discrepancies in self- and observer-rated anxiety scales. A comparison between the self- and observer-rated MarksSheehan scales. European Archives of Psychiatry and Clinical Neuroscience 1990;240(2):96-102.

Andersch S, Hanson L, Hallstrom T. Panic disorder: a five-year follow-up study in 52 patients. European Journal of Psychiatry 1997;11(3):145-55.

Andersch S, Hetta J. A 15-year follow-up study of patients with panic disorder. European Psychiatry 2003;18(8):401-08.
Andersch S, Rosenberg NK, Kullingsjö H, Ottosson JO, Bech P, Bruun-Hansen J, et al. Efficacy and safety of alprazolam, imipramine and placebo in treating panic disorder. A Scandinavian multicenter study. Acta Psychiatrica Scandinavica. Supplementum 1991;365:18-27.

Berlanga C, Canetti A, Chavez E, De La Fuente R, del Carmen Lara M, Leon C, et al. Pharmacologic treatment of panic disorders: Comparative report on the efficacy and safety of alprazolam and imipramine in a controlled study [Tratamiento farmacologico de las crisis de angustia Reporte comparativo de la eficacia y seguridad del alprazolam y la imipramina en un estudio controlado]. Salud Mental 1991;14(1):1-5.

Buller R, Maier W, Goldenberg IM, Lavori PW, Benkert O. Chronology of panic and avoidance, age of onset in panic disorder, and prediction of treatment response. A report from the Cross-National Collaborative Panic Study. European Archives of Psychiatry and Clinical Neuroscience 1991;240(3):163-8.

Cassano GB, Toni C, Petracca A, Deltito J, Benkert O, Curtis G, et al. Adverse effects associated with the short-term treatment of panic disorder with imipramine, alprazolam or placebo. European Neuropsychopharmacology 1994;4(1):47-53.

* Cross National Collaborative Panic Study Second Phase Investigators. Drug treatment of panic disorder: Comparative efficacy of alprazolam, imipramine, and placebo. British Journal of Psychiatry 1992;160:191-202.

Curtis GC, Massana J, Udina C, Ayuso JL, Cassano GB, Perugi G. Maintenance drug therapy of panic disorder. Journal of Psychiatry Research 1993;27(Suppl 1):127-42.

Deltito JA, Argyle N, Buller R, Nutzinger D, Ottosson JO, Brandon $\mathrm{S}$, et al. The sequence of improvement of the symptoms encountered in patients with panic disorder. Comprehensive Psychiatry 1991;32(2):120-9.

Deltito JA, Argyle N, Klerman GL. Patients with panic disorder unaccompanied by depression improve with alprazolam and imipramine treatment. Journal of Clinical Psychiatry 1991;52(3):121-7.

Green MA, Curtis GC. Personality disorders in panic patients: Response to termination of antipanic medication. Journal of Personality Disorders 1988;2(4):303-14.

Katschnig H, Amering M, Stolk JM, Klerman GL, Ballenger JC, Briggs $\mathrm{A}$, et al. Long-term follow-up after a drug trial for panic disorder. British Journal of Psychiatry 1995;167(4):487-94.

Klerman GL. Depression and panic anxiety: the effect of depressive co-morbidity on response to drug treatment of patients with panic disorder and agoraphobia. Journal of Psychiatric Research 1990;24(Suppl 2):27-41.

Klerman GL, Coleman JH, Purpura RP. The design and conduct of the Upjohn Cross-National Collaborative Panic Study. Psychopharmacology Bulletin 1986;22(1):59-64. 
Lavori PW, Dawson R, Shera D. A multiple imputation strategy for clinical trials with truncation of patient data. Statistics in Medicine 1995;14(17):1913-25.

León CA, De Arango MV, Arevalo W, Calvo A, Montoya A, León A. Comparison of the effect of alprazolam, imipramine and placebo in the treatment of panic disorders in Cali, Colombia [Comparacion del efecto del alprazolam, la imipramina y placebo en el tratamiento del trastorno de panico en Cali, Colombia]. Acta Psiquiatrica y Psicologica de America Latina 1990;36(1-2):59-72.

Maier W, Albus M, Buller R, Nutzinger D, Shera D, Bech P. Selfand observer assessment in anxiolytic drug trials: a comparison of their validity. European Archives of Psychiatry and Clinical Neuroscience 1990;240(2):103-8.

Maier W, Roth SM, Argyle N, Buller R, Lavori P, Brandon S, et al. Avoidance behaviour: a predictor of the efficacy of pharmacotherapy in panic disorder?. European Archives of Psychiatry and Clinical Neuroscience 1991;241(3):151-8.

Maier WA, Roth M, Buller R, Argyle N, Rosenberg R, Sydney B, et al. Agoraphobia in panic disorder: An indicator of the severity of panic disorder or a distinct diagnostic entity?. Psychiatric Annals 1991;21(6):374-81.

Rifkin A. The sequence of improvement of the symptoms encountered in patients with panic disorder. Comprehensive Psychiatry 1991;32(6):559-60.

Rosenberg NK, Mellergård M, Rosenberg R, Beck P, Ottosson JO. Characteristics of panic disorder patients responding to placebo. Acta Psychiatrica Scandinavica Supplementum 1991;365:33-8.

Rosenberg R, Bech P, Mellergård M, Ottosson JO. Alprazolam, imipramine and placebo treatment of panic disorder: predicting therapeutic response. Acta Psychiatrica Scandinavica. Supplementum 1991;365:46-52.

Swoboda H, Amering M, Windhaber J, Katschnig H. The longterm course of panic disorder--an 11 year follow-up. Journal of Anxiety Disorders 2003;17(2):223-32.

\section{Den Boer 1988 \{published data only\}}

Den Boer JA, Westenberg HGM. Effect of a serotonin and noradrenaline uptake inhibitor in panic disorder; a doubleblind comparative study with fluvoxamine and maprotiline. International Clinical Psychopharmacology 1988;3(1):59-74.

\section{Den Boer 1990 \{published data only\}}

* Den Boer JA, Westenberg HGM. Serotonin function in panic disorder: a double blind placebo controlled study with fluvoxamine and ritanserin. Psychopharmacology 1990;102:85-94.

Westenberg HG, den Boer JA. Selective monoamine uptake inhibitors and a serotonin antagonist in the treatment of panic disorder. Psychopharmacology Bulletin 1989;25(1):119-23.

\section{Garvey 1989 \{published data only\}}

Garvey M, Noyes R Jr, Cook B, Tollefson G. The relationship of panic disorder and its treatment outcome to 24-hour urinary MHPG levels. Psychiatry Research 1989;30(1):53-61.

\section{Gentil 1993 \{published data only\}}

* Gentil V, Lotufo-Neto F, Andrade L, Cordás T, Bernik M, Ramos R, et al. Clomipramine, a better reference drug for panic/ agoraphobia. I. Effectiveness comparison with imipramine. Journal of Psychopharmacology 1993;7(4):316-24.

Marcourakis T, Gorenstein C, Gentil V. Clomipramine, a better reference drug for panic/agoraphobia. II. Psychomotor and cognitive effects. Journal of Psychopharmacology 1993; $7(4): 325-30$

\section{GSK-29060/1 \{unpublished data only\}}

GSK. A double-blind, multicenter, flexible-dose study of paroxetine, alprazolam and placebo in the treatment of panic disorder. GSK Clinical Studies Register [http://www.gskclinicalstudyregister.com/].

\section{GSK-29060/525 \{unpublished data only\}}

GSK. A double blind, multicenter randomized drug-controlled study to assess the efficacy and tolerance of paroxetine compared with clomipramine in treatment of panic disorder. GSK Clinical Studies Register [http://www.gskclinicalstudyregister.com/].

\section{Holland 1999 \{published data only\}}

Holland R, Musch B, Hindmarch I. Specific effects of benzodiazepines and tricyclic antidepressants in panic disorder: comparisons of clomipramine with alprazolam SR and adinazolam SR. Human Psychopharmacology Clinical Experimental 1999;14:119-24.

\section{Krueger 1999 \{published data only\}}

Krueger MB, Dahl AA. The efficacy and safety of moclobemide compared to clomipramine in the treatment of panic disorder. European Archives of Psychiatry and Clinical Neuroscience 1999;249(Suppl 1):S19-S24

\section{Lecrubier 1997 \{published data only\}}

GlaxoSmithKline. A double-blind placebo controlled comparative study of paroxetine and clomipramine in the treatment of panic disorder. GSK-ClinicalStudy Register (www.gsk-clinicalstudyregister.com) 1993/11/12.

* Lecrubier Y, Bakker A, Dunbar G, Judge R and the Collaborative Panic Study Investigators. A comparison of paroxetine, clomipramine and placebo in the treatment of panic disorder. Acta Psychiatrica Scandinavica 1997;95:145-52.

Lecrubier Y, Judge R and the Collaborative Paroxetine Panic Study Investigators. Long-term evaluation of paroxetine, clomipramine and placebo in panic disorder. Acta Psychiatrica Scandinavica 1997;95:153-60.

Lepola 1990 \{published data only\}

* Lepola U, Heikkinen H, Rimon R, Riekkinen P. Clinical evaluation of alprazolam in patients with panic disorder; 
a double-blind comparison with imipramine. Human

Psychopharmacology 1990;5:159-63.

Rimon R. Spectral electroencephalogram and clinical follow-up during alprazolam and imipramine treatment in panic disorder. Nordic Journal of Psychiatry 1998;52(3):245-9.

Meco 1989 \{published data only\}

Meco G, Capriani C, Bonifati U. Etizolam: a new therapeutic possibility in the treatment of panic disorder. Advances in Therapy 1989;6(4):196-206.

\section{Nair 1996 \{published data only\}}

Bakish D, Hooper CL, Filteau MJ, Charbonneau Y, Fraser G, West DL, et al. A double-blind placebo-controlled trial comparing fluvoxamine and imipramine in the treatment of panic disorder with or without agoraphobia. Psychopharmacology Bulletin 1996;32(1):135-41.

* Nair NP, Bakish D, Saxena B, Amin M, Schwartz G, West TE. Comparison of fluvoxamine, imipramine, and placebo in the treatment of outpatients with panic disorder. Anxiety 1996;2(4):192-8.

\section{Noyes 1996 \{published data only\}}

Coryell W, Noyes R Jr, Schlechte J. The significance of HPA axis disturbance in panic disorder. Biological Psychiatry 1989;25(8):989-1002.

Lopez AL, Kathol RG, Noyes R Jr. Reduction in urinary free cortisol during benzodiazepine treatment of panic disorder. Psychoneuroendocrinology 1990;15(1):23-8.

* Noyes R Jr, Burrows GD, Reich JH, Judd FK, Garvey MJ, Norman TR, et al. Diazepam versus alprazolam for the treatment of panic disorder. Journal of Clinical Psychiatry 1996;57(8):349-55.

Noyes R Jr, Garvey MJ, Cook B, Suelzer M. Controlled discontinuation of benzodiazepine treatment for patients with panic disorder. American Journal of Psychiatry 1991;148(4):517-23.

\section{Pfizer 2008 \{unpublished data only\}}

Pfizer. A randomized, double-blind, multicenter study of sertraline compared with paroxetine In the treatment of panic disorder. http://clinicaltrials.gov/show/NCT00677352 2008.

Pollack 2007a \{published data only\}

* Pollack M, Mangano R, Entsuah R, Tzanis E, Simon NM. A randomized controlled trial of venlafaxine $E R$ and paroxetine in the treatment of outpatients with panic disorder. Psychopharmacology 2007;194:233-42.

Pollack M, Whitaker T, Mangano R, Gao B. A comparison of venlafaxine $X R$ and paroxetine in the treatment of outpatients with panic disorder. ACNP 2004 Annual Meeting S201-S202.

\section{Pollack 2007b \{published data only\}}

Pollack MH, Lepola U, Koponen H, Simon NM, Worthington JJ, Emilien G, et al. A double-blind study of the efficacy of venlafaxine extended-release, paroxetine and placebo in the treatment of panic disorder. Depression and Anxiety 2007;24:1-14.

Ribeiro 2001 \{published data only\}

Ribeiro L, Busnello JV, Kauer-Sant'Anna M, Madruga M, Quevedo J, Busnello EAD, et al. Mirtazapine versus fluoxetine in the treatment of panic disorder. Brazilian Journal of Medical and Biological Research 2001;34:1303-7.

\section{Sasson 1999 \{published data only\}}

Sasson Y, Iancu I, Fux M, Taub M, Dannon P, Zohar J. A double-blind crossover comparison of clomipramine and desipramine in the treatment of panic disorder. European Neuropsychopharmacology 1999;9:191-6.

\section{Schweizer 1993 \{published data only\}}

Rickels K, Schweizer E. Panic disorder: Long-term pharmacotherapy and discontinuation. Journal of Clinical Psychopharmacology 1998;18(6 Suppl 2):12-8.

Rickels K, Schweizer E, Weiss S, Zavodnick S. Maintenance drug treatment for panic disorder. II. Short- and long-term outcome after drug taper. Archives of General Psychiatry 1993;50:61-8.

* Schweizer E, Rickels K, Weiss S, Zavodnick S. Maintenance drug treatment of panic disorder. I. Results of a prospective, placebo-controlled comparison of alprazolam and imipramine. Archives of General Psychiatry 1993;50:51-60.

\section{Sheikh 1999 \{published data only\}}

Sheikh JI, Swales PJ. Treatment of panic disorder in older adults: a pilot study comparison of alprazolam, imipramine, and placebo. International Journal of Psychiatry in Medicine 1999;29(1):107-17.

\section{Stahl 2003 \{published data only\}}

Bandelow B, Stein DJ, Dolberg OT, Andersen HF, Baldwin DS. Improvement of quality of life in panic disorder with escitalopram, citalopram, or placebo. Pharmacopsychiatry 2007;40:152-6.

Forest Laboratories. Flexible-dose comparison of the safety and efficacy of Lu 26-054 (escitalopram), citalopram and placebo in the treatment of panic disorder. Forest Laboratories Clinical Study Register [www.forestclinicaltrials.com].

* Stahl S, Gergel I, Li D. Escitalopram in the treatment of panic disorder: a randomized, double-blind, placebo-controlled trial. Journal of Clinical Psychiatry 2003;64:1322-7.

\section{Taylor 1990 \{published data only\}}

Clark DB, Taylor CB, Roth WT, Hayward C, Ehlers A, Margraf J, et al. Surreptitious drug use by patients in a panic disorder study. American Journal of Psychiatry 1990;147(4):507-9.

Margraf J, Ehlers A, Roth WT, Clark DB, Sheikh J, Agras WS, et al. How "blind" are double-blind studies?. Journal of Consulting and Clinical Psychology 1991;59(1):184-7.

* Taylor CB, Hayward C, King R, Ehlers A, Margraf J, Maddock R, et al. Cardiovascular and symptomatic reduction effects of alprazolam and imipramine in patients with panic disorder: 
results of a double-blind, placebo-controlled trial. Journal of Clinical Psychopharmacology 1990;10(2):112-8.

\section{Tesar 1991 \{published data only\}}

Fava M, Rosenbaum JF, MacLaughlin RA, Tesar GE, Pollack MH, Cohen LS, et al. Dehydroepiandrosterone-sulfate/cortisol ratio in panic disorder. Psychiatry Research 1989;28(3):345-50.

Labbate LA, Pollack MH, Otto MW, Tesar GM, Rosenbaum JF. The relationship of alprazolam and clonazepam dose to steady-state concentration in plasma. Journal of Clinical Psychopharmacology 1994;14(4):274-6.

Pollack MH, Otto MW, Tesar GE, Cohen LS, Meltzer-Brody S, Rosenbaum JF. Long-term outcome after acute treatment with alprazolam or clonazepam for panic disorder. Journal of Clinical Psychopharmacology 1993;13(4):257-63.

Tesar GE, Rosenbaum JF, Pollack MH, Herman JB, Sachs GS, Mahoney EM, et al. Clonazepam versus alprazolam in the treatment of panic disorder: interim analysis of data from a prospective, double-blind, placebo-controlled trial. Journal of Clinical Psychiatry 1987;48(Suppl 10):16-21.

* Tesar GE, Rosenbaum JF, Pollack MH, Otto MW, Sachs GS, Herman JB, et al. Double-blind, placebo-controlled comparison of clonazepam and alprazolam for panic disorder. Journal of Clinical Psychiatry 1991;52(2):69-76.

\section{Tiller 1999 \{published data only\}}

Tiller JWG, Bouwer C, Behnke K. Moclobemide and fluoxetine for panic disorder. European Archives of Psychiatry and Clinical Neuroscience 1999;249(Suppl1):S7-S10.

\section{Uhlenhuth 1989 \{published data only\}}

Uhlenhuth EH, Matuzas W, Glass RM, Easton C. Response of panic disorder to fixed doses of alprazolam or imipramine. Journal of Affective Disorders 1989;17(3):261-70.

\section{Van Vliet 1996 \{published data only\}}

Slaap BR, van Vliet IM, Westenberg HGM, den Boer JA. Phobic symptoms as predictors of nonresponse to drug therapy in panic disorder patients (a preliminary report). Journal of Affective Disorders 1995;33(1):31-8.

* van Vliet IM, den Boer JA, Westenberg HGM, Slaap BR. A double-blind comparative study of brofaromine and fluvoxamine in outpatients with panic disorder. Journal of Clinical Psychopharmacology 1996;16(4):299-306.

\section{Wade 1997 \{published data only\}}

Leinonen E, Lepola U, Koponen H, Turtonen J, Wade A, Lehto H. Citalopram controls phobic symptoms in patients with panic disorder: randomized controlled trial. Journal of Psychiatry and Neuroscience 2000;25(1):24-32.

Lepola U, Wade AG, Leinonen EV, Koponen HJ, Frazer J, Sjoedin I, et al. A controlled, prospective, 1-year trial of citalopram in the treatment of panic disorder. Journal of Clinical Psychiatry 1998;59:528-34.
Wade A, Overe KF, Lemming $\mathrm{O}$. Weight monitoring during two long-term trials of citalopram. European Neuropsychopharmacology 1999;9 Suppl 5:S221.

* Wade AG, Lepola U, Koponen HJ, Pedersen V, Pedersen T. The effect of citalopram in panic disorder. British Journal of Psychiatry 1997;170:549-53.

Westenberg 1989 \{published data only\}

* Westenberg HG, den Boer JA. Selective monoamine uptake inhibitors and a serotonin antagonist in the treatment of panic disorder. Psychopharmacology Bulletin 1989;25(1):119-23.

Zhang 2000 \{published data only\}

Zhang HY, Zhao QP, Ma C. Paroxetine versus clomipramine for the treatment of panic disorders: a double-blind randomised study. Chinese Mental Health Journal 2000;14(6):410-3.

\section{References to studies excluded from this review}

Bakish 1994 \{published data only\}

Bakish $D$. The use of the reversible monoamine oxidase-A inhibitor brofaromine in social phobia complicated by panic disorder with or without agoraphobia. Journal of Clinical Psychopharmacology 1994;14(1):74-5.

Baldini Rossi 2000 \{published data only\}

Baldini Rossi NA, Cassano PA, Dell'Osso LA, Ciapparelli AA, Bandettini di Poggio AA, Russo AA, et al. Depression comorbid with panic disorder or other anxiety disorders: a 16-week multicentre randomised parallel-group trial of moclobemide versus paroxetine. European Neuropsychopharmacology 2000;10(Suppl 2):S52-S53.

Balon 1991 \{published data only\}

Balon R, Pohl R, Yeragani VK, Ramesh C, Glitz DA. The changes of thyroid hormone during pharmacological treatment of panic disorder patients. Progress in Neuropsychopharmacology and Biological Psychiatry 1991;15(5):595-600.

Balon 1993 \{published data only\}

Balon R, Yeragani VK, Pohl R, Merlos B, Sherwood P. Changes in appetite and weight during the pharmacological treatment of patients with panic disorder. Canadian Journal of Psychiatry 1993;38(1):19-22

Bystritsky 1990 \{published data only\}

Bystritsky AA, Pasnau RO. Initial reaction and subsequent response to antidepressants in panic patients. American Journal of Psychiatry 1990;147(11):1575.

Charney 1986 \{published data only\}

Charney DS, Woods SW, Goodman WK, Rifkin B, Kinch M, Aiken B, et al. Drug treatment of panic disorder: the comparative efficacy of imipramine, alprazolam, and trazodone. Journal of Clinical Psychiatry 1986;47(12):580-6.

Davis 1981 \{published data only\}

Davis JM, Nasr S, Spira N, Vogel C. Anxiety: differential diagnosis and treatment from a biologic perspective. Journal of Clinical Psychiatry 1981;42(11 pt 2):4-14. 
de Jonghe 1989 \{published data only\}

de Jonghe F, Swinkels J, Tuynman-Qua H, Jonkers F. A comparative study of suriclone, lorazepam and placebo in anxiety disorder. Pharmacopsychiatry 1989;22(6):266-71.

\section{Dell'Erba 2006 \{published data only\}}

* Dell'Erba GL, Nuzzo E. Effectiveness treatment for panic and agoraphobia in comparison between drug and specialized psychological treatment [Il trattamento efficace nella pratica del disturbo di panico e agorafobia in una valutazione comparativa tra psicofarmacologia e trattamento psicologico specifico]. Rivista di Psichiatria 2006;41(6):397-403.

\section{den Boer 1987 \{published data only\}}

den Boer JA, Westenberg HG, Kamerbeek WD, Verhoeven WM, Kahn RS. Effect of serotonin uptake inhibitors in anxiety disorders; a double-blind comparison of clomipramine and fluvoxamine. International Clinical Psychopharmacology 1987;2(1):21-32.

\section{Dunner 1986 \{published data only\}}

Dunner DL, Ishiki D, Avery DH, Wilson LG, Hyde TS. Effect of alprazolam and diazepam on anxiety and panic attacks in panic disorder: a controlled study. Journal of Clinical Psychiatry 1986;47(9):458-60.

\section{Dyukova 1992 \{published data only\}}

Dyukova GM, Shepeleva IP, Vorob'eva OV. Treatment of negative crises (panic attacks). Neuroscience and Behavioral Physiology 1992;22(4):343-5.

\section{Evans 1986 \{published data only\}}

Evans L, Kenardy J, Schneider P, Hoey H. Effect of a selective serotonin uptake inhibitor in agoraphobia with panic attacks. A double-blind comparison of zimeldine, imipramine and placebo. Acta Psychiatrica Scandinavica 1986;73(1):49-53.

\section{Fahy 1992 \{published data only\}}

Fahy TJ, O'Rourke D, Brophy J, Schazmann W, Sciascia S. Clomipramine and lofepramine in DSM III-R panic disorder: a placebo controlled trial. Clinical Neuropharmacology 1992;15(1 pt B):94.

* Fahy TJ, O'Rourke D, Brophy J, Schazmann W, Sciascia S. The Galway Study of Panic Disorder. I: Clomipramine and lofepramine in DSM III-R panic disorder: a placebo controlled trial. Journal of Affective Disorders 1992;25(1):63-75.

\section{Franulic 1989 \{published data only\}}

Franulic AM, Sanchez GV, O'Ryan FG, Gladic DM, Barahona MC, Gloger SK. Clomipramine and diazepam plasma levels in panic disorder and agoraphobia. Preliminary findings [Concentraciones plasmaticas de clomipramina y diazepam en Desorden de Panico y Agoraphobia. Un estudio preliminar]. Revista Chilena de Neuro-Psiquiatria 1989;27:101-10.

\section{Furukawa 2009 \{published data only\}}

Furukawa TA, Katherine Shear M, Barlow DH, Gorman JM, Woods SW, Money R, et al. Evidence-based guidelines for interpretation of the Panic Disorder Severity Scale. Depression and Anxiety 2009;26(10):922-9.
Grilo 1998 \{published data only\}

Grilo CM, Money R, Barlow DH, Goddard AW, Gorman JM, Hofmann SG, et al. Pretreatment patient factors predicting attrition from a multicenter randomized controlled treatment study for panic disorder. Comprehensive Psychiatry 1998;39(6):323-32.

\section{Keller 1993 \{published data only\}}

* Keller MB, Lavori PW, Goldenberg IM, Baker LA, Pollack MH, Sachs GS, et al. Influence of depression on the treatment of panic disorder with imipramine, alprazolam and placebo. Journal of Affective Disorders 1993;28(1):27-38.

Leon AC, Shear MK, Portera L, Klerman GL. Effect size as a measure of symptom-specific drug change in clinical trials. Psychopharmacology Bulletin. 1993;29(2):163-7.

Pollack MH, Otto MW, Sachs GS, Leon A, Shear MK, Deltito JA, et al. Anxiety psychopathology predictive of outcome in patients with panic disorder and depression treated with imipramine, alprazolam and placebo. Journal of Affective Disorders 1994;30(4):273-81.

Shear MK, Leon AC, Pollack MH, Rosenbaum JF, Keller MB. Pattern of placebo response in panic disorder. Psychopharmacology Bulletin 1995;31(2):273-8.

\section{Lepola 1989 \{published data only\}}

Lepola U, Jolkkonen J, Rimón R, Riekkinen P. Long-term effects of alprazolam and imipramine on cerebrospinal fluid monoamine metabolites and neuropeptides in panic disorder. Neuropsychobiology 1989;21(4):182-6.

Mavissakalian 2003 \{published data only\}

Mavissakalian MR. Imipramine vs. sertraline in panic disorder: 24-week treatment completers. Annals of Clinical Psychiatry 2003;15(3):171-80.

\section{McHugh 2007 \{published data only\}}

McHugh RK, Otto MW, Barlow DH, Gorman JM, Shear MK, Woods SW. Cost-efficacy of individual and combined treatments for panic disorder. Journal of Clinical Psychiatry 2007;68(7):1038-44.

\section{Miretzky 1992 \{published data only\}}

Miretzky A, Horn R, Koehler K, Moeller HJ. Combination of alprazolam, antidepressive drugs and cognitive behavior therapy in the treatment of panic disorder. Clinical Neuropharmacology 1992;15(1 pt B):536.

\section{Nair 1982 \{published data only\}}

Nair NP, Singh AN, Lapierre Y, Saxena BM, Nestoros JN, Schwartz G. Ketazolam in the treatment of anxiety: a standard and placebo controlled study. Current Therapeutic Research, Clinical \& Experimental 1982;31(5):679-91.

\section{Nardi 2011 \{published data only\}}

Nardi AE, Freire RC, Mochcovitch MD, Amrein R, Levitan MN, King AL, et al. A randomized, naturalistic, parallel-group study for the long-term treatment of panic disorder with clonazepam or paroxetine. Journal of Clinical Psychopharmacology 2012;32(1):120-6. 
Nardi AE, Valença AM, Freire RC, Amrein R, Sardinha A, Levitan MN, et al. Randomized, open naturalistic, acute treatment of panic disorder with clonazepam or paroxetine. Journal of Clinical Psychopharmacology 2011;31(2):259-61.

* Nardi AE, Valença AM, Freire RC, Mochcovitch MD, Amrein R, Sardinha A, et al. Psychopharmacotherapy of panic disorder: 8-week randomized trial with clonazepam and paroxetine. Brazilian Journal of Medical and Biological Research 2011;44(4):366-73

\section{Pareek 2014 \{published data only\}}

Ipca Laboratories Ltd. Comparative Evaluation of Efficacy and Safety of Clonazepam-CR and Conventional Clonazepam in Patients with Panic Disorder. http://www.ctri.nic.in.

Pfizer 2002 \{unpublished data only\}

Pfizer. A double-blind, placebo-controlled, parallel-group comparison of venlafaxine extended-release capsules and paroxetine in outpatients with panic disorder. http:// clinicaltrials.gov/show/NCT00044772 2002.

\section{Pfizer 2005 \{unpublished data only\}}

Pfizer. Pilot study of venlafaxine extended release (XR) in the treatment of panic disorder (PD) in comparison to paroxetine. http://clinicaltrials.gov/show/NCT00195598 2005.

\section{Pohl 1989 \{published data only\}}

* Pohl R, Rickels K, Charney D. Clinical results on the use of lorazepam to treat panic attacks [Risultati clinici sull'uso del lorazepam nel disturbo da attacchi di panico]. Rivista di Psichiatria 1989;24(2):99-100.

Schweizer E, Fox I, Case G, Rickels K. Lorazepam vs. alprazolam in the treatment of panic disorder. Psychopharmacol Bulletin 1988;24(2):224-7.

Schweizer E, Pohl R, Balon R, Fox I, Rickels K, Yeragani VK. Lorazepam vs. alprazolam in the treatment of panic disorder. Pharmacopsychiatry 1990;23(2):90-3.

\section{Pollack 2003 \{published data only\}}

Pollack MH, Simon NM, Worthington JJ, Doyle AL, Peters P, Toshkov F, et al. Combined paroxetine and clonazepam treatment strategies compared to paroxetine monotherapy for panic disorder. Journal of Psychopharmacology 2003;17(3):276-82.

\section{Pyke 1989 \{published data only\}}

Pyke RE, Greenberg HS. Double-blind comparison of alprazolam and adinazolam for panic and phobic disorders. Journal of Clinical Psychopharmacology 1989;9(1):15-21.

\section{Raffaele 2002 \{published data only\}}

Raffaele R, Vecchio I, Malaguarnera M, Rampello L, Ruggieri M, Nicoletti F. Therapy of panic attacks in the elderly. Archives of Gerontology and Geriatrics. Supplement 2002;8:295-301.

\section{Roll 2004 \{published data only\}}

Roll D, Ray SE, Marcus SM, Passarelli V, Money R, Barlow DH, et al. Independent evaluator knowledge of treatment in a multicenter comparative treatment study of panic disorder. Neuropsychopharmacology 2004;29(3):612-8.

Roy-Byrne 2001 \{published data only\}

Roy-Byrne PP, Katon W, Cowley DS, Russo J. A randomized effectiveness trial of collaborative care for patients with panic disorder in primary care. Archives of General Psychiatry 2001;58(9):869-76.

\section{Sheehan 1980 \{published data only\}}

Sheehan DV, Ballenger J, Jacobsen G. Treatment of endogenous anxiety with phobic, hysterical, and hypochondriacal symptoms. Archives of General Psychiatry 1980;37(1):51-9.

\section{Surman 1986 \{published data only\}}

Surman OS, Williams J, Sheehan DV, Strom TB, Jones KJ, Coleman J. Immunological response to stress in agoraphobia and panic attacks. Biological Psychiatry 1986;21(8-9):768-74.

Svebak 1990 \{published data only\}

Svebak S, Cameron A, Levander S. Clonazepam and imipramine in the treatment of panic attacks: a double-blind comparison of efficacy and side effects. Journal of Clinical Psychiatry 1990;51(Suppl 5):14-17.

\section{Tsutsui 2000 \{published data only\}}

Tsutsui S. Clinical evaluation of paroxetine $\mathrm{HCl}$, a selective serotonin reuptake inhibitor, in the treatment of panic disorder: Phase III double-blind, parallel group study [選択的セロト. ニン再取り込み阻害薬塩酸パロキセチンのパニック障害 対する臨床評価] . Japanese Pharmacology \& Therapeutics 2000;28(Suppl 1):S295-S314.

\section{Tyrer 1988 \{published data only\}}

Tyrer P, Seivewright N, Ferguson B, Murphy S, Darling C, Brothwell J, et al. The Nottingham Study of Neurotic Disorder: relationship between personality status and symptoms. Psychological Medicine 1990;20(2):423-31.

Tyrer P, Seivewright N, Ferguson B, Murphy S, Johnson AL. The Nottingham study of neurotic disorder. Effect of personality status on response to drug treatment, cognitive therapy and self-help over two years. British Journal of Psychiatry 1993;162:219-26.

* Tyrer P, Seivewright N, Murphy S, Ferguson B, Kingdon D, Barczak $\mathrm{P}$, et al. The Nottingham study of neurotic disorder: comparison of drug and psychological treatments. Lancet 1988;2(8605):235-40.

\section{van Apeldoorn 2008 \{published data only\}}

van Apeldoorn FJ, van Hout WJ, Mersch PP, Huisman M, Slaap BR, Hale WW 3rd, et al. Is a combined therapy more effective than either CBT or SSRI alone? Results of a multicenter trial on panic disorder with or without agoraphobia. Acta Psychiatrica Scandinavica 2008;117(4):260-70.

\section{Yang 2005 \{published data only\}}

Yang H, Yu C, Gao H. The control study of mirtazapine in treating patients with panic disorder. Medical Journal of Chinese People Health 2005;17(3):133-5. 
Yang 2006 \{published data only\}

Yang $\mathrm{H}, \mathrm{Gao} \mathrm{H}$, Yu C. Effect of citalopram in the treatment of panic disorder. Shandong Archives of Psychiatry 2006;19(2):186-7.

\section{Yeragani 1992 \{published data only\}}

Yeragani VK, Pohl R, Balon R, Ramesh C, Weinberg P. Imipramine-induced jitteriness and decreased serum iron levels. Neuropsychobiology 1992;25(1):8-10.

\section{Zajecka 1996 \{published data only\}}

Zajecka JM. The effect of nefazodone on comorbid anxiety symptoms associated with depression: experience in family practice and psychiatric outpatient settings. Journal of Clinical Psychiatry 1996;57(Suppl 2):10-4.

\section{References to studies awaiting assessment}

Minutentag 2001 \{published data only\}

Minutentag NW, Bernik MA. Comparative study of citalopram and imipramine in the treatment of panic disorder [Estudo comparativo entre o citalopram e a imiprimina no tratamento do transtorno de panico]. Revista de Psiquiatria Clinica 2001;28(2):44-51.

\section{Additional references}

\section{Als-Nielsen 2003}

Als-Nielsen B, Chen W, Gluud C, Kjaergard LL. Association of funding and conclusions in randomized drug trials: a reflection of treatment effect or adverse events?. JAMA 2003;290:921-8.

\section{Altman 1996}

Altman DG, Bland MJ. Detecting skewness for summary information. BMJ 1996;313:1200.

\section{APA 1980}

American Psychiatric Association. Diagnostic and Statistical Manual of Mental Disorders (DSM-III). Third Edition. Washington, DC: American Psychiatric Association, 1980.

\section{APA 1994}

American Psychiatric Association. Diagnostic and Statistical Manual of Mental Disorders (DSM-IV). Fourth. Washington, DC: American Psychiatric Association, 1994.

\section{APA 2009}

American Psychiatric Association. Practice Guideline for the Treatment of Patients With Panic Disorder. Second Edition. 2009. Available from http://psychiatryonline.org/pb/assets/ raw/sitewide/practice_guidelines/guidelines/panicdisorder.pdf.

\section{APA 2013a}

American Psychiatric Association. Diagnostic and Statistical Manual of Mental Disorders (DSM-5). Fifth. Washington, DC: American Psychiatric Association, 2013.

\section{APA 2013b}

American Psychiatric Association. Highlights of Changes from DSM-IV-TR to DSM-5. 2013. Available from http://
www.dsm5.org/Documents/changes $\% 20$ from $\% 20$ dsm-iv-tr \%20to\%20dsm-5.pdf.

\section{Bakker 2002}

Bakker A, van Balkom AJ, Spinhoven P. SSRIs vs. TCAs in the treatment of panic disorder: a meta-analysis. Acta Psychiatrica Scandinavica 2002;106:163-7.

\section{Ballenger 1998}

Ballenger JC, Davidson JR, Lecrubier Y, Nutt DJ, Baldwin DS, den Boer JA, et al. Consensus statement on panic disorder from the International Consensus Group on Depression and Anxiety. Journal of Clinical Psychiatry 1998;59(Suppl 8):47-54.

\section{Bandelow 2006}

Bandelow B, Baldwin DS, Dolberg OT, Andersen HF, Stein DJ. What is the threshold for symptomatic response and remission for major depressive disorder, panic disorder, social anxiety disorder, and generalized anxiety disorder?. Journal of Clinical Psychiatry 2006;67:1428-34.

\section{BAP 2005}

Baldwin DS, Anderson IM, Nutt DJ, Bandelow B, Bond A, Davidson JR, et al. Evidence-based guidelines for the pharmacological treatment of anxiety disorders: recommendations from the British Association for Psychopharmacology. Journal of Psychopharmacology 2005;19:567-96.

\section{Batelaan 2012}

Batelaan NM, Van Balkom AJ, Stein DJ. Evidence-based pharmacotherapy of panic disorder: an update. International Journal of Neuropsychopharmacology 2012;15(3):403-15.

\section{Bhandari 2004}

Bhandari M, Busse JW, Jackowski D, Montori VM, Schünemann H, Sprague S, et al. Association between industry funding and statistically significant pro-industry findings in medical and surgical randomized trials. Canadian Medical Association Journal 2004;170:477-80.

\section{Bijl 1998}

Bijl RV, Ravelli A, van Zessen G. Prevalence of psychiatric disorder in the general population: results of The Netherlands Mental Health Survey and Incidence Study (NEMESIS). Social Psychiatry and Psychiatric Epidemiology 1998;33:587-95.

\section{Boissel 1999}

Boissel JP, Cucherat M, Li W, Chatellier G, Gueyffier F, Buyse M, et al. The problem of therapeutic efficacy indices. Comparison of the indices and their use. Therapie 1999;54:405-11.

\section{Briley 1993}

Briley M, Moret C. Neurobiological mechanisms involved in antidepressant therapies. Clinical Neuropharmacology 1993;16:387-400

\section{Bruce 2003}

Bruce SE, Vasile RG, Goisman RM, Salzman C, Spencer M, Machan JT, et al. Are benzodiazepines still the medication 
of choice for patients with panic disorder with or without agoraphobia?. American Journal of Psychiatry 2003;160:1432-8.

\section{Buchkowsky 2004}

Buchkowsky SS, Jewesson PJ. Industry sponsorship and authorship of clinical trials over 20 years. Annals of Pharmacotherapy 2004;38:579-85.

\section{Buscemi 2006}

Buscemi N, Hartling L, Vandermeer B, Tjosvold L, Klassen TP. Single data extraction generated more errors than double data extraction in systematic reviews. Journal of Clinical Epidemiology 2006;59(7):697-703.

\section{Cameron 2007}

Cameron OG, Huang GC, Nichols T, Koeppe RA, Minoshima S, Rose $D$, et al. Reduced gamma-aminobutyric $\operatorname{acid}(A)$ benzodiazepine binding sites in insular cortex of individuals with panic disorder. Archives of General Psychiatry 2007;64:793-800.

\section{Deeks 2002}

Deeks JJ. Issues in the selection of a summary statistic for metaanalysis of clinical trials with binary outcomes. Statistics in Medicine 2002;21(11):1575-600.

\section{Eaton 1994}

Eaton WW, Kessler RC, Wittchen H-U, Magee WJ. Panic and panic disorder in the United States. American Journal of Psychiatry 1994;151:413-20.

\section{Elbourne 2002}

Elbourne DR, Altman DG, Higgins JP, Curtin F, Worthington HV, Vail A. Meta-analyses involving cross-over trials: methodological issues. International Journal of Epidemiology 2002;31:140-9.

\section{Furukawa 2002}

Furukawa TA, Guyatt GH, Griffith LE. Can we individualize the 'number needed to treat'? An empirical study of summary effect measures in meta-analyses. International Journal of Epidemiology 2002;31:72-6.

\section{Furukawa 2005}

Furukawa TA, Cipriani A, Barbui C, Brambilla P, Watanabe N. Imputing response rates from means and standard deviations in meta-analysis. International Clinical Psychopharmacology 2005;20:49-52.

\section{Furukawa 2006}

Furukawa TA, Barbui C, Cipriani A, Brambilla P, Watanabe N. Imputing missing standard deviations in meta-analyses can provide accurate results. Journal of Clinical Epidemiology 2000;59:7-10.

\section{Furukawa 2007}

Furukawa TA, Watanabe N, Churchill R. Combined psychotherapy plus antidepressants for panic disorder with or without agoraphobia. Cochrane Database of Systematic Reviews 2007, Issue 1. [DOI: 10.1002/14651858.CD004364.pub2]

\section{Goisman 1995}

Goisman RM, Warshaw MG, Steketee GS, Fierman EJ, Rogers MP, Goldenberg I, et al. DSM-IV and the disappearance of agoraphobia without a history of panic disorder: new data on a controversial diagnosis. American Journal of Psychiatry 1995;152:1438-43.

\section{Gorman 2000}

Gorman JM, Kent JM, Sullivan GM, Coplan JD. Neuroanatomical hypothesis of panic disorder, revised. American Journal of Psychiatry 2000;157:493-505.

\section{Grant 2006}

Grant BF, Hasin DS, Stinson FS, Dawson DA, Goldstein RB, Smith S, et al. The epidemiology of DSM-IV panic disorder and agoraphobia in the United States: results from the National Epidemiologic Survey on Alcohol and Related Conditions. Journal of Clinical Psychiatry 2006;67:363-74.

\section{Guaiana 2013a}

Guaiana G, Barbui C, Chiodo D, Cipriani A, Davies SJC, Koesters M. Benzodiazepines versus placebo for panic disorder in adults. Cochrane Database of Systematic Reviews 2013, Issue 7. [DOI: 10.1002/14651858.CD010677]

\section{Guaiana 2013b}

Guaiana G, Barbui C, Chiodo D, Cipriani A, Davies SJC, Koesters M. Antidepressants versus placebo for panic disorder in adults. Cochrane Database of Systematic Reviews 2013, Issue 7. [DOI: 10.1002/14651858.CD010676]

\section{Guaiana 2014}

Guaiana G, Barbui C, Caldwell D, Davies SJC, Furukawa T, Imai H, et al. Antidepressants, benzodiazepines and azapirones for panic disorder in adults: a network meta-analysis. Cochrane Database of Systematic Reviews 2014 (Data on file).

\section{Higgins 2011}

Higgins JPT, Green S (editors). Cochrane Handbook for Systematic Reviews of Interventions Version 5.1.0 [updated March 2011]. The Cochrane Collaboration, 2011. Available from www.cochrane-handbook.org.

\section{Kessler 2006}

Kessler RC, Chiu WT, Jin R, Ruscio AM, Shear K, Walters EE. The epidemiology of panic attacks, panic disorder, and agoraphobia in the National Comorbidity Survey Replication. Archives of General Psychiatry 2006;63:415-24.

\section{King 2008}

King M, Nazareth I, Levy G, Walker C, Morris R, Weich S, et al. Prevalence of common mental disorders in general practice attendees across Europe. British Journal of Psychiatry 2008;192:362-7.

\section{Klein 1964}

Klein DF. Delineation of two drug-responsive anxiety syndromes. Psychopharmacologia 1964;5:397-408. 


\section{Lexchin 2003}

Lexchin J, Bero LA, Djulbegovic B, Clark O. Pharmaceutical industry sponsorship and research outcome and quality: systematic review. BMJ 2003;326:1167-70.

\section{Malizia 1998}

Malizia AL, Cunningham VJ, Bell CJ, Liddle PF, Jones T, Nutt DJ. Decreased brain GABA(A)-benzodiazepine receptor binding in panic disorder: preliminary results from a quantitative PET study. Archives of General Psychiatry 1998;55:715-20.

\section{Moher 2009}

Moher D, Liberati A, Tetzlaff J, Altman DG, The PRISMA group. Preferred Reporting Items for Systematic Reviews and Meta-Analyses: The PRISMA Statement. PLoS Medicine 2009;6(6):e1000097.

\section{Nash 2008}

Nash JR, Sargent PA, Rabiner EA, Hood SD, Argyropoulos SV, Potokar JP, et al. Serotonin 5-HT1A receptor binding in people with panic disorder: positron emission tomography study. British Journal of Psychiatry 2008;1993:229-34.

\section{NICE 2011}

National Institute for Health and Care Excellence. Generalised anxiety disorder and panic disorder (with or without agoraphobia) in adults: Management in primary, secondary and community care. NICE guidelines [CG113]. Published date: January 2011. Available from http://www.nice.org.uk/guidance/ cg113/chapter/guidance.

\section{Offidani 2013}

Offidani E, Guidi J, Tomba E, Fava GA. Efficacy and tolerability of benzodiazepines versus antidepressants in anxiety disorders: a systematic review and meta-analysis. Psychotherapy and Psychosomatics 2013;82:355-62.

\section{Otto 2001}

Otto MW, Tuby KS, Gould RA, McLean RY, Pollack MH. An effectsize analysis of the relative efficacy and tolerability of serotonin selective reuptake inhibitors for panic disorder. American Journal of Psychiatry 2000;158:1989-92.

\section{Pompoli 2016}

Pompoli A, Furukawa TA, Imai H, Tajika A, Efthimiou O, Salanti G. Psychological therapies for panic disorder with or without agoraphobia in adults: a network meta-analysis. Cochrane Database of Systematic Reviews 2016, Issue 4. [DOI: 10.1002/14651858.CD011004.pub2]

\section{Sanchez-Meca 2010}

Sánchez-Meca J, Rosa-Alcázar Al, Marín-Martínez F, GómezConesa A. Psychological treatment of panic disorder with or

\section{CHARACTERISTICS OF STUDIES}

\section{Characteristics of included studies [ordered by study ID]}

without agoraphobia: a meta-analysis. Clinical Psychology Review 2010;30:37-50.

\section{Starcevic 2009}

Starcevic V. Anxiety Disorders in Adults: A Clinical Guide. New York: Oxford University Press, 2009.

\section{Starcevic 2014}

Starcevic V. The reappraisal of benzodiazepines in the treatment of anxiety and related disorders. Expert Review of Neurotherapeutics 2014;14(11):1275-1286.

\section{Stein 2010}

Stein M, Steckler T, Lightfoot JD, Hay E, Goddard AW. Pharmacologic treatment of panic disorder. Current Topics in Behavioral Neurosciences 2010;2:469-85.

\section{Wade 1999}

Wade AG. Antidepressants in panic disorder. International Clinical Psychopharmacology 1999;14(Suppl 2):13-7.

\section{Watanabe 2009}

Watanabe N, Churchill R, Furukawa TA. Combined psychotherapy plus benzodiazepines for panic disorder. Cochrane Database of Systematic Reviews 2009, Issue 1. [DOI: 10.1002/14651858.CD005335.pub2]

\section{WHO 2003}

World Health Organization. Introduction to drug utilization research. WHO International Working Group for Drug Statistics Methodology; WHO Collaborating Centre for Drug Statistics Methodology; WHO Collaborating Centre for Drug Utilization Research and Clinical Pharmacological Services. 2003. Available from http://www.who.int/medicines/areas/quality_safety/ safety_efficacy/utilization/en/.

\section{Wilkinson 1991}

Wilkinson G, Balestrieri M, Ruggeri M, Bellantuono C. Meta-analysis of double-blind placebo-controlled trials of antidepressants and benzodiazepines for patients with panic disorders. Psychological Medicine 1991;21:991-8.

\section{References to other published versions of this review \\ Guaiana 2015}

Guaiana G, Barbui C, Bighelli I, Trespidi C, Chiodo D, Cipriani A, et al. Antidepressants and benzodiazepines for panic disorder in adults. Cochrane Database of Systematic Reviews 2015, Issue Issue 3. [DOI: 10.1002/14651858.CD011567]

* Indicates the major publication for the study 
Amore 1999

\begin{tabular}{|c|c|}
\hline Methods & Study design: Randomised controlled trial \\
\hline \multirow[t]{7}{*}{ Participants } & Diagnosis: DSM-IV Panic Disorder with or without agoraphobia \\
\hline & Method of diagnosis: Not stated \\
\hline & Age: for fluoxetine, $M=37.0(S D=7.1)$; for imipramine, $M=37.2(S D=8.2)$ \\
\hline & Sex: for fluoxetine, $57.89 \%$ women, $42.11 \%$ men; for imipramine $36.84 \%$ women, $63.16 \%$ men \\
\hline & Location: Italy; setting unclear. \\
\hline & $\begin{array}{l}\text { Co-morbidities: patients with history of psychosis, current major depression, organic brain syndromes } \\
\text { or significant neurological disorders, seizures, clinically relevant cardiovascular, hepatic, renal or } \\
\text { haematological diseases were excluded }\end{array}$ \\
\hline & $\begin{array}{l}\text { Rescue medication: Oxazepam (up to a maximum daily dose of } 30 \mathrm{mg} \text { ) permitted during first four } \\
\text { weeks of double-blind treatment }\end{array}$ \\
\hline \multirow[t]{7}{*}{ Interventions } & Participants were randomly assigned to either: \\
\hline & (1) fluoxetine arm ( $n=19)$ \\
\hline & $\begin{array}{l}\text { Duration: } 24 \text { weeks of active treatment (acute and continuation phase), } 6 \text { months maintenance phase } \\
\text { for responders }\end{array}$ \\
\hline & Treatment Protocol: flexible dosage; range $=10-50 \mathrm{mg}, \mathrm{M}=20 \mathrm{mg} / \mathrm{day}(\mathrm{SD}=10)$ (responder group) \\
\hline & (2) imipramine arm $(n=19)$ \\
\hline & $\begin{array}{l}\text { Duration: } 24 \text { weeks of active treatment (acute and continuation phase), } 6 \text { months maintenance phase } \\
\text { for responders }\end{array}$ \\
\hline & Treatment Protocol: flexible dosage; range $=25-250 \mathrm{mg}, \mathrm{M}=150 \mathrm{mg} / \mathrm{day}(\mathrm{SD}=25)($ responder group) \\
\hline \multirow[t]{6}{*}{ Outcomes } & $\begin{array}{l}\text { Time points for assessment: baseline and weekly for } 16 \text { weeks, every two weeks between week } 17 \\
\text { and } 24 \text {, later monthly }\end{array}$ \\
\hline & Outcomes: \\
\hline & 1. Panic-Associated Symptoms Scale (PASS) \\
\hline & 2. Hamilton Rating Scale for Anxiety (HAMA) \\
\hline & 3. Hamilton Rating Scale for Depression (HRSD) \\
\hline & 4. Clinical Global Impression (CGI) \\
\hline
\end{tabular}

\begin{tabular}{lll}
\hline Notes & Date of study: Not stated & Funding source: Not stated \\
& Declarations of interest among the primary researchers: Not stated \\
\hline Risk of bias & \\
\hline Bias & Authors' judgement & Support for judgement \\
\hline $\begin{array}{lll}\text { Random sequence genera- } \\
\text { tion (selection bias) }\end{array}$ & Unclear risk & $\begin{array}{l}\text { Quote: "they were randomly assigned to fluoxetine or imipramine treatment". } \\
\text { No further details. }\end{array}$ \\
\hline $\begin{array}{l}\text { Allocation concealment } \\
\text { (selection bias) }\end{array}$ & Unclear risk & No information provided. \\
\hline
\end{tabular}


Amore 1999 (Continued)

Blinding of participants Unclear risk Quote: "double blind". No further details. and personnel (performance bias)

All outcomes

Blinding of outcome as-
sessment (detection bias) $\quad$ Unclear risk Quote: "double blind". No further details.

All outcomes

Incomplete outcome data Unclear risk

No clear information on incomplete outcome data management.

(attrition bias)

Noclear information on incomplete outcome data management.

All outcomes

Selective reporting (re- High risk Data on the scales CGI, PASS and HRSD not reported at endpoint.
porting bias)

Other bias Unclear risk Sponsorship bias cannot be ruled out.

Amore 1999 bis

Methods Study design: Randomised controlled trial

Participants

Diagnosis: DSM-IV Panic Disorder with or without agoraphobia

Method of diagnosis: Not stated

Age: for fluoxetine, $M=37.2(S D=7.0)$; for citalopram, $M=36.7(S D=7.4)$

Sex: for fluoxetine, $57.1 \%$ women, $42.9 \%$ men; for imipramine $61.9 \%$ women, $38.1 \%$ men

Location: Italy; setting unclear

Co-morbidities: patients with history of psychosis, current major depression, organic brain syndromes or significant neurological disorders, seizures, clinically relevant cardiovascular, hepatic, renal or haematological diseases, alcohol or drugs abuse were excluded

Rescue medication: Oxazepam (up to a maximum daily dose of $30 \mathrm{mg}$ ) permitted

Interventions Participants were randomly assigned to either:

(1) fluoxetine arm $(n=21)$

Duration: 24 weeks of active treatment (acute and continuation phase), 6 months maintenance phase for responders

Treatment Protocol: flexible dosage; range $=10-50 \mathrm{mg}, \mathrm{M}=20 \mathrm{mg} / \mathrm{day}(\mathrm{SD}=10)$

(2) citalopram arm $(n=21)$

Duration: 24 weeks of active treatment (acute and continuation phase), 6 months maintenance phase for responders

Treatment Protocol: flexible dosage; range $=20-60 \mathrm{mg}, \mathrm{M}=40 \mathrm{mg} /$ day $(\mathrm{SD}=10)$

Outcomes

Time points for assessment: baseline and weekly for 16 weeks, every two weeks between week 17 and 24 , later monthly

\section{Outcomes:}

1. Panic-Associated Symptoms Scale (PASS) 
Amore 1999 bis (Continued)
2. Hamilton Rating Scale for Anxiety (HAMA)
3. Clinical Global Impression (CGI)
4. Dosage Records and Treatment-Emergent Symptoms Scale (DOTES)

Dotes of study: Not stated
Funding source: Not stated
Declarations of interest among the primary researchers: Not stated

\section{Risk of bias}

\begin{tabular}{lll}
\hline Bias & Authors' judgement & Support for judgement \\
\hline $\begin{array}{l}\text { Random sequence genera- } \\
\text { tion (selection bias) }\end{array}$ & Unclear risk & Quote: "they were randomly assigned". No further details. \\
\hline $\begin{array}{l}\text { Allocation concealment } \\
\text { (selection bias) }\end{array}$ & Unclear risk & No information provided. \\
\hline $\begin{array}{l}\text { Blinding of participants } \\
\text { and personnel (perfor- } \\
\text { mance bias) }\end{array}$ & Unclear risk & Quote: "double blind". No further details. \\
$\begin{array}{l}\text { All outcomes } \\
\text { Blinding of outcome as- } \\
\text { sessment (detection bias) } \\
\text { All outcomes }\end{array}$ & Unclear risk & Quote: "double blind". No further details. \\
\hline $\begin{array}{l}\text { Incomplete outcome data } \\
\text { (attrition bias) } \\
\text { All outcomes }\end{array}$ & Unclear risk & No clear information on incomplete outcome data management. \\
\hline $\begin{array}{l}\text { Selective reporting (re- } \\
\text { porting bias) }\end{array}$ & High risk & Data on the scales CGl, PASS and HAMA not reported at endpoint. \\
\hline \begin{tabular}{l} 
Other bias \\
\hline
\end{tabular} & Sponsorship bias cannot be ruled out. \\
\hline
\end{tabular}

\section{Bakish 1993}

\begin{tabular}{ll}
\hline Methods & Study design: Randomised controlled trial \\
\hline Participants & Diagnosis: DSM-III panic disorder with or without agoraphobia \\
Method of diagnosis: Not stated \\
Age: No information provided \\
Sex: No information provided \\
Location: Canada; setting: outpatients \\
Co-morbidities: Not stated \\
Rescue medication: Chloral hydrate, up to $1 \mathrm{~g}$ at night \\
\hline Interventions & Participants were randomly assigned to either: \\
\hline
\end{tabular}


Bakish 1993 (Continued)

(1) brofaromine arm $(n=47)$

Duration: 8 weeks

Treatment Protocol: flexible dosage; range $=50-150 \mathrm{mg}, \mathrm{M}$ and SD not provided

(2) clomipramine $\operatorname{arm}(n=46)$

Duration: 8 weeks

Treatment Protocol: flexible dosage; range $=25-75 \mathrm{mg}, \mathrm{M}$ and SD not provided

Time points for assessment: baseline, every two weeks
Outcomes:
1. number of panic attacks per week
2. Hamilton Rating Scale for Anxiety (HAMA)
3. Hamilton Rating Scales for Depression (HAM-D)
4. Clinician Rated Impairment and Disability Scale (CRIDS)
5. Clinician Rated Global Change Scale (CRGCS)
6. Patient Rating Impairment Disability Scale (PRIDS)
7. Patient Rated Anxiety Scale (PRAS)
8. Marks Matthews Phobia Scale
9. Patient Rated Global Change Scale (PRGCS)
10. Daily Panic Inventory (DPI)

Notes

Date of study: Not stated

Funding source: Not stated

Declarations of interest among the primary researchers: Not stated

\section{Risk of bias}

\begin{tabular}{lll}
\hline Bias & Authors' judgement & Support for judgement \\
\hline $\begin{array}{l}\text { Random sequence genera- } \\
\text { tion (selection bias) }\end{array}$ & Unclear risk & Quote: "randomised". No further details. \\
\hline $\begin{array}{l}\text { Allocation concealment } \\
\text { (selection bias) }\end{array}$ & Unclear risk & No information provided. \\
\hline
\end{tabular}

Blinding of participants Unclear risk Quote: "double blind". No further details.

and personnel (perfor-

mance bias)

All outcomes

Blinding of outcome as- Unclear risk $\quad$ Quote: "double blind". No further details.
sessment (detection bias)
All outcomes

Incomplete outcome data Unclear risk $\quad$ No clear information on incomplete outcome data management.
(attrition bias)


Bakish 1993 (Continued)

All outcomes

Selective reporting (re- High risk porting bias)
Data on the scales HAMD, CRIDS, CRGCS, PRIDS, PRAS, PRCGS, DPI not reported at endpoint; data on the scales HAMA and Mark Matthews Phobia Scale are reported only in graphs; number of patients evaluated not specified.

Other bias Unclear risk Sponsorship bias cannot be ruled out.

Bandelow 2004

\begin{tabular}{ll}
\hline Methods $\quad$ Study design: Randomised controlled trial \\
\hline
\end{tabular}

Participants

Diagnosis: DSM-IV and ICD-10 diagnosis of panic disorder with or without agoraphobia

Method of diagnosis: Not stated

Age: for sertraline, $M=39.6(S D=11.7)$; for paroxetine, $M=38.1(S D=11.7)$

Sex: for sertraline, $60 \%$ women, $40 \%$ men; for paroxetine $66 \%$ women, $34 \%$ men

Location: 5 centres in Denmark, 22 centres in Germany, 2 centres in the Netherlands, 2 centres in Switzerland, 2 centres in Turkey; setting: outpatients

Co-morbidities: patients with clinically significant and unstable medical illness, bipolar disorder, schizophrenic disorder, delusional disorder, epilepsy, major depressive disorder (MDD), obsessive-compulsive disorder (OCD), social phobia, history of alcoholism or drug abuse were excluded

Rescue medication: chloral hydrate, zolpidem or zopiclone allowed if necessary to treat severe insomnia, less than 3 times per week

(1) sertraline arm $(n=112)$

Duration: 12 weeks

Treatment Protocol: flexible dosage; range $=25-150 \mathrm{mg}, \mathrm{M}=84.5, \mathrm{SD}=39.1$

(2) paroxetine arm $(n=113)$

Duration: 12 weeks

Treatment Protocol: flexible dosage; range $=10-60 \mathrm{mg}, \mathrm{M}=48.1, \mathrm{SD}=11.2$

\section{Outcomes}

Time points for assessment: baseline, week 1, 2, 4, 6, 8, 12 and 15

\section{Outcomes:}

1. Panic and Agoraphobia Scale (PAS)

2. agoraphobia/avoidance behaviour

3. anticipatory anxiety

4. disability

5. health worries

6. Clinical Global Impression-Severity of IIIness (CGI-S)

7. Clinical Global Impression-Improvement (CGI-I) 
Bandelow 2004 (Continued)

8. Hamilton Rating Scale for Anxiety (HAMA)

9. Montgomery-Åsberg Depression Rating Scale (MADRS)

10. Sertraline Quality of Life Battery

11. Digit Symbol Substitution Task

12. Digit Span

13. Patient Global Impression (PGI)

Notes

Date of study: data were collected from January 2000 to June 2001

Funding source: Funded by Pfizer Inc, New York

Declarations of interest among the primary researchers: Dr Bandelow has received grant/research support from GlaxoSmithKline

\section{Risk of bias}

\begin{tabular}{|c|c|c|}
\hline Bias & Authors' judgement & Support for judgement \\
\hline $\begin{array}{l}\text { Random sequence genera- } \\
\text { tion (selection bias) }\end{array}$ & Unclear risk & Quote: "they were randomly assigned". No further details. \\
\hline $\begin{array}{l}\text { Allocation concealment } \\
\text { (selection bias) }\end{array}$ & Unclear risk & No information provided. \\
\hline $\begin{array}{l}\text { Blinding of participants } \\
\text { and personnel (perfor- } \\
\text { mance bias) } \\
\text { All outcomes }\end{array}$ & Unclear risk & Quote: "double blind". No further details. \\
\hline $\begin{array}{l}\text { Blinding of outcome as- } \\
\text { sessment (detection bias) } \\
\text { All outcomes }\end{array}$ & Unclear risk & Quote: "double blind". No further details. \\
\hline $\begin{array}{l}\text { Incomplete outcome data } \\
\text { (attrition bias) } \\
\text { All outcomes }\end{array}$ & Low risk & $\begin{array}{l}\text { "[...] a secondary analysis was performed on the ITT population, which consist- } \\
\text { ed of all patients who were randomly assigned to study drug and for whom at } \\
\text { least one post baseline PAS assessment was available" }\end{array}$ \\
\hline $\begin{array}{l}\text { Selective reporting (re- } \\
\text { porting bias) }\end{array}$ & Low risk & All outcomes were reported. \\
\hline Other bias & High risk & $\begin{array}{l}\text { Sponsored by Pfizer; the role of the funder in planning, conducting and writing } \\
\text { the study is not discussed. }\end{array}$ \\
\hline
\end{tabular}

\section{Bystritsky 1995}

\begin{tabular}{ll}
\hline Methods & Study design: Randomised controlled trial \\
\hline Participants & Diagnosis: DSM-III panic disorder with or without agoraphobia \\
Method of diagnosis: Not stated \\
Age: average age of 37 years, no between-group differences \\
Sex: 12 males and 9 females, no between-group differences \\
\hline
\end{tabular}


Location: USA; setting unclear

Co-morbidities: lack of significant drug or alcohol history or significant medical illness; patients that had an additional diagnosis of major depression (MD) or generalised anxiety disorder (GAD) were allowed to participate only if they presented a predominant picture of panic disorder and if panic symptoms preceded the onset of the current episode of MD or GAD

Rescue medication: Not stated.

Interventions Participants were randomly assigned to either:

(1) desipramine arm $(n=11)$

Duration: 10 weeks

Treatment Protocol: flexible dosage; range $=10-300 \mathrm{mg}, \mathrm{M}=110, \mathrm{SD}=49$

(2) fluoxetine arm $(n=11)$

Duration: 10 weeks

Treatment Protocol: flexible dosage; range $=2.5-60 \mathrm{mg}, \mathrm{M}=19, \mathrm{SD}=10$

Time points for assessment: weekly
Outcomes:
1. Hamilton Rating Scale for Anxiety (HAMA)
2. Hamilton Rating Scales for Depression (HAM-D)
3. Four Dimensional Anxiety Scale
4. Clinical Global Impression-Severity of IIIness (CGI-S)
5. Clinical Global Impression-Improvement (CGI-I)

Funding source: this research has been supported in part by NIMH grant MH 45342-02 and by an NPI Opportunity Grant

Declarations of interest among the primary researchers: None.

\section{Risk of bias}

\begin{tabular}{|c|c|c|}
\hline Bias & Authors' judgement & Support for judgement \\
\hline $\begin{array}{l}\text { Random sequence genera- } \\
\text { tion (selection bias) }\end{array}$ & Unclear risk & Quote: "they were assigned randomly". No further details. \\
\hline $\begin{array}{l}\text { Allocation concealment } \\
\text { (selection bias) }\end{array}$ & Unclear risk & No information provided. \\
\hline $\begin{array}{l}\text { Blinding of participants } \\
\text { and personnel (perfor- } \\
\text { mance bias) } \\
\text { All outcomes }\end{array}$ & Low risk & $\begin{array}{l}\text { Quote: "both patients and investigators were blind to the assignment"; "pa- } \\
\text { tients were administered identical capsules labeled A, B or C: Capsules A, con- } \\
\text { taining } 2,5 \mathrm{mg} \text { of fluoxetine or } 10 \mathrm{mg} \text { of desipramine were administered for } \\
\text { one week [...], capsules B (containing) } 25 \mathrm{mg} \text { of desipramine or } 5 \mathrm{mg} \text { of fluoxe- } \\
\text { tine, (capsules) C (containing) } 50 \mathrm{mg} \text { of desipramine or } 10 \mathrm{mg} \text { of fluoxetine". }\end{array}$ \\
\hline
\end{tabular}

$\begin{aligned} & \text { Blinding of outcome as- } \\ & \text { sessment (detection bias) }\end{aligned} \quad$ Low risk Quote: "both patients and investigators were blind to the assignment"


Bystritsky 1995 (Continued)

All outcomes

Incomplete outcome data Unclear risk $\quad$ No clear information on incomplete outcome data management.
(attrition bias)

(attrition bias)

All outcomes

\begin{tabular}{lll}
\hline $\begin{array}{l}\text { Selective reporting (re- } \\
\text { porting bias) }\end{array}$ & Low risk & All outcomes were reported. \\
\hline Other bias & Unclear risk & $\begin{array}{l}\text { Quote: "this research has been supported in part by NIMH grant MH } 45342-02 \\
\text { and by an NPI Opportunity Grant". }\end{array}$ \\
\hline
\end{tabular}

\section{CNCPS 1992}

\begin{tabular}{ll}
\hline Methods & Study design: Randomised controlled trial \\
\hline Participants & $\begin{array}{l}\text { Diagnosis: DSM-III panic disorder with limited or extensive phobic avoidance (panic attacks with ago- } \\
\text { raphobia) }\end{array}$
\end{tabular}

Method of diagnosis: "patients were evaluated by Structured Clinical Interview for DSM-III Diagnosis, Upjohn (SCID-UP)

Age: $M=34, S D$ not provided

Sex: $62 \%$ female, $38 \%$ male

Location: 12 centres in USA, Spain, Denmark, Germany, England, Italy, Brazil, Mexico, France, Colombia, Austria, Sweden, Canada, Belgium; setting: inpatients and outpatients

Co-morbidities: patients with psychotic disorders, dementia, bipolar disorder, alcoholism or drug abuse within the last six months or significant medical problems were excluded. Patients with current major depression were excluded unless the depression was judged to be secondary to the anxiety disorder and did not have melancholic or psychotic features.

Rescue medication: Quote "patients taking CNS drugs, including benzodiazepines, were excluded from the study. During the washout period, blood was drawn for benzodiazepines screening".

Interventions

\section{Participants were randomly assigned to either:}

(1) imipramine $\operatorname{arm}(\mathrm{n}=391)$

Duration: 8 weeks

Treatment Protocol: flexible dosage; range $=25-250 \mathrm{mg}, \mathrm{M}=155$, SD not provided

(2) alprazolam arm $(n=386)$

Duration: 8 weeks

Treatment Protocol: flexible dosage; range $=1-10 \mathrm{mg}, \mathrm{M}=5.7$, SD not provided

(3) placebo arm $(n=391)$

Duration: 8 weeks

Outcomes

Time points for assessment: baseline, weekly, endpoint

\section{Outcomes:}

1. Physician's and patient's global improvement scales 
CNCPS 1992 (Continued)
2. Panic Attack Scale, patient's diary
3. Overall Phobia Scale (Marks \& Matthews), Phobic Anxiety Factor of the Symptom Check List (SCL-90)
4. anticipatory anxiety
5. Hamilton Rating Scale for Anxiety (HAMA)
6. social functioning, five-point scale
7. Hamilton Rating Scale for Depression (HRSD)
8. Hopkins SCL-90 patient self-rating scale for presence and intensity of symptoms

$\begin{array}{ll}\text { Notes } & \text { Date of study: Data collection: } 1984-1987 \\ \text { Funding source: sponsored by Upjohn Company, Kalamazoo, Michigan } \\ \text { Declarations of interest among the primary researchers: Not stated. }\end{array}$

\section{Risk of bias}

Bias Authors' judgement Support for judgement

Random sequence genera- Unclear risk tion (selection bias)
Quote: "randomly assigned"; "alprazolam, imipramine or placebo were assigned in 12 randomization blocks of the basic three cell random-assignment, parallel treatment-design. [...] At each center patients were blindly and randomly assigned to alprazolam, imipramine or placebo treatment, based on a table of random numbers [...]. Patients removed from the protocol before three weeks had to be replaced; after three weeks, non-completers were not replaced."

Allocation concealment Unclear risk $\quad$ No information provided.
(selection bias)

Blinding of participants Unclear risk Quote "double-blind design". No further details.
and personnel (perfor-
mance bias)
All outcomes

\begin{tabular}{lll}
\hline $\begin{array}{l}\text { Blinding of outcome as- } \\
\text { sessment (detection bias) } \\
\text { All outcomes }\end{array}$ & Unclear risk & Quote "double-blind design". No further details. \\
\hline $\begin{array}{l}\text { Incomplete outcome data } \\
\text { (attrition bias) } \\
\text { All outcomes }\end{array}$ & Unclear risk & Quote: "of 1168 patients randomized, 1122 met criteria for ITT". \\
\hline $\begin{array}{l}\text { Selective reporting (re- } \\
\text { porting bias) }\end{array}$ & High risk & $\begin{array}{l}\text { In the primary publication, data on Panic Attack scale are not reported; data } \\
\text { on Physician's global Improvement scale are only partially reported, and with- } \\
\text { out the number of patients evaluated; data on other continuous outcomes } \\
\text { (HAMA, HRSD) are reported without number of patients evaluated. Other data } \\
\text { are partially reported in secondary publication of this study. }\end{array}$ \\
\hline Other bias & High risk & $\begin{array}{l}\text { Sponsored by Upjohn Company, Kalamazoo, Michigan; the role of the funder } \\
\text { in planning, conducting and writing the study is not discussed. }\end{array}$ \\
\hline
\end{tabular}


Den Boer 1988

\begin{tabular}{|c|c|}
\hline Methods & Study design: Randomised controlled trial \\
\hline \multirow[t]{7}{*}{ Participants } & $\begin{array}{l}\text { Diagnosis: DSM-III panic disorder without phobic avoidance or panic disorder with severe phobic } \\
\text { avoidance behaviour }\end{array}$ \\
\hline & Method of diagnosis: Not stated \\
\hline & Age: for maprotiline, $M=35.0$ (SD = 7.4); for fluvoxamine, $M=37.3(S D=10.6)$ \\
\hline & Sex: for maprotiline, 4 males and 20 females; for fluvoxamine 5 males and 15 females \\
\hline & Location: the Netherlands; setting: outpatients \\
\hline & $\begin{array}{l}\text { Co-morbidities: patients with major affective disorders, schizophrenia, other psychotic disorder or sig- } \\
\text { nificant medical problems were excluded }\end{array}$ \\
\hline & Rescue medication: Not stated \\
\hline \multirow[t]{7}{*}{ Interventions } & Participants were randomly assigned to either: \\
\hline & (1) maprotiline arm ("24 patients were included in the maprotiline group") \\
\hline & Duration: 6 weeks \\
\hline & Treatment Protocol: flexible dosage; range $=50-150 \mathrm{mg}, \mathrm{M}$ and SD not provided \\
\hline & (2) fluvoxamine arm ("20 patients were included in the fluvoxamine group") \\
\hline & Duration: 6 weeks \\
\hline & Treatment Protocol: flexible dosage; range $=50-150 \mathrm{mg}, \mathrm{M}$ and SD not provided \\
\hline \multirow[t]{9}{*}{ Outcomes } & Time points for assessment: baseline and weekly \\
\hline & Outcomes: \\
\hline & 1. SCL-90 \\
\hline & 2. State Anxiety Inventory (A-STATE) \\
\hline & 3. Self Rating Depression Scale (SDS) \\
\hline & 4. Hamilton Anxiety Scale (HAS) \\
\hline & 5. Hamilton Depression Scale (HDS) \\
\hline & 6. panic attack inventory \\
\hline & 7. side-effects scale \\
\hline
\end{tabular}

Notes

Date of study: Not stated

Funding source: Not stated

Declarations of interest among the primary researchers: Not stated.

\section{Risk of bias}

\begin{tabular}{lll}
\hline Bias & Authors' judgement & Support for judgement \\
\hline $\begin{array}{l}\text { Random sequence genera- } \\
\text { tion (selection bias) }\end{array}$ & Unclear risk & Quote: "they were randomly allocated". No further details.
\end{tabular}


Den Boer 1988 (Continued)

Allocation concealment $\quad$ Unclear risk $\quad$ No information provided.
(selection bias)

Blinding of participants Unclear risk Quote "double-blind treatment". No further details.
and personnel (perfor-
mance bias)
All outcomes

\begin{tabular}{ll}
\hline Blinding of outcome as- & Unclear risk
\end{tabular} Quote "double-blind treatment". No further details.

All outcomes

\begin{tabular}{lll}
\hline $\begin{array}{l}\text { Incomplete outcome data } \\
\text { (attrition bias) } \\
\text { All outcomes }\end{array}$ & High risk & $\begin{array}{l}\text { Number of patients randomised per group not reported (number of total ran- } \\
\text { domised patients }=47 \text { ); only number of patients evaluated per group was } \\
\text { available, respectively 24 in maprotiline group and 20 in fluvoxamine. }\end{array}$ \\
\hline $\begin{array}{l}\text { Selective reporting (re- } \\
\text { porting bias) }\end{array}$ & High risk & Continuous outcome data are reported only in graphs. \\
\hline Other bias & Unclear risk & Sponsorship bias cannot be ruled out. \\
\hline
\end{tabular}

\section{Den Boer 1990}

\begin{tabular}{ll}
\hline Methods & Study design: Randomised controlled trial \\
\hline Participants & Diagnosis: DSM-III-R \\
Method of diagnosis: not stated \\
Age: for fluvoxamine $M=37$, for ritanserin $M=35$, for placebo $M=37$ \\
Sex: the female to male ratio was almost 3 to 1 in all groups \\
Location: the Netherlands; setting: outpatients \\
Co-morbidities: patients with a primary diagnosis other than panic disorder were excluded \\
Rescue medication: none
\end{tabular}

\section{Interventions $\quad$ Participants were randomly assigned to either:}

(1) fluvoxamine arm

\section{Duration: 8 weeks}

Treatment Protocol: fixed dosage $=150 \mathrm{mg}$

(2) ritanserin arm

\section{Duration: 8 weeks}

Treatment Protocol: fixed dosage $=20 \mathrm{mg}$

(3) placebo arm

Duration: 8 weeks

Total number of randomised patients $=60$. The number of patients randomised for each arm is not provided. 
Den Boer 1990 (Continued)

Time points for assessment: baseline, weekly
Outcomes:
1. SCL-90
2. Hamilton Rating Scale for Anxiety (HAMA)
3. State-Trait Anxiety Inventory (STAI)
4. Fear Questionnaire (FQ)
5. panic inventory

Notes

Date of study: not stated

Funding source: not stated

Declarations of interest among the primary researchers: not stated.

\section{Risk of bias}

\begin{tabular}{|c|c|c|}
\hline Bias & Authors' judgement & Support for judgement \\
\hline $\begin{array}{l}\text { Random sequence genera- } \\
\text { tion (selection bias) }\end{array}$ & Unclear risk & Quote: "randomised". \\
\hline $\begin{array}{l}\text { Allocation concealment } \\
\text { (selection bias) }\end{array}$ & Unclear risk & No information provided. \\
\hline $\begin{array}{l}\text { Blinding of participants } \\
\text { and personnel (perfor- } \\
\text { mance bias) } \\
\text { All outcomes }\end{array}$ & Unclear risk & Quote: "double blind". No further information provided. \\
\hline $\begin{array}{l}\text { Blinding of outcome as- } \\
\text { sessment (detection bias) } \\
\text { All outcomes }\end{array}$ & Unclear risk & Quote: "double blind". No further information provided. \\
\hline $\begin{array}{l}\text { Incomplete outcome data } \\
\text { (attrition bias) } \\
\text { All outcomes }\end{array}$ & Unclear risk & No information provided about management of incomplete outcome data. \\
\hline $\begin{array}{l}\text { Selective reporting (re- } \\
\text { porting bias) }\end{array}$ & High risk & Data are reported in graphs (HAMA, FQ); other data only partially reported. \\
\hline Other bias & Unclear risk & Sponsorship bias cannot be ruled out. \\
\hline
\end{tabular}

Garvey 1989

\begin{tabular}{ll}
\hline Methods & Study design: Randomised controlled trial \\
\hline Participants & Diagnosis: DSM-III panic disorder with agoraphobia \\
& Method of diagnosis: Not stated \\
& Age: $\mathrm{M}=40.0(\mathrm{SD}=12)$ \\
& Sex: $61 \%$ women \\
\hline
\end{tabular}


Location: USA; setting unclear

Co-morbidities: patients with MDD, alcoholism during the previous six months, current or past mania or psychotic disorder, major medical problems were excluded

Rescue medication: Not stated

Interventions

\section{Participants were randomly assigned to either:}

(1) alprazolam arm

Duration: 3 weeks

Treatment Protocol: dosage gradually increased to a maximum of $6 \mathrm{mg}$

(2) diazepam arm

Duration: 3 weeks

Treatment Protocol: dosage gradually increased to a maximum of $60 \mathrm{mg}$

(3) placebo arm

Duration: 3 weeks

Total number of randomised patients $=30$; number of patients per arm not provided.

Outcomes

The study is about urinary MHPG levels in relation to Panic Disorder treatment.

Time points for assessment: before the beginning of treatment and after 3 weeks of treatment

\section{Outcomes:}

1. MHPG urinary levels

2. Hamilton Rating Scale for Anxiety (HAMA)

Date of study: Not stated
Funding source: Not stated
Declarations of interest among the primary researchers: Not stated.

\section{Risk of bias}

\begin{tabular}{lll}
\hline Bias & Authors' judgement & Support for judgement \\
\hline $\begin{array}{l}\text { Random sequence genera- } \\
\text { tion (selection bias) }\end{array}$ & Unclear risk & $\begin{array}{l}\text { Quote: "thirty consecutive patients meeting study criteria were randomly as- } \\
\text { signed" }\end{array}$ \\
\hline $\begin{array}{l}\text { Allocation concealment } \\
\text { (selection bias) }\end{array}$ & Unclear risk & No information provided. \\
\hline $\begin{array}{l}\text { Blinding of participants } \\
\text { and personnel (perfor- } \\
\text { mance bias) }\end{array}$ & Unclear risk & Quote "double-blind treatment". No further details. \\
$\begin{array}{l}\text { All outcomes } \\
\text { Blinding of outcome as- } \\
\text { sessment (detection bias) } \\
\begin{array}{l}\text { All outcomes } \\
\end{array}\end{array}$ & Unclear risk & Quote "double-blind treatment". No further details. \\
\hline
\end{tabular}


Garvey 1989 (Continued)
Incomplete outcome data
Unclear risk
No information provided.
(attrition bias)

All outcomes

\begin{tabular}{|c|c|c|}
\hline $\begin{array}{l}\text { Selective reporting (re- } \\
\text { porting bias) }\end{array}$ & Unclear risk & $\begin{array}{l}\text { Study on urinary MHPG levels; HAMA mentioned in methods section, but no re- } \\
\text { sults reported. }\end{array}$ \\
\hline
\end{tabular}

Other bias Unclear risk Sponsorship bias cannot be ruled out.

Gentil 1993

Methods Study design: Randomised controlled trial

Participants

Diagnosis: DSM-III-R panic disorder with or without agoraphobia

Method of diagnosis: semi-structured interview

Age: for imipramine, $\mathrm{M}=36.35$ (SEM $=2.12)$; for clomipramine, $\mathrm{M}=34.1(\mathrm{SEM}=1.89)$

Sex: for imipramine, 70\% women, 30\% men; for clomipramine 50\% women, 50\% men

Location: Brazil; setting: outpatients

Co-morbidities: patients with other medical condition, drug abuse, OCD, primary major depression or psychoses were excluded; major depression without melancholia, secondary to panic disorder, could still be included

Rescue medication: Not stated

\section{Participants were randomly assigned to either:}

(1) imipramine arm $(n=20)$

Duration: 8 weeks

Treatment Protocol: flexible dosage; range $=25-200 \mathrm{mg}, \mathrm{M}=113.8, \mathrm{SD}=9.5$

(2) clomipramine arm $(\mathrm{n}=20)$

Duration: 8 weeks

Treatment Protocol: flexible dosage; range $=10-80 \mathrm{mg}, \mathrm{M}=50, \mathrm{SD}=4.2$

(3) placebo arm (propantheline) $(n=20)$

Duration: 8 weeks

Treatment Protocol: flexible dosage; $M=85.5, S D=5.7$

\section{Outcomes}

\section{Time points for assessment: baseline, week 2, 4, 6 and 8}

\section{Outcomes:}

1. Clinical Global Impression Scale (CGI)

2. Sheehan Anxiety Scales

3. Hamilton Rating Scale for Depression (HRSD)

4. Beck Depression Inventory (BDI) 
Gentil 1993 (Continued)

Notes

Date of study: Not stated

Funding source: grants from FAPESP and FINEP, donations from Rhodia SA, Metalurgica Matarazzo, Itautec, Soft Consultoria an Industrias Bardella SPA, Fundacao Zerbini and Fundacao Faculdade de Medicina

Declarations of interest among the primary researchers: Not stated

\section{Risk of bias}

\begin{tabular}{|c|c|c|}
\hline Bias & Authors' judgement & Support for judgement \\
\hline $\begin{array}{l}\text { Random sequence genera- } \\
\text { tion (selection bias) }\end{array}$ & Unclear risk & $\begin{array}{l}\text { Quote: "they were randomly allocated". Dropouts before completing the } \\
\text { fourth week of treatment were replaced (therefore we considered only data } \\
\text { before replacing: number of dropouts at fourth week). }\end{array}$ \\
\hline $\begin{array}{l}\text { Allocation concealment } \\
\text { (selection bias) }\end{array}$ & Unclear risk & No information provided. \\
\hline $\begin{array}{l}\text { Blinding of participants } \\
\text { and personnel (perfor- } \\
\text { mance bias) } \\
\text { All outcomes }\end{array}$ & Low risk & $\begin{array}{l}\text { Quote "double-blind treatment"; "Capsules were in the hospital pharmacy } \\
\text { with tablets of the commercially available TCAs or propanteline (placebo) and } \\
\text { filled up with lactose. The dose range of propanteline was selected to give mild } \\
\text { to moderate peripheral anticholinergic effects". }\end{array}$ \\
\hline $\begin{array}{l}\text { Blinding of outcome as- } \\
\text { sessment (detection bias) } \\
\text { All outcomes }\end{array}$ & Low risk & $\begin{array}{l}\text { Quote "double-blind treatment"; "Capsules were in the hospital pharmacy } \\
\text { with tablets of the commercially available TCAs or propanteline (placebo) and } \\
\text { filled up with lactose. The dose range of propanteline was selected to give mild } \\
\text { to moderate peripheral anticholinergic effects". }\end{array}$ \\
\hline $\begin{array}{l}\text { Incomplete outcome data } \\
\text { (attrition bias) } \\
\text { All outcomes }\end{array}$ & Unclear risk & $\begin{array}{l}15 \text { patients left the trial before completing the first four weeks of treatment } \\
\text { and were replaced. No information provided on incomplete outcome data } \\
\text { management. }\end{array}$ \\
\hline $\begin{array}{l}\text { Selective reporting (re- } \\
\text { porting bias) }\end{array}$ & High risk & $\begin{array}{l}\text { Data on the scales HAMD and BDI not reported at endpoint. Data on the scales } \\
\mathrm{CGI} \text { and Sheehan are reported only in graphs; number of patients evaluated } \\
\text { not specified. }\end{array}$ \\
\hline Other bias & Low risk & $\begin{array}{l}\text { Quote: "this study was not supported by the manufacturers of the drugs test- } \\
\text { ed". }\end{array}$ \\
\hline
\end{tabular}

\section{GSK-29060/1}

\begin{tabular}{ll}
\hline Methods & Study design: Randomised controlled trial \\
\hline Participants & Diagnosis: Panic disorder according to the DSM - III criteria \\
Method of diagnosis: Structured Clinical Interview (SCID) for DSM - III \\
Age: for paroxetine, M = 39.1 (SD = 11.1); for alprazolam, M = 39.5 (SD = 12.5) \\
Sex: for paroxetine, 49 women, 28 men; for alprazolam 48 women, 28 men \\
Location: USA; setting: outpatients \\
Co-morbidities: patients with Axis I disorders other than panic disorder, current major depression (un- \\
less panic disorder dominated the clinical picture and preceded affective symptoms chronologically), \\
any severe or uncontrolled medical condition were excluded
\end{tabular}


(1) paroxetine arm $(n=77)$

Duration: 10 weeks

Treatment Protocol: flexible dosage; range $=10-60 \mathrm{mg}, \mathrm{M}$ and SD not provided

(2) alprazolam arm $(n=77)$

Duration: 10 weeks

Treatment Protocol: flexible dosage; range $=1-6 \mathrm{mg}, \mathrm{M}$ and SD not provided

(3) placebo arm $(n=72)$

Duration: 10 weeks

Time points for assessment: baseline, endpoint (10 weeks)

\section{Outcomes:}

1. $\%$ of patients having zero panic attacks

2. mean change from baseline in the number of panic attacks

3. $\%$ of participants with $a>50 \%$ reduction from baseline in the number of full panic attacks

4. Clinical Global Impression Severity of Illness Score (CGI-S)

5. number of full and limited panic attacks

6. intensity of panic attacks

7. anticipatory anxiety

8. Marks Sheehan Phobia Scale

9. Clinical Global Impression Improvement Score (CGI-I)

10. Hamilton Rating Scale for Anxiety (HAMA)

11. Montgomery-Åsberg Depression Rating Scale (MADRS)

12. Sheehan Disability Scale (SDS)

13. Social Adjustment Self-Report Questionnaire

Funding source: GSK

Declarations of interest among the primary researchers: Not stated.

\section{Risk of bias}

\begin{tabular}{lll}
\hline Bias & Authors' judgement & Support for judgement \\
\hline $\begin{array}{l}\text { Random sequence genera- } \\
\text { tion (selection bias) }\end{array}$ & Unclear risk & Quote: "randomized". \\
\hline
\end{tabular}




\section{GSK-29060/1 (Continued)}

Allocation concealment $\quad$ Unclear risk
(selection bias)

Blinding of participants

Unclear risk

Quote: "double-blind". No further details.

and personnel (perfor-

mance bias)

All outcomes

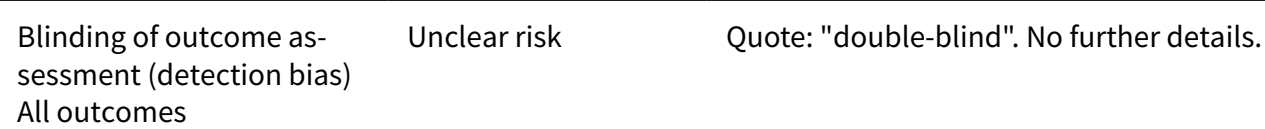

All outcomes

Incomplete outcome data Low risk

(attrition bias)

Quote: "All subjects in the ITT population (which included all subjects who re-

All outcomes ceived any double blind medication) for whom at least one valid post efficacy evaluation was available were included in the ITT efficacy analysis. All subjects randomized were included in the safety analysis".

Selective reporting (re- Low risk All outcomes were reported.

porting bias)

Other bias High risk Sponsored by GSK; the role of the funder in planning, conducting and writing the study is not discussed.

\section{GSK-29060/525}

\begin{tabular}{ll}
\hline Methods & Study design: Randomised controlled trial \\
\hline Participants & Diagnosis: Panic disorder; no further details provided \\
Method of diagnosis: Not stated \\
Age: for paroxetine, $M=37.12$ (SD = 9.92); for clomipramine, M = 40.13 (SD = 11.34) \\
Sex: for paroxetine, 14 women, 23 men, 1 unknown; for clomipramine 17 women, 14 men \\
Location: China; setting unclear \\
Co-morbidities: patients with current major depression were excluded. No other co-morbidities men- \\
tioned \\
Rescue medication: Not stated
\end{tabular}

\section{Interventions $\quad$ Participants were randomly assigned to either:}

(1) paroxetine $\operatorname{arm}(n=38)$

Duration: 10 weeks

Treatment Protocol: flexible dosage; range $=10-50 \mathrm{mg}, \mathrm{M}$ and SD not provided

(2) clomipramine arm $(n=35)$

Duration: 10 weeks

Treatment Protocol: flexible dosage; range $=50-100 \mathrm{mg}, \mathrm{M}$ and SD not provided

Outcomes Time points for assessment: baseline, endpoint (10 weeks)

\section{Outcomes:}


2. Hamilton Rating Scale for Anxiety (HAMA)

3. Panic Associated Symptoms Scale

4. Clinical Global Impression Severity of Illness Score (CGI-S)

5. Patient Global Evaluation (PGE)

\section{Notes}

Date of study: September 1998 to September 1999

Funding source: GSK

Declarations of interest among the primary researchers: Not stated.

\section{Risk of bias}

\begin{tabular}{|c|c|c|}
\hline Bias & Authors' judgement & Support for judgement \\
\hline $\begin{array}{l}\text { Random sequence genera- } \\
\text { tion (selection bias) }\end{array}$ & Unclear risk & Quote: "randomized". \\
\hline $\begin{array}{l}\text { Allocation concealment } \\
\text { (selection bias) }\end{array}$ & Unclear risk & No information provided. \\
\hline $\begin{array}{l}\text { Blinding of participants } \\
\text { and personnel (perfor- } \\
\text { mance bias) } \\
\text { All outcomes }\end{array}$ & Unclear risk & Quote: "double-blind". No further details. \\
\hline $\begin{array}{l}\text { Blinding of outcome as- } \\
\text { sessment (detection bias) } \\
\text { All outcomes }\end{array}$ & Unclear risk & Quote: "double-blind". No further details. \\
\hline $\begin{array}{l}\text { Incomplete outcome data } \\
\text { (attrition bias) } \\
\text { All outcomes }\end{array}$ & Unclear risk & $\begin{array}{l}\text { Quote: "ITT population consisted of all subjects who received treatment and } \\
\text { have one post treatment evaluation". }\end{array}$ \\
\hline $\begin{array}{l}\text { Selective reporting (re- } \\
\text { porting bias) }\end{array}$ & Low risk & All outcomes were reported. \\
\hline Other bias & High risk & $\begin{array}{l}\text { Sponsored by GSK; the role of the funder in planning, conducting and writing } \\
\text { the study is not discussed. }\end{array}$ \\
\hline
\end{tabular}

Holland 1999

\begin{tabular}{ll}
\hline Methods & Study design: Randomised controlled trial \\
\hline Participants & Diagnosis: DSM - III - R panic disorder with or without agoraphobia
\end{tabular}

Method of diagnosis: Not stated

Age: for adinazolam, $M=36.5$; for clomipramine, $M=35.8$; SD not provided

Sex: for adinazolam, 36\% male; for clomipramine $38 \%$ male

Location: UK; setting unclear 
Holland 1999 (Continued)

Co-morbidities: patients with psychiatric co-morbidities were excluded

Rescue medication: Not stated

Interventions

Participants were randomly assigned to either:

(1) adinazolam arm $(n=166)$

Duration: 24 weeks

Treatment Protocol: flexible dosage; range $=30-90 \mathrm{mg}, \mathrm{M}$ and SD not provided

(2) clomipramine arm $(n=149)$

Duration: 24 weeks

Treatment Protocol: flexible dosage; range $=50-150 \mathrm{mg}, \mathrm{M}$ and SD not provided

Outcomes

Time points for assessment: weeks $1,2,4,8,12,16,20$ and 24

\section{Outcomes:}

1. total number of panic attacks (Panic Attack and Anticipatory Anxiety scale)

2. Clinical Global Impression Improvement Score (CGI-I)

3. $\mathrm{SCL}$ - 90, Phobic Anxiety Dimension

4. Sheehan Disability Scale

Notes

Date of study: Not stated

Funding source: Not stated

Declarations of interest among the primary researchers: Not stated.

\section{Risk of bias}

\begin{tabular}{lll}
\hline Bias & Authors' judgement & Support for judgement \\
\hline $\begin{array}{l}\text { Random sequence genera- } \\
\text { tion (selection bias) }\end{array}$ & Unclear risk & Quote: "randomized". \\
\hline $\begin{array}{l}\text { Allocation concealment } \\
\text { (selection bias) }\end{array}$ & Unclear risk & No information provided. \\
\hline $\begin{array}{l}\text { Blinding of participants } \\
\text { and personnel (perfor- } \\
\text { mance bias) }\end{array}$ & Unclear risk & Quote: "double-blind". No further details. \\
All outcomes & \\
\hline
\end{tabular}

\begin{tabular}{|c|c|c|}
\hline $\begin{array}{l}\text { Blinding of outcome as- } \\
\text { sessment (detection bias) } \\
\text { All outcomes }\end{array}$ & Unclear risk & Quote: "double-blind". No further details. \\
\hline $\begin{array}{l}\text { Incomplete outcome data } \\
\text { (attrition bias) } \\
\text { All outcomes }\end{array}$ & High risk & LOCF data are reported, but without specifying number of patients evaluated. \\
\hline $\begin{array}{l}\text { Selective reporting (re- } \\
\text { porting bias) }\end{array}$ & Unclear risk & $\begin{array}{l}\text { All outcomes were reported, but without specifying number of patients evalu- } \\
\text { ated. }\end{array}$ \\
\hline
\end{tabular}


Holland 1999 (Continued)

Other bias Unclear risk Authors' affiliations refer to pharmaceutical companies.

Krueger 1999

Methods Study design: Randomised controlled trial

Participants

Diagnosis: DSM - III - R panic disorder with or without agoraphobia

Method of diagnosis: SCID Axis I, Roche edition

Age: for moclobemide, $M=35.0(S D=8.9)$; for clomipramine, $M=36.0(S D=9.5)$

Sex: for moclobemide, $41.8 \%$ males, 58.2 females; for clomipramine $39.7 \%$ males, $60.3 \%$ females

Location: Norway, Sweden, the Netherlands; setting unclear

Co-morbidities: none, except of generalised anxiety disorders and social phobia of less than moderate severity

Rescue medication: chloral hydrate as an occasional night time hypnotic

Interventions

\section{Participants were randomly assigned to either:}

(1) moclobemide arm $(n=67)$

Duration: 8 weeks

Treatment Protocol: fixed-flexible dosage, range $=300-600 \mathrm{mg}, \mathrm{M}$ and SD not provided

(2) clomipramine $\operatorname{arm}(\mathrm{n}=68)$

Duration: 8 weeks

Treatment Protocol: fixed-flexible dosage, range $=100-200 \mathrm{mg}, \mathrm{M}$ and SD not provided

Outcomes

Time points for assessment: week 1, 2, 4, and 8

\section{Outcomes:}

1. number of panic attacks

2. Patients' Clinical Global Impression of Change (P-CGI-C)

3. Investigators' rating of Clinical Global Impression of the Severity of the patients' panic disorder (I(GI-S)

4. Patients' rating of Clinical Global Impression of Severity (P-CGI-S)

6. Sheehan Disability Scale (SDS)

7. Hamilton Rating Scale for Anxiety (HAMA)

8. Montgomery-Åsberg Depression Rating Scale (MADRS)

Notes

Date of study: Not stated

Funding source: Hoffmann - La Roche

Declarations of interest among the primary researchers: Not stated.

\section{Risk of bias}

Antidepressants and benzodiazepines for panic disorder in adults (Review) 
Krueger 1999 (Continued)

\begin{tabular}{lll} 
Bias & Authors' judgement & Support for judgement \\
\hline $\begin{array}{l}\text { Random sequence genera- } \\
\text { tion (selection bias) }\end{array}$ & Unclear risk & Quote: "randomized". \\
\hline
\end{tabular}

Allocation concealment Unclear risk No information provided.

(selection bias)

Blinding of participants Unclear risk $\quad$ Quote: "double-blind". No further details.
and personnel (perfor-
mance bias)
All outcomes

Blinding of outcome as- $\quad$ Unclear risk $\quad$ Quote: "double-blind". No further details.
sessment (detection bias)
All outcomes

\begin{tabular}{|c|c|c|}
\hline $\begin{array}{l}\text { Incomplete outcome data } \\
\text { (attrition bias) } \\
\text { All outcomes }\end{array}$ & Low risk & $\begin{array}{l}\text { Quote: "it was estimated that the ITT population with two-sided significance } \\
\text { level of } 0.05 \text { and a power of at least } 0.8 \text { had to be at least } 66 \text { patients in each } \\
\text { treatment group"; "the ITT population comprised } 135 \text { patients who had re- } \\
\text { ceived treatment and at least one assessment after baseline". }\end{array}$ \\
\hline $\begin{array}{l}\text { Selective reporting (re- } \\
\text { porting bias) }\end{array}$ & Unclear risk & All outcomes were reported. \\
\hline Other bias & High risk & $\begin{array}{l}\text { Sponsored by Hoffmann-La Roche; the role of the funder in planning, conduct- } \\
\text { ing and writing the study is not discussed. }\end{array}$ \\
\hline
\end{tabular}

Lecrubier 1997

\begin{tabular}{|c|c|}
\hline Methods & Study design: Randomised controlled trial \\
\hline Participants & $\begin{array}{l}\text { Diagnosis: DSM-III-R panic disorder with or without agoraphobia } \\
\text { Method of diagnosis: not stated } \\
\text { Age: for paroxetine, } M=34.7(\mathrm{SD}=9.3) \text {; for clomipramine, } \mathrm{M}=35.1 \text { (SD = 9.2) } \\
\text { Sex: for paroxetine, } 53 \text { males, } 70 \text { females; for clomipramine } 46 \text { males, } 75 \text { females } \\
\text { Location: } 39 \text { centres in Belgium, Denmark, France, Hungary, Ireland, Israel, Italy, the Netherlands, Nor- } \\
\text { way, Spain, Switzerland, UK, Yugoslavia; setting: outpatients } \\
\text { Co-morbidities: none } \\
\text { Rescue medication: chloral hydrate for night time sedation allowed }\end{array}$ \\
\hline
\end{tabular}

Interventions

\section{Participants were randomly assigned to either:}

(1) paroxetine arm $(n=123)$

Duration: 12 weeks

Treatment Protocol: flexible dosage, range $=10-60 \mathrm{mg}, \mathrm{M}$ and SD not provided

(2) clomipramine arm $(n=122)$

Duration: 12 weeks 
(3) placebo arm $(n=123)$

Duration: 12 weeks

Time points for assessment: weeks $3,6,9,12$
Outcomes:
1. change in number of panic attacks
2. proportion of subjects with zero panic attacks
3. proportion of subjects with a > 50\% reduction in the number of panic attacks
4. change in intensity of panic attacks
5. Hamilton Rating Scale for Anxiety (HAMA)
6. Clinical Global Impression Scale (CGI)
7. Montgomery-Åsberg Depression Rating Scale (MADRS)
8. Mark Sheehan Phobia Scale
9. Patient Global Evaluation (PGE)
10. Sheehan Disability Scale

Funding source: Sponsored by GSK

Declarations of interest among the primary researchers: Department of Clinical Research, Development and Medical Affairs, SmithKline Beecham Pharmaceuticals

\section{Risk of bias}

\begin{tabular}{|c|c|c|}
\hline Bias & Authors' judgement & Support for judgement \\
\hline $\begin{array}{l}\text { Random sequence genera- } \\
\text { tion (selection bias) }\end{array}$ & Unclear risk & Quote: "randomized". \\
\hline $\begin{array}{l}\text { Allocation concealment } \\
\text { (selection bias) }\end{array}$ & Unclear risk & No information provided. \\
\hline $\begin{array}{l}\text { Blinding of participants } \\
\text { and personnel (perfor- } \\
\text { mance bias) } \\
\text { All outcomes }\end{array}$ & Unclear risk & Quote: "double-blind". No further details. \\
\hline $\begin{array}{l}\text { Blinding of outcome as- } \\
\text { sessment (detection bias) } \\
\text { All outcomes }\end{array}$ & Unclear risk & Quote: "double-blind". No further details. \\
\hline $\begin{array}{l}\text { Incomplete outcome data } \\
\text { (attrition bias) } \\
\text { All outcomes }\end{array}$ & Unclear risk & $\begin{array}{l}\text { Quote: "the primary and secondary efficacy analysis were performed on the } \\
\text { ITT population, which included all subjects who were randomized, who re- } \\
\text { ceived their randomized treatment and for whom at least one assessment was } \\
\text { available after active treatment. Safety assessment were performed on the ITT } \\
\text { population. Dropouts rates were around } 30 \% \text { in both treatment arms. }\end{array}$ \\
\hline
\end{tabular}


Lecrubier 1997 (Continued)

Selective reporting (re- Unclear risk $\quad$ All outcomes were reported. porting bias)

Other bias

High risk

Sponsored by GSK; the role of the funder in planning, conducting and writing the study is not discussed.

Lepola 1990

Methods Study design: Randomised controlled trial

Participants

Diagnosis: DMS-III panic disorder with or without agoraphobia

Method of diagnosis: Not stated

Age: $M=37.4$, SD not provided

Sex: not stated

Location: Finland; setting: inpatients

Co-morbidities: patients with psychiatric co-morbidities were excluded; medical co-morbidities are not mentioned; six patients suspected cases of epilepsy

Rescue medication: "the patients did not receive any other treatment during the trial period"

Interventions

\section{Participants were randomly assigned to either:}

(1) alprazolam arm $(n=27)$

Duration: 9 weeks

Treatment Protocol: flexible dosage, range $=1.5-8 \mathrm{mg}, \mathrm{M}=4.9$, SD not provided

(2) imipramine arm $(n=28)$

Duration: 9 weeks

Treatment Protocol: flexible dosage, range $=30-225 \mathrm{mg}, \mathrm{M}=130$, SD not provided

Outcomes

Time points for assessment: baseline, 3 weeks, 9 weeks

Outcomes:

1. panic attack frequency

2. Hamilton Rating Scale for Anxiety (HAMA)

3. Montgomery-Åsberg Depression Rating Scale (MADRS)

4. seven-point evaluation scale of the clinical state (not better specified)

Notes

Date of study: Not stated

Funding source: Not stated

Declarations of interest among the primary researchers: None (but authors' affiliations refer to pharmaceutical companies).

Risk of bias 
Lepola 1990 (Continued)

\begin{tabular}{lll} 
Bias & Authors' judgement & Support for judgement \\
\hline $\begin{array}{l}\text { Random sequence genera- } \\
\text { tion (selection bias) }\end{array}$ & Unclear risk & Quote: "randomized". \\
\hline
\end{tabular}

Allocation concealment Unclear risk No information provided.

(selection bias)

Blinding of participants Unclear risk Quote: "double-blind". No further details.

and personnel (perfor-

mance bias)

All outcomes

\begin{tabular}{|c|c|c|}
\hline $\begin{array}{l}\text { Blinding of outcome as- } \\
\text { sessment (detection bias) } \\
\text { All outcomes }\end{array}$ & Unclear risk & Quote: "double-blind". No further details. \\
\hline
\end{tabular}

\begin{tabular}{lll}
\hline $\begin{array}{l}\text { Incomplete outcome data } \\
\text { (attrition bias) } \\
\text { All outcomes }\end{array}$ & Unclear risk & No information provided about management of incomplete outcome data. \\
\hline $\begin{array}{l}\text { Selective reporting (re- } \\
\text { porting bias) }\end{array}$ & Low risk & All relevant outcomes were reported. \\
\hline Other bias & Unclear risk & Authors' affiliations refer to pharmaceutical companies. \\
\hline
\end{tabular}

Meco 1989

Methods Study design: Randomised controlled trial

Participants

Diagnosis: DSM III-R panic disorder with agoraphobia

Method of diagnosis: not stated

Age: for etizolam, $M=49(S D=12.7)$; for alprazolam, $M=47.8(S D=12.5)$

Sex: for etizolam, 9 females and 6 males; for alprazolam, 8 females and 7 males

Location: Italy; setting: outpatients

Co-morbidities: patients with psychotic or epilepsy disorders, organic cerebral disorders, and severe medical diseases were excluded

Rescue medication: not stated

\section{Interventions Participants were randomly assigned to either:}

(1) etizolam arm $(n=15)$

Duration: 6 weeks

Treatment Protocol: fixed dosage; range $=1-1.5 \mathrm{mg}, \mathrm{M}$ and SD not provided

(2) alprazolam arm $(n=15)$

Duration: 6 weeks

Treatment Protocol: fixed dosage; range $=1-1.5 \mathrm{mg}, \mathrm{M}$ and SD not provided 
Meco 1989 (Continued)

Outcomes

Time points for assessment: 3, 6 weeks

\section{Outcomes:}

1. Hamilton Anxiety Rating Scale (HARS)

2. number of panic attack

3. Side Effect Check List

\section{Date of study: Not stated}

Funding source: Not stated

Declarations of interest among the primary researchers: None.

\section{Risk of bias}

\begin{tabular}{lll}
\hline Bias & Authors' judgement & Support for judgement \\
\hline $\begin{array}{l}\text { Random sequence genera- } \\
\text { tion (selection bias) }\end{array}$ & Unclear risk & Quote: "randomized". \\
\hline
\end{tabular}

Allocation concealment Unclear risk No information provided.

(selection bias)

Blinding of participants Unclear risk Quote: "double-blind". No further details.
and personnel (performance bias)

All outcomes

Blinding of outcome as- $\quad$ Unclear risk Quote: "double-blind". No further details.
sessment (detection bias)
All outcomes

\begin{tabular}{lll}
\hline $\begin{array}{l}\text { Incomplete outcome data } \\
\text { (attrition bias) } \\
\text { All outcomes }\end{array}$ & Unclear risk & No information provided about management of incomplete outcome data. \\
\hline $\begin{array}{l}\text { Selective reporting (re- } \\
\text { porting bias) }\end{array}$ & High risk & $\begin{array}{l}\text { Data are reported only in graphs; number of patients evaluated not specified; } \\
\text { data on HAMA are reported only for each single item. }\end{array}$ \\
\hline Other bias & Unclear risk & Sponsorship bias cannot be ruled out. \\
\hline
\end{tabular}

Nair 1996

Methods Study design: Randomised controlled trial

Participants

Diagnosis: DSM-III-R panic disorder with or without agoraphobia

Method of diagnosis: not stated

Age: for fluvoxamine, $M=34.5$; for imipramine, $M=34.5$, SD not provided

Sex: for fluvoxamine $56 \%$ females $44 \%$ males; for imipramine $50 \%$ females $50 \%$ males

Location: Canada; setting: outpatients 
Nair 1996 (Continued)

Co-morbidities: patients with a history of bipolar disorder, organic brain syndrome, schizophrenia or other psychotic disorders were excluded

Rescue medication: oxazepam up to $60 \mathrm{mg}$ daily or chloral hydrate up to $2000 \mathrm{mg}$ daily were permitted during first four weeks of treatment

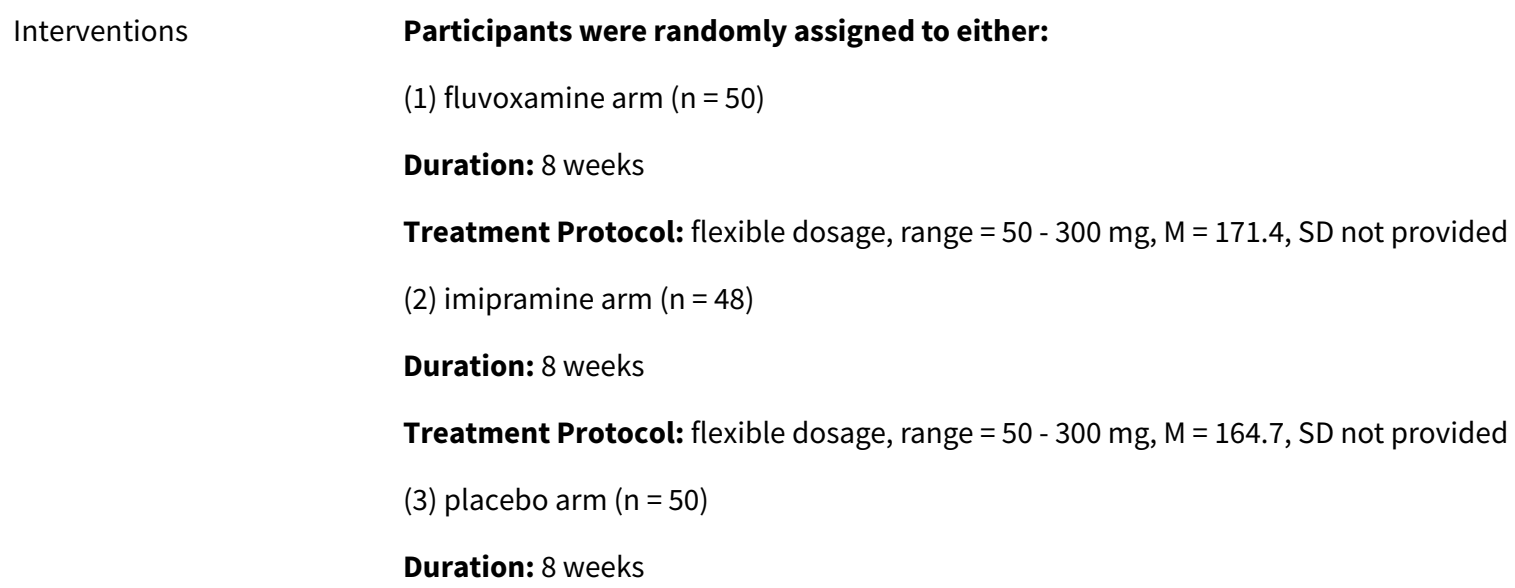

(1) fluvoxamine arm $(n=50)$

Duration: 8 weeks

Treatment Protocol: flexible dosage, range $=50-300 \mathrm{mg}, \mathrm{M}=171.4$, SD not provided

(2) imipramine arm $(n=48)$

Duration: 8 weeks

Treatment Protocol: flexible dosage, range $=50-300 \mathrm{mg}, \mathrm{M}=164.7$, SD not provided

(3) placebo arm $(n=50)$

Duration: 8 weeks

Time points for assessment: weekly
Outcomes:
1. Sheehan Panic and Anticipatory Anxiety Scale
2. Clinical Global Impression Scale (CGI)
3. Montgomery-Åsberg Depression Rating Scale (MADRS)
4. Sheehan Disability Scale (SDS)
5. Sheehan Panic Attack Diary (intensity and number of panic attacks)
6. Sheehan Phobia Scale
7. Hopkins Symptom Checklist

Notes

Date of study: Not stated

Funding source: Orto McNeil Ltd.

Declarations of interest among the primary researchers: Not stated.

\section{Risk of bias}

\begin{tabular}{|c|c|c|}
\hline Bias & Authors' judgement & Support for judgement \\
\hline $\begin{array}{l}\text { Random sequence genera- } \\
\text { tion (selection bias) }\end{array}$ & Unclear risk & Quote: "randomized". \\
\hline $\begin{array}{l}\text { Allocation concealment } \\
\text { (selection bias) }\end{array}$ & Unclear risk & No information provided. \\
\hline $\begin{array}{l}\text { Blinding of participants } \\
\text { and personnel (perfor- } \\
\text { mance bias) } \\
\text { All outcomes }\end{array}$ & Low risk & $\begin{array}{l}\text { Quote: "the study medication was in the form of identically appearing cap- } \\
\text { sules each containing either placebo, } 50 \mathrm{mg} \text { of fluvoxamine or } 50 \mathrm{mg} \text { of } \\
\text { imipramine". }\end{array}$ \\
\hline
\end{tabular}


Nair 1996 (Continued)

Blinding of outcome as- Low risk Q Q $\quad$ Quote: "the study medication was in the form of identically appearing capsessment (detection bias) sules each containing either placebo, 50 mg of fluvoxamine or 50 mg of

All outcomes imipramine".

Incomplete outcome data Low risk (attrition bias)

Quote: "two patient samples were identified for analysis and reporting purpos-

All outcomes es prior to unblinding: an all patients analysis and an ITT. The all patients sample was defined as those randomised to double blind treatment and who provided at least some drug safety and tolerance data [...] the main efficacy analysis of the study was based on the LOCF of the ITT sample".

Selective reporting (re- Low risk All outcomes were reported.
porting bias)

Other bias and writing the study is not discussed.

Noyes 1996

\begin{tabular}{ll}
\hline Methods & Study design: Randomised controlled trial \\
\hline Participants & Diagnosis: DSM-III panic disorder or agoraphobia with panic attacks \\
& Method of diagnosis: Structured Clinical Interview for DSM-III, Upjohn Version
\end{tabular}

Age: $M=36.6 ; S D=10.5$

Sex: women $=157$, men $=84$

Location: USA, Australia; setting: outpatients

Co-morbidities: patients with major psychiatric co-morbidities, head trauma or seizures were excluded

Rescue medication: none

\section{Interventions $\quad$ Participants were randomly assigned to either:}

(1) diazepam arm $(n=81)$

Duration: 8 weeks

Treatment Protocol: flexible dosage, range $=10-100 \mathrm{mg}, \mathrm{M}=43$, SD not provided

(2) alprazolam arm $(n=78)$

Duration: 8 weeks

Treatment Protocol: flexible dosage, range $=1-10 \mathrm{mg}, \mathrm{M}=4.9$, SD not provided

(3) placebo arm $(n=79)$

Duration: 8 weeks

\section{Outcomes:}

1. frequency of panic attacks

2. Sheehan Self Rated Scale for Anxiety 
Noyes 1996 (Continued)

3. Hamilton Rating Scale for Anxiety (HAMA)

4. Marks and Mathews Agoraphobia Scale

5. Profile of Mood States

6. Hamilton Rating Scale for Depression (HRSD)

7. Work and Social Disability Scale

8. Systematic Assessment for Treatment-Emergent Events

Date of study: not stated
Funding source: supported by a grant from the Upjohn Company
Declarations of interest among the primary researchers: Not stated.

\section{Risk of bias}

\begin{tabular}{|c|c|c|}
\hline Bias & Authors' judgement & Support for judgement \\
\hline $\begin{array}{l}\text { Random sequence genera- } \\
\text { tion (selection bias) }\end{array}$ & Unclear risk & Quote: "randomized". \\
\hline $\begin{array}{l}\text { Allocation concealment } \\
\text { (selection bias) }\end{array}$ & Unclear risk & No information provided. \\
\hline $\begin{array}{l}\text { Blinding of participants } \\
\text { and personnel (perfor- } \\
\text { mance bias) } \\
\text { All outcomes }\end{array}$ & Unclear risk & Quote: "double-blind". No further details. \\
\hline $\begin{array}{l}\text { Blinding of outcome as- } \\
\text { sessment (detection bias) } \\
\text { All outcomes }\end{array}$ & Unclear risk & Quote: "double-blind". No further details. \\
\hline $\begin{array}{l}\text { Incomplete outcome data } \\
\text { (attrition bias) } \\
\text { All outcomes }\end{array}$ & Low risk & $\begin{array}{l}\text { Quote: "to examine differences in treatment groups over time we completed } \\
\text { ITT analysis using logistic regression procedures. The results of analysis using } \\
\text { the completer sample were very similar to those using the III subjects". }\end{array}$ \\
\hline $\begin{array}{l}\text { Selective reporting (re- } \\
\text { porting bias) }\end{array}$ & Low risk & All outcomes were reported. \\
\hline Other bias & High risk & $\begin{array}{l}\text { Supported by a grant from the Upjohn Company; the role of the funder in plan- } \\
\text { ning, conducting and writing the study is not discussed. }\end{array}$ \\
\hline
\end{tabular}

Pfizer 2008

\begin{tabular}{ll}
\hline Methods & Study design: Randomised controlled trial \\
\hline Participants & Diagnosis: Panic disorder with or without agoraphobia according to DSM IV \\
Method of diagnosis: no information provided \\
Age: range $=18-64$ years, mean and SD not provided \\
Sex: sertraline: female $=113$, male $=44 ;$ paroxetine: female $=109$, male $=53$
\end{tabular}


Pfizer 2008 (Continued)

Location: Japan; setting unclear

Co-morbidities: "patients with bipolar disorder, schizophrenia, delusional disorder, epilepsy, MDD, OCD, seasonal affective disorder or GAD were excluded; patients who concurrently have depression/depressive state, anxiety disorder and generalized anxiety disorder may be included if the primary diagnosis is identified to be panic disorder"

Rescue medication: none

Interventions Participants were randomly assigned to either:

(1) sertraline arm $(n=157)$

Duration: 12 weeks

Treatment Protocol: flexible dosage, range $=25-100 \mathrm{mg}$

(2) paroxetine arm $(n=164)$

Duration: 12 weeks

Treatment Protocol: flexible dosage, range $=10-30 \mathrm{mg}$

Time points for assessment:
Outcomes:
1. Panic and Agoraphobia Scale
2. Clinical Global Impression Improvement Score (CGI-I)
3. frequency of panic attacks
4. Hamilton Rating Scale for Anxiety (HAMA)

Notes

Date of study: May 2008 - February 2010

Funding source: Pfizer

Declarations of interest among the primary researchers: not stated.

\section{Risk of bias}

\begin{tabular}{lll}
\hline Bias & Authors' judgement & Support for judgement \\
\hline $\begin{array}{l}\text { Random sequence genera- } \\
\text { tion (selection bias) }\end{array}$ & Unclear risk & Quote: "Allocation: randomized" \\
\hline $\begin{array}{l}\text { Allocation concealment } \\
\text { (selection bias) }\end{array}$ & Unclear risk & No information provided. \\
\hline $\begin{array}{l}\text { Blinding of participants } \\
\begin{array}{l}\text { and personnel (perfor- } \\
\text { mance bias) } \\
\text { All outcomes }\end{array}\end{array}$ & Low risk & Quote: "Masking: double-blind (subject, investigator)" \\
\hline
\end{tabular}

\begin{tabular}{lll}
\hline $\begin{array}{l}\text { Blinding of outcome as- } \\
\text { sessment (detection bias) }\end{array}$ & Low risk & Quote: "Masking: double-blind (subject, investigator)" \\
All outcomes & \\
\hline $\begin{array}{l}\text { Incomplete outcome data } \\
\text { (attrition bias) }\end{array}$ & Unclear risk & "Last Obsevation Carried Forward"
\end{tabular}


Pfizer 2008 (Continued)

All outcomes

Selective reporting (re- Low risk $\quad$ All outcomes were reported.
porting bias)

\begin{tabular}{ll}
\hline Other bias $\quad$ High risk & $\begin{array}{l}\text { Sponsored by Pfizer; the role of the funder in planning, conducting and writing } \\
\text { the study is not discussed. }\end{array}$
\end{tabular}

\section{Pollack 2007a}

\begin{tabular}{ll}
\hline Methods & Study design: Randomised controlled trial \\
\hline Participants & Diagnosis: DSM-IV panic disorder with or without agoraphobia \\
& Method of diagnosis: Mini-International Neuropsychiatric Interview \\
& Age: for venlafaxine $75 \mathrm{mg}, \mathrm{M}=35.8, \mathrm{SD}=9.97$; for venlafaxine $225 \mathrm{mg}, \mathrm{M}=37.1, \mathrm{SD}=11.8$, for paroxe- \\
& tine $\mathrm{M}=37.5, \mathrm{SD}=11$ \\
& Sex: for venlafaxine $75 \mathrm{mg}$, females $=65 \%$, males $=35 \%$; for venlafaxine $225 \mathrm{mg}$, females $=68 \%$, males \\
& $=33 \%$; for paroxetine females $=68 \%$, males $=32 \%$ \\
& Location: Argentina, Mexico, Chile, Costa Rica; setting: outpatients \\
& Co-morbidities: patients with other predominant Axis I or II disorders and important medical condi- \\
tions were excluded & \\
& Rescue medication: zaleplon or zolpidem permitted up to 3 times per week for the first 2 weeks of ran- \\
domised treatment
\end{tabular}

\section{Participants were randomly assigned to either:}

(1) venlafaxine $75 \mathrm{mg}$ arm $(n=163)$

Duration: 12 weeks

Treatment Protocol: fixed dosage $=75 \mathrm{mg} /$ day

(2) venlafaxine $225 \mathrm{mg}$ arm $(\mathrm{n}=167)$

Duration: 12 weeks

Treatment Protocol: fixed dosage $=225 \mathrm{mg} /$ day

(3) paroxetine arm $(n=161)$

Duration: 12 weeks

Treatment Protocol: fixed dosage $=40 \mathrm{mg} /$ day

(4) placebo arm $(n=162)$

Duration: 12 weeks

Outcomes

Time points for assessment: baseline, weeks 1, 2, 3, 4, 6, 8, 10, 12

\section{Outcomes:}

1. patients free of panic attacks at endpoint

2. Panic Disorder Severity Scale (PDSS) 
Pollack 2007a (Continued)

3. panic attacks frequency

4. Clinical Global Impression Improvement Score (CGI-I)

Date of study: not stated
Funding source: Wyeth Research, Collegeville, Pennsylvania
Declarations of interest among the primary researchers: members of advisory boards, and research
support received by many pharmaceutical companies, including AstraZeneca, GlaxoSmithKline, Eli Lil-
ly, Pfizer, Roche, Wyeth

\section{Risk of bias}

\begin{tabular}{lll}
\hline Bias & Authors' judgement & Support for judgement \\
\hline $\begin{array}{l}\text { Random sequence genera- } \\
\text { tion (selection bias) }\end{array}$ & Unclear risk & Quote: "randomized". \\
\hline $\begin{array}{l}\text { Allocation concealment } \\
\text { (selection bias) }\end{array}$ & Unclear risk & No information provided. \\
\hline $\begin{array}{l}\text { Blinding of participants } \\
\text { and personnel (perfor- } \\
\text { mance bias) } \\
\text { All outcomes }\end{array}$ & Low risk & $\begin{array}{l}\text { Quote: "...they were randomly assigned to receive venlafaxine } 75 \text { mg/day, ven- } \\
\text { lafaxine } 225 \mathrm{mg} / \text { day, paroxetine or placebo once daily in identically appearing } \\
\text { capsules". }\end{array}$ \\
\hline
\end{tabular}

\begin{tabular}{ll}
\hline $\begin{array}{l}\text { Blinding of outcome as- } \\
\text { sessment (detection bias) }\end{array}$ & Low risk
\end{tabular}$\quad \begin{aligned} & \text { Quote: "...they were randomly assigned to receive venlafaxine } 75 \mathrm{mg} / \mathrm{day}, \text { ven- } \\
& \text { lafaxine } 225 \mathrm{mg} / \mathrm{day}, \text { paroxetine or placebo once daily in identically appearing } \\
& \text { capsules". }\end{aligned}$

\begin{tabular}{ll}
\hline $\begin{array}{l}\text { Incomplete outcome data } \\
\text { (attrition bias) }\end{array}$ & Low risk \\
All outcomes & $\begin{array}{l}\text { Quote: "statystical analysis on the primary and secondary outcome measures } \\
\text { were performed for an ITT population of patients who had at least one post } \\
\text { randomisation visit on therapy using LOCF values". }\end{array}$
\end{tabular}

\begin{tabular}{lll}
\hline $\begin{array}{l}\text { Selective reporting (re- } \\
\text { porting bias) }\end{array}$ & Unclear risk & Continuous data at endpoint are reported only in graphs. \\
\hline Other bias & High risk & $\begin{array}{l}\text { Sponsored by Wyeth; the role of the funder in planning, conducting and writ- } \\
\text { ing the study is not discussed. }\end{array}$ \\
\hline
\end{tabular}

\section{Pollack 2007b}

\begin{tabular}{ll}
\hline Methods & Study design: Randomised controlled trial \\
\hline Participants & Diagnosis: DSM-IV panic disorder with or without agoraphobia \\
& Method of diagnosis: Mini-International Neuropsychiatric Interview \\
& Age: for venlafaxine $75 \mathrm{mg}, \mathrm{M}=36.2, \mathrm{SD}=10.7$; for venlafaxine $150 \mathrm{mg}, \mathrm{M}=37.7, \mathrm{SD}=11.5$, for paroxe- \\
tine $\mathrm{M}=37.6, \mathrm{SD}=10.5$ \\
Sex: for venlafaxine $75 \mathrm{mg}$, females $=66 \%$, males $=34 \%$; for venlafaxine $150 \mathrm{mg}$, females $=70 \%$, males \\
$=30 \%$; for paroxetine females $=64 \%$, males $=36 \%$
\end{tabular}

Location: Europe; setting: outpatients 
Pollack 2007b (Continued)

Co-morbidities: patients with other predominant Axis I or II disorders and important medical conditions were excluded

Rescue medication: zaleplon or zolpidem permitted up to 3 times per week for the first 2 weeks of randomised treatment

Interventions

Participants were randomly assigned to either:

(1) venlafaxine $75 \mathrm{mg}$ arm $(\mathrm{n}=166)$

Duration: 12 weeks

Treatment Protocol: fixed dosage $=75 \mathrm{mg} /$ day

(2) venlafaxine $150 \mathrm{mg}$ arm $(n=168)$

Duration: 12 weeks

Treatment Protocol: fixed dosage $=150 \mathrm{mg} /$ day

(3) paroxetine arm $(n=166)$

Duration: 12 weeks

Treatment Protocol: fixed dosage $=40 \mathrm{mg} /$ day

(4) placebo arm $(n=163)$

Duration: 12 weeks

Outcomes

Time points for assessment: baseline, weeks 1, 2, 3, 4, 6, 8, 10, 12

\section{Outcomes:}

1. frequency of panic attacks from the Panic and Anticipatory Anxiety Scale

2. patients free of panic attacks at endpoint

3. Panic Disorder Severity Scale (PDSS)

4. PDSS: anticipatory anxiety

5. Phobia Scale

6. Hamilton Rating Scale for Anxiety (HAMA)

7. Sheehan Disability Scale (SDS)

8. Quality of Life Enjoyment and Satisfaction Questionnaire

9. Clinical Global Impression Improvement Score (CGI-I)
Date of study: not stated

Funding source: sponsored by Wyeth Research

Declarations of interest among the primary researchers: members of advisory boards, and research support received by many pharmaceutical companies, including AstraZeneca, GlaxoSmithKline, Eli Lilly, Pfizer, Roche, Wyeth; some authors' affiliations refer to Wyeth.

\section{Risk of bias}

Bias Authors' judgement Support for judgement


Pollack 2007b (Continued)

Random sequence genera- Unclear risk Quote: "randomized". tion (selection bias)

Allocation concealment $\quad$ Unclear risk $\quad$ No information provided.
(selection bias)

\begin{tabular}{|c|c|c|}
\hline $\begin{array}{l}\text { Blinding of participants } \\
\text { and personnel (perfor- } \\
\text { mance bias) } \\
\text { All outcomes }\end{array}$ & Low risk & $\begin{array}{l}\text { Quote: "Study medication was provided as identical appearing capsules and } \\
\text { was to be taken once daily with food". }\end{array}$ \\
\hline
\end{tabular}

\begin{tabular}{|c|c|c|}
\hline $\begin{array}{l}\text { Blinding of outcome as- } \\
\text { sessment (detection bias) }\end{array}$ & Low risk & $\begin{array}{l}\text { Quote: "Study medication was provided as identical appearing capsules and } \\
\text { was to be taken once daily with food". }\end{array}$ \\
\hline
\end{tabular}

All outcomes

\begin{tabular}{|c|c|c|}
\hline $\begin{array}{l}\text { Incomplete outcome data } \\
\text { (attrition bias) } \\
\text { All outcomes }\end{array}$ & Unclear risk & $\begin{array}{l}\text { Quote: "statystical analysis on the primary and secondary outcome measures } \\
\text { were performed for an ITT population of patients who had at least one post } \\
\text { randomisation visit on therapy using LOCF values". }\end{array}$ \\
\hline
\end{tabular}

All outcomes randomisation visit on therapy using LOCF values".

\begin{tabular}{lll}
\hline $\begin{array}{l}\text { Selective reporting (re- } \\
\text { porting bias) }\end{array}$ & Low risk & All outcomes were reported. \\
\hline Other bias & High risk & $\begin{array}{l}\text { Sponsored by Wyeth; the role of the funder in planning, conducting and writ- } \\
\text { ing the study is not discussed. }\end{array}$ \\
\hline
\end{tabular}

Ribeiro 2001

Methods Study design: Randomised controlled trial

Participants

Diagnosis: DSM-IV panic disorder with or without agoraphobia

Method of diagnosis: not stated

Age: for mirtazapine, $M=36.1, S D=10.9$; for fluoxetine, $M=36.4, S D=10.1$

Sex: for mirtazapine, $86.7 \%$ females, for fluoxetine $66.7 \%$ females

Location: Brasil; setting: outpatients

Co-morbidities: patients with psychiatric and physical disorders were excluded

Rescue medication: none

Interventions

\section{Participants were randomly assigned to either:}

(1) mirtazapine arm ( $n=15)$

Duration: 8 weeks

Treatment Protocol: flexible dosage, range $=15-30 \mathrm{mg}, \mathrm{M}=17.9, \mathrm{SD}=4.3$

(2) fluoxetine arm ( $n=15)$

Duration: 8 weeks

Treatment Protocol: flexible dosage, range $=10-20 \mathrm{mg}, \mathrm{M}=13.1, \mathrm{SD}=3.2$ 
Ribeiro 2001 (Continued)

\section{Outcomes:}

1. Panic Diary

2. Clinical Global Impression Severity of Illness Score (CGI-S)

3. Clinical Global Impression Improvement Score (CGI-I)

4. Hamilton Rating Scale for Anxiety (HAMA)

5. Sheehan Phobic Scale

\section{Notes}

Date of study: November 1998 - March 1999

Funding source: research supported by FIPE-HCPA (FUNDO DE INCENTIVO À PESQUISA E EVENTOS)

Declarations of interest among the primary researchers: Not stated.

\section{Risk of bias}

\begin{tabular}{lll}
\hline Bias & Authors' judgement & Support for judgement \\
\hline $\begin{array}{l}\text { Random sequence genera- } \\
\text { tion (selection bias) }\end{array}$ & Low risk & $\begin{array}{l}\text { Quote: "patients were randomised to mirtazapine or fluoxetine using a com- } \\
\text { puter program which assigned 15 patients to each group". }\end{array}$ \\
\hline $\begin{array}{l}\text { Allocation concealment } \\
\text { (selection bias) }\end{array}$ & Low risk & $\begin{array}{l}\text { Quote: "a person who was not participating in the study labeled flasks contain- } \\
\text { ing enough medications for periods between visits". }\end{array}$ \\
\hline $\begin{array}{l}\text { Blinding of participants } \\
\text { and personnel (perfor- } \\
\text { mance bias) } \\
\text { All outcomes }\end{array}$ & Unclear risk & Quote: "double-blind". No further details. \\
\hline $\begin{array}{l}\text { Blinding of outcome as- } \\
\text { sessment (detection bias) } \\
\text { All outcomes }\end{array}$ & Unclear risk & Quote: "double-blind". No further details. \\
\hline $\begin{array}{l}\text { Incomplete outcome data } \\
\text { (attrition bias) } \\
\text { All outcomes }\end{array}$ & Unclear risk & $\begin{array}{l}\text { Quote: "the analysis included all patients who took at least one dose of med- } \\
\text { ication during the double-blind phase and who provided any follow-up data". }\end{array}$ \\
\hline $\begin{array}{l}\text { Selective reporting (re- } \\
\text { porting bias) }\end{array}$ & Low risk & All outcomes were reported. \\
\hline \begin{tabular}{l} 
Other bias \\
\hline
\end{tabular} & Unclear risk & \begin{tabular}{l} 
Organon Pharmaceutical kindly provided mirtazapine for the trial. \\
\hline
\end{tabular} \\
\hline
\end{tabular}

Sasson 1999

\begin{tabular}{ll} 
Methods & Study design: Randomised cross-over trial \\
\hline Participants & Diagnosis: DSM-III-R panic disorder \\
Method of diagnosis: not stated \\
Age: $M=36, \mathrm{SD}=8$ \\
Sex: males $=6$, females $=11$ \\
Location: Israel; setting unclear
\end{tabular}


Co-morbidities: patients were free of significant medical problems, mental retardation, psychosis, drug abuse, organic lesions and primary mood disorders

Rescue medication: not stated

Interventions

\section{Participants were randomly assigned to either:}

(1) clomipramine arm $(n=17)$

Duration: 6 weeks

Treatment Protocol: flexible dosage, range $=75-200 \mathrm{mg}, \mathrm{M}=140, \mathrm{SD}=52$

(2) desipramine arm $(n=17)$

Duration: 6 weeks

Treatment Protocol: flexible dosage, range $=50-300 \mathrm{mg}, \mathrm{M}=190, \mathrm{SD}=69$

According to the cross-over design, patients received placebo for 2 weeks; active treatment for 6 weeks; placebo for 2 weeks; switch to the other active treatment for 6 weeks.

Data on the active treatment phases are provided together.

Time points for assessment: baseline, every two weeks
Outcomes:
1. Hamilton Rating Scale for Anxiety (HAMA)
2. NIMH Global Scales for anxiety, depression and impairment
3. Zung Anxiety Scale
4. State-Trait Anxiety Inventory (STAI)
5. number of panic attacks

Notes Date of study: not stated

Funding source: not stated

Declarations of interest among the primary researchers: not stated.

\section{Risk of bias}

\begin{tabular}{lll}
\hline Bias & Authors' judgement & Support for judgement \\
\hline $\begin{array}{l}\text { Random sequence genera- } \\
\text { tion (selection bias) }\end{array}$ & Unclear risk & Quote: "randomized". \\
\hline $\begin{array}{l}\text { Allocation concealment } \\
\text { (selection bias) }\end{array}$ & Unclear risk & No information provided. \\
\hline $\begin{array}{l}\text { Blinding of participants } \\
\text { and personnel (perfor- } \\
\text { mance bias) } \\
\text { All outcomes }\end{array}$ & Unclear risk & Quote: "double blind". No further information provided. \\
\hline
\end{tabular}

Blinding of outcome as- Unclear risk Quote: "double blind". No further information provided. sessment (detection bias)

All outcomes 
Sasson 1999 (Continued)

Incomplete outcome data Low risk No missing outcome data.
(attrition bias)

All outcomes

Selective reporting (re- Low risk $\quad$ All outcomes were reported. porting bias)

Other bias Unclear risk Sponsorship bias cannot be ruled out.

Schweizer 1993

\begin{tabular}{ll}
\hline Methods & Study design: Randomised controlled trial \\
\hline Participants & Diagnosis: DSM - III panic disorder
\end{tabular}

Participants

Method of diagnosis: Structured Clinical Interview for DSM-III, Upjohn Version

Age: $M=33, S D=7$

Sex: female $=75 \%$, male $=25 \%$

Location: USA; setting: in and outpatients

Co-morbidities: none

Rescue medication: Quote: "no concomitant centrally active medication therapy was permitted during the study"

Interventions

\section{Participants were randomly assigned to either:}

(1) alprazolam arm $(n=37)$

Duration: 8 weeks short term, 32 weeks long term

Treatment Protocol: flexible dosage, range $=2-10 \mathrm{mg}, \mathrm{M}=5.4, \mathrm{SD}=2.1$

(2) imipramine arm $(n=34)$

Duration: 8 weeks short term, 32 weeks long term

Treatment Protocol: flexible dosage, range $=50-250 \mathrm{mg}, \mathrm{M}=152, \mathrm{SD}=65$

(3) placebo arm $(n=35)$

Duration: 8 weeks short term, 32 weeks long term

Outcomes

Time points for assessment: weekly until week 6 , week 8 , monthly for 6 months

\section{Outcomes:}

1. panic attack frequency and severity

2. Hamilton Rating Scale for Anxiety (HAMA)

3. phobias

4. disability resulting from the phobic anxiety

5. global assessment of improvement

6. safety questionnaire (SAFTEE) 
Schweizer 1993 (Continued)

7. benzodiazepines plasma levels

Notes Date of study: not stated

Funding source: sponsored by Upjohn Co.

Declarations of interest among the primary researchers: not stated.

\section{Risk of bias}

\begin{tabular}{|c|c|c|}
\hline Bias & Authors' judgement & Support for judgement \\
\hline $\begin{array}{l}\text { Random sequence genera- } \\
\text { tion (selection bias) }\end{array}$ & Unclear risk & Quote: "randomized". \\
\hline $\begin{array}{l}\text { Allocation concealment } \\
\text { (selection bias) }\end{array}$ & Unclear risk & No information provided. \\
\hline $\begin{array}{l}\text { Blinding of participants } \\
\text { and personnel (perfor- } \\
\text { mance bias) } \\
\text { All outcomes }\end{array}$ & Low risk & $\begin{array}{l}\text { Quote: "patients were dispensed identical capsules containing either } 1 \mathrm{mg} \text { of } \\
\text { alprazolam or } 25 \mathrm{mg} \text { of imipramine". }\end{array}$ \\
\hline $\begin{array}{l}\text { Blinding of outcome as- } \\
\text { sessment (detection bias) } \\
\text { All outcomes }\end{array}$ & Low risk & $\begin{array}{l}\text { Quote: "patients were dispensed identical capsules containing either } 1 \mathrm{mg} \text { of } \\
\text { alprazolam or } 25 \mathrm{mg} \text { of imipramine". }\end{array}$ \\
\hline $\begin{array}{l}\text { Incomplete outcome data } \\
\text { (attrition bias) } \\
\text { All outcomes }\end{array}$ & Low risk & $\begin{array}{l}\text { Quote: "ITT endpoint analysis, including all patients with at least one week of } \\
\text { treatment and 'evaluable patients' or 'decreasing N' analysis, using only those } \\
\text { patients available at each visit, were the primary set of analysis conducted. } \\
\text { Supplementary completers analysis using only patients who completed either } \\
8 \text { weeks or } 32 \text { weeks of treatment were also conducted". } \\
\text { "While the high attrition rate in the imipramine and placebo treatment groups } \\
\text { posed a problem for the statystical analysis of the various outcome measures, } \\
\text { attrition rates themselves constituted an important and independent outcome } \\
\text { measures. Survival analysis was performed for on-study treatment". }\end{array}$ \\
\hline
\end{tabular}

\begin{tabular}{ll}
\hline $\begin{array}{l}\text { Selective reporting (re- } \\
\text { porting bias) }\end{array}$ Low risk & All relevant outcomes were reported.
\end{tabular}
porting bias)

Other bias High risk Sponsored by Upjohn Co; the role of the funder in planning, conducting and
writing the study is not discussed.

Sheikh 1999

\begin{tabular}{ll}
\hline Methods & Study design: Randomised controlled trial \\
\hline Participants & Diagnosis: DSM-III-R panic disorder with or without agoraphobia \\
& Method of diagnosis: Structured Clinical Interview for DSM-III-R-Patient Version (SCID-P), Mini-Mental \\
State Exam (MMSE) & Age: M $=61.64, \mathrm{SD}=5.27$ \\
& Sex: 23 females, 2 males \\
& Location: USA; setting: outpatients
\end{tabular}


Sheikh 1999 (Continued)

Co-morbidities: patients with bipolar disorder, schizophrenia or psychosis, borderline personality disorder, obsessive-compulsive disorder alcohol or substance abuse, unstable medical, metabolic or cardiopulmonary condition were excluded

Rescue medication: none

Participants were randomly assigned to either:
(1) alprazolam $\operatorname{arm}(n=8)$
Duration: 8 weeks
Treatment Protocol: flexible dosage, range $=1-6 \mathrm{mg}, \mathrm{M}=2.87, \mathrm{SD}=1.66$
(2) imipramine arm $(\mathrm{n}=10)$
Duration: 8 weeks
Treatment Protocol: flexible dosage, range $=10-200 \mathrm{mg}, \mathrm{M}=77.5, \mathrm{SD}=59.4$
(3) placebo arm $(\mathrm{n}=7)$
Duration: 8 weeks

Outcomes

Time points for assessment: weeks 1, 2, 3, 4, 6, 8

Outcomes:

1. panic diary

2. Hamilton Rating Scale for Anxiety (HAMA)

3. Hamilton Rating Scale for Depression (HAM-D)

4. Physician's Global Impression (PGI)

Notes

Date of study: 1988-1990

Funding source: sponsored by Upjohn Co.

Declarations of interest among the primary researchers: not stated.

\begin{tabular}{lll}
\hline Risk of bias & & \\
\hline Bias & Authors' judgement & Support for judgement \\
\hline $\begin{array}{l}\text { Random sequence genera- } \\
\text { tion (selection bias) }\end{array}$ & Unclear risk & Quote: "randomized". \\
\hline $\begin{array}{l}\text { Allocation concealment } \\
\text { (selection bias) }\end{array}$ & Unclear risk & No information provided. \\
\hline $\begin{array}{l}\text { Blinding of participants } \\
\text { and personnel (perfor- } \\
\text { mance bias) }\end{array}$ & Low risk & $\begin{array}{l}\text { Quote: "medication for this double blinding protocol were provided by Upjohn } \\
\text { All outcomes }\end{array}$ \\
\hline $\begin{array}{l}\text { Blinding of outcome as- } \\
\text { sessment (detection bias) } \\
\text { All outcomes }\end{array}$ & Low risk & $\begin{array}{l}\text { Quote: "medication for this double blinding protocol were provided by Upjohn } \\
\text { Co. in the form of identical looking capsules". }\end{array}$ \\
\hline
\end{tabular}


Sheikh 1999 (Continued)

Incomplete outcome data Unclear risk (attrition bias)

All outcomes
Quote: "this is a pilot study compromised by small sample size and six of seven of the seven subjects in the placebo groups withdrawing early in the treatment course. This limitation prevent statystical analysis between the three groups".

Selective reporting (re- Low risk $\quad$ All outcomes were reported.
porting bias)

\begin{tabular}{ll}
\hline Other bias $\quad$ High risk & $\begin{array}{l}\text { Sponsored by Upjohn Co; the role of the funder in planning, conducting and } \\
\text { writing the study is not discussed. }\end{array}$
\end{tabular}

\section{Stahl 2003}

\begin{tabular}{ll}
\hline Methods & Study design: Randomised controlled trial \\
\hline Participants & Diagnosis: DSM - IV panic disorder \\
Method of diagnosis: not stated \\
Age: for escitalopram, M $=37.5$, for citalopram M $=37.1$ \\
Sex: for escitalopram $57.6 \%$ female, for citalopram $61.6 \%$ female \\
Location: USA; setting: outpatients \\
Co-morbidities: patients with bipolar disorder, schizophrenia, obsessive-compulsive disorder or other \\
psychotic disorder, \\
psychoactive substance use disorder, clinically significant abnormalities in laboratory evaluations or \\
electrocardiographic readings were excluded \\
Rescue medication: zolpidem
\end{tabular}

Interventions

\section{Participants were randomly assigned to either:}

(1) escitalopram arm $(n=129)$

Duration: 10 weeks

Treatment Protocol: flexible dosage, range $=5-20 \mathrm{mg}, \mathrm{M}=10.8 \mathrm{SD}$ not provided

(2) citalopram arm $(n=126)$

Duration: 10 weeks

Treatment Protocol: flexible dosage, range $=10-40 \mathrm{mg}, \mathrm{M}=21.3$, SD not provided

(3) placebo arm $(n=125)$

Duration: 10 weeks

Outcomes

Time points for assessment: baseline, weeks 1, 2, 4, 6, 8 and 10

\section{Outcomes:}

1. Panic and Anticipatory Anxiety Scale (PAAS)

2. panic attack frequency

3. Panic \& Agoraphobia Scale

4. Clinical Global Impression Improvement Score (CGI-I) 
Stahl 2003 (Continued)

5. Clinical Global Impression Severity of Illness Score (CGI-S)

6. Hamilton Rating Scale for Anxiety (HAMA)

7. Patient Global Evaluation (PGE)

8. Quality of Life Enjoyment and Satisfaction Questionnaire (Q-LES-Q)

9. Hamilton Rating Scale for Depression (HAM-D)

Notes

Date of study: $1999-2001$

Funding source: sponsored by Forest Laboratories

Declarations of interest among the primary researchers: one of the authors has received research support from many drug companies; other authors are employees of Forest Laboratories.

\section{Risk of bias}

\begin{tabular}{lll}
\hline Bias & Authors' judgement & Support for judgement \\
\hline $\begin{array}{l}\text { Random sequence genera- } \\
\text { tion (selection bias) }\end{array}$ & Unclear risk & Quote: "randomized". \\
\hline $\begin{array}{l}\text { Allocation concealment } \\
\text { (selection bias) }\end{array}$ & Unclear risk & No information provided. \\
\hline
\end{tabular}

Blinding of participants Unclear risk Quote: "double blind". No further information provided.

and personnel (perfor-

mance bias)

All outcomes

\begin{tabular}{|c|c|c|}
\hline $\begin{array}{l}\text { Blinding of outcome as- } \\
\text { sessment (detection bias) } \\
\text { All outcomes }\end{array}$ & Unclear risk & Quote: "double blind". No further information provided \\
\hline
\end{tabular}

\begin{tabular}{|c|c|c|}
\hline $\begin{array}{l}\text { Incomplete outcome data } \\
\text { (attrition bias) } \\
\text { All outcomes }\end{array}$ & Unclear risk & $\begin{array}{l}\text { Quote: "The ITT set consisted of } 351 \text { patients, } 125 \text { treated with escitalopram, } \\
112 \text { with citalopram and } 114 \text { with placebo". } \\
\text { Dropout rates were different between treatment groups (escitalopram = } \\
24.2 \% \text {, citalopram }=31.9 \% \text { ). }\end{array}$ \\
\hline $\begin{array}{l}\text { Selective reporting (re- } \\
\text { porting bias) }\end{array}$ & Low risk & All outcomes were reported. \\
\hline Other bias & High risk & $\begin{array}{l}\text { Sponsored by Forest Laboratories; the role of the funder in planning, conduct- } \\
\text { ing and writing the study is not discussed. }\end{array}$ \\
\hline
\end{tabular}

\section{Taylor 1990}

\begin{tabular}{ll}
\hline Methods & Study design: Randomised controlled trial \\
\hline Participants & Diagnosis: 79 patients with panic disorder. \\
& Method of diagnosis: Structured Clinical Interview for Diagnoses-Upjohn version (SCID-UP). \\
& Age: Alprazolam: M = 35.0; Imipramine: M=34.1; Placebo: M = 34.9 \\
& Sex: Alprazolam: Male $=19 \%$, Imipramine: $30 \%$, Placebo: $31 \%$.
\end{tabular}


Taylor 1990 (Continued)

Location: USA; setting: outpatients

Co-morbidities: none

Rescue medication: none

Interventions

Participants were randomly assigned to either:

(1) alprazolam $(n=26)$

Duration: 8 weeks

Treatment Protocol: flexible dosage; range $=1-8 \mathrm{mg}, \mathrm{M}=3.7$

(2) imipramine $(n=27)$

Duration: 8 weeks

Treatment Protocol: flexible dosage; range $=30-270 \mathrm{mg}, \mathrm{M}=147$

(3) placebo $(n=26)$

Duration: 8 weeks

\section{Outcomes:}

(1) Frequency/Intensity of panic attacks: panic diary

(2) Anxiety: Hamilton Anxiety rating scale (HAMA)

(3) Depression: Beck Depression Inventory (BDI)

(4) Overall psychiatric symptomatology: Symptom Check List- 90 (SCL-90)

(5) Global improvement: 7-point scale.

(6) Work and social disability: 5-point scale

(7) Avoidance: Marks/Mathews Fear Questionnaire

(8) Adverse effects: SAFTEE-UP

\section{Notes}

\section{Date of study: not stated}

Funding source: this research was supported in part by NIMH grant 40118 and by a gift from the Upjohn Company.

Declarations of interest among the primary researchers: not stated

\section{Risk of bias}

\begin{tabular}{lll}
\hline Bias & Authors' judgement & Support for judgement \\
\hline $\begin{array}{l}\text { Random sequence genera- } \\
\text { tion (selection bias) }\end{array}$ & Unclear risk & Quote: "randomized". \\
\hline $\begin{array}{l}\text { Allocation concealment } \\
\text { (selection bias) }\end{array}$ & Unclear risk & No information provided. \\
\hline
\end{tabular}

Blinding of participants $\quad$ Unclear risk $\quad$ "Double blind": no further information provided.
and personnel (perfor-
mance bias) 
Taylor 1990 (Continued)

All outcomes

Blinding of outcome as-
sessment (detection bias) $\quad$ Unclear risk $\quad$ See above.

sessment (detection bias)

All outcomes

\begin{tabular}{|c|c|c|}
\hline $\begin{array}{l}\text { ncomplete outcome data } \\
\text { (attrition bias) }\end{array}$ & High risk & $\begin{array}{l}\text { Completer analysis only, unequal drop-out rate (Alprazolam: } 8 \%, \text { In } \\
19 \% \text { ) }\end{array}$ \\
\hline
\end{tabular}

All outcomes

$19 \%)$

Selective reporting (re- High risk

porting bias)

Almost all the efficacy outcome measures described in the methods are reported in the results, but data are incomplete (standard deviations are not always presented). Furthermore, SAFTEE-UP event form is not reported.

Other bias High risk

This research was supported in part by NIMH grant 40118 and by a gift from the Upjohn Company. The role of the funder in planning, conducting and writing the study is not discussed.

Tesar 1991

Methods Study design: Randomised controlled trial

Participants

Diagnosis: DSM-III panic disorder with or without limited or extensive phobic avoidance

Method of diagnosis: Structured Clinical Interview for DSM-III-Upjohn version (SCID-Up)

Age: for alprazolam, $\mathrm{M}=32.8, \mathrm{SD}=8.9$; for clonazepam $\mathrm{M}=30.5, \mathrm{SD}=6.5$

Sex: for alprazolam 58 female, 42 male; for clonazepam 58 female, 42 male

Location: USA; setting unclear

Co-morbidities: patients with bipolar disorder, OCD, psychosis, dementia, substance abuse or major medical disorders were excluded; a concurrent diagnosis of major depression was permissible as long as it was judged to be secondary to panic disorder

Rescue medication: not stated

\section{Interventions Participants were randomly assigned to either:}

(1) alprazolam arm $(n=24)$

Duration: 6 weeks

Treatment Protocol: flexible dosage, range $=1-10 \mathrm{mg}, \mathrm{M}=5.39 \mathrm{SD}=2.89$

(2) clonazepam arm $(n=26)$

Duration: 6 weeks

Treatment Protocol: flexible dosage, range $=0.5-5 \mathrm{mg}, \mathrm{M}=2.5 \mathrm{SD}=0.94$

(3) placebo arm $(n=22)$

Duration: 6 weeks

Outcomes Time points for assessment: weekly

Outcomes:

1. Clinical Global Impression Scale (CGI) 
Tesar 1991 (Continued)
2. Patient Global Assessment (PGI)
3. panic attacks frequency
4. phobias
5. Work and Social Disability Scale
6. Beck Depression Inventory (BDI)

Date of study: not stated
Funding source: sponsored by Upjohn Co.
Declarations of interest among the primary researchers: none.

\section{Risk of bias}

\begin{tabular}{|c|c|c|}
\hline Bias & Authors' judgement & Support for judgement \\
\hline $\begin{array}{l}\text { Random sequence genera- } \\
\text { tion (selection bias) }\end{array}$ & Unclear risk & Quote: "randomly". \\
\hline $\begin{array}{l}\text { Allocation concealment } \\
\text { (selection bias) }\end{array}$ & Unclear risk & No information provided. \\
\hline $\begin{array}{l}\text { Blinding of participants } \\
\text { and personnel (perfor- } \\
\text { mance bias) } \\
\text { All outcomes }\end{array}$ & Low risk & $\begin{array}{l}\text { Quote: "the study drugs were administered in identical capsules according to a } \\
\text { standardised but flexible dosing schedule". }\end{array}$ \\
\hline $\begin{array}{l}\text { Blinding of outcome as- } \\
\text { sessment (detection bias) } \\
\text { All outcomes }\end{array}$ & Low risk & $\begin{array}{l}\text { Quote: "the study drugs were administered in identical capsules according to a } \\
\text { standardised but flexible dosing schedule". }\end{array}$ \\
\hline $\begin{array}{l}\text { Incomplete outcome data } \\
\text { (attrition bias) } \\
\text { All outcomes }\end{array}$ & Low risk & $\begin{array}{l}\text { Both endpoint analysis based on LOCF and completers analysis were per- } \\
\text { formed. }\end{array}$ \\
\hline $\begin{array}{l}\text { Selective reporting (re- } \\
\text { porting bias) }\end{array}$ & Low risk & All outcomes were reported. \\
\hline Other bias & High risk & $\begin{array}{l}\text { Supported in part by a grant from the Upjohn Co.; the role of the funder in } \\
\text { planning, conducting and writing the study is not discussed. }\end{array}$ \\
\hline
\end{tabular}

Tiller 1999

\begin{tabular}{ll}
\hline Methods & Study design: Randomised controlled trial \\
\hline Participants & Diagnosis: DSM-III-R panic disorder \\
Method of diagnosis: Structured Clinical Interview (SCID) \\
Age: M $=35$ \\
Sex: $67 \%$ female \\
Location: not stated; setting: unclear
\end{tabular}


Tiller 1999 (Continued)

Co-morbidities: not stated

Rescue medication: not stated; "there was not extensive co-prescription of hypnotics, sedatives or beta-blockers".

Interventions

\section{Participants were randomly assigned to either:}

(1) moclobemide arm $(n=182)$

Duration: 8 weeks

Treatment Protocol: flexible dosage, range $=300-600 \mathrm{mg}, \mathrm{M}=498, \mathrm{SD}=68$

(2) fluoxetine arm $(n=184)$

Duration: 8 weeks

Treatment Protocol: flexible dosage, range $=10-30 \mathrm{mg}, \mathrm{M}=20.5, \mathrm{SD}=2.7$

Time points for assessment:
Outcomes:
1. number of adverse events
2. severe adverse events
3. clinical global impression of tolerability
4. panic-free patients
5. Clinical Global Impression Scale (CGI)

Notes

Date of study: not stated

Funding source: sponsored by Hoffmann-La Roche

Declarations of interest among the primary researchers: none.

\section{Risk of bias}

\begin{tabular}{|c|c|c|}
\hline Bias & Authors' judgement & Support for judgement \\
\hline $\begin{array}{l}\text { Random sequence genera- } \\
\text { tion (selection bias) }\end{array}$ & Unclear risk & Quote: "randomly allocated" \\
\hline $\begin{array}{l}\text { Allocation concealment } \\
\text { (selection bias) }\end{array}$ & Unclear risk & No information provided. \\
\hline $\begin{array}{l}\text { Blinding of participants } \\
\text { and personnel (perfor- } \\
\text { mance bias) } \\
\text { All outcomes }\end{array}$ & Unclear risk & Quote: "double blind". No further information provided. \\
\hline $\begin{array}{l}\text { Blinding of outcome as- } \\
\text { sessment (detection bias) } \\
\text { All outcomes }\end{array}$ & Unclear risk & Quote: "double blind". No further information provided. \\
\hline $\begin{array}{l}\text { Incomplete outcome data } \\
\text { (attrition bias) } \\
\text { All outcomes }\end{array}$ & High risk & $\begin{array}{l}\text { No information provided about management of incomplete outcome data; } \\
\text { number of total dropouts not reported. }\end{array}$ \\
\hline
\end{tabular}


Tiller 1999 (Continued)

Selective reporting (re- Unclear risk $\quad$ All relevant outcomes mentioned in the methods section were reported. porting bias)

Other bias

High risk

Sponsored by Hoffmann-La Roche; the role of the funder in planning, conducting and writing the study is not discussed.

Uhlenhuth 1989

\begin{tabular}{ll} 
Methods & Study design: Randomised controlled trial \\
\hline Participants & Diagnosis: DSM - III panic disorder or agoraphobia with panic attacks
\end{tabular}

Method of diagnosis: SCID-UP

Age: $M=31.54, S D=7.12$

Sex: $58 \%$ female

Location: USA; setting: outpatients

Co-morbidities: patients with another primary psychiatric disorder or a physical disorder judged likely to interfere with the study were excluded

Rescue medication: not stated

(1) alprazolam $2 \mathrm{mg}$ arm $(\mathrm{n}=20)$

\section{Duration: 8 weeks}

Treatment Protocol: fixed dosage $2 \mathrm{mg}$

(2) alprazolam $6 \mathrm{mg}$ arm $(\mathrm{n}=21)$

Duration: 8 weeks

Treatment Protocol: fixed dosage $6 \mathrm{mg}$

(3) imipramine $\operatorname{arm}(\mathrm{n}=20)$

Duration: 8 weeks

Treatment Protocol: fixed dosage $225 \mathrm{mg}$

(4) placebo arm $(n=20)$

Duration: 8 weeks

\section{Outcomes:}

1. number of panic attacks (major, spontaneous, minor, situational)

2. Marks \& Matthews Phobia Scale

3. disability

4. Hamilton Rating Scale for Anxiety (HAMA)

5. Hamilton Rating Scale for Depression (HRSD) 
Uhlenhuth 1989 (Continued)

6. SAFTEE-UP for adverse effects

Notes Date of study: not stated

Funding source: sponsored by Upjohn Company

Declarations of interest among the primary researchers: not stated.

\section{Risk of bias}

\begin{tabular}{|c|c|c|}
\hline Bias & Authors' judgement & Support for judgement \\
\hline $\begin{array}{l}\text { Random sequence genera- } \\
\text { tion (selection bias) }\end{array}$ & Unclear risk & Quote: "random". \\
\hline $\begin{array}{l}\text { Allocation concealment } \\
\text { (selection bias) }\end{array}$ & Unclear risk & No information provided. \\
\hline $\begin{array}{l}\text { Blinding of participants } \\
\text { and personnel (perfor- } \\
\text { mance bias) } \\
\text { All outcomes }\end{array}$ & Low risk & $\begin{array}{l}\text { Quote: "All patients received two identical appearing capsules four times dai- } \\
\text { ly". }\end{array}$ \\
\hline $\begin{array}{l}\text { Blinding of outcome as- } \\
\text { sessment (detection bias) } \\
\text { All outcomes }\end{array}$ & Low risk & $\begin{array}{l}\text { Quote: "All patients received two identical appearing capsules four times dai- } \\
\text { ly". }\end{array}$ \\
\hline $\begin{array}{l}\text { Incomplete outcome data } \\
\text { (attrition bias) } \\
\text { All outcomes }\end{array}$ & Low risk & $\begin{array}{l}\text { Quote: "two sets of outcome analysis were employed; one included all } 81 \text { pa- } \\
\text { tients who entered treatment, and the other included only the } 63 \text { patients who } \\
\text { completed at least } 4 \text { weeks of treatment. Both sets of analysis presented here } \\
\text { were based on the final (last available) clinical score for each patient (endpoint } \\
\text { analysis). Patterns of dropout by treatment were analysed by survival analysis } \\
\text { using the actuarial life table method." }\end{array}$ \\
\hline $\begin{array}{l}\text { Selective reporting (re- } \\
\text { porting bias) }\end{array}$ & Low risk & All outcomes were reported. \\
\hline Other bias & High risk & $\begin{array}{l}\text { Sponsored by Upjohn Company; the role of the funder in planning, conducting } \\
\text { and writing the study is not discussed. }\end{array}$ \\
\hline
\end{tabular}

Van Vliet 1996

Methods Study design: Randomised controlled trial

Participants

Diagnosis: DSM-III-R panic disorder with or without agoraphobia

Method of diagnosis: open interview

Age: $M=35, S D=7.46$

Sex: 26 women, 6 men

Location: the Netherlands; setting: outpatients

Co-morbidities: patients with another anxiety disorder, major affective disorders or psychotic disorder, alcohol or drug abuse and medical problems were excluded

Rescue medication: oxazepam maximum $30 \mathrm{mg}$ daily 
Van Vliet 1996 (Continued)

Interventions
Participants were randomly assigned to either:

(1) brofaromine arm $(n=15)$

Duration: 12 weeks

Treatment Protocol: fixed dosage $150 \mathrm{mg}$

(2) fluvoxamine arm $(n=15)$

Duration: 12 weeks

Treatment Protocol: fixed dosage $150 \mathrm{mg}$

\section{Time points for assessment: weekly}

\section{Outcomes:}

1. Hamilton Rating Scale for Anxiety (HAMA)

2. Montgomery-Åsberg Depression Rating Scale (MADRS)

3. Fear Questionnaire

4. number of panic attacks

5. Hamilton Rating Scale for Depression (HAM-D)

6. SCL-90

\section{Notes}

Date of study: not stated

Funding source: not stated

Declarations of interest among the primary researchers: not stated.

\section{Risk of bias}

\begin{tabular}{lll}
\hline Bias & Authors' judgement & Support for judgement \\
\hline $\begin{array}{l}\text { Random sequence genera- } \\
\text { tion (selection bias) }\end{array}$ & Unclear risk & Quote: "randomly". \\
\hline
\end{tabular}

Allocation concealment Unclear risk No information provided.

(selection bias)

Blinding of participants Unclear risk Quote: "double blind". No further information provided.
and personnel (performance bias)

All outcomes

\begin{tabular}{|c|c|c|}
\hline $\begin{array}{l}\text { Blinding of outcome as- } \\
\text { sessment (detection bias) } \\
\text { All outcomes }\end{array}$ & Unclear risk & Quote: "double blind". No further information provided. \\
\hline
\end{tabular}

Incomplete outcome data Unclear risk No information provided about management of incomplete outcome data.
(attrition bias)
All outcomes


Van Vliet 1996 (Continued)

Selective reporting (re- High risk Continuous outcomes are reported incompletely (number of evaluated paporting bias) tients is not reported), so that they cannot be entered in a meta-analysis; Fear Questionnaire data for agoraphobia are only reported in graphs.

Other bias Unclear risk Sponsorship bias cannot be ruled out.

Wade 1997

Methods Study design: Randomised controlled trial

Participants

Diagnosis: DSM-III-R panic disorder

Method of diagnosis: not stated

Age: $M=38, S D$ not provided

Sex: $70 \%$ female, $30 \%$ male

Location: not stated; setting unclear

Co-morbidities: patients with depression, organic brain damage, drug/alcohol misuse and other severe psychiatric or somatic disorders were excluded

Rescue medication: treatment with oxazepam was permitted during weeks 1 and 2 (maximum dose 20 mg daily), discontinued during weeks 3 and 4 , and prohibited during weeks 5 to 8 .

\section{Participants were randomly assigned to either:}

(1) citalopram 10-15 mg arm ( $\mathrm{n}=97)$

Duration: 8 weeks

Treatment Protocol: $10 \mathrm{mg}$, with the option of increasing to $15 \mathrm{mg}$ if efficacy was not seen

(2) citalopram 20-30 mg arm ( $\mathrm{n}=95)$

Duration: 8 weeks

Treatment Protocol: $20 \mathrm{mg}$, with the option of increasing to $30 \mathrm{mg}$ if efficacy was not seen

(3) citalopram 40-60 arm ( $\mathrm{n}=89)$

Duration: 8 weeks

Treatment Protocol: $40 \mathrm{mg}$, with the option of increasing to $60 \mathrm{mg}$ if efficacy was not seen

(4) clomipramine $(n=98)$

Duration: 8 weeks

Treatment Protocol: $60 \mathrm{mg}$, with the option of increasing to $90 \mathrm{mg}$ if efficacy was not seen

(5) placebo $(n=96)$

Outcomes

Time points for assessment: baseline, last assessment (no further details provided)

\section{Outcomes:}

1. number of panic attacks - Clinical Anxiety Scale (CAS)

2. general improvement (Physician's Global Improvement Scale, Patient's Global Improvement Scale) 
Wade 1997 (Continued)

3. Hamilton Anxiety Rating Scale (HAS)

4. Montgomery-Åsberg Depression Rating Scale (MADRS)

Date of study: not stated
$\begin{aligned} & \text { Funding source: not stated } \\ & \text { Declarations of interest among the primary researchers: None (but authors' affiliations refer to } \\ & \text { pharmaceutical companies). }\end{aligned}$

\section{Risk of bias}

\begin{tabular}{lll}
\hline Bias & Authors' judgement & Support for judgement \\
\hline $\begin{array}{l}\text { Random sequence genera- } \\
\text { tion (selection bias) }\end{array}$ & Unclear risk & Quote: "randomised". \\
\hline
\end{tabular}

Allocation concealment Unclear risk No information provided.

(selection bias)

Blinding of participants Unclear risk Quote: "double blind". No further information provided.
and personnel (performance bias)

All outcomes

Blinding of outcome as- $\quad$ Unclear risk Quote: "double blind". No further information provided.
sessment (detection bias)
All outcomes

\begin{tabular}{lll}
\hline $\begin{array}{l}\text { Incomplete outcome data } \\
\text { (attrition bias) } \\
\text { All outcomes }\end{array}$ & Low risk & $\begin{array}{l}\text { Quote: "the primary analysis of efficacy was based upon the relative number of } \\
\text { responding patients for the ITT population and by use of the LOCF". }\end{array}$ \\
\hline $\begin{array}{l}\text { Selective reporting (re- } \\
\text { porting bias) }\end{array}$ & Unclear risk & All outcomes were reported; data on CAS are reported only in graphs. \\
\hline Other bias & Unclear risk & One of the authors' affiliation refer to Lundbeck. \\
\hline
\end{tabular}

Westenberg 1989

\begin{tabular}{ll}
\hline Methods & Study design: Randomised controlled trial \\
\hline Participants & Diagnosis: DSM-III-R \\
Method of diagnosis: not stated \\
Age: not stated \\
Sex: 38 females, 9 males \\
Location: the Netherlands; setting: outpatients \\
Co-morbidities: patients with a primary diagnosis other than panic disorder were excluded \\
Rescue medication: none \\
Participants were randomly assigned to either:
\end{tabular}


Westenberg 1989 (Continued)

(1) fluvoxamine arm

Duration: 6 weeks

Treatment Protocol: dose gradually increased to $150 \mathrm{mg}$

(2) maprotiline arm

Duration: 6 weeks

Treatment Protocol: dose gradually increased to $150 \mathrm{mg}$

Total number of randomised patients $=47$. The number of patients randomised for each arm is not provided.

Time points for assessment: baseline, week
Outcomes:
1. SCL-90
2. Hamilton Rating Scale for Anxiety (HAMA)
3. State-Trait Anxiety Inventory (STAI)
4. Self Rating Depression Scale
5. number of panic attacks per week

Notes

Date of study: not stated

Funding source: not stated

Declarations of interest among the primary researchers: not stated.

\section{Risk of bias}

\begin{tabular}{lll}
\hline Bias & Authors' judgement & Support for judgement \\
\hline $\begin{array}{l}\text { Random sequence genera- } \\
\text { tion (selection bias) }\end{array}$ & Unclear risk & Quote: "randomised". \\
\hline $\begin{array}{l}\text { Allocation concealment } \\
\text { (selection bias) }\end{array}$ & Unclear risk & No information provided. \\
\hline
\end{tabular}

Blinding of participants Unclear risk Quote: "double blind". No further information provided.
and personnel (perfor-

mance bias)

All outcomes

Blinding of outcome as- Unclear risk Quote: "double blind". No further information provided.
sessment (detection bias)
All outcomes

\begin{tabular}{lll}
\hline $\begin{array}{l}\text { Incomplete outcome data } \\
\text { (attrition bias) } \\
\text { All outcomes }\end{array}$ & Unclear risk & No information provided about management of incomplete outcome data. \\
\hline $\begin{array}{l}\text { Selective reporting (re- } \\
\text { porting bias) }\end{array}$ & High risk & The only available and comparable data are reported in a graph (HAMA). \\
\hline Other bias & Unclear risk & Sponsorship bias cannot be ruled out. \\
\hline \hline
\end{tabular}


Zhang 2000

\begin{tabular}{ll}
\hline Methods & Study design: Randomised controlled trial \\
\hline Participants & Diagnosis: DSM III - R \\
Method of diagnosis: no available information \\
Age: no available information \\
Sex: no available information \\
Location: China; setting: in and outpatients \\
Co-morbidities: none \\
Rescue medication: not stated \\
\hline
\end{tabular}

Interventions

Participants were randomly assigned to either:

(1) paroxetine $\operatorname{arm}(n=38)$

Duration: 10 weeks

Treatment Protocol: week 1: 20 mg, week 2: 30 mg, week 3: 40 mg, week 4-10: 40-50 mg; M = 43.5, SD = 4.8

(2) clomipramine arm $(n=35)$

Duration: 10 weeks

Treatment Protocol: week 1: $50 \mathrm{mg}$, week 2: $100 \mathrm{mg}$, week 3: $150 \mathrm{mg}$, week 4-10: 150-200 mg; M = 159.7, SD = 20.1

Outcomes Time points for assessment: no available information

Outcomes: no available information

\begin{tabular}{|c|c|c|}
\hline Notes & \multicolumn{2}{|c|}{$\begin{array}{l}\text { Date of study: no available information } \\
\text { Funding source: sponsored by the drug company marketing the drug } \\
\text { Declarations of interest among the primary researchers: no available information. }\end{array}$} \\
\hline \multicolumn{3}{|l|}{ Risk of bias } \\
\hline Bias & Authors' judgement & Support for judgement \\
\hline $\begin{array}{l}\text { Random sequence genera- } \\
\text { tion (selection bias) }\end{array}$ & Unclear risk & Insufficient information to assess whether an important risk of bias exists. \\
\hline $\begin{array}{l}\text { Allocation concealment } \\
\text { (selection bias) }\end{array}$ & Unclear risk & Insufficient information to assess whether an important risk of bias exists. \\
\hline $\begin{array}{l}\text { Blinding of participants } \\
\text { and personnel (perfor- } \\
\text { mance bias) } \\
\text { All outcomes }\end{array}$ & Unclear risk & Insufficient information to assess whether an important risk of bias exists. \\
\hline
\end{tabular}

\begin{tabular}{ll}
\hline Blinding of outcome as- \\
sessment (detection bias)
\end{tabular}$\quad$ Unclear risk Insufficient information to assess whether an important risk of bias exists.


Zhang 2000 (Continued)

All outcomes

\begin{tabular}{lll}
\hline $\begin{array}{l}\text { Incomplete outcome data } \\
\text { (attrition bias) } \\
\text { All outcomes }\end{array}$ & Unclear risk & Insufficient information to assess whether an important risk of bias exists. \\
\hline $\begin{array}{l}\text { Selective reporting (re- } \\
\text { porting bias) }\end{array}$ & Unclear risk & Insufficient information to assess whether an important risk of bias exists. \\
\hline Other bias & High risk & $\begin{array}{l}\text { Sponsored by the drug company marketing the drug; the role of the funder in } \\
\text { planning, conducting and writing the study is not discussed. }\end{array}$ \\
\hline
\end{tabular}

DSM III/IV: Diagnostic and Statistical Manual of Mental Disorders (third/fourth revision)

GAD: generalised anxiety disorder

ICD-10: International Statistical Classification of Diseases and Related Health Problems, 10th revision

ITT: intention-to-treat

LOCF: last observation carried forward

M: mean

MDD: major depressive disorder

MHPG: 3-methoxy-4-hydroxyphenylglycol

OCD: obsessive compulsive disorder

SCL-90: Anxiety Subscale of Symptom Checklist-90-Revised

SD: standard deviation

SEM: standard error of the mean

TCAs: tricyclic antidepressants

Characteristics of excluded studies [ordered by study ID]

\begin{tabular}{|c|c|}
\hline Study & Reason for exclusion \\
\hline Bakish 1994 & Study design (single case). \\
\hline Baldini Rossi 2000 & Participants were not primarily diagnosed with panic disorder. \\
\hline Balon 1991 & Study design (panicogenic). \\
\hline Balon 1993 & Study design (panicogenic). \\
\hline Bystritsky 1990 & Study design (not double-blind). \\
\hline Charney 1986 & Study design (not randomised). \\
\hline Davis 1981 & Study design (it is not a study). \\
\hline de Jonghe 1989 & $\begin{array}{l}\text { Participants were diagnosed with anxiety disorders including panic disorder, but the randomisa- } \\
\text { tion was not stratified by diagnosis; wrong comparison (lorazepam versus suriclone). }\end{array}$ \\
\hline Dell'Erba 2006 & Study design (not randomised); combined therapy with cognitive behaviour therapy. \\
\hline den Boer 1987 & $\begin{array}{l}\text { Participants were diagnosed with anxiety disorders including panic disorder, but the randomisa- } \\
\text { tion was not stratified by diagnosis. }\end{array}$ \\
\hline Dunner 1986 & $\begin{array}{l}\text { Participants were diagnosed with anxiety disorders including panic disorder, but the randomisa- } \\
\text { tion was not stratified by diagnosis. }\end{array}$ \\
\hline Dyukova 1992 & Study design (not randomised); participants were not diagnosed with panic disorder. \\
\hline
\end{tabular}




\begin{tabular}{|c|c|}
\hline Study & Reason for exclusion \\
\hline Evans 1986 & Combined therapy with psychotherapy. \\
\hline Fahy 1992 & Combined therapy with psychotherapy. \\
\hline Franulic 1989 & Study design (not randomised). \\
\hline Furukawa 2009 & Study design (it is not a study); combined therapy with cognitive behaviour therapy. \\
\hline Grilo 1998 & Combined therapy with cognitive behaviour therapy. \\
\hline Keller 1993 & Participants were not primarily diagnosed with panic disorder. \\
\hline Lepola 1989 & Study design (not randomised). \\
\hline Mavissakalian 2003 & Study design (long-term phase of a discontinuation/maintenance open-label study). \\
\hline McHugh 2007 & Combined therapy with cognitive behaviour therapy. \\
\hline Miretzky 1992 & Combined therapy with cognitive behaviour therapy. \\
\hline Nair 1982 & $\begin{array}{l}\text { Participants were diagnosed with anxiety disorders including panic disorder, but the randomisa- } \\
\text { tion was not stratified by diagnosis. }\end{array}$ \\
\hline Nardi 2011 & Study design (not double-blind). \\
\hline Pareek 2014 & Wrong comparison (clonazepam-CR versus clonazepam). \\
\hline Pfizer 2002 & Wrong comparison (venlafaxine versus placebo). \\
\hline Pfizer 2005 & Study design (not double-blind). \\
\hline Pohl 1989 & Combined therapy with psychotherapy. \\
\hline Pollack 2003 & Combined therapy with different drugs. \\
\hline Pyke 1989 & Study design (panicogenic). \\
\hline Raffaele 2002 & Wrong comparison (citalopram versus placebo). \\
\hline Roll 2004 & Combined therapy with cognitive behaviour therapy. \\
\hline Roy-Byrne 2001 & Wrong comparison (paroxetine versus usual care). \\
\hline Sheehan 1980 & Participants were not diagnosed with panic disorder. \\
\hline Surman 1986 & Study design (not randomised). \\
\hline Svebak 1990 & Study design (not randomised). \\
\hline Tsutsui 2000 & Wrong comparison (paroxetine versus placebo). \\
\hline Tyrer 1988 & Participants with different diagnosis, and randomisation was not stratified according to diagnosis. \\
\hline van Apeldoorn 2008 & Combined therapy with cognitive behaviour therapy. \\
\hline
\end{tabular}




\begin{tabular}{ll}
\hline Study & Reason for exclusion \\
\hline Yang 2005 & Study design (not double-blind). \\
\hline Yang 2006 & Study design (not double-blind). \\
\hline Yeragani 1992 & Study design (panicogenic). \\
\hline Zajecka 1996 & Participants were not diagnosed with panic disorder. \\
\hline
\end{tabular}

Characteristics of studies awaiting assessment [ordered by study ID]

Minutentag 2001

\begin{tabular}{ll}
\hline Methods & 18 weeks randomised trial. \\
\hline Participants & 39 patients with panic disorder. \\
\hline Interventions & Imipramine and citalopram. \\
\hline Outcomes & Bandelow Panic and Agoraphobia Scale, CGI-S. Sheehan Disability Scale. \\
\hline Notes & Waiting for translation from Portuguese to English. \\
\hline
\end{tabular}

CGI-S: Clinical Global Impression Severity of Illness Score

\section{DATA AND ANALYSES}

\section{Comparison 1. Antidepressants versus benzodiazepines}

\begin{tabular}{|c|c|c|c|c|}
\hline $\begin{array}{l}\text { Outcome or subgroup } \\
\text { title }\end{array}$ & No. of studies & $\begin{array}{l}\text { No. of partici- } \\
\text { pants }\end{array}$ & Statistical method & Effect size \\
\hline 1 Failure to respond & 2 & 215 & Risk Ratio (M-H, Random, 95\% Cl) & $0.99[0.67,1.47]$ \\
\hline 1.1 TCAs versus BDZs & 1 & 61 & Risk Ratio (M-H, Random, 95\% Cl) & $1.03[0.63,1.67]$ \\
\hline $1.2 \mathrm{SSRIs}$ versus BDZs & 1 & 154 & Risk Ratio (M-H, Random, 95\% Cl) & $0.93[0.48,1.80]$ \\
\hline $1.3 \mathrm{MAOIs}$ versus BDZs & 0 & 0 & Risk Ratio (M-H, Random, 95\% Cl) & $0.0[0.0,0.0]$ \\
\hline 1.4 SNRIs versus BDZs & 0 & 0 & Risk Ratio (M-H, Random, 95\% Cl) & $0.0[0.0,0.0]$ \\
\hline $1.5 \mathrm{NaSSAs}$ versus BDZs & 0 & 0 & Risk Ratio (M-H, Random, 95\% Cl) & $0.0[0.0,0.0]$ \\
\hline 1.6 NDRIs versus BDZs & 0 & 0 & Risk Ratio (M-H, Random, 95\% Cl) & $0.0[0.0,0.0]$ \\
\hline 1.7 NRIs versus BDZs & 0 & 0 & Risk Ratio (M-H, Random, 95\% Cl) & $0.0[0.0,0.0]$ \\
\hline $\begin{array}{l}1.8 \text { Other ADs versus } \\
\text { BDZs }\end{array}$ & 0 & 0 & Risk Ratio (M-H, Random, 95\% Cl) & $0.0[0.0,0.0]$ \\
\hline
\end{tabular}




\begin{tabular}{|c|c|c|c|c|}
\hline $\begin{array}{l}\text { Outcome or subgroup } \\
\text { title }\end{array}$ & No. of studies & $\begin{array}{l}\text { No. of partici- } \\
\text { pants }\end{array}$ & Statistical method & Effect size \\
\hline $\begin{array}{l}2 \text { Total number of } \\
\text { dropouts }\end{array}$ & 7 & 1449 & Risk Ratio (M-H, Random, 95\% Cl) & $1.64[1.03,2.63]$ \\
\hline 2.1 TCAs versus BDZs & 6 & 1295 & Risk Ratio (M-H, Random, 95\% Cl) & $1.67[0.93,2.99]$ \\
\hline 2.2 SSRIs versus BDZs & 1 & 154 & Risk Ratio (M-H, Random, 95\% Cl) & $1.71[1.03,2.84]$ \\
\hline $2.3 \mathrm{MAOIs}$ versus $\mathrm{BDZs}$ & 0 & 0 & Risk Ratio (M-H, Random, 95\% Cl) & $0.0[0.0,0.0]$ \\
\hline 2.4 SNRIs versus BDZs & 0 & 0 & Risk Ratio (M-H, Random, 95\% Cl) & $0.0[0.0,0.0]$ \\
\hline 2.5 NaSSAs versus BDZs & 0 & 0 & Risk Ratio (M-H, Random, 95\% Cl) & $0.0[0.0,0.0]$ \\
\hline 2.6 NDRIs versus BDZs & 0 & 0 & Risk Ratio (M-H, Random, 95\% Cl) & $0.0[0.0,0.0]$ \\
\hline 2.7 NRIs versus BDZs & 0 & 0 & Risk Ratio (M-H, Random, 95\% Cl) & $0.0[0.0,0.0]$ \\
\hline $\begin{array}{l}2.8 \text { Other ADs versus } \\
\text { BDZs }\end{array}$ & 0 & 0 & Risk Ratio (M-H, Random, 95\% Cl) & $0.0[0.0,0.0]$ \\
\hline 3 Failure to remit & 3 & 1002 & Risk Ratio (M-H, Random, 95\% Cl) & $1.18[1.01,1.37]$ \\
\hline 3.1 TCAs versus BDZs & 2 & 848 & Risk Ratio (M-H, Random, 95\% Cl) & $1.19[1.00,1.41]$ \\
\hline 3.2 SSRIs versus BDZs & 1 & 154 & Risk Ratio (M-H, Random, 95\% Cl) & $1.12[0.79,1.59]$ \\
\hline 3.3 MAOIs versus BDZs & 0 & 0 & Risk Ratio (M-H, Random, 95\% Cl) & $0.0[0.0,0.0]$ \\
\hline 3.4 SNRIs versus BDZs & 0 & 0 & Risk Ratio (M-H, Random, 95\% Cl) & $0.0[0.0,0.0]$ \\
\hline 3.5 NaSSAs versus BDZs & 0 & 0 & Risk Ratio (M-H, Random, 95\% Cl) & $0.0[0.0,0.0]$ \\
\hline 3.6 NDRIs versus BDZs & 0 & 0 & Risk Ratio (M-H, Random, 95\% Cl) & $0.0[0.0,0.0]$ \\
\hline 3.7 NRIs versus BDZs & 0 & 0 & Risk Ratio (M-H, Random, 95\% Cl) & $0.0[0.0,0.0]$ \\
\hline $\begin{array}{l}3.8 \text { Other ADs versus } \\
\text { BDZs }\end{array}$ & 0 & 0 & Risk Ratio (M-H, Random, 95\% Cl) & $0.0[0.0,0.0]$ \\
\hline $\begin{array}{l}4 \text { Panic symptoms - end- } \\
\text { point score }\end{array}$ & 3 & 1144 & Mean Difference (IV, Random, 95\% Cl) & $-0.13[-1.06,0.80]$ \\
\hline 4.1 TCAs versus BDZs & 2 & 998 & Mean Difference (IV, Random, 95\% Cl) & $-0.25[-1.72,1.22]$ \\
\hline 4.2 SSRIs versus BDZs & 1 & 146 & Mean Difference (IV, Random, 95\% Cl) & $0.10[-0.34,0.54]$ \\
\hline 4.3 MAOIs versus BDZs & 0 & 0 & Mean Difference (IV, Random, 95\% Cl) & $0.0[0.0,0.0]$ \\
\hline 4.4 SNRIs versus BDZs & 0 & 0 & Mean Difference (IV, Random, 95\% CI) & $0.0[0.0,0.0]$ \\
\hline 4.5 NaSSAs versus BDZs & 0 & 0 & Mean Difference (IV, Random, 95\% Cl) & $0.0[0.0,0.0]$ \\
\hline 4.6 NDRIs versus BDZs & 0 & 0 & Mean Difference (IV, Random, 95\% CI) & $0.0[0.0,0.0]$ \\
\hline
\end{tabular}




\begin{tabular}{|c|c|c|c|c|}
\hline $\begin{array}{l}\text { Outcome or subgroup } \\
\text { title }\end{array}$ & No. of studies & $\begin{array}{l}\text { No. of partici- } \\
\text { pants }\end{array}$ & Statistical method & Effect size \\
\hline 4.7 NRIs versus BDZs & 0 & 0 & Mean Difference (IV, Random, 95\% CI) & $0.0[0.0,0.0]$ \\
\hline $\begin{array}{l}4.8 \text { Other ADs versus } \\
\text { BDZs }\end{array}$ & 0 & 0 & Mean Difference (IV, Random, 95\% CI) & $0.0[0.0,0.0]$ \\
\hline $\begin{array}{l}5 \text { Panic symptoms - } \\
\text { mean change }\end{array}$ & 1 & 44 & Mean Difference (IV, Random, 95\% CI) & $0.40[-0.83,1.63]$ \\
\hline 5.1 TCAs versus BDZs & 1 & 44 & Mean Difference (IV, Random, 95\% CI) & $0.40[-0.83,1.63]$ \\
\hline 5.2 SSRIs versus BDZs & 0 & 0 & Mean Difference (IV, Random, 95\% CI) & $0.0[0.0,0.0]$ \\
\hline 5.3 MAOIs versus BDZs & 0 & 0 & Mean Difference (IV, Random, 95\% CI) & $0.0[0.0,0.0]$ \\
\hline 5.4 SNRIs versus BDZs & 0 & 0 & Mean Difference (IV, Random, 95\% CI) & $0.0[0.0,0.0]$ \\
\hline $5.5 \mathrm{NaSSAs}$ versus BDZs & 0 & 0 & Mean Difference (IV, Random, 95\% CI) & $0.0[0.0,0.0]$ \\
\hline 5.6 NDRIs versus BDZs & 0 & 0 & Mean Difference (IV, Random, 95\% CI) & $0.0[0.0,0.0]$ \\
\hline 5.7 NRIs versus BDZs & 0 & 0 & Mean Difference (IV, Random, 95\% CI) & $0.0[0.0,0.0]$ \\
\hline $\begin{array}{l}5.8 \text { Other ADs versus } \\
\text { BDZs }\end{array}$ & 0 & 0 & Mean Difference (IV, Random, 95\% CI) & $0.0[0.0,0.0]$ \\
\hline $\begin{array}{l}6 \text { Frequency of panic at- } \\
\text { tacks }\end{array}$ & 7 & 595 & Mean Difference (IV, Random, 95\% CI) & $0.33[-0.72,1.38]$ \\
\hline 6.1 TCAs versus BDZs & 6 & 458 & Mean Difference (IV, Random, 95\% CI) & $0.30[-0.81,1.41]$ \\
\hline $6.2 \mathrm{SSRIs}$ versus $\mathrm{BDZs}$ & 1 & 137 & Mean Difference (IV, Random, 95\% CI) & $1.20[-3.31,5.71]$ \\
\hline 6.3 MAOIs versus BDZs & 0 & 0 & Mean Difference (IV, Random, 95\% CI) & $0.0[0.0,0.0]$ \\
\hline 6.4 SNRIs versus BDZs & 0 & 0 & Mean Difference (IV, Random, 95\% CI) & $0.0[0.0,0.0]$ \\
\hline 6.5 NaSSAs versus BDZs & 0 & 0 & Mean Difference (IV, Random, 95\% CI) & $0.0[0.0,0.0]$ \\
\hline 6.6 NDRIs versus BDZs & 0 & 0 & Mean Difference (IV, Random, 95\% CI) & $0.0[0.0,0.0]$ \\
\hline $6.7 \mathrm{NRIs}$ versus $\mathrm{BDZs}$ & 0 & 0 & Mean Difference (IV, Random, 95\% CI) & $0.0[0.0,0.0]$ \\
\hline $\begin{array}{l}6.8 \text { Other ADs versus } \\
\text { BDZs }\end{array}$ & 0 & 0 & Mean Difference (IV, Random, 95\% CI) & $0.0[0.0,0.0]$ \\
\hline 7 Agoraphobia & 5 & 1061 & Std. Mean Difference (IV, Random, 95\% CI) & $0.04[-0.08,0.16]$ \\
\hline 7.1 TCAs versus BDZs & 4 & 949 & Std. Mean Difference (IV, Random, 95\% CI) & $0.04[-0.08,0.17]$ \\
\hline 7.2 SSRIs versus BDZs & 1 & 112 & Std. Mean Difference (IV, Random, 95\% CI) & $0.0[-0.37,0.37]$ \\
\hline 7.3 MAOIs versus BDZs & 0 & 0 & Std. Mean Difference (IV, Random, 95\% CI) & $0.0[0.0,0.0]$ \\
\hline 7.4 SNRIs versus BDZs & 0 & 0 & Std. Mean Difference (IV, Random, 95\% CI) & $0.0[0.0,0.0]$ \\
\hline
\end{tabular}




\begin{tabular}{|c|c|c|c|c|}
\hline $\begin{array}{l}\text { Outcome or subgroup } \\
\text { title }\end{array}$ & No. of studies & $\begin{array}{l}\text { No. of partici- } \\
\text { pants }\end{array}$ & Statistical method & Effect size \\
\hline $7.5 \mathrm{NaSSAs}$ versus BDZs & 0 & 0 & Std. Mean Difference (IV, Random, 95\% CI) & $0.0[0.0,0.0]$ \\
\hline 7.6 NDRIs versus BDZs & 0 & 0 & Std. Mean Difference (IV, Random, 95\% CI) & $0.0[0.0,0.0]$ \\
\hline 7.7 NRIs versus BDZs & 0 & 0 & Std. Mean Difference (IV, Random, 95\% CI) & $0.0[0.0,0.0]$ \\
\hline $\begin{array}{l}\text { 7.8 Other ADs versus } \\
\text { BDZs }\end{array}$ & 0 & 0 & Std. Mean Difference (IV, Random, 95\% CI) & $0.0[0.0,0.0]$ \\
\hline 8 General anxiety & 8 & 1365 & Std. Mean Difference (IV, Random, 95\% CI) & $0.06[-0.25,0.37]$ \\
\hline 8.1 TCAs versus BDZs & 7 & 1238 & Std. Mean Difference (IV, Random, 95\% CI) & $0.08[-0.28,0.45]$ \\
\hline 8.2 SSRIs versus BDZs & 1 & 127 & Std. Mean Difference (IV, Random, 95\% CI) & $-0.08[-0.42,0.27]$ \\
\hline 8.3 MAOIs versus BDZs & 0 & 0 & Std. Mean Difference (IV, Random, 95\% CI) & $0.0[0.0,0.0]$ \\
\hline 8.4 SNRIs versus BDZs & 0 & 0 & Std. Mean Difference (IV, Random, 95\% CI) & $0.0[0.0,0.0]$ \\
\hline 8.5 NaSSAs versus BDZs & 0 & 0 & Std. Mean Difference (IV, Random, 95\% CI) & $0.0[0.0,0.0]$ \\
\hline 8.6 NDRIs versus BDZs & 0 & 0 & Std. Mean Difference (IV, Random, 95\% CI) & $0.0[0.0,0.0]$ \\
\hline 8.7 NRIs versus BDZs & 0 & 0 & Std. Mean Difference (IV, Random, 95\% CI) & $0.0[0.0,0.0]$ \\
\hline $\begin{array}{l}8.8 \text { Other ADs versus } \\
\text { BDZs }\end{array}$ & 0 & 0 & Std. Mean Difference (IV, Random, 95\% CI) & $0.0[0.0,0.0]$ \\
\hline 9 Depression & 6 & 892 & Std. Mean Difference (IV, Random, 95\% CI) & $-0.14[-0.29,0.02]$ \\
\hline 9.1 TCAs versus BDZs & 5 & 765 & Std. Mean Difference (IV, Random, 95\% CI) & $-0.08[-0.22,0.06]$ \\
\hline 9.2 SSRIs versus BDZs & 1 & 127 & Std. Mean Difference (IV, Random, 95\% CI) & $-0.46[-0.81,-0.11]$ \\
\hline 9.3 MAOIs versus BDZs & 0 & 0 & Std. Mean Difference (IV, Random, 95\% CI) & $0.0[0.0,0.0]$ \\
\hline 9.4 SNRIs versus BDZs & 0 & 0 & Std. Mean Difference (IV, Random, 95\% CI) & $0.0[0.0,0.0]$ \\
\hline 9.5 NaSSAs versus BDZs & 0 & 0 & Std. Mean Difference (IV, Random, 95\% CI) & $0.0[0.0,0.0]$ \\
\hline 9.6 NDRIs versus BDZs & 0 & 0 & Std. Mean Difference (IV, Random, 95\% CI) & $0.0[0.0,0.0]$ \\
\hline 9.7 NRIs versus BDZs & 0 & 0 & Std. Mean Difference (IV, Random, 95\% CI) & $0.0[0.0,0.0]$ \\
\hline $\begin{array}{l}\text { 9.8 Other ADs versus } \\
\text { BDZs }\end{array}$ & 0 & 0 & Std. Mean Difference (IV, Random, 95\% CI) & $0.0[0.0,0.0]$ \\
\hline 10 Social functioning & 4 & 1007 & Std. Mean Difference (IV, Random, 95\% CI) & $0.16[-0.12,0.44]$ \\
\hline 10.1 TCAs versus BDZs & 3 & 882 & Std. Mean Difference (IV, Random, 95\% CI) & $0.23[0.03,0.42]$ \\
\hline 10.2 SSRIs versus BDZs & 1 & 125 & Std. Mean Difference (IV, Random, 95\% CI) & $-0.22[-0.57,0.13]$ \\
\hline $10.3 \mathrm{MAOIs}$ versus BDZs & 0 & 0 & Std. Mean Difference (IV, Random, 95\% Cl) & $0.0[0.0,0.0]$ \\
\hline
\end{tabular}




\begin{tabular}{|c|c|c|c|c|}
\hline $\begin{array}{l}\text { Outcome or subgroup } \\
\text { title }\end{array}$ & No. of studies & $\begin{array}{l}\text { No. of partici- } \\
\text { pants }\end{array}$ & Statistical method & Effect size \\
\hline 10.4 SNRIs versus BDZs & 0 & 0 & Std. Mean Difference (IV, Random, 95\% CI) & $0.0[0.0,0.0]$ \\
\hline $10.5 \mathrm{NaSSAs}$ versus BDZs & 0 & 0 & Std. Mean Difference (IV, Random, 95\% CI) & $0.0[0.0,0.0]$ \\
\hline 10.6 NDRIs versus BDZs & 0 & 0 & Std. Mean Difference (IV, Random, 95\% CI) & $0.0[0.0,0.0]$ \\
\hline 10.7 NRIs versus BDZs & 0 & 0 & Std. Mean Difference (IV, Random, 95\% CI) & $0.0[0.0,0.0]$ \\
\hline $\begin{array}{l}10.8 \text { Other ADs versus } \\
\text { BDZs }\end{array}$ & 0 & 0 & Std. Mean Difference (IV, Random, 95\% CI) & $0.0[0.0,0.0]$ \\
\hline 11 Quality of life & 0 & 0 & Std. Mean Difference (IV, Random, 95\% CI) & $0.0[0.0,0.0]$ \\
\hline 11.1 TCAs versus BDZs & 0 & 0 & Std. Mean Difference (IV, Random, 95\% CI) & $0.0[0.0,0.0]$ \\
\hline 11.2 SSRIs versus BDZs & 0 & 0 & Std. Mean Difference (IV, Random, 95\% CI) & $0.0[0.0,0.0]$ \\
\hline $11.3 \mathrm{MAOIs}$ versus $\mathrm{BDZs}$ & 0 & 0 & Std. Mean Difference (IV, Random, 95\% CI) & $0.0[0.0,0.0]$ \\
\hline 11.4 SNRIs versus BDZs & 0 & 0 & Std. Mean Difference (IV, Random, 95\% CI) & $0.0[0.0,0.0]$ \\
\hline $11.5 \mathrm{NaSSAs}$ versus BDZs & 0 & 0 & Std. Mean Difference (IV, Random, 95\% CI) & $0.0[0.0,0.0]$ \\
\hline 11.6 NDRIs versus BDZs & 0 & 0 & Std. Mean Difference (IV, Random, 95\% CI) & $0.0[0.0,0.0]$ \\
\hline $11.7 \mathrm{NRI}$ s versus BDZs & 0 & 0 & Std. Mean Difference (IV, Random, 95\% CI) & $0.0[0.0,0.0]$ \\
\hline $\begin{array}{l}11.8 \text { Other ADs versus } \\
\text { BDZs }\end{array}$ & 0 & 0 & Std. Mean Difference (IV, Random, 95\% CI) & $0.0[0.0,0.0]$ \\
\hline 12 Patient satisfaction & 0 & 0 & Std. Mean Difference (IV, Random, 95\% CI) & $0.0[0.0,0.0]$ \\
\hline 12.1 TCAs versus BDZs & 0 & 0 & Std. Mean Difference (IV, Random, 95\% CI) & $0.0[0.0,0.0]$ \\
\hline 12.2 SSRIs versus BDZs & 0 & 0 & Std. Mean Difference (IV, Random, 95\% CI) & $0.0[0.0,0.0]$ \\
\hline $12.3 \mathrm{MAOIs}$ versus $\mathrm{BDZs}$ & 0 & 0 & Std. Mean Difference (IV, Random, 95\% CI) & $0.0[0.0,0.0]$ \\
\hline 12.4 SNRIs versus BDZs & 0 & 0 & Std. Mean Difference (IV, Random, 95\% CI) & $0.0[0.0,0.0]$ \\
\hline $12.5 \mathrm{NaSSAs}$ versus BDZs & 0 & 0 & Std. Mean Difference (IV, Random, 95\% CI) & $0.0[0.0,0.0]$ \\
\hline 12.6 NDRIs versus BDZs & 0 & 0 & Std. Mean Difference (IV, Random, 95\% CI) & $0.0[0.0,0.0]$ \\
\hline 12.7 NRIs versus BDZs & 0 & 0 & Std. Mean Difference (IV, Random, 95\% CI) & $0.0[0.0,0.0]$ \\
\hline $\begin{array}{l}12.8 \text { Other ADs versus } \\
\text { BDZs }\end{array}$ & 0 & 0 & Std. Mean Difference (IV, Random, 95\% CI) & $0.0[0.0,0.0]$ \\
\hline 13 Economic costs & 0 & 0 & Std. Mean Difference (IV, Random, 95\% CI) & $0.0[0.0,0.0]$ \\
\hline 13.1 TCAs versus BDZs & 0 & 0 & Std. Mean Difference (IV, Random, 95\% CI) & $0.0[0.0,0.0]$ \\
\hline 13.2 SSRIs versus BDZs & 0 & 0 & Std. Mean Difference (IV, Random, 95\% CI) & $0.0[0.0,0.0]$ \\
\hline
\end{tabular}




\begin{tabular}{|c|c|c|c|c|}
\hline $\begin{array}{l}\text { Outcome or subgroup } \\
\text { title }\end{array}$ & No. of studies & $\begin{array}{l}\text { No. of partici- } \\
\text { pants }\end{array}$ & Statistical method & Effect size \\
\hline 13.3 MAOIs versus BDZs & 0 & 0 & Std. Mean Difference (IV, Random, 95\% CI) & $0.0[0.0,0.0]$ \\
\hline 13.4 SNRIs versus BDZs & 0 & 0 & Std. Mean Difference (IV, Random, 95\% CI) & $0.0[0.0,0.0]$ \\
\hline 13.5 NaSSAs versus BDZs & 0 & 0 & Std. Mean Difference (IV, Random, 95\% CI) & $0.0[0.0,0.0]$ \\
\hline 13.6 NDRIs versus BDZs & 0 & 0 & Std. Mean Difference (IV, Random, 95\% CI) & $0.0[0.0,0.0]$ \\
\hline 13.7 NRIs versus BDZs & 0 & 0 & Std. Mean Difference (IV, Random, 95\% CI) & $0.0[0.0,0.0]$ \\
\hline $\begin{array}{l}13.8 \text { Other ADs versus } \\
\text { BDZs }\end{array}$ & 0 & 0 & Std. Mean Difference (IV, Random, 95\% CI) & $0.0[0.0,0.0]$ \\
\hline $\begin{array}{l}14 \text { Number of dropouts } \\
\text { due to adverse effects }\end{array}$ & 3 & 1002 & Risk Ratio (M-H, Random, 95\% Cl) & $1.72[1.03,2.87]$ \\
\hline 14.1 TCAs versus BDZs & 2 & 848 & Risk Ratio (M-H, Random, 95\% Cl) & $2.10[1.13,3.93]$ \\
\hline 14.2 SSRIs versus BDZs & 1 & 154 & Risk Ratio (M-H, Random, 95\% Cl) & $1.2[0.55,2.61]$ \\
\hline 14.3 MAOIs versus BDZs & 0 & 0 & Risk Ratio (M-H, Random, 95\% Cl) & $0.0[0.0,0.0]$ \\
\hline 14.4 SNRIs versus BDZs & 0 & 0 & Risk Ratio (M-H, Random, 95\% Cl) & $0.0[0.0,0.0]$ \\
\hline 14.5 NaSSAs versus BDZs & 0 & 0 & Risk Ratio (M-H, Random, 95\% Cl) & $0.0[0.0,0.0]$ \\
\hline 14.6 NDRIs versus BDZs & 0 & 0 & Risk Ratio (M-H, Random, 95\% Cl) & $0.0[0.0,0.0]$ \\
\hline 14.7 NRIs versus BDZs & 0 & 0 & Risk Ratio (M-H, Random, 95\% Cl) & $0.0[0.0,0.0]$ \\
\hline $\begin{array}{l}14.8 \text { Other ADs versus } \\
\text { BDZs }\end{array}$ & 0 & 0 & Risk Ratio (M-H, Random, 95\% Cl) & $0.0[0.0,0.0]$ \\
\hline $\begin{array}{l}15 \text { Number of patients } \\
\text { experiencing at least one } \\
\text { adverse effect }\end{array}$ & 1 & 154 & Risk Ratio (M-H, Random, 95\% Cl) & $1.03[0.92,1.15]$ \\
\hline 15.1 TCAs versus BDZs & 0 & 0 & Risk Ratio (M-H, Random, 95\% Cl) & $0.0[0.0,0.0]$ \\
\hline 15.2 SSRIs versus BDZs & 1 & 154 & Risk Ratio (M-H, Random, 95\% Cl) & $1.03[0.92,1.15]$ \\
\hline 15.3 MAOls versus BDZs & 0 & 0 & Risk Ratio (M-H, Random, 95\% Cl) & $0.0[0.0,0.0]$ \\
\hline 15.4 SNRIs versus BDZs & 0 & 0 & Risk Ratio (M-H, Random, 95\% Cl) & $0.0[0.0,0.0]$ \\
\hline 15.5 NaSSAs versus BDZs & 0 & 0 & Risk Ratio (M-H, Random, 95\% Cl) & $0.0[0.0,0.0]$ \\
\hline 15.6 NDRIs versus BDZs & 0 & 0 & Risk Ratio (M-H, Random, 95\% Cl) & $0.0[0.0,0.0]$ \\
\hline 15.7 NRIs versus BDZs & 0 & 0 & Risk Ratio (M-H, Random, 95\% Cl) & $0.0[0.0,0.0]$ \\
\hline $\begin{array}{l}\text { 15.8 Other ADs versus } \\
\text { BDZs }\end{array}$ & 0 & 0 & Risk Ratio (M-H, Random, 95\% Cl) & $0.0[0.0,0.0]$ \\
\hline
\end{tabular}


Analysis 1.1. Comparison 1 Antidepressants versus benzodiazepines, Outcome 1 Failure to respond.

$\begin{array}{ccccc}\text { Study or subgroup } & \begin{array}{c}\text { Antide- } \\ \text { pressants }\end{array} & \begin{array}{c}\text { Benzodi- } \\ \text { azepines }\end{array} & \text { Risk Ratio } & \text { Weight }\end{array}$

$n / N \quad n / N$

M-H, Random, $95 \% \mathrm{CI}$

M-H, Random, 95\% Cl

\subsubsection{TCAs versus BDZs}

Uhlenhuth 1989

$11 / 20$

$\mathrm{n} / \mathrm{N}$

$64.4 \%$

$1.02[0.63,1.67]$

Subtotal $(95 \% \mathrm{CI})$

20

$22 / 41$

41

$64.4 \%$

$1.02[0.63,1.67]$

Total events: 11 (Antidepressants), 22 (Benzodiazepines)

Heterogeneity: Not applicable

Test for overall effect: $\mathrm{Z}=0.1(\mathrm{P}=0.92)$

\subsubsection{SSRIs versus BDZs}

GSK-29060/1

$14 / 77$

77

$15 / 77$

Total events: 14 (Antidepressants), 15 (Benzodiazepines)

Heterogeneity: Not applicable

Test for overall effect: $Z=0.21(P=0.84)$

\subsubsection{MAOIs versus BDZs}

Total events: 0 (Antidepressants), 0 (Benzodiazepines)

Heterogeneity: Not applicable

Test for overall effect: Not applicable

\subsubsection{SNRIs versus BDZs}

Total events: 0 (Antidepressants), 0 (Benzodiazepines)

Heterogeneity: Not applicable

Test for overall effect: Not applicable

\subsubsection{NaSSAs versus BDZs}

Total events: 0 (Antidepressants), 0 (Benzodiazepines)

Heterogeneity: Not applicable

Test for overall effect: Not applicable

\subsubsection{NDRIs versus BDZs}

Total events: 0 (Antidepressants), 0 (Benzodiazepines)

Heterogeneity: Not applicable

Test for overall effect: Not applicable

\subsubsection{NRIs versus BDZs}

Total events: 0 (Antidepressants), 0 (Benzodiazepines)

Heterogeneity: Not applicable

Test for overall effect: Not applicable

1.1.8 Other ADs versus BDZs

Total events: 0 (Antidepressants), 0 (Benzodiazepines) 


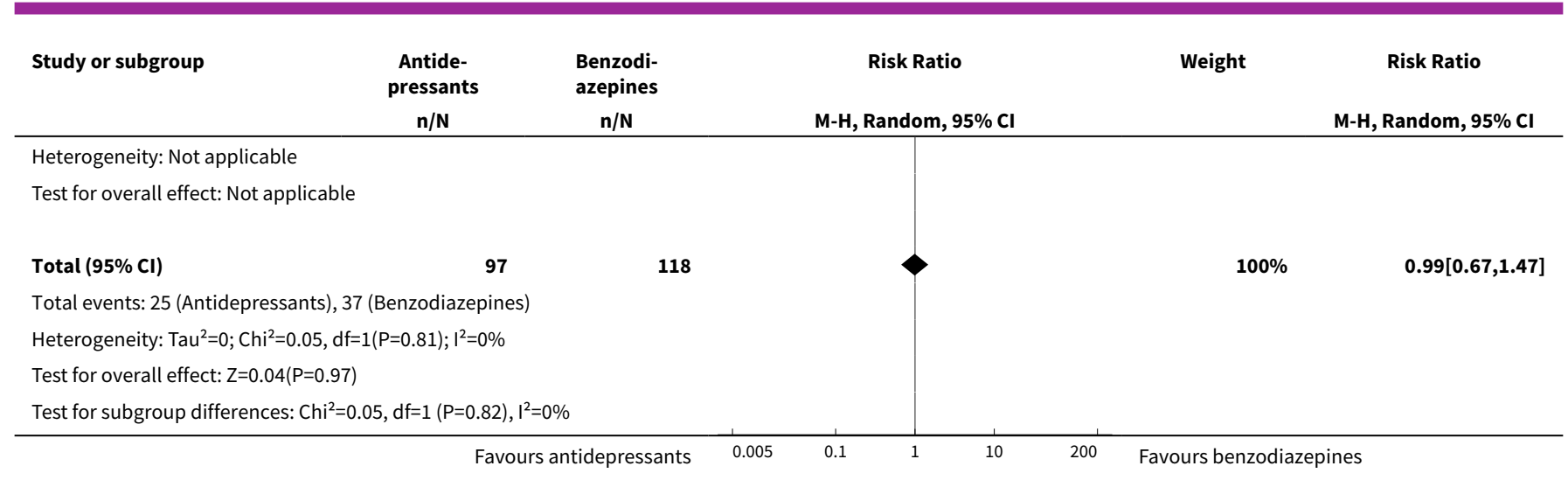

Analysis 1.2. Comparison 1 Antidepressants versus benzodiazepines, Outcome 2 Total number of dropouts.

\begin{tabular}{ccccc} 
Study or subgroup & $\begin{array}{c}\text { Antide- } \\
\text { pressants } \\
n / N\end{array}$ & $\begin{array}{c}\text { Benzodi- } \\
\text { azepines } \\
n / N\end{array}$ & Risk Ratio & Risk Ratio \\
\hline
\end{tabular}

\begin{tabular}{lrr}
\hline 1.2.1 TCAs versus BDZs & \\
CNCPS 1992 & $118 / 391$ & $67 / 386$ \\
Holland 1999 & $36 / 149$ & $58 / 166$ \\
Schweizer 1993 & $14 / 34$ & $4 / 37$ \\
Sheikh 1999 & $1 / 10$ & $0 / 8$ \\
Taylor 1990 & $5 / 27$ & $2 / 26$ \\
Uhlenhuth 1989 & $10 / 20$ & $10 / 41$ \\
Subtotal (95\% Cl) & $\mathbf{6 3 1}$ & $\mathbf{6 6 4}$
\end{tabular}

Total events: 184 (Antidepressants), 141 (Benzodiazepines) Heterogeneity: $\mathrm{Tau}^{2}=0.31 ; \mathrm{Chi}^{2}=23.52, \mathrm{df}=5(\mathrm{P}=0) ; \mathrm{I}^{2}=78.74 \%$ Test for overall effect: $\mathrm{Z}=1.72(\mathrm{P}=0.08)$

\subsubsection{SSRIs versus BDZs}

GSK-29060/1

Subtotal $(95 \% \mathrm{Cl})$

$29 / 77$

77

Total events: 29 (Antidepressants), 17 (Benzodiazepines)

Heterogeneity: Not applicable

Test for overall effect: $Z=2.06(P=0.04)$

\subsubsection{MAOIs versus BDZs}

Subtotal $(95 \% \mathrm{Cl})$

0

0

Not estimable

Total events: 0 (Antidepressants), 0 (Benzodiazepines)

Heterogeneity: Not applicable

Test for overall effect: Not applicable

\subsubsection{SNRIs versus BDZs}

Subtotal $(95 \% \mathrm{Cl})$

0

Total events: 0 (Antidepressants), 0 (Benzodiazepines)

Heterogeneity: Not applicable

Test for overall effect: Not applicable

1.2.5 NaSSAs versus BDZs

Subtotal $(95 \% \mathrm{Cl})$

0

0
$1.74[1.33,2.27]$

$0.69[0.49,0.98]$

$3.81[1.39,10.45]$

$2.45[0.11,53.25]$

$2.41[0.51,11.33]$

$2.05[1.02,4.11]$

$1.67[0.93,2.99]$

Total events: 0 (Antidepressants), 0 (Benzodiazepines) 


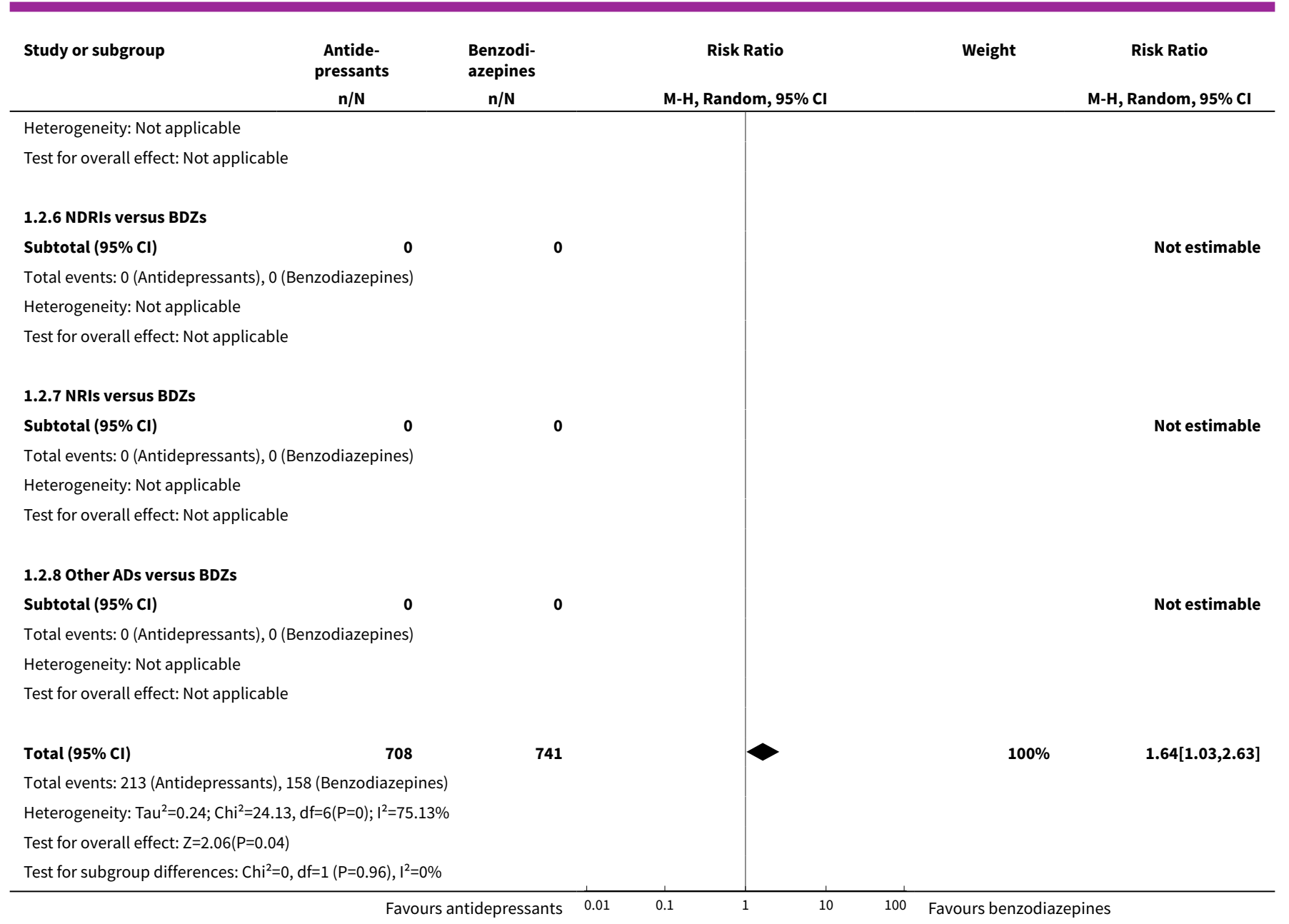

Analysis 1.3. Comparison 1 Antidepressants versus benzodiazepines, Outcome 3 Failure to remit.

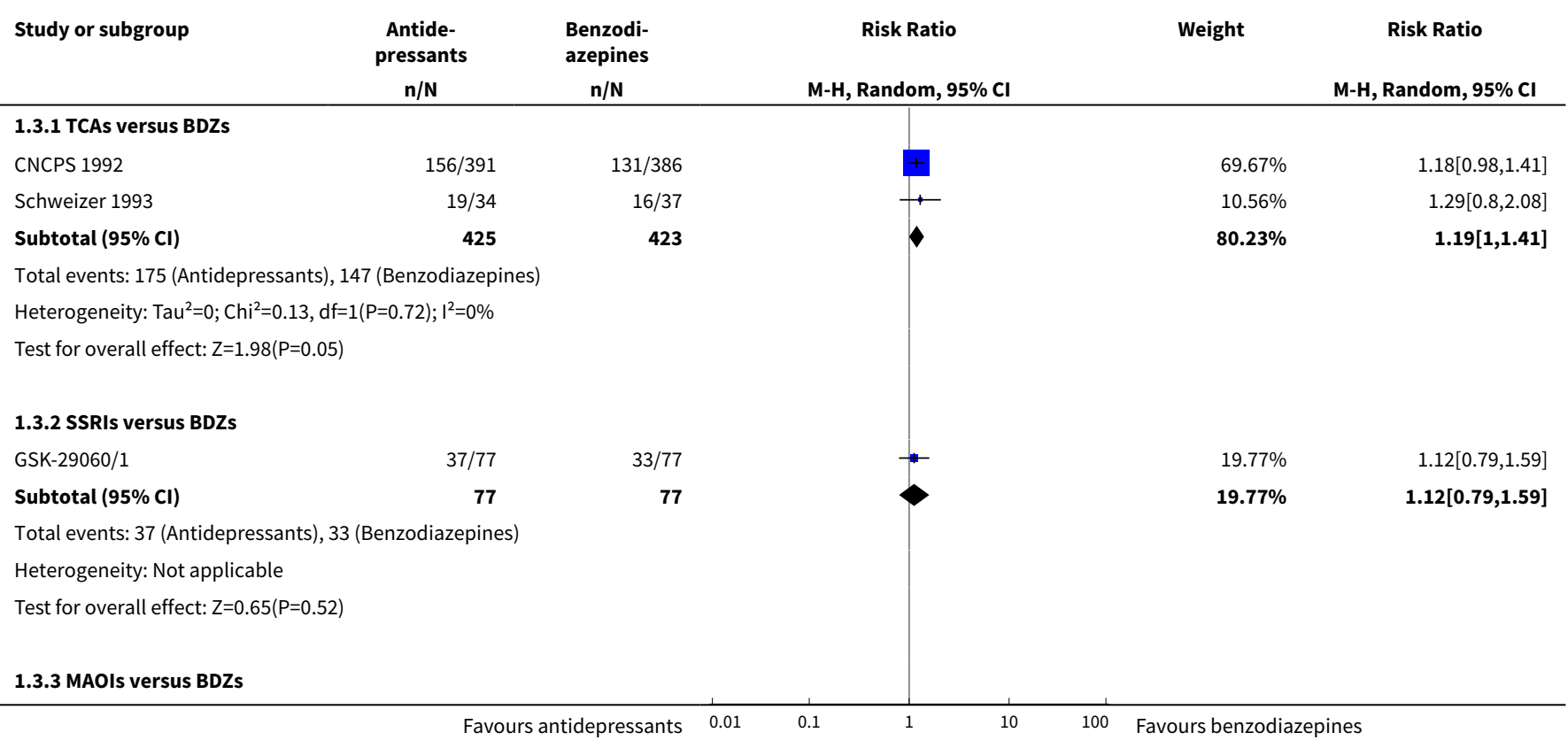




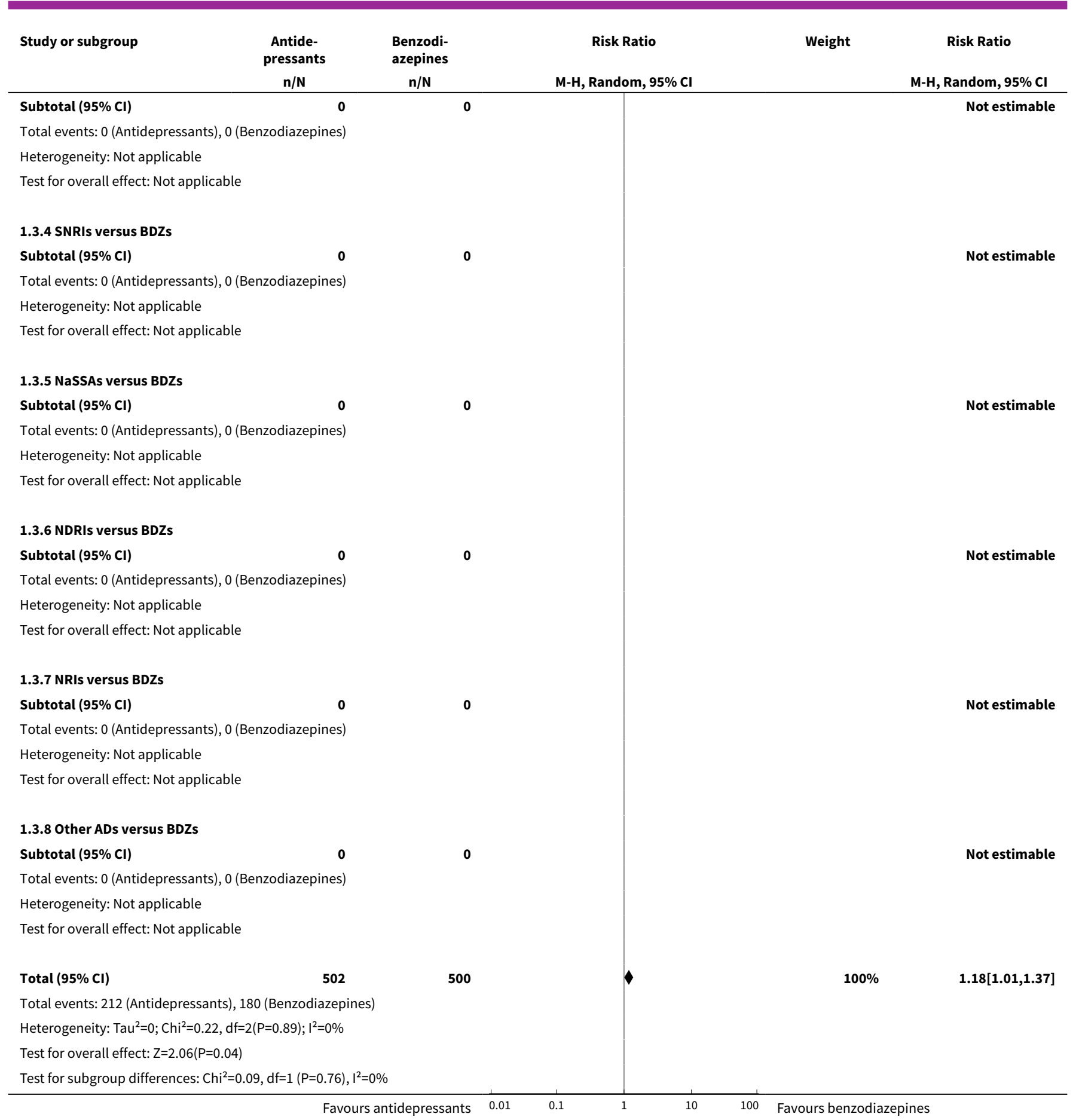

Analysis 1.4. Comparison 1 Antidepressants versus benzodiazepines, Outcome 4 Panic symptoms - endpoint score.

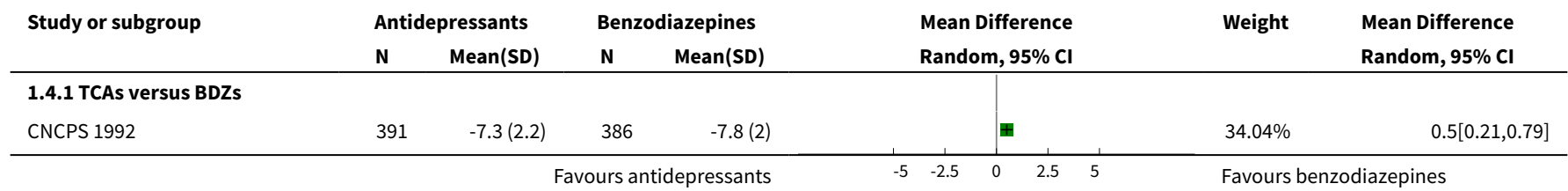




\begin{tabular}{llrrrr}
\multirow{2}{*}{ Study or subgroup } & \multicolumn{2}{c}{ Antidepressants } & \multicolumn{2}{c}{ Benzodiazepine } \\
& N & Mean(SD) & N & Mean(SD) \\
\hline Holland 1999 & 113 & $1.7(1.1)$ & 108 & $2.7(1.7)$ \\
Subtotal $\star \star \star$ & $\mathbf{5 0 4}$ & & $\mathbf{4 9 4}$ &
\end{tabular}

\section{(20)}

Heterogeneity: Tau $^{2}=1.1 ; \mathrm{Chi}^{2}=38.44, \mathrm{df}=1(\mathrm{P}<0.0001) ; \mathrm{I}^{2}=97.4 \%$

Test for overall effect: $Z=0.33(P=0.74)$

\subsubsection{SSRIs versus BDZs}

$\begin{array}{llll}\text { GSK-29060/1 } & 73 & 2(0.9) & 73 \\ \text { Subtotal } \star \star \star & 73 & & 73 \\ \text { Heterogeneity: Not applicable } & & & \end{array}$

(SD)

Mean Difference
Random, 95\%

3

$32.65 \%$

Test for overall effect: $Z=0.45(P=0.65)$

\subsubsection{MAOIs versus BDZs}

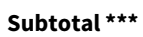

0

0

Not estimable

Heterogeneity: Not applicable

Test for overall effect: Not applicable

\subsubsection{SNRIs versus BDZs}

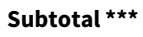

0

0

Not estimable

Heterogeneity: Not applicable

Test for overall effect: Not applicable

\subsubsection{NaSSAs versus BDZs}

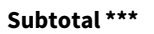

0

0

Not estimable

Heterogeneity: Not applicable

Test for overall effect: Not applicable

\subsubsection{NDRIs versus BDZs}

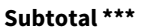

0

0

Not estimable

Heterogeneity: Not applicable

Test for overall effect: Not applicable

\subsubsection{NRIs versus BDZs}

Subtotal $* \star \star$

0

Not estimable

Heterogeneity: Not applicable

Test for overall effect: Not applicable

\subsubsection{Other ADs versus BDZs}

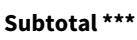

0

0

Not estimable

Heterogeneity: Not applicable

Test for overall effect: Not applicable

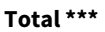

577

567

$100 \%$

$-0.13[-1.06,0.8]$

Heterogeneity: $\mathrm{Tau}^{2}=0.63 ; \mathrm{Chi}^{2}=38.86, \mathrm{df}=2(\mathrm{P}<0.0001) ; \mathrm{I}^{2}=94.85 \%$

Test for overall effect: $\mathrm{Z}=0.28(\mathrm{P}=0.78)$

Test for subgroup differences: $\mathrm{Chi}^{2}=0.19, \mathrm{df}=1(\mathrm{P}=0.66), \mathrm{I}^{2}=0 \%$ 
Analysis 1.5. Comparison 1 Antidepressants versus benzodiazepines, Outcome 5 Panic symptoms - mean change.

Study or subgroup

\begin{tabular}{lrlr}
\multicolumn{2}{c}{ Antidepressants } & \multicolumn{2}{c}{ Benzodiazepines } \\
N & Mean(SD) & N & Mean(SD)
\end{tabular}

Weight

Mean Difference

1.5.1 TCAs versus BDZs

Taylor 1990

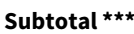

20

$-1.9(2.2)$

24

Heterogeneity: Not applicable

Test for overall effect: $\mathrm{Z}=0.64(\mathrm{P}=0.52)$

1.5.2 SSRIs versus BDZs

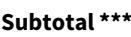

Heterogeneity: Not applicable

Test for overall effect: Not applicable

1.5.3 MAOIs versus BDZs

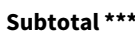

Heterogeneity: Not applicable

Test for overall effect: Not applicable

1.5.4 SNRIs versus BDZs

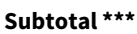

Heterogeneity: Not applicable

Test for overall effect: Not applicable

1.5.5 NaSSAs versus BDZs

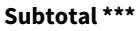

0

Heterogeneity: Not applicable

Test for overall effect: Not applicable

1.5.6 NDRIs versus BDZs

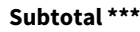

0

Random, $95 \% \mathrm{Cl}$

Heterogeneity: Not applicable

Test for overall effect: Not applicable

\subsubsection{NRIs versus BDZs}

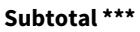

0

0

Heterogeneity: Not applicable

Test for overall effect: Not applicable

1.5.8 Other ADs versus BDZs

Subtotal $* \star \star$

0

Heterogeneity: Not applicable

Test for overall effect: Not applicable

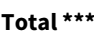

Heterogeneity: Not applicable

Test for overall effect: $\mathrm{Z}=0.64(\mathrm{P}=0.52)$

Test for subgroup differences: Not applicable
$100 \%$

Not estimable

Not estimable

$\begin{array}{rrr}100 \% & 0.4[-0.83,1.63] \\ 10.00 \% & \mathbf{0 . 4}[-\mathbf{0 . 8 3 , 1 . 6 3}]\end{array}$

Not estimable

Not estimable

Not estimable

Not estimable

Not estimable

$0.4[-0.83,1.63]$ 
Analysis 1.6. Comparison 1 Antidepressants versus benzodiazepines, Outcome 6 Frequency of panic attacks.

\begin{tabular}{|c|c|c|c|c|}
\hline \multirow[t]{2}{*}{ Study or subgroup } & \multicolumn{2}{|c|}{ Antidepressants } & \multicolumn{2}{|c|}{ Benzodiazepines } \\
\hline & $\mathbf{N}$ & $\operatorname{Mean}(\mathrm{SD})$ & $\mathbf{N}$ & Mean(SD) \\
\hline \multicolumn{5}{|c|}{ 1.6.1 TCAs versus BDZs } \\
\hline Holland 1999 & 113 & $1.5(5.3)$ & 108 & $3.1(6.5$ \\
\hline Lepola 1990 & 22 & $2.4(6.6)$ & 26 & $4.7(14.6$ \\
\hline Schweizer 1993 & 30 & $1.3(2.1)$ & 37 & $0.9(1.9$ \\
\hline Sheikh 1999 & 9 & $0.1(0.4)$ & 8 & $0(0$ \\
\hline Taylor 1990 & 20 & $1.2(4.5)$ & 24 & $1.3(7.7$ \\
\hline Uhlenhuth 1989 & 20 & $8.9(4.6)$ & 41 & $4.1(5.6$ \\
\hline 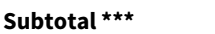 & 214 & & 244 & \\
\hline
\end{tabular}

Heterogeneity: Tau $^{2}=0.98 ; \mathrm{Chi}^{2}=17.11, \mathrm{df}=5(\mathrm{P}=0) ; \mathrm{I}^{2}=70.78 \%$

Test for overall effect: $Z=0.52(P=0.6)$

\subsubsection{SSRIs versus BDZs}

\begin{tabular}{|c|c|c|c|}
\hline GSK-29060/1 & 67 & $-10.1(10.6)$ & 70 \\
\hline Subtotal $* \star \star$ & 67 & & 70 \\
\hline
\end{tabular}

Heterogeneity: Not applicable

Test for overall effect: $Z=0.52(P=0.6)$

1.6.3 MAOIs versus BDZs

Heterogeneity: Not applicable

Test for overall effect: Not applicable

\subsubsection{SNRIs versus BDZs}

Heterogeneity: Not applicable

Test for overall effect: Not applicable

\subsubsection{NaSSAs versus BDZs}

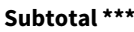

Heterogeneity: Not applicable

Test for overall effect: Not applicable

\subsubsection{NDRIs versus BDZs}

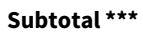

Heterogeneity: Not applicable

Test for overall effect: Not applicable

\subsubsection{NRIs versus BDZs}

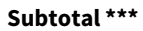

Heterogeneity: Not applicable

Test for overall effect: Not applicable

\subsubsection{Other ADs versus BDZs}

Heterogeneity: Not applicable

Test for overall effect: Not applicable

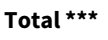




\begin{tabular}{|c|c|c|c|c|c|c|c|c|}
\hline \multirow[t]{2}{*}{ Study or subgroup } & \multicolumn{2}{|c|}{ Antidepressants } & Benzodiazepines & \multirow{2}{*}{\multicolumn{3}{|c|}{$\begin{array}{l}\text { Mean Difference } \\
\text { Random, } 95 \% \mathrm{CI}\end{array}$}} & \multirow[t]{2}{*}{ Weight } & \multirow{2}{*}{$\begin{array}{l}\text { Mean Difference } \\
\text { Random, } 95 \% \mathrm{Cl}\end{array}$} \\
\hline & $\mathbf{N}$ & Mean(SD) & $\operatorname{Mean}(S D)$ & & & & & \\
\hline \multicolumn{9}{|c|}{ Test for subgroup differences: $\mathrm{Chi}^{2}=0.15, \mathrm{df}=1(\mathrm{P}=0.7), \mathrm{I}^{2}=0 \%$} \\
\hline
\end{tabular}

\section{Analysis 1.7. Comparison 1 Antidepressants versus benzodiazepines, Outcome 7 Agoraphobia.}

\begin{tabular}{lrrrr} 
Study or subgroup & \multicolumn{2}{c}{ Antidepressants } & \multicolumn{2}{c}{ Benzodiazepines } \\
& N & Mean(SD) & \multicolumn{1}{c}{ N } & Mean(SD) \\
\hline 1.7.1 TCAs versus BDZs & & & & \\
CNCPS 1992 & 391 & $2.6(2.8)$ & 386 & $2.6(3.1)$ \\
Schweizer 1993 & 30 & $1.7(0.7)$ & 37 & $1.5(0.5)$ \\
Taylor 1990 & 20 & $4.9(6.7)$ & 24 & $3.4(5)$ \\
Uhlenhuth 1989 & 20 & $5(8.3)$ & 41 & $3.7(7.3)$ \\
Subtotal *** & $\mathbf{4 6 1}$ & & $\mathbf{4 8 8}$ &
\end{tabular}

\author{
Std. Mean Difference \\ Random, 95\% Cl
}

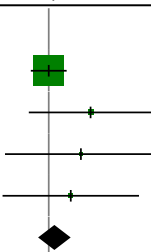

Heterogeneity: $\mathrm{Tau}^{2}=0 ; \mathrm{Chi}^{2}=2.39, \mathrm{df}=3(\mathrm{P}=0.5) ; \mathrm{I}^{2}=0 \%$

Test for overall effect: $\mathrm{Z}=0.68(\mathrm{P}=0.5)$

\subsubsection{SSRIs versus BDZs}

GSK-29060/1

Subtotal ${ }^{\star \star \star}$

$\begin{array}{lll}54 & 1.5(1.5) & 58 \\ \mathbf{5 4} & & \mathbf{5 8}\end{array}$

$1.5(1.5)$

Heterogeneity: Not applicable

Test for overall effect: Not applicable

1.7.3 MAOIs versus BDZs

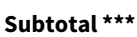

0

0

Heterogeneity: Not applicable

Test for overall effect: Not applicable

1.7.4 SNRIs versus BDZs

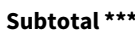

Heterogeneity: Not applicable

Test for overall effect: Not applicable

1.7.5 NaSSAs versus BDZs

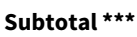

Not estimable

Not estimable

Heterogeneity: Not applicable

Test for overall effect: Not applicable

1.7.6 NDRIs versus BDZs

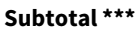

Heterogeneity: Not applicable

Test for overall effect: Not applicable

1.7.7 NRIs versus BDZs

Subtotal ***

0

Heterogeneity: Not applicable

Test for overall effect: Not applicable

1.7.8 Other ADs versus BDZs

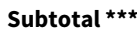

0

Favours antidepressants 


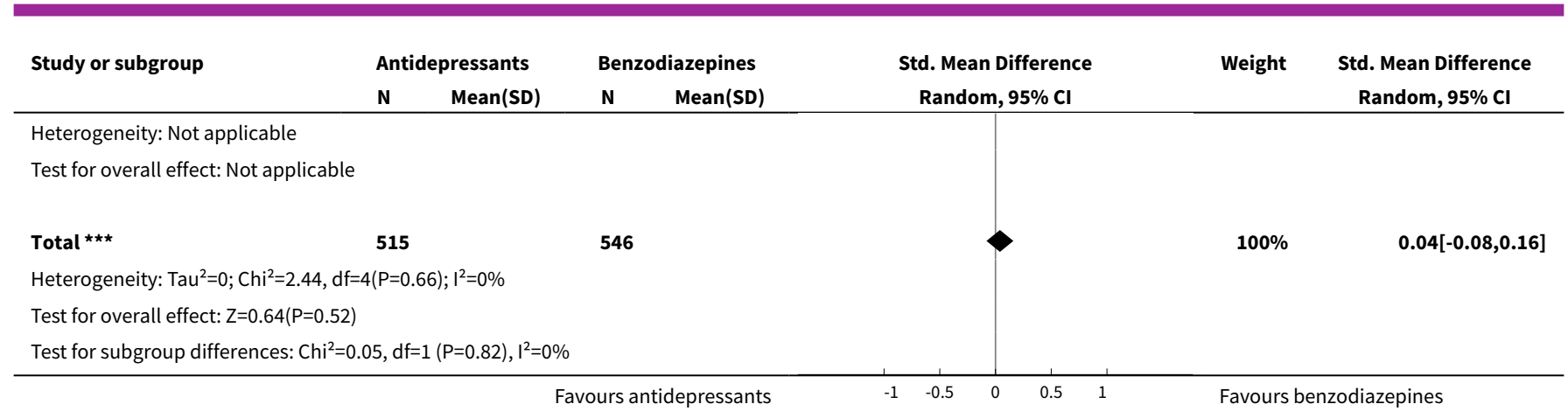

\section{Analysis 1.8. Comparison 1 Antidepressants versus benzodiazepines, Outcome 8 General anxiety.}

\begin{tabular}{lrrrr} 
Study or subgroup & \multicolumn{2}{c}{ Antidepressants } & \multicolumn{2}{c}{ Benzodiazepines } \\
& N & Mean(SD) & \multicolumn{1}{c}{ N } & Mean(SD) \\
\hline 1.8.1 TCAs versus BDZs & & & & \\
CNCPS 1992 & 391 & $12.1(9.1)$ & 386 & $10.3(8.1)$ \\
Holland 1999 & 113 & $3.7(5)$ & 108 & $7.8(7.2)$ \\
Lepola 1990 & 24 & $9.5(9.1)$ & 27 & $9.3(9.2)$ \\
Schweizer 1993 & 30 & $9.4(8.8)$ & 37 & $6.3(6.7)$ \\
Sheikh 1999 & 9 & $5.3(3.7)$ & 8 & $3.9(5.6)$ \\
Taylor 1990 & 20 & $6.8(8)$ & 24 & $5.7(8.5)$ \\
Uhlenhuth 1989 & 20 & $16.1(8.3)$ & 41 & $12.7(7.4)$ \\
Subtotal $\star \star \star$ & $\mathbf{6 0 7}$ & & $\mathbf{6 3 1}$ &
\end{tabular}

Heterogeneity: $\mathrm{Tau}^{2}=0.18 ; \mathrm{Chi}^{2}=35.88, \mathrm{df}=6(\mathrm{P}<0.0001) ; \mathrm{I}^{2}=83.28 \%$

Test for overall effect: $Z=0.45(P=0.65)$

\subsubsection{SSRIs versus BDZs}

\begin{tabular}{|c|c|c|c|}
\hline GSK-29060/1 & 61 & $10.1(8.3)$ & 66 \\
\hline 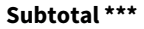 & 61 & & 66 \\
\hline
\end{tabular}

Heterogeneity: Not applicable

Test for overall effect: $Z=0.43(P=0.67)$

\subsubsection{MAOIs versus BDZs}

Subtotal $* \star \star$

Std. Mean Difference Random, $95 \% \mathrm{Cl}$

Heterogeneity: Not applicable

Test for overall effect: Not applicable

\subsubsection{SNRIs versus BDZs}

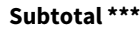

Heterogeneity: Not applicable

Test for overall effect: Not applicable

\subsubsection{NaSSAs versus BDZs}

Subtotal ${ }^{\star \star \star}$

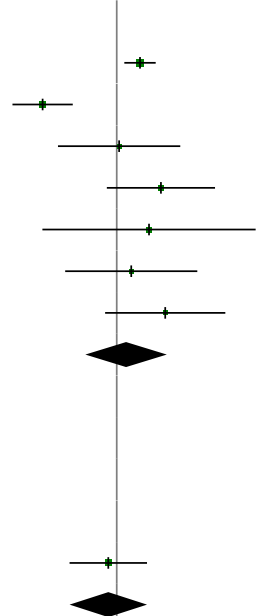

$14.52 \%$

$-0.08[-0.42,0.27]$

$14.52 \%$

Not estimable

Not estimable

Not estimable

Heterogeneity: Not applicable

Test for overall effect: Not applicable

1.8.6 NDRIs versus BDZs

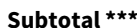

Heterogeneity: Not applicable 


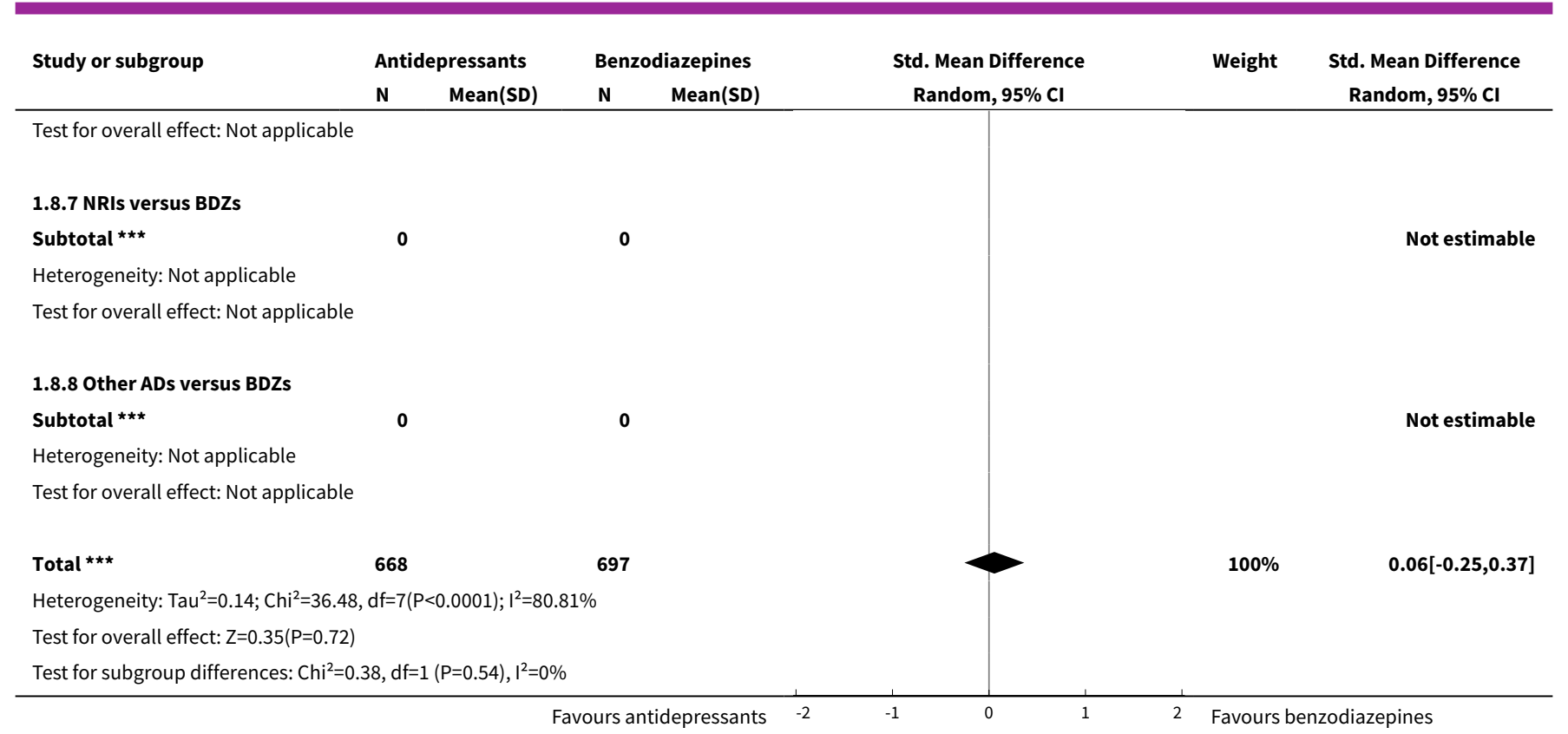

Analysis 1.9. Comparison 1 Antidepressants versus benzodiazepines, Outcome 9 Depression.

\begin{tabular}{|c|c|c|c|c|}
\hline \multirow[t]{2}{*}{ Study or subgroup } & \multicolumn{2}{|c|}{ Antidepressants } & \multicolumn{2}{|c|}{ Benzodiazepines } \\
\hline & $\mathbf{N}$ & Mean(SD) & $\mathbf{N}$ & Mean(SD) \\
\hline \multicolumn{5}{|c|}{ 1.9.1 TCAs versus BDZs } \\
\hline CNCPS 1992 & 273 & $6.8(4.7)$ & 319 & $7.4(6.1)$ \\
\hline Lepola 1990 & 24 & $3.3(5)$ & 27 & $4.3(5.6)$ \\
\hline Sheikh 1999 & 9 & $3.2(2.4)$ & 8 & $3.9(6.1)$ \\
\hline Taylor 1990 & 20 & $5.4(5.6)$ & 24 & $4.3(6.9)$ \\
\hline Uhlenhuth 1989 & 20 & $9.8(4.7)$ & 41 & $9(6.1)$ \\
\hline Subtotal $\star \star \star$ & 346 & & 419 & \\
\hline
\end{tabular}

Heterogeneity: $\mathrm{Tau}^{2}=0 ; \mathrm{Chi}^{2}=1.61, \mathrm{df}=4(\mathrm{P}=0.81) ; \mathrm{I}^{2}=0 \%$

Test for overall effect: $\mathrm{Z}=1.11(\mathrm{P}=0.27)$

\subsubsection{SSRIs versus BDZs}

$\begin{array}{lllll}\text { GSK-29060/1 } & 61 & 5.4(9.6) & 66 & 9(5.6) \\ \text { Subtotal } & & \mathbf{6} & \mathbf{6 6} & \end{array}$

Std. Mean Difference Random, $95 \% \mathrm{Cl}$

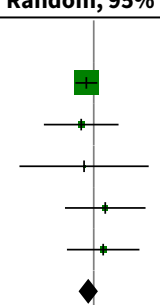

Weight Std. Mean Difference Random, $95 \% \mathrm{Cl}$

$$
\begin{array}{r}
-0.11[-0.27,0.05] \\
-0.18[-0.74,0.37] \\
-0.14[-1.1,0.81] \\
0.17[-0.42,0.76] \\
0.14[-0.4,0.67] \\
-\mathbf{0 . 0 8}[-\mathbf{0 . 2 2}, \mathbf{0 . 0 6}]
\end{array}
$$

$58.89 \%$

$2.54 \%$

$6.39 \%$

$7.82 \%$

$83.02 \%$

Heterogeneity: Tau $^{2}=0 ; \mathrm{Chi}^{2}=0, \mathrm{df}=0(\mathrm{P}<0.0001) ; \mathrm{I}^{2}=100 \%$

Test for overall effect: $Z=2.55(P=0.01)$

\subsubsection{MAOIs versus BDZs}

Heterogeneity: Not applicable

Test for overall effect: Not applicable

\subsubsection{SNRIs versus BDZs}

Heterogeneity: Not applicable

Test for overall effect: Not applicable

\subsubsection{NaSSAs versus BDZs}




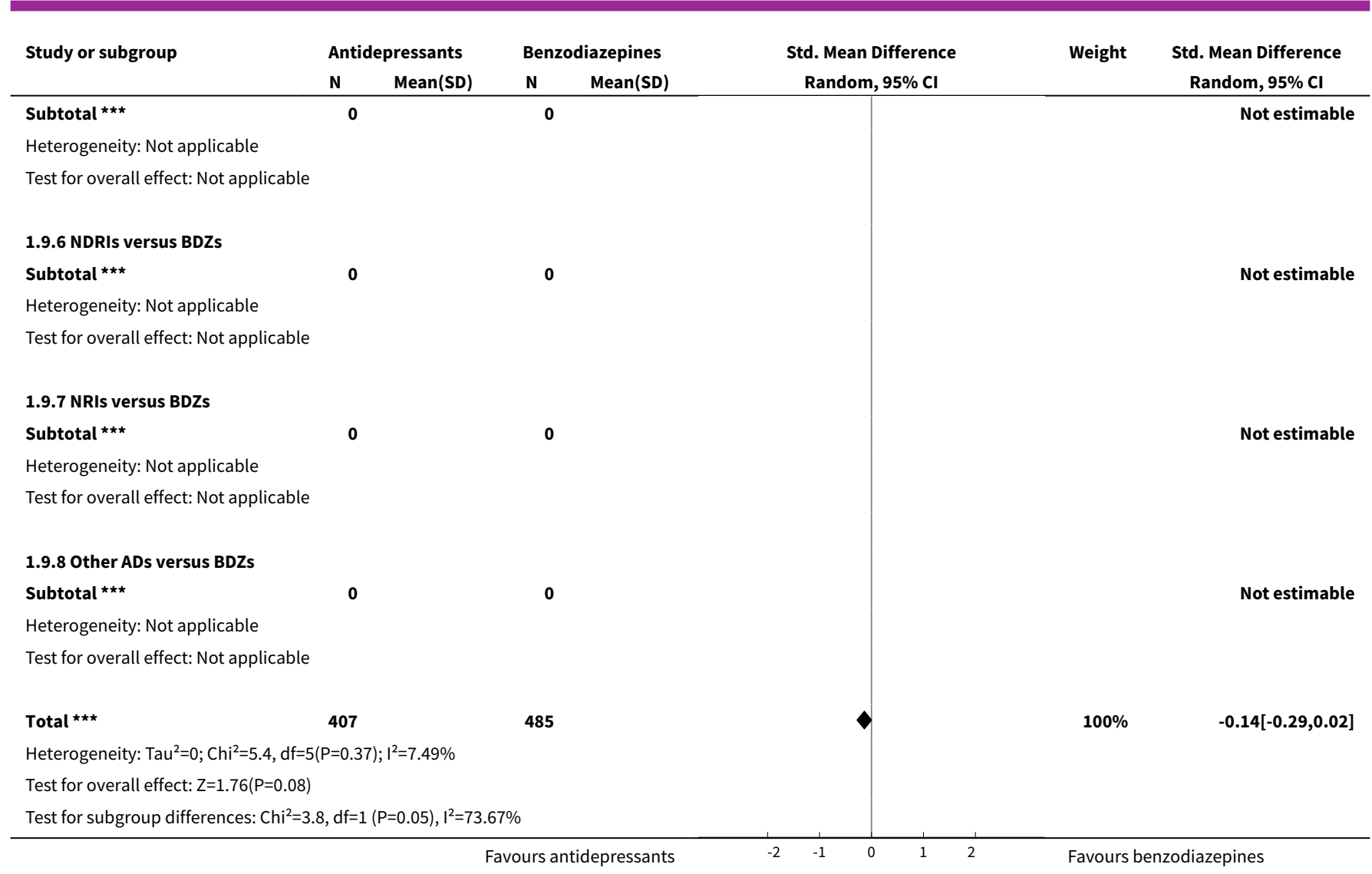

Analysis 1.10. Comparison 1 Antidepressants versus benzodiazepines, Outcome 10 Social functioning.

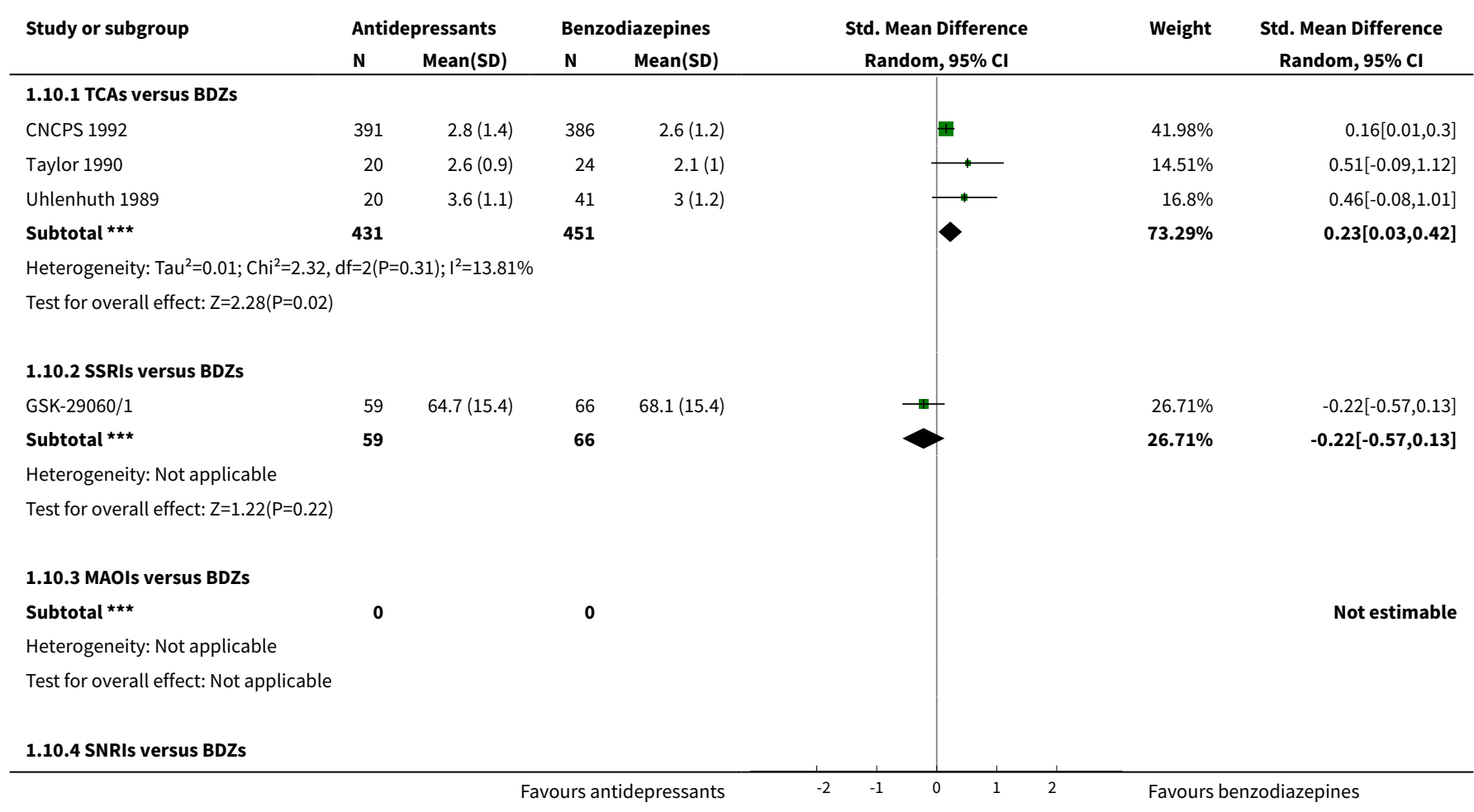




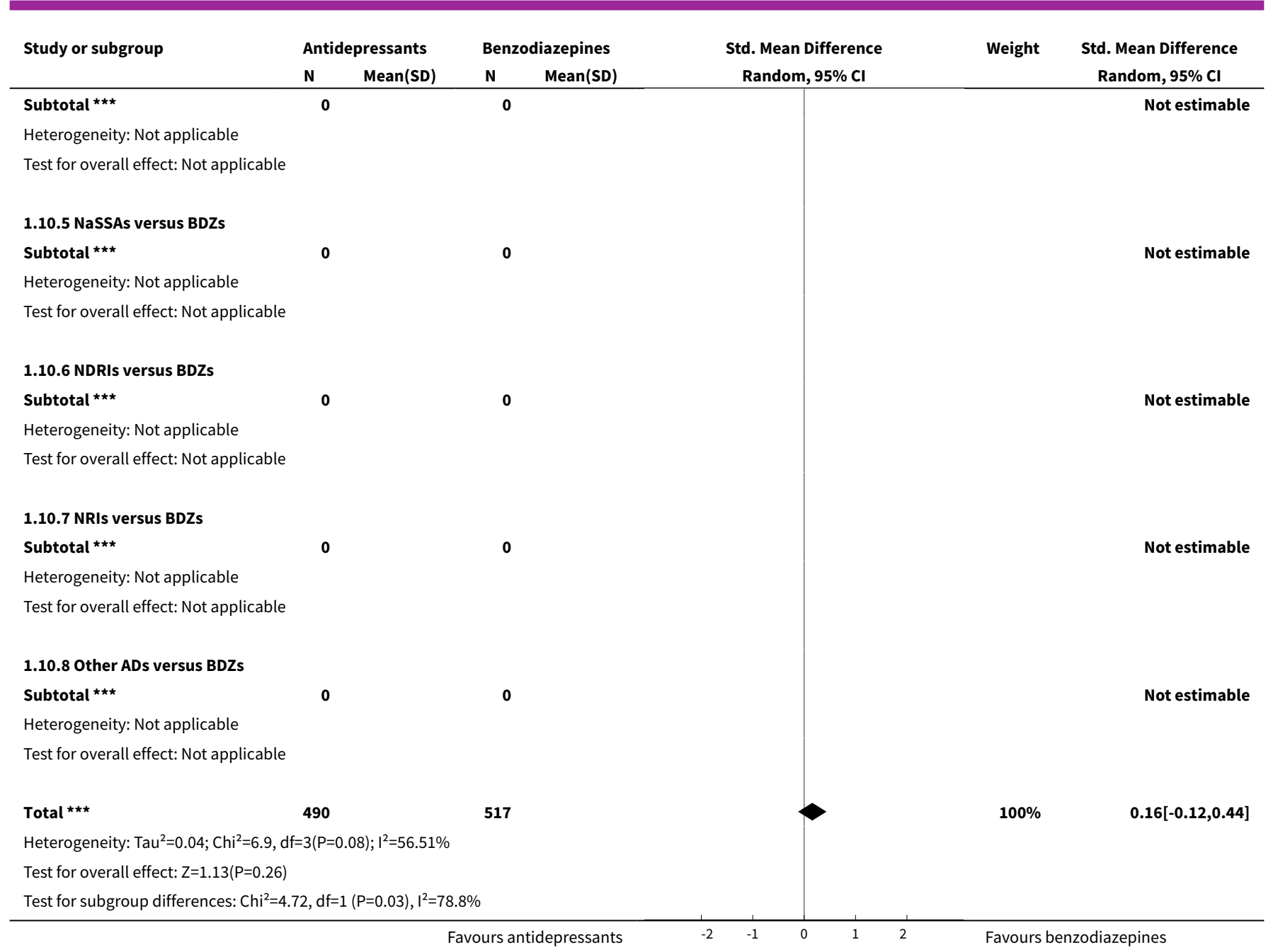

Analysis 1.14. Comparison 1 Antidepressants versus benzodiazepines, Outcome 14 Number of dropouts due to adverse effects.

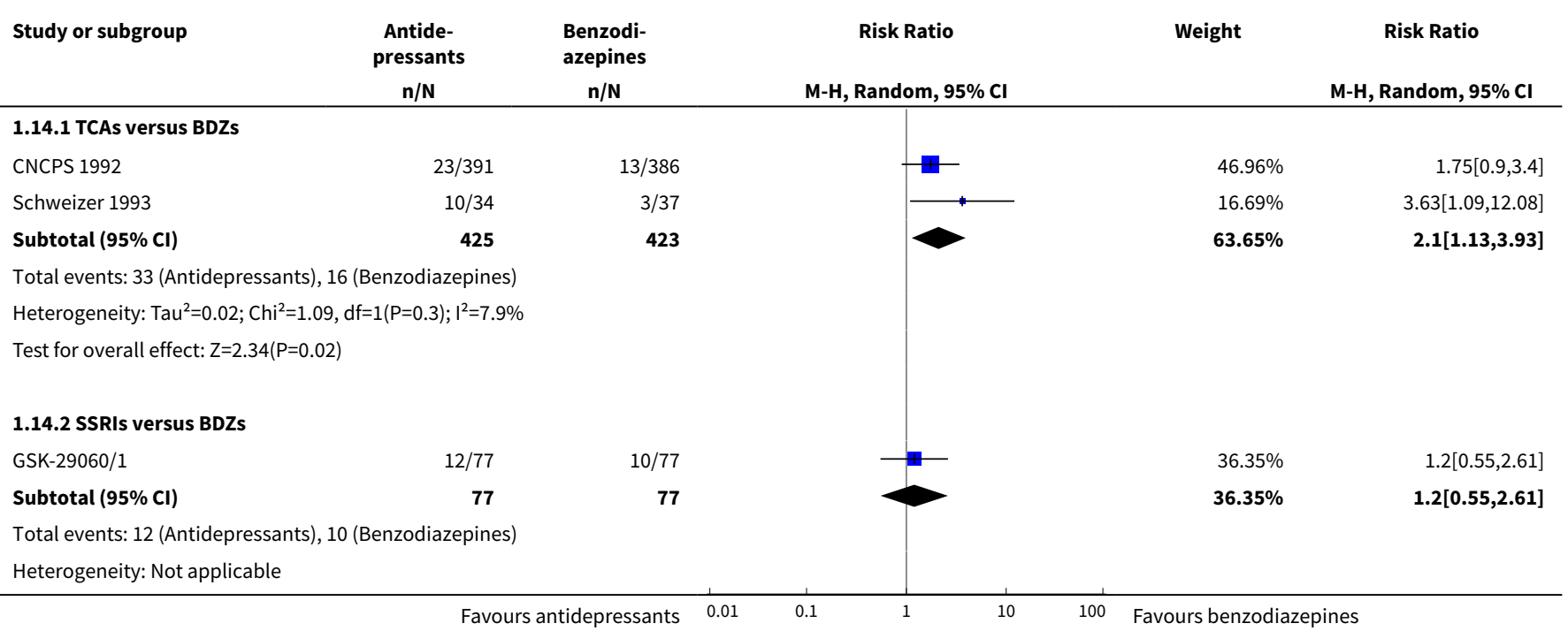




\begin{tabular}{ccccc} 
Study or subgroup & $\begin{array}{c}\text { Antide- } \\
\text { pressants }\end{array}$ & $\begin{array}{c}\text { Benzodi- } \\
\text { azepines } \\
n / N\end{array}$ & $\mathrm{n} / \mathrm{N}$ & Risk Ratio \\
\hline
\end{tabular}

Test for overall effect: $\mathrm{Z}=0.46(\mathrm{P}=0.65)$

1.14.3 MAOIs versus BDZs

Total events: 0 (Antidepressants), 0 (Benzodiazepines)

Heterogeneity: Not applicable

Test for overall effect: Not applicable

\subsubsection{SNRIs versus BDZs}

Total events: 0 (Antidepressants), 0 (Benzodiazepines)

Heterogeneity: Not applicable

Test for overall effect: Not applicable

\subsubsection{NaSSAs versus BDZs}

Total events: 0 (Antidepressants), 0 (Benzodiazepines)

Heterogeneity: Not applicable

Test for overall effect: Not applicable

\subsubsection{NDRIs versus BDZs}

Total events: 0 (Antidepressants), 0 (Benzodiazepines)

Heterogeneity: Not applicable

Test for overall effect: Not applicable

\subsubsection{NRIs versus BDZs}

Total events: 0 (Antidepressants), 0 (Benzodiazepines)

Heterogeneity: Not applicable

Test for overall effect: Not applicable

\subsubsection{Other ADs versus BDZs}

Total events: 0 (Antidepressants), 0 (Benzodiazepines)

Heterogeneity: Not applicable

Test for overall effect: Not applicable

Total events: 45 (Antidepressants), 26 (Benzodiazepines) Heterogeneity: $\mathrm{Tau}^{2}=0.03 ; \mathrm{Chi}^{2}=2.31, \mathrm{df}=2(\mathrm{P}=0.31) ; \mathrm{I}^{2}=13.47 \%$

Test for overall effect: $Z=2.09(P=0.04)$

Test for subgroup differences: $\mathrm{Chi}^{2}=1.22, \mathrm{df}=1(\mathrm{P}=0.27), \mathrm{I}^{2}=17.98 \%$ 
Analysis 1.15. Comparison 1 Antidepressants versus benzodiazepines,

Outcome 15 Number of patients experiencing at least one adverse effect.

\begin{tabular}{|c|c|c|c|c|c|}
\hline \multirow[t]{2}{*}{ Study or subgroup } & $\begin{array}{c}\text { Antide- } \\
\text { pressants }\end{array}$ & $\begin{array}{l}\text { Benzodi- } \\
\text { azepines }\end{array}$ & Risk Ratio & Weight & Risk Ratio \\
\hline & $n / N$ & $n / N$ & M-H, Random, $95 \% \mathrm{CI}$ & & M-H, Random, 95\% Cl \\
\hline
\end{tabular}

1.15.1 TCAs versus BDZs

Subtotal $(95 \% \mathrm{Cl})$

Total events: 0 (Antidepressants), 0 (Benzodiazepines)

Heterogeneity: Not applicable

Test for overall effect: Not applicable

\subsubsection{SSRIs versus BDZs}

GSK-29060/1 70/77

Subtotal $(95 \% \mathrm{CI})$

Total events: 70 (Antidepressants), 68 (Benzodiazepines) Heterogeneity: Not applicable

Test for overall effect: $Z=0.53(P=0.6)$

\subsubsection{MAOIs versus BDZs}

Total events: 0 (Antidepressants), 0 (Benzodiazepines) Heterogeneity: Not applicable

Test for overall effect: Not applicable

\subsubsection{SNRIs versus BDZs}

Total events: 0 (Antidepressants), 0 (Benzodiazepines) Heterogeneity: Not applicable

Test for overall effect: Not applicable

\subsubsection{NaSSAs versus BDZs}

Total events: 0 (Antidepressants), 0 (Benzodiazepines) Heterogeneity: Not applicable

Test for overall effect: Not applicable

\subsubsection{NDRIs versus BDZs}

Total events: 0 (Antidepressants), 0 (Benzodiazepines) Heterogeneity: Not applicable

Test for overall effect: Not applicable

\subsubsection{NRIs versus BDZs}

Total events: 0 (Antidepressants), 0 (Benzodiazepines) Heterogeneity: Not applicable

Test for overall effect: Not applicable

Total events: 0 (Antidepressants), 0 (Benzodiazepines) 


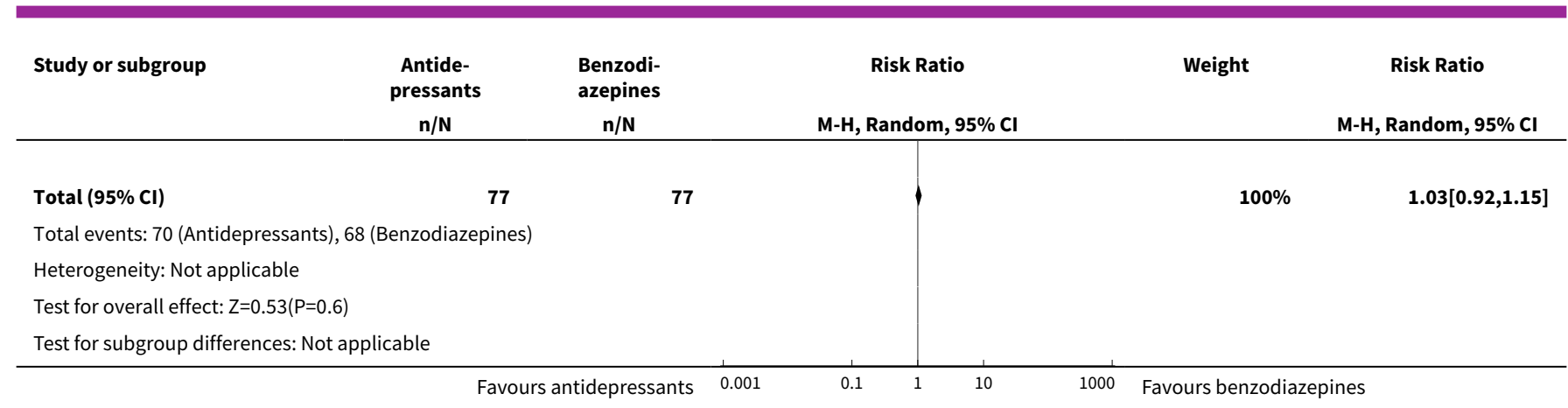

\section{Comparison 2. TCAs versus benzodiazepines}

\begin{tabular}{|c|c|c|c|c|}
\hline Outcome or subgroup title & No. of studies & $\begin{array}{l}\text { No. of partici- } \\
\text { pants }\end{array}$ & Statistical method & Effect size \\
\hline 1 Failure to respond & 1 & 61 & Risk Ratio (M-H, Random, 95\% Cl) & $1.03[0.63,1.67]$ \\
\hline 1.1 Imipramine versus BDZs & 1 & 61 & Risk Ratio (M-H, Random, 95\% Cl) & $1.03[0.63,1.67]$ \\
\hline 2 Total number of dropouts & 6 & 1295 & Risk Ratio (M-H, Random, 95\% Cl) & $1.67[0.93,2.99]$ \\
\hline 2.1 Imipramine versus BDZs & 5 & 980 & Risk Ratio (M-H, Random, 95\% Cl) & $1.87[1.47,2.37]$ \\
\hline $\begin{array}{l}2.2 \text { Clomipramine versus } \\
\text { BDZs }\end{array}$ & 1 & 315 & Risk Ratio (M-H, Random, 95\% Cl) & $0.69[0.49,0.98]$ \\
\hline 3 Failure to remit & 2 & 848 & Risk Ratio (M-H, Random, 95\% Cl) & $1.19[1.00,1.41]$ \\
\hline 3.1 Imipramine versus BDZs & 2 & 848 & Risk Ratio (M-H, Random, 95\% Cl) & $1.19[1.00,1.41]$ \\
\hline $\begin{array}{l}4 \text { Panic symptoms - end- } \\
\text { point score }\end{array}$ & 2 & 998 & Mean Difference (IV, Random, 95\% CI) & $-0.25[-1.72,1.22]$ \\
\hline 4.1 Imipramine versus BDZs & 1 & 777 & Mean Difference (IV, Random, 95\% CI) & $0.5[0.21,0.79]$ \\
\hline $\begin{array}{l}4.2 \text { Clomipramine versus } \\
\text { BDZs }\end{array}$ & 1 & 221 & Mean Difference (IV, Random, 95\% CI) & $-1.00[-1.37,-0.63]$ \\
\hline $\begin{array}{l}5 \text { Panic symptoms - mean } \\
\text { change }\end{array}$ & 1 & 44 & Mean Difference (IV, Random, 95\% Cl) & $0.40[-0.83,1.63]$ \\
\hline 5.1 Imipramine versus BDZs & 1 & 44 & Mean Difference (IV, Random, 95\% CI) & $0.40[-0.83,1.63]$ \\
\hline $\begin{array}{l}6 \text { Frequency of panic at- } \\
\text { tacks }\end{array}$ & 6 & 458 & Mean Difference (IV, Random, 95\% CI) & $0.30[-0.81,1.41]$ \\
\hline 6.1 Imipramine versus BDZs & 5 & 237 & Mean Difference (IV, Random, 95\% CI) & $0.75[-0.44,1.94]$ \\
\hline $\begin{array}{l}6.2 \text { Clomipramine versus } \\
\text { BDZs }\end{array}$ & 1 & 221 & Mean Difference (IV, Random, 95\% CI) & $-1.6[-3.17,-0.03]$ \\
\hline 7 Agoraphobia & 4 & 949 & $\begin{array}{l}\text { Std. Mean Difference (IV, Random, } \\
95 \% \mathrm{Cl} \text { ) }\end{array}$ & $0.04[-0.08,0.17]$ \\
\hline
\end{tabular}




\begin{tabular}{|c|c|c|c|c|}
\hline Outcome or subgroup title & No. of studies & $\begin{array}{l}\text { No. of partici- } \\
\text { pants }\end{array}$ & Statistical method & Effect size \\
\hline 7.1 Imipramine versus BDZs & 4 & 949 & $\begin{array}{l}\text { Std. Mean Difference (IV, Random, } \\
95 \% \mathrm{CI} \text { ) }\end{array}$ & $0.04[-0.08,0.17]$ \\
\hline 8 General anxiety & 7 & 1238 & $\begin{array}{l}\text { Std. Mean Difference (IV, Random, } \\
95 \% \mathrm{Cl} \text { ) }\end{array}$ & $0.08[-0.28,0.45]$ \\
\hline 8.1 Imipramine versus BDZs & 6 & 1017 & $\begin{array}{l}\text { Std. Mean Difference (IV, Random, } \\
95 \% \mathrm{CI} \text { ) }\end{array}$ & $0.22[0.10,0.35]$ \\
\hline $\begin{array}{l}8.2 \text { Clomipramine versus } \\
\text { BDZs }\end{array}$ & 1 & 221 & $\begin{array}{l}\text { Std. Mean Difference (IV, Random, } \\
95 \% \mathrm{CI} \text { ) }\end{array}$ & $-0.67[-0.94,-0.40]$ \\
\hline 9 Depression & 5 & 765 & $\begin{array}{l}\text { Std. Mean Difference (IV, Random, } \\
95 \% \mathrm{CI})\end{array}$ & $-0.08[-0.22,0.06]$ \\
\hline 9.1 Imipramine versus BDZs & 5 & 765 & $\begin{array}{l}\text { Std. Mean Difference (IV, Random, } \\
95 \% \mathrm{Cl} \text { ) }\end{array}$ & $-0.08[-0.22,0.06]$ \\
\hline 10 Social functioning & 3 & 882 & Mean Difference (IV, Random, 95\% Cl) & $0.25[0.09,0.42]$ \\
\hline $\begin{array}{l}10.1 \text { Imipramine versus } \\
\text { BDZs }\end{array}$ & 3 & 882 & Mean Difference (IV, Random, 95\% Cl) & $0.25[0.09,0.42]$ \\
\hline 11 Quality of life & 0 & 0 & $\begin{array}{l}\text { Std. Mean Difference (IV, Random, } \\
95 \% \mathrm{CI} \text { ) }\end{array}$ & $0.0[0.0,0.0]$ \\
\hline 12 Patient satisfaction & 0 & 0 & $\begin{array}{l}\text { Std. Mean Difference (IV, Random, } \\
95 \% \mathrm{CI} \text { ) }\end{array}$ & $0.0[0.0,0.0]$ \\
\hline 13 Economic costs & 0 & 0 & $\begin{array}{l}\text { Std. Mean Difference (IV, Random, } \\
95 \% \mathrm{CI} \text { ) }\end{array}$ & $0.0[0.0,0.0]$ \\
\hline $\begin{array}{l}14 \text { Number of dropouts due } \\
\text { to adverse effects }\end{array}$ & 2 & 848 & Risk Ratio (M-H, Random, 95\% Cl) & $2.10[1.13,3.93]$ \\
\hline $\begin{array}{l}14.1 \text { Imipramine versus } \\
\text { BDZs }\end{array}$ & 2 & 848 & Risk Ratio (M-H, Random, 95\% Cl) & $2.10[1.13,3.93]$ \\
\hline $\begin{array}{l}15 \text { Number of patients ex- } \\
\text { periencing at least one ad- } \\
\text { verse effect }\end{array}$ & 0 & 0 & Risk Ratio (M-H, Random, 95\% Cl) & $0.0[0.0,0.0]$ \\
\hline
\end{tabular}

Analysis 2.1. Comparison 2 TCAs versus benzodiazepines, Outcome 1 Failure to respond.

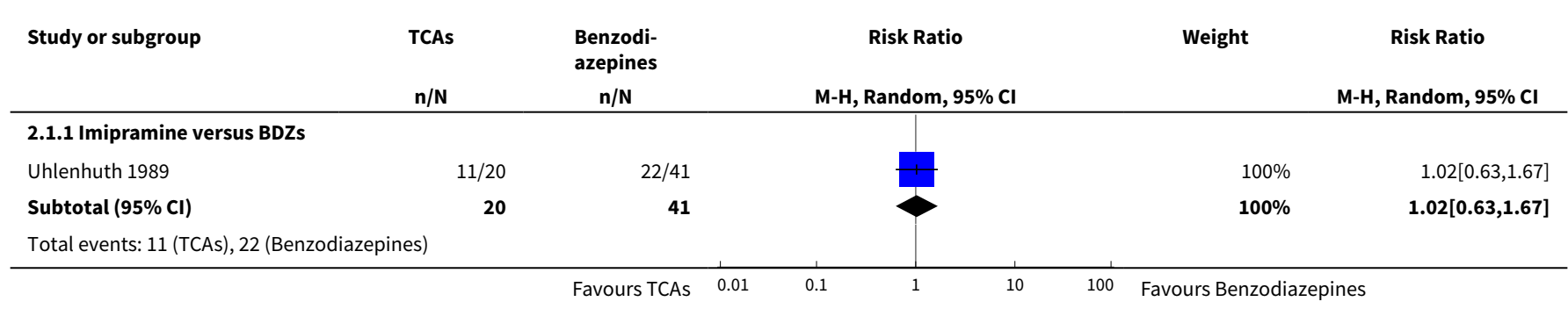




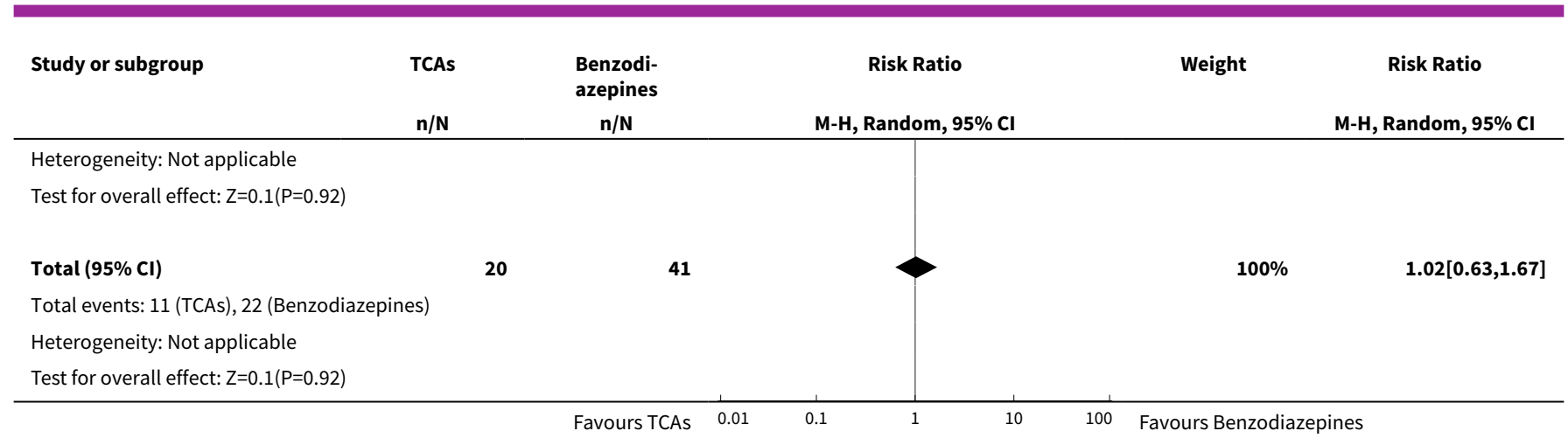

Analysis 2.2. Comparison 2 TCAs versus benzodiazepines, Outcome 2 Total number of dropouts.

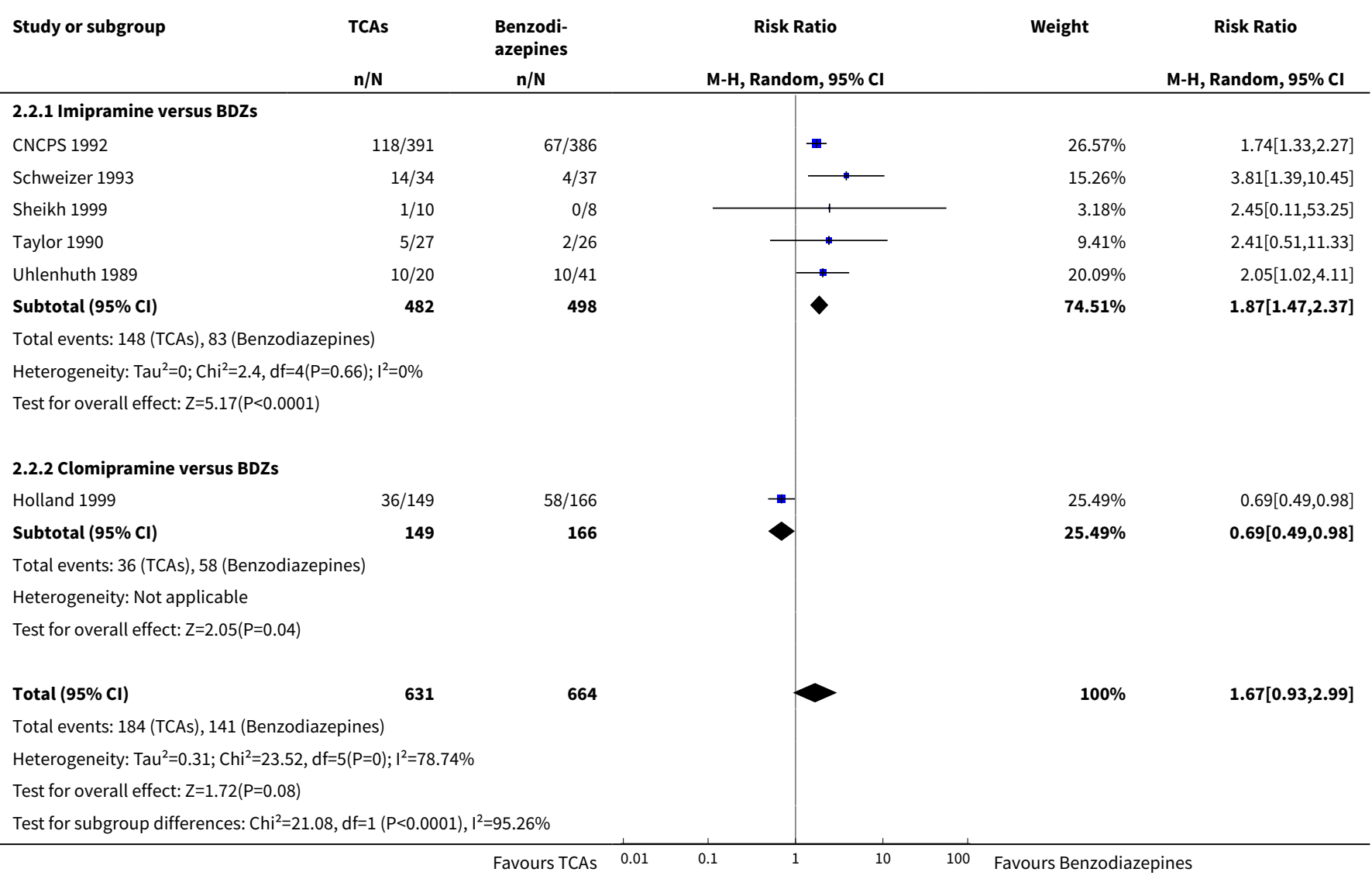

Analysis 2.3. Comparison 2 TCAs versus benzodiazepines, Outcome 3 Failure to remit.

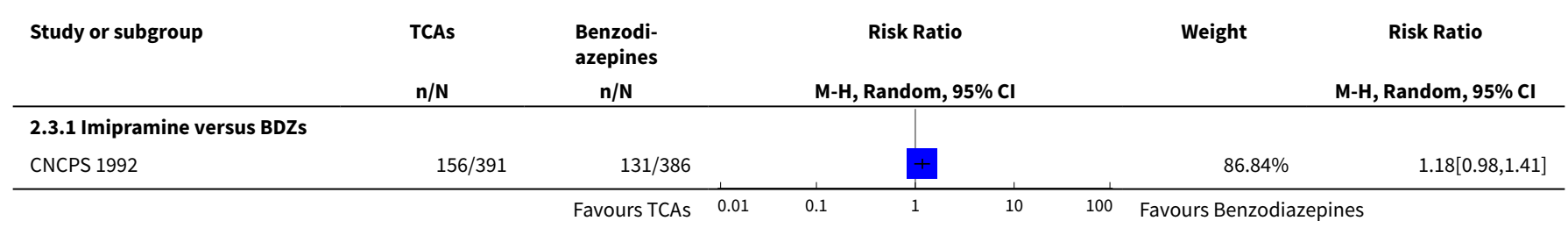




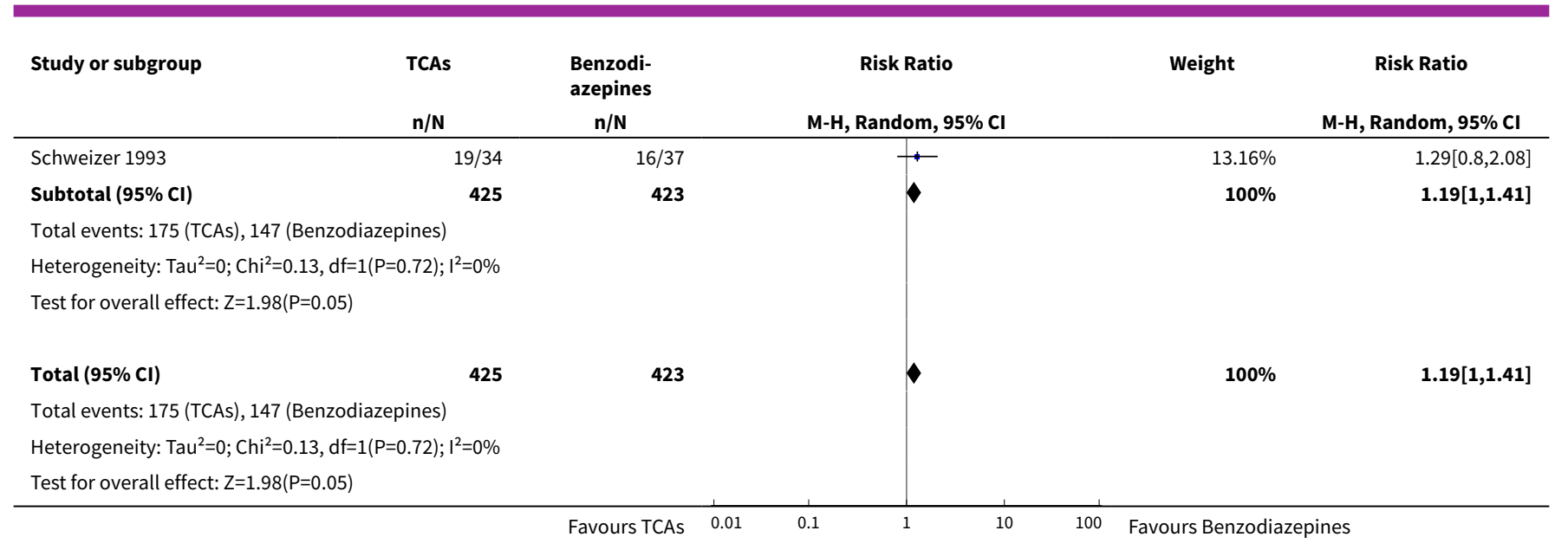

Analysis 2.4. Comparison 2 TCAs versus benzodiazepines, Outcome 4 Panic symptoms - endpoint score.

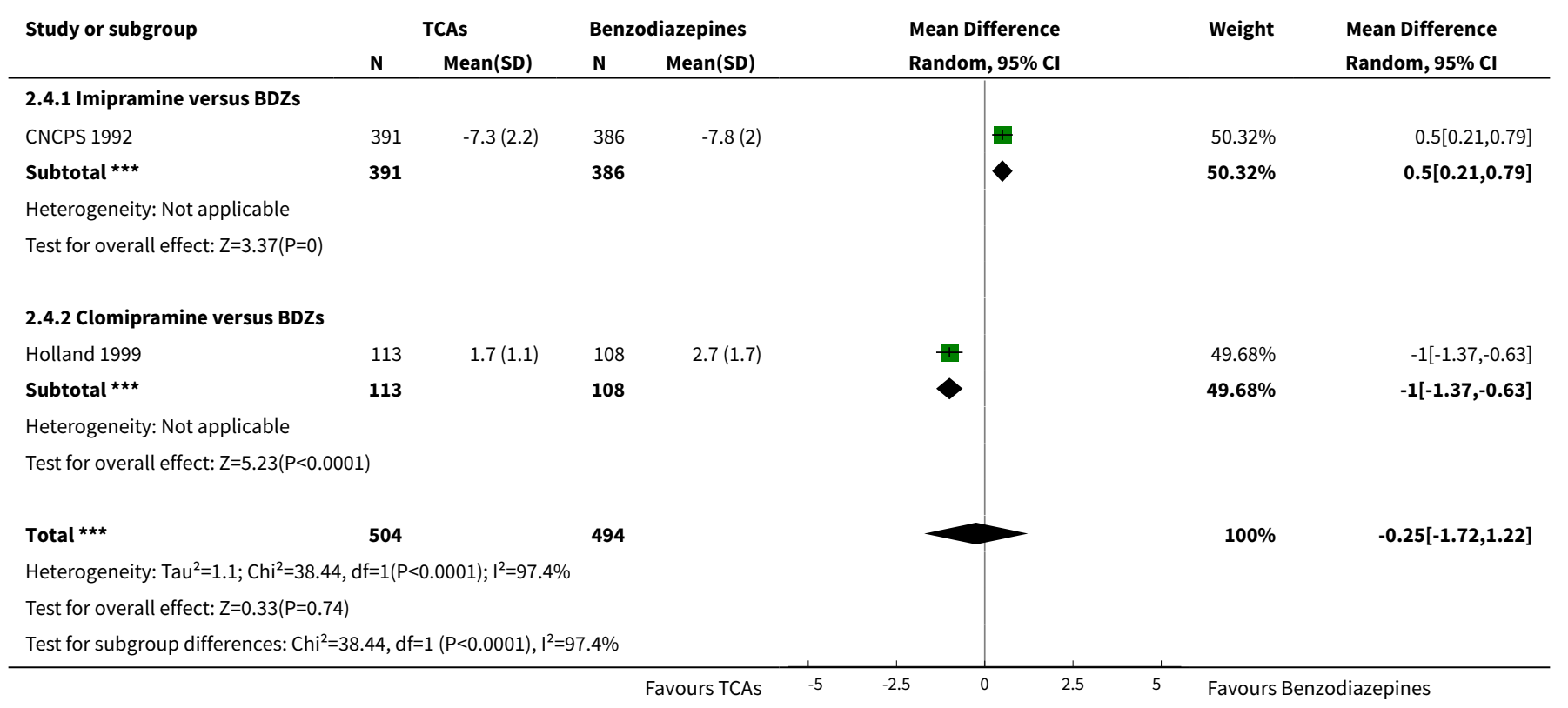

Analysis 2.5. Comparison 2 TCAs versus benzodiazepines, Outcome 5 Panic symptoms - mean change.

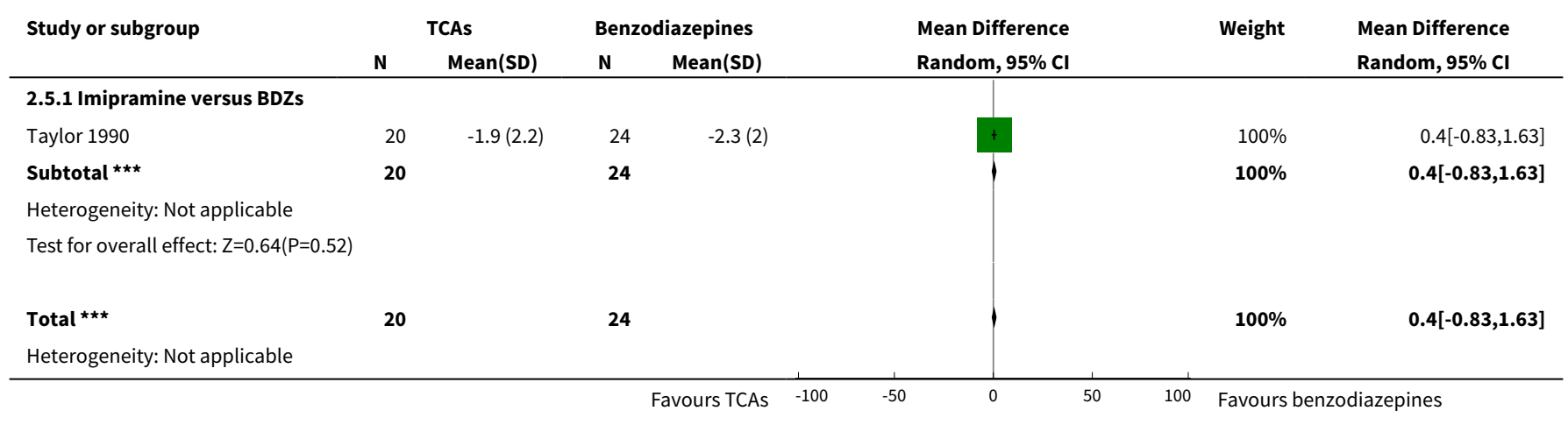




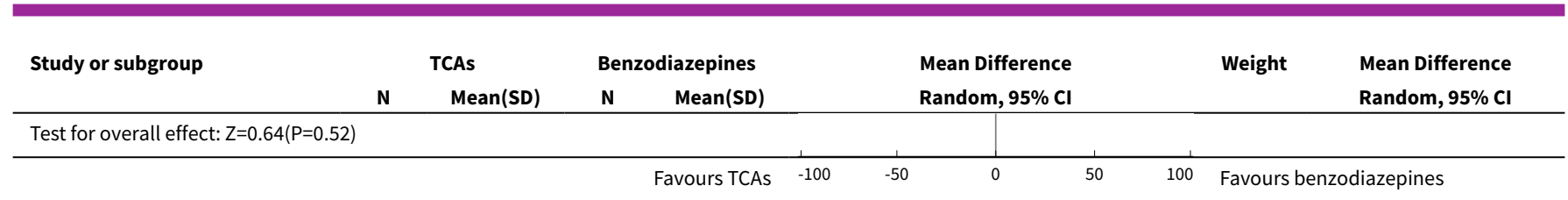

\section{Analysis 2.6. Comparison 2 TCAs versus benzodiazepines, Outcome 6 Frequency of panic attacks.}

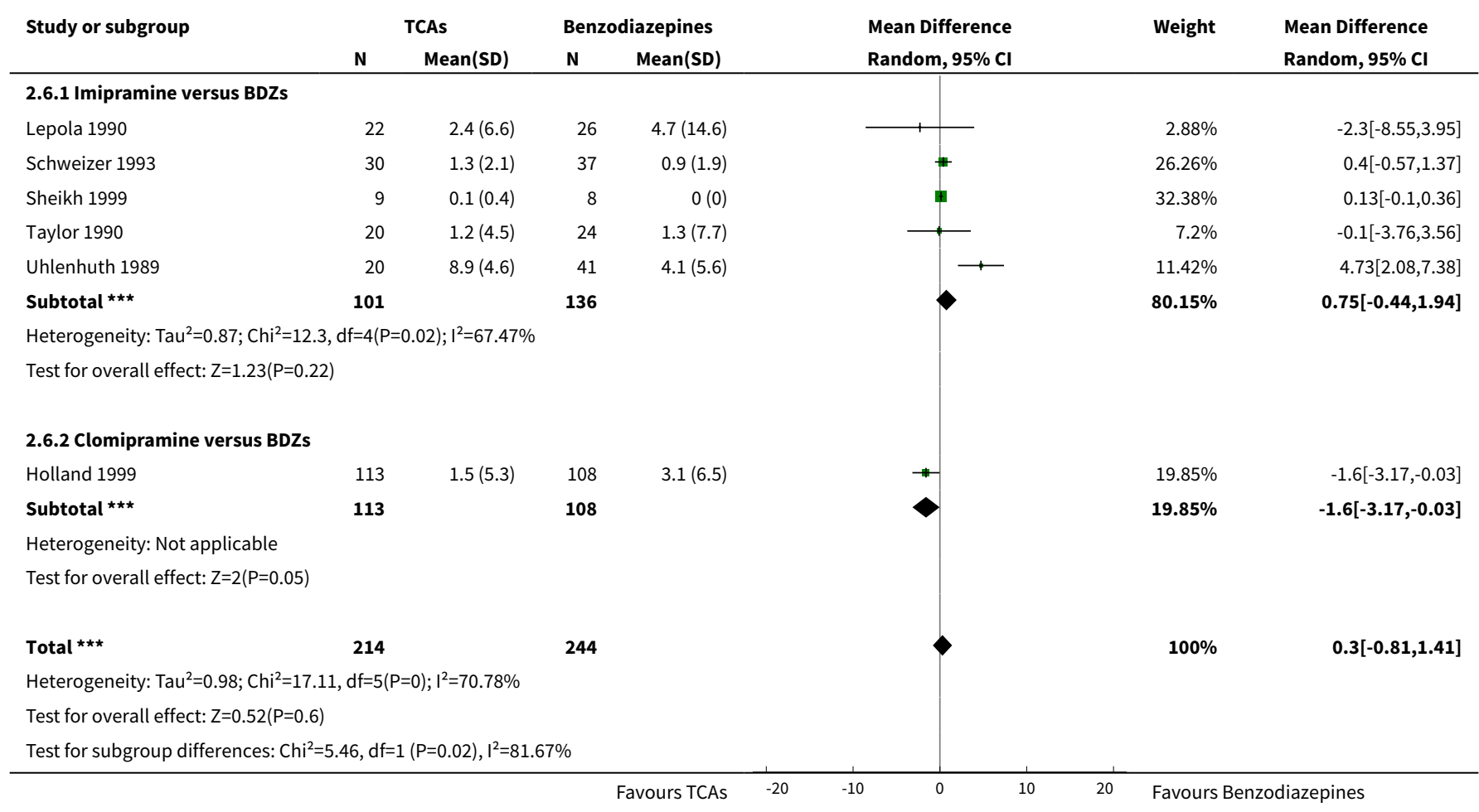

Analysis 2.7. Comparison 2 TCAs versus benzodiazepines, Outcome 7 Agoraphobia.

\begin{tabular}{|c|c|c|c|c|c|c|c|}
\hline \multirow[t]{2}{*}{ Study or subgroup } & \multicolumn{2}{|c|}{ TCAs } & \multicolumn{2}{|c|}{ Benzodiazepines } & \multirow{2}{*}{$\begin{array}{c}\text { Std. Mean Difference } \\
\text { Random, } 95 \% \mathrm{Cl}\end{array}$} & \multirow[t]{2}{*}{ Weight } & \multirow{2}{*}{$\begin{array}{c}\text { Std. Mean Difference } \\
\text { Random, } 95 \% \mathrm{Cl}\end{array}$} \\
\hline & $\mathbf{N}$ & Mean(SD) & $\mathbf{N}$ & Mean(SD) & & & \\
\hline \multicolumn{8}{|c|}{ 2.7.1 Imipramine versus BDZs } \\
\hline CNCPS 1992 & 391 & $2.6(2.8)$ & 386 & $2.6(3.1)$ & & $82.73 \%$ & $0[-0.14,0.14]$ \\
\hline Schweizer 1993 & 30 & $1.7(0.7)$ & 37 & $1.5(0.5)$ & & $6.96 \%$ & $0.33[-0.16,0.81]$ \\
\hline Taylor 1990 & 20 & $4.9(6.7)$ & 24 & $3.4(5)$ & $1+$ & $4.61 \%$ & $0.25[-0.34,0.85]$ \\
\hline Uhlenhuth 1989 & 20 & $5(8.3)$ & 41 & $3.7(7.3)$ & $\longrightarrow$ & $5.71 \%$ & $0.17[-0.36,0.71]$ \\
\hline Subtotal *** & 461 & & 488 & & 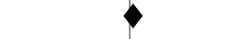 & $100 \%$ & $0.04[-0.08,0.17]$ \\
\hline \multicolumn{8}{|c|}{ Heterogeneity: $\mathrm{Tau}^{2}=0 ; \mathrm{Chi}^{2}=2.39, \mathrm{df}=3(\mathrm{P}=0.5) ; \mathrm{I}^{2}=0 \%$} \\
\hline \multicolumn{8}{|c|}{ Test for overall effect: $\mathrm{Z}=0.68(\mathrm{P}=0.5)$} \\
\hline Total $\star \star \star$ & 461 & & 488 & & 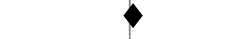 & $100 \%$ & $0.04[-0.08,0.17]$ \\
\hline \multicolumn{8}{|c|}{ Heterogeneity: $\mathrm{Tau}^{2}=0 ; \mathrm{Chi}^{2}=2.39, \mathrm{df}=3(\mathrm{P}=0.5) ; \mathrm{I}^{2}=0 \%$} \\
\hline \multicolumn{8}{|c|}{ Test for overall effect: $Z=0.68(P=0.5)$} \\
\hline & & & & avours TCAs & -1 & Favours & zodiazepines \\
\hline
\end{tabular}


Analysis 2.8. Comparison 2 TCAs versus benzodiazepines, Outcome 8 General anxiety.

\begin{tabular}{|c|c|c|c|c|c|c|c|c|}
\hline \multirow[t]{2}{*}{ Study or subgroup } & \multicolumn{2}{|c|}{ TCAs } & \multicolumn{2}{|c|}{ Benzodiazepines } & \multirow{2}{*}{\multicolumn{2}{|c|}{$\begin{array}{c}\text { Std. Mean Difference } \\
\text { Random, } 95 \% \mathrm{Cl}\end{array}$}} & \multirow[t]{2}{*}{ Weight } & \multirow{2}{*}{$\begin{array}{c}\text { Std. Mean Difference } \\
\text { Random, } 95 \% \mathrm{Cl}\end{array}$} \\
\hline & $\mathbf{N}$ & $\operatorname{Mean}(S D)$ & $\mathbf{N}$ & $\operatorname{Mean}(S D)$ & & & & \\
\hline \multicolumn{9}{|c|}{ 2.8.1 Imipramine versus BDZs } \\
\hline CNCPS 1992 & 391 & $12.1(9.1)$ & 386 & $10.3(8.1)$ & & $\#$ & $19.06 \%$ & $0.21[0.07,0.35]$ \\
\hline Lepola 1990 & 24 & $9.5(9.1)$ & 27 & $9.3(9.2)$ & & - & $13.6 \%$ & $0.02[-0.53,0.57]$ \\
\hline Schweizer 1993 & 30 & $9.4(8.8)$ & 37 & $6.3(6.7)$ & & $\rightarrow$ & $14.57 \%$ & $0.4[-0.09,0.88]$ \\
\hline Sheikh 1999 & 9 & $5.3(3.7)$ & 8 & $3.9(5.6)$ & & + & $8.37 \%$ & $0.29[-0.67,1.25]$ \\
\hline Uhlenhuth 1989 & 20 & $16.1(8.3)$ & 41 & $12.7(7.4)$ & & $\longrightarrow$ & $13.74 \%$ & $0.44[-0.1,0.98]$ \\
\hline Subtotal ${ }^{\star \star \star}$ & 494 & & 523 & & & $\downarrow$ & $82.29 \%$ & $0.22[0.1,0.35]$ \\
\hline \multicolumn{9}{|c|}{ Heterogeneity: $\mathrm{Tau}^{2}=0 ; \mathrm{Chi}^{2}=1.76, \mathrm{df}=5(\mathrm{P}=0.88) ; \mathrm{I}^{2}=0 \%$} \\
\hline \multicolumn{9}{|c|}{ Test for overall effect: $\mathrm{Z}=3.51(\mathrm{P}=0)$} \\
\hline \multicolumn{9}{|c|}{ 2.8.2 Clomipramine versus BDZs } \\
\hline Subtotal $\star \star \star ~$ & 113 & & 108 & & & & $17.71 \%$ & $-0.67[-0.94,-0.4]$ \\
\hline \multicolumn{9}{|c|}{ Heterogeneity: Not applicable } \\
\hline \multicolumn{9}{|c|}{ Test for overall effect: $Z=4.82(P<0.0001)$} \\
\hline Total $* \star \star$ & 607 & & 631 & & & & $100 \%$ & $0.08[-0.28,0.45]$ \\
\hline \multicolumn{9}{|c|}{ Heterogeneity: $\mathrm{Tau}^{2}=0.18 ; \mathrm{Chi}^{2}=35.88, \mathrm{df}=6(\mathrm{P}<0.0001) ; \mathrm{I}^{2}=83.28 \%$} \\
\hline \multicolumn{9}{|c|}{ Test for overall effect: $Z=0.45(P=0.65)$} \\
\hline \multicolumn{9}{|c|}{ Test for subgroup differences: $\mathrm{Chi}^{2}=34.13, \mathrm{df}=1(\mathrm{P}<0.0001), \mathrm{I}^{2}=97.07 \%$} \\
\hline & & & & vours TCAs & -2 & 2 & Favours & diazepines \\
\hline
\end{tabular}

Analysis 2.9. Comparison 2 TCAs versus benzodiazepines, Outcome 9 Depression.

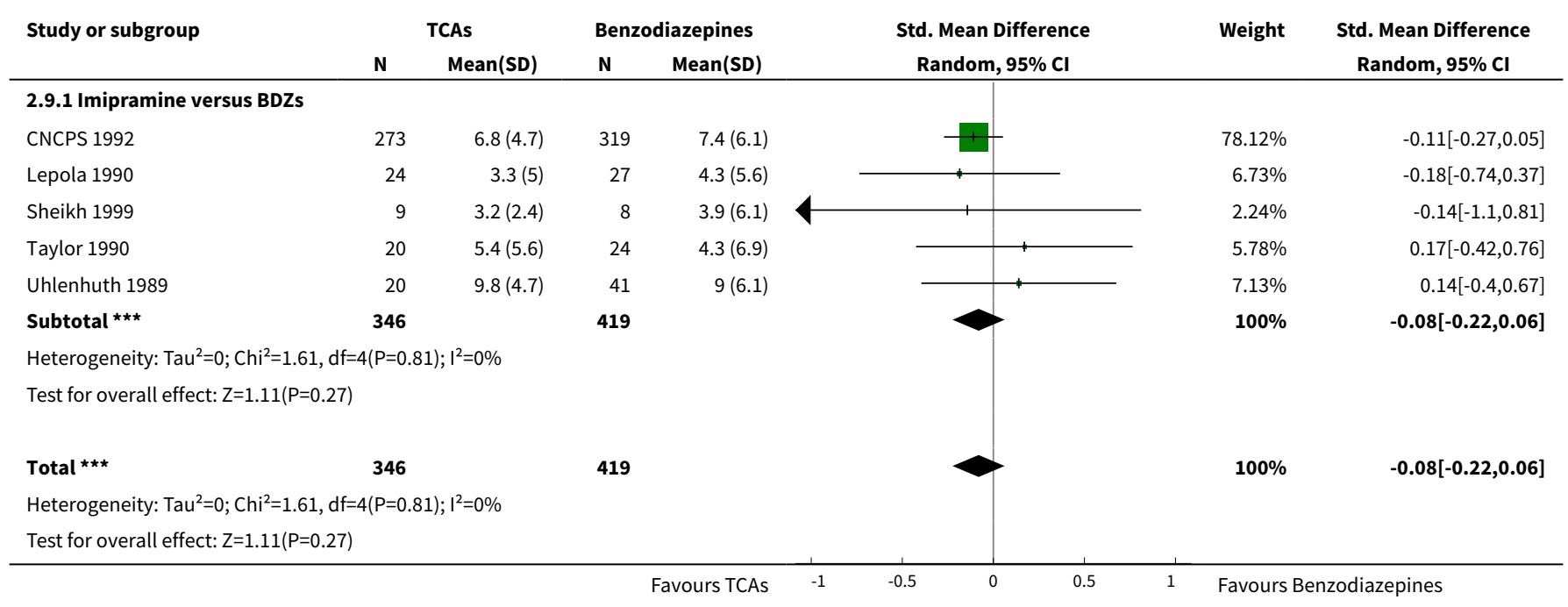


Analysis 2.10. Comparison 2 TCAs versus benzodiazepines, Outcome 10 Social functioning.

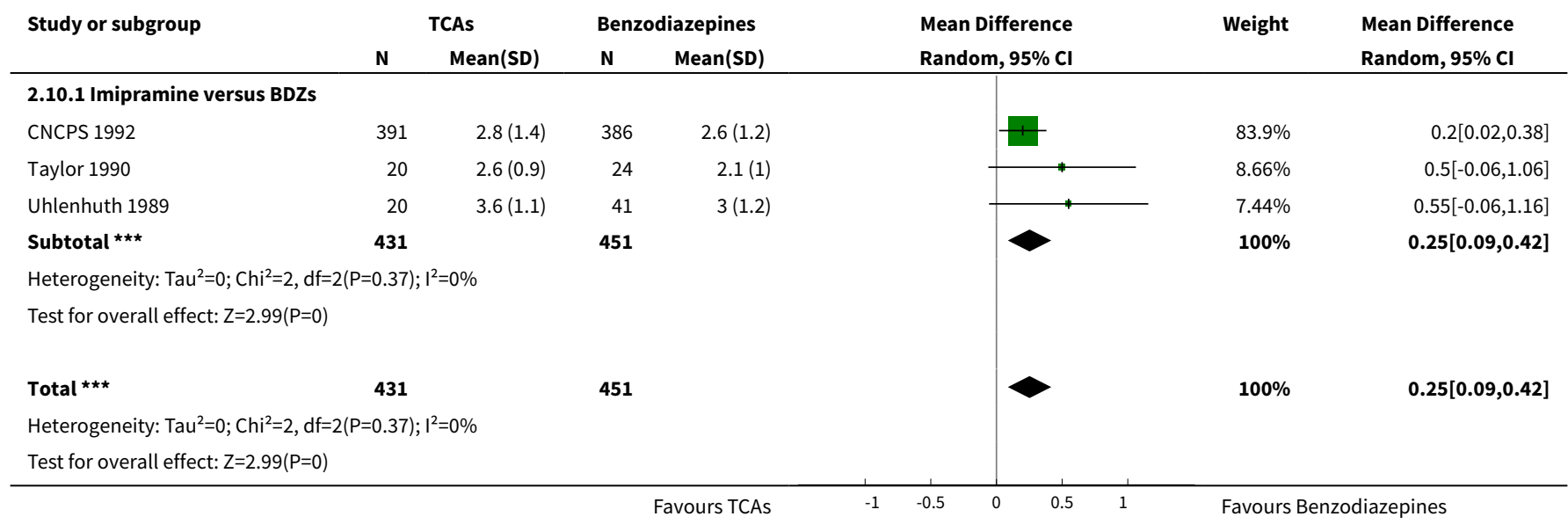

Analysis 2.14. Comparison 2 TCAs versus benzodiazepines, Outcome 14 Number of dropouts due to adverse effects.

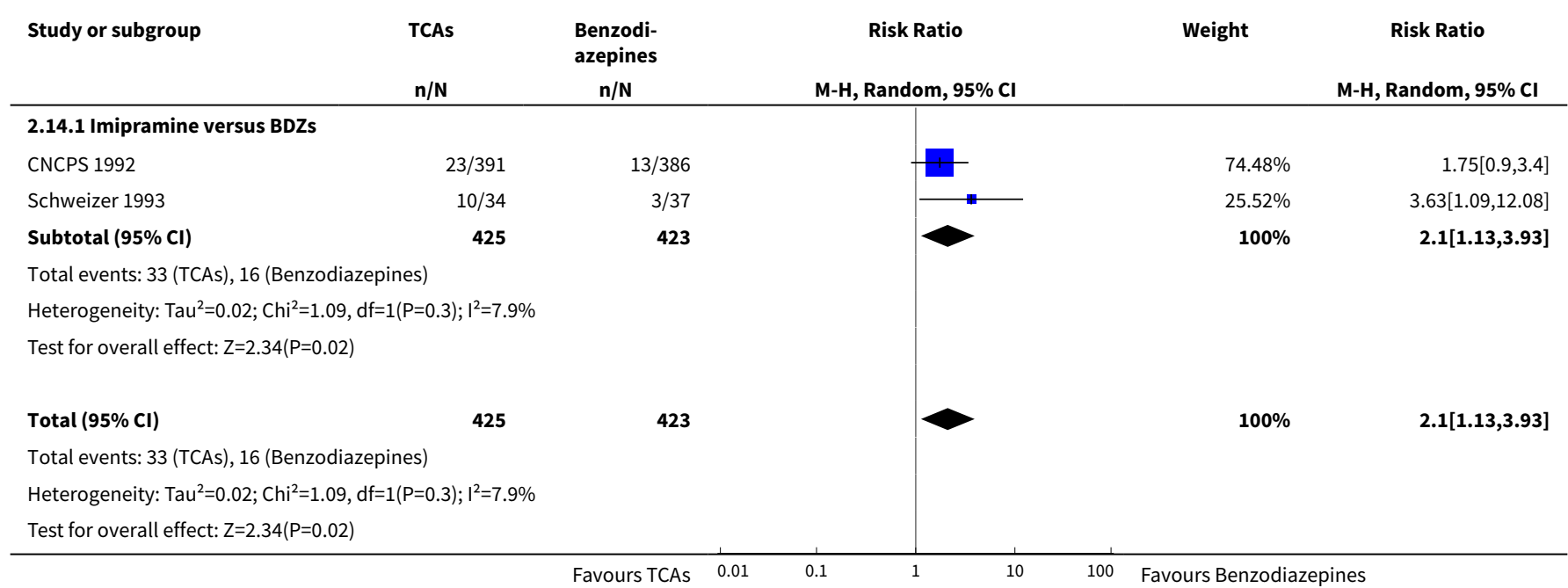

\section{Comparison 3. SSRIs versus benzodiazepines}

\begin{tabular}{llllll}
\hline Outcome or subgroup title & No. of studies & $\begin{array}{l}\text { No. of partici- } \\
\text { pants }\end{array}$ & Statistical method & Effect size \\
\hline 1 Failure to respond & 1 & 154 & Risk Ratio (M-H, Random, 95\% Cl) & $0.93[0.48,1.80]$ \\
\hline 1.1 Paroxetine versus BDZs & 1 & 154 & Risk Ratio (M-H, Random, 95\% Cl) & $0.93[0.48,1.80]$ \\
\hline 2 Total number of dropouts & 1 & 154 & Risk Ratio (M-H, Random, 95\% Cl) & $1.71[1.03,2.84]$ \\
\hline 2.1 Paroxetine versus BDZs & 1 & 154 & Risk Ratio (M-H, Random, 95\% Cl) & $1.71[1.03,2.84]$ \\
\hline 3 Failure to remit & 1 & 154 & Risk Ratio (M-H, Random, 95\% Cl) & $1.12[0.79,1.59]$ \\
\hline
\end{tabular}




\begin{tabular}{|c|c|c|c|c|}
\hline Outcome or subgroup title & No. of studies & $\begin{array}{l}\text { No. of partici- } \\
\text { pants }\end{array}$ & Statistical method & Effect size \\
\hline 3.1 Paroxetine versus BDZs & 1 & 154 & Risk Ratio (M-H, Random, 95\% Cl) & $1.12[0.79,1.59]$ \\
\hline 4 Panic symptoms & 1 & 146 & $\begin{array}{l}\text { Mean Difference (IV, Random, 95\% } \\
\mathrm{CI})\end{array}$ & $0.10[-0.34,0.54]$ \\
\hline 4.1 Paroxetine versus BDZs & 1 & 146 & $\begin{array}{l}\text { Mean Difference (IV, Random, 95\% } \\
\mathrm{CI} \text { ) }\end{array}$ & $0.10[-0.34,0.54]$ \\
\hline $\begin{array}{l}5 \text { Frequency of panic at- } \\
\text { tacks }\end{array}$ & 1 & 137 & $\begin{array}{l}\text { Mean Difference (IV, Random, 95\% } \\
\mathrm{CI})\end{array}$ & $1.20[-3.31,5.71]$ \\
\hline 5.1 Paroxetine versus BDZs & 1 & 137 & $\begin{array}{l}\text { Mean Difference (IV, Random, 95\% } \\
\mathrm{CI} \text { ) }\end{array}$ & $1.20[-3.31,5.71]$ \\
\hline 6 Agoraphobia & 1 & 112 & $\begin{array}{l}\text { Std. Mean Difference (IV, Random, } \\
95 \% \mathrm{CI})\end{array}$ & $0.0[-0.37,0.37]$ \\
\hline 6.1 Paroxetine versus BDZs & 1 & 112 & $\begin{array}{l}\text { Std. Mean Difference (IV, Random, } \\
95 \% \mathrm{CI})\end{array}$ & $0.0[-0.37,0.37]$ \\
\hline 7 General anxiety & 1 & 127 & $\begin{array}{l}\text { Mean Difference (IV, Random, 95\% } \\
\mathrm{Cl} \text { ) }\end{array}$ & $-0.60[-3.35,2.15]$ \\
\hline 7.1 Paroxetine versus BDZs & 1 & 127 & $\begin{array}{l}\text { Mean Difference (IV, Random, 95\% } \\
\mathrm{CI})\end{array}$ & $-0.60[-3.35,2.15]$ \\
\hline 8 Depression & 1 & 127 & $\begin{array}{l}\text { Mean Difference (IV, Random, 95\% } \\
\mathrm{CI} \text { ) }\end{array}$ & $-3.60[-6.36,-0.84]$ \\
\hline 8.1 Paroxetine versus BDZs & 1 & 127 & $\begin{array}{l}\text { Mean Difference (IV, Random, 95\% } \\
\mathrm{CI} \text { ) }\end{array}$ & $-3.60[-6.36,-0.84]$ \\
\hline 9 Social functioning & 1 & 125 & $\begin{array}{l}\text { Mean Difference (IV, Random, 95\% } \\
\mathrm{Cl} \text { ) }\end{array}$ & $-3.40[-8.81,2.01]$ \\
\hline 9.1 Paroxetine versus BDZs & 1 & 125 & $\begin{array}{l}\text { Mean Difference (IV, Random, 95\% } \\
\mathrm{Cl} \text { ) }\end{array}$ & $-3.40[-8.81,2.01]$ \\
\hline 10 Quality of life & 0 & 0 & $\begin{array}{l}\text { Std. Mean Difference (IV, Random, } \\
95 \% \mathrm{CI})\end{array}$ & $0.0[0.0,0.0]$ \\
\hline 11 Patient satisfaction & 0 & 0 & $\begin{array}{l}\text { Std. Mean Difference (IV, Random, } \\
95 \% \mathrm{CI})\end{array}$ & $0.0[0.0,0.0]$ \\
\hline 12 Economic costs & 0 & 0 & $\begin{array}{l}\text { Std. Mean Difference (IV, Random, } \\
95 \% \mathrm{CI})\end{array}$ & $0.0[0.0,0.0]$ \\
\hline $\begin{array}{l}13 \text { Number of dropouts due } \\
\text { to adverse effects }\end{array}$ & 1 & 154 & Risk Ratio (M-H, Random, 95\% Cl) & $1.2[0.55,2.61]$ \\
\hline 13.1 Paroxetine versus BDZs & 1 & 154 & Risk Ratio (M-H, Random, 95\% Cl) & $1.2[0.55,2.61]$ \\
\hline $\begin{array}{l}14 \text { Number of patients ex- } \\
\text { periencing at least one ad- } \\
\text { verse effect }\end{array}$ & 1 & 154 & Risk Ratio (M-H, Random, 95\% Cl) & $1.03[0.92,1.15]$ \\
\hline
\end{tabular}




\begin{tabular}{lllll}
\hline Outcome or subgroup title & No. of studies & $\begin{array}{l}\text { No. of partici- } \\
\text { pants }\end{array}$ & Statistical method & Effect size \\
\hline 14.1 Paroxetine versus BDZs & 1 & 154 & Risk Ratio (M-H, Random, 95\% Cl) & $1.03[0.92,1.15]$ \\
\hline
\end{tabular}

Analysis 3.1. Comparison 3 SSRIs versus benzodiazepines, Outcome 1 Failure to respond.

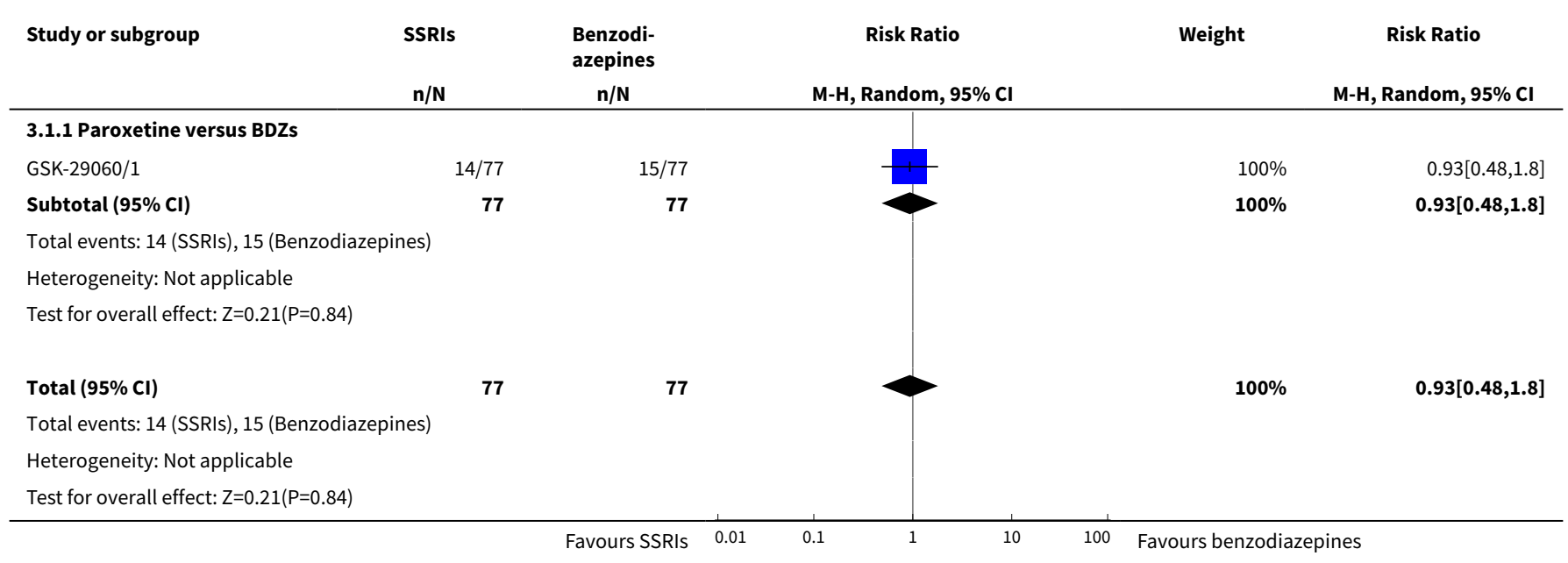

Analysis 3.2. Comparison 3 SSRIs versus benzodiazepines, Outcome 2 Total number of dropouts.

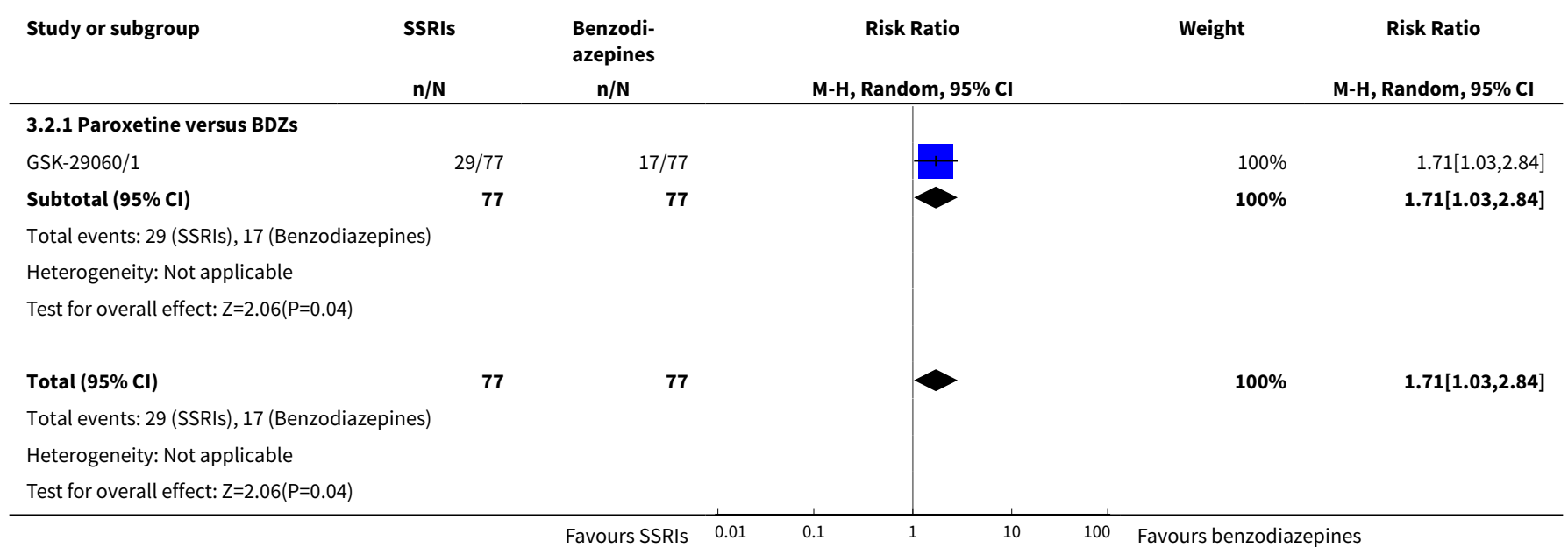

Analysis 3.3. Comparison 3 SSRIs versus benzodiazepines, Outcome 3 Failure to remit.

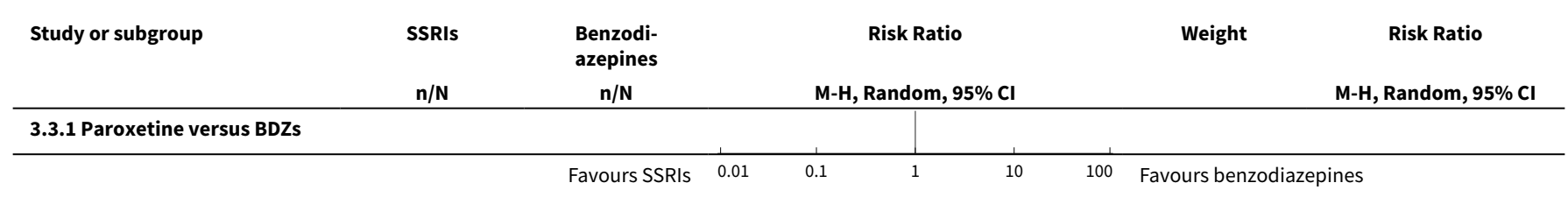




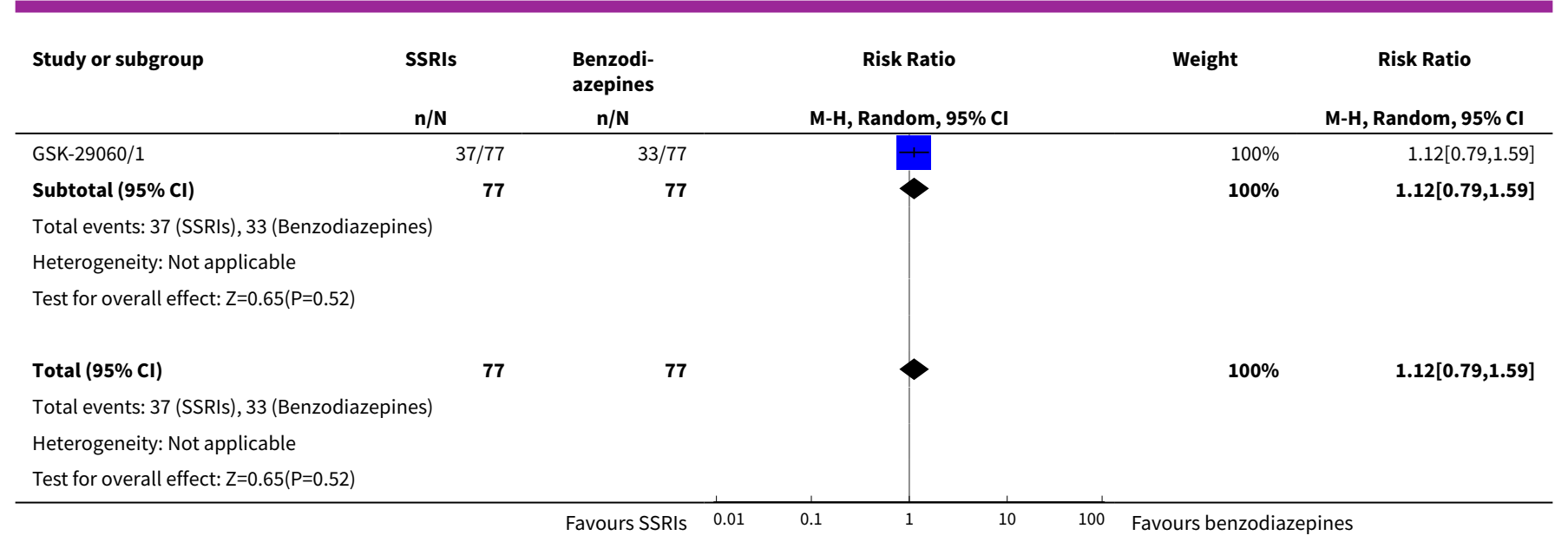

Analysis 3.4. Comparison 3 SSRIs versus benzodiazepines, Outcome 4 Panic symptoms.

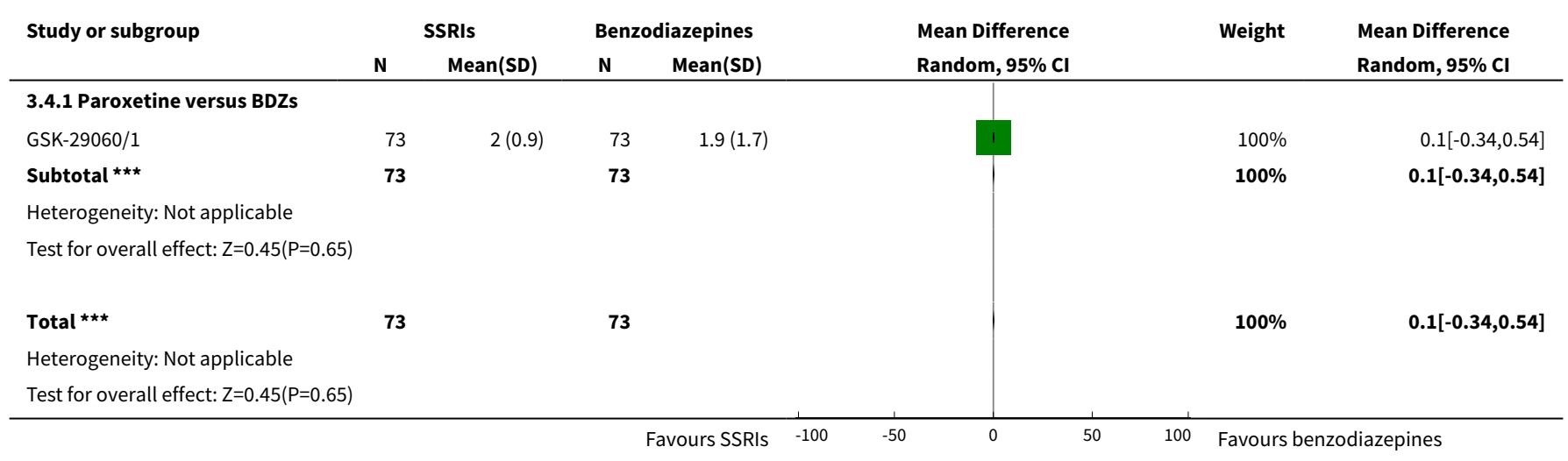

\section{Analysis 3.5. Comparison 3 SSRIs versus benzodiazepines, Outcome 5 Frequency of panic attacks.}

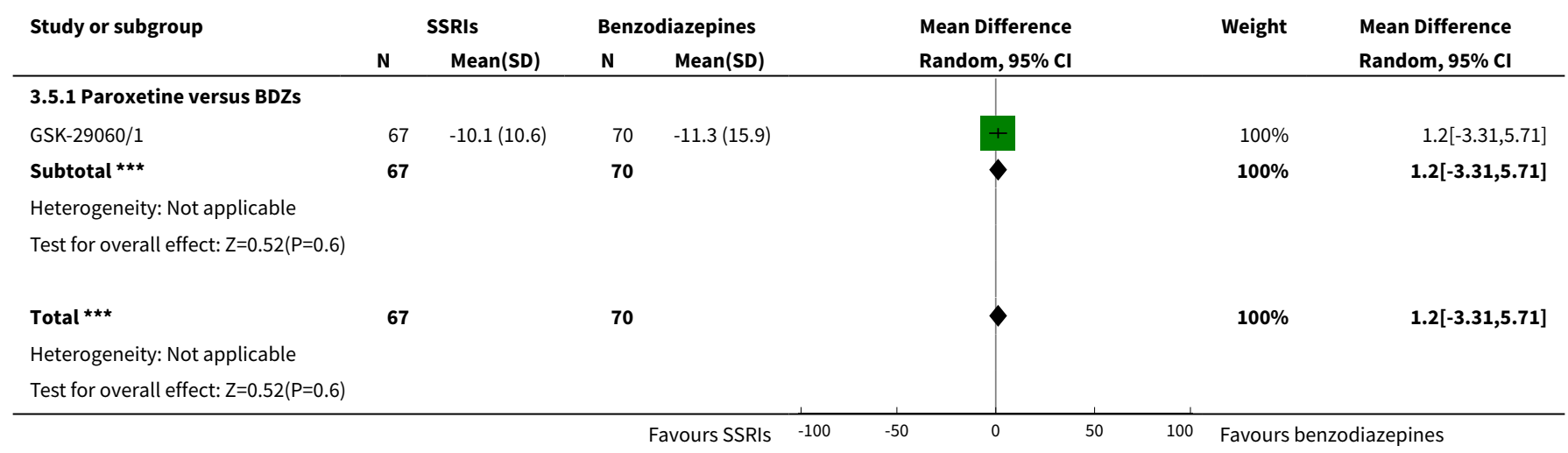


Analysis 3.6. Comparison 3 SSRIs versus benzodiazepines, Outcome 6 Agoraphobia.

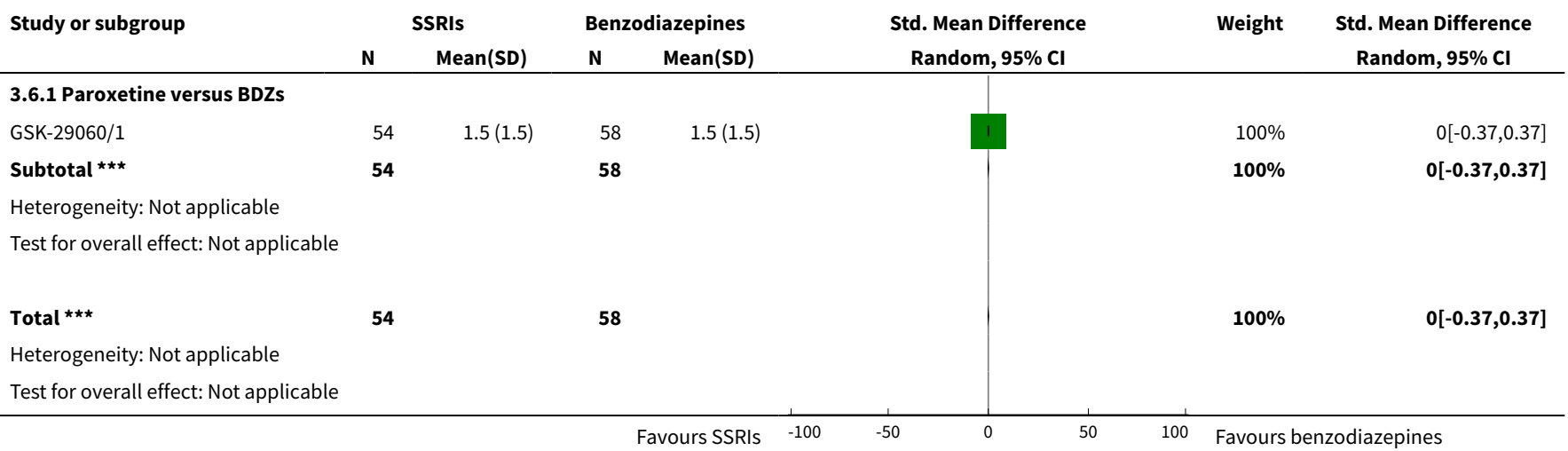

Analysis 3.7. Comparison 3 SSRIs versus benzodiazepines, Outcome 7 General anxiety.

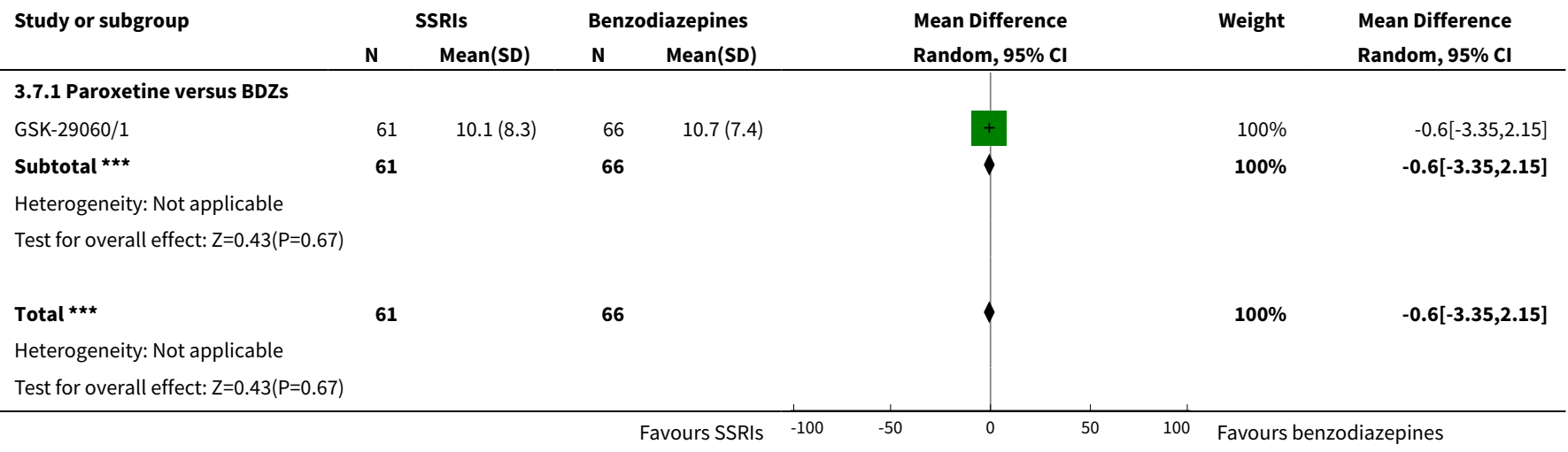

Analysis 3.8. Comparison 3 SSRIs versus benzodiazepines, Outcome 8 Depression.

\begin{tabular}{|c|c|c|c|c|c|c|c|c|}
\hline \multirow[t]{2}{*}{ Study or subgroup } & \multicolumn{2}{|c|}{ SSRIS } & \multicolumn{2}{|c|}{ Benzodiazepines } & & \multirow{2}{*}{$\begin{array}{l}\text { Mean Difference } \\
\text { Random, } 95 \% \mathrm{Cl}\end{array}$} & \multirow[t]{2}{*}{ Weight } & \multirow{2}{*}{$\begin{array}{l}\text { Mean Difference } \\
\text { Random, } 95 \% \mathrm{Cl}\end{array}$} \\
\hline & $\mathbf{N}$ & Mean(SD) & $\mathbf{N}$ & Mean(SD) & & & & \\
\hline \multicolumn{9}{|l|}{ 3.8.1 Paroxetine versus BDZs } \\
\hline GSK-29060/1 & 61 & $5.4(9.6)$ & 66 & $9(5.6)$ & & & $100 \%$ & $-3.6[-6.36,-0.84]$ \\
\hline Subtotal $\star \star \star$ & 61 & & 66 & & & & $100 \%$ & $-3.6[-6.36,-0.84]$ \\
\hline \multicolumn{9}{|l|}{ Heterogeneity: Not applicable } \\
\hline \multicolumn{9}{|l|}{ Test for overall effect: $Z=2.55(P=0.01)$} \\
\hline 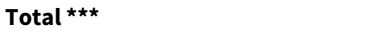 & 61 & & 66 & & & & $100 \%$ & $-3.6[-6.36,-0.84]$ \\
\hline \multicolumn{9}{|l|}{ Heterogeneity: Not applicable } \\
\hline Test for overall effect: $Z=2.55(P=0.01)$ & & & & & & & & \\
\hline & & & & ours SSRIs & -10 & -5 & Favours & azepines \\
\hline
\end{tabular}


Analysis 3.9. Comparison 3 SSRIs versus benzodiazepines, Outcome 9 Social functioning.

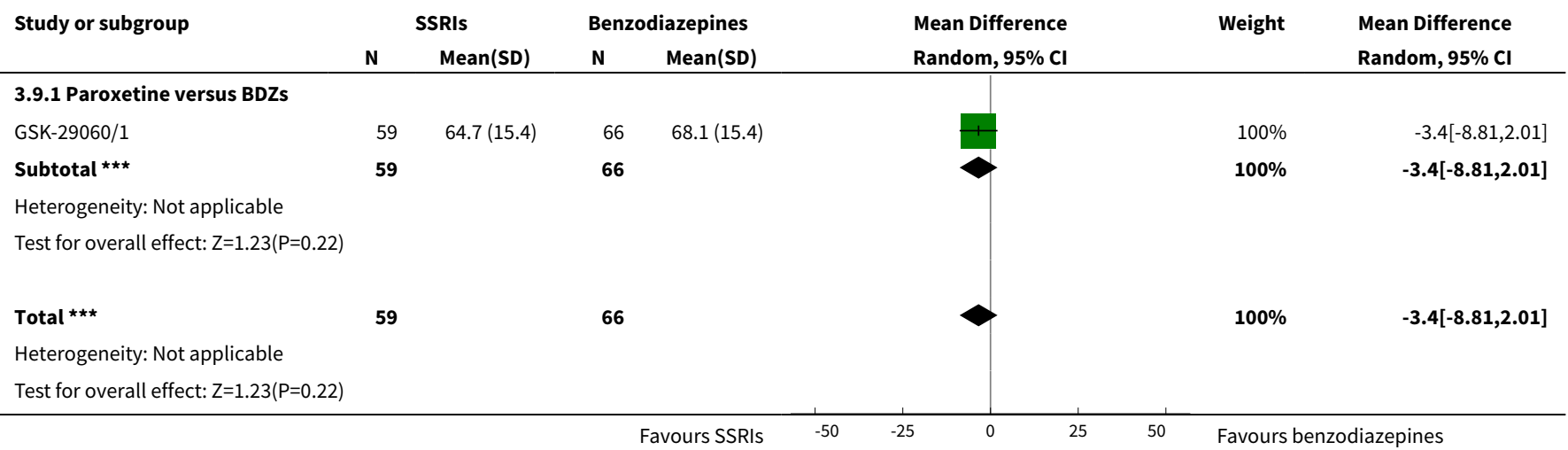

Analysis 3.13. Comparison 3 SSRIs versus benzodiazepines, Outcome 13 Number of dropouts due to adverse effects.

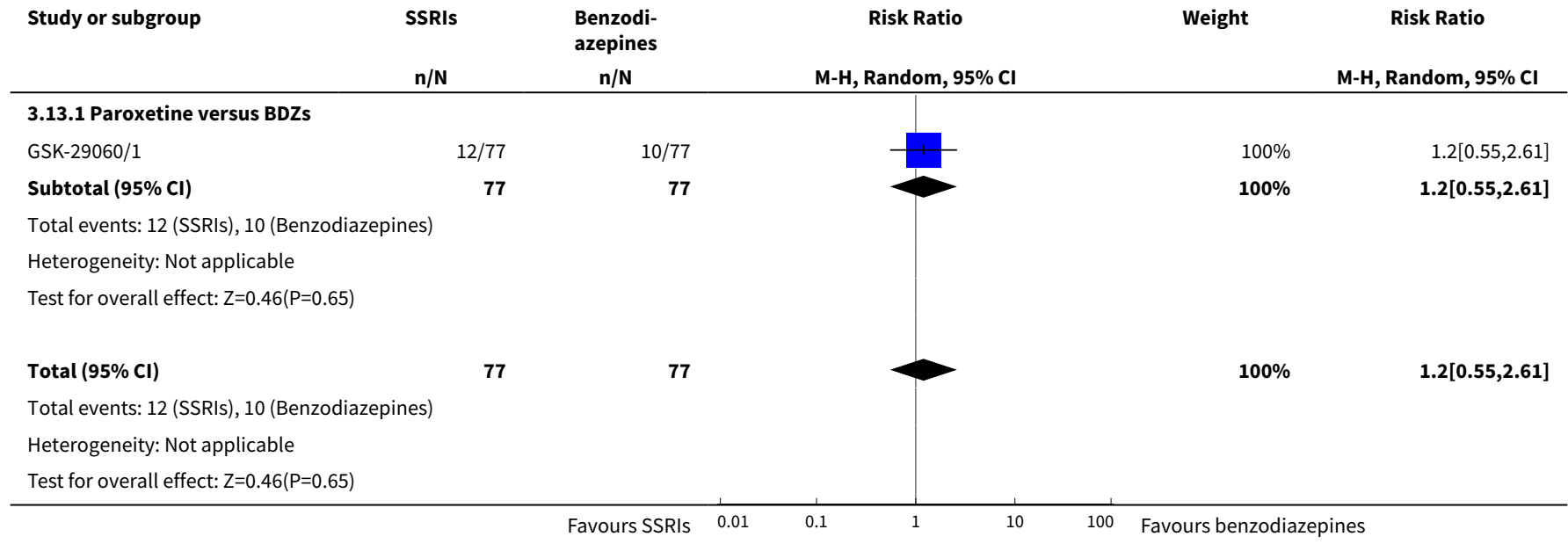

Analysis 3.14. Comparison 3 SSRIs versus benzodiazepines, Outcome 14 Number of patients experiencing at least one adverse effect.

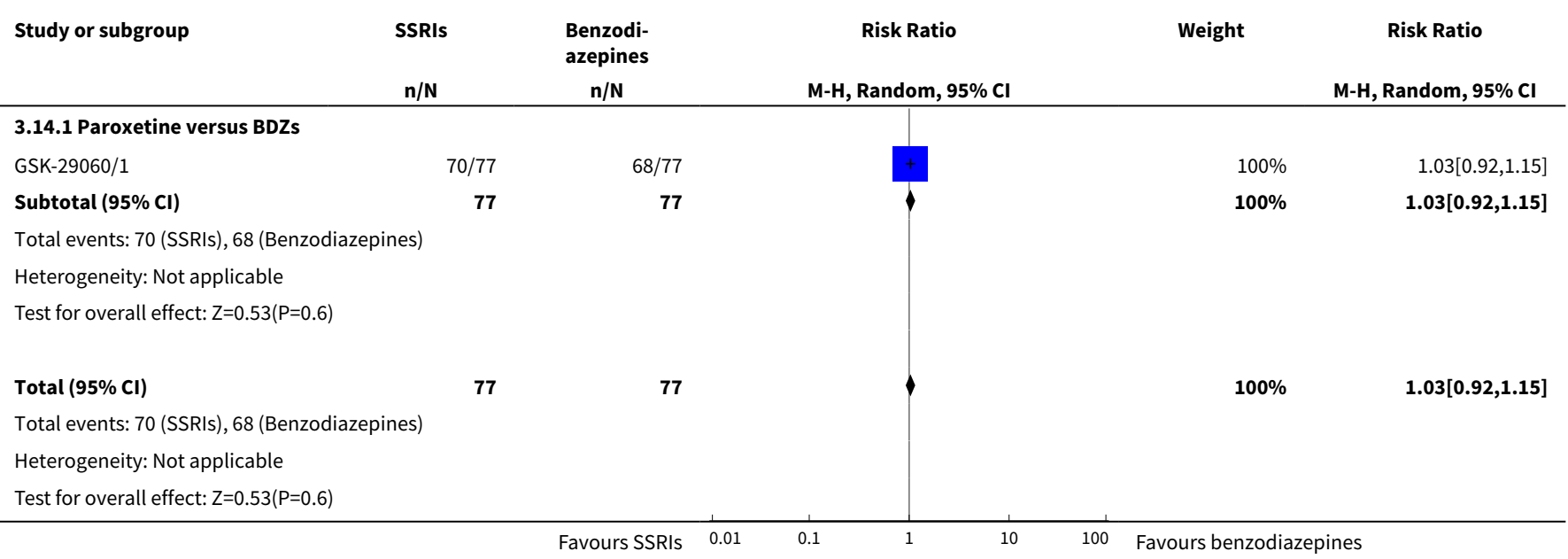


Comparison 4. TCAs versus SSRIs

\begin{tabular}{|c|c|c|c|c|}
\hline Outcome or subgroup title & No. of studies & $\begin{array}{l}\text { No. of partici- } \\
\text { pants }\end{array}$ & Statistical method & Effect size \\
\hline 1 Failure to respond & 4 & 438 & Risk Ratio (M-H, Random, 95\% Cl) & $1.08[0.71,1.66]$ \\
\hline 2 Total number of dropouts & 7 & 928 & Risk Ratio (M-H, Random, 95\% Cl) & $0.98[0.65,1.48]$ \\
\hline 3 Failure to remit & 5 & 475 & Risk Ratio (M-H, Random, 95\% Cl) & $0.89[0.63,1.26]$ \\
\hline 4 Panic symptoms & 4 & 243 & Mean Difference (IV, Random, 95\% CI) & $-0.20[-0.88,0.48]$ \\
\hline $\begin{array}{l}5 \text { Frequency of panic at- } \\
\text { tacks }\end{array}$ & 3 & 329 & Mean Difference (IV, Random, 95\% CI) & $2.30[-0.11,4.71]$ \\
\hline 6 Agoraphobia & 1 & 226 & Mean Difference (IV, Random, 95\% CI) & $0.10[-0.68,0.88]$ \\
\hline $\begin{array}{l}7 \text { General anxiety - endpoint } \\
\text { score }\end{array}$ & 7 & 668 & $\begin{array}{l}\text { Std. Mean Difference (IV, Random, } \\
95 \% \mathrm{CI})\end{array}$ & $0.09[-0.27,0.45]$ \\
\hline $\begin{array}{l}8 \text { General anxiety - mean } \\
\text { change }\end{array}$ & 2 & 309 & Mean Difference (IV, Random, 95\% CI) & $0.99[-1.39,3.36]$ \\
\hline $\begin{array}{l}9 \text { Depression - endpoint } \\
\text { score }\end{array}$ & 5 & 597 & $\begin{array}{l}\text { Std. Mean Difference (IV, Random, } \\
95 \% \mathrm{CI})\end{array}$ & $-0.04[-0.26,0.17]$ \\
\hline $\begin{array}{l}10 \text { Depression - mean } \\
\text { change }\end{array}$ & 1 & 212 & $\begin{array}{l}\text { Std. Mean Difference (IV, Random, } \\
95 \% \mathrm{CI})\end{array}$ & $-0.04[-0.31,0.23]$ \\
\hline 11 Social functioning & 0 & 0 & $\begin{array}{l}\text { Std. Mean Difference (IV, Random, } \\
95 \% \mathrm{CI} \text { ) }\end{array}$ & $0.0[0.0,0.0]$ \\
\hline 12 Quality of life & 0 & 0 & $\begin{array}{l}\text { Std. Mean Difference (IV, Random, } \\
95 \% \mathrm{CI} \text { ) }\end{array}$ & $0.0[0.0,0.0]$ \\
\hline 13 Patient satisfaction & 0 & 0 & $\begin{array}{l}\text { Std. Mean Difference (IV, Random, } \\
95 \% \mathrm{CI})\end{array}$ & $0.0[0.0,0.0]$ \\
\hline 14 Economic costs & 0 & 0 & $\begin{array}{l}\text { Std. Mean Difference (IV, Random, } \\
95 \% \mathrm{CI})\end{array}$ & $0.0[0.0,0.0]$ \\
\hline $\begin{array}{l}15 \text { Number of dropouts due } \\
\text { to adverse effects }\end{array}$ & 5 & 476 & Risk Ratio (M-H, Random, 95\% Cl) & $1.43[0.82,2.48]$ \\
\hline $\begin{array}{l}16 \text { Number of patients ex- } \\
\text { periencing at least one ad- } \\
\text { verse effect }\end{array}$ & 4 & 489 & Risk Ratio (M-H, Random, 95\% Cl) & $1.29[1.02,1.65]$ \\
\hline
\end{tabular}


Analysis 4.1. Comparison 4 TCAs versus SSRIs, Outcome 1 Failure to respond.

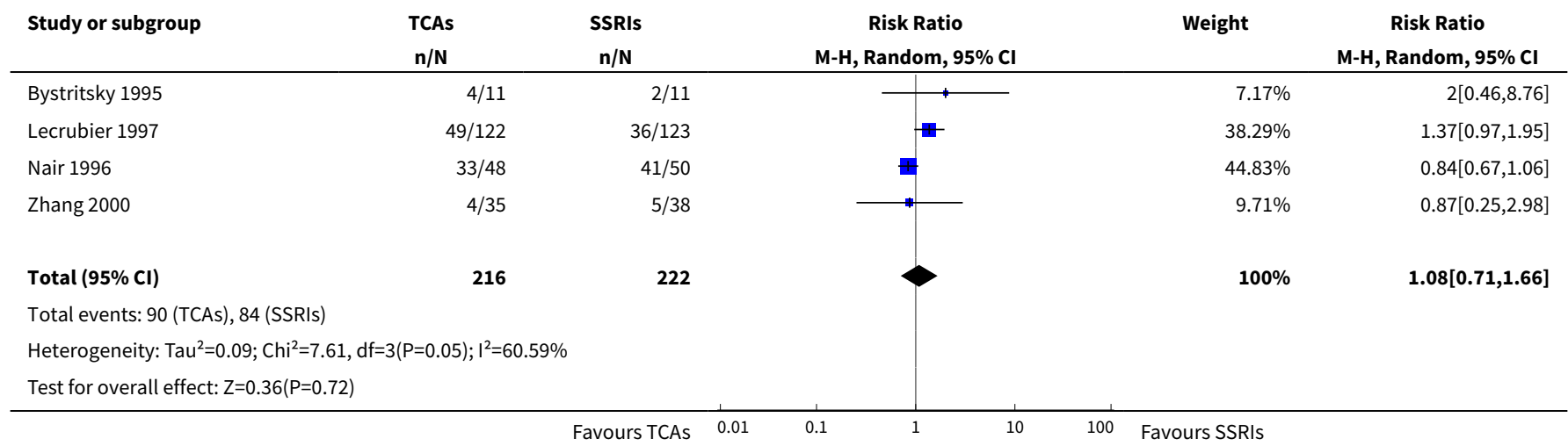

Analysis 4.2. Comparison 4 TCAs versus SSRIs, Outcome 2 Total number of dropouts.

\begin{tabular}{|c|c|c|c|c|c|}
\hline Study or subgroup & $\begin{array}{l}\text { TCAs } \\
\mathrm{n} / \mathrm{N}\end{array}$ & $\begin{array}{c}\text { SSRIs } \\
\mathrm{n} / \mathrm{N}\end{array}$ & $\begin{array}{c}\text { Risk Ratio } \\
\text { M-H, Random, } 95 \% \mathrm{Cl}\end{array}$ & Weight & $\begin{array}{c}\text { Risk Ratio } \\
\text { M-H, Random, } 95 \% \mathrm{Cl}\end{array}$ \\
\hline Amore 1999 & $2 / 19$ & $1 / 19$ & 1 & $2.99 \%$ & $2[0.2,20.24]$ \\
\hline Bystritsky 1995 & $2 / 11$ & $1 / 11$ & 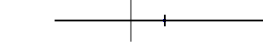 & $3.15 \%$ & $2[0.21,18.98]$ \\
\hline GSK-29060/525 & $4 / 35$ & $1 / 38$ & 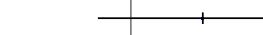 & $3.44 \%$ & $4.34[0.51,37.01]$ \\
\hline Lecrubier 1997 & $33 / 122$ & $36 / 123$ & $\rightarrow$ & $29.72 \%$ & $0.92[0.62,1.38]$ \\
\hline Nair 1996 & $16 / 48$ & $31 / 50$ & $\rightarrow-$ & $27.51 \%$ & $0.54[0.34,0.85]$ \\
\hline Zhang 2000 & $4 / 35$ & $1 / 38$ & 1 & $3.44 \%$ & $4.34[0.51,37.01]$ \\
\hline Total $(95 \% \mathrm{CI})$ & 368 & 560 & & $100 \%$ & $0.98[0.65,1.48]$ \\
\hline \multicolumn{6}{|c|}{ Total events: 86 (TCAs), 136 (SSRIs) } \\
\hline \multicolumn{6}{|c|}{ Heterogeneity: $\mathrm{Tau}^{2}=0.11 ; \mathrm{Chi}^{2}=11.27, \mathrm{df}=6(\mathrm{P}=0.08) ; \mathrm{I}^{2}=46.76 \%$} \\
\hline Test for overall effect & & & & & \\
\hline
\end{tabular}

Analysis 4.3. Comparison 4 TCAs versus SSRIs, Outcome 3 Failure to remit.

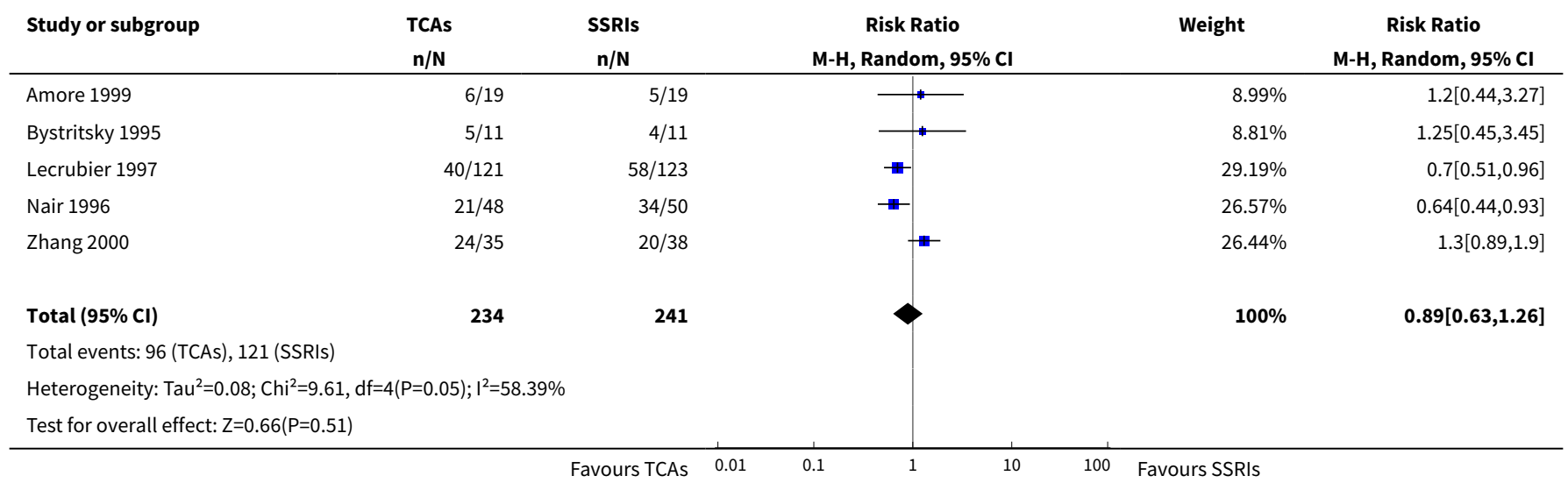


Analysis 4.4. Comparison 4 TCAs versus SSRIs, Outcome 4 Panic symptoms.

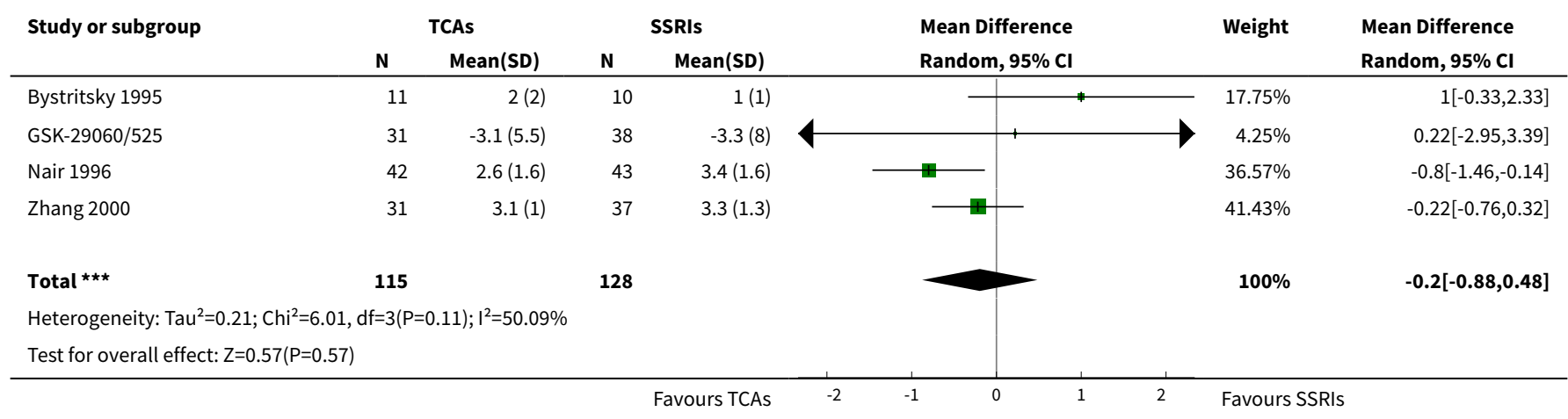

Analysis 4.5. Comparison 4 TCAs versus SSRIs, Outcome 5 Frequency of panic attacks.

\begin{tabular}{|c|c|c|c|c|c|c|c|}
\hline \multirow[t]{2}{*}{ Study or subgroup } & \multicolumn{2}{|c|}{ TCAs } & \multicolumn{2}{|c|}{ SSRIS } & \multirow{2}{*}{$\begin{array}{l}\text { Mean Difference } \\
\text { Random, 95\% Cl }\end{array}$} & \multirow[t]{2}{*}{ Weight } & \multirow{2}{*}{$\begin{array}{l}\text { Mean Difference } \\
\text { Random, } 95 \% \mathrm{Cl}\end{array}$} \\
\hline & $\mathbf{N}$ & Mean(SD) & $\mathbf{N}$ & $\operatorname{Mean}(S D)$ & & & \\
\hline Den Boer 1988 & 24 & $5(2)$ & 20 & $1.6(1.8)$ & \# & $46.12 \%$ & $3.4[2.28,4.52]$ \\
\hline GSK-29060/525 & 31 & $0.2(2.5)$ & 38 & $0.4(8.1)$ & $\longrightarrow$ & $31.07 \%$ & $-0.22[-2.93,2.49]$ \\
\hline Lecrubier 1997 & 107 & $-8.7(12.7)$ & 109 & $-12.2(15.4)$ & & $22.81 \%$ & $3.5[-0.26,7.26]$ \\
\hline 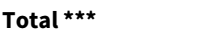 & 162 & & 167 & & & $100 \%$ & $2.3[-0.11,4.71]$ \\
\hline Test for overall effect & & & & & & & \\
\hline
\end{tabular}

Analysis 4.6. Comparison 4 TCAs versus SSRIs, Outcome 6 Agoraphobia.

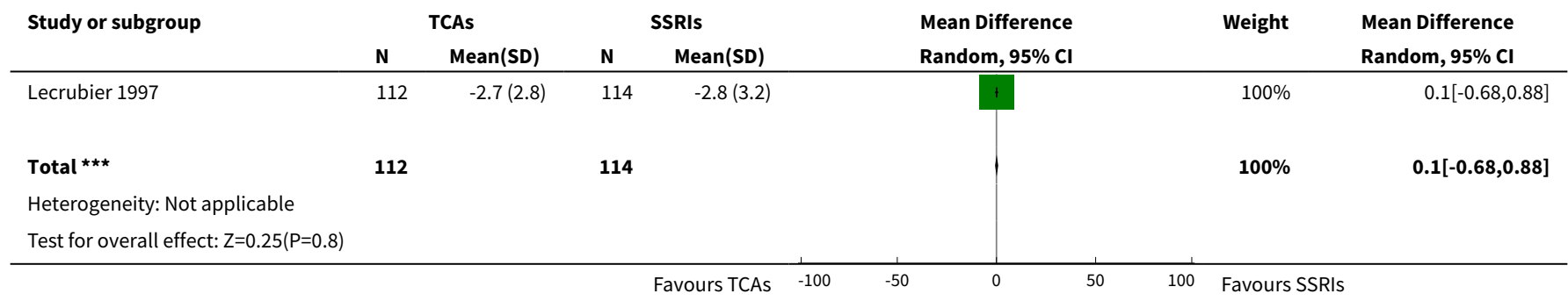

Analysis 4.7. Comparison 4 TCAs versus SSRIs, Outcome 7 General anxiety - endpoint score.

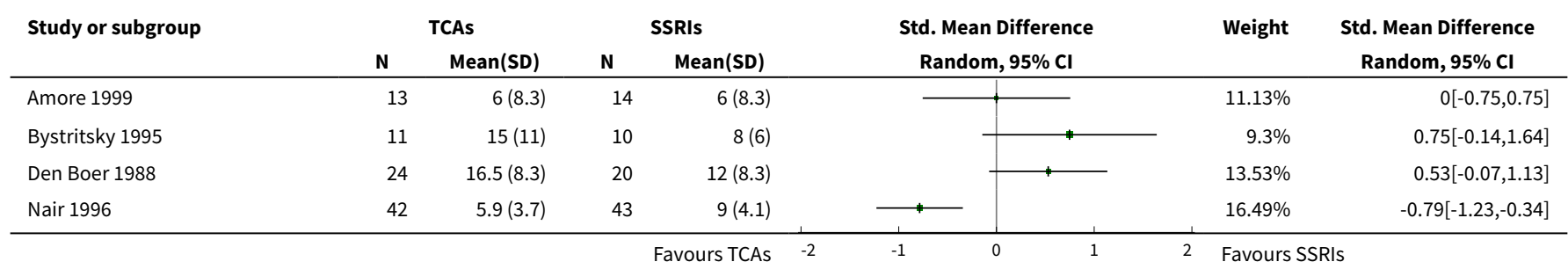




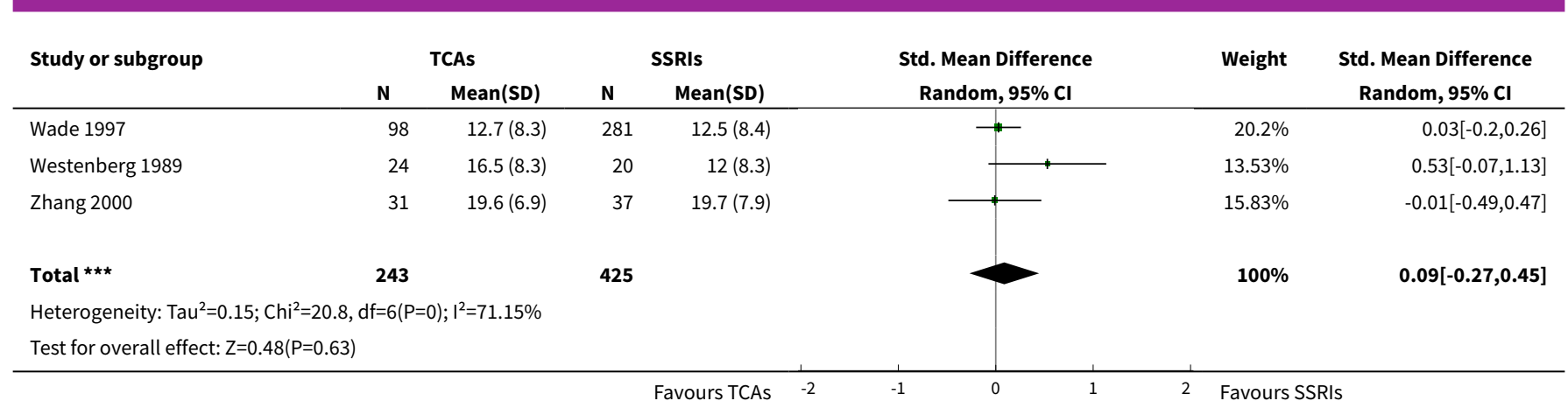

Analysis 4.8. Comparison 4 TCAs versus SSRIs, Outcome 8 General anxiety - mean change.

\begin{tabular}{|c|c|c|c|c|c|c|c|}
\hline \multirow[t]{2}{*}{ Study or subgroup } & \multicolumn{2}{|c|}{ TCAs } & \multicolumn{2}{|c|}{ SSRIS } & \multirow{2}{*}{$\begin{array}{l}\text { Mean Difference } \\
\text { Random, 95\% Cl }\end{array}$} & \multirow[t]{2}{*}{ Weight } & \multirow{2}{*}{$\begin{array}{l}\text { Mean Difference } \\
\text { Random, } 95 \% \mathrm{Cl}\end{array}$} \\
\hline & $\mathbf{N}$ & Mean(SD) & $\mathbf{N}$ & Mean(SD) & & & \\
\hline GSK-29060/525 & 31 & $-19.6(38.3)$ & 38 & $-19.7(48.8)$ & & $1.34 \%$ & $0.07[-20.48,20.62]$ \\
\hline Lecrubier 1997 & 121 & $-9.1(9.8)$ & 119 & $-10.1(9.1)$ & & $98.66 \%$ & $1[-1.39,3.39]$ \\
\hline 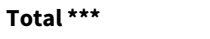 & 152 & & 157 & & & $100 \%$ & $0.99[-1.39,3.36]$ \\
\hline \multicolumn{8}{|c|}{ Heterogeneity: $\mathrm{Tau}^{2}=0 ; \mathrm{Chi}^{2}=0.01, \mathrm{df}=1(\mathrm{P}=0.93) ; \mathrm{I}^{2}=0 \%$} \\
\hline \multicolumn{8}{|c|}{ Test for overall effect: $Z=0.81(P=0.42)$} \\
\hline
\end{tabular}

Analysis 4.9. Comparison 4 TCAs versus SSRIs, Outcome 9 Depression - endpoint score.

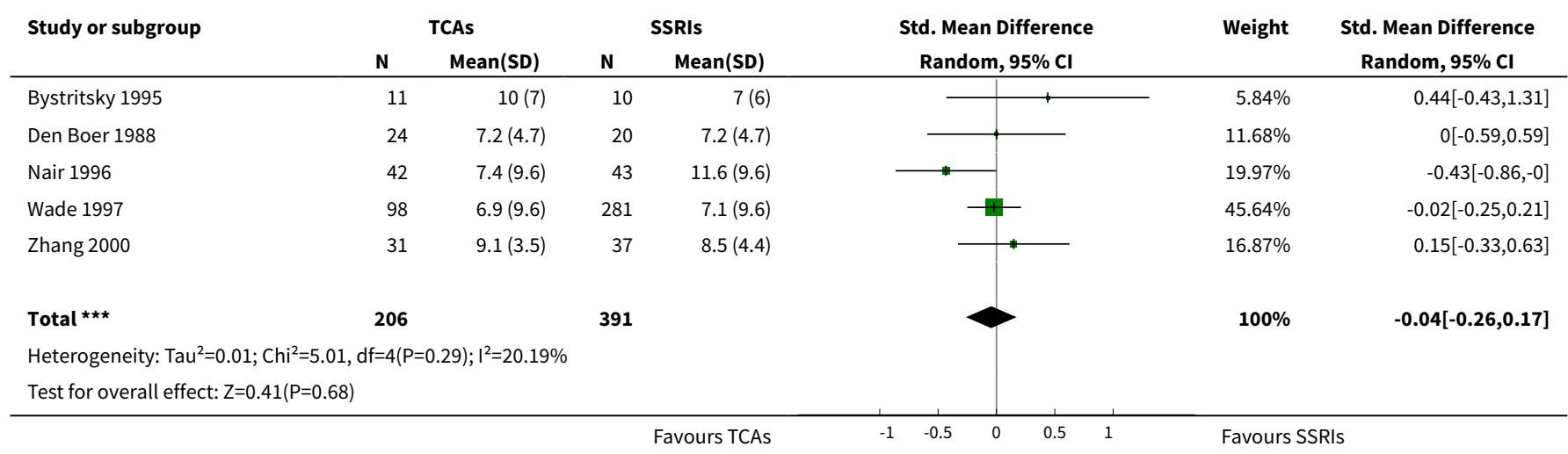

Analysis 4.10. Comparison 4 TCAs versus SSRIs, Outcome 10 Depression - mean change.

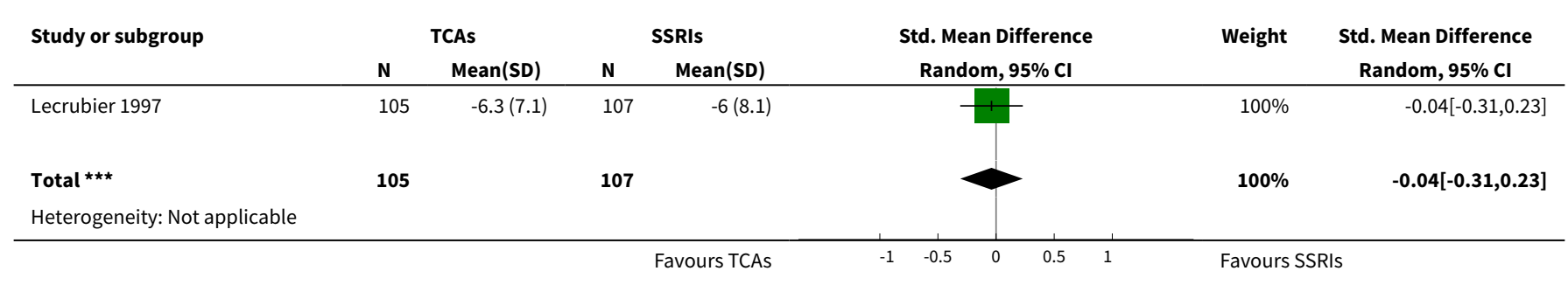




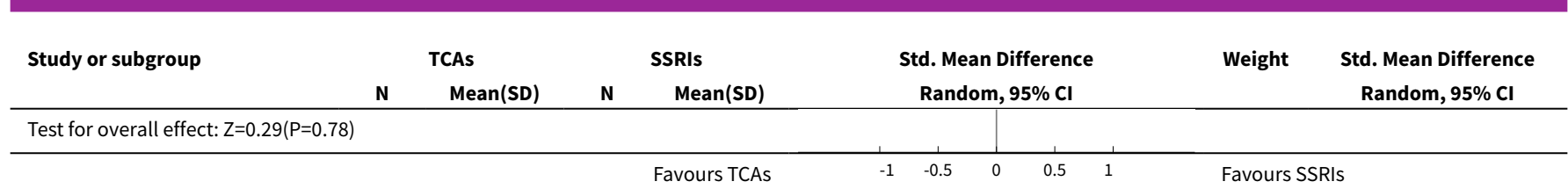

Analysis 4.15. Comparison 4 TCAs versus SSRIs, Outcome 15 Number of dropouts due to adverse effects.

\begin{tabular}{|c|c|c|c|c|c|}
\hline Study or subgroup & $\begin{array}{l}\text { TCAs } \\
\mathbf{n} / \mathbf{N}\end{array}$ & $\begin{array}{c}\text { SSRIs } \\
\mathrm{n} / \mathrm{N}\end{array}$ & $\begin{array}{c}\text { Risk Ratio } \\
\text { M-H, Random, } 95 \% \mathrm{CI}\end{array}$ & Weight & $\begin{array}{c}\text { Risk Ratio } \\
\text { M-H, Random, } 95 \% \text { Cl }\end{array}$ \\
\hline Amore 1999 & $2 / 19$ & $1 / 19$ & 1 & $5.49 \%$ & $2[0.2,20.24]$ \\
\hline Bystritsky 1995 & $2 / 11$ & $1 / 11$ & 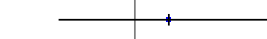 & $5.79 \%$ & $2[0.21,18.98]$ \\
\hline GSK-29060/525 & $4 / 35$ & $1 / 38$ & $\rightarrow$ & $6.37 \%$ & $4.34[0.51,37.01]$ \\
\hline Lecrubier 1997 & $18 / 122$ & 9/123 & - & $39.66 \%$ & $2.02[0.94,4.31]$ \\
\hline Nair 1996 & $10 / 48$ & $13 / 50$ & $\rightarrow$ & $42.7 \%$ & $0.8[0.39,1.65]$ \\
\hline Total $(95 \% \mathrm{Cl})$ & 235 & 241 & & $100 \%$ & $1.43[0.82,2.48]$ \\
\hline \multicolumn{6}{|c|}{ Total events: 36 (TCAs), 25 (SSRIs) } \\
\hline \multicolumn{6}{|c|}{ Heterogeneity: $\operatorname{Tau}^{2}=0.05 ; \mathrm{Chi}^{2}=4.5, \mathrm{df}=4(\mathrm{P}=0.34) ; \mathrm{I}^{2}=11.17 \%$} \\
\hline \multicolumn{6}{|c|}{ Test for overall effect: $Z=1.26(P=0.21)$} \\
\hline
\end{tabular}

Analysis 4.16. Comparison 4 TCAs versus SSRIs, Outcome 16 Number of patients experiencing at least one adverse effect.

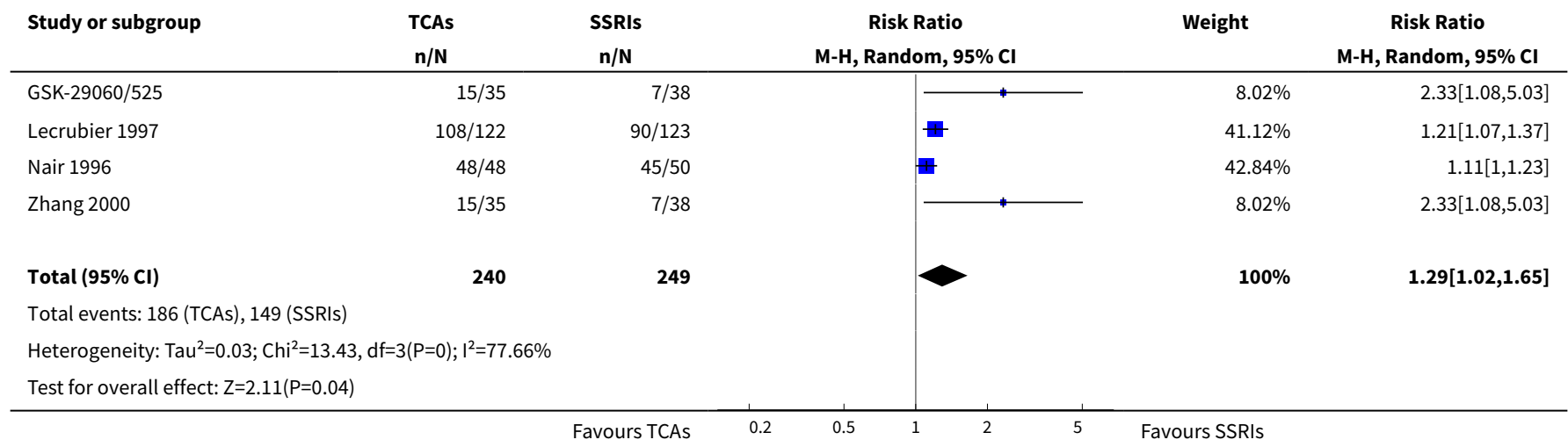

\section{Comparison 5. TCAs versus MAOIs}

\begin{tabular}{lllll}
\hline $\begin{array}{l}\text { Outcome or subgroup ti- } \\
\text { tle }\end{array}$ & No. of studies & $\begin{array}{l}\text { No. of partici- } \\
\text { pants }\end{array}$ & Statistical method & Effect size \\
\hline 1 Failure to respond & 1 & 135 & Risk Ratio (M-H, Random, 95\% Cl) & $0.59[0.28,1.26]$ \\
\hline $\begin{array}{l}\text { 2 Total number of } \\
\text { dropouts }\end{array}$ & 2 & 228 & Risk Ratio (M-H, Random, 95\% Cl) & $0.84[0.61,1.17]$ \\
\hline 3 Failure to remit & 0 & 0 & Risk Ratio (M-H, Random, 95\% Cl) & $0.0[0.0,0.0]$ \\
\hline \hline
\end{tabular}

Antidepressants and benzodiazepines for panic disorder in adults (Review) 


\begin{tabular}{|c|c|c|c|c|}
\hline $\begin{array}{l}\text { Outcome or subgroup ti- } \\
\text { tle }\end{array}$ & No. of studies & $\begin{array}{l}\text { No. of partici- } \\
\text { pants }\end{array}$ & Statistical method & Effect size \\
\hline 4 Panic symptoms & 1 & 135 & Mean Difference (IV, Random, 95\% CI) & $-0.20[-0.75,0.35]$ \\
\hline $\begin{array}{l}5 \text { Frequency of panic at- } \\
\text { tacks }\end{array}$ & 1 & 135 & Mean Difference (IV, Random, 95\% CI) & $-0.30[-3.35,2.75]$ \\
\hline 6 Agoraphobia & 0 & 0 & $\begin{array}{l}\text { Std. Mean Difference (IV, Random, 95\% } \\
\text { CI) }\end{array}$ & $0.0[0.0,0.0]$ \\
\hline 7 General anxiety & 1 & 135 & Mean Difference (IV, Random, 95\% CI) & $0.30[-3.03,3.63]$ \\
\hline 8 Depression & 1 & 135 & Mean Difference (IV, Random, 95\% CI) & $-4.1[-8.14,-0.06]$ \\
\hline 9 Social functioning & 0 & 0 & $\begin{array}{l}\text { Std. Mean Difference (IV, Random, 95\% } \\
\text { CI) }\end{array}$ & $0.0[0.0,0.0]$ \\
\hline 10 Quality of life & 0 & 0 & $\begin{array}{l}\text { Std. Mean Difference (IV, Random, 95\% } \\
\text { CI) }\end{array}$ & $0.0[0.0,0.0]$ \\
\hline 11 Patient satisfaction & 0 & 0 & $\begin{array}{l}\text { Std. Mean Difference (IV, Random, 95\% } \\
\text { CI) }\end{array}$ & $0.0[0.0,0.0]$ \\
\hline 12 Economic costs & 0 & 0 & $\begin{array}{l}\text { Std. Mean Difference (IV, Random, 95\% } \\
\mathrm{CI} \text { ) }\end{array}$ & $0.0[0.0,0.0]$ \\
\hline $\begin{array}{l}13 \text { Number of dropouts } \\
\text { due to adverse effects }\end{array}$ & 1 & 135 & Risk Ratio (M-H, Random, 95\% Cl) & $1.27[0.50,3.21]$ \\
\hline $\begin{array}{l}14 \text { Number of patients ex- } \\
\text { periencing at least one ad- } \\
\text { verse effect }\end{array}$ & 1 & 135 & Risk Ratio (M-H, Random, 95\% Cl) & $1.24[1.06,1.45]$ \\
\hline
\end{tabular}

Analysis 5.1. Comparison 5 TCAs versus MAOIs, Outcome 1 Failure to respond.

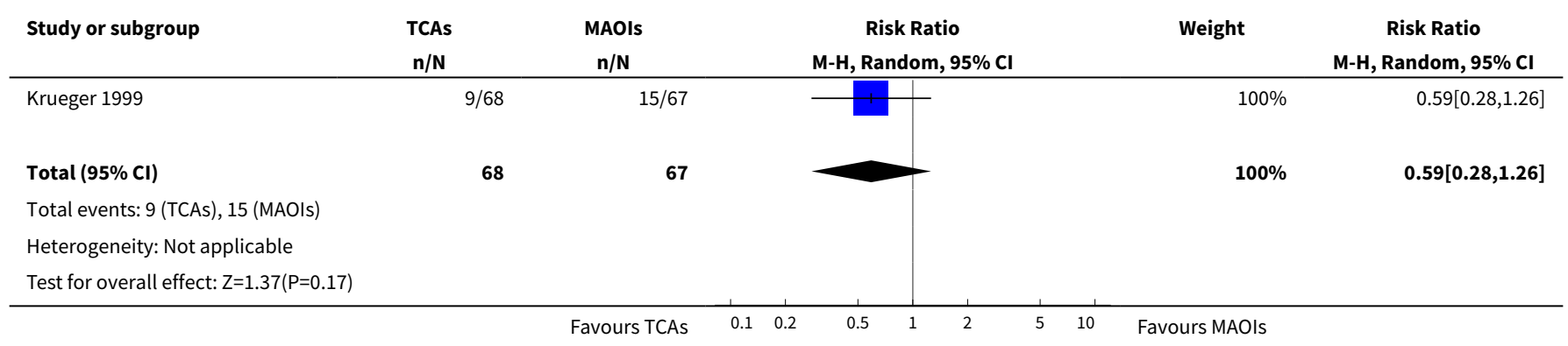


Analysis 5.2. Comparison 5 TCAs versus MAOIs, Outcome 2 Total number of dropouts.

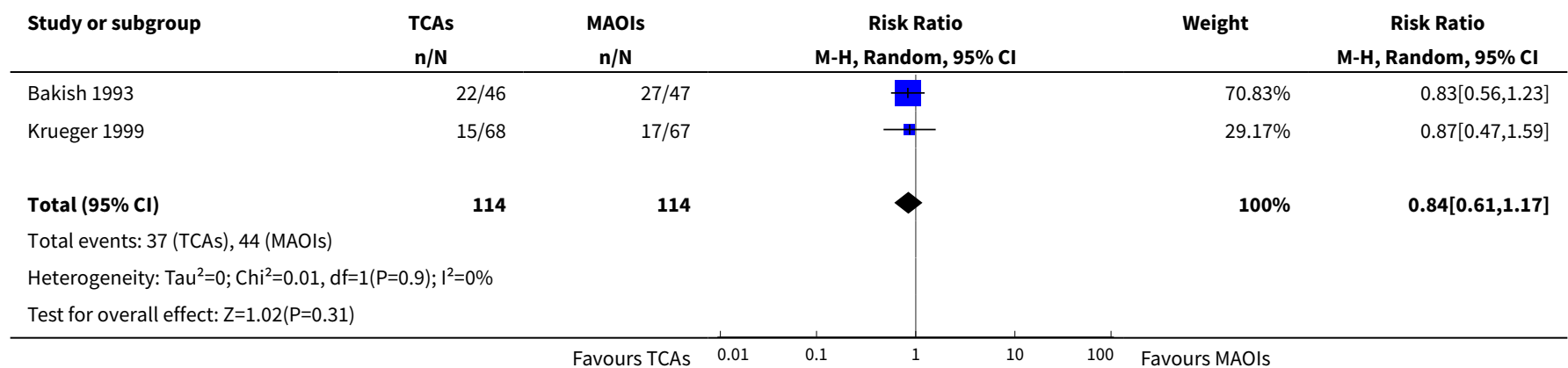

Analysis 5.4. Comparison 5 TCAs versus MAOIs, Outcome 4 Panic symptoms.

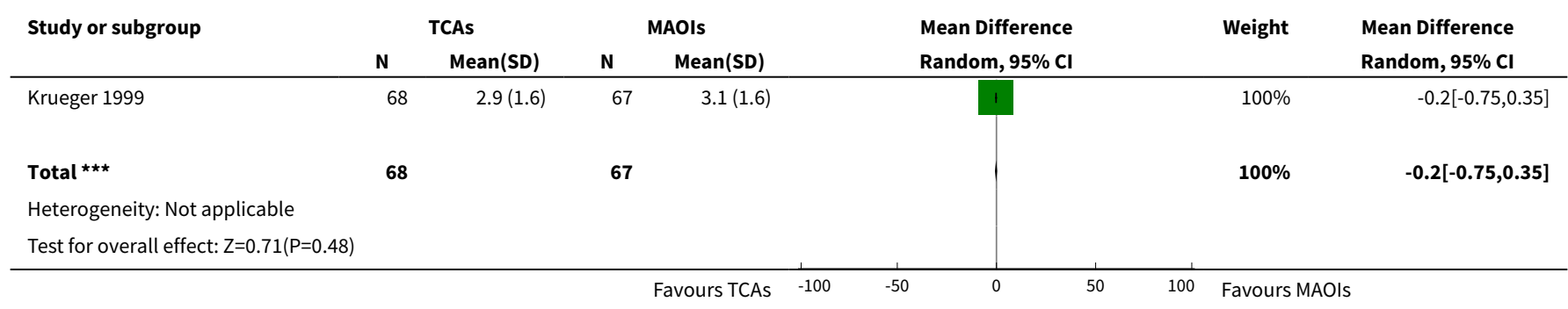

Analysis 5.5. Comparison 5 TCAs versus MAOIs, Outcome 5 Frequency of panic attacks.

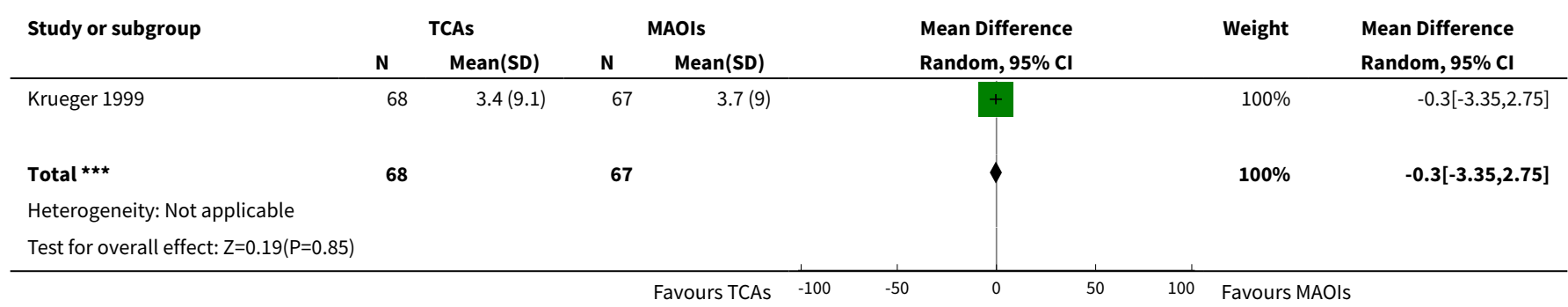

Analysis 5.7. Comparison 5 TCAs versus MAOIs, Outcome 7 General anxiety.

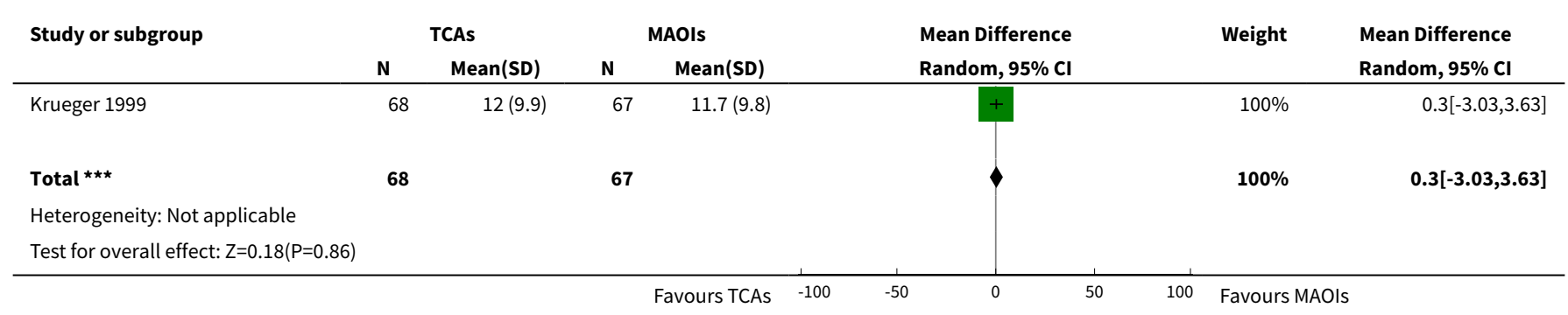


Analysis 5.8. Comparison 5 TCAs versus MAOIs, Outcome 8 Depression.

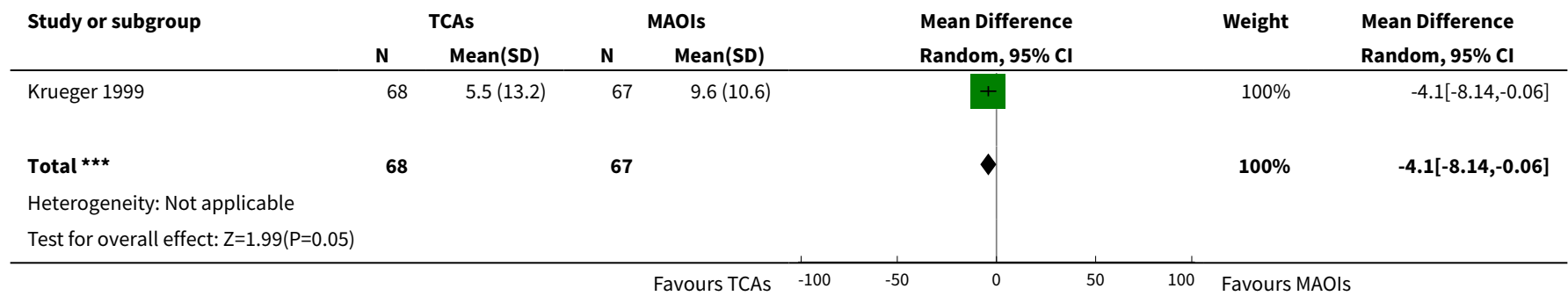

Analysis 5.13. Comparison 5 TCAs versus MAOIs, Outcome 13 Number of dropouts due to adverse effects.

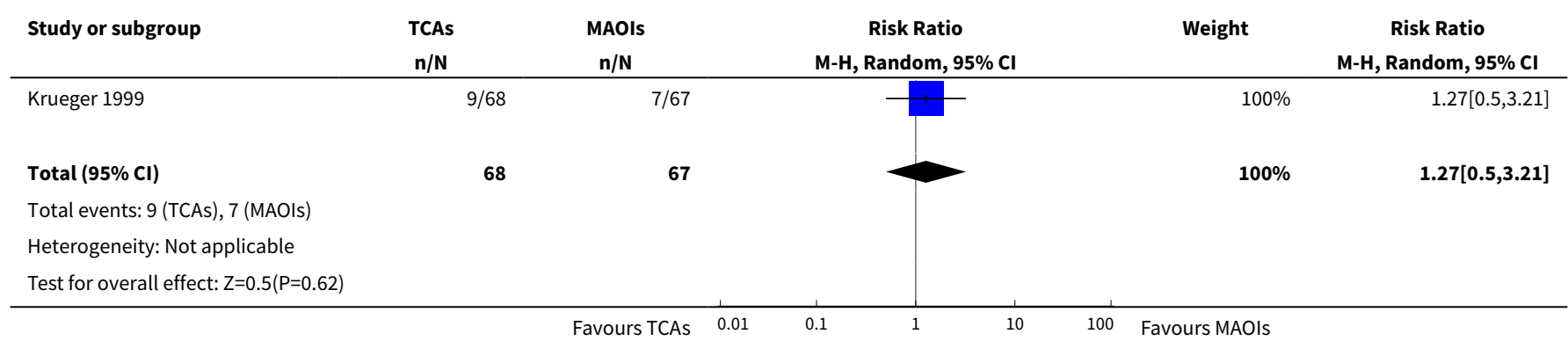

Analysis 5.14. Comparison 5 TCAs versus MAOIs, Outcome 14 Number of patients experiencing at least one adverse effect.

\begin{tabular}{|c|c|c|c|c|c|}
\hline Study or subgroup & $\begin{array}{l}\text { TCAs } \\
\mathrm{n} / \mathrm{N}\end{array}$ & $\begin{array}{c}\text { MAOIs } \\
\mathrm{n} / \mathrm{N}\end{array}$ & $\begin{array}{c}\text { Risk Ratio } \\
\text { M-H, Random, 95\% Cl }\end{array}$ & Weight & $\begin{array}{c}\text { Risk Ratio } \\
\text { M-H, Random, } 95 \% \mathrm{CI}\end{array}$ \\
\hline Krueger 1999 & $63 / 68$ & $50 / 67$ & & $100 \%$ & $1.24[1.06,1.45]$ \\
\hline Total $(95 \% \mathrm{CI})$ & 68 & 67 & $\checkmark$ & $100 \%$ & $1.24[1.06,1.45]$ \\
\hline \multicolumn{6}{|c|}{ Total events: 63 (TCAs), 50 (MAOIs) } \\
\hline \multicolumn{6}{|c|}{ Heterogeneity: Not applicable } \\
\hline
\end{tabular}

\section{Comparison 6. SSRIs versus MAOIs}

\begin{tabular}{lllll}
\hline $\begin{array}{l}\text { Outcome or subgroup ti- } \\
\text { tle }\end{array}$ & No. of studies & $\begin{array}{l}\text { No. of partici- } \\
\text { pants }\end{array}$ & Statistical method & Effect size \\
\hline $\begin{array}{lllll}\text { 1 Failure to respond } \\
\text { 2 Total number of }\end{array}$ & 2 & 396 & Risk Ratio (M-H, Random, 95\% Cl) & $1.12[0.83,1.52]$ \\
\hline $\begin{array}{l}\text { dropouts } \\
\text { 3 Failure to remit }\end{array}$ & 1 & 30 & Risk Ratio (M-H, Random, 95\% Cl) & $1.0[0.07,14.55]$ \\
\hline
\end{tabular}




\begin{tabular}{|c|c|c|c|c|}
\hline $\begin{array}{l}\text { Outcome or subgroup ti- } \\
\text { tle }\end{array}$ & No. of studies & $\begin{array}{l}\text { No. of partici- } \\
\text { pants }\end{array}$ & Statistical method & Effect size \\
\hline 4 Panic symptoms & 0 & 0 & $\begin{array}{l}\text { Std. Mean Difference (IV, Random, 95\% } \\
\mathrm{CI} \text { ) }\end{array}$ & $0.0[0.0,0.0]$ \\
\hline $\begin{array}{l}5 \text { Frequency of panic at- } \\
\text { tacks }\end{array}$ & 0 & 0 & $\begin{array}{l}\text { Std. Mean Difference (IV, Random, 95\% } \\
\mathrm{Cl} \text { ) }\end{array}$ & $0.0[0.0,0.0]$ \\
\hline 6 Agoraphobia & 1 & 30 & Mean Difference (IV, Random, 95\% CI) & $1.5[-4.47,7.47]$ \\
\hline 7 General anxiety & 1 & 30 & Mean Difference (IV, Random, 95\% CI) & $-0.60[-1.10,-0.10]$ \\
\hline 8 Depression & 0 & 0 & $\begin{array}{l}\text { Std. Mean Difference (IV, Random, 95\% } \\
\mathrm{Cl} \text { ) }\end{array}$ & $0.0[0.0,0.0]$ \\
\hline 9 Social functioning & 0 & 0 & $\begin{array}{l}\text { Std. Mean Difference (IV, Random, 95\% } \\
\mathrm{Cl} \text { ) }\end{array}$ & $0.0[0.0,0.0]$ \\
\hline 10 Quality of life & 0 & 0 & $\begin{array}{l}\text { Std. Mean Difference (IV, Random, 95\% } \\
\mathrm{Cl} \text { ) }\end{array}$ & $0.0[0.0,0.0]$ \\
\hline 11 Patient satisfaction & 0 & 0 & $\begin{array}{l}\text { Std. Mean Difference (IV, Random, 95\% } \\
\mathrm{CI} \text { ) }\end{array}$ & $0.0[0.0,0.0]$ \\
\hline 12 Economic costs & 0 & 0 & $\begin{array}{l}\text { Std. Mean Difference (IV, Random, 95\% } \\
\mathrm{CI} \text { ) }\end{array}$ & $0.0[0.0,0.0]$ \\
\hline $\begin{array}{l}13 \text { Number of dropouts } \\
\text { due to adverse effects }\end{array}$ & 1 & 366 & Risk Ratio (M-H, Random, 95\% Cl) & $1.26[0.59,2.70]$ \\
\hline $\begin{array}{l}14 \text { Number of patients ex- } \\
\text { periencing at least one ad- } \\
\text { verse effect }\end{array}$ & 1 & 366 & Risk Ratio (M-H, Random, 95\% Cl) & $1.09[0.94,1.27]$ \\
\hline
\end{tabular}

Analysis 6.1. Comparison 6 SSRIs versus MAOIs, Outcome 1 Failure to respond.

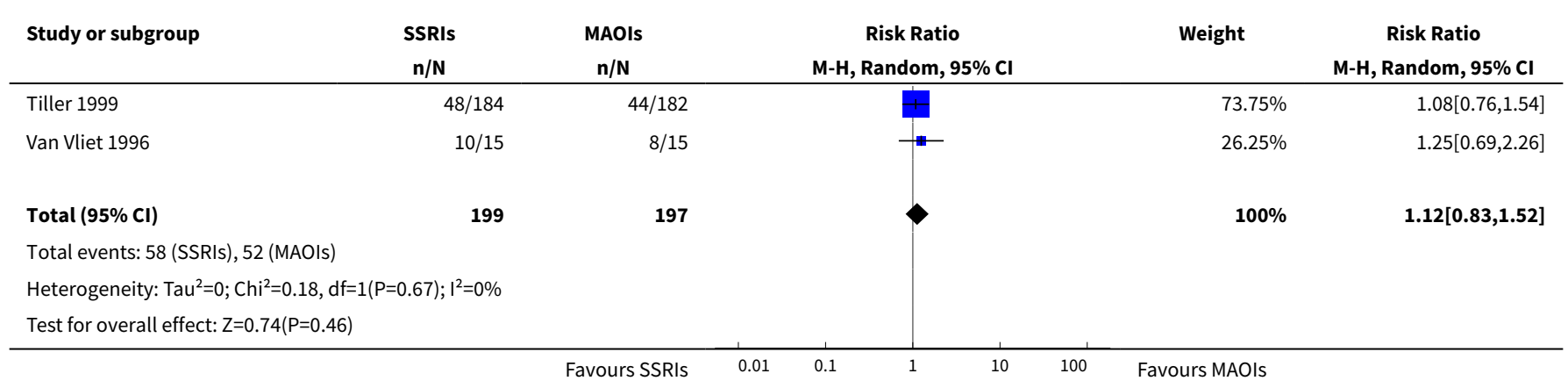


Analysis 6.2. Comparison 6 SSRIs versus MAOIs, Outcome 2 Total number of dropouts.

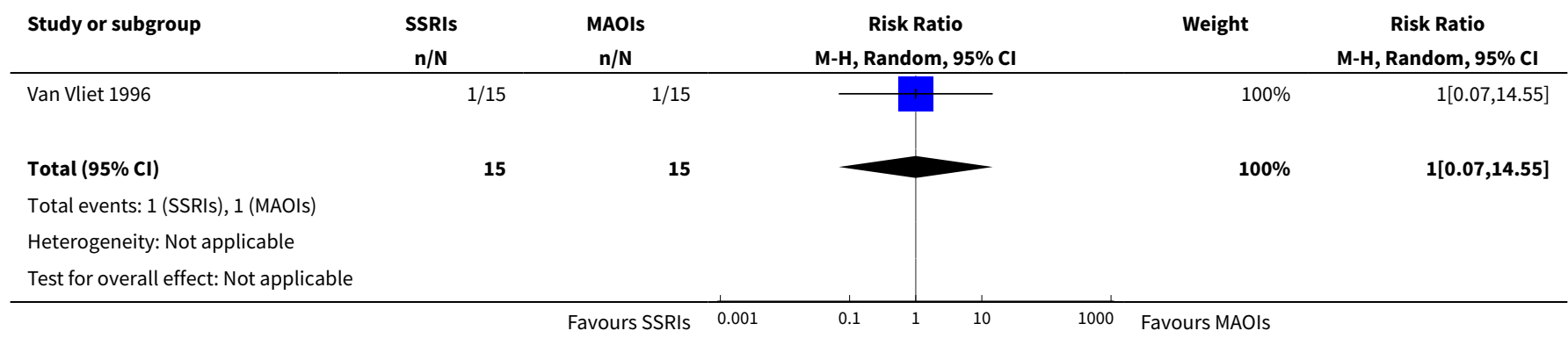

Analysis 6.3. Comparison 6 SSRIs versus MAOIs, Outcome 3 Failure to remit.

\begin{tabular}{|c|c|c|c|c|c|}
\hline Study or subgroup & $\begin{array}{c}\text { SSRIs } \\
\mathrm{n} / \mathrm{N}\end{array}$ & $\begin{array}{c}\text { MAOIs } \\
\mathrm{n} / \mathrm{N}\end{array}$ & $\begin{array}{c}\text { Risk Ratio } \\
\text { M-H, Random, 95\% Cl }\end{array}$ & Weight & $\begin{array}{c}\text { Risk Ratio } \\
\text { M-H, Random, 95\% Cl }\end{array}$ \\
\hline Tiller 1999 & $80 / 184$ & $85 / 182$ & & $100 \%$ & $0.93[0.74,1.17]$ \\
\hline Total $(95 \% \mathrm{Cl})$ & 184 & 182 & & $100 \%$ & $0.93[0.74,1.17]$ \\
\hline \multicolumn{6}{|c|}{ Total events: 80 (SSRIs), 85 (MAOIs) } \\
\hline \multicolumn{6}{|c|}{ Heterogeneity: Not applicable } \\
\hline \multicolumn{6}{|c|}{ Test for overall effect: $Z=0.62(P=0.54)$} \\
\hline
\end{tabular}

Analysis 6.6. Comparison 6 SSRIs versus MAOIs, Outcome 6 Agoraphobia.

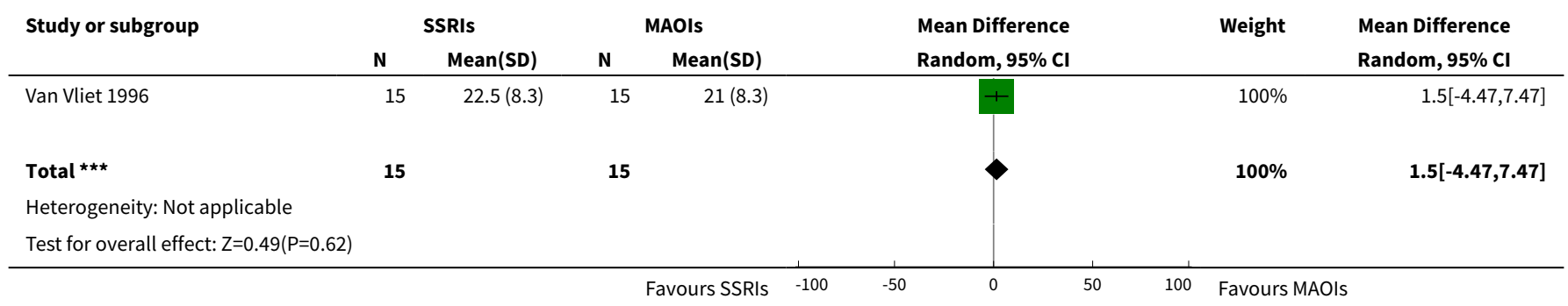

Analysis 6.7. Comparison 6 SSRIs versus MAOIs, Outcome 7 General anxiety.

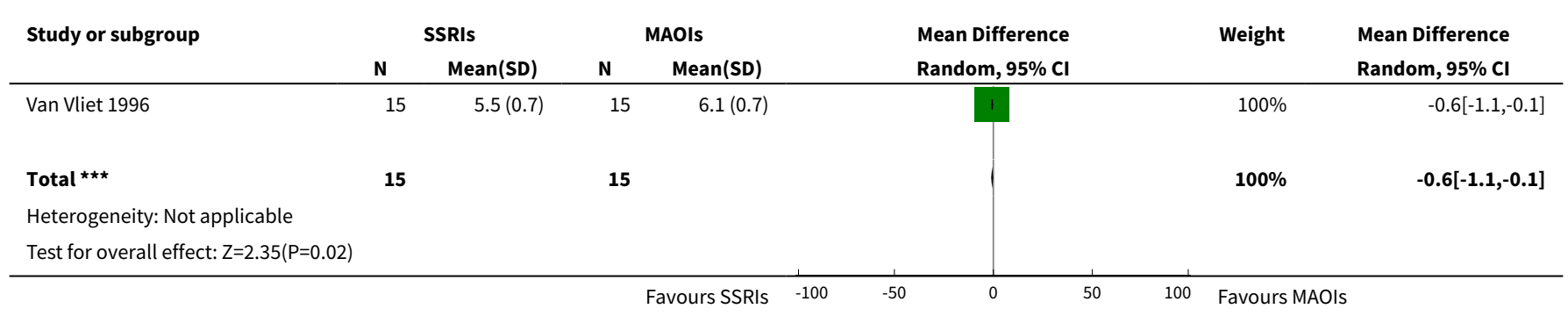


Analysis 6.13. Comparison 6 SSRIs versus MAOIs, Outcome 13 Number of dropouts due to adverse effects.

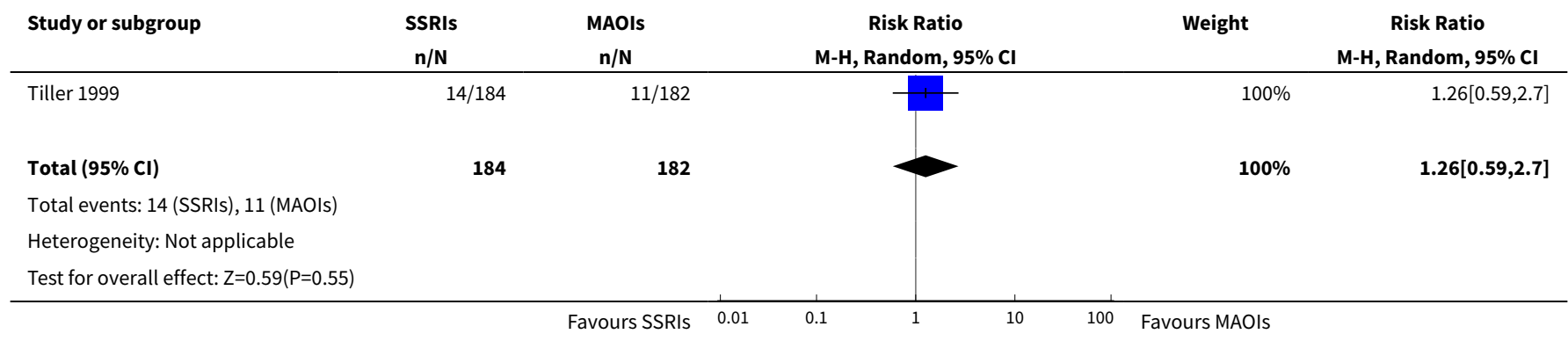

Analysis 6.14. Comparison 6 SSRIs versus MAOIs, Outcome 14 Number of patients experiencing at least one adverse effect.

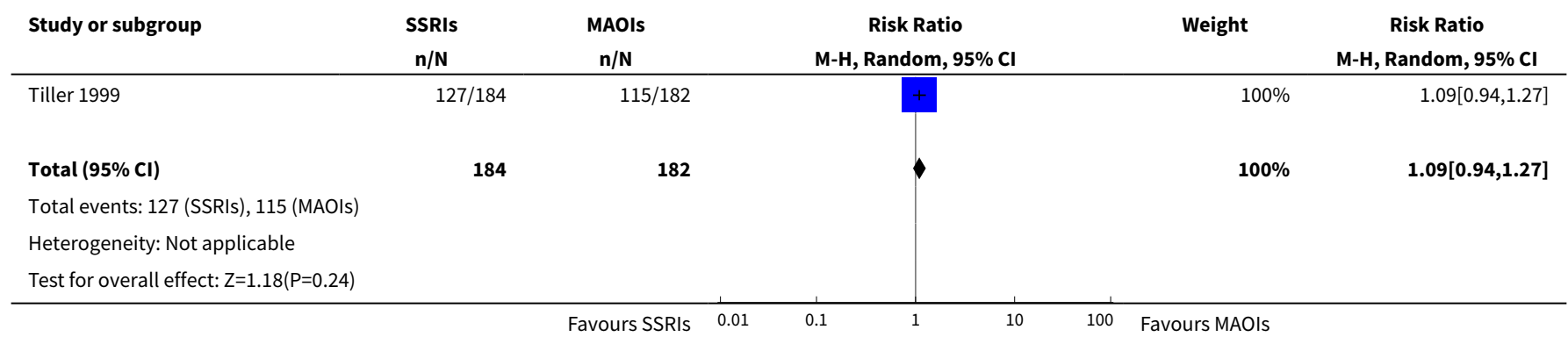

\section{Comparison 7. SSRIs versus SNRIs}

\begin{tabular}{|c|c|c|c|c|}
\hline $\begin{array}{l}\text { Outcome or subgroup ti- } \\
\text { tle }\end{array}$ & No. of studies & $\begin{array}{l}\text { No. of partici- } \\
\text { pants }\end{array}$ & Statistical method & Effect size \\
\hline 1 Failure to respond & 2 & 991 & Risk Ratio (M-H, Random, 95\% Cl) & $0.96[0.75,1.23]$ \\
\hline $\begin{array}{l}2 \text { Total number of } \\
\text { dropouts }\end{array}$ & 2 & 991 & Risk Ratio (M-H, Random, 95\% Cl) & $1.11[0.74,1.65]$ \\
\hline 3 Failure to remit & 2 & 991 & Risk Ratio (M-H, Random, 95\% Cl) & $1.05[0.91,1.21]$ \\
\hline 4 Panic symptoms & 2 & 945 & Mean Difference (IV, Random, 95\% CI) & $-0.12[-0.33,0.10]$ \\
\hline $\begin{array}{l}5 \text { Frequency of panic at- } \\
\text { tacks }\end{array}$ & 0 & 0 & $\begin{array}{l}\text { Std. Mean Difference (IV, Random, 95\% } \\
\mathrm{CI} \text { ) }\end{array}$ & $0.0[0.0,0.0]$ \\
\hline 6 Agoraphobia & 1 & 478 & $\begin{array}{l}\text { Std. Mean Difference (IV, Random, 95\% } \\
\mathrm{Cl} \text { ) }\end{array}$ & $0.00[-0.19,0.19]$ \\
\hline 7 General anxiety & 1 & 478 & Mean Difference (IV, Random, 95\% CI) & $-0.25[-1.83,1.33]$ \\
\hline 8 Depression & 0 & 0 & $\begin{array}{l}\text { Std. Mean Difference (IV, Random, 95\% } \\
\mathrm{CI} \text { ) }\end{array}$ & $0.0[0.0,0.0]$ \\
\hline 9 Social functioning & 1 & 478 & Mean Difference (IV, Random, 95\% CI) & $0.15[-0.71,1.01]$ \\
\hline
\end{tabular}




\begin{tabular}{lllll}
\hline $\begin{array}{l}\text { Outcome or subgroup ti- } \\
\text { tle }\end{array}$ & No. of studies & $\begin{array}{l}\text { No. of partici- } \\
\text { pants }\end{array}$ & Statistical method & Effect size \\
\hline 10 Quality of life & 1 & 478 & Mean Difference (IV, Random, 95\% Cl) & 0.06 [-2.11, 2.23] \\
\hline 11 Patient satisfaction & 0 & 0 & $\begin{array}{l}\text { Std. Mean Difference (IV, Random, 95\% } \\
\text { Cl) }\end{array}$ & 0.0 [0.0, 0.0] \\
\hline 12 Economic costs & 0 & 0 & $\begin{array}{l}\text { Std. Mean Difference (IV, Random, 95\% } \\
\text { Cl) }\end{array}$ & 0.0 [0.0, 0.0] \\
\hline $\begin{array}{l}13 \text { Number of dropouts } \\
\text { due to adverse effects }\end{array}$ & 2 & 991 & Risk Ratio (M-H, Random, 95\% Cl) & $1.86[0.49,7.05]$ \\
\hline $\begin{array}{l}14 \text { Number of patients ex- } \\
\text { periencing at least one ad- } \\
\text { verse effect }\end{array}$ & 2 & 991 & Risk Ratio (M-H, Random, 95\% Cl) & $0.97[0.88,1.08]$ \\
\hline
\end{tabular}

Analysis 7.1. Comparison 7 SSRIs versus SNRIs, Outcome 1 Failure to respond.

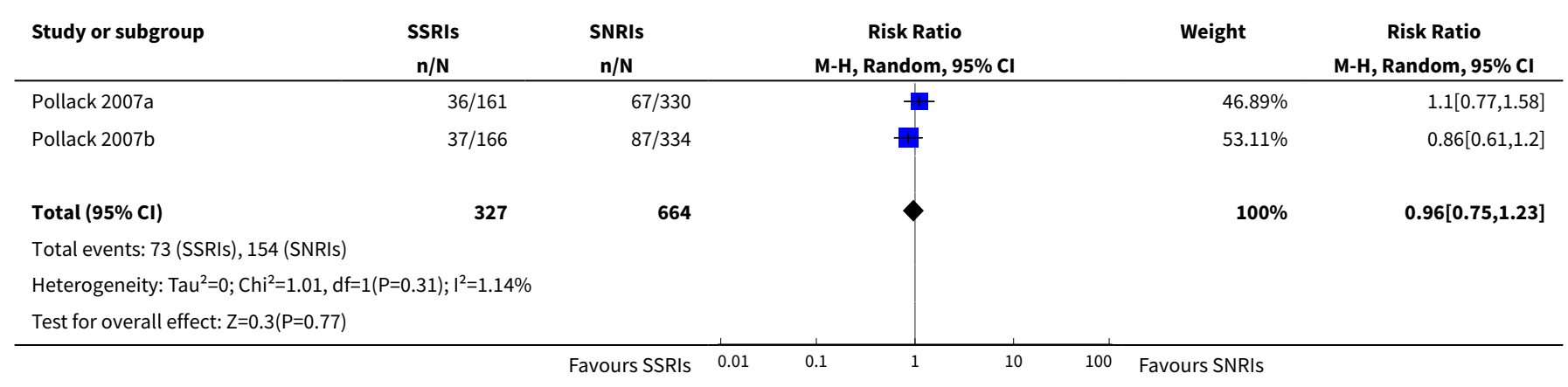

Analysis 7.2. Comparison 7 SSRIs versus SNRIs, Outcome 2 Total number of dropouts.

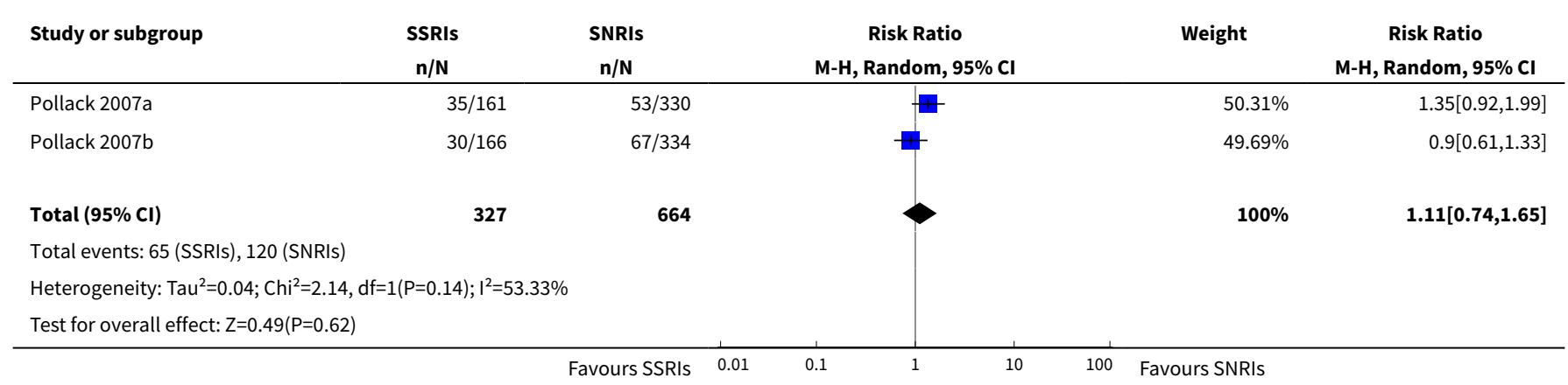


Analysis 7.3. Comparison 7 SSRIs versus SNRIs, Outcome 3 Failure to remit.

\begin{tabular}{|c|c|c|c|c|c|}
\hline Study or subgroup & $\begin{array}{c}\text { SSRIs } \\
n / N\end{array}$ & $\begin{array}{c}\text { SNRIS } \\
\mathrm{n} / \mathrm{N}\end{array}$ & $\begin{array}{c}\text { Risk Ratio } \\
\text { M-H, Random, } 95 \% \mathrm{CI}\end{array}$ & Weight & $\begin{array}{c}\text { Risk Ratio } \\
\text { M-H, Random, } 95 \% \mathrm{CI}\end{array}$ \\
\hline Pollack 2007a & $102 / 161$ & $186 / 330$ & - & $51.49 \%$ & $1.12[0.97,1.31]$ \\
\hline Pollack 2007b & $95 / 166$ & $197 / 334$ & & $48.51 \%$ & $0.97[0.83,1.14]$ \\
\hline Total $(95 \% \mathrm{Cl})$ & 327 & 664 & & $100 \%$ & $1.05[0.91,1.21]$ \\
\hline \multicolumn{6}{|c|}{ Total events: 197 (SSRIs), 383 (SNRIs) } \\
\hline \multicolumn{6}{|c|}{ Test for overall effect: $Z=0.62(P=0.54)$} \\
\hline
\end{tabular}

Analysis 7.4. Comparison 7 SSRIs versus SNRIs, Outcome 4 Panic symptoms.

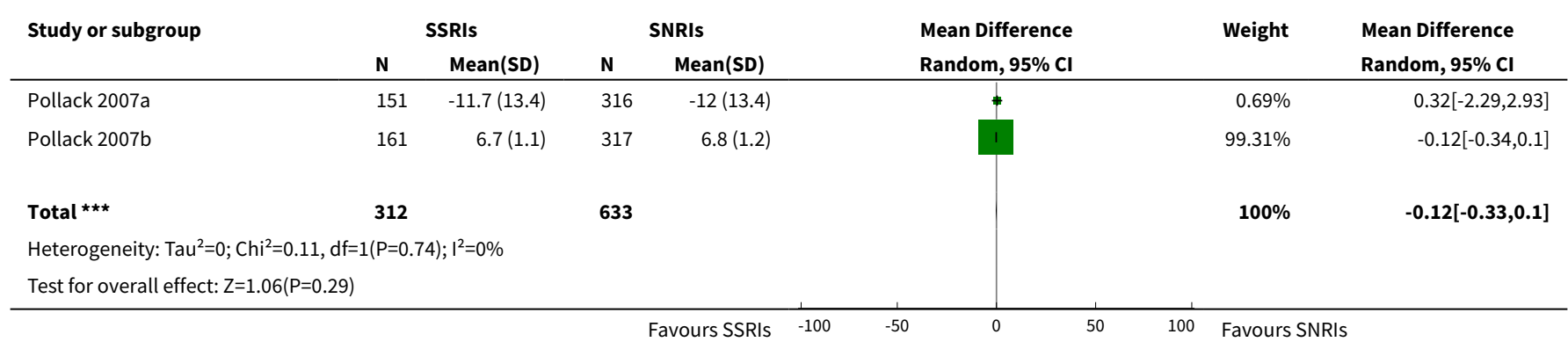

Analysis 7.6. Comparison 7 SSRIs versus SNRIs, Outcome 6 Agoraphobia.

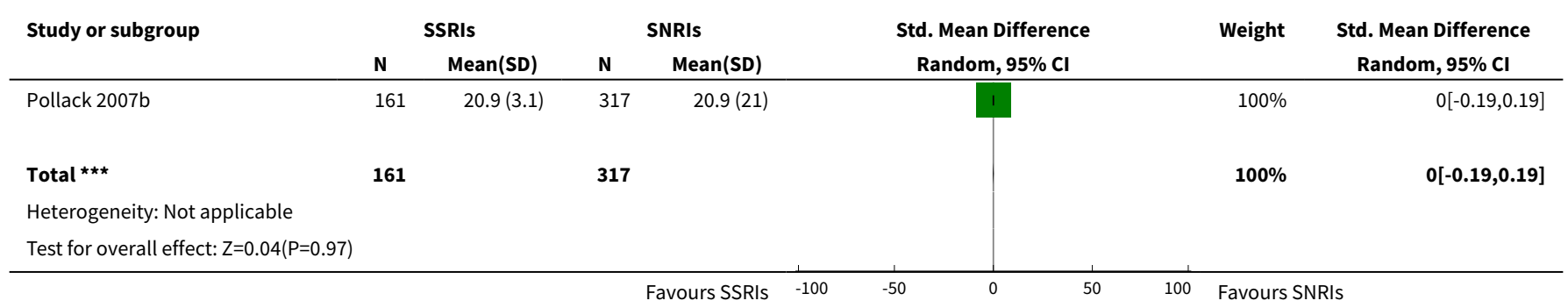

Analysis 7.7. Comparison 7 SSRIs versus SNRIs, Outcome 7 General anxiety.

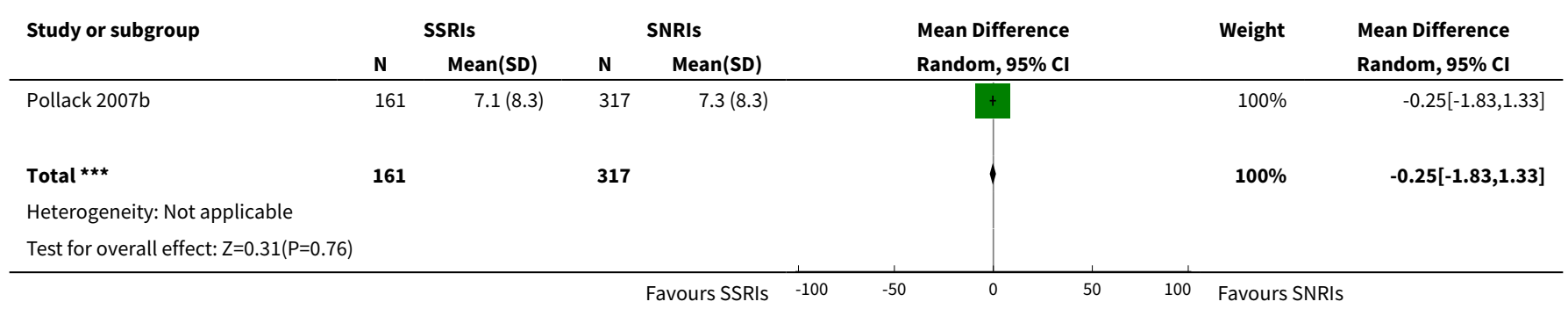


Analysis 7.9. Comparison 7 SSRIs versus SNRIs, Outcome 9 Social functioning.

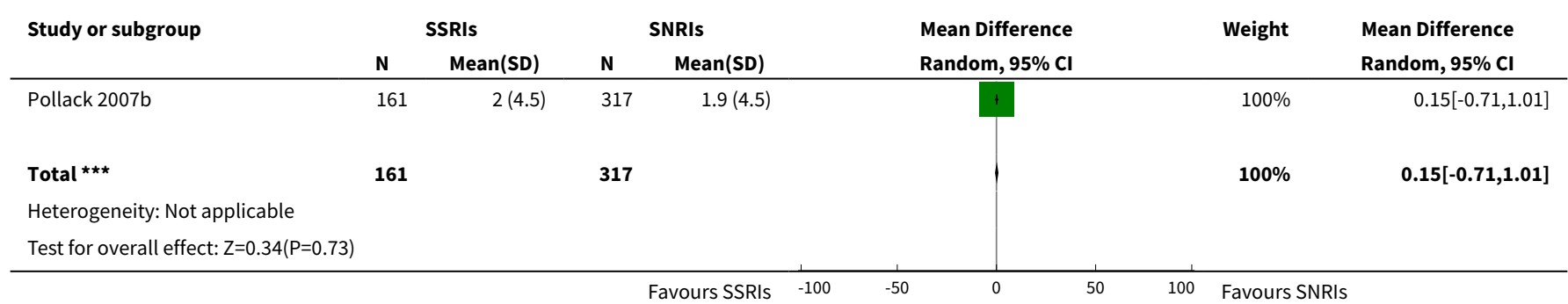

Analysis 7.10. Comparison 7 SSRIs versus SNRIs, Outcome 10 Quality of life.

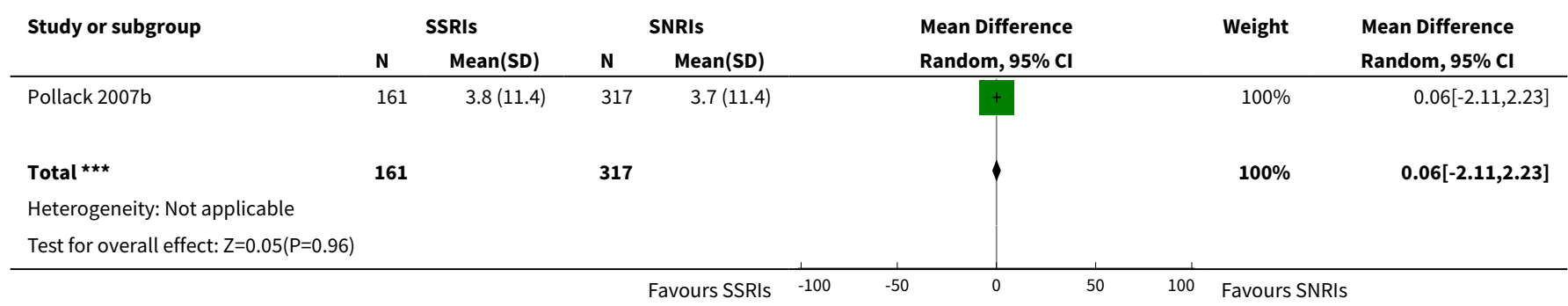

Analysis 7.13. Comparison 7 SSRIs versus SNRIs, Outcome 13 Number of dropouts due to adverse effects.

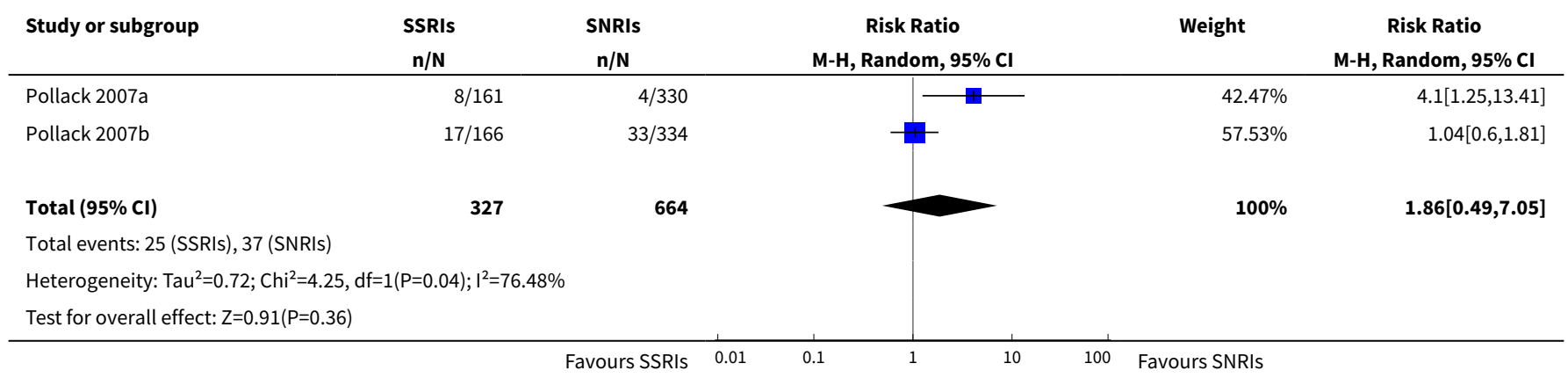

Analysis 7.14. Comparison 7 SSRIs versus SNRIs, Outcome 14 Number of patients experiencing at least one adverse effect.

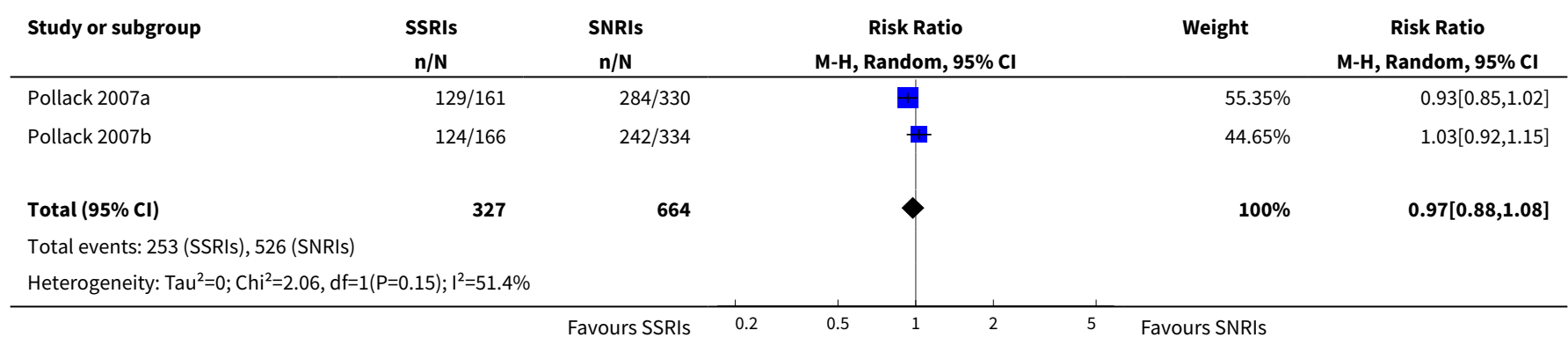




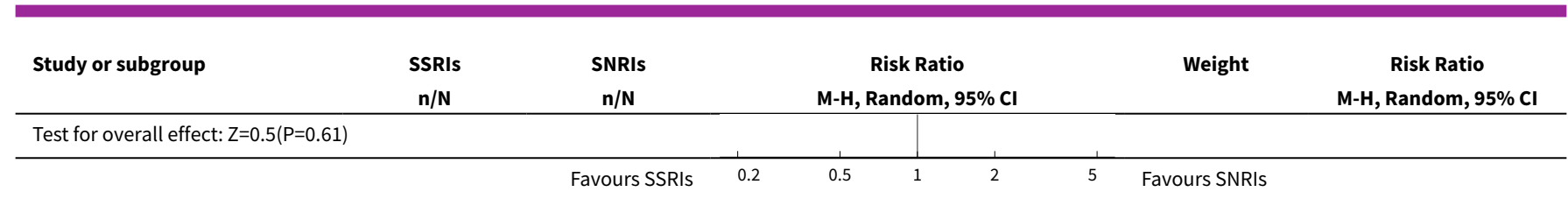

\section{Comparison 8. SSRIs versus NaSSAs}

\begin{tabular}{|c|c|c|c|c|}
\hline $\begin{array}{l}\text { Outcome or subgroup ti- } \\
\text { tle }\end{array}$ & No. of studies & $\begin{array}{l}\text { No. of partici- } \\
\text { pants }\end{array}$ & Statistical method & Effect size \\
\hline 1 Failure to respond & 0 & 0 & Risk Ratio (M-H, Random, 95\% Cl) & $0.0[0.0,0.0]$ \\
\hline $\begin{array}{l}2 \text { Total number of } \\
\text { dropouts }\end{array}$ & 1 & 30 & Risk Ratio (M-H, Random, 95\% Cl) & $1.5[0.29,7.73]$ \\
\hline 3 Failure to remit & 0 & 0 & Risk Ratio (M-H, Random, 95\% Cl) & $0.0[0.0,0.0]$ \\
\hline 4 Panic symptoms & 1 & 22 & Mean Difference (IV, Random, 95\% Cl) & $0.30[-0.49,1.09]$ \\
\hline $\begin{array}{l}5 \text { Frequency of panic at- } \\
\text { tacks }\end{array}$ & 0 & 0 & $\begin{array}{l}\text { Std. Mean Difference (IV, Random, 95\% } \\
\mathrm{CI})\end{array}$ & $0.0[0.0,0.0]$ \\
\hline 6 Agoraphobia & 0 & 0 & $\begin{array}{l}\text { Std. Mean Difference (IV, Random, 95\% } \\
\mathrm{CI})\end{array}$ & $0.0[0.0,0.0]$ \\
\hline 7 General anxiety & 1 & 27 & Mean Difference (IV, Random, 95\% Cl) & $1.10[-6.04,8.24]$ \\
\hline 8 Depression & 0 & 0 & $\begin{array}{l}\text { Std. Mean Difference (IV, Random, 95\% } \\
\mathrm{CI})\end{array}$ & $0.0[0.0,0.0]$ \\
\hline 9 Social functioning & 0 & 0 & $\begin{array}{l}\text { Std. Mean Difference (IV, Random, 95\% } \\
\mathrm{CI} \text { ) }\end{array}$ & $0.0[0.0,0.0]$ \\
\hline 10 Quality of life & 0 & 0 & $\begin{array}{l}\text { Std. Mean Difference (IV, Random, 95\% } \\
\mathrm{CI})\end{array}$ & $0.0[0.0,0.0]$ \\
\hline 11 Patient satisfaction & 0 & 0 & $\begin{array}{l}\text { Std. Mean Difference (IV, Random, 95\% } \\
\mathrm{CI})\end{array}$ & $0.0[0.0,0.0]$ \\
\hline 12 Economic costs & 0 & 0 & $\begin{array}{l}\text { Std. Mean Difference (IV, Random, 95\% } \\
\mathrm{CI} \text { ) }\end{array}$ & $0.0[0.0,0.0]$ \\
\hline $\begin{array}{l}13 \text { Number of dropouts } \\
\text { due to adverse effects }\end{array}$ & 1 & 30 & Risk Ratio (M-H, Random, 95\% Cl) & $1.5[0.29,7.73]$ \\
\hline $\begin{array}{l}14 \text { Number of patients ex- } \\
\text { periencing at least one ad- } \\
\text { verse effect }\end{array}$ & 0 & 0 & Risk Ratio (M-H, Random, 95\% Cl) & $0.0[0.0,0.0]$ \\
\hline
\end{tabular}


Analysis 8.2. Comparison 8 SSRIs versus NaSSAs, Outcome 2 Total number of dropouts.

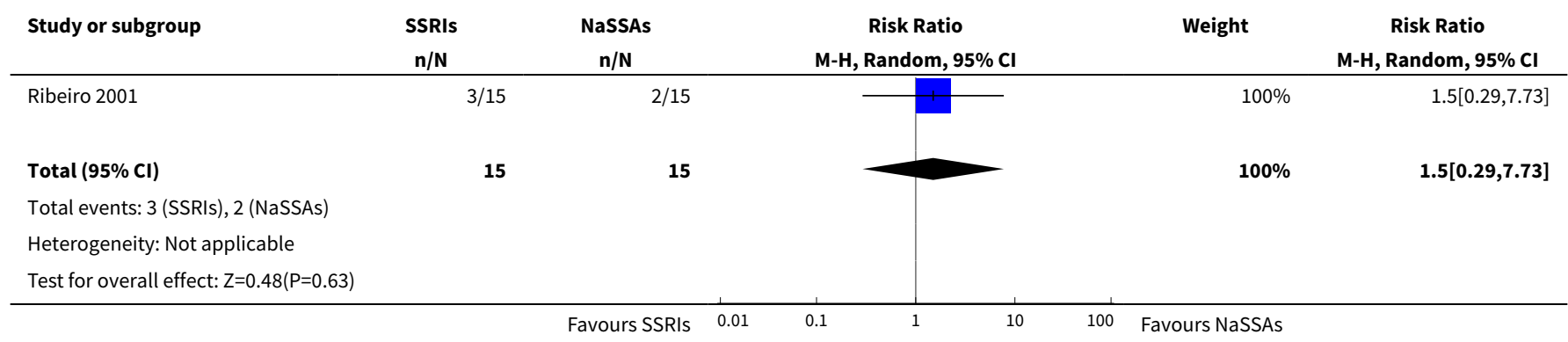

Analysis 8.4. Comparison 8 SSRIs versus NaSSAs, Outcome 4 Panic symptoms.

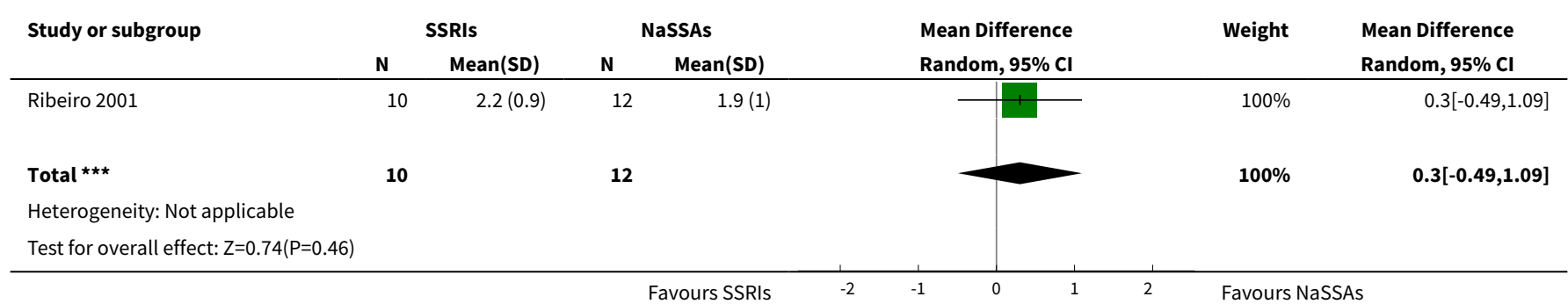

Analysis 8.7. Comparison 8 SSRIs versus NaSSAs, Outcome 7 General anxiety.

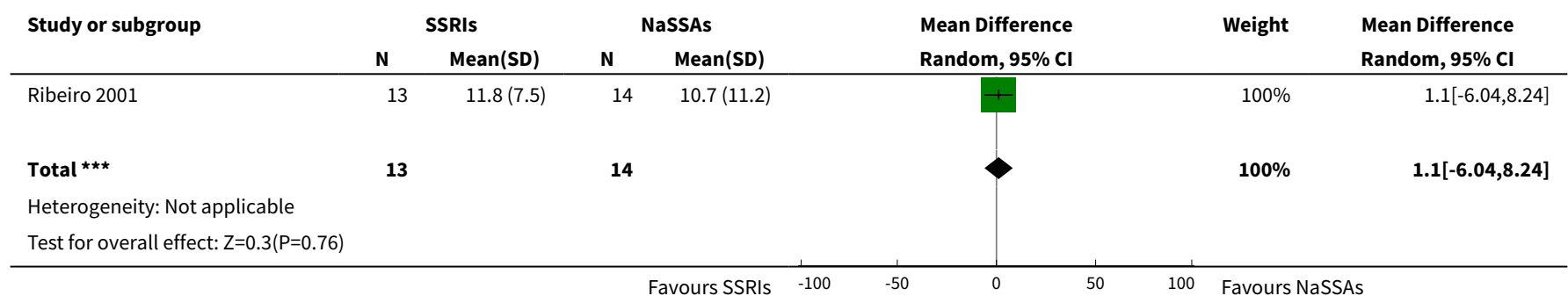

Analysis 8.13. Comparison 8 SSRIs versus NaSSAs, Outcome 13 Number of dropouts due to adverse effects.

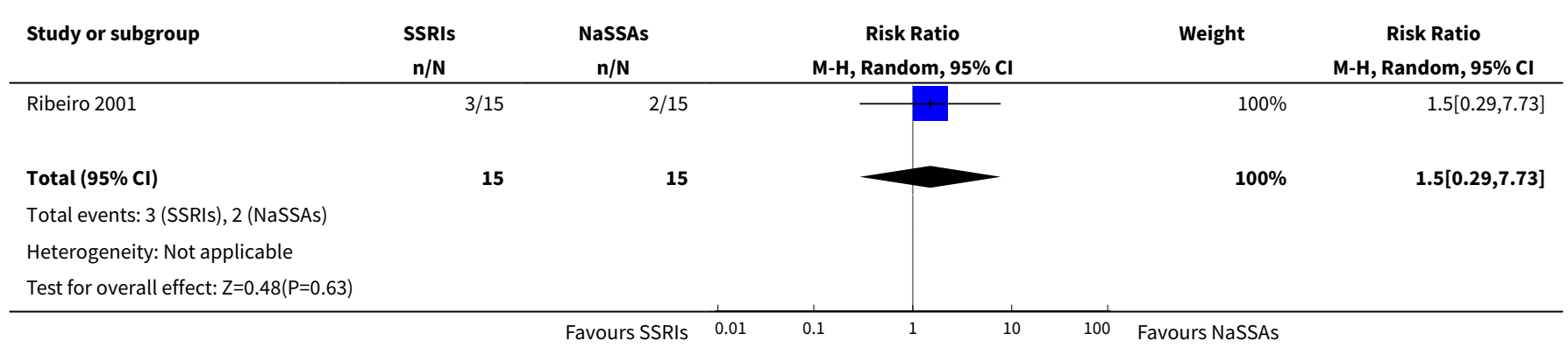


Comparison 9. SSRIs versus Other Antidepressants

\begin{tabular}{|c|c|c|c|c|}
\hline $\begin{array}{l}\text { Outcome or subgroup ti- } \\
\text { tle }\end{array}$ & No. of studies & $\begin{array}{l}\text { No. of partici- } \\
\text { pants }\end{array}$ & Statistical method & Effect size \\
\hline 1 Failure to respond & 1 & 40 & Risk Ratio (M-H, Random, 95\% Cl) & $0.28[0.13,0.60]$ \\
\hline $\begin{array}{l}2 \text { Total number of } \\
\text { dropouts }\end{array}$ & 1 & 40 & Risk Ratio (M-H, Random, 95\% Cl) & $0.0[0.0,0.0]$ \\
\hline 3 Failure to remit & 0 & 0 & Risk Ratio (M-H, Random, 95\% Cl) & $0.0[0.0,0.0]$ \\
\hline 4 Panic symptoms & 0 & 0 & $\begin{array}{l}\text { Std. Mean Difference (IV, Random, 95\% } \\
\mathrm{Cl} \text { ) }\end{array}$ & $0.0[0.0,0.0]$ \\
\hline $\begin{array}{l}5 \text { Frequency of panic at- } \\
\text { tacks }\end{array}$ & 0 & 0 & $\begin{array}{l}\text { Std. Mean Difference (IV, Random, 95\% } \\
\mathrm{CI})\end{array}$ & $0.0[0.0,0.0]$ \\
\hline 6 Agoraphobia & 1 & 40 & Mean Difference (IV, Random, 95\% Cl) & $\begin{array}{l}-7.35[-13.73 \\
-0.97]\end{array}$ \\
\hline 7 General anxiety & 1 & 40 & Mean Difference (IV, Random, 95\% Cl) & $-3.55[-5.07,-2.03]$ \\
\hline 8 Depression & 1 & 40 & Mean Difference (IV, Random, 95\% CI) & $-5.38[-7.90,-2.86]$ \\
\hline 9 Social functioning & 0 & 0 & $\begin{array}{l}\text { Std. Mean Difference (IV, Random, 95\% } \\
\mathrm{CI})\end{array}$ & $0.0[0.0,0.0]$ \\
\hline 10 Quality of life & 0 & 0 & $\begin{array}{l}\text { Std. Mean Difference (IV, Random, 95\% } \\
\mathrm{CI})\end{array}$ & $0.0[0.0,0.0]$ \\
\hline 11 Patient satisfaction & 0 & 0 & $\begin{array}{l}\text { Std. Mean Difference (IV, Random, 95\% } \\
\mathrm{CI})\end{array}$ & $0.0[0.0,0.0]$ \\
\hline 12 Economic costs & 0 & 0 & $\begin{array}{l}\text { Std. Mean Difference (IV, Random, 95\% } \\
\mathrm{CI})\end{array}$ & $0.0[0.0,0.0]$ \\
\hline $\begin{array}{l}13 \text { Number of dropouts } \\
\text { due to adverse effects }\end{array}$ & 1 & 40 & Risk Ratio (M-H, Random, 95\% Cl) & $0.0[0.0,0.0]$ \\
\hline $\begin{array}{l}14 \text { Number of patients ex- } \\
\text { periencing at least one ad- } \\
\text { verse effect }\end{array}$ & 0 & 0 & Risk Ratio (M-H, Random, 95\% Cl) & $0.0[0.0,0.0]$ \\
\hline
\end{tabular}

Analysis 9.1. Comparison 9 SSRIs versus Other Antidepressants, Outcome 1 Failure to respond.

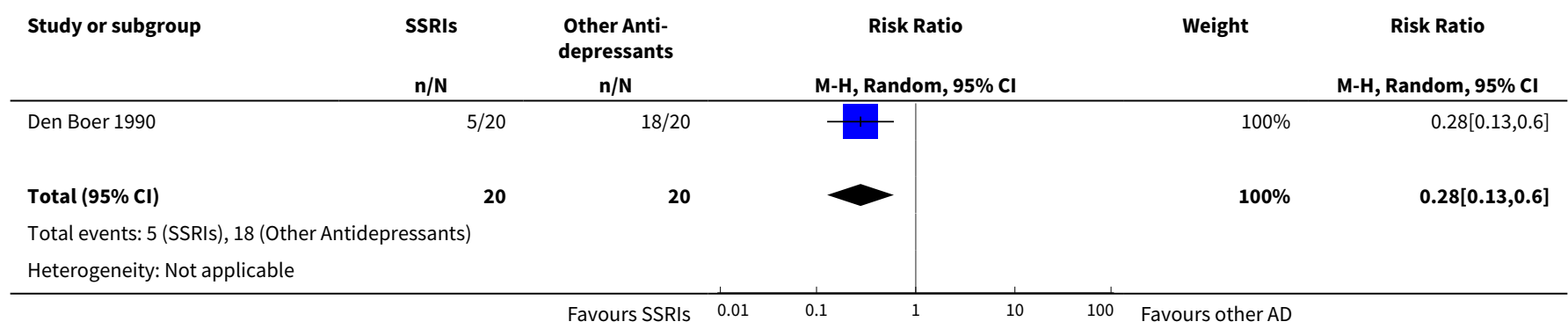




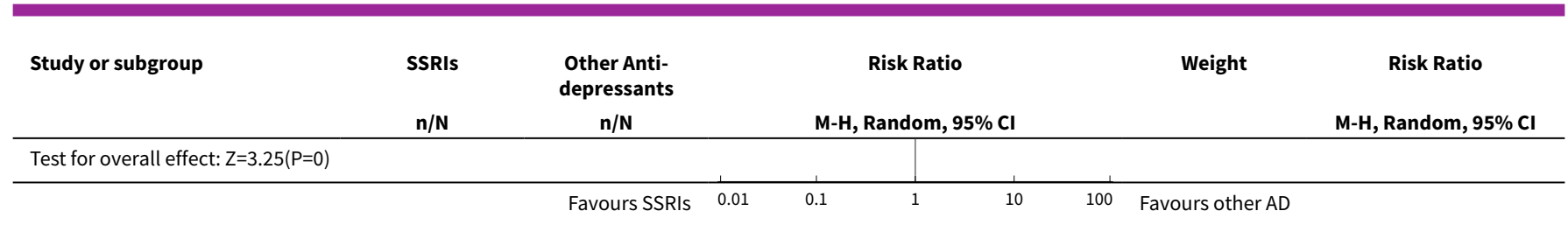

Analysis 9.2. Comparison 9 SSRIs versus Other Antidepressants, Outcome 2 Total number of dropouts.

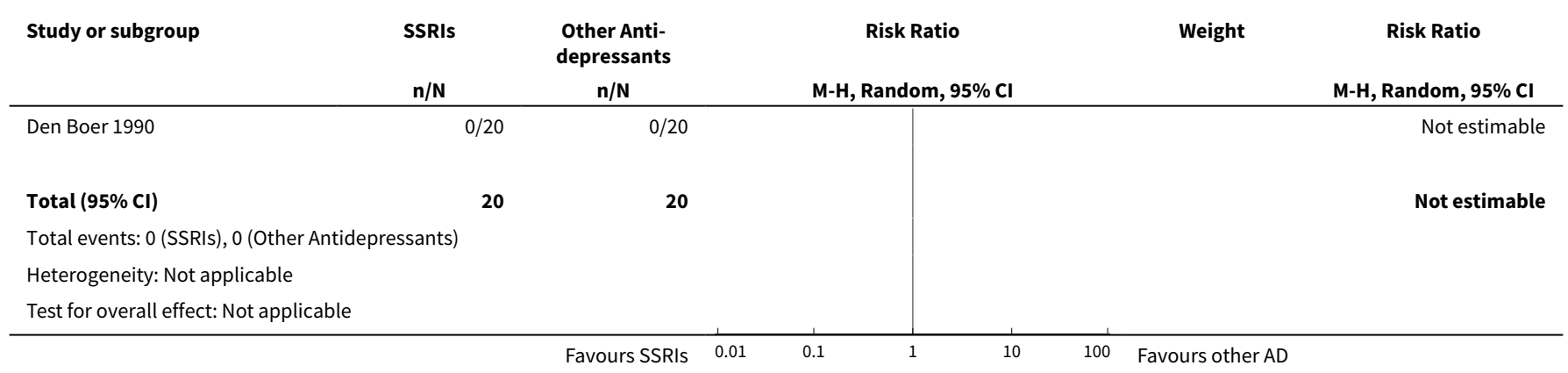

Analysis 9.6. Comparison 9 SSRIs versus Other Antidepressants, Outcome 6 Agoraphobia.

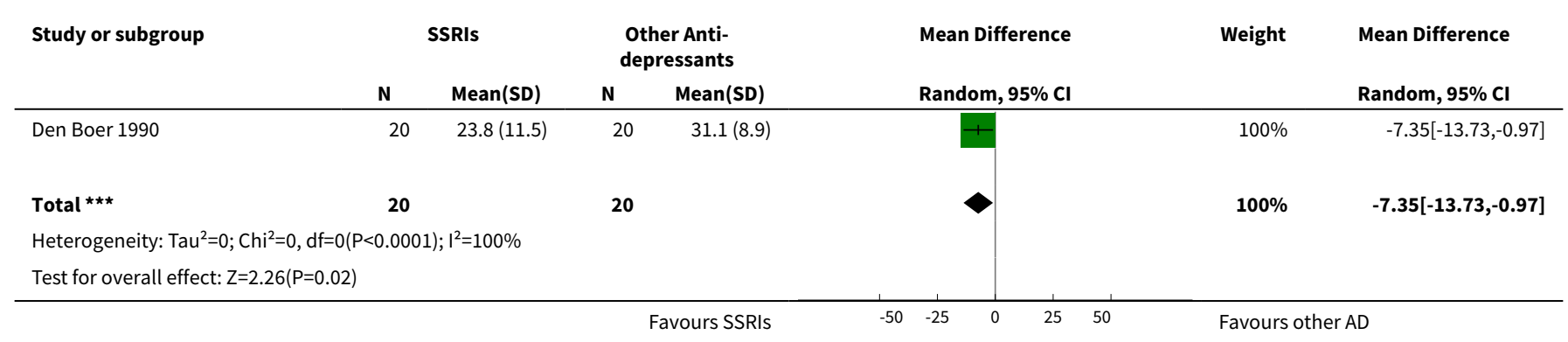

Analysis 9.7. Comparison 9 SSRIs versus Other Antidepressants, Outcome 7 General anxiety.

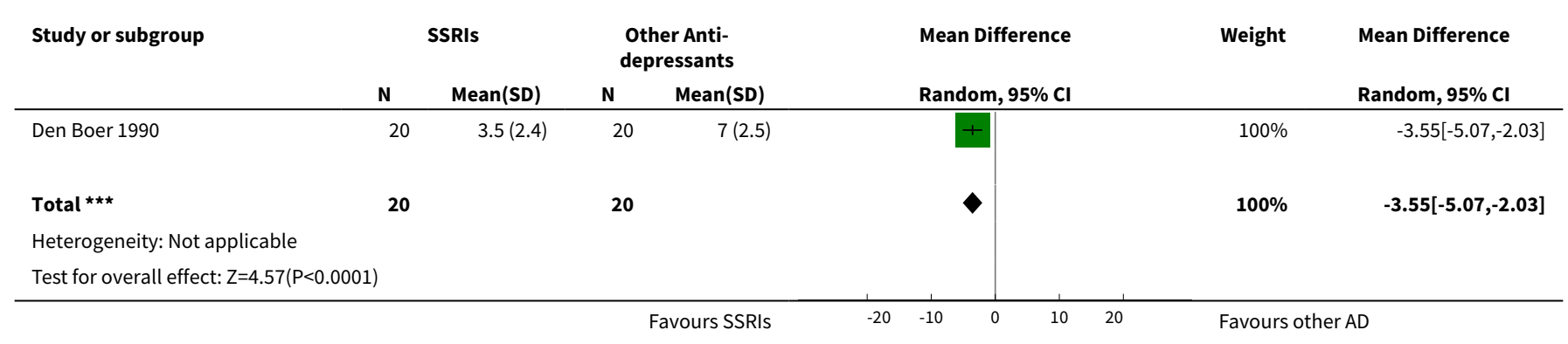


Analysis 9.8. Comparison 9 SSRIs versus Other Antidepressants, Outcome 8 Depression.

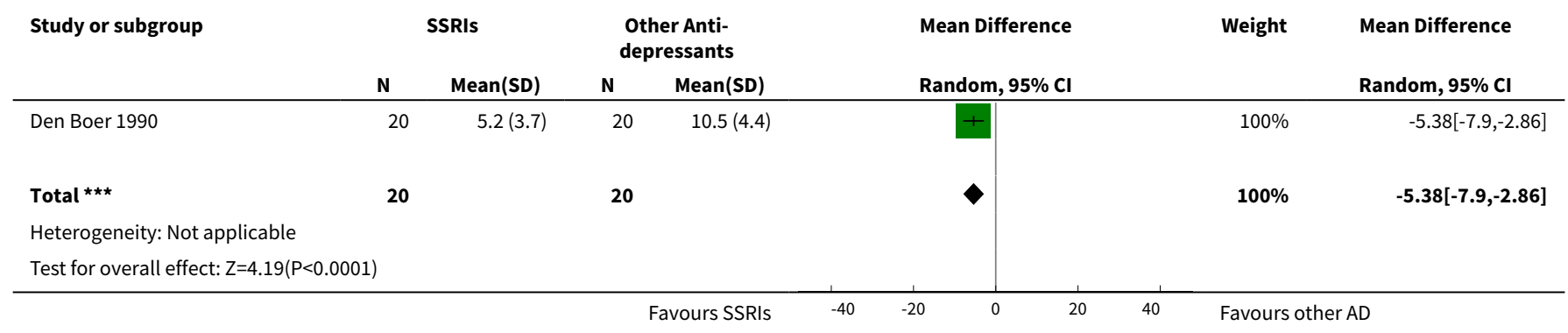

Analysis 9.13. Comparison 9 SSRIs versus Other Antidepressants, Outcome 13 Number of dropouts due to adverse effects.

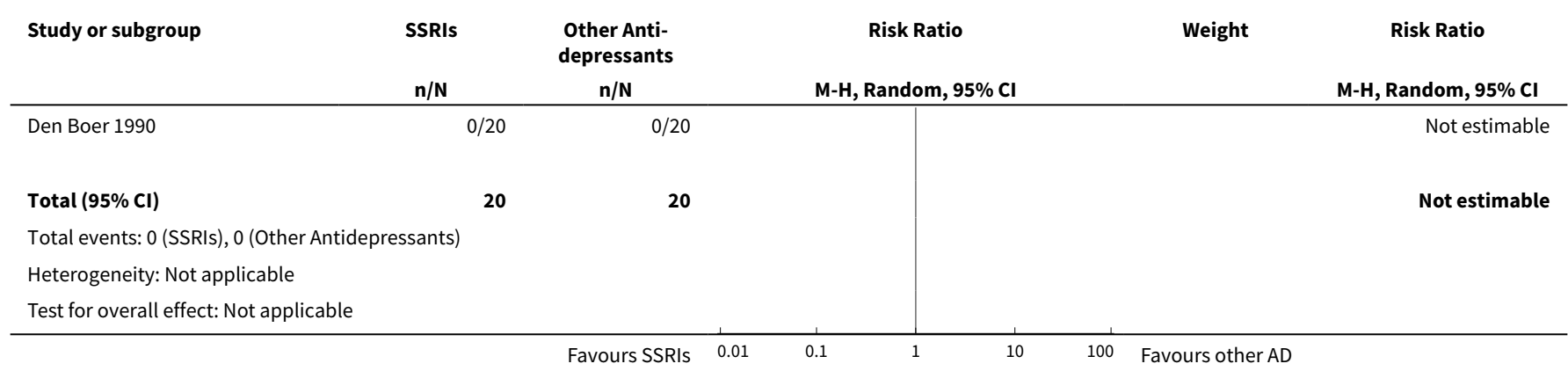

Comparison 10. Individual Antidepressants versus another antidepressant within the same class

\begin{tabular}{|c|c|c|c|c|}
\hline Outcome or subgroup title & No. of studies & $\begin{array}{l}\text { No. of partici- } \\
\text { pants }\end{array}$ & Statistical method & Effect size \\
\hline 1 Failure to respond & 4 & & Risk Ratio (M-H, Random, 95\% Cl) & Subtotals only \\
\hline $\begin{array}{l}\text { 1.1 Escitalopram versus citalo- } \\
\text { pram }\end{array}$ & 1 & 255 & Risk Ratio (M-H, Random, 95\% Cl) & $0.92[0.79,1.06]$ \\
\hline $\begin{array}{l}\text { 1.2 Fluoxetine versus Citalo- } \\
\text { pram }\end{array}$ & 1 & 42 & Risk Ratio (M-H, Random, 95\% Cl) & $0.57[0.20,1.66]$ \\
\hline $\begin{array}{l}1.3 \text { Sertraline versus paroxe- } \\
\text { tine }\end{array}$ & 2 & 546 & Risk Ratio (M-H, Random, 95\% Cl) & $0.97[0.80,1.17]$ \\
\hline 2 Total number of dropouts & 5 & & Risk Ratio (M-H, Random, 95\% Cl) & Subtotals only \\
\hline $\begin{array}{l}\text { 2.1 Escitalopram versus Citalo- } \\
\text { pram }\end{array}$ & 1 & 255 & Risk Ratio (M-H, Random, 95\% Cl) & $0.80[0.53,1.20]$ \\
\hline $\begin{array}{l}\text { 2.2 Fluoxetine versus Citalo- } \\
\text { pram }\end{array}$ & 1 & 42 & Risk Ratio (M-H, Random, 95\% Cl) & $1.0[0.07,14.95]$ \\
\hline $\begin{array}{l}\text { 2.3 Sertraline versus Paroxe- } \\
\text { tine }\end{array}$ & 2 & 546 & Risk Ratio (M-H, Random, 95\% Cl) & $0.74[0.55,0.99]$ \\
\hline
\end{tabular}




\begin{tabular}{|c|c|c|c|c|}
\hline Outcome or subgroup title & No. of studies & $\begin{array}{l}\text { No. of partici- } \\
\text { pants }\end{array}$ & Statistical method & Effect size \\
\hline $\begin{array}{l}2.4 \text { Imipramine versus } \\
\text { Clomipramine }\end{array}$ & 1 & 40 & Risk Ratio (M-H, Random, 95\% Cl) & $0.86[0.35,2.10]$ \\
\hline 3 Failure to remit & 1 & 255 & Risk Ratio (M-H, Random, 95\% Cl) & $0.90[0.71,1.15]$ \\
\hline $\begin{array}{l}\text { 3.1 Escitalopram versus Citalo- } \\
\text { pram }\end{array}$ & 1 & 255 & Risk Ratio (M-H, Random, 95\% Cl) & $0.90[0.71,1.15]$ \\
\hline 4 Panic symptoms & 3 & & $\begin{array}{l}\text { Std. Mean Difference (IV, Random, } \\
95 \% \mathrm{Cl} \text { ) }\end{array}$ & Subtotals only \\
\hline $\begin{array}{l}\text { 4.1 Escitalopram versus Citalo- } \\
\text { pram }\end{array}$ & 1 & 237 & $\begin{array}{l}\text { Std. Mean Difference (IV, Random, } \\
95 \% \mathrm{Cl} \text { ) }\end{array}$ & $-0.09[-0.35,0.16]$ \\
\hline $\begin{array}{l}\text { 4.2 Sertraline versus Paroxe- } \\
\text { tine }\end{array}$ & 2 & 473 & $\begin{array}{l}\text { Std. Mean Difference (IV, Random, } \\
95 \% \mathrm{CI} \text { ) }\end{array}$ & $-0.05[-0.23,0.13]$ \\
\hline 5 Frequency of panic attacks & 2 & & $\begin{array}{l}\text { Mean Difference (IV, Random, 95\% } \\
\mathrm{CI})\end{array}$ & Subtotals only \\
\hline $\begin{array}{l}5.1 \text { Sertraline versus Paroxe- } \\
\text { tine }\end{array}$ & 2 & 461 & $\begin{array}{l}\text { Mean Difference (IV, Random, 95\% } \\
\text { CI) }\end{array}$ & $0.48[-1.02,1.98]$ \\
\hline 6 Agoraphobia & 1 & & $\begin{array}{l}\text { Mean Difference (IV, Random, 95\% } \\
\text { Cl) }\end{array}$ & Subtotals only \\
\hline $\begin{array}{l}\text { 6.1 Escitalopram versus Citalo- } \\
\text { pram }\end{array}$ & 1 & 237 & $\begin{array}{l}\text { Mean Difference (IV, Random, 95\% } \\
\mathrm{CI} \text { ) }\end{array}$ & $-1.10[-3.46,1.26]$ \\
\hline 7 General anxiety & 3 & & $\begin{array}{l}\text { Mean Difference (IV, Random, 95\% } \\
\mathrm{CI})\end{array}$ & Subtotals only \\
\hline $\begin{array}{l}\text { 7.1 Escitalopram versus Citalo- } \\
\text { pram }\end{array}$ & 1 & 237 & $\begin{array}{l}\text { Mean Difference (IV, Random, 95\% } \\
\mathrm{CI})\end{array}$ & $-1.20[-3.14,0.74]$ \\
\hline $\begin{array}{l}\text { 7.2 Sertraline versus Paroxe- } \\
\text { tine }\end{array}$ & 2 & 454 & $\begin{array}{l}\text { Mean Difference (IV, Random, 95\% } \\
\mathrm{CI})\end{array}$ & $-0.72[-2.42,0.99]$ \\
\hline 8 Depression & 2 & & $\begin{array}{l}\text { Std. Mean Difference (IV, Random, } \\
95 \% \mathrm{CI})\end{array}$ & Subtotals only \\
\hline $\begin{array}{l}\text { 8.1 Escitalopram versus Citalo- } \\
\text { pram }\end{array}$ & 1 & 237 & $\begin{array}{l}\text { Std. Mean Difference (IV, Random, } \\
95 \% \mathrm{CI})\end{array}$ & $-0.11[-0.36,0.15]$ \\
\hline $\begin{array}{l}\text { 8.2 Sertraline versus Paroxe- } \\
\text { tine }\end{array}$ & 1 & 189 & $\begin{array}{l}\text { Std. Mean Difference (IV, Random, } \\
95 \% \mathrm{CI})\end{array}$ & $0.00[-0.28,0.29]$ \\
\hline 9 Social functioning & 0 & 0 & $\begin{array}{l}\text { Std. Mean Difference (IV, Random, } \\
95 \% \mathrm{CI})\end{array}$ & $0.0[0.0,0.0]$ \\
\hline 10 Quality of life & 1 & & $\begin{array}{l}\text { Mean Difference (IV, Random, 95\% } \\
\mathrm{CI} \text { ) }\end{array}$ & Subtotals only \\
\hline $\begin{array}{l}10.1 \text { Escitalopram versus } \\
\text { Citalopram }\end{array}$ & 1 & 237 & $\begin{array}{l}\text { Mean Difference (IV, Random, 95\% } \\
\mathrm{CI} \text { ) }\end{array}$ & $1.40[-1.51,4.31]$ \\
\hline
\end{tabular}




\begin{tabular}{|c|c|c|c|c|}
\hline Outcome or subgroup title & No. of studies & $\begin{array}{l}\text { No. of partici- } \\
\text { pants }\end{array}$ & Statistical method & Effect size \\
\hline 11 Patient satisfaction & 0 & 0 & $\begin{array}{l}\text { Std. Mean Difference (IV, Random, } \\
95 \% \mathrm{Cl})\end{array}$ & $0.0[0.0,0.0]$ \\
\hline 12 Economic costs & 0 & 0 & $\begin{array}{l}\text { Std. Mean Difference (IV, Random, } \\
95 \% \mathrm{Cl})\end{array}$ & $0.0[0.0,0.0]$ \\
\hline $\begin{array}{l}13 \text { Number of dropouts due to } \\
\text { adverse effects }\end{array}$ & 5 & & Risk Ratio (M-H, Random, 95\% Cl) & Subtotals only \\
\hline $\begin{array}{l}\text { 13.1 Escitalopram versus } \\
\text { Citalopram }\end{array}$ & 1 & 255 & Risk Ratio (M-H, Random, 95\% Cl) & $0.78[0.32,1.92]$ \\
\hline $\begin{array}{l}\text { 13.2 Fluoxetine versus Citalo- } \\
\text { pram }\end{array}$ & 1 & 42 & Risk Ratio (M-H, Random, 95\% Cl) & $1.0[0.07,14.95]$ \\
\hline $\begin{array}{l}\text { 13.3 Sertraline versus Paroxe- } \\
\text { tine }\end{array}$ & 2 & 546 & Risk Ratio (M-H, Random, 95\% Cl) & $0.66[0.42,1.04]$ \\
\hline $\begin{array}{l}13.4 \text { Imipramine versus } \\
\text { Clomipramine }\end{array}$ & 1 & 40 & Risk Ratio (M-H, Random, 95\% Cl) & $0.33[0.08,1.46]$ \\
\hline $\begin{array}{l}14 \text { Number of patients experi- } \\
\text { encing at least one adverse ef- } \\
\text { fect }\end{array}$ & 2 & & Risk Ratio (M-H, Random, 95\% Cl) & Subtotals only \\
\hline $\begin{array}{l}\text { 14.1 Escitalopram versus } \\
\text { Citalopram }\end{array}$ & 1 & 255 & Risk Ratio (M-H, Random, 95\% Cl) & $0.95[0.85,1.06]$ \\
\hline $\begin{array}{l}14.2 \text { Sertraline versus Paroxe- } \\
\text { tine }\end{array}$ & 1 & 321 & Risk Ratio (M-H, Random, 95\% Cl) & $0.99[0.89,1.10]$ \\
\hline
\end{tabular}

\section{Analysis 10.1. Comparison 10 Individual Antidepressants versus another antidepressant within the same class, Outcome 1 Failure to respond.}

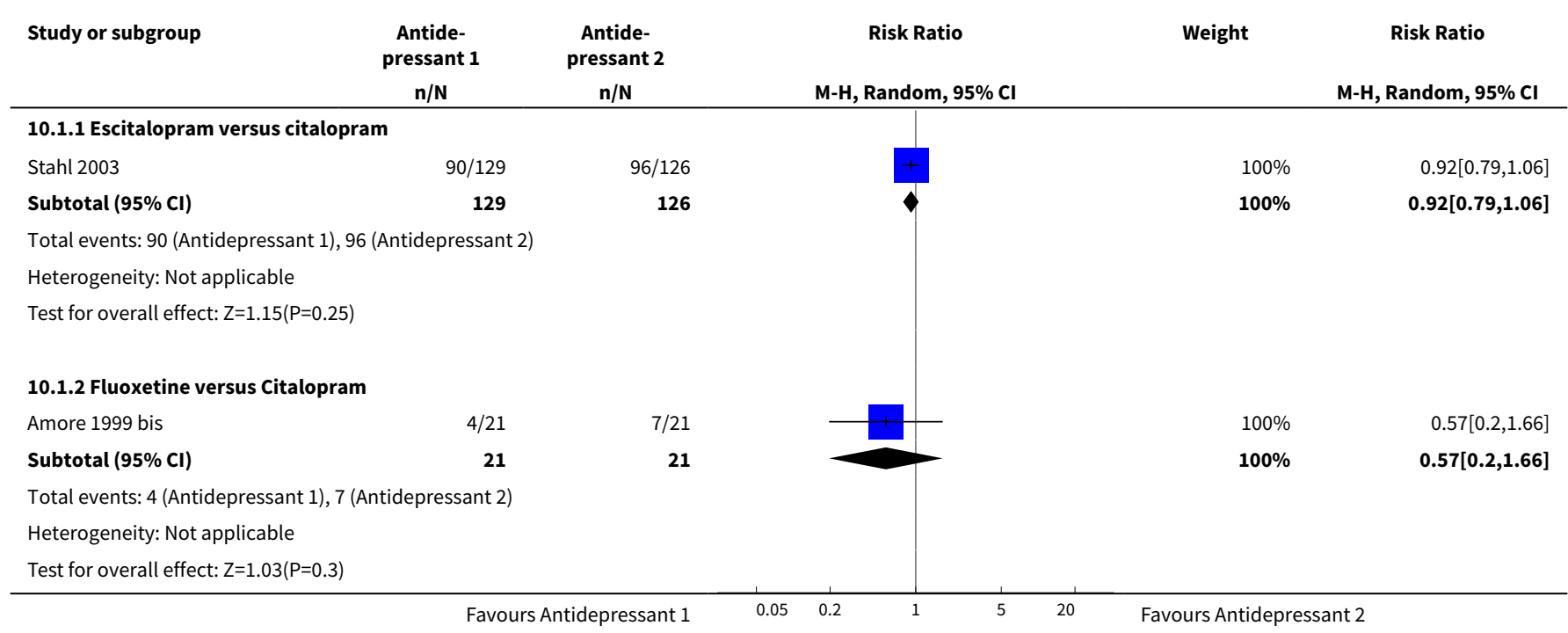




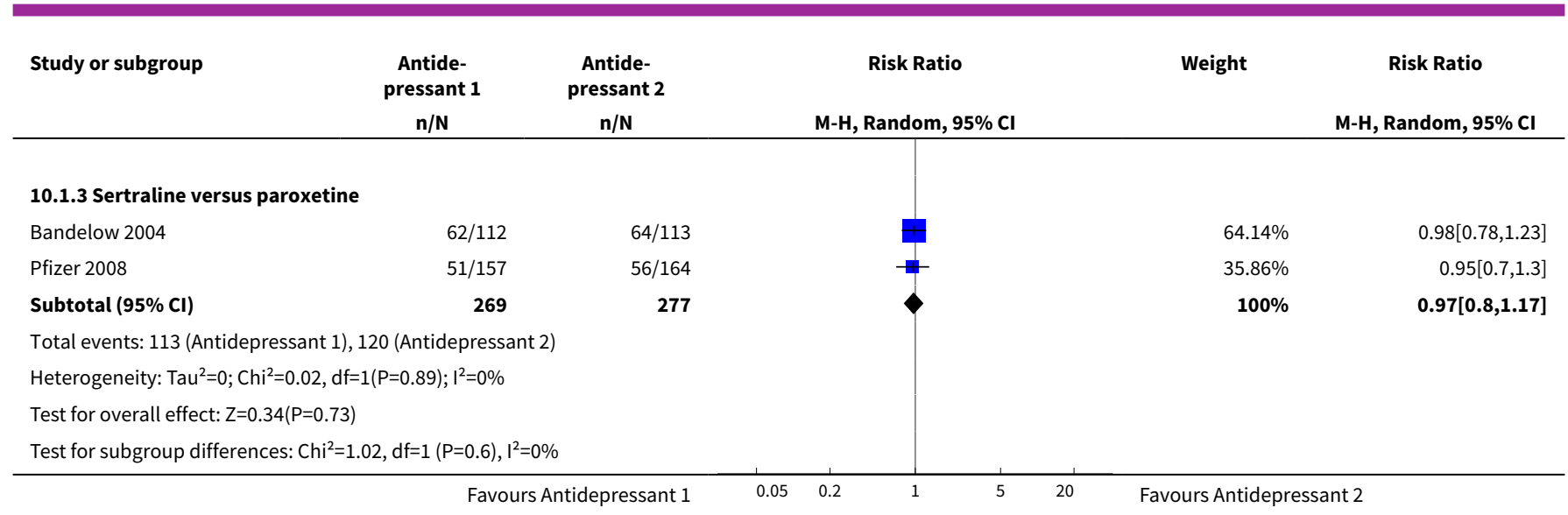

Analysis 10.2. Comparison 10 Individual Antidepressants versus another antidepressant within the same class, Outcome 2 Total number of dropouts.

\begin{tabular}{|c|c|c|c|c|c|}
\hline Study or subgroup & $\begin{array}{c}\text { Antide- } \\
\text { pressant } 1 \\
\mathrm{n} / \mathrm{N} \\
\end{array}$ & $\begin{array}{c}\text { Antide- } \\
\text { pressant } 2 \\
\mathrm{n} / \mathrm{N} \\
\end{array}$ & $\begin{array}{c}\text { Risk Ratio } \\
\text { M-H, Random, } 95 \% \mathrm{Cl}\end{array}$ & Weight & $\begin{array}{c}\text { Risk Ratio } \\
\text { M-H, Random, } 95 \% \mathrm{Cl}\end{array}$ \\
\hline \multicolumn{6}{|c|}{ 10.2.1 Escitalopram versus Citalopram } \\
\hline Stahl 2003 & $31 / 129$ & $38 / 126$ & & $100 \%$ & $0.8[0.53,1.2]$ \\
\hline Subtotal $(95 \% \mathrm{Cl})$ & 129 & 126 & & $100 \%$ & $0.8[0.53,1.2]$ \\
\hline \multicolumn{6}{|c|}{ Total events: 31 (Antidepressant 1), 38 (Antidepressant 2) } \\
\hline \multicolumn{6}{|c|}{ Test for overall effect: $Z=1.1(P=0.27)$} \\
\hline \multicolumn{6}{|c|}{ 10.2.2 Fluoxetine versus Citalopram } \\
\hline Amore 1999 bis & $1 / 21$ & $1 / 21$ & & $100 \%$ & $1[0.07,14.95]$ \\
\hline Subtotal $(95 \% \mathrm{Cl})$ & 21 & 21 & & $100 \%$ & $1[0.07,14.95]$ \\
\hline \multicolumn{6}{|c|}{ Total events: 1 (Antidepressant 1), 1 (Antidepressant 2) } \\
\hline \multicolumn{6}{|c|}{ Test for overall effect: Not applicable } \\
\hline \multicolumn{6}{|c|}{ 10.2.3 Sertraline versus Paroxetine } \\
\hline Bandelow 2004 & $31 / 112$ & $37 / 113$ & & $55.18 \%$ & $0.85[0.57,1.26]$ \\
\hline Pfizer 2008 & $25 / 157$ & $42 / 164$ & & $44.82 \%$ & $0.62[0.4,0.97]$ \\
\hline Subtotal $(95 \% \mathrm{Cl})$ & 269 & 277 & & $100 \%$ & $0.74[0.55,0.99]$ \\
\hline \multicolumn{6}{|c|}{ Total events: 56 (Antidepressant 1), 79 (Antidepressant 2) } \\
\hline \multicolumn{6}{|c|}{ Heterogeneity: $\mathrm{Tau}^{2}=0 ; \mathrm{Chi}^{2}=1.02, \mathrm{df}=1(\mathrm{P}=0.31) ; \mathrm{I}^{2}=2.26 \%$} \\
\hline \multicolumn{6}{|c|}{ Test for overall effect: $Z=1.99(P=0.05)$} \\
\hline \multicolumn{6}{|c|}{ 10.2.4 Imipramine versus Clomipramine } \\
\hline Gentil 1993 & $6 / 20$ & $7 / 20$ & & $100 \%$ & $0.86[0.35,2.1]$ \\
\hline Subtotal $(95 \% \mathrm{Cl})$ & 20 & 20 & & $100 \%$ & $0.86[0.35,2.1]$ \\
\hline \multicolumn{6}{|c|}{ Heterogeneity: Not applicable } \\
\hline \multicolumn{6}{|c|}{ Test for overall effect: $Z=0.34(P=0.74)$} \\
\hline Test for subgroup diff & $\mathrm{df}=1(\mathrm{P}=0.98), \mathrm{I}$ & & & & \\
\hline
\end{tabular}


Analysis 10.3. Comparison 10 Individual Antidepressants versus another antidepressant within the same class, Outcome 3 Failure to remit.

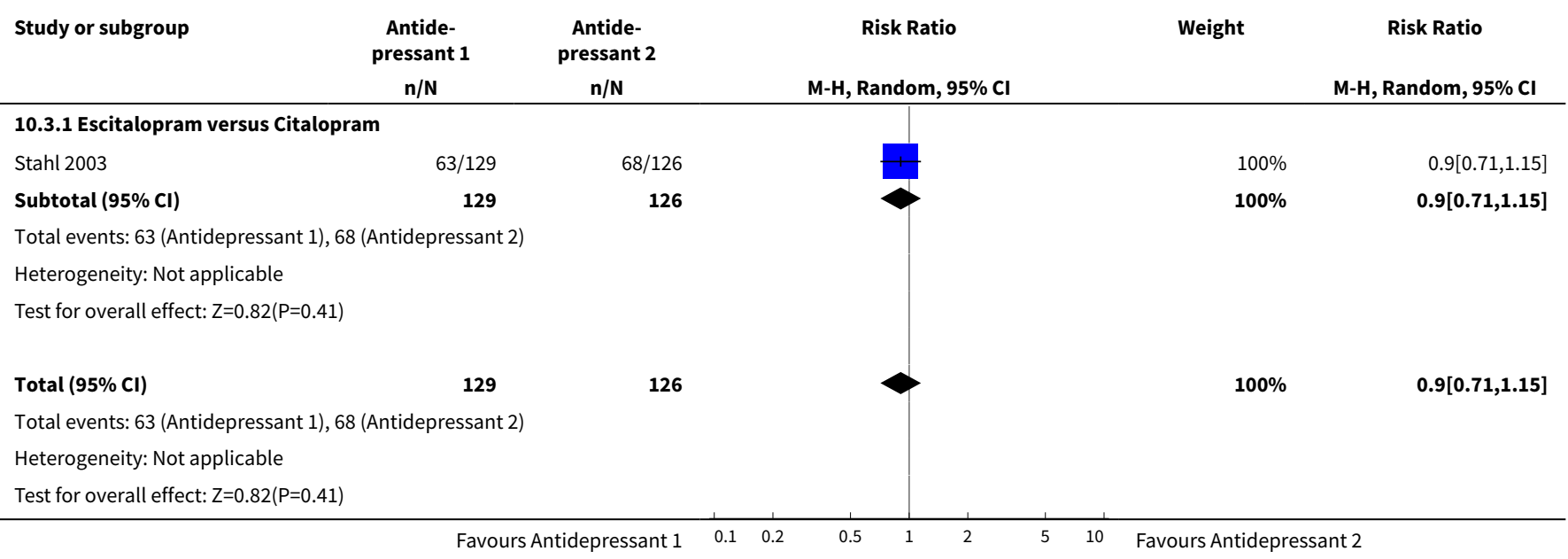

Analysis 10.4. Comparison 10 Individual Antidepressants versus another antidepressant within the same class, Outcome 4 Panic symptoms.

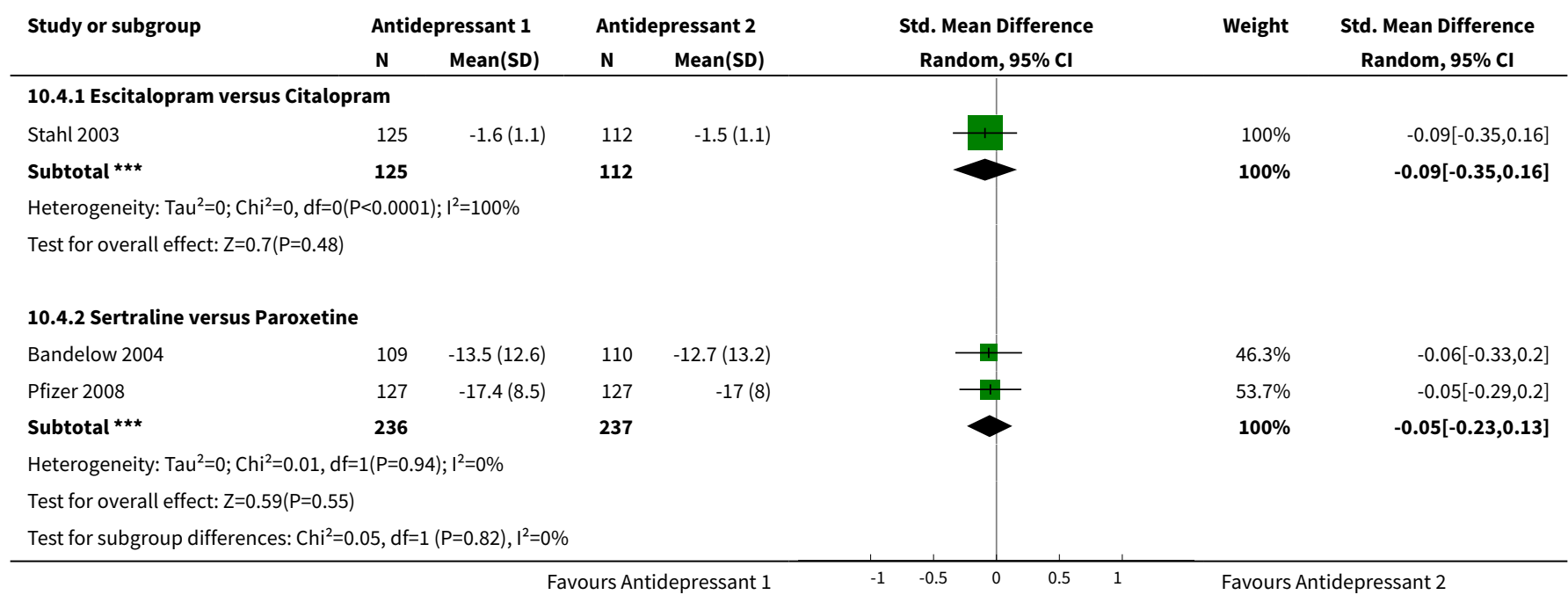

Analysis 10.5. Comparison 10 Individual Antidepressants versus another antidepressant within the same class, Outcome 5 Frequency of panic attacks.

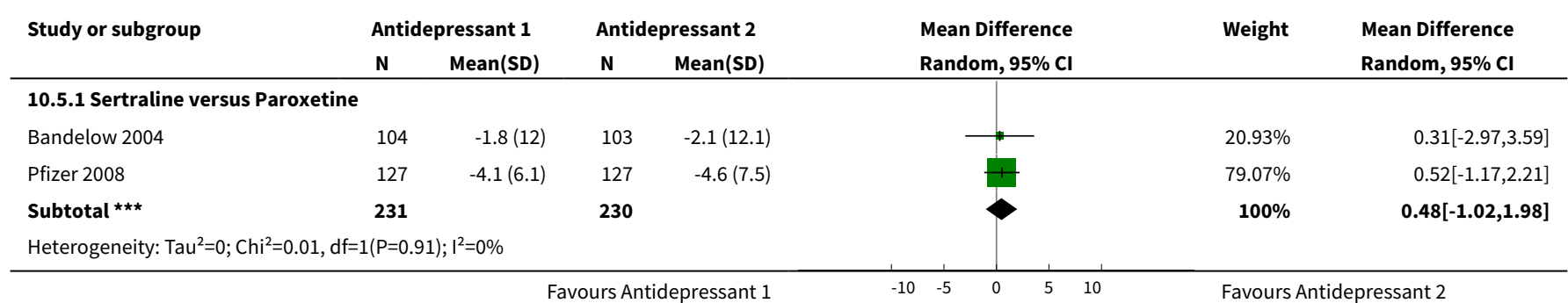




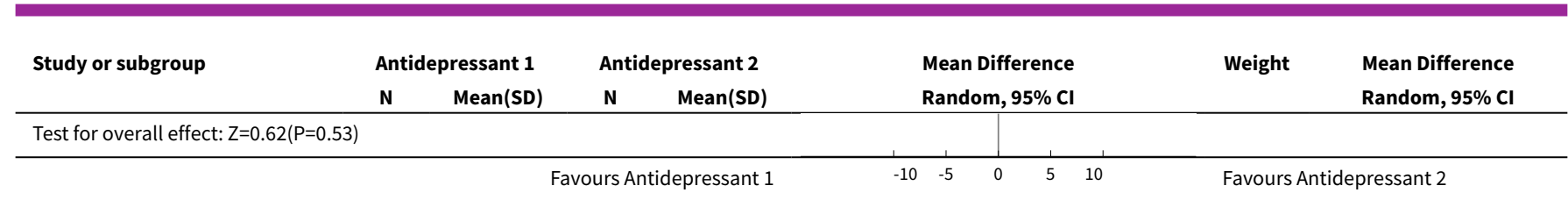

Analysis 10.6. Comparison 10 Individual Antidepressants versus another antidepressant within the same class, Outcome 6 Agoraphobia.

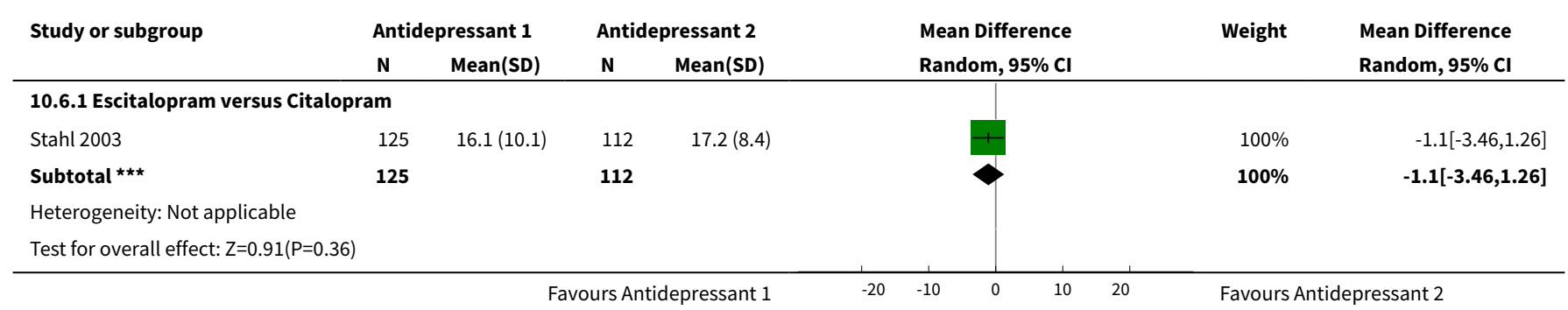

\section{Analysis 10.7. Comparison 10 Individual Antidepressants versus another} antidepressant within the same class, Outcome 7 General anxiety.

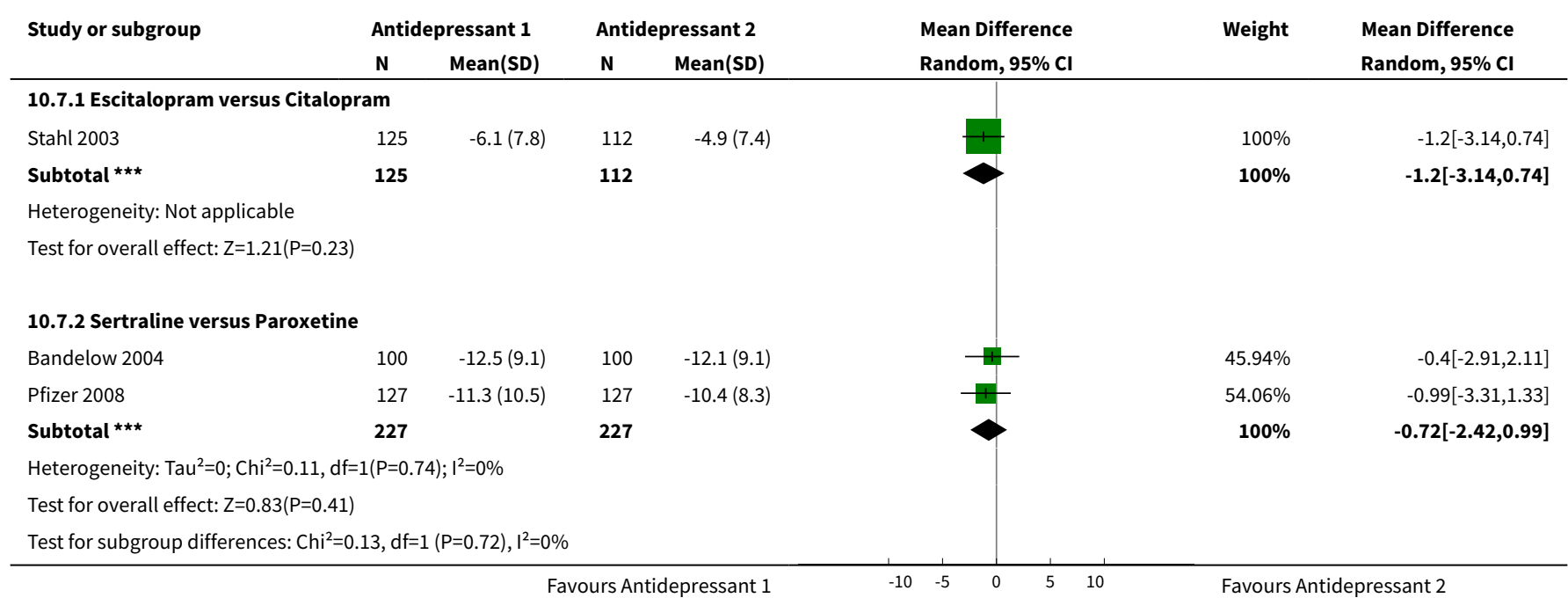

Analysis 10.8. Comparison 10 Individual Antidepressants versus another antidepressant within the same class, Outcome 8 Depression.

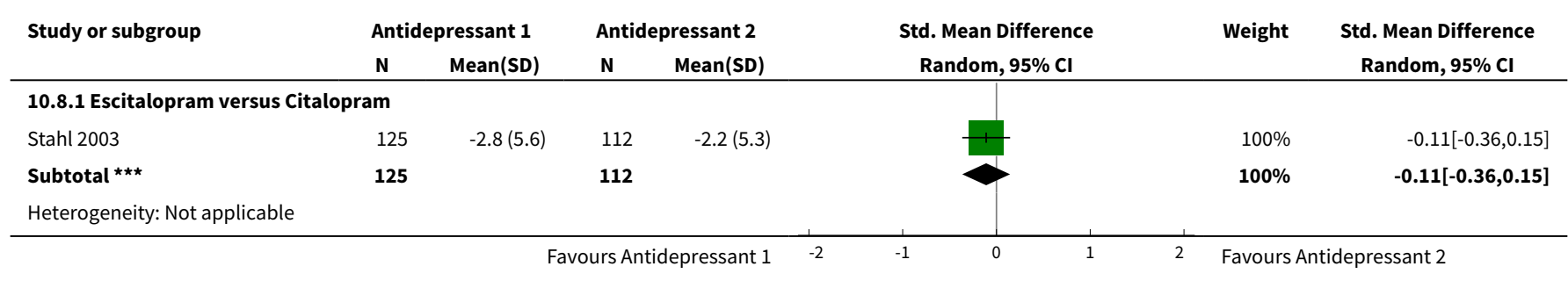




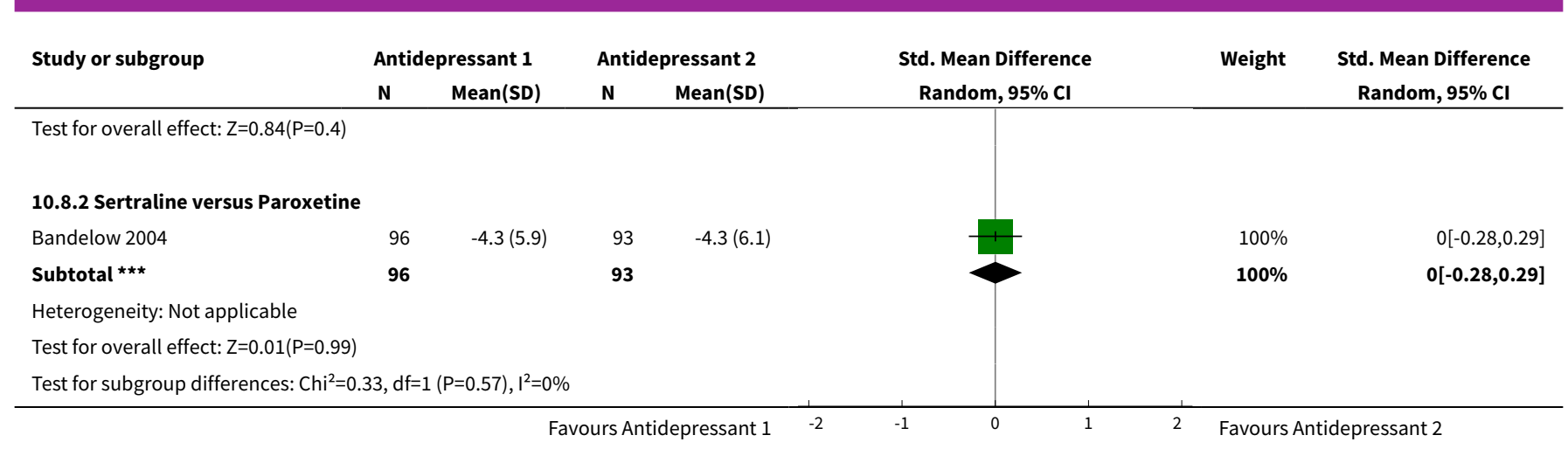

Analysis 10.10. Comparison 10 Individual Antidepressants versus another antidepressant within the same class, Outcome 10 Quality of life.

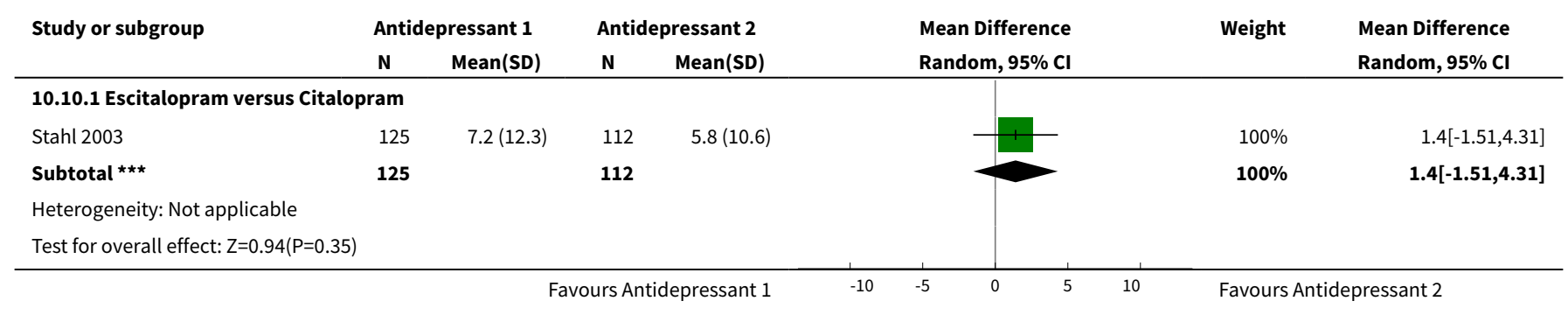

\section{Analysis 10.13. Comparison 10 Individual Antidepressants versus another antidepressant} within the same class, Outcome 13 Number of dropouts due to adverse effects.

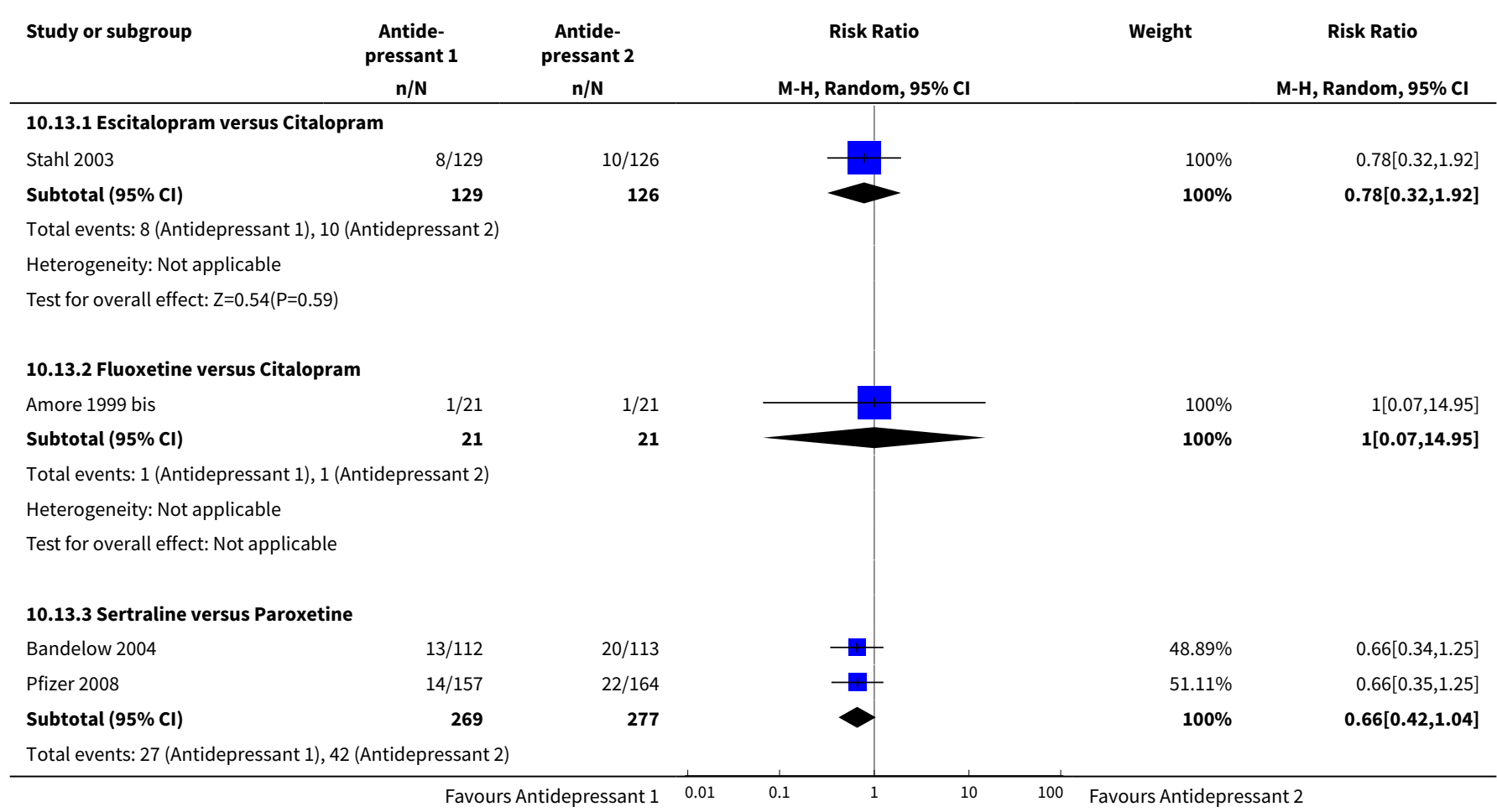




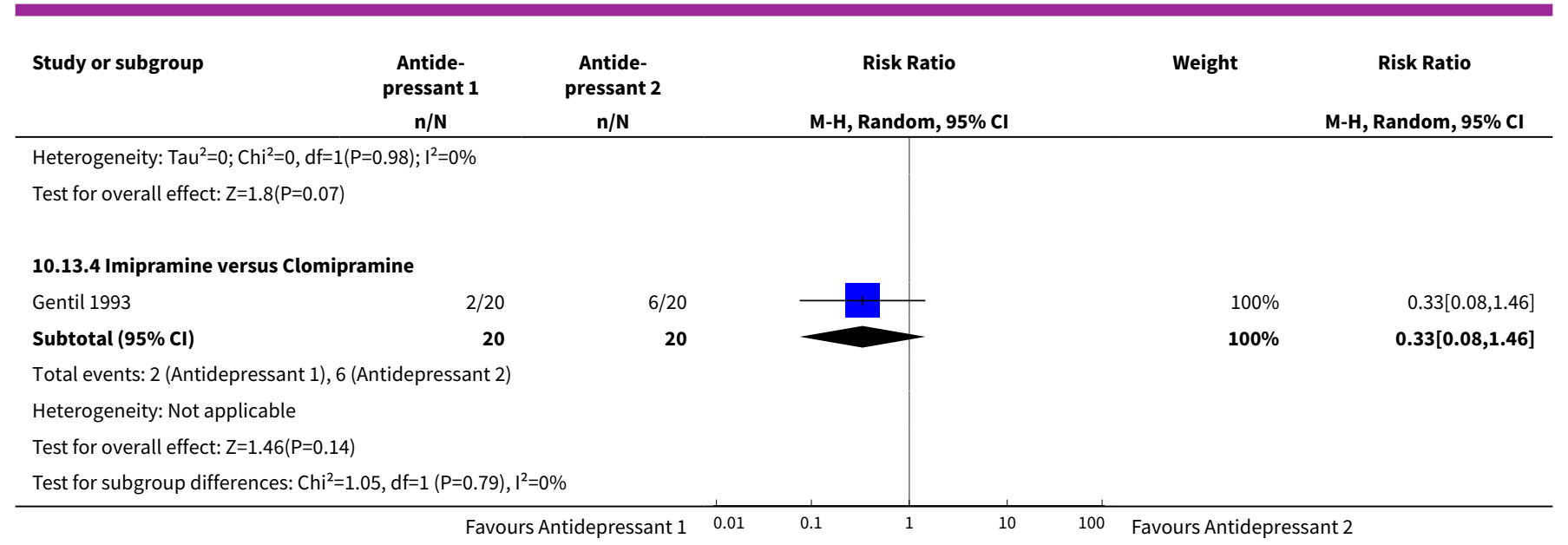

Analysis 10.14. Comparison 10 Individual Antidepressants versus another antidepressant
within the same class, Outcome 14 Number of patients experiencing at least one adverse effect.

\begin{tabular}{|c|c|c|c|c|c|}
\hline Study or subgroup & $\begin{array}{c}\text { Antide- } \\
\text { pressant } 1 \\
\mathrm{n} / \mathrm{N} \\
\end{array}$ & $\begin{array}{c}\text { Antide- } \\
\text { pressant } 2 \\
\mathrm{n} / \mathrm{N} \\
\end{array}$ & $\begin{array}{c}\text { Risk Ratio } \\
\text { M-H, Random, } 95 \% \mathrm{Cl} \\
\end{array}$ & Weight & $\begin{array}{c}\text { Risk Ratio } \\
\text { M-H, Random, } 95 \% \mathrm{CI}\end{array}$ \\
\hline \multicolumn{6}{|c|}{ 10.14.1 Escitalopram versus Citalopram } \\
\hline Stahl 2003 & $104 / 129$ & $107 / 126$ & & $100 \%$ & $0.95[0.85,1.06]$ \\
\hline Subtotal $(95 \% \mathrm{Cl})$ & 129 & 126 & 1 & $100 \%$ & $0.95[0.85,1.06]$ \\
\hline \multicolumn{6}{|c|}{ Total events: 104 (Antidepressant 1), 107 (Antidepressant 2) } \\
\hline \multicolumn{6}{|c|}{ Test for overall effect: $Z=0.91(P=0.36)$} \\
\hline \multicolumn{6}{|c|}{ 10.14.2 Sertraline versus Paroxetine } \\
\hline Pfizer 2008 & $127 / 157$ & $134 / 164$ & & $100 \%$ & $0.99[0.89,1.1]$ \\
\hline Subtotal $(95 \% \mathrm{Cl})$ & 157 & 164 & 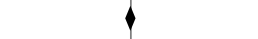 & $100 \%$ & $0.99[0.89,1.1]$ \\
\hline \multicolumn{6}{|c|}{ Total events: 127 (Antidepressant 1), 134 (Antidepressant 2) } \\
\hline \multicolumn{6}{|c|}{ Test for overall effect: $Z=0.19(P=0.85)$} \\
\hline Test for subgroup dif & 9, $d f=1(P=0.59)$, & & & & \\
\hline
\end{tabular}

Comparison 11. Individual benzodiazepines versus another benzodiazepine

\begin{tabular}{|c|c|c|c|c|}
\hline Outcome or subgroup title & No. of studies & $\begin{array}{l}\text { No. of partici- } \\
\text { pants }\end{array}$ & Statistical method & Effect size \\
\hline 1 Failure to respond & 1 & & Risk Ratio (M-H, Random, 95\% Cl) & Subtotals only \\
\hline $\begin{array}{l}\text { 1.1 Alprazolam versus di- } \\
\text { azepam }\end{array}$ & 1 & 159 & Risk Ratio (M-H, Random, 95\% Cl) & $0.94[0.63,1.40]$ \\
\hline 2 Total number of dropouts & 2 & 209 & Risk Ratio (M-H, Random, 95\% Cl) & $1.01[0.49,2.08]$ \\
\hline $\begin{array}{l}\text { 2.1 Alprazolam versus di- } \\
\text { azepam }\end{array}$ & 1 & 159 & Risk Ratio (M-H, Random, 95\% Cl) & $0.84[0.44,1.64]$ \\
\hline
\end{tabular}




\begin{tabular}{|c|c|c|c|c|}
\hline Outcome or subgroup title & No. of studies & $\begin{array}{l}\text { No. of partici- } \\
\text { pants }\end{array}$ & Statistical method & Effect size \\
\hline $\begin{array}{l}\text { 2.2 Alprazolam versus clon- } \\
\text { azepam }\end{array}$ & 1 & 50 & Risk Ratio (M-H, Random, 95\% Cl) & $2.17[0.44,10.78]$ \\
\hline 3 Failure to remit & 2 & 209 & Risk Ratio (M-H, Random, 95\% Cl) & $0.96[0.58,1.57]$ \\
\hline $\begin{array}{l}\text { 3.1 Alprazolam versus di- } \\
\text { azepam }\end{array}$ & 1 & 159 & Risk Ratio (M-H, Random, 95\% Cl) & $0.76[0.48,1.20]$ \\
\hline $\begin{array}{l}\text { 3.2 Alprazolam versus clon- } \\
\text { azepam }\end{array}$ & 1 & 50 & Risk Ratio (M-H, Random, 95\% Cl) & $1.26[0.73,2.17]$ \\
\hline 4 Panic symptoms & 2 & 209 & $\begin{array}{l}\text { Mean Difference (IV, Random, 95\% } \\
\mathrm{CI})\end{array}$ & $0.16[-0.62,0.93]$ \\
\hline $\begin{array}{l}\text { 4.1 Alprazolam versus di- } \\
\text { azepam }\end{array}$ & 1 & 159 & $\begin{array}{l}\text { Mean Difference (IV, Random, 95\% } \\
\mathrm{Cl} \text { ) }\end{array}$ & $-0.20[-0.70,0.30]$ \\
\hline $\begin{array}{l}\text { 4.2 Alprazolam versus clon- } \\
\text { azepam }\end{array}$ & 1 & 50 & $\begin{array}{l}\text { Mean Difference (IV, Random, 95\% } \\
\mathrm{Cl} \text { ) }\end{array}$ & $0.60[-0.12,1.32]$ \\
\hline 5 Frequency of panic attacks & 2 & 209 & $\begin{array}{l}\text { Mean Difference (IV, Random, 95\% } \\
\mathrm{CI})\end{array}$ & $0.39[-1.29,2.08]$ \\
\hline $\begin{array}{l}5.1 \text { Alprazolam versus di- } \\
\text { azepam }\end{array}$ & 1 & 159 & $\begin{array}{l}\text { Mean Difference (IV, Random, 95\% } \\
\mathrm{CI})\end{array}$ & $0.40[-1.34,2.14]$ \\
\hline $\begin{array}{l}5.2 \text { Alprazolam versus clon- } \\
\text { azepam }\end{array}$ & 1 & 50 & $\begin{array}{l}\text { Mean Difference (IV, Random, 95\% } \\
\mathrm{CI})\end{array}$ & $0.30[-6.75,7.35]$ \\
\hline 6 Agoraphobia & 0 & 0 & $\begin{array}{l}\text { Std. Mean Difference (IV, Random, } \\
95 \% \mathrm{Cl} \text { ) }\end{array}$ & $0.0[0.0,0.0]$ \\
\hline 7 General anxiety & 1 & & $\begin{array}{l}\text { Mean Difference (IV, Random, 95\% } \\
\mathrm{Cl} \text { ) }\end{array}$ & Subtotals only \\
\hline $\begin{array}{l}\text { 7.1 Alprazolam versus di- } \\
\text { azepam }\end{array}$ & 1 & 159 & $\begin{array}{l}\text { Mean Difference (IV, Random, 95\% } \\
\mathrm{CI})\end{array}$ & $0.20[-2.28,2.68]$ \\
\hline 8 Depression & 1 & & $\begin{array}{l}\text { Mean Difference (IV, Random, 95\% } \\
\mathrm{CI})\end{array}$ & Subtotals only \\
\hline $\begin{array}{l}8.1 \text { Alprazolam versus clon- } \\
\text { azepam }\end{array}$ & 1 & 44 & $\begin{array}{l}\text { Mean Difference (IV, Random, 95\% } \\
\mathrm{CI} \text { ) }\end{array}$ & $2.3[-2.83,7.43]$ \\
\hline 9 Social functioning & 1 & & $\begin{array}{l}\text { Mean Difference (IV, Random, 95\% } \\
\mathrm{CI})\end{array}$ & Subtotals only \\
\hline $\begin{array}{l}\text { 9.1 Alprazolam versus di- } \\
\text { azepam }\end{array}$ & 1 & 159 & $\begin{array}{l}\text { Mean Difference (IV, Random, 95\% } \\
\mathrm{Cl} \text { ) }\end{array}$ & $-0.10[-0.47,0.27]$ \\
\hline 10 Quality of life & 1 & & $\begin{array}{l}\text { Mean Difference (IV, Random, 95\% } \\
\mathrm{Cl} \text { ) }\end{array}$ & Subtotals only \\
\hline $\begin{array}{l}\text { 10.1 Alprazolam versus clon- } \\
\text { azepam }\end{array}$ & 1 & 44 & $\begin{array}{l}\text { Mean Difference (IV, Random, 95\% } \\
\mathrm{Cl} \text { ) }\end{array}$ & $-0.30[-1.07,0.47]$ \\
\hline
\end{tabular}




\begin{tabular}{|c|c|c|c|c|}
\hline Outcome or subgroup title & No. of studies & $\begin{array}{l}\text { No. of partici- } \\
\text { pants }\end{array}$ & Statistical method & Effect size \\
\hline 11 Patient satisfaction & 0 & 0 & $\begin{array}{l}\text { Std. Mean Difference (IV, Random, } \\
95 \% \mathrm{CI} \text { ) }\end{array}$ & $0.0[0.0,0.0]$ \\
\hline 12 Economic costs & 0 & 0 & $\begin{array}{l}\text { Std. Mean Difference (IV, Random, } \\
95 \% \mathrm{CI} \text { ) }\end{array}$ & $0.0[0.0,0.0]$ \\
\hline $\begin{array}{l}13 \text { Number of dropouts due } \\
\text { to adverse effects }\end{array}$ & 2 & 209 & Risk Ratio (M-H, Random, 95\% Cl) & $1.69[0.33,8.58]$ \\
\hline $\begin{array}{l}\text { 13.1 Alprazolam versus di- } \\
\text { azepam }\end{array}$ & 1 & 159 & Risk Ratio (M-H, Random, 95\% Cl) & $1.04[0.15,7.19]$ \\
\hline $\begin{array}{l}13.2 \text { Alprazolam versus clon- } \\
\text { azepam }\end{array}$ & 1 & 50 & Risk Ratio (M-H, Random, 95\% Cl) & $5.4[0.27,107.09]$ \\
\hline $\begin{array}{l}14 \text { Number of patients expe- } \\
\text { riencing at least one adverse } \\
\text { effect }\end{array}$ & 0 & 0 & Risk Ratio (M-H, Random, 95\% Cl) & $0.0[0.0,0.0]$ \\
\hline
\end{tabular}

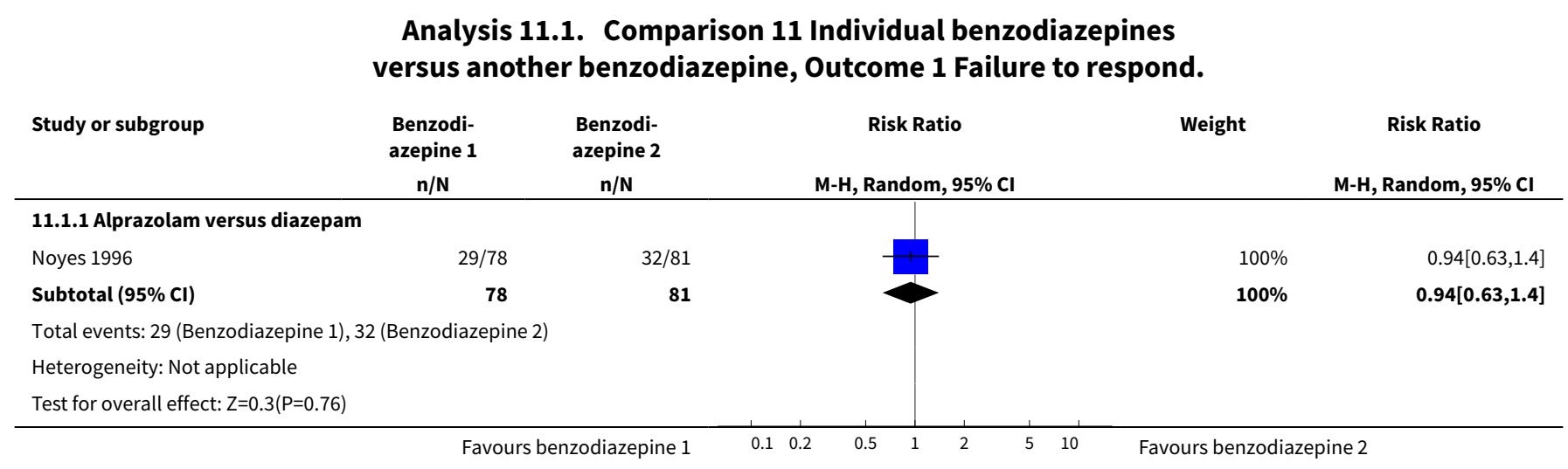

\section{Analysis 11.2. Comparison 11 Individual benzodiazepines versus another benzodiazepine, Outcome 2 Total number of dropouts.}

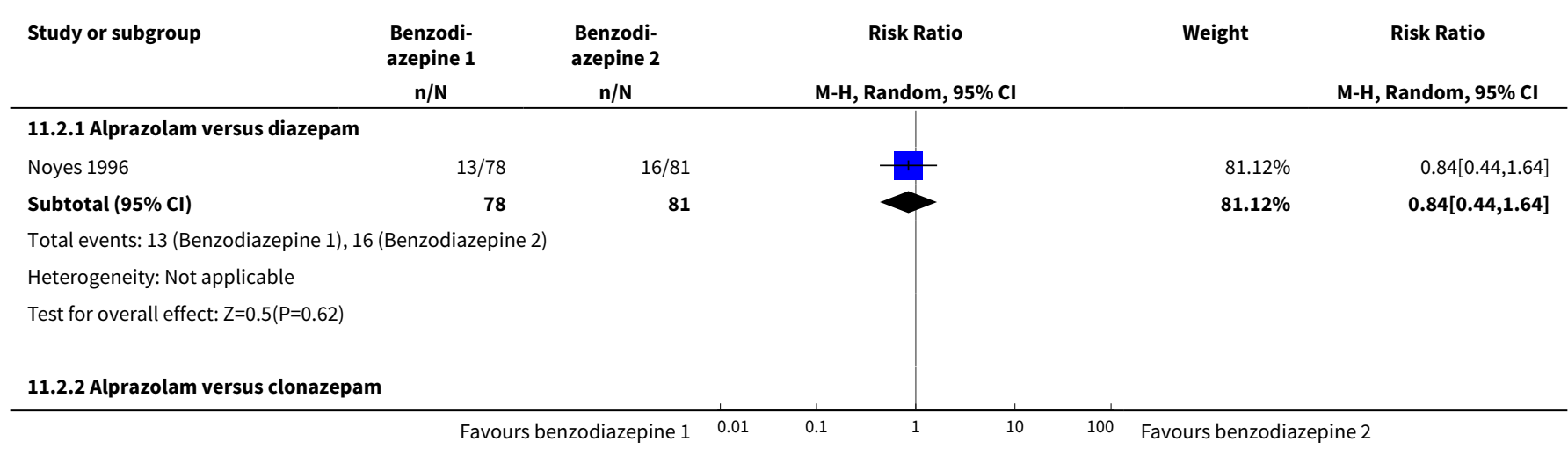




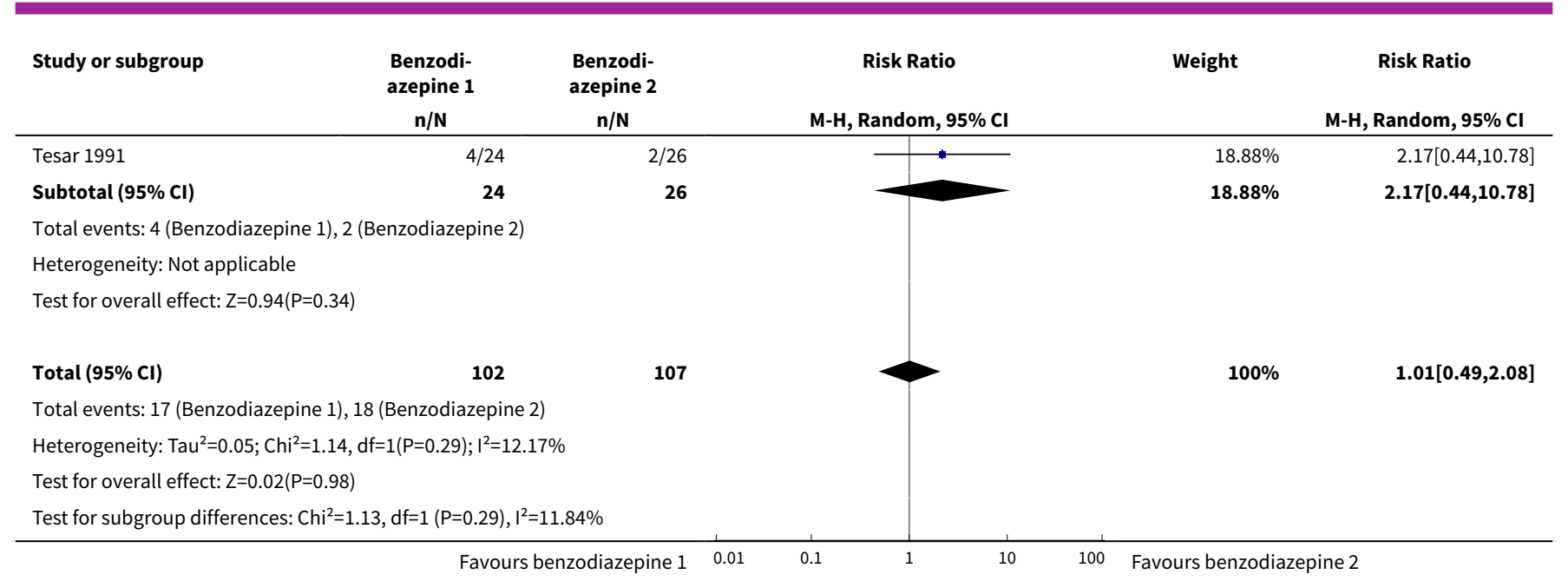

\section{Analysis 11.3. Comparison 11 Individual benzodiazepines versus another benzodiazepine, Outcome 3 Failure to remit.}

$\begin{array}{ccccc}\text { Study or subgroup } & \begin{array}{c}\text { Benzodi- } \\ \text { azepine } 1\end{array} & \begin{array}{c}\text { Benzodi- } \\ \text { azepine } 2\end{array} & \text { Risk Ratio } & \text { Weight } \\ \mathrm{n} / \mathrm{N} & \mathrm{n} / \mathrm{N} & \mathrm{M}-\mathrm{H}, \mathrm{Random}, 95 \% \mathrm{Cl} & \mathrm{M}-\mathrm{H}, \mathrm{Random}, 95 \% \mathrm{Cl}\end{array}$

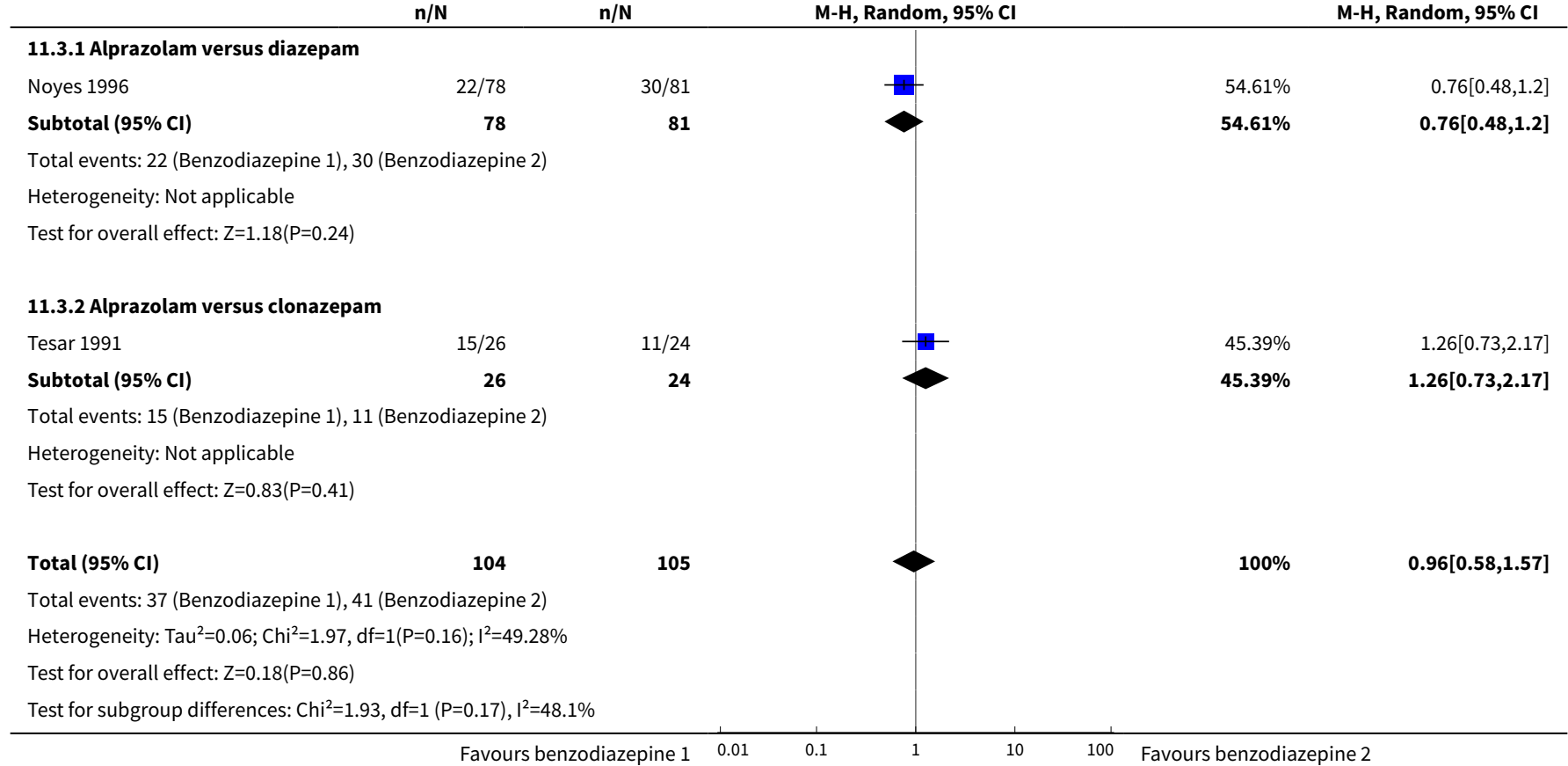

Analysis 11.4. Comparison 11 Individual benzodiazepines versus another benzodiazepine, Outcome 4 Panic symptoms.

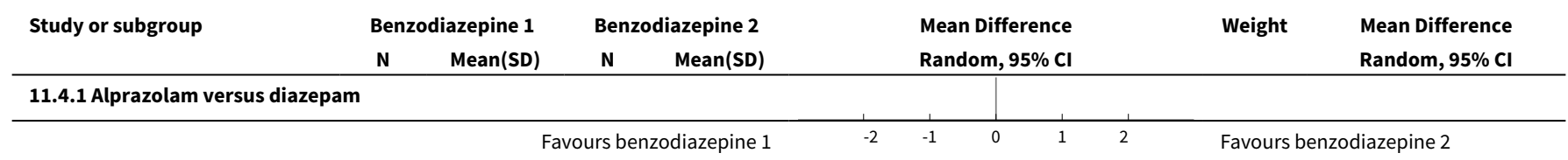




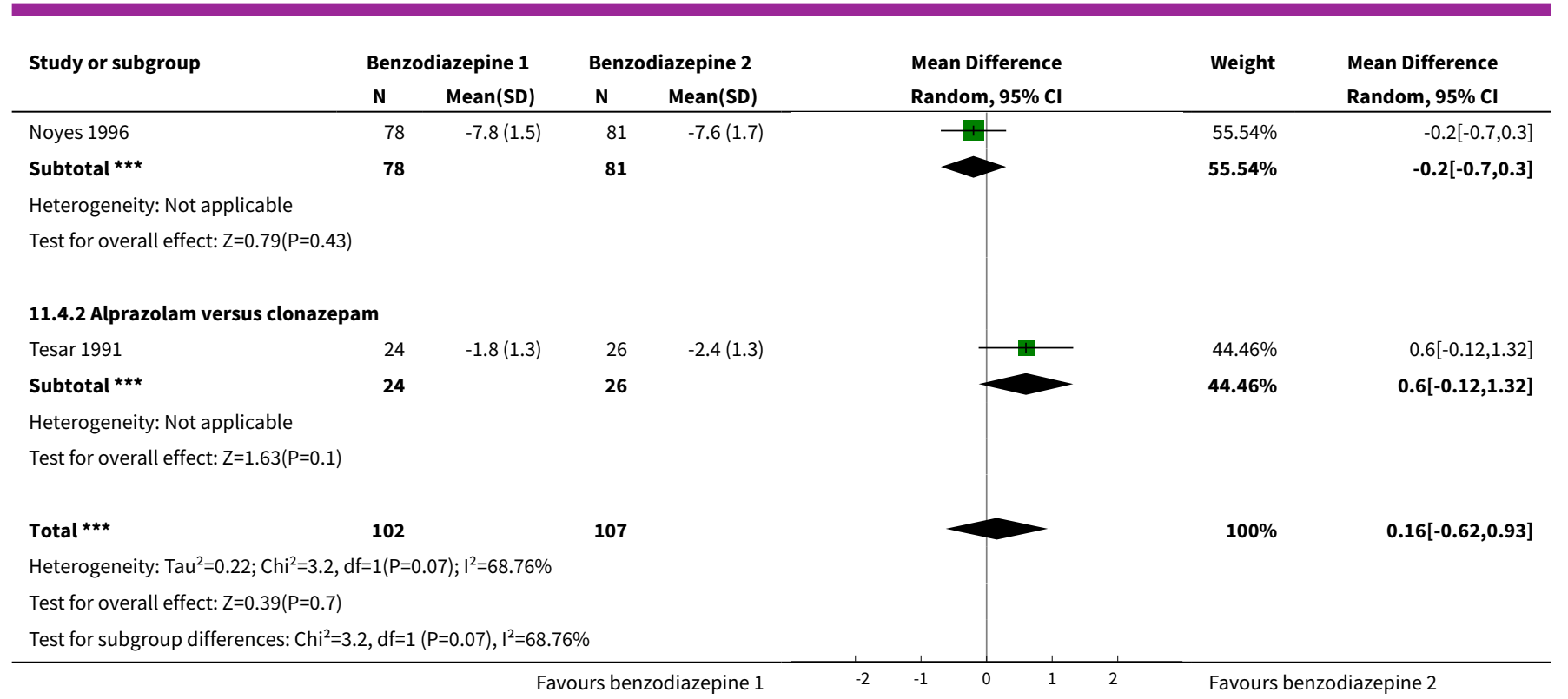

Analysis 11.5. Comparison 11 Individual benzodiazepines versus another benzodiazepine, Outcome 5 Frequency of panic attacks.

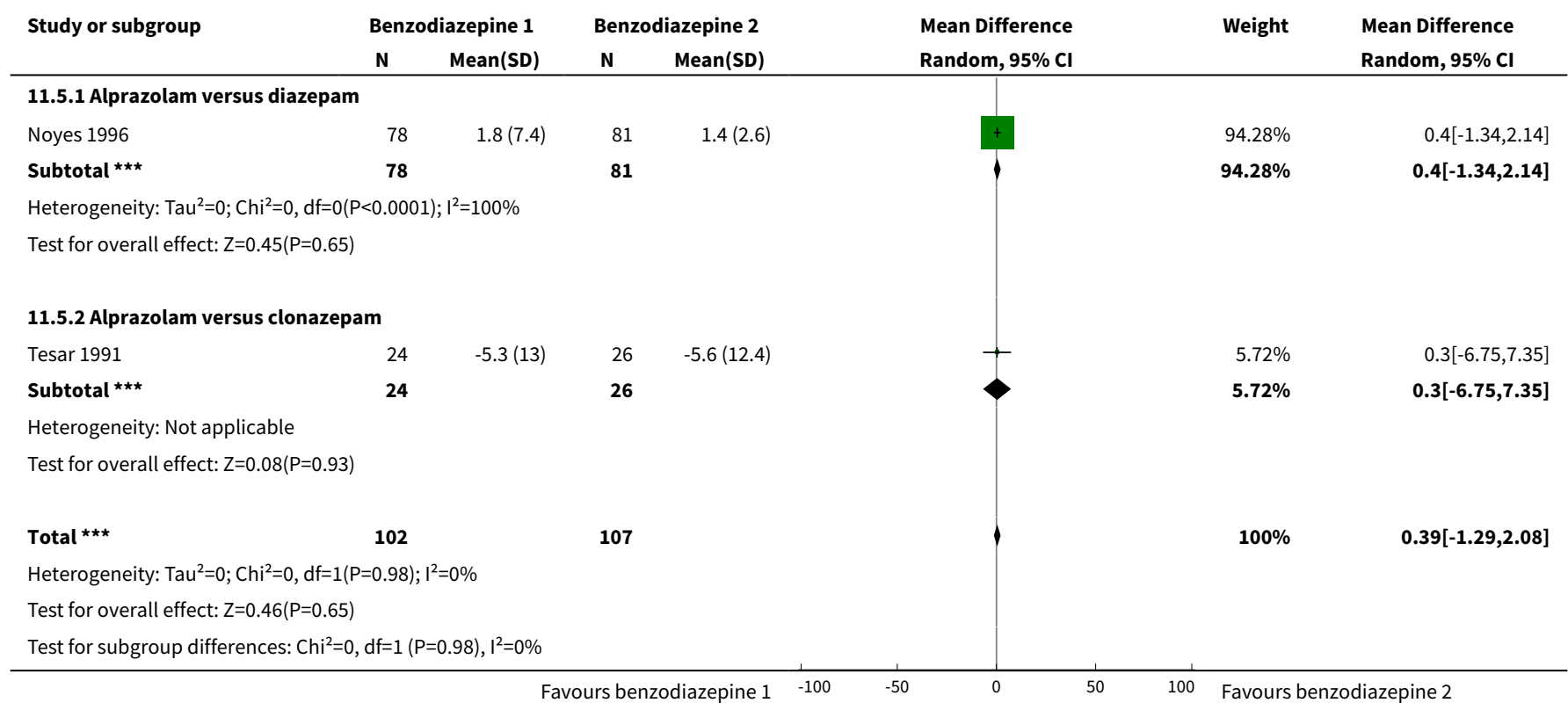

\section{Analysis 11.7. Comparison 11 Individual benzodiazepines versus another benzodiazepine, Outcome 7 General anxiety.}

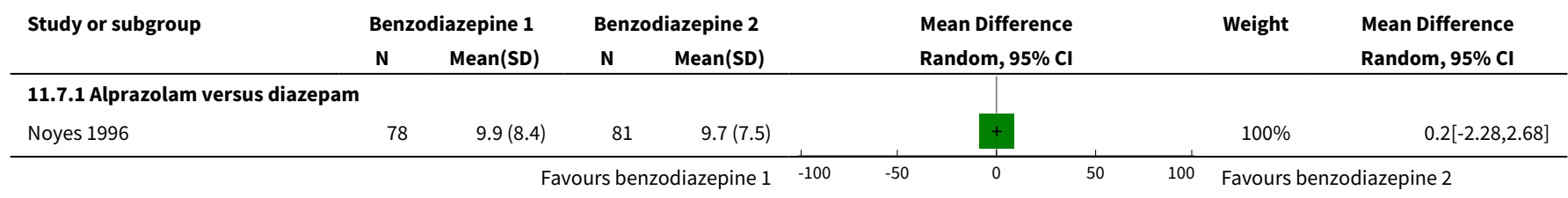




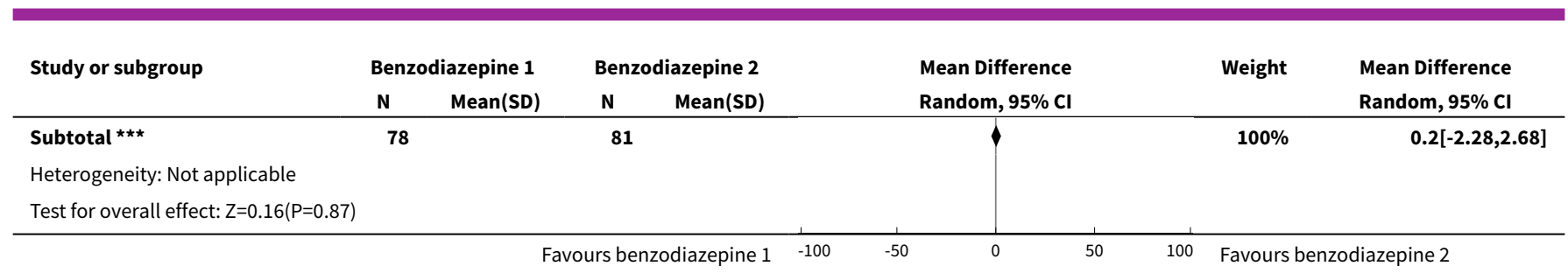

Analysis 11.8. Comparison 11 Individual benzodiazepines versus another benzodiazepine, Outcome 8 Depression.

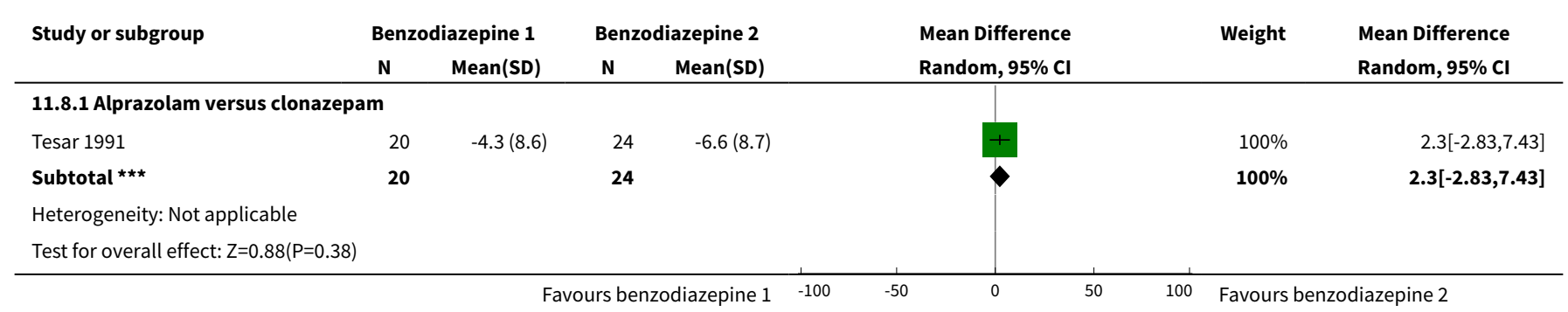

Analysis 11.9. Comparison 11 Individual benzodiazepines versus another benzodiazepine, Outcome 9 Social functioning.

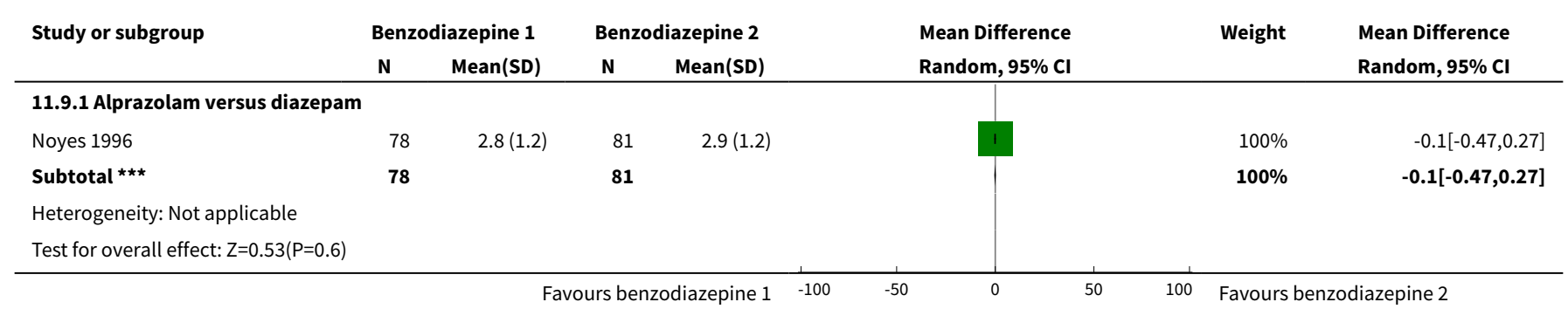

Analysis 11.10. Comparison 11 Individual benzodiazepines

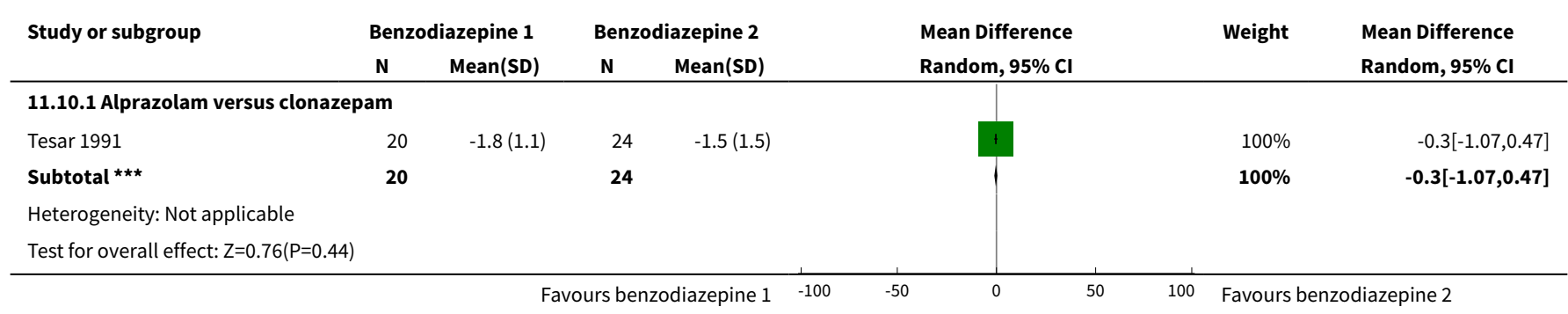


Analysis 11.13. Comparison 11 Individual benzodiazepines versus another benzodiazepine, Outcome 13 Number of dropouts due to adverse effects.

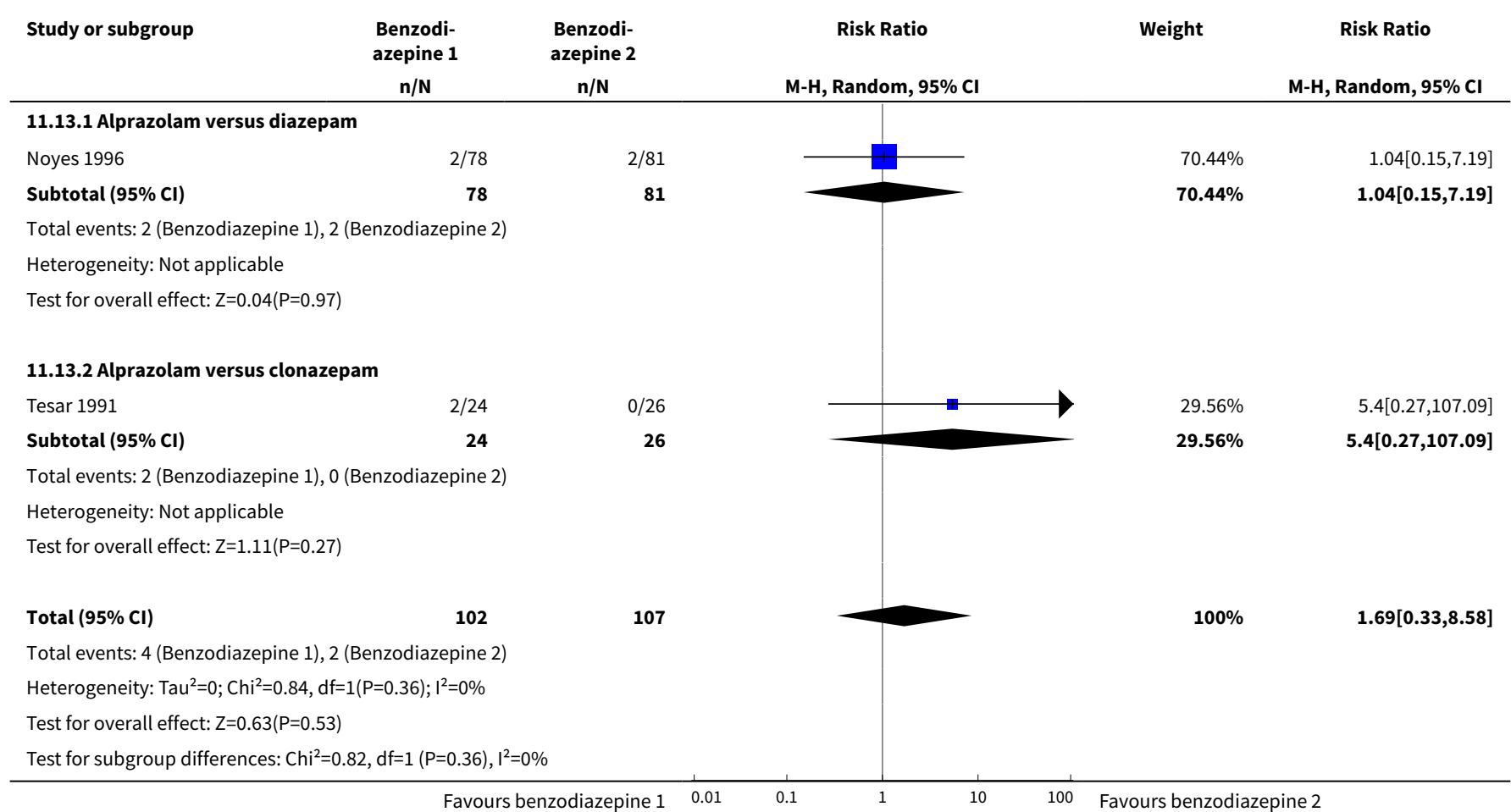

\section{Comparison 12. High risk of bias excluded - Antidepressants versus benzodiazepines}

\begin{tabular}{|c|c|c|c|c|}
\hline $\begin{array}{l}\text { Outcome or subgroup ti- } \\
\text { tle }\end{array}$ & No. of studies & $\begin{array}{l}\text { No. of partici- } \\
\text { pants }\end{array}$ & Statistical method & Effect size \\
\hline $\begin{array}{l}1 \text { Total number of } \\
\text { dropouts }\end{array}$ & 5 & 619 & Risk Ratio (M-H, Random, 95\% Cl) & $1.63[0.82,3.23]$ \\
\hline $1.1 \mathrm{TCAs}$ versus BDZs & 4 & 465 & Risk Ratio (M-H, Random, 95\% Cl) & $1.67[0.64,4.36]$ \\
\hline $1.2 \mathrm{SSRIs}$ versus $\mathrm{BDZs}$ & 1 & 154 & Risk Ratio (M-H, Random, 95\% Cl) & $1.71[1.03,2.84]$ \\
\hline $1.3 \mathrm{MAOIs}$ versus $\mathrm{BDZs}$ & 0 & 0 & Risk Ratio (M-H, Random, 95\% Cl) & $0.0[0.0,0.0]$ \\
\hline 1.4 SNRIs versus BDZs & 0 & 0 & Risk Ratio (M-H, Random, 95\% Cl) & $0.0[0.0,0.0]$ \\
\hline $1.5 \mathrm{NaSSAs}$ versus BDZs & 0 & 0 & Risk Ratio (M-H, Random, 95\% Cl) & $0.0[0.0,0.0]$ \\
\hline 1.6 NDRIs versus BDZs & 0 & 0 & Risk Ratio (M-H, Random, 95\% Cl) & $0.0[0.0,0.0]$ \\
\hline $1.7 \mathrm{NRIs}$ versus $\mathrm{BDZs}$ & 0 & 0 & Risk Ratio (M-H, Random, 95\% Cl) & $0.0[0.0,0.0]$ \\
\hline 1.8 Other ADs versus BDZs & 0 & 0 & Risk Ratio (M-H, Random, 95\% Cl) & $0.0[0.0,0.0]$ \\
\hline
\end{tabular}


Analysis 12.1. Comparison 12 High risk of bias excluded - Antidepressants versus benzodiazepines, Outcome 1 Total number of dropouts.

\begin{tabular}{lrr} 
Study or subgroup & $\begin{array}{c}\text { Antide- } \\
\text { pressants } \\
\mathbf{n} / \mathbf{N}\end{array}$ & $\begin{array}{c}\text { Benzodi- } \\
\text { azepines } \\
\mathbf{n} / \mathbf{N}\end{array}$ \\
\hline $\mathbf{1 2 . 1 . 1}$ TCAs versus BDZs & & \\
Holland 1999 & $36 / 149$ & $58 / 166$ \\
Schweizer 1993 & $14 / 34$ & $4 / 37$ \\
Sheikh 1999 & $1 / 10$ & $0 / 8$ \\
Uhlenhuth 1989 & $10 / 20$ & $10 / 41$ \\
Subtotal (95\% Cl) & $\mathbf{2 1 3}$ & $\mathbf{2 5 2}$
\end{tabular}

Subtotal $(95 \% \mathrm{Cl})$

213

252

Total events: 61 (Antidepressants), 72 (Benzodiazepines) Heterogeneity: $\mathrm{Tau}^{2}=0.65 ; \mathrm{Chi}^{2}=15.43, \mathrm{df}=3(\mathrm{P}=0) ; \mathrm{I}^{2}=80.56 \%$

Test for overall effect: $Z=1.04(P=0.3)$

\subsubsection{SSRIs versus BDZs}

GSK-29060/1

Total events: 29 (Antidepressants), 17 (Benzodiazepines)

Heterogeneity: Not applicable

Test for overall effect: $Z=2.06(P=0.04)$

12.1.3 MAOIs versus BDZs

Total events: 0 (Antidepressants), 0 (Benzodiazepines)

Heterogeneity: Not applicable

Test for overall effect: Not applicable

\subsubsection{SNRIs versus BDZs}

$\rightarrow-$

Total events: 0 (Antidepressants), 0 (Benzodiazepines)

Heterogeneity: Not applicable

Test for overall effect: Not applicable

\subsubsection{NaSSAs versus BDZs}

\begin{tabular}{rrr} 
& $18.35 \%$ & $3.81[1.39,10.45]$ \\
\hline & $4.28 \%$ & $2.45[0.11,53.25]$ \\
\hline & $23.18 \%$ & $2.05[1.02,4.11]$ \\
\hline & $73.95 \%$ & $\mathbf{1 . 6 7}[\mathbf{0 . 6 4 , 4 . 3 6}]$
\end{tabular}

Total events: 0 (Antidepressants), 0 (Benzodiazepines)

Heterogeneity: Not applicable

Test for overall effect: Not applicable

\subsubsection{NDRIs versus BDZs}

Total events: 0 (Antidepressants), 0 (Benzodiazepines)

Heterogeneity: Not applicable

Test for overall effect: Not applicable

\subsubsection{NRIs versus BDZs}




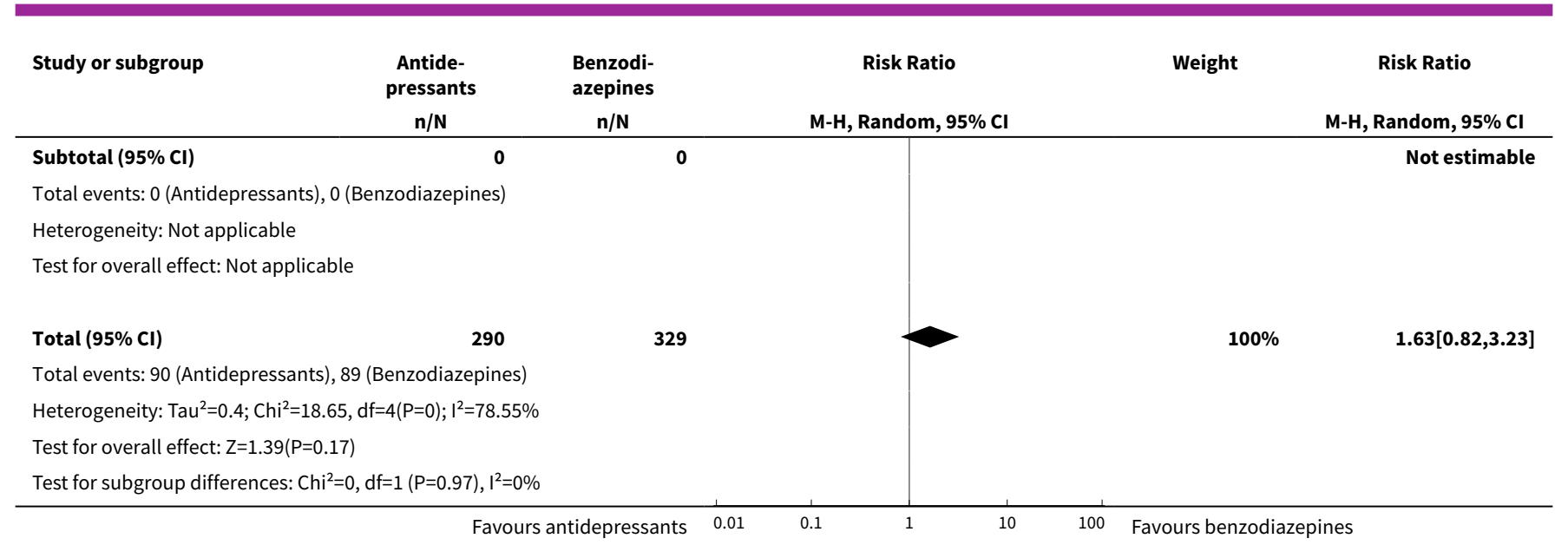

\section{Comparison 13. High risk of bias excluded - TCAs versus benzodiazepines}

\begin{tabular}{lllll}
\hline Outcome or subgroup title & No. of studies & $\begin{array}{l}\text { No. of partici- } \\
\text { pants }\end{array}$ & Statistical method & Effect size \\
\hline 1 Total number of dropouts & 4 & 465 & Risk Ratio (M-H, Random, 95\% Cl) & $1.67[0.64,4.36]$ \\
\hline 1.1 Imipramine versus BDZs & 3 & 150 & Risk Ratio (M-H, Random, 95\% Cl) & $2.50[1.42,4.39]$ \\
\hline 1.2 Clomipramine versus BDZs & 1 & 315 & Risk Ratio (M-H, Random, 95\% Cl) & $0.69[0.49,0.98]$ \\
\hline
\end{tabular}

Analysis 13.1. Comparison 13 High risk of bias excluded - TCAs versus benzodiazepines, Outcome 1 Total number of dropouts.

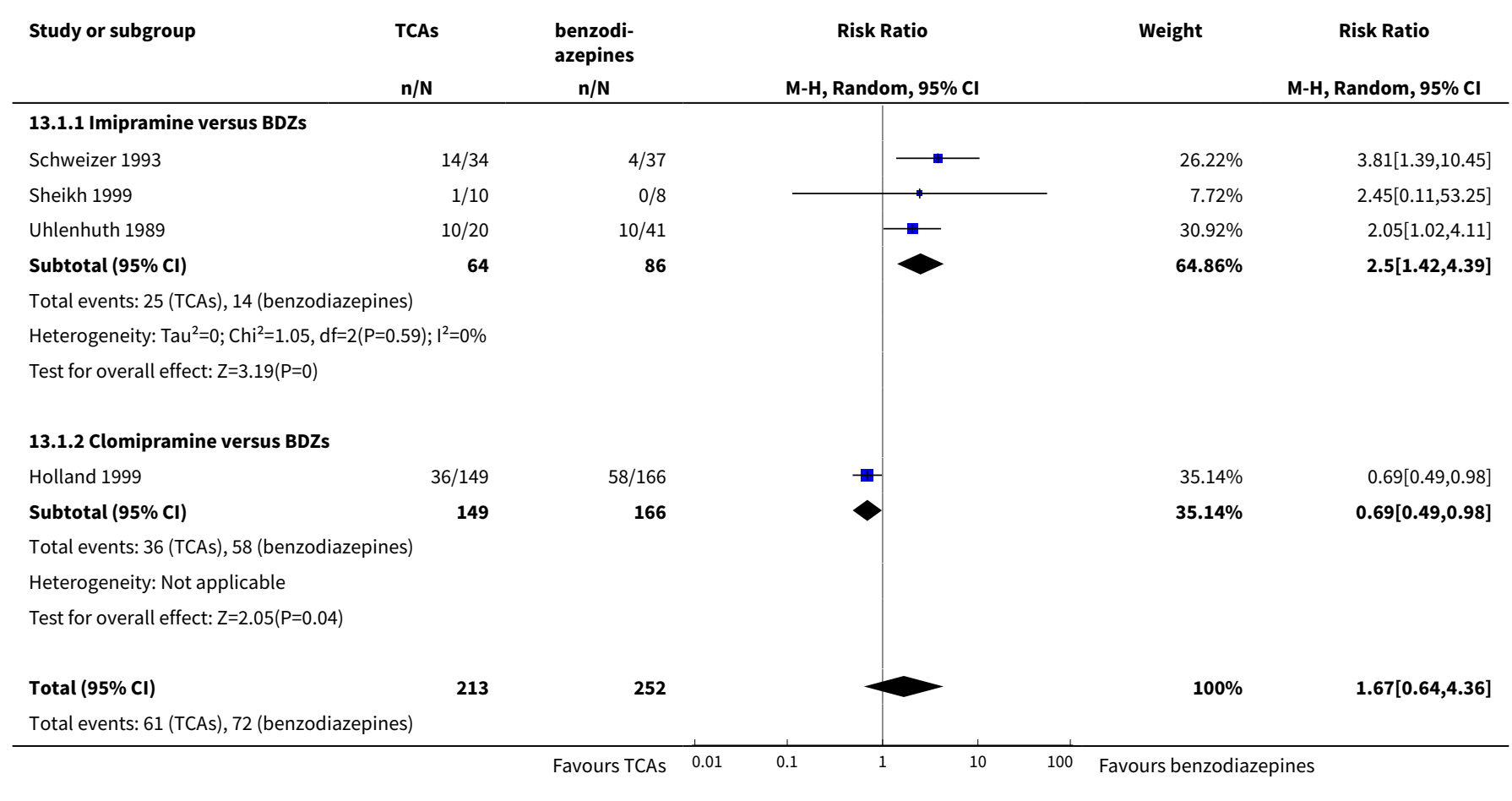




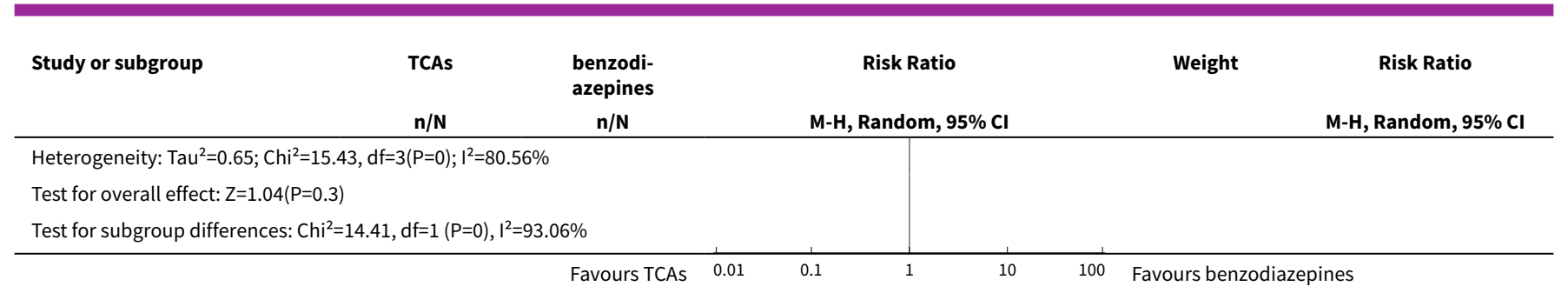

Comparison 14. High risk of bias excluded - TCAs versus SSRIs

\begin{tabular}{lllll}
\hline Outcome or subgroup title & No. of studies & $\begin{array}{l}\text { No. of partici- } \\
\text { pants }\end{array}$ & Statistical method & Effect size \\
\hline 1 Total number of dropouts & 6 & 890 & $\begin{array}{l}\text { Risk Ratio (M-H, Random, 95\% } \\
\text { Cl) }\end{array}$ & $0.97[0.62,1.50]$ \\
\hline
\end{tabular}

Analysis 14.1. Comparison 14 High risk of bias excluded - TCAs versus SSRIs, Outcome 1 Total number of dropouts.

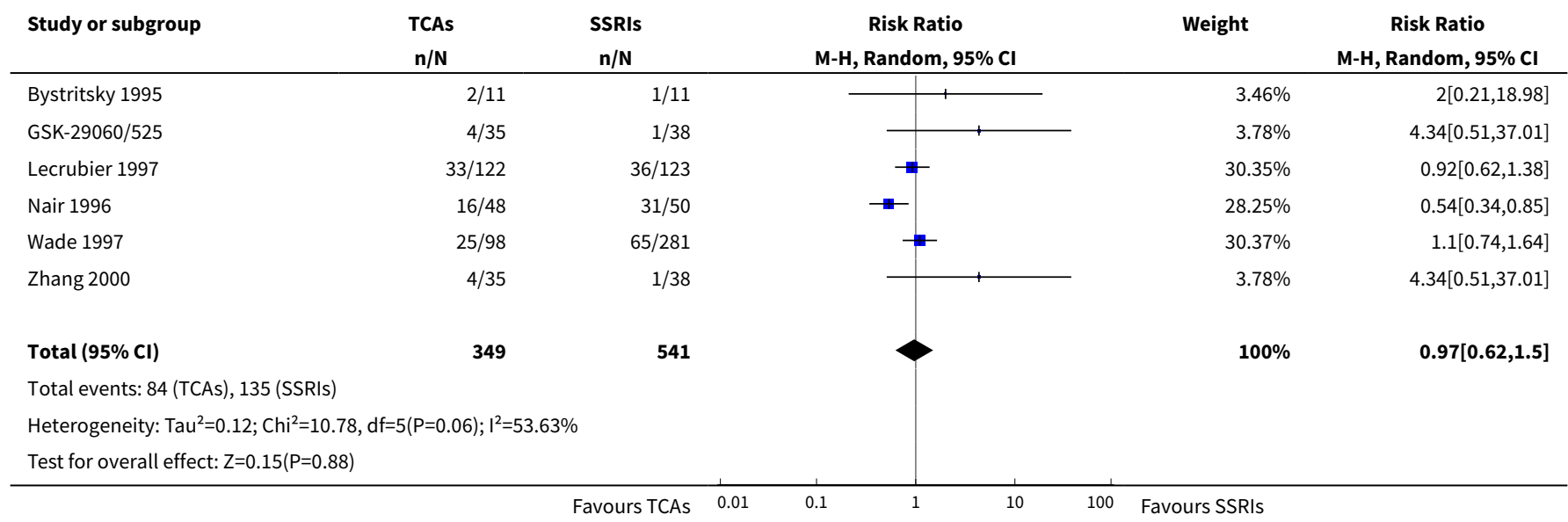

Comparison 15. High risk of bias excluded - TCAs versus MAOIs

\begin{tabular}{lllll}
\hline Outcome or subgroup title & No. of studies & $\begin{array}{l}\text { No. of partici- } \\
\text { pants }\end{array}$ & Statistical method & Effect size \\
\hline 1 Total number of dropouts & 1 & 135 & $\begin{array}{l}\text { Risk Ratio (M-H, Random, 95\% } \\
\text { Cl) }\end{array}$ & $0.87[0.47,1.59]$ \\
\hline
\end{tabular}


Analysis 15.1. Comparison 15 High risk of bias excluded - TCAs versus MAOIs, Outcome 1 Total number of dropouts.

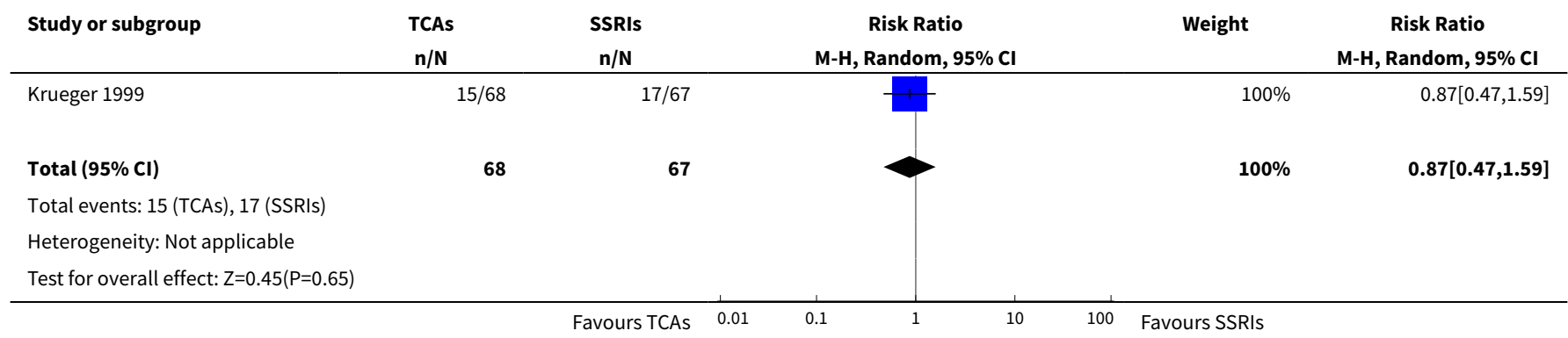

Comparison 16. High risk of bias excluded - SSRIs versus MAOIs

\begin{tabular}{lllll}
\hline Outcome or subgroup title & No. of studies & $\begin{array}{l}\text { No. of partici- } \\
\text { pants }\end{array}$ & Statistical method & Effect size \\
\hline 1 Failure to respond & 1 & 366 & Risk Ratio (M-H, Random, 95\% Cl) & $1.08[0.76,1.54]$ \\
\hline
\end{tabular}

Analysis 16.1. Comparison 16 High risk of bias excluded - SSRIs versus MAOIs, Outcome 1 Failure to respond.

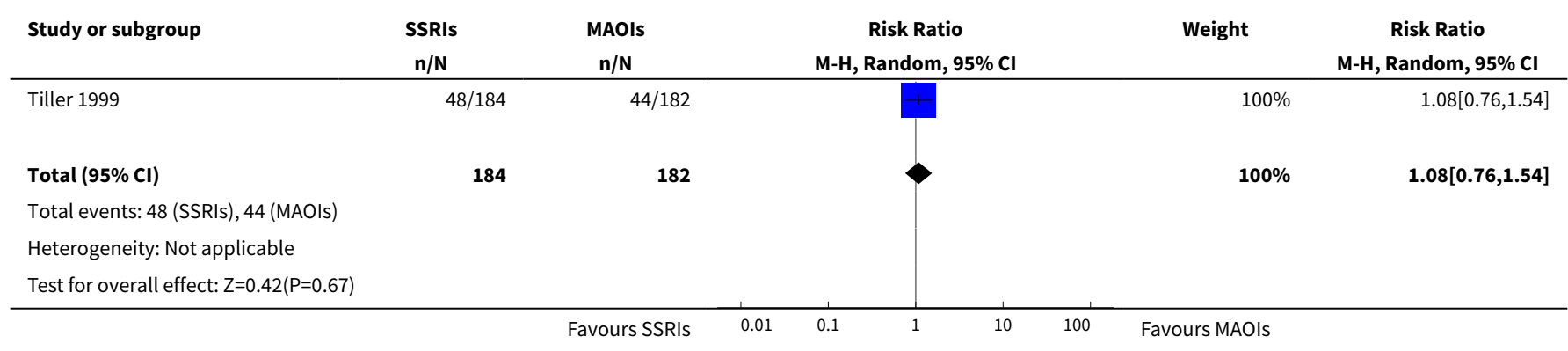

\section{Comparison 17. High dropout rates excluded - Antidepressants versus benzodiazepines}

\begin{tabular}{llllll}
\hline Outcome or subgroup title & No. of studies & $\begin{array}{l}\text { No. of partici- } \\
\text { pants }\end{array}$ & Statistical method & Effect size \\
\hline 1 Total number of dropouts & 2 & 71 & Risk Ratio (M-H, Random, 95\% Cl) & $2.42[0.61,9.64]$ \\
\hline 1.1 TCAs versus BDZs & 2 & 71 & Risk Ratio (M-H, Random, 95\% Cl) & $2.42[0.61,9.64]$ \\
\hline 1.2 SSRIs versus BDZs & 0 & 0 & Risk Ratio (M-H, Random, 95\% Cl) & $0.0[0.0,0.0]$ \\
\hline 1.3 MAOIs versus BDZs & 0 & 0 & Risk Ratio (M-H, Random, 95\% Cl) & $0.0[0.0,0.0]$ \\
\hline 1.4 SNRIs versus BDZs & 0 & 0 & Risk Ratio (M-H, Random, 95\% Cl) & $0.0[0.0,0.0]$ \\
\hline 1.5 NaSSAs versus BDZs & 0 & 0 & Risk Ratio (M-H, Random, 95\% Cl) & $0.0[0.0,0.0]$ \\
\hline 1.6 NDRIs versus BDZs & 0 & 0 & Risk Ratio (M-H, Random, 95\% Cl) & $0.0[0.0,0.0]$ \\
\hline \hline
\end{tabular}




\begin{tabular}{llllll}
\hline Outcome or subgroup title & No. of studies & $\begin{array}{l}\text { No. of partici- } \\
\text { pants }\end{array}$ & Statistical method & Effect size \\
\hline 1.7 NRIs versus BDZs & 0 & 0 & Risk Ratio (M-H, Random, 95\% Cl) & $0.0[0.0,0.0]$ \\
\hline 1.8 Other ADs versus BDZs & 0 & 0 & Risk Ratio (M-H, Random, 95\% Cl) & $0.0[0.0,0.0]$ \\
\hline
\end{tabular}

Analysis 17.1. Comparison 17 High dropout rates excluded - Antidepressants versus benzodiazepines, Outcome 1 Total number of dropouts.

\begin{tabular}{ccccc} 
Study or subgroup & $\begin{array}{c}\text { Antide- } \\
\text { pressants } \\
\mathrm{n} / \mathrm{N}\end{array}$ & $\begin{array}{c}\text { Benzodi- } \\
\text { azepines } \\
\mathrm{n} / \mathrm{N}\end{array}$ & Risk Ratio & Weight \\
\hline
\end{tabular}

\subsubsection{TCAs versus BDZs}

Sheikh 1999

Taylor 1990

Subtotal $(95 \% \mathrm{Cl})$

$1 / 10 \quad 0 / 8$

Total events: 6 (Antidepressants), 2 (Benzodiazepines) Heterogeneity: $\mathrm{Tau}^{2}=0 ; \mathrm{Chi}^{2}=0, \mathrm{df}=1(\mathrm{P}=0.99) ; \mathrm{I}^{2}=0 \%$

Test for overall effect: $Z=1.25(P=0.21)$

\subsubsection{SSRIs versus BDZs}

\subsubsection{MAOIs versus BDZs}

\subsubsection{SNRIs versus BDZs}

\subsubsection{NaSSAs versus BDZs}

Total events: 0 (Antidepressants), 0 (Benzodiazepines) Heterogeneity: Not applicable

Test for overall effect: Not applicable

\subsubsection{NDRIs versus BDZs}

Total events: 0 (Antidepressants), 0 (Benzodiazepines)

Heterogeneity: Not applicable

Test for overall effect: Not applicable 


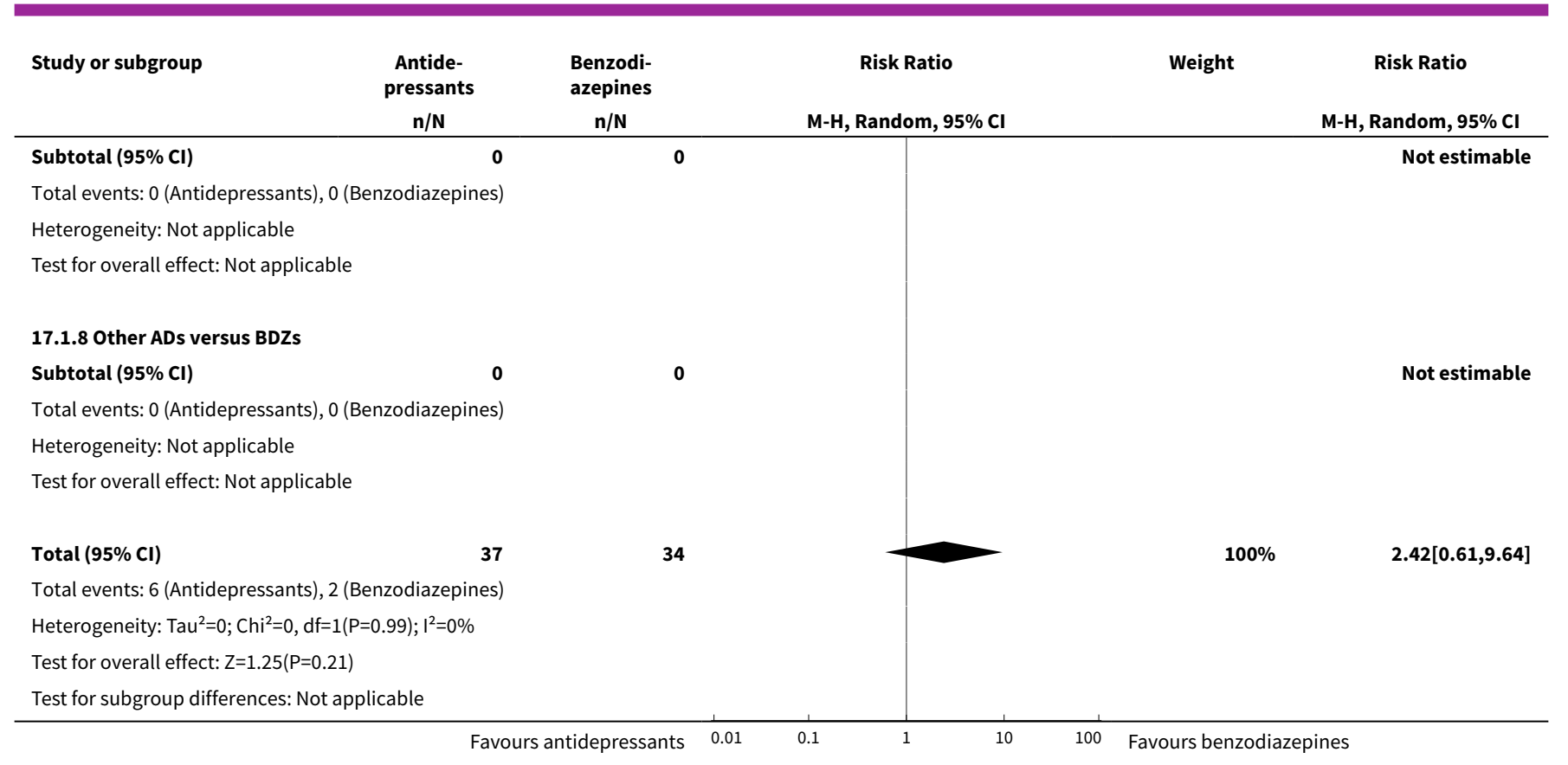

\section{Comparison 18. High dropout rates excluded - TCAs versus benzodiazepines}

\begin{tabular}{llllll}
\hline Outcome or subgroup title & No. of studies & $\begin{array}{l}\text { No. of partici- } \\
\text { pants }\end{array}$ & Statistical method & Effect size \\
\hline 1 Total number of dropouts & 2 & 71 & Risk Ratio (M-H, Random, 95\% Cl) & $2.42[0.61,9.64]$ \\
\hline 1.1 Imipramine versus BDZs & 2 & 71 & Risk Ratio (M-H, Random, 95\% Cl) & $2.42[0.61,9.64]$ \\
\hline 1.2 Clomipramine versus BDZs & 0 & 0 & Risk Ratio (M-H, Random, 95\% Cl) & $0.0[0.0,0.0]$ \\
\hline
\end{tabular}

Analysis 18.1. Comparison 18 High dropout rates excluded - TCAs versus benzodiazepines, Outcome 1 Total number of dropouts.

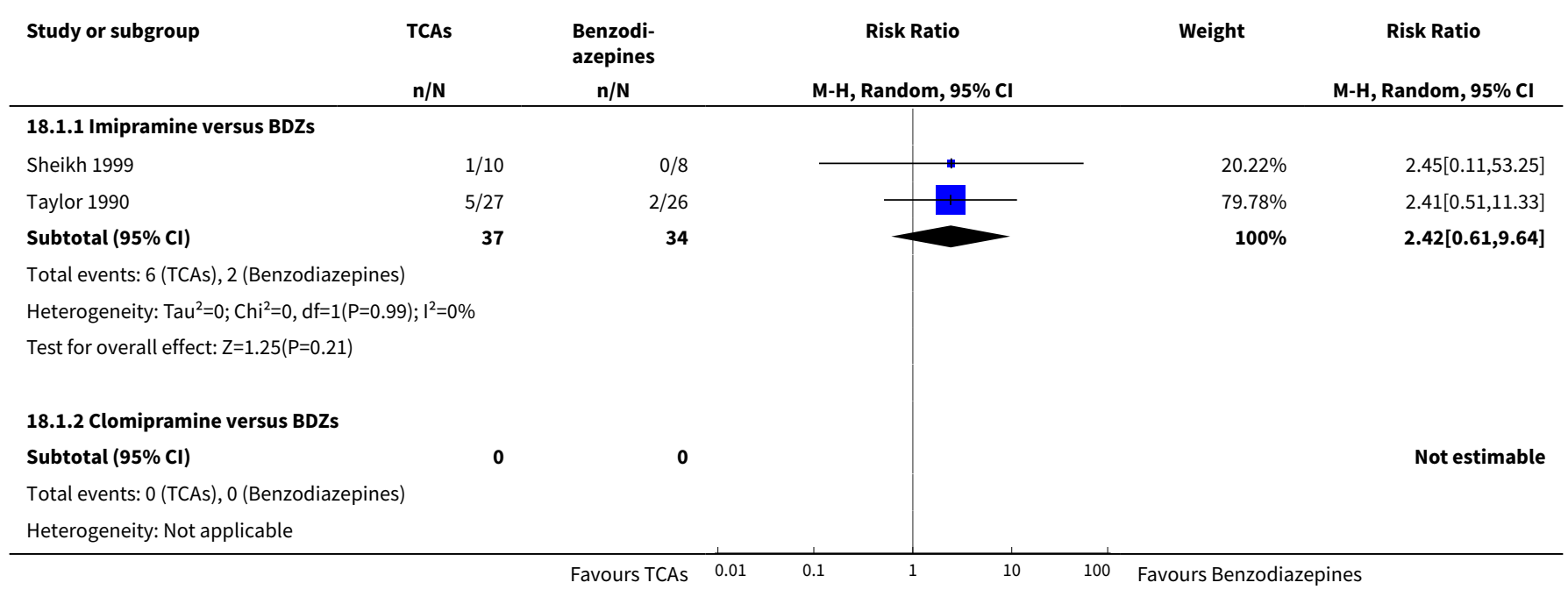




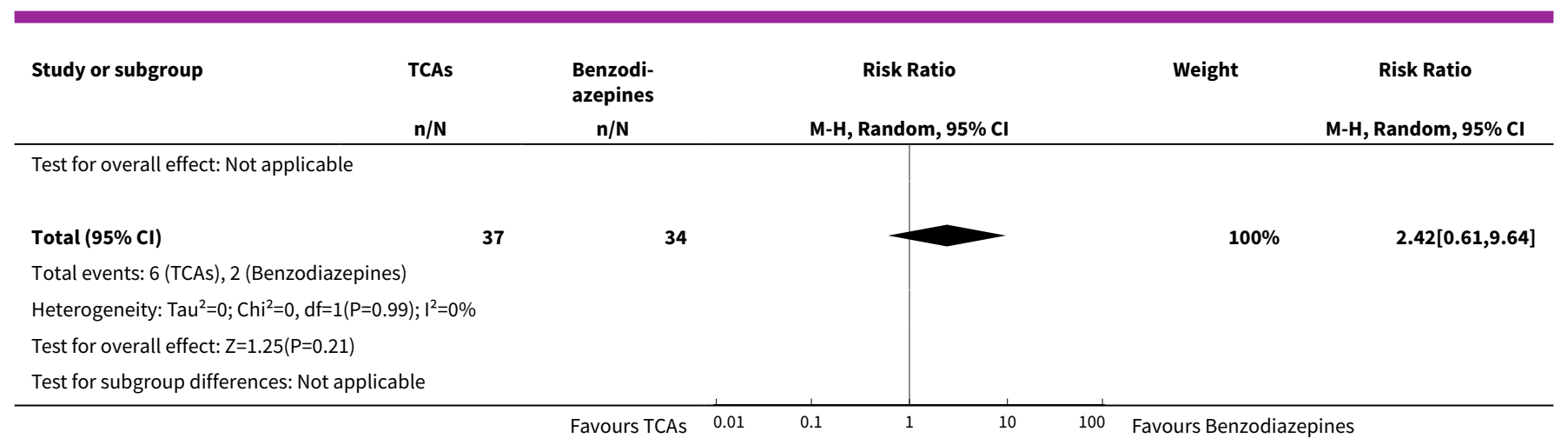

\section{Comparison 19. High dropout rates excluded - TCAs versus SSRIs}

\begin{tabular}{llllll}
\hline Outcome or subgroup title & No. of studies & $\begin{array}{l}\text { No. of partici- } \\
\text { pants }\end{array}$ & Statistical method & Effect size \\
\hline 1 Failure to respond & 2 & 95 & Risk Ratio (M-H, Random, 95\% Cl) & $1.22[0.47,3.15]$ \\
\hline 2 Total number of dropouts & 4 & 206 & Risk Ratio (M-H, Random, 95\% Cl) & $3.02[1.00,9.11]$ \\
\hline
\end{tabular}

\section{Analysis 19.1. Comparison 19 High dropout rates excluded - TCAs versus SSRIs, Outcome 1 Failure to respond.}

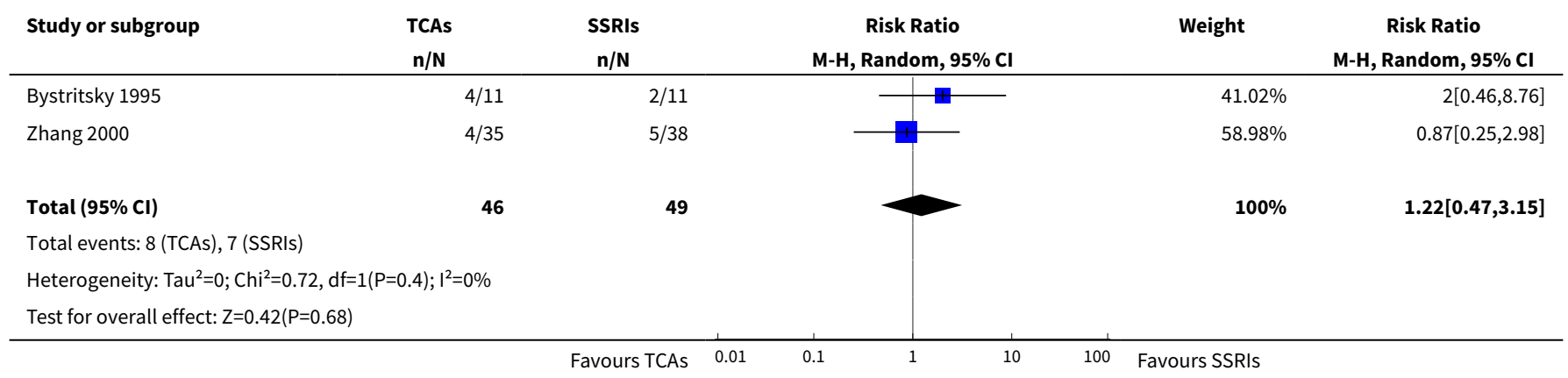

Analysis 19.2. Comparison 19 High dropout rates excluded - TCAs versus SSRIs, Outcome 2 Total number of dropouts.

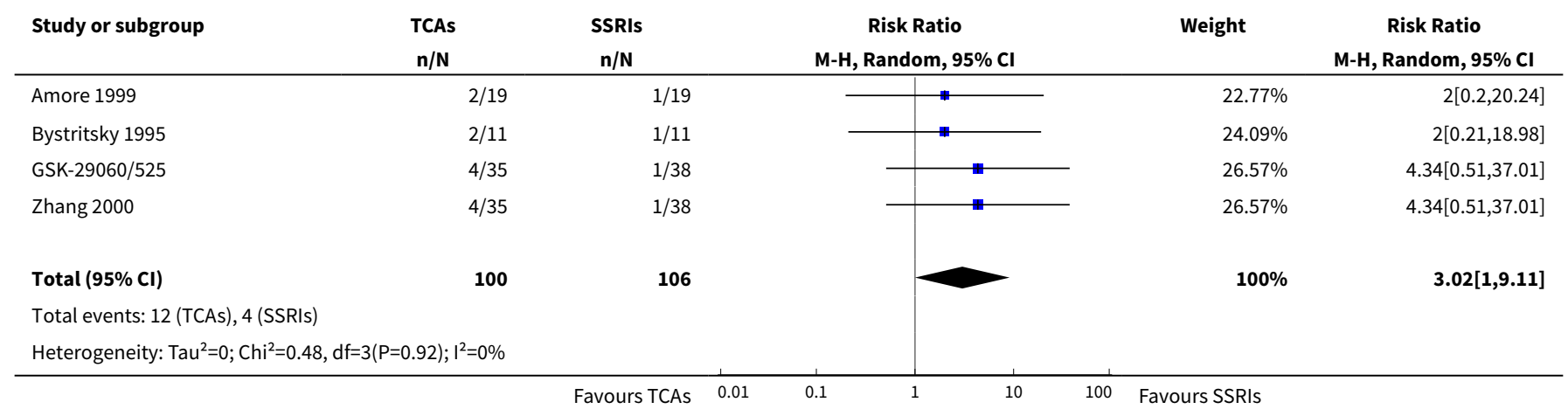




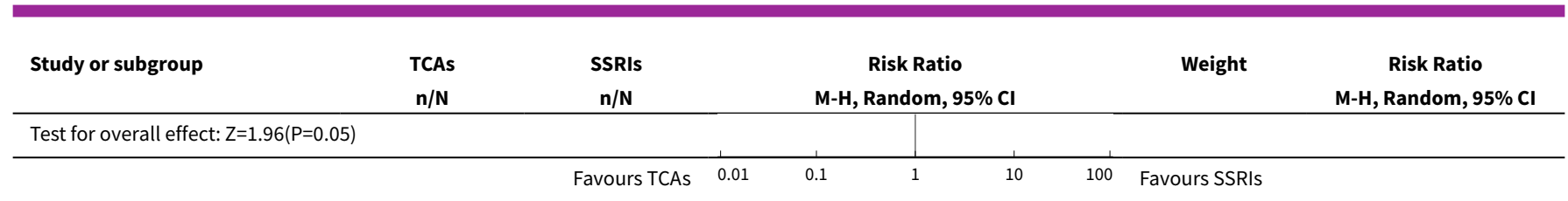

\section{Comparison 20. Funded excluded - Antidepressants versus benzodiazepines}

\begin{tabular}{|c|c|c|c|c|}
\hline $\begin{array}{l}\text { Outcome or subgroup ti- } \\
\text { tle }\end{array}$ & No. of studies & $\begin{array}{l}\text { No. of partici- } \\
\text { pants }\end{array}$ & Statistical method & Effect size \\
\hline $\begin{array}{l}1 \text { Total number of } \\
\text { dropouts }\end{array}$ & 2 & 469 & Risk Ratio (M-H, Random, 95\% Cl) & $1.07[0.44,2.58]$ \\
\hline 1.1 TCAs versus BDZs & 1 & 315 & Risk Ratio (M-H, Random, 95\% Cl) & $0.69[0.49,0.98]$ \\
\hline 1.2 SSRIs versus BDZs & 1 & 154 & Risk Ratio (M-H, Random, 95\% Cl) & $1.71[1.03,2.84]$ \\
\hline $1.3 \mathrm{MAOIs}$ versus BDZs & 0 & 0 & Risk Ratio (M-H, Random, 95\% Cl) & $0.0[0.0,0.0]$ \\
\hline 1.4 SNRIs versus BDZs & 0 & 0 & Risk Ratio (M-H, Random, 95\% Cl) & $0.0[0.0,0.0]$ \\
\hline $1.5 \mathrm{NaSSAs}$ versus BDZs & 0 & 0 & Risk Ratio (M-H, Random, 95\% Cl) & $0.0[0.0,0.0]$ \\
\hline 1.6 NDRIs versus BDZs & 0 & 0 & Risk Ratio (M-H, Random, 95\% Cl) & $0.0[0.0,0.0]$ \\
\hline $1.7 \mathrm{NRIs}$ versus $\mathrm{BDZs}$ & 0 & 0 & Risk Ratio (M-H, Random, 95\% Cl) & $0.0[0.0,0.0]$ \\
\hline 1.8 Other ADs versus BDZs & 0 & 0 & Risk Ratio (M-H, Random, 95\% Cl) & $0.0[0.0,0.0]$ \\
\hline
\end{tabular}

\section{Analysis 20.1. Comparison 20 Funded excluded - Antidepressants versus benzodiazepines, Outcome 1 Total number of dropouts.}

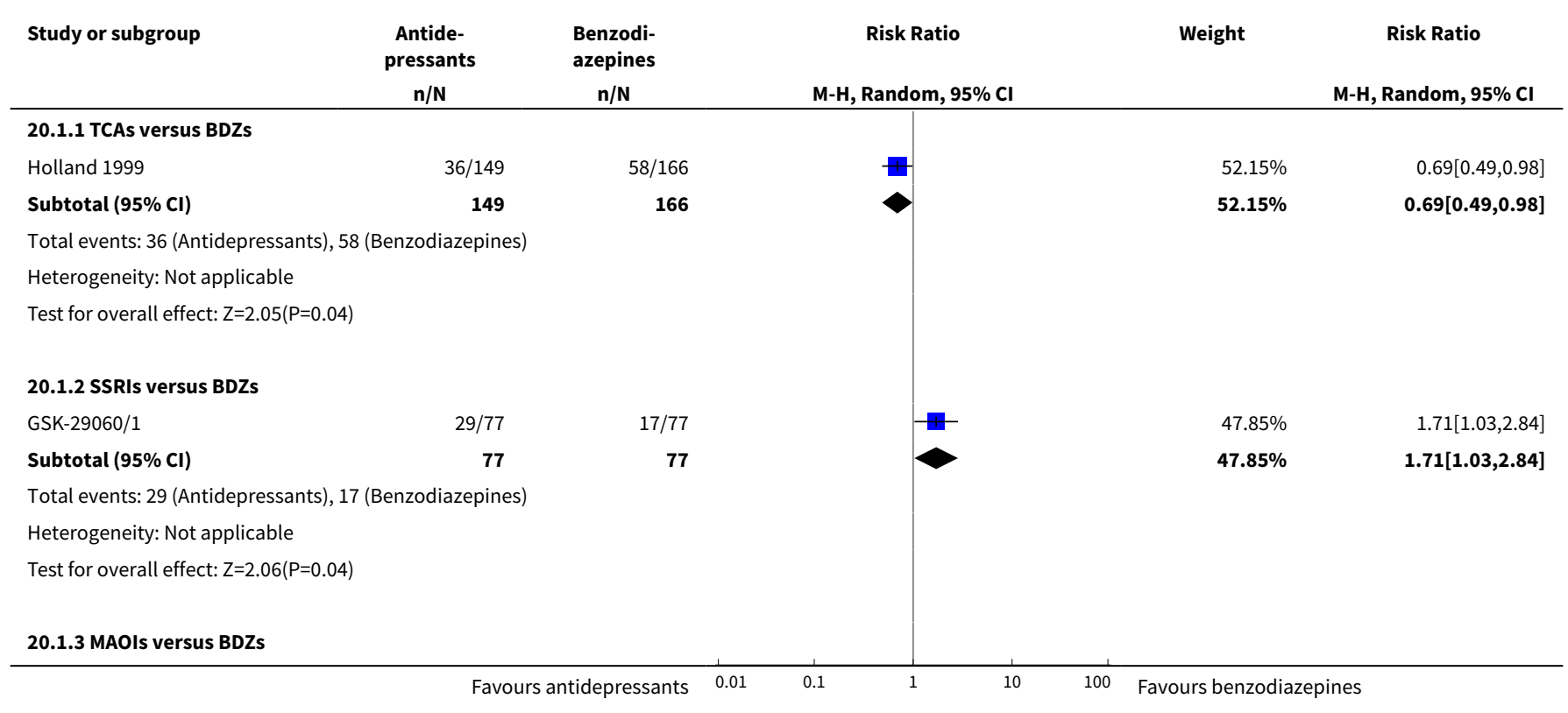




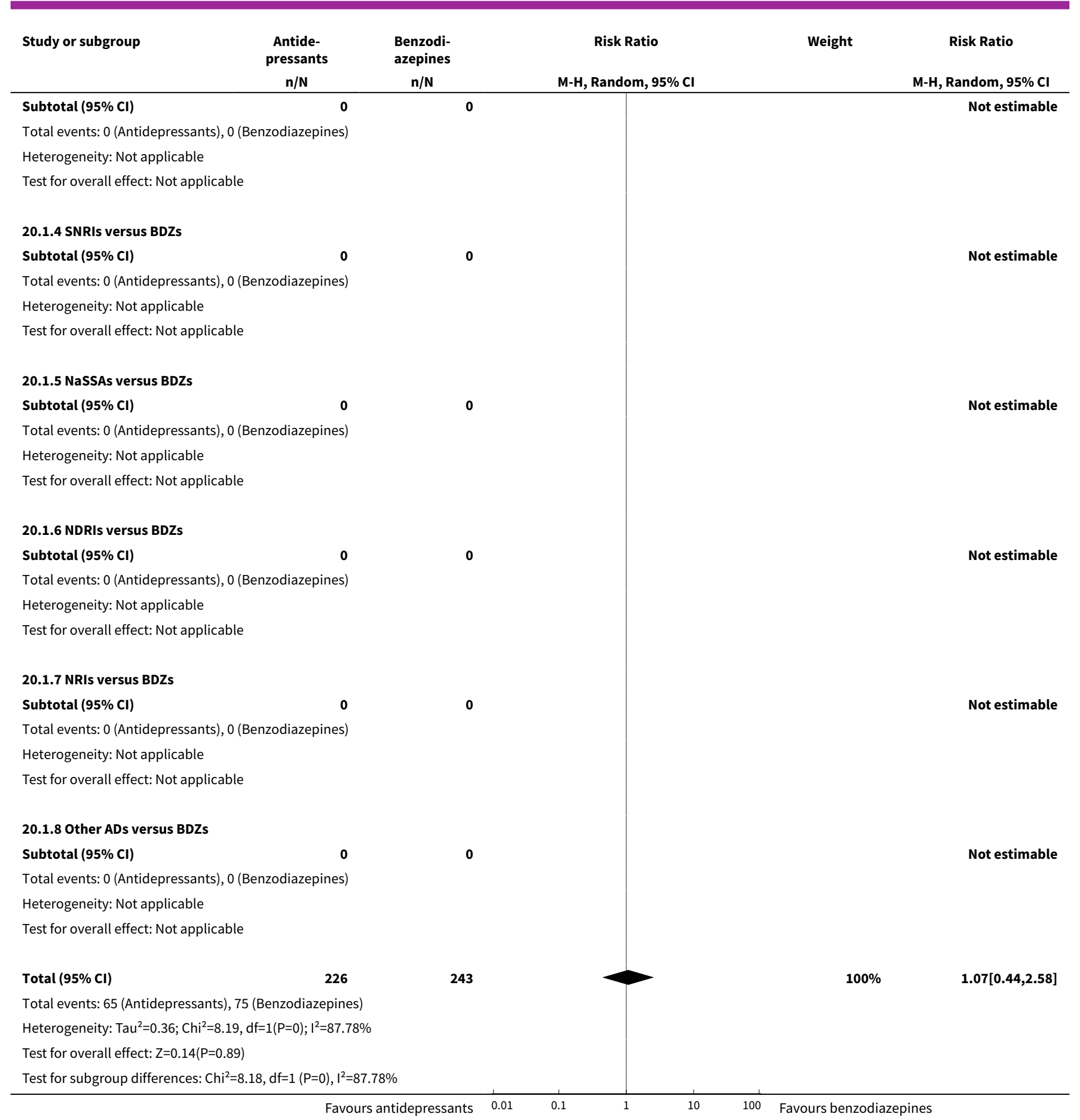

\section{Comparison 21. Funded excluded - TCAs versus benzodiazepines}

\begin{tabular}{llllll}
\hline Outcome or subgroup title & No. of studies & $\begin{array}{l}\text { No. of partici- } \\
\text { pants }\end{array}$ & Statistical method & Effect size \\
\hline 1 Total number of dropouts & 1 & 315 & Risk Ratio (M-H, Random, 95\% Cl) & $0.69[0.49,0.98]$ \\
\hline 1.1 Imipramine versus BDZs & 0 & 0 & Risk Ratio (M-H, Random, 95\% Cl) & $0.0[0.0,0.0]$ \\
\hline \hline
\end{tabular}

Antidepressants and benzodiazepines for panic disorder in adults (Review) 


\begin{tabular}{lllll}
\hline Outcome or subgroup title & No. of studies & $\begin{array}{l}\text { No. of partici- } \\
\text { pants }\end{array}$ & Statistical method & Effect size \\
\hline 1.2 Clomipramine versus BDZs & 1 & 315 & Risk Ratio (M-H, Random, 95\% Cl) & $0.69[0.49,0.98]$ \\
\hline
\end{tabular}

\section{Analysis 21.1. Comparison 21 Funded excluded - TCAs versus benzodiazepines, Outcome 1 Total number of dropouts.}

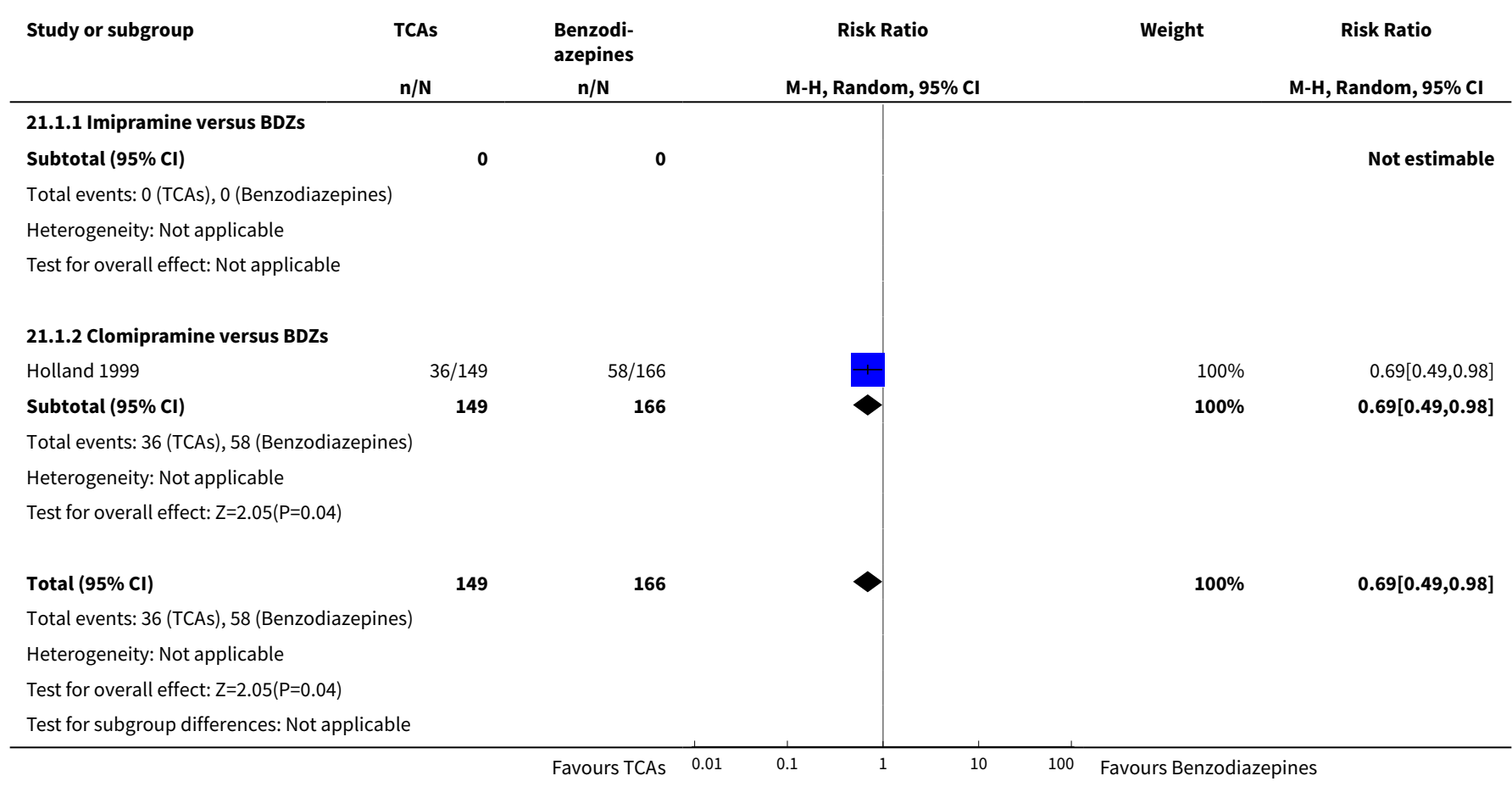

\section{Comparison 22. Funded excluded - TCAs versus SSRIS}

\begin{tabular}{lllll}
\hline Outcome or subgroup title & No. of studies & $\begin{array}{l}\text { No. of partici- } \\
\text { pants }\end{array}$ & Statistical method & Effect size \\
\hline 1 Failure to respond & 1 & 22 & Risk Ratio (M-H, Random, 95\% Cl) & $2.0[0.46,8.76]$ \\
\hline 2 Total number of dropouts & 3 & 439 & Risk Ratio (M-H, Random, 95\% Cl) & $1.14[0.77,1.68]$ \\
\hline
\end{tabular}

Analysis 22.1. Comparison 22 Funded excluded - TCAs versus SSRIs, Outcome 1 Failure to respond.

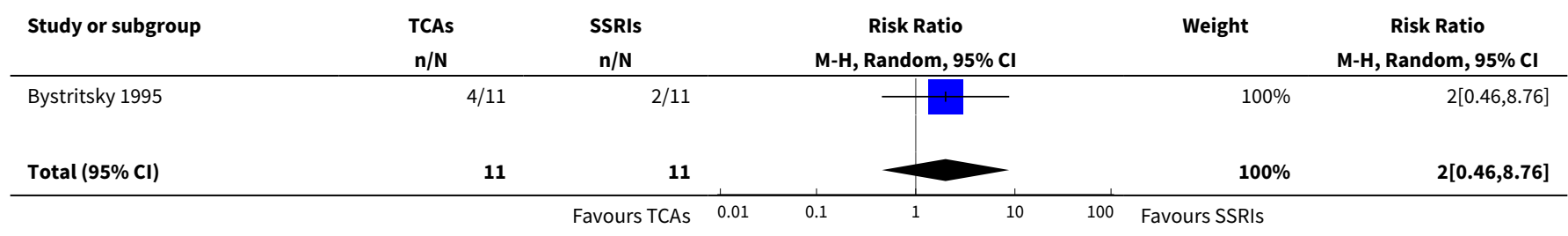




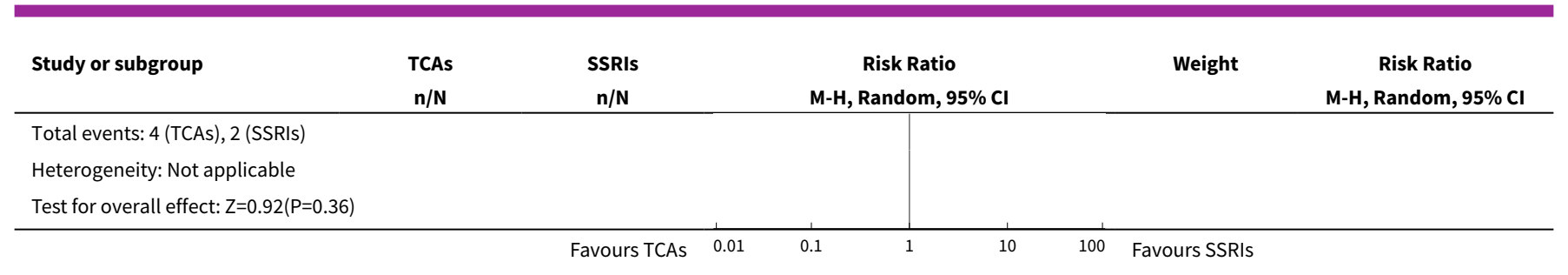

Analysis 22.2. Comparison 22 Funded excluded - TCAs versus SSRIs, Outcome 2 Total number of dropouts.

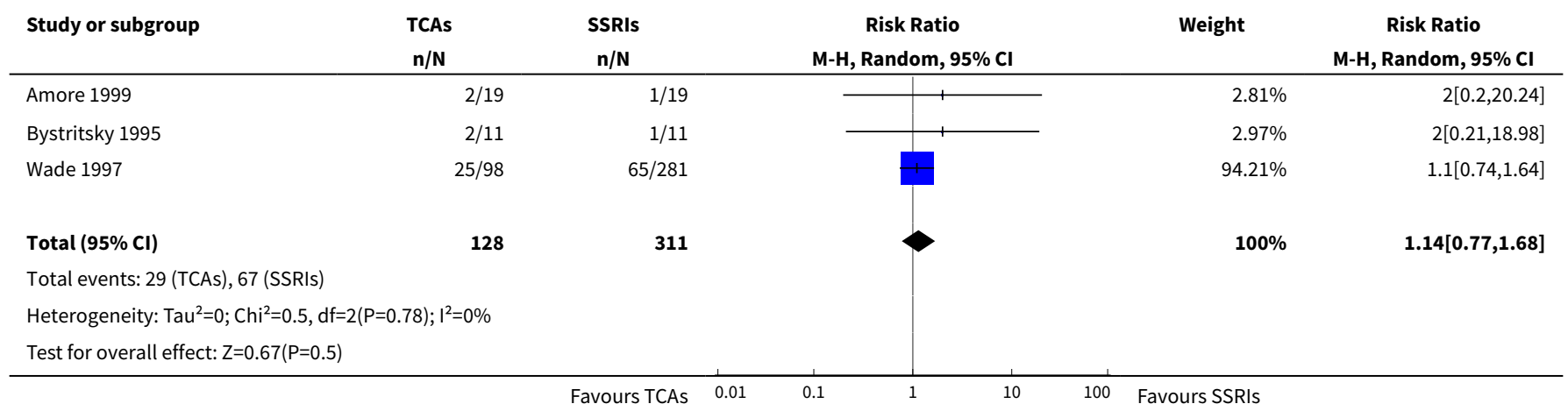

\section{Comparison 23. Funded excluded - SSRIs versus MAOIs}

\begin{tabular}{lllll}
\hline Outcome or subgroup title & No. of studies & $\begin{array}{l}\text { No. of partici- } \\
\text { pants }\end{array}$ & Statistical method & Effect size \\
\hline 1 Failure to respond & 1 & 30 & Risk Ratio (M-H, Random, 95\% Cl) & $1.25[0.69,2.26]$ \\
\hline
\end{tabular}

Analysis 23.1. Comparison 23 Funded excluded - SSRIs versus MAOIs, Outcome 1 Failure to respond.

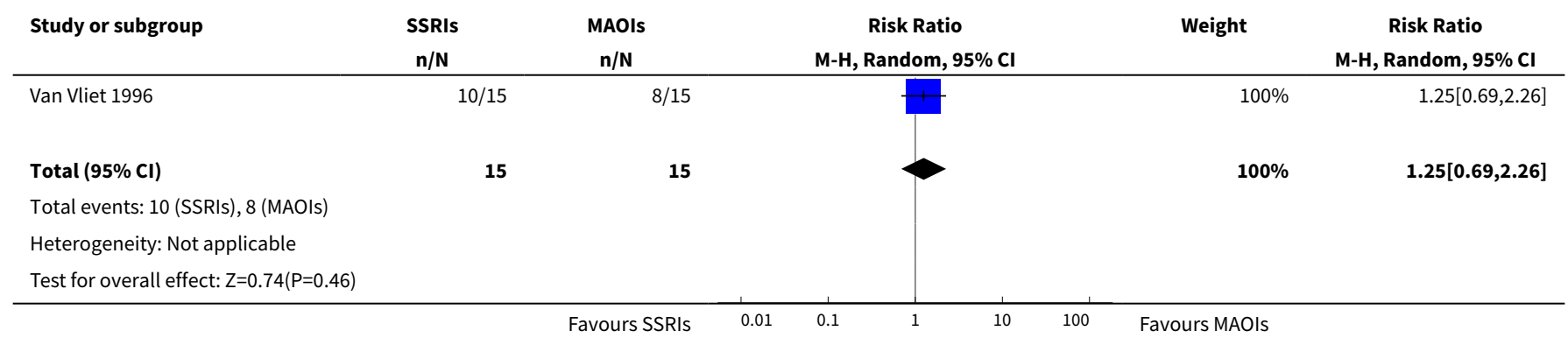


Comparison 24. Psychiatric comorbidities excluded - Antidepressants versus benzodiazepines

\begin{tabular}{|c|c|c|c|c|}
\hline $\begin{array}{l}\text { Outcome or subgroup ti- } \\
\text { tle }\end{array}$ & No. of studies & $\begin{array}{l}\text { No. of partici- } \\
\text { pants }\end{array}$ & Statistical method & Effect size \\
\hline 1 Failure to respond & 1 & 61 & Risk Ratio (M-H, Random, 95\% Cl) & $1.03[0.63,1.67]$ \\
\hline 1.1 TCAs versus BDZs & 1 & 61 & Risk Ratio (M-H, Random, 95\% Cl) & $1.03[0.63,1.67]$ \\
\hline 1.2 SSRIs versus BDZs & 0 & 0 & Risk Ratio (M-H, Random, 95\% Cl) & $0.0[0.0,0.0]$ \\
\hline $1.3 \mathrm{MAOIs}$ versus BDZs & 0 & 0 & Risk Ratio (M-H, Random, 95\% Cl) & $0.0[0.0,0.0]$ \\
\hline 1.4 SNRIs versus BDZs & 0 & 0 & Risk Ratio (M-H, Random, 95\% Cl) & $0.0[0.0,0.0]$ \\
\hline $1.5 \mathrm{NaSSAs}$ versus BDZs & 0 & 0 & Risk Ratio (M-H, Random, 95\% Cl) & $0.0[0.0,0.0]$ \\
\hline 1.6 NDRIs versus BDZs & 0 & 0 & Risk Ratio (M-H, Random, 95\% Cl) & $0.0[0.0,0.0]$ \\
\hline 1.7 NRIs versus BDZs & 0 & 0 & Risk Ratio (M-H, Random, 95\% Cl) & $0.0[0.0,0.0]$ \\
\hline 1.8 Other ADs versus BDZs & 0 & 0 & Risk Ratio (M-H, Random, 95\% Cl) & $0.0[0.0,0.0]$ \\
\hline $\begin{array}{l}2 \text { Total number of } \\
\text { dropouts }\end{array}$ & 4 & 465 & Risk Ratio (M-H, Random, 95\% Cl) & $1.67[0.64,4.36]$ \\
\hline 2.1 TCAs versus BDZs & 4 & 465 & Risk Ratio (M-H, Random, 95\% Cl) & $1.67[0.64,4.36]$ \\
\hline 2.2 SSRIs versus BDZs & 0 & 0 & Risk Ratio (M-H, Random, 95\% Cl) & $0.0[0.0,0.0]$ \\
\hline $2.3 \mathrm{MAOIs}$ versus BDZs & 0 & 0 & Risk Ratio (M-H, Random, 95\% Cl) & $0.0[0.0,0.0]$ \\
\hline 2.4 SNRIs versus BDZs & 0 & 0 & Risk Ratio (M-H, Random, 95\% Cl) & $0.0[0.0,0.0]$ \\
\hline 2.5 NaSSAs versus BDZs & 0 & 0 & Risk Ratio (M-H, Random, 95\% Cl) & $0.0[0.0,0.0]$ \\
\hline 2.6 NDRIs versus BDZs & 0 & 0 & Risk Ratio (M-H, Random, 95\% Cl) & $0.0[0.0,0.0]$ \\
\hline 2.7 NRIs versus BDZs & 0 & 0 & Risk Ratio (M-H, Random, 95\% Cl) & $0.0[0.0,0.0]$ \\
\hline 2.8 Other ADs versus BDZs & 0 & 0 & Risk Ratio (M-H, Random, 95\% Cl) & $0.0[0.0,0.0]$ \\
\hline
\end{tabular}

Analysis 24.1. Comparison 24 Psychiatric comorbidities excluded Antidepressants versus benzodiazepines, Outcome 1 Failure to respond.

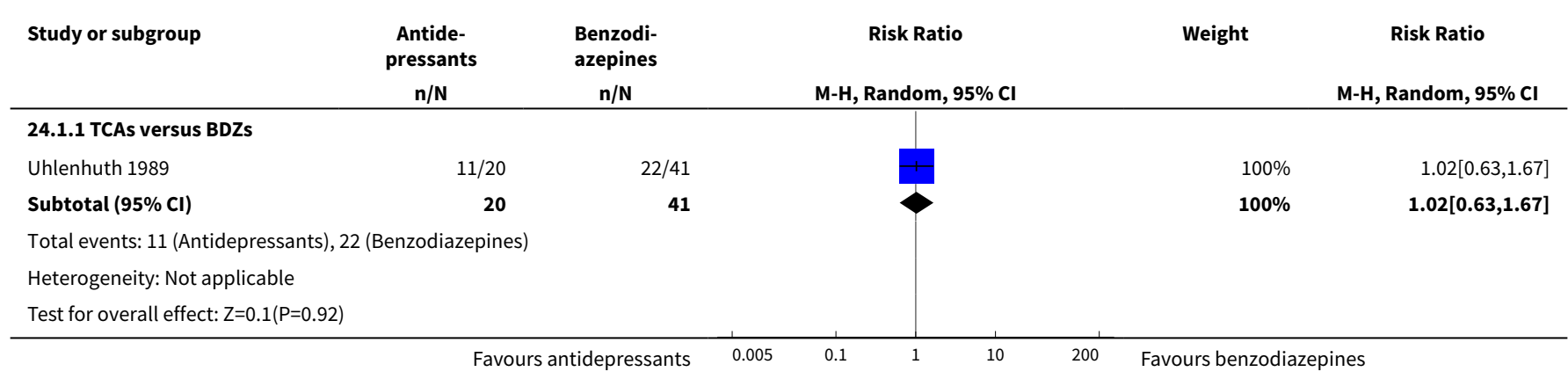




\begin{tabular}{|c|c|c|c|c|c|}
\hline Study or subgroup & $\begin{array}{c}\text { Antide- } \\
\text { pressants }\end{array}$ & $\begin{array}{l}\text { Benzodi- } \\
\text { azepines }\end{array}$ & Risk Ratio & Weight & Risk Ratio \\
\hline & $n / N$ & $n / N$ & M-H, Random, 95\% Cl & & M-H, Random, 95\% Cl \\
\hline
\end{tabular}

\subsubsection{SSRIs versus BDZs}

\subsubsection{MAOIs versus BDZs}

Total events: 0 (Antidepressants), 0 (Benzodiazepines) Heterogeneity: Not applicable

Test for overall effect: Not applicable

\subsubsection{SNRIs versus BDZs} Heterogeneity: Not applicable

Test for overall effect: Not applicable

\subsubsection{NaSSAs versus BDZs}

Total events: 0 (Antidepressants), 0 (Benzodiazepines) Heterogeneity: Not applicable

Test for overall effect: Not applicable

\subsubsection{NDRIs versus BDZs}

Total events: 0 (Antidepressants), 0 (Benzodiazepines) Heterogeneity: Not applicable

Test for overall effect: Not applicable

\subsubsection{NRIs versus BDZs}

Total events: 0 (Antidepressants), 0 (Benzodiazepines) Heterogeneity: Not applicable

Test for overall effect: Not applicable

\subsubsection{Other ADs versus BDZs}

Total events: 0 (Antidepressants), 0 (Benzodiazepines)

Heterogeneity: Not applicable

Test for overall effect: Not applicable 
Analysis 24.2. Comparison 24 Psychiatric comorbidities excluded Antidepressants versus benzodiazepines, Outcome 2 Total number of dropouts.

\begin{tabular}{lrr} 
Study or subgroup & $\begin{array}{c}\text { Antide- } \\
\text { pressants } \\
\text { n/N }\end{array}$ & $\begin{array}{c}\text { Benzodi- } \\
\text { azepines } \\
\text { n/N }\end{array}$ \\
\hline 24.2.1 TCAs versus BDZs & & \\
Holland 1999 & $36 / 149$ & $58 / 166$ \\
Schweizer 1993 & $14 / 34$ & $4 / 37$ \\
Sheikh 1999 & $1 / 10$ & $0 / 8$ \\
Uhlenhuth 1989 & $10 / 20$ & $10 / 41$ \\
Subtotal (95\% Cl) & $\mathbf{2 1 3}$ & $\mathbf{2 5 2}$
\end{tabular}

Risk Ratio

M-H, Random, $95 \% \mathrm{CI}$

Weight

Risk Ratio

M-H, Random, $95 \% \mathrm{Cl}$

Total events: 61 (Antidepressants), 72 (Benzodiazepines) Heterogeneity: $\mathrm{Tau}^{2}=0.65 ; \mathrm{Chi}^{2}=15.43, \mathrm{df}=3(\mathrm{P}=0) ; \mathrm{I}^{2}=80.56 \%$

Test for overall effect: $Z=1.04(P=0.3)$

\subsubsection{SSRIs versus BDZs}

Test for overall effect: Not applicable

\subsubsection{MAOIs versus BDZs}

Total events: 0 (Antidepressants), 0 (Benzodiazepines) Heterogeneity: Not applicable

Test for overall effect: Not applicable

\subsubsection{SNRIs versus BDZs} Heterogeneity: Not applicable

Test for overall effect: Not applicable

\subsubsection{NaSSAs versus BDZs} Heterogeneity: Not applicable

Test for overall effect: Not applicable

24.2.6 NDRIs versus BDZs

Total events: 0 (Antidepressants), 0 (Benzodiazepines) Heterogeneity: Not applicable

Test for overall effect: Not applicable

\subsubsection{NRIs versus BDZs}

Total events: 0 (Antidepressants), 0 (Benzodiazepines)

Heterogeneity: Not applicable

Test for overall effect: Not applicable

\subsubsection{Other ADs versus BDZs}




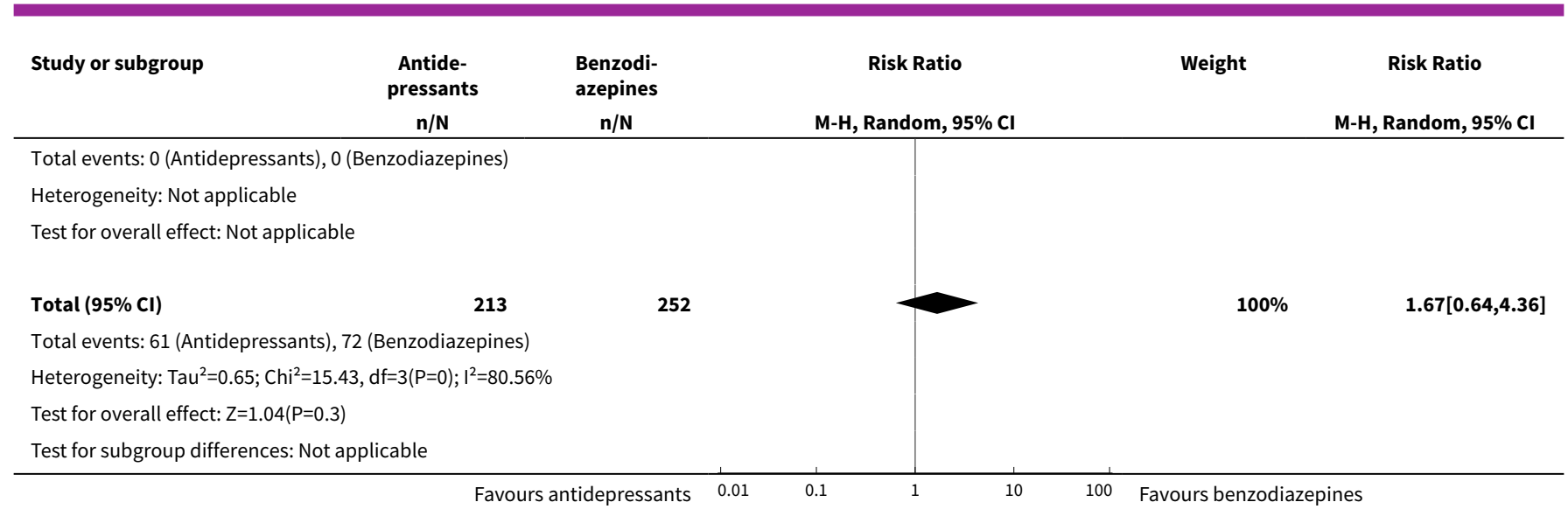

\section{Comparison 25. Psychiatric comorbidities excluded - TCAs versus benzodiazepines}

\begin{tabular}{llllll}
\hline Outcome or subgroup title & No. of studies & $\begin{array}{l}\text { No. of partici- } \\
\text { pants }\end{array}$ & Statistical method & Effect size \\
\hline 1 Total number of dropouts & 4 & 465 & Risk Ratio (M-H, Random, 95\% Cl) & $1.67[0.64,4.36]$ \\
\hline 1.1 Imipramine versus BDZs & 3 & 150 & Risk Ratio (M-H, Random, 95\% Cl) & $2.50[1.42,4.39]$ \\
\hline 1.2 Clomipramine versus BDZs & 1 & 315 & Risk Ratio (M-H, Random, 95\% Cl) & $0.69[0.49,0.98]$ \\
\hline
\end{tabular}

Analysis 25.1. Comparison 25 Psychiatric comorbidities excluded -

\section{TCAs versus benzodiazepines, Outcome 1 Total number of dropouts.}

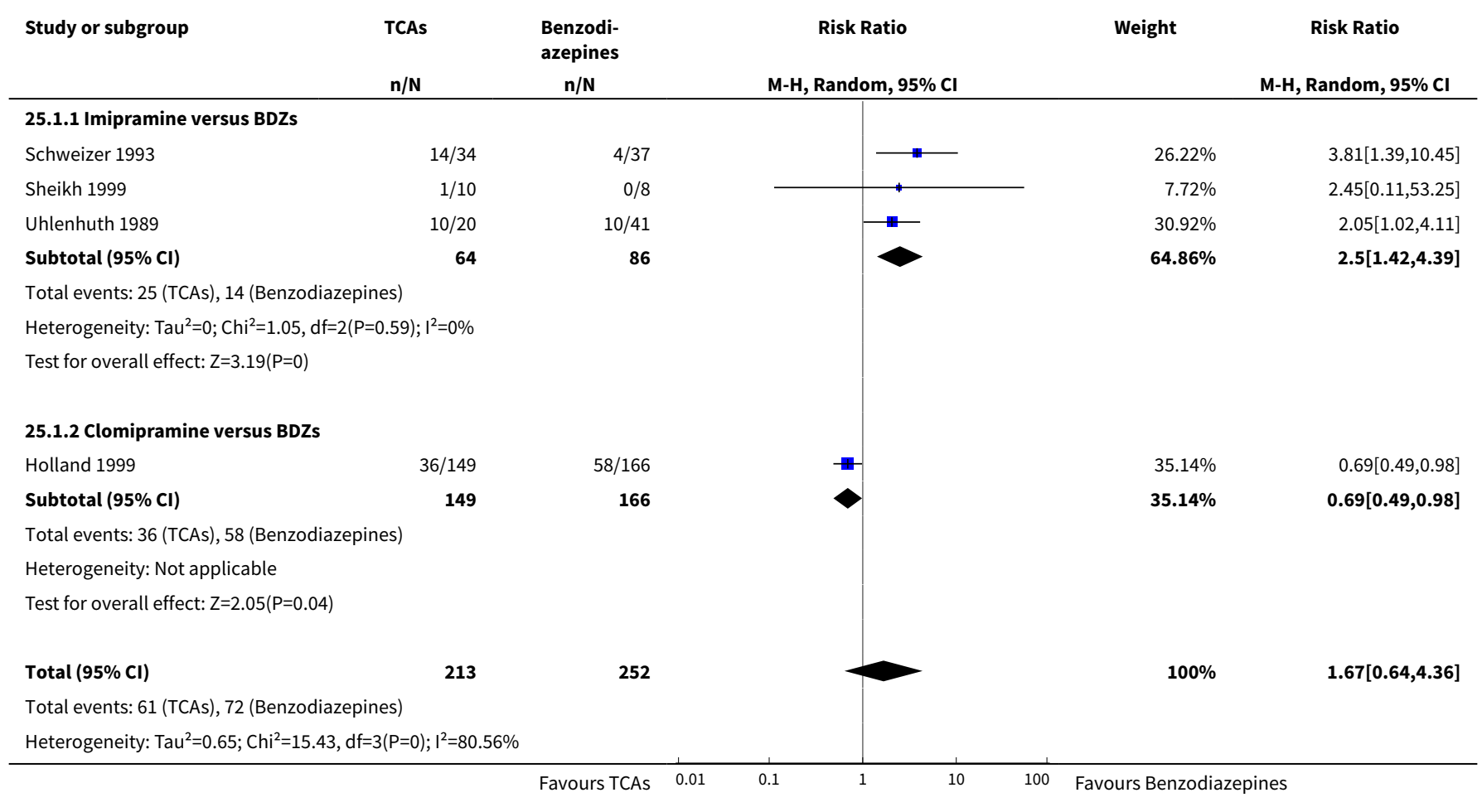




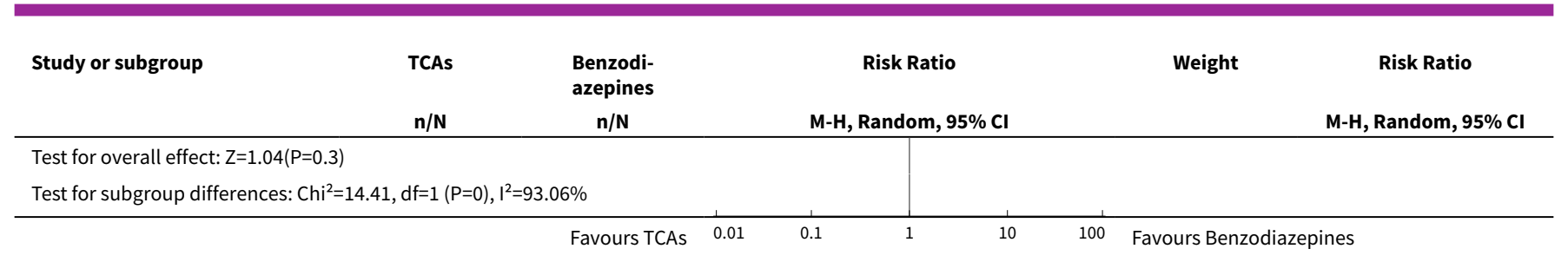

Comparison 26. Psychiatric comorbidities excluded - TCAs versus SSRIS

\begin{tabular}{lllll}
\hline Outcome or subgroup title & No. of studies & $\begin{array}{l}\text { No. of partici- } \\
\text { pants }\end{array}$ & Statistical method & Effect size \\
\hline 1 Failure to respond & 3 & 416 & Risk Ratio (M-H, Random, 95\% Cl) & $1.03[0.66,1.61]$ \\
\hline 2 Total number of dropouts & 6 & 906 & Risk Ratio (M-H, Random, 95\% Cl) & $0.96[0.62,1.49]$ \\
\hline
\end{tabular}

Analysis 26.1. Comparison 26 Psychiatric comorbidities excluded - TCAs versus SSRIs, Outcome 1 Failure to respond.

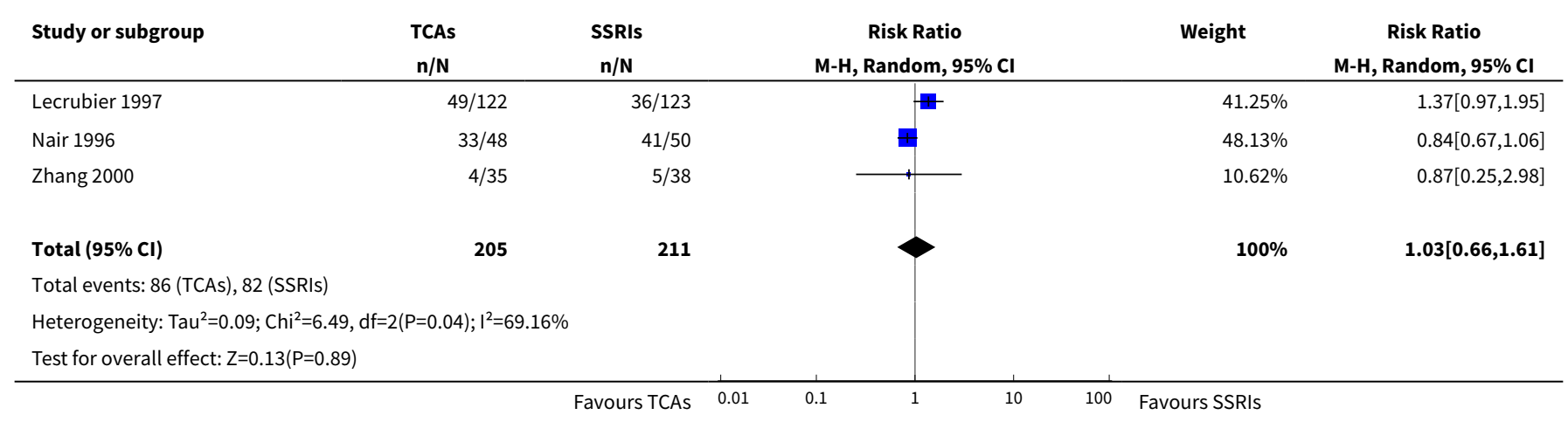

Analysis 26.2. Comparison 26 Psychiatric comorbidities excluded - TCAs versus SSRIs, Outcome 2 Total number of dropouts.

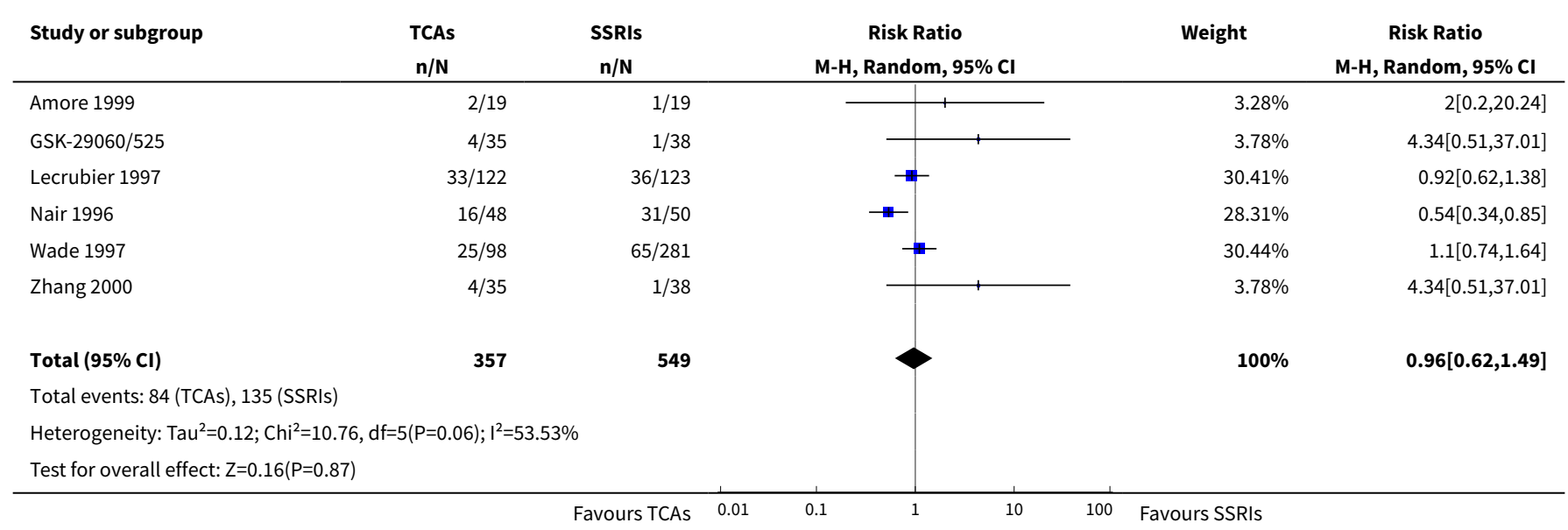


Comparison 27. Psychiatric comorbidities excluded - Individual antidepressants versus individual antidepressants (within the same class)

\begin{tabular}{|c|c|c|c|c|}
\hline Outcome or subgroup title & No. of studies & $\begin{array}{l}\text { No. of partici- } \\
\text { pants }\end{array}$ & Statistical method & Effect size \\
\hline 1 Failure to respond & 3 & & $\begin{array}{l}\text { Risk Ratio (M-H, Random, 95\% } \\
\text { Cl) }\end{array}$ & Subtotals only \\
\hline $\begin{array}{l}\text { 1.1 Escitalopram versus citalo- } \\
\text { pram }\end{array}$ & 1 & 255 & $\begin{array}{l}\text { Risk Ratio (M-H, Random, 95\% } \\
\text { Cl) }\end{array}$ & $0.92[0.79,1.06]$ \\
\hline 1.2 Fluoxetine versus Citalopram & 1 & 42 & $\begin{array}{l}\text { Risk Ratio (M-H, Random, 95\% } \\
\mathrm{Cl} \text { ) }\end{array}$ & $0.57[0.20,1.66]$ \\
\hline 1.3 Sertraline versus paroxetine & 1 & 225 & $\begin{array}{l}\text { Risk Ratio (M-H, Random, 95\% } \\
\text { Cl) }\end{array}$ & $0.98[0.78,1.23]$ \\
\hline 2 Total number of dropouts & 3 & & $\begin{array}{l}\text { Risk Ratio (M-H, Random, 95\% } \\
\text { Cl) }\end{array}$ & Subtotals only \\
\hline $\begin{array}{l}2.1 \text { Escitalopram versus Citalo- } \\
\text { pram }\end{array}$ & 1 & 255 & $\begin{array}{l}\text { Risk Ratio (M-H, Random, 95\% } \\
\text { CI) }\end{array}$ & $0.80[0.53,1.20]$ \\
\hline 2.2 Fluoxetine versus Citalopram & 1 & 42 & $\begin{array}{l}\text { Risk Ratio (M-H, Random, 95\% } \\
\text { Cl) }\end{array}$ & $1.0[0.07,14.95]$ \\
\hline 2.3 Sertraline versus Paroxetine & 1 & 225 & $\begin{array}{l}\text { Risk Ratio (M-H, Random, 95\% } \\
\text { Cl) }\end{array}$ & $0.85[0.57,1.26]$ \\
\hline $\begin{array}{l}2.4 \text { Imipramine versus } \\
\text { Clomipramine }\end{array}$ & 0 & 0 & $\begin{array}{l}\text { Risk Ratio (M-H, Random, 95\% } \\
\mathrm{Cl} \text { ) }\end{array}$ & $0.0[0.0,0.0]$ \\
\hline
\end{tabular}

\section{Analysis 27.1. Comparison 27 Psychiatric comorbidities excluded - Individual antidepressants versus individual antidepressants (within the same class), Outcome 1 Failure to respond.}

\begin{tabular}{|c|c|c|c|c|c|}
\hline Study or subgroup & $\begin{array}{c}\text { Antide- } \\
\text { pressant } 1 \\
\text { n/N }\end{array}$ & $\begin{array}{c}\text { Antide- } \\
\text { pressant } 2 \\
\text { n/N }\end{array}$ & $\begin{array}{c}\text { Risk Ratio } \\
\text { M-H, Random, } 95 \% \mathrm{CI}\end{array}$ & Weight & $\begin{array}{c}\text { Risk Ratio } \\
\text { M-H, Random, 95\% Cl }\end{array}$ \\
\hline \multicolumn{6}{|c|}{ 27.1.1 Escitalopram versus citalopram } \\
\hline Stahl 2003 & $90 / 129$ & $96 / 126$ & & $100 \%$ & $0.92[0.79,1.06]$ \\
\hline Subtotal $(95 \% \mathrm{Cl})$ & 129 & 126 & $\checkmark$ & $100 \%$ & $0.92[0.79,1.06]$ \\
\hline \multicolumn{6}{|c|}{ Total events: 90 (Antidepressant 1), 96 (Antidepressant 2) } \\
\hline \multicolumn{6}{|c|}{ Test for overall effect: $Z=1.15(P=0.25)$} \\
\hline \multicolumn{6}{|c|}{ 27.1.2 Fluoxetine versus Citalopram } \\
\hline Amore 1999 bis & $4 / 21$ & $7 / 21$ & & $100 \%$ & $0.57[0.2,1.66]$ \\
\hline Subtotal $(95 \% \mathrm{Cl})$ & 21 & 21 & & $100 \%$ & $0.57[0.2,1.66]$ \\
\hline \multicolumn{6}{|c|}{ Total events: 4 (Antidepressant 1), 7 (Antidepressant 2) } \\
\hline Test for overall effect & & & & & \\
\hline
\end{tabular}




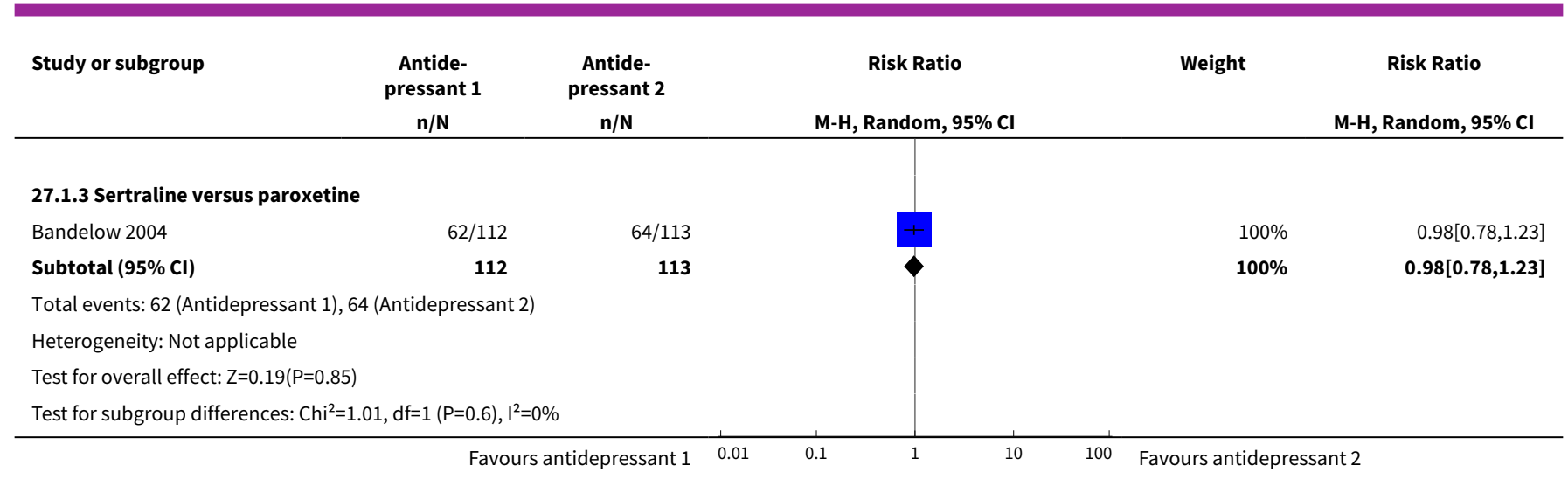

Analysis 27.2. Comparison 27 Psychiatric comorbidities excluded - Individual antidepressants versus individual antidepressants (within the same class), Outcome 2 Total number of dropouts.

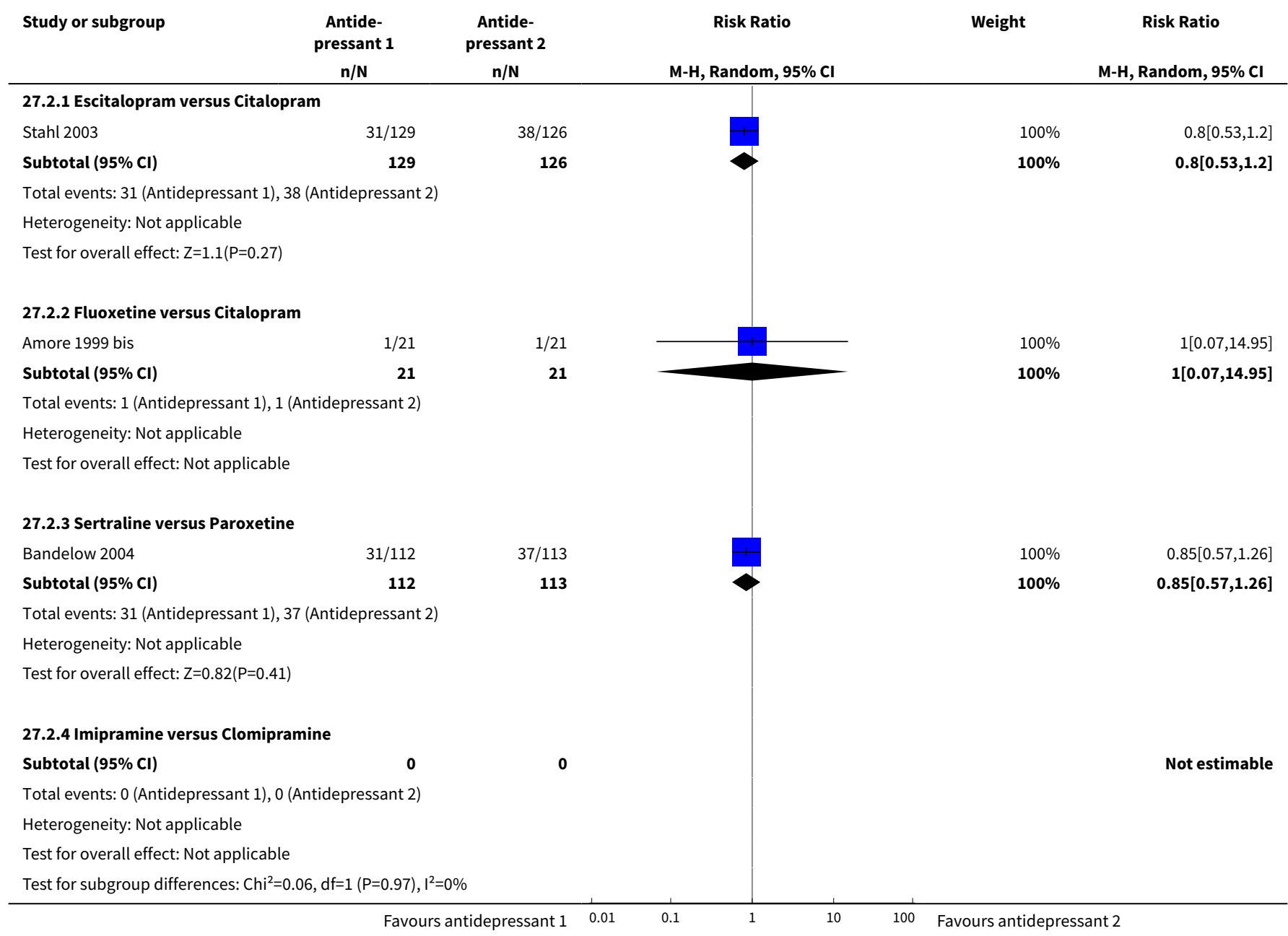


Comparison 28. Psychiatric comorbidities excluded - Individual benzodiazepines versus individual benzodiazepines

\begin{tabular}{|c|c|c|c|c|}
\hline Outcome or subgroup title & No. of studies & $\begin{array}{l}\text { No. of partici- } \\
\text { pants }\end{array}$ & Statistical method & Effect size \\
\hline 1 Total number of dropouts & 1 & 159 & Risk Ratio (M-H, Random, 95\% Cl) & $0.84[0.44,1.64]$ \\
\hline 1.1 Alprazolam versus diazepam & 1 & 159 & Risk Ratio (M-H, Random, 95\% Cl) & $0.84[0.44,1.64]$ \\
\hline $\begin{array}{l}1.2 \text { Alprazolam versus clon- } \\
\text { azepam }\end{array}$ & 0 & 0 & Risk Ratio (M-H, Random, 95\% Cl) & $0.0[0.0,0.0]$ \\
\hline
\end{tabular}

Analysis 28.1. Comparison 28 Psychiatric comorbidities excluded - Individual benzodiazepines versus individual benzodiazepines, Outcome 1 Total number of dropouts.

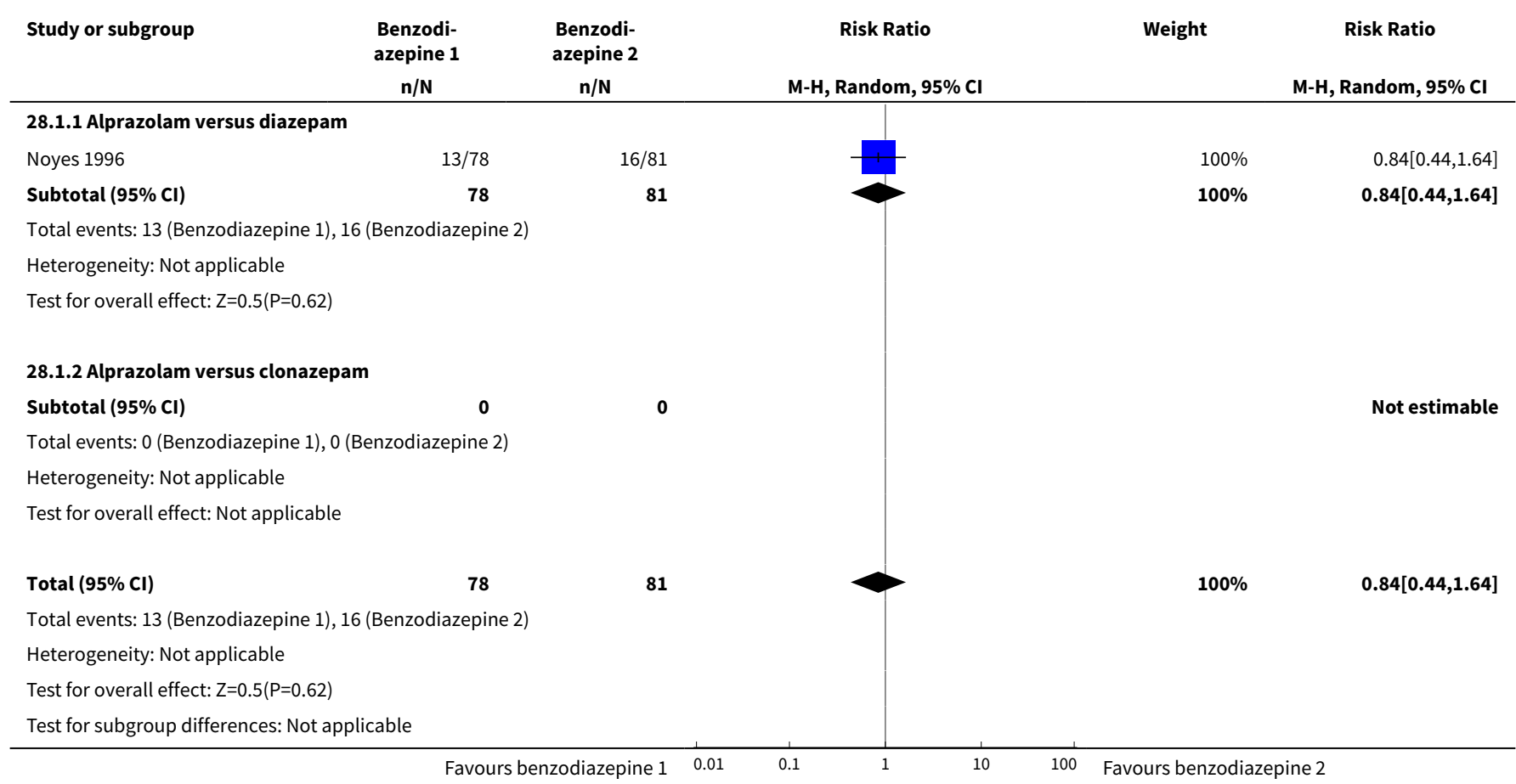

\section{Comparison 29. Imputation excluded - TCAs versus SSRIs}

\begin{tabular}{llllll}
\hline Outcome or subgroup title & No. of studies & $\begin{array}{l}\text { No. of partici- } \\
\text { pants }\end{array}$ & Statistical method & Effect size \\
\hline 1 Failure to respond & 2 & 318 & Risk Ratio (M-H, Random, 95\% Cl) & $1.33[0.95,1.86]$ \\
\hline 2 Total number of dropouts & 5 & 808 & Risk Ratio (M-H, Random, 95\% Cl) & $1.08[0.80,1.45]$ \\
\hline
\end{tabular}


Analysis 29.1. Comparison 29 Imputation excluded - TCAs versus SSRIs, Outcome 1 Failure to respond.

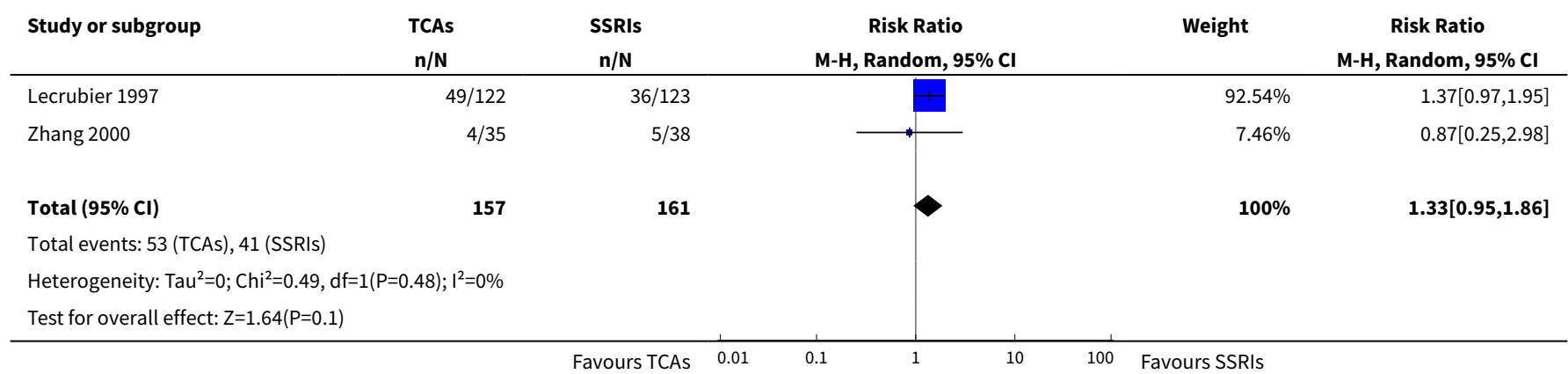

Analysis 29.2. Comparison 29 Imputation excluded - TCAs versus SSRIs, Outcome 2 Total number of dropouts.

\begin{tabular}{|c|c|c|c|c|c|}
\hline Study or subgroup & $\begin{array}{l}\text { TCAs } \\
\mathrm{n} / \mathrm{N}\end{array}$ & $\begin{array}{l}\text { SSRIs } \\
\mathrm{n} / \mathrm{N}\end{array}$ & $\begin{array}{c}\text { Risk Ratio } \\
\text { M-H, Random, } 95 \% \mathrm{Cl}\end{array}$ & Weight & $\begin{array}{c}\text { Risk Ratio } \\
\text { M-H, Random, } 95 \% \mathrm{CI}\end{array}$ \\
\hline Amore 1999 & $2 / 19$ & $1 / 19$ & 1 & $1.62 \%$ & $2[0.2,20.24]$ \\
\hline GSK-29060/525 & $4 / 35$ & $1 / 38$ & & $1.89 \%$ & $4.34[0.51,37.01]$ \\
\hline Lecrubier 1997 & $33 / 122$ & $36 / 123$ & \# & $47.23 \%$ & $0.92[0.62,1.38]$ \\
\hline Wade 1997 & $25 / 98$ & $65 / 281$ & + & $47.36 \%$ & $1.1[0.74,1.64]$ \\
\hline Zhang 2000 & $4 / 35$ & $1 / 38$ & $\longrightarrow$ & $1.89 \%$ & $4.34[0.51,37.01]$ \\
\hline Total $(95 \% \mathrm{Cl})$ & 309 & 499 & 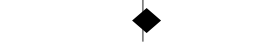 & $100 \%$ & $1.08[0.8,1.45]$ \\
\hline \multicolumn{6}{|c|}{ Total events: 68 (TCAs), 104 (SSRIs) } \\
\hline \multicolumn{6}{|c|}{ Heterogeneity: $\mathrm{Tau}^{2}=0.01 ; \mathrm{Chi}^{2}=4.18, \mathrm{df}=4(\mathrm{P}=0.38) ; \mathrm{I}^{2}=4.23 \%$} \\
\hline \multicolumn{6}{|c|}{ Test for overall effect: $Z=0.5(P=0.61)$} \\
\hline
\end{tabular}

Comparison 30. Imputation excluded - Individual antidepressants versus individual antidepressants (within the same class)

\begin{tabular}{|c|c|c|c|c|}
\hline Outcome or subgroup title & No. of studies & $\begin{array}{l}\text { No. of partici- } \\
\text { pants }\end{array}$ & Statistical method & Effect size \\
\hline 1 Failure to respond & 2 & & Risk Ratio (M-H, Random, 95\% Cl) & Subtotals only \\
\hline $\begin{array}{l}\text { 1.1 Escitalopram versus citalo- } \\
\text { pram }\end{array}$ & 0 & 0 & Risk Ratio (M-H, Random, 95\% Cl) & $0.0[0.0,0.0]$ \\
\hline $\begin{array}{l}\text { 1.2 Fluoxetine versus Citalo- } \\
\text { pram }\end{array}$ & 1 & 42 & Risk Ratio (M-H, Random, 95\% Cl) & $0.57[0.20,1.66]$ \\
\hline 1.3 Sertraline versus paroxetine & 1 & 321 & Risk Ratio (M-H, Random, 95\% Cl) & $0.95[0.70,1.30]$ \\
\hline 2 Total number of dropouts & 3 & & Risk Ratio (M-H, Random, 95\% Cl) & Subtotals only \\
\hline $\begin{array}{l}2.1 \text { Escitalopram versus Citalo- } \\
\text { pram }\end{array}$ & 0 & 0 & Risk Ratio (M-H, Random, 95\% Cl) & $0.0[0.0,0.0]$ \\
\hline
\end{tabular}




\begin{tabular}{lllll}
\hline Outcome or subgroup title & No. of studies & $\begin{array}{l}\text { No. of partici- } \\
\text { pants }\end{array}$ & Statistical method & Effect size \\
\hline $\begin{array}{l}\text { 2.2 Fluoxetine versus Citalo- } \\
\text { pram }\end{array}$ & 1 & 42 & Risk Ratio (M-H, Random, 95\% Cl) & $1.0[0.07,14.95]$ \\
\hline $\begin{array}{l}2.3 \text { Sertraline versus Paroxetine } \\
\text { 2.4 Imipramine versus }\end{array}$ & 1 & 321 & Risk Ratio (M-H, Random, 95\% Cl) & $0.62[0.40,0.97]$ \\
\hline \begin{tabular}{l} 
Clomipramine \\
\hline
\end{tabular} & 1 & 40 & Risk Ratio (M-H, Random, 95\% Cl) & $0.86[0.35,2.10]$ \\
\hline
\end{tabular}

\section{Analysis 30.1. Comparison 30 Imputation excluded - Individual antidepressants versus individual antidepressants (within the same class), Outcome 1 Failure to respond.}

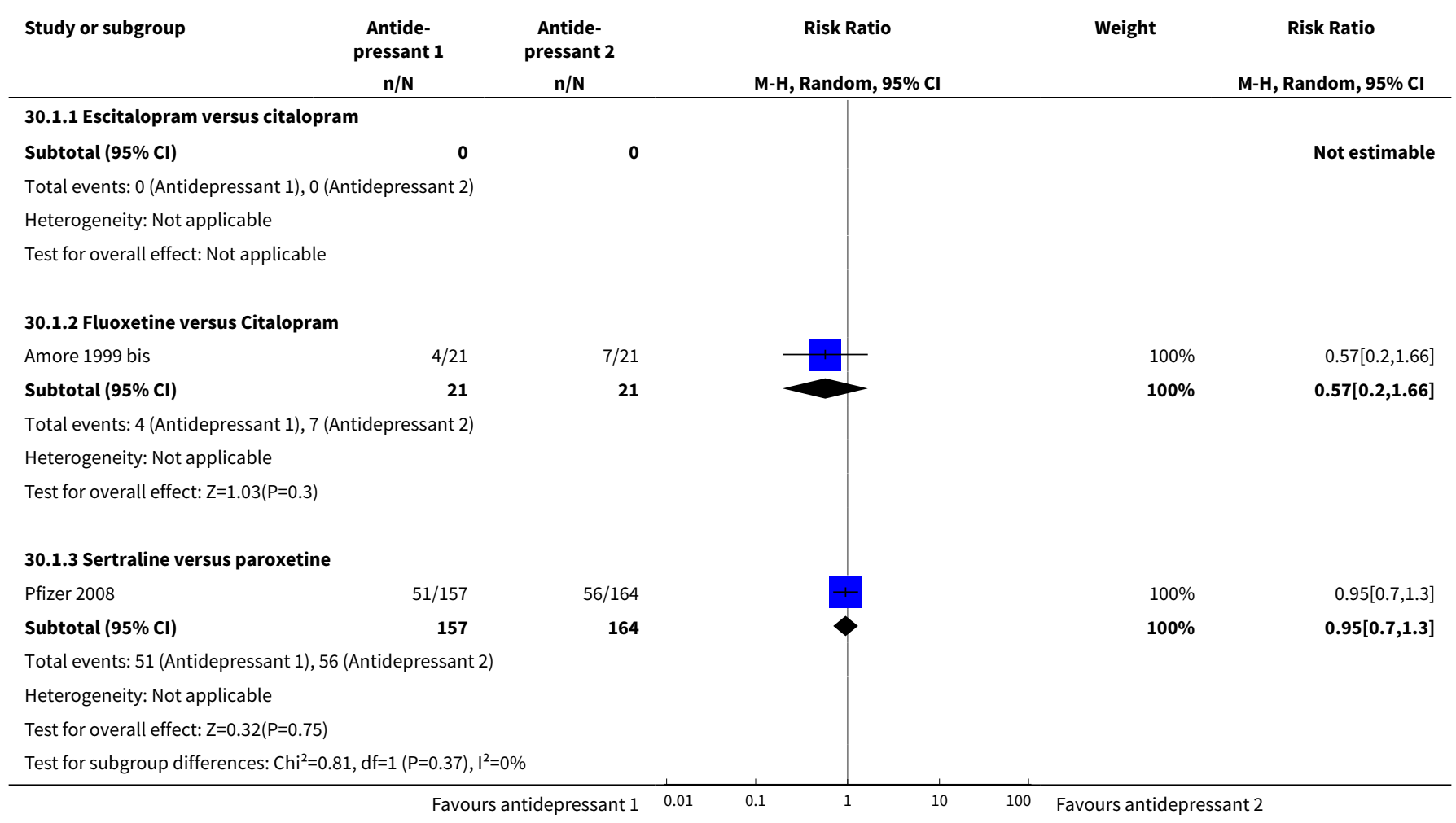

Analysis 30.2. Comparison 30 Imputation excluded - Individual antidepressants versus individual antidepressants (within the same class), Outcome 2 Total number of dropouts.

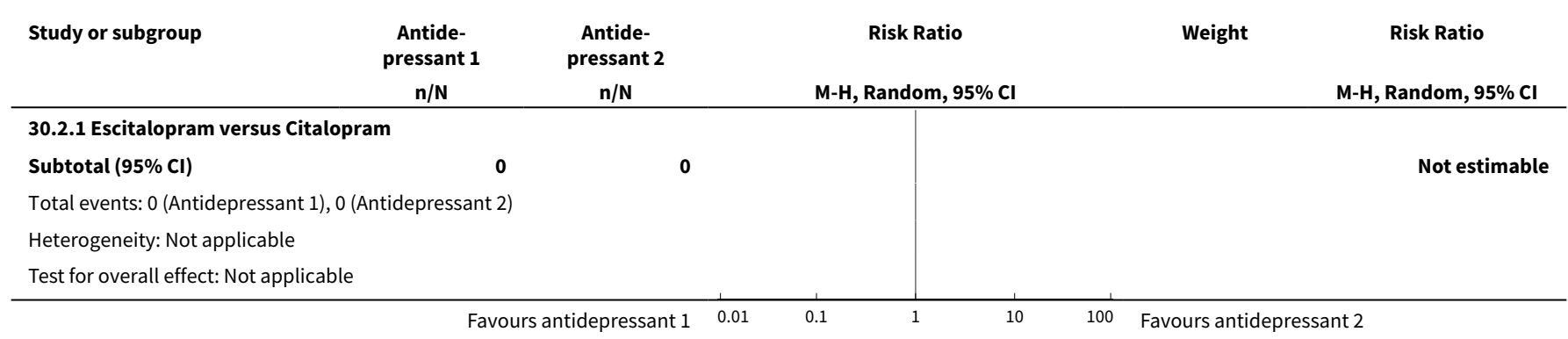




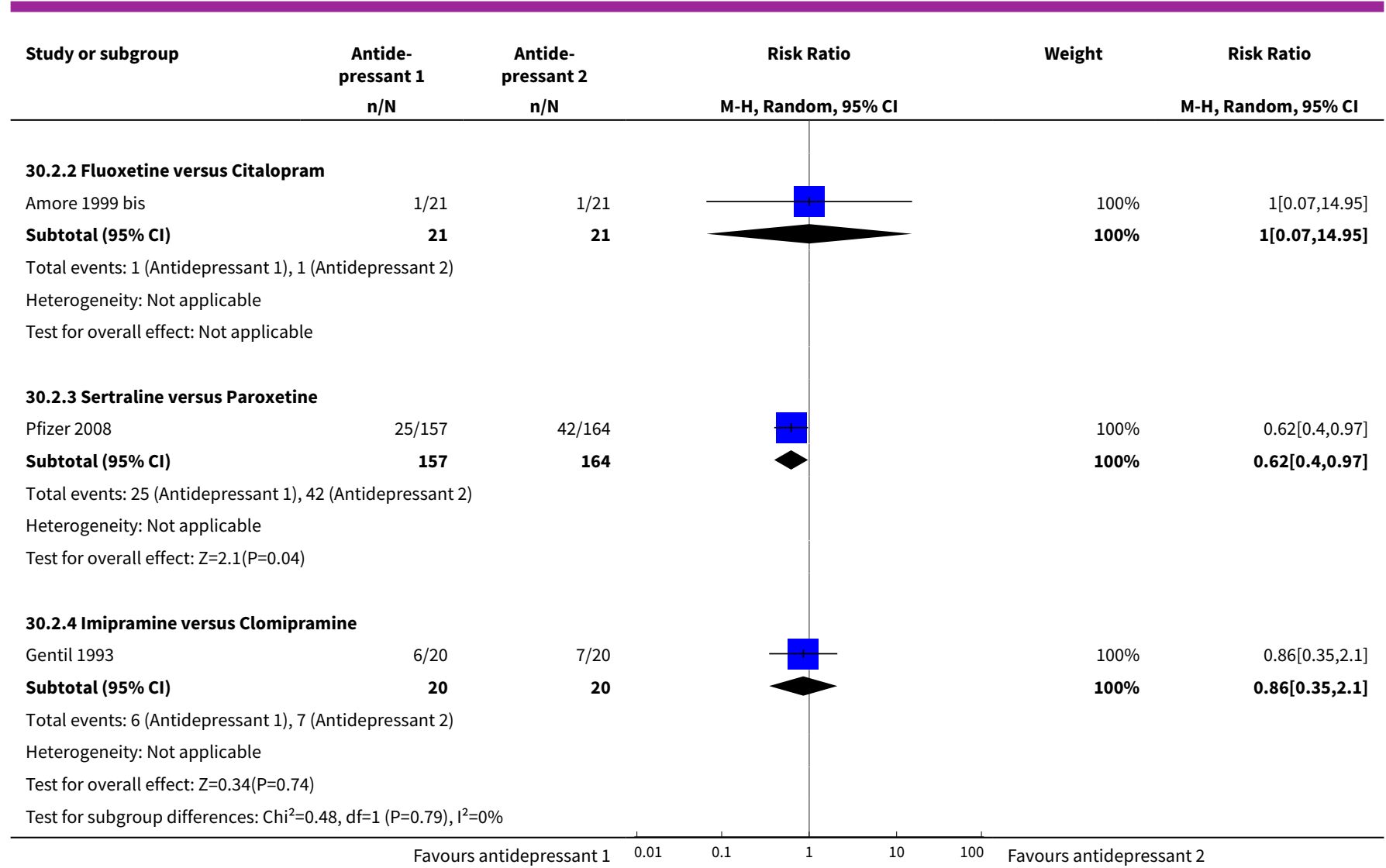

Comparison 31. Irregular benzodiazepines use excluded - TCAs versus SSRIs

\begin{tabular}{llllll}
\hline Outcome or subgroup title & No. of studies & $\begin{array}{l}\text { No. of partici- } \\
\text { pants }\end{array}$ & Statistical method & Effect size \\
\hline 1 Failure to respond & 2 & 95 & Risk Ratio (M-H, Random, 95\% Cl) & $1.22[0.47,3.15]$ \\
\hline 2 Total number of dropouts & 5 & 585 & Risk Ratio (M-H, Random, 95\% Cl) & $1.24[0.85,1.81]$ \\
\hline
\end{tabular}

Analysis 31.1. Comparison 31 Irregular benzodiazepines use excluded - TCAs versus SSRIs, Outcome 1 Failure to respond.

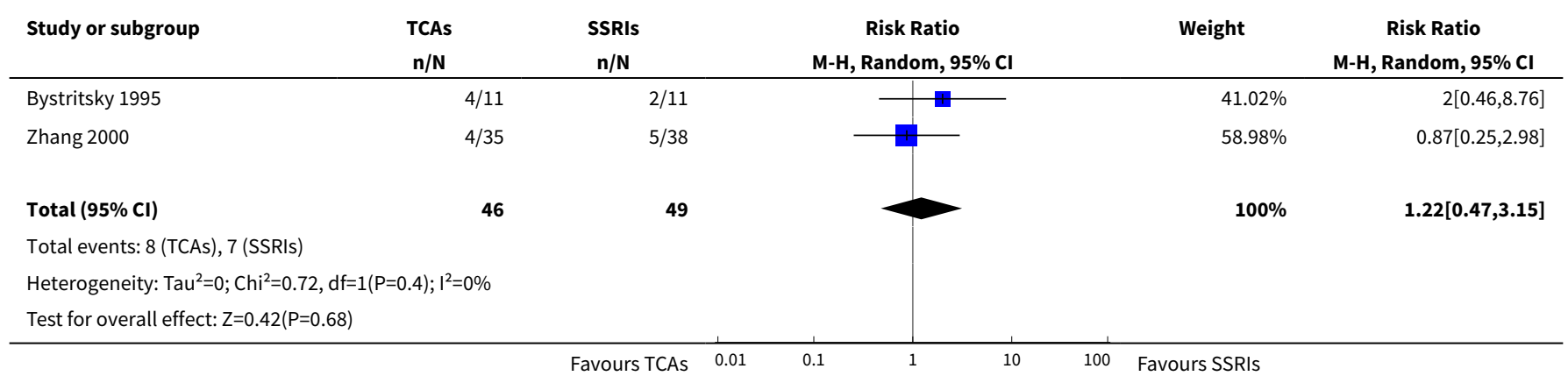


Analysis 31.2. Comparison 31 Irregular benzodiazepines use excluded - TCAs versus SSRIs, Outcome 2 Total number of dropouts.

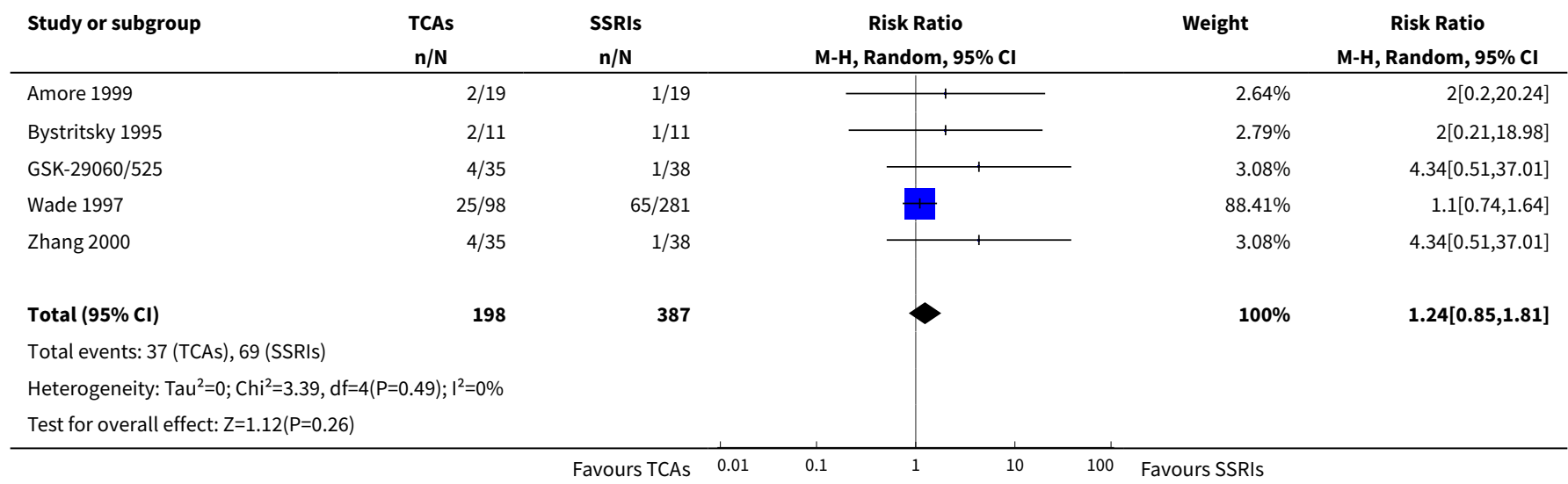

Comparison 32. Irregular benzodiazepines use excluded - SSRIs versus MAOIs

\begin{tabular}{lllll}
\hline Outcome or subgroup title & No. of studies & $\begin{array}{l}\text { No. of partici- } \\
\text { pants }\end{array}$ & Statistical method & Effect size \\
\hline 1 Failure to respond & 1 & 366 & Risk Ratio (M-H, Random, 95\% Cl) & $1.08[0.76,1.54]$ \\
\hline
\end{tabular}

Analysis 32.1. Comparison 32 Irregular benzodiazepines use excluded - SSRIs versus MAOIs, Outcome 1 Failure to respond.

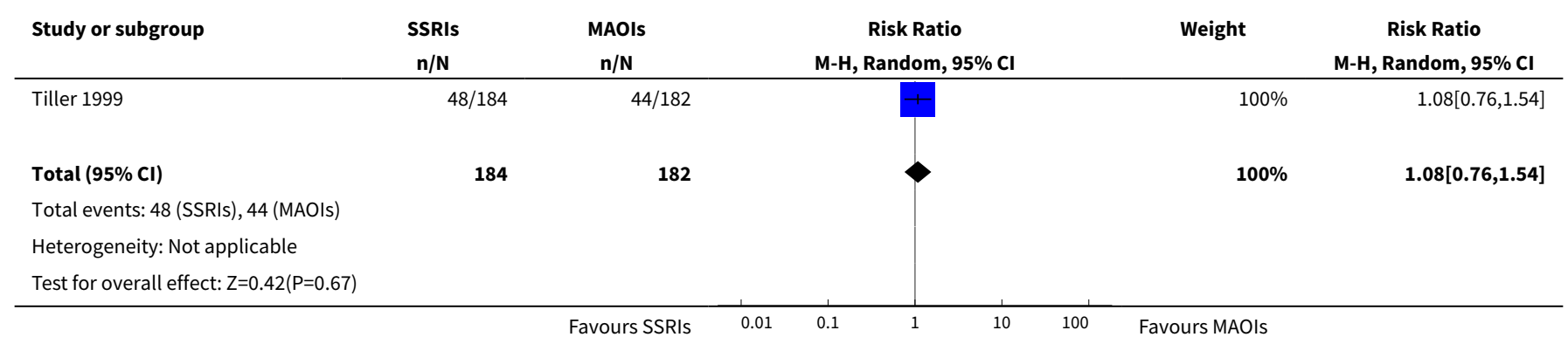

Comparison 33. Irregular benzodiazepines use excluded - Individual antidepressants versus individual antidepressants (within the same class)

\begin{tabular}{llll}
\hline Outcome or subgroup title & No. of studies & $\begin{array}{l}\text { No. of partici- } \\
\text { pants }\end{array}$ & Statistical method \\
\hline 1 Failure to respond & 2 & Risk Ratio (M-H, Random, 95\% Cl) $\quad$ Subtotals only & Effect size \\
\hline
\end{tabular}




\begin{tabular}{|c|c|c|c|c|}
\hline Outcome or subgroup title & No. of studies & $\begin{array}{l}\text { No. of partici- } \\
\text { pants }\end{array}$ & Statistical method & Effect size \\
\hline $\begin{array}{l}1.1 \text { Escitalopram versus citalo- } \\
\text { pram }\end{array}$ & 0 & 0 & Risk Ratio (M-H, Random, 95\% Cl) & $0.0[0.0,0.0]$ \\
\hline $\begin{array}{l}\text { 1.2 Fluoxetine versus Citalo- } \\
\text { pram }\end{array}$ & 1 & 42 & Risk Ratio (M-H, Random, 95\% Cl) & $0.57[0.20,1.66]$ \\
\hline 1.3 Sertraline versus paroxetine & 1 & 321 & Risk Ratio (M-H, Random, 95\% Cl) & $0.95[0.70,1.30]$ \\
\hline 2 Total number of dropouts & 3 & & Risk Ratio (M-H, Random, 95\% Cl) & Subtotals only \\
\hline $\begin{array}{l}2.1 \text { Escitalopram versus Citalo- } \\
\text { pram }\end{array}$ & 0 & 0 & Risk Ratio (M-H, Random, 95\% Cl) & $0.0[0.0,0.0]$ \\
\hline $\begin{array}{l}\text { 2.2 Fluoxetine versus Citalo- } \\
\text { pram }\end{array}$ & 1 & 42 & Risk Ratio (M-H, Random, 95\% Cl) & $1.0[0.07,14.95]$ \\
\hline 2.3 Sertraline versus Paroxetine & 1 & 321 & Risk Ratio (M-H, Random, 95\% Cl) & $0.62[0.40,0.97]$ \\
\hline $\begin{array}{l}2.4 \text { Imipramine versus } \\
\text { Clomipramine }\end{array}$ & 1 & 40 & Risk Ratio (M-H, Random, 95\% Cl) & $0.86[0.35,2.10]$ \\
\hline
\end{tabular}

\section{Analysis 33.1. Comparison 33 Irregular benzodiazepines use excluded - Individual antidepressants versus individual antidepressants (within the same class), Outcome 1 Failure to respond.}

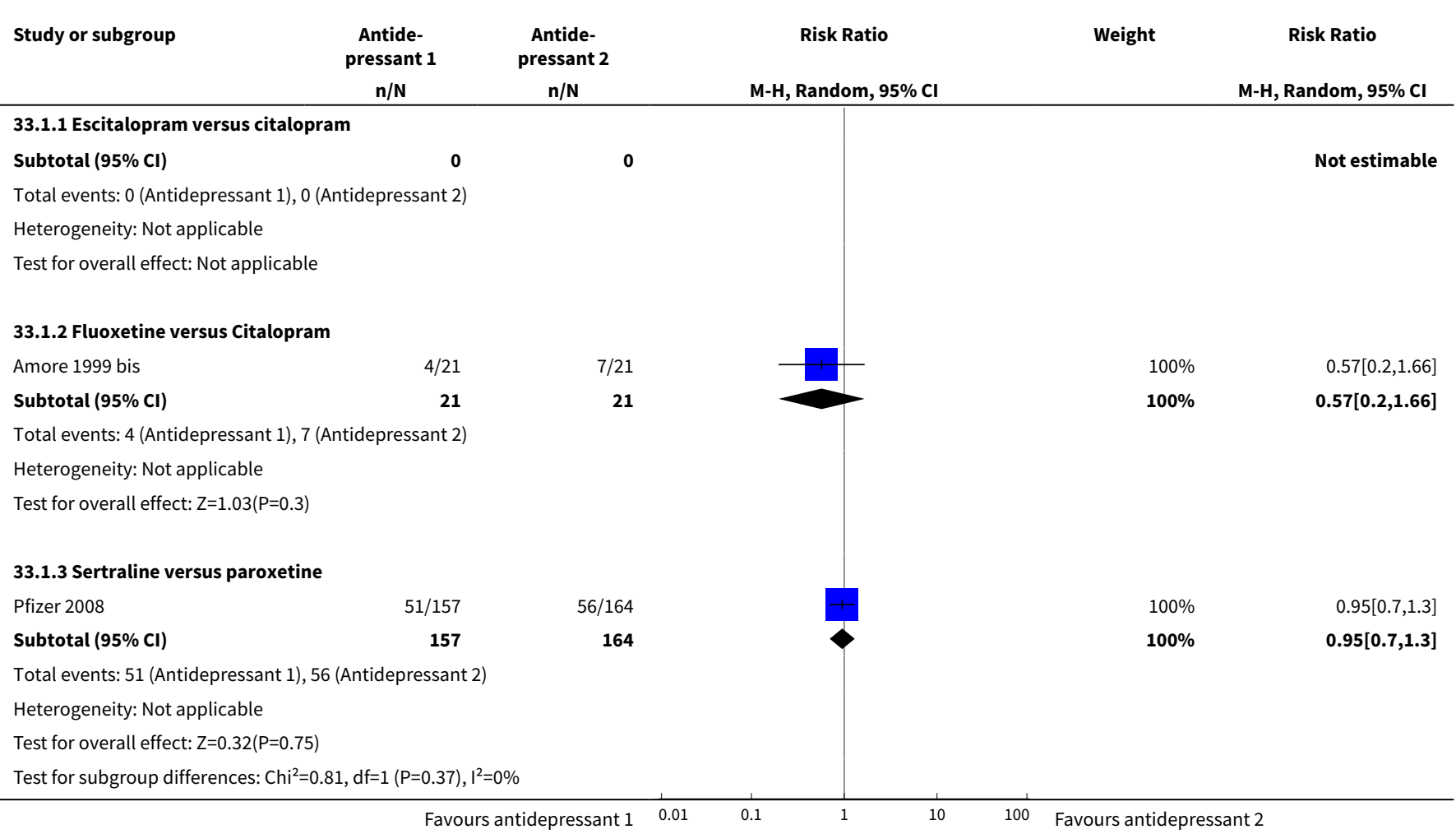




\section{Analysis 33.2. Comparison 33 Irregular benzodiazepines use excluded - Individual antidepressants} versus individual antidepressants (within the same class), Outcome 2 Total number of dropouts.

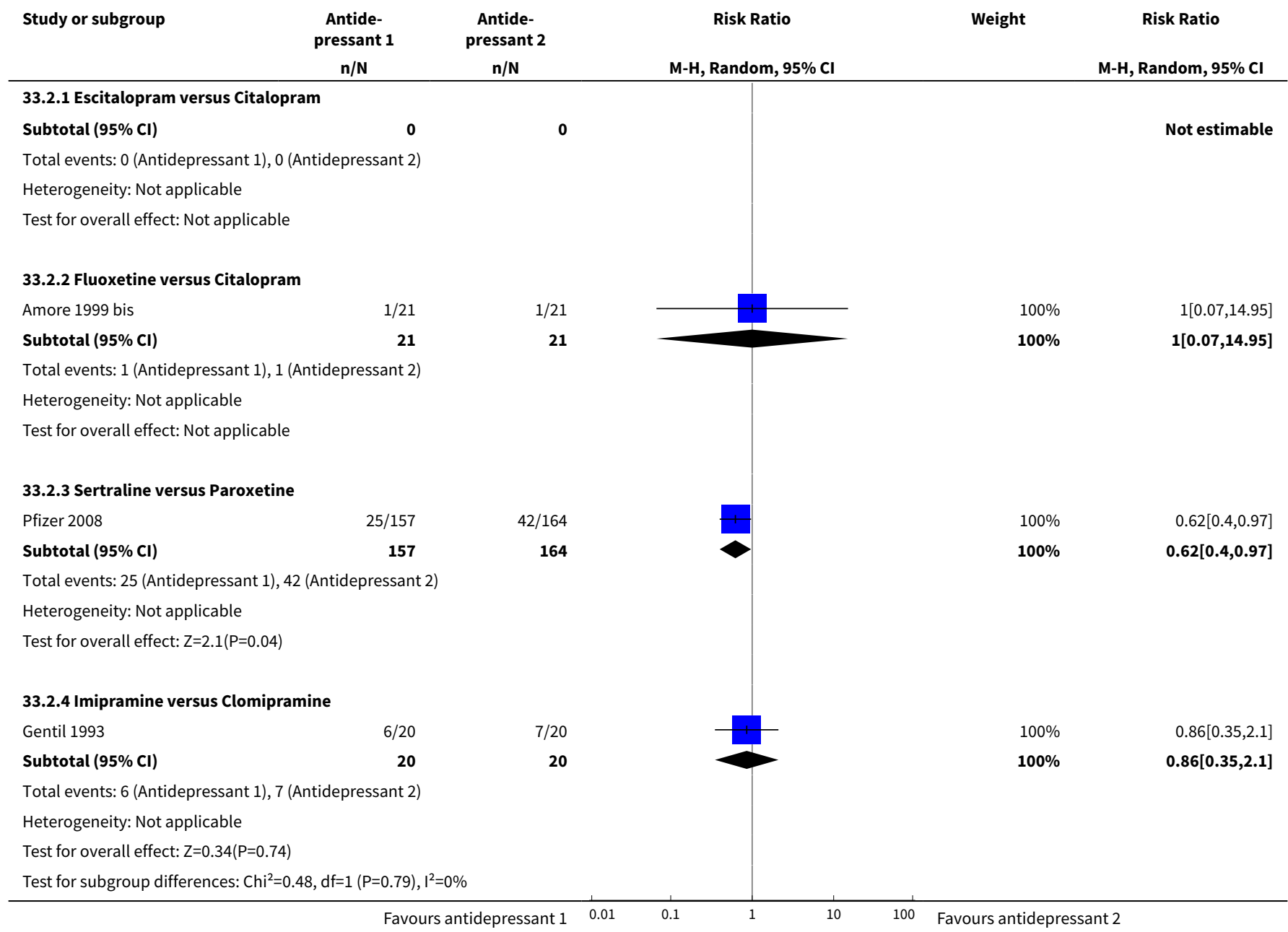

\section{APPENDICES}

\section{Appendix 1. Cochrane Specialised Register - core MEDLINE search strategy}

Core search strategy used to inform the Cochrane Common Mental Disorders Group's specialised register: OVID MEDLINE A weekly search alert based on condition + RCT filter only

1. [MeSH Headings]:

eating disorders/ or anorexia nervosa/ or binge-eating disorder/ or bulimia nervosa/ or female athlete triad syndrome/ or pica/ or hyperphagia/ or bulimia/ or self-injurious behavior/ or self mutilation/ or suicide/ or suicidal ideation/ or suicide, attempted/ or mood disorders/ or affective disorders, psychotic/ or bipolar disorder/ or cyclothymic disorder/ or depressive disorder/ or depression, postpartum/ or depressive disorder, major/ or depressive disorder, treatment-resistant/ or dysthymic disorder/ or seasonal affective disorder/ or neurotic disorders/ or depression/ or adjustment disorders/ or exp antidepressive agents/ or anxiety disorders/ or agoraphobia/ or neurocirculatory asthenia/ or obsessive-compulsive disorder/ or obsessive hoarding/ or panic disorder/ or phobic disorders/ or stress disorders, traumatic/ or combat disorders/ or stress disorders, post-traumatic/ or stress disorders, traumatic, acute/ or anxiety/ or anxiety, castration/ or koro/ or anxiety, separation/ or panic/ or exp anti-anxiety agents/or somatoform disorders/ or body dysmorphic disorders/ or conversion disorder/ or hypochondriasis/ or neurasthenia/ or hysteria/ or munchausen syndrome by proxy/ or munchausen syndrome/ or fatigue syndrome, chronic/ or obsessive behavior/ or compulsive behavior/ or behavior, addictive/ or impulse control disorders/ or firesetting behavior/ or gambling/ or trichotillomania/ or stress, psychological/ or burnout, professional/ or sexual dysfunctions, psychological/ or vaginismus/ or Anhedonia/ or Affective Symptoms/ or *Mental Disorders/ 
2. [Title/Author Keywords]:

(eating disorder ${ }^{\star}$ or anorexia nervosa or bulimi* or binge eat* or (self adj (injur* or mutilat*)) or suicide* or suicidal or parasuicid ${ }^{\star}$ or mood disorder ${ }^{\star}$ or affective disorder ${ }^{\star}$ or bipolar i or bipolar ii or (bipolar and (affective or disorder $\left.{ }^{\star}\right)$ ) or mania or manic or cyclothymic ${ }^{\star}$ or depression or depressive or dysthymi ${ }^{\star}$ or neurotic or neurosis or adjustment disorder* or antidepress* or anxiety disorder ${ }^{\star}$ or agoraphobia or obsess ${ }^{\star}$ or compulsi ${ }^{\star}$ or panic or phobi* or ptsd or posttrauma* or post trauma* or combat or somatoform or somati\#ation or medical ${ }^{\star}$ unexplained or body dysmorphi* or conversion disorder or hypochondria* or neurastheni* or hysteria or munchausen or chronic fatigue* or gambling or trichotillomania or vaginismus or anhedoni* or affective symptoms or mental disorder* or mental health).ti,kf.

\section{3. [RCT filter]:}

(controlled clinical trial.pt. or randomized controlled trial.pt. or (randomi\#ed or randomi\#ation).ab,ti. or randomly.ab. or (random* adj3

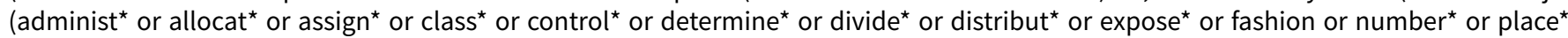
or recruit* or subsitut ${ }^{\star}$ or treat $\left.{ }^{\star}\right)$ ).ab. or placebo ${ }^{\star}$.ab,ti. or drug therapy.fs. or trial.ab,ti. or groups.ab. or (control ${ }^{\star}$ adj3 (trial $^{\star}$ or study or $^{\star}$ studies)).ab,ti. or ((singl ${ }^{*}$ or doubl* or tripl* or trebl*) adj3 (blind ${ }^{\star}$ or mask ${ }^{*}$ or dummy $\left.\left.{ }^{\star}\right)\right) . m p$. or clinical trial, phase ii/ or clinical trial, phase iii/ or clinical trial, phase iv/ or randomized controlled trial/ or pragmatic clinical trial/ or (quasi adj (experimental or random*)).ti,ab. or ((waitlist ${ }^{\star}$ or wait* list $^{\star}$ or treatment as usual or TAU) adj3 (control or group)).ab.)

4. (1 and 2 and 3)

Records are screened for reports of RCTs within the scope of the Cochrane Common Mental Disorders Group. Secondary reports of RCTs are tagged to the appropriate study record.

Similar weekly search alerts are also conducted on OVID Embase and PsycINFO, using relevant subject headings (controlled vocabularies) and search syntax, appropriate to each resource.

\section{Appendix 2. Review search: CCDANCTR-References Register}

\#1 panic

\#2 (antidepress* or anti-depress* or "anti depress" or noradrenaline or neurotransmitter* or dopamin ${ }^{\star}$ ) NEAR (uptake or reuptake or re-uptake or "re uptake")) or SSRI* or SNRI* or NARI* or $\mathrm{SARI}^{\star}$ or $\mathrm{NDRI}^{\star}$ or $\mathrm{TCA}^{\star}$ or tricyclic ${ }^{*}$ or tetracyclic $\left.{ }^{\star}\right)$

\#3 (Agomelatine or Alaproclate or Amoxapine or Amineptine or Amitriptylin* or Amitriptylinoxide or Atomoxetine or Befloxatone or Benactyzine or Binospirone or Brofaromine or (Buproprion or Amfebutamone) or Butriptyline or Caroxazone or Cianopramine or Cilobamine or Cimoxatone or Citalopram or (Chlorimipramin* or Clomipramin* or Chlomipramin* or Clomipramine) or Clorgyline or Clovoxamine or (CX157 or Tyrima) or Demexiptiline or Deprenyl or (Desipramine* or Pertofrane) or Desvenlafaxine or Dibenzepin or Diclofensine or Dimetacrin* or Dosulepin or Dothiepin or Doxepin or Duloxetine or Desvenlafaxine or DVS-233)

\#4 (Escitalopram or Etoperidone or Femoxetine or Fluotracen or Fluoxetine or Fluvoxamine or (Hyperforin or Hypericum or "St John*”) or Imipramin* or Iprindole or Iproniazid* or Ipsapirone or Isocarboxazid" or Levomilnacipran or Lofepramine* or ("Lu AA21004" or Vortioxetine) or "Lu AA24530" or (LY2216684 or Edivoxetine) or Maprotiline or Melitracen or Metapramine or Mianserin or Milnacipran or Minaprine or Mirtazapine or Moclobemide or Nefazodone or Nialamide or Nitroxazepine or Nomifensine or Norfenfluramine or Nortriptylin* or Noxiptilin*)

\#5 (Opipramol or Oxaflozane or Paroxetine or Phenelzine or Pheniprazine or Pipofezine or Pirlindole or Pivagabine or Pizotyline or Propizepine or Protriptylin* or Quinupramine or Reboxetine or Rolipram or Scopolamine or Selegiline or Sertraline or Setiptiline or Teciptiline or Thozalinone or Tianeptin* or Toloxatone or Tranylcypromin* or Trazodone or Trimipramine or Venlafaxine or Viloxazine or Vilazodone or Viqualine or Zalospirone)

\#6 (\#2 or \#3 or \#4 or \#5)

\#7 (Benzodiazepin* or BZD or Abecarnil or Adinazolam or Alprazolam or Arfendazam or Bentazepam or Bretazenil or Bromazepam or Brotizolam or Camazepam or Chlordiazepoxide or Chlordesmethyldiazepam or Cinolazepam or Clobazam or Clonazepam or Clorazepate or Chlorazepate or Clotiazepam or Cloxazolam or Delorazepam or Demoxepam or Desmethyldiazepam or Desoxydemoxepam or Devazepide or Diazepam or Doxefazepam or Estazolam or "ethyl loflazepate" or "CM 6912" or CM-6912 or Etizolam or Fludiazepam or Flunitrazepam or Flurazepam or dealkylflurazepam or Flutoprazepam or Fosazepam or Gidazepam or Girisopam or Halazepam or Haloxazolam or Ketazolam or Loflazepate or Loprazolam or Lorazepam or Lormetazepam or Meclonazepam or Medazepam or Metaclazepam or Mexazolam or Midazolam or Nerisopam or Nimetazepam or Nitrazepam or Norchlordiazepoxide or Norclobazam or Nordazepam or Norfludiazepam or Norflunitrazepam or Oxazepam or "WY 3498" or WY-3498 or Oxazolam or Phenazepam or Pinazepam or Prazepam or Premazepam or Propazepam or Quazepam or Ripazepam or Serazepine or Sograzepide or Talampanel or Tarazepide or Temazepam or Tetrazepam or Tofisopam or Triazolam or (Zolazepam or Zaleplon or Zolpidem or Zopiclone or Eszopiclone or Z-Drugs or “Z Drugs")) \#8 (\#1 and (\#6 or \#7))

\#9 ("anxiety disorder*" and not (agoraphobi* or panic or (social and (anxi* or phobi $\left.{ }^{\star}\right)$ ) or generalised or generalized or obsessive or compulsive or OCD or PTSD or post-trauma* or "post trauma*" or posttrauma*)):ti,ab,kw,ky,emt,mh,mc

$\# 10$ (\#9 and (\#6 or \#7))

\#11 (\#8 or \#10) 


\section{CONTRIBUTIONS OF AUTHORS}

GG devised the idea for the review. GG, IB, and CB worked on the first draft of the protocol. $C T$, $A C$, and MK provided suggestions. IB and CT collected the data; IB and CB ran the analyses; MC, AC, TF, FG, GG, MK provided suggestions and input; IB and CB drafted and critically revised the manuscript; all authors reviewed and approved the final version of the review.

\section{DECLARATIONS OF INTEREST}

IB: none

CT: none

MC: none

AC is supported by the NIHR Oxford Cognitive Health Clinical Research Facility and was expert witness for Accord Healthcare for a patent issue about quetiapine extended release.

FG: none

TAF has received lecture fees from Eli Lilly, Meiji, Mochida, MSD, Otsuka, Pfizer and Tanabe-Mitsubishi, and consultancy fees from Sekisui Chemicals and Takeda Science Foundation. He has received royalties from Igaku-Shoin, Seiwa-Shoten and Nihon Bunka Kagaku-sha publishers. He has received grant or research support from the Japanese Ministry of Education, Science, and Technology, the Japanese Ministry of Health, Labour and Welfare, the Japan Society for the Promotion of Science, the Japan Foundation for Neuroscience and Mental Health, Mochida and Tanabe-Mitsubishi. He is a diplomate of the Academy of Cognitive Therapy.

GG: none

MK: none

CB: none.

\section{SOURCES OF SUPPORT}

\section{Internal sources}

- None, Not specified.

\section{External sources}

- None, Not specified.

\section{DIFFERENCES BETWEEN PROTOCOL AND REVIEW}

The intended primary outcome was originally defined 'rate of response' at the protocol stage. However, in order to have consistency in the direction of forest plots, data were extracted as number of patients who failed to meet improvement criteria, as defined by the authors of each study. Therefore, the primary outcome 'rate of response' was renamed 'failure to respond'. This allowed us to present results in such a way that the area to the left of the line of no effect always indicated a favourable outcome for antidepressants. The same reasoning was applied for the outcome 'failure to remit', that was originally defined as 'rates of remission' in the protocol; data on lack of remission were extracted from the studies and presented in forest plots in such a way that the area to the left of the line of no effect always indicated a favourable outcome for antidepressants.

In addition to the planned overall antidepressants versus benzodiazepines comparison (stratified by class), we added separate comparisons of individual classes of antidepressants versus benzodiazepines, in order to present data also about single drugs belonging to each class of antidepressants versus benzodiazepines. This level of information is the most relevant from a clinician's point of view.

For the comparison antidepressants versus antidepressants (comparison B, see Methods), we made the decision to conduct separate comparisons for each class of antidepressant rather than stratify an overall comparison of antidepressants versus antidepressants according to class of drug. We also added individual antidepressants versus another antidepressant of the same class in order to comprehensively address the objectives of the review.

In the protocol we planned to calculate agreement in the selection of studies using Cohen's Kappa (K). However, discordances were much less, and were resolved reaching a consensus through discussion between authors, so we decided not to include a formal measure of agreement. 


\section{NOTES}

This review is one of a number of separate reviews examining the efficacy and tolerability of pharmacological and non-pharmacological treatments for panic disorders. These individual reviews will then be combined in a multiple-treatment meta-analysis using multipletreatments model methodology (protocol published in the Cochrane Database of Systematic Reviews). Please note that the majority of the text in the Methods sections for these protocols is identical since the full reviews will be following the same methodology. 